\title{
Hydrogen Assisted Cracking of Ultra-High Strength Steels
}

\author{
A Thesis Presented to the Faculty of \\ The School of Engineering and Applied Science \\ University of Virginia \\ In partial fulfillment of the requirements for the degree \\ Doctor of Philosophy in Materials Science and Engineering
}

Greger L. Pioszak

August, 2015 


\section{Approval Sheet}

This dissertation is submitted in partial fulfillment of the requirements for the degree of Doctor of Philosophy in Materials Science and Engineering

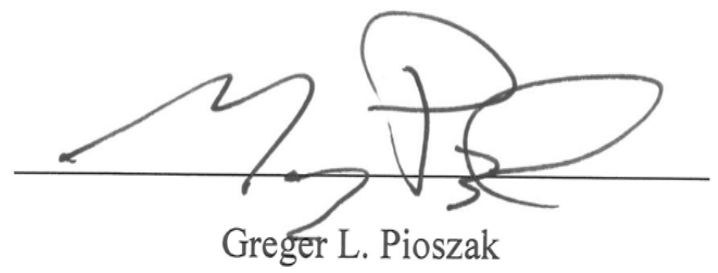

The dissertation has been read and approved by the examining committee:
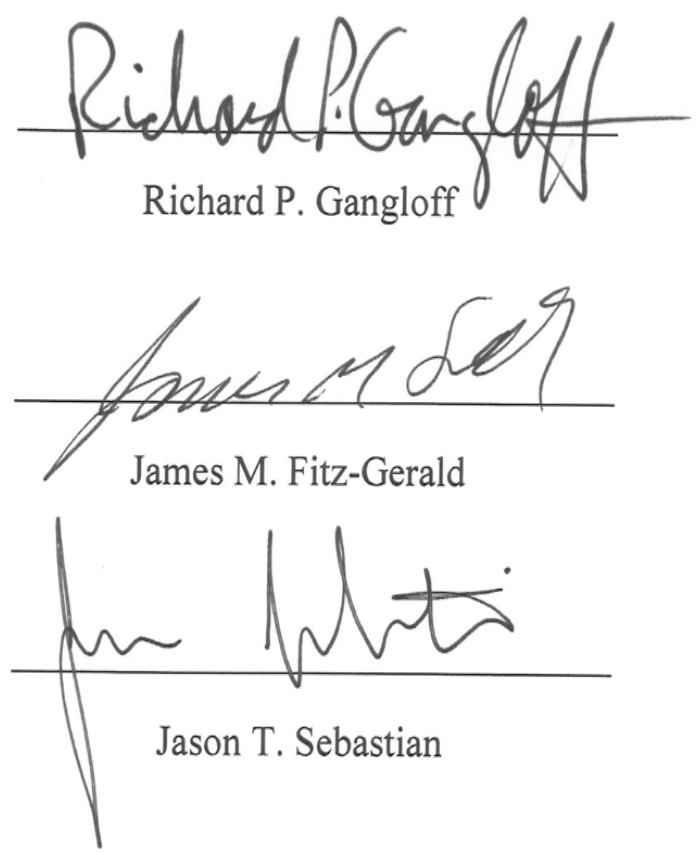
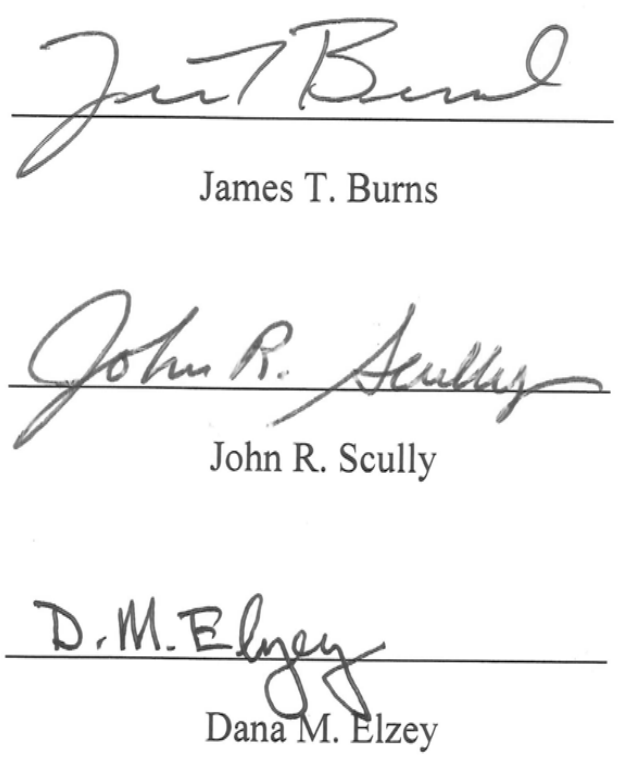

Accepted for the School of Engineering and Applied Science

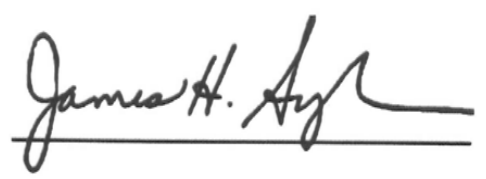

James H. Aylor

August, 2015 


\section{$\underline{\text { Abstract }}$}

Modern martensitic ultra-high strength steels (UHSS) are used in aerospace structural and other high performance applications due to their exceptionally high combination of tensile strength and fracture toughness. High strength martensitic microstructures are susceptible to severe stress corrosion cracking (SCC) via a hydrogen environment assisted cracking (HEAC) mechanism when exposed to service environments that produce atomic hydrogen near ambient temperature. Hydrogen-driven degradation of fracture and fatigue properties in UHSS alloys experiencing SCC is typically severe, limiting operating stress levels and component life. Mechanism-based micromechanical models of HEAC properties allow the accurate prediction of component life. However, the predictive accuracy of such models is severely limited by a lack of quantitative crack growth kinetics in modern UHSS. Further, the microstructural dependences of key variables in these micromechanical models have not been sufficiently investigated and tied to physical quantities. The objective of this research is to: (a) quantitatively measure high-resolution subcritical crack growth kinetics for modern UHSS, and (b) further the fundamental understanding of the electrochemical and microstructural basis for key variables in micro-mechanical models of SCC-HEAC properties.

The stress corrosion cracking resistance of a systematically selected, multi-generational, range of UHSS and martensitic stainless UHSS (UHSSS) is characterized via slow-rising stress intensity (K) loading in full immersion $0.6 \mathrm{M} \mathrm{NaCl}$ solution for a wide range of applied-cathodic potentials $\left(\mathrm{E}_{\mathrm{app}}\right)$. Quantitative crack growth kinetics and HEAC fracture morphology are correlated with a detailed high resolution EBSD analysis of the complex sub-micron martensitic microstructure for each steel, including feature size/morphology and interface crystallographic characteristics. Results are interpreted in the context of quantitative micromechanical models of the threshold stress intensity for HEAC growth $\left(\mathrm{K}_{\mathrm{TH}}\right)$ and diffusion-limited Stage II crack growth rate (da/dt II). The results establish that all alloys are susceptible to severe HEAC, characterized by low $\mathrm{K}_{\mathrm{TH}}(\sim 10$ $\mathrm{MPa} \sqrt{\mathrm{m}}$ ) when polarized at substantial cathodic $\mathrm{E}_{\text {app }}$ in aqueous chloride immersion. However, all modern steels are highly resistant to HEAC when stressed within a limited range of $E_{a p p}$, mildly cathodic to the open circuit condition, which confers elevated $\mathrm{K}_{\mathrm{TH}}$ and reduced da/dt $\mathrm{II}$. Decohesion based models of HEAC provide consistent and accurate predictions of the observed $E_{\text {app }}$ dependence of $\mathrm{K}_{\mathrm{TH}}$ and $\mathrm{da} / \mathrm{dt}_{\mathrm{II}}$ in legacy $300 \mathrm{M}$, AerMet 100 , and Ferrium M54. Modeling efforts establish the dominating-beneficial effect of increased alloy purity and precipitation of nano scale carbides in reducing HEAC susceptibility of modern UHSS. The HEAC fracture path in modern UHSS is dominated by an identical transgranular morphology established as cracking along $\{110\}_{\alpha}$, martensite block interface planes. HEAC of UHSS(S) is consistent with a modern and microstructurally sensitive, interactive hydrogen enhanced localized plasticity (HELP) hydrogen enhanced decohesion (HEDE) mechanism.

This dissertation provides: (a) a comprehensive database of HEAC kinetics for modern UHSS(S) with sub-micrometer microstructure and interface characterization, (b) fundamental understanding gained from semi-quantitative mechanism-based crack tip damage modeling, and (c) guidance pertaining to the important roles of alloy purity and strengthening precipitates in metallurgical design of next generation UHSS. 


\section{$\underline{\text { Executive Summary }}$}

Modern martensitic ultra-high strength steels (UHSS) are used in aerospace structural and other high performance applications due to their exceptionally high combination of tensile strength and fracture toughness. High strength martensitic microstructures are susceptible to severe stress corrosion cracking (SCC) via a hydrogen environment assisted cracking (HEAC) mechanism when exposed to service environments that produce atomic hydrogen near ambient temperature. Hydrogen-driven degradation of fracture and fatigue properties in UHSS alloys experiencing SCC is typically severe, limiting operating stress levels and component life. Significant advances have been made to the measurement and modeling of alloy-environment dependent SCC-HEAC cracking kinetics, paving the way for damage tolerant SCC management in structural components. Mechanism-based micromechanical models of HEAC properties allow the accurate prediction of component life. However, the predictive accuracy of such models is severely limited by a lack of quantitative crack growth kinetics for modern UHSS. Further, the microstructural dependences of key variables in these micromechanical models have not been sufficiently investigated and tied to physical quantities. The objective of this dissertation is to: (a) quantitatively measure highresolution subcritical crack growth kinetics for modern UHSS, and (b) further the fundamental understanding of the electrochemical and microstructural basis for key variables in micromechanical models of SCC-HEAC properties. Toward this objective this dissertation is structured into four chapters. Chapters 1 and 2 address the necessary background and experimental procedures for the remaining two chapters. Chapter 3 focuses on integrating quantitative EBSDbased characterization of UHSS microstructure, fracture morphology, and HEAC kinetics to enhance the current state of the art in HEAC modeling. Chapter 4 focuses on the microstructural origins of SCC-HEAC resistance in martensitic stainless UHSS as a function of aging condition and alloy composition.

In Chapter 3, the stress corrosion cracking resistance of a systematically selected, multigenerational, range of ultra-high strength martensitic steels is characterized via slow-rising stress intensity $(\mathrm{K})$ loading in full immersion $0.6 \mathrm{M} \mathrm{NaCl}$ solution for a wide range of applied-cathodic potentials ( $E_{\text {app }}$ ). Quantitative crack growth kinetics and HEAC fracture morphology are correlated with a high resolution EBSD analysis of complex sub-micron martensitic microstructure for each 
steel, including feature size/morphology and interface crystallographic characteristics. The resolution of EBSD-based microstructure characterization is unprecedented in hydrogen assisted cracking literature. Results are interpreted in the context of quantitative micromechanical models of HEAC properties, with predictions of the threshold stress intensity for crack growth $\left(\mathrm{K}_{\mathrm{TH}}\right)$ and diffusion-limited Stage II crack growth rate (da/dtII). The results establish that legacy $300 \mathrm{M}$, experimental low alloy CrNiMoWV, AerMet 100, Ferrium M54, and Ferrium S53 steels aged to ultra-high strength are susceptible to severe HEAC, characterized by low $\mathrm{K}_{\mathrm{TH}}(\sim 10 \mathrm{MPa} \sqrt{\mathrm{m}})$ when polarized near open circuit potential (OCP) or at substantial cathodic $\mathrm{E}_{\text {app }}$ in aqueous chloride immersion. All modern UHSS (experimental CrMoWV, AerMet 100, Ferrium M54, and Ferrium S53) are highly resistant to hydrogen assisted cracking when stressed within a limited range of $\mathrm{E}_{\mathrm{app}}$, mildly cathodic to OCP, which confers elevated $\mathrm{K}_{\mathrm{TH}}$ and reduced da/dt $\mathrm{dI}_{\text {II }}$ The breath of this protective-potential window is correlated to low diffusible hydrogen concentration $\left(\mathrm{C}_{\mathrm{H}-\mathrm{DIFF}}\right)$ owed to a minimum in crack tip hydrolytic acidification and overpotential for hydrogen production. Hydrogen-decohesion mechanism based micromechanical models of crack tip HEAC provide consistent and accurate predictions of the observed $\mathrm{E}_{\mathrm{app}}$ dependence of $\mathrm{K}_{\mathrm{TH}}$ and da/dt $\mathrm{dI}$ in legacy 300M, AerMet 100, and Ferrium M54 using published electrochemical measurements for AerMet 100 by Kehler and Scully. Comparison of predicted and measured HEAC properties among these alloys demonstrates the dominating-beneficial effect of increased alloy purity and precipitation of nano scale carbides in reducing HEAC susceptibility of modern UHSS.

As a second important result in Chapter 3, the HEAC crack path in modern UHSS is predominantly transgranular (TG) and dominated by a quasi-cleavage (QC) fracture morphology indicative of decohesion-based cracking along $\{110\}_{\alpha}$, martensite block interface planes. Close matching of fracture surface features with literature FIB/TEM characterization of $\mathrm{H}$-assisted fracture morphology by Robertson and coworkers indicates that HEAC in UHSS is likely governed by the conjoint action of hydrogen enhanced localized plasticity (HELP) and hydrogen enhanced decohesion (HEDE) mechanisms. With regard to the HELP contribution, H-enhanced dislocation movement along $\{110\}_{\alpha}$, martensite slip planes redistributes $H$ and severely work hardens the microstructure local to the slip system-martensite/austenite boundary intersection. When sufficient local stress is reached, a microcrack initiates on the H-embrittled boundary and propagates along the martensite block interface via decohesion, constituting the HEDE contribution. This 
mechanism is supported by EBSD measurements of martensite interface crystallography, which indicate that a network of random high energy interfaces is present in each UHSS. Randomly oriented high energy martensite interfaces form the majority of boundaries (59-67\%) in each alloy and exhibit high connectivity. High energy interfaces speculatively block slip and trap significantly more hydrogen during HEAC providing an interconnected $\mathrm{H}$-embrittled crack path along block interfaces through the microstructure. While coincident site lattice interfaces are present in each martensitic microstructure, results do not demonstrate a correlation with HEAC properties.

From an alloy design perspective, the results of Chapter 3 indicate four key points. First, extensive TG cracking is unique to high purity UHSS compositions with a substantial rare earth addition. Second, the strong SCC-HEAC resistance and slow da/dt of modern UHSS is controlled by a distribution of nanoscale strengthening precipitates. Third, reduced $\mathrm{Cr}$ and Ni in Ferrium M54 and CrNiMoWV did not adversely impact HEAC resistance, indicating the possibility of low-cost alternatives to high alloy AerMet 100. Finally, the addition of $10 \mathrm{wt} \% \mathrm{Cr}$ to an AerMet 100 base (Ferrium S53) had no effect on HEAC kinetics despite a hypothesized-deleterious influence of $\mathrm{Cr}$ hydrolysis at the crack tip.

Chapter 4 furthers understanding of the interactive role of electrochemical and microstructural variables on HEAC properties by extending the interpretations of Chapter 3 to ultra-high strength martensitic stainless steels (UHSSS). The stress corrosion cracking resistance of two compositionally similar UHSSS, Custom 465 and next generation Ferrium PH48S, are characterized for slow-rising $\mathrm{K}$ loading in $0.6 \mathrm{M} \mathrm{NaCl}$ for a wide range of cathodic $\mathrm{E}_{\text {app. }}$. Emphasis is placed on very high strength aging conditions of these steels, not typically studied or employed commercially due to severe SCC-HEAC susceptibility. This sharp decline in the HEAC resistance of precipitation strengthened martensitic stainless steels at ultra-high strengths is investigated through detailed characterization of four systematically chosen aging conditions of Ferrium PH48S (H860, H900, H950, H968). The results establish that Custom 465 and Ferrium PH48S, aged to several ultra-high strength levels, is each susceptible to severe SCC-HEAC, characterized by low $\mathrm{K}_{\mathrm{TH}}(\sim 10 \mathrm{MPa} \sqrt{\mathrm{m}})$ and rapid da/dt $\mathrm{dI}$, when stressed in $3.5 \% \mathrm{NaCl}$ under moderate-heavy cathodic polarization. Exclusively intergranular HEAC along prior austenite grain boundaries is observed in Custom 465, while exclusively transgranular HEAC is observed in Ferrium PH48S, at all SCCsusceptible $\mathrm{E}_{\text {app. }}$. Intergranular fracture in Custom 465 is traced to a significant reduction in grain 
boundary cohesion sourced at the synergistic interaction of metalloid impurities and hydrogen local to the grain boundary. Matching of HEAC fracture surface features with EBSD measured martensite microstructure indicates that HEAC primarily proceeds along $\{110\}_{\alpha}$ martensite block boundary planes in Ferrium PH48S, identical to TG HEAC of UHSS. Close agreement in the morphology of quasi-cleavage facets observed on the TG fracture surfaces of AerMet 100, Ferrium M54, CrNiMoWV, Ferrium S53, and Ferrium PH48S indicates that an identical HEAC mechanism is active in each alloy. The HELP/HEDE HEAC mechanism provides a reasonable set of mechanistic steps to explain the HEAC fracture morphology of UHSS and UHSSS.

Micromechanical modeling based on the HEDE mechanism is employed in Chapters 3 and 4 to predict the $\mathrm{E}_{\text {app }}$ dependencies of $\mathrm{K}_{\mathrm{TH}}$ and da/dtII. For UHSS (Chapter 3), known crack tip $\mathrm{H}$ solubility versus $E_{a p p}$ provides a necessary input for these predictions applied to AerMet 100, Ferrium M54 and 300M. Good agreement is observed using a single-adjustable parameter ( $\alpha$ or $\mathrm{C}_{\mathrm{H \sigma}-\mathrm{CRIT}}$ ) for micromechanical models of $\mathrm{K}_{\mathrm{TH}}$ and da/dt $\mathrm{dI}_{\mathrm{II}}$, respectively. The role of low crack tip overpotential for $\mathrm{H}$ production on HEAC resistance in the modest-cathodic polarization regime is established. In Chapter 4 the HEDE-based $\mathrm{K}_{\mathrm{TH}}$ model is used to predict the unknown crack tip $\mathrm{H}$ solubility for the UHSSS (Custom 465, Ferrium PH48S, and Ferrium S53), which is used to predict the $E_{\text {app }}$ dependence of diffusion limited da/dtII. The form of da/dtII versus $E_{\text {app }}$ predictions is reasonable and demonstrates the aging dependence of HEAC for Ferrium PH48S. The combined modeling effort provides a basis for future work on the steel composition dependence of crack tip $\mathrm{H}$ solubility and diffusivity, as well as the microstructural dependence of the critical distance parameter.

The contributions of this $\mathrm{PhD}$ research are as follows. Results establish a comprehensive data base of high resolution HEAC crack growth kinetics, unprecedented high resolution martensite microstructure and crystallography characterization, and HEAC fracture morphology for a systematically chosen group of commercial and experimental ultra-high strength steels. Cracking kinetics are critical for component lifetime modeling, and the present work validates the use of current micromechanical models for predicting HEAC properties in UHSS(S) alloys. Microstructural and fractographic characterizations indicate that an identical, microstructurally sensitive HELP/HEDE HEAC mechanism is active in all modern UHSS(S), further strengthening the use of decohesion based micromechanical models. Finally, the present results establish the 
dominating-beneficial effect of increased alloy purity and precipitation of nano-scale strengthening precipitates in reducing HEAC susceptibility of UHSS(S). 


\section{Acknowledgements}

This dissertation would simply not have been possible without the help of so many peers, friends and family. First and foremost, I would like to thank my advisor, Dr. Richard Gangloff. His dogged pursuit of fundamental understanding has served as an inspiration and helped me grow immensely as an engineer during my time at UVa. I would especially like to thank him for indulging an endless barrage of impromptu material science discussions. I am deeply grateful for his mentorship and support during my graduate career. Further, I would like to express my gratitude to to my committee and QuesTek Innovations LLC for their technical support during my graduate career.

I would like to acknowledge the faculty, students, and staff of the Materials Science Department at UVa; I am deeply appreciative of your help at every point of my career. Mary Lyn Lim and Jay Srinivasan have selflessly helped me pursue the electrochemical aspects of my work. All of the TDS measurements were conducted by Rebecca Schaller who graciously donated her time to advance this dissertation. Thank you to MSE staff members Barry Baber, Kim Fitzhugh-Higgins, Eric Newsome, and Richard White who have given their tireless support over the years.

Graduate school would not have been possible without the support of my family. For enduring the endless frazzled phone calls, shortened holiday visits, and long drives to Virginia, thank you.

Finally, I am deeply grateful for the support and camaraderie of my friends, both new and old. To Brad Richards, Marcel Mibus, Amber Lass, Ryan Donahue, Justin Dolph, and all the other regulars of game night, your friendship has kept me sane on a long road. Without you all I would have graduated two years earlier and been infinitely miserable doing it, thank you. To Justin Dallal and Laura Garrison for each providing countless hours of skype therapy and irreplaceable backpacking trips. I wouldn't have made it without either of you.

This research was supported by the DoD Office of Corrosion Policy and Oversight, as administered by the United States Air Force Academy through the Center for Aircraft Structural Life Extension. 


\section{Table of Contents}

$\begin{array}{llr}1.1 & \text { Problem Statement } & 1\end{array}$

1.2 MeChANISM OF HEAC $\quad 2$

1.2.1 MicRomeChanicAl MOdelS OF SCC-HEAC 2

$\begin{array}{lll}1.3 & \text { Metallurgical Design of UHSS } & 4\end{array}$

1.3.1 KeY DEVELOPMENTS OF MODERN UHSS 4

1.3.2 DeVelopment of NeXt Generation M $\mathrm{M}_{2}$ C Strengthened UhSS

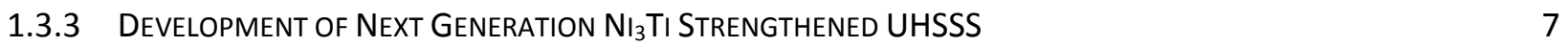

$\begin{array}{llr}1.4 & \text { The Role OF Grain Boundary Character } & 8\end{array}$

$\begin{array}{lll}1.5 & \text { Objectives } & 10\end{array}$

1.5.1 Chapter 2: Experimental Procedures 11

1.5.2 Chapter 3: Measurement AND Modeling of HEAC in Modern UHSS 11

1.5.3 Chapter 4: ORIGINS OF HEAC ReSistance In MOdern UHSSS 12

$\underline{2}$ EXPERIMENTAL METHODS 13

$\begin{array}{lll}2.1 & \text { Material Composition and Heat Treatment } & 14\end{array}$

$\begin{array}{lll}2.1 .1 & \text { UHSS MICROSTRUCTURE IN LITERATURE } & 17\end{array}$

$\begin{array}{lll}2.1 .2 & 300 \mathrm{M} & 17\end{array}$

$\begin{array}{llr}\text { 2.1.3 } & \text { AerMet } 100 & 18\end{array}$

$\begin{array}{lll}\text { 2.1.4 } & \text { FERRIUM M54 } & 18\end{array}$

$\begin{array}{llr}2.1 .5 & \text { CRNIMOWV } & 19\end{array}$

$\begin{array}{llr}\text { 2.1.6 } & \text { FERRIUM S53 } & 19\end{array}$

$\begin{array}{ll}\text { 2.1.7 } & 19\end{array}$

$\begin{array}{llr}2.1 .8 & \text { FERRIUM PH48S } & 20\end{array}$

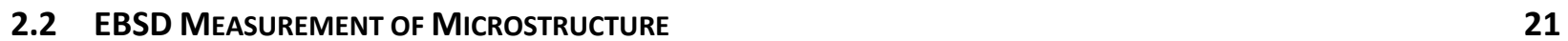

$\begin{array}{lll}2.2 .1 & \text { SAMPle Preparation } & 21\end{array}$

2.2.2 Characterization OF Grain Boundary Character 22 
$\begin{array}{lll}\text { 2.3.1 SENT SPECIMEN DESIGN AND PRECRACKING } & 24\end{array}$

2.3.2 Electrochemical Control and Loading 24

2.3.3 Crack Length Measurement

$\begin{array}{lll}2.3 .4 & \text { HEAC TEST ANALYSIS } & 26\end{array}$

$\begin{array}{ll}\text { 2.3.5 Resolution LIMIT OF THE DCPD METHOD } & 27\end{array}$

2.3.6 ANOMALOUS DA/DT AT LOW K

2.3.7 DETERMINATION OF $\mathrm{K}_{\text {TH }}$

2.3.8 DETERMINATION OF DA/DTII

2.4 Fracture SURface ANALYSIS

2.5 HydRogen UPTAKE / OUtgassing BehaVIOR OF UHSSS 33

2.6 Thermal Desorption SPECtroscopy (TDS) OF H-TRAP StATES IN UHSSS 34

\section{$\underline{3}$ MEASUREMENT AND MODELING OF HEAC IN MODERN}

$\begin{array}{lll}3.1 & \text { Problem Statement } & 35\end{array}$

$\begin{array}{lll}3.2 & \text { RESULTS } & 36\end{array}$

3.2.1 EBSD Measured Microstructure 36

3.2.2 HEAC CRACK GROWTH KINETICS

$\begin{array}{lll}\text { 3.2.3 } & \text { EFFECT OF E } E_{\text {APP }} \text { ON HEAC KINETICS } & 67\end{array}$

3.2.4 FRACTAL ANALYSIS OF MARTENSITE INTERFACE CONNECTIVITY

$\begin{array}{lll}3.3 & \text { DISCUSSION } & 73\end{array}$

3.3.1 TG HEAC MECHANISM IN UHSS

3.3.2 Effect of Grain Boundary Character Distribution On HEAC ON UHSS 80

3.3.3 Measurement of Precipitated Austenite in UHSS 82

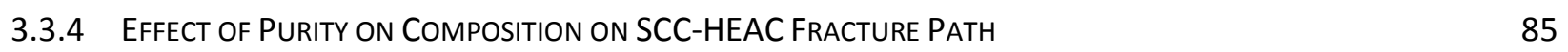

3.3.5 MOdeling the EfFeCt Of E E $_{\text {APP }}$ ON HEAC KINETICS 91

3.3.6 MICROSTRUCTURAL BASIS FOR X XRIT IN TG QUASI-CLEAVAGE OF MODERN UHSS 115

$\begin{array}{lll}3.4 & \text { CONCLUSIONS } & 119\end{array}$ 


\section{$4 \underline{\text { CHARACTERIZATION OF HEAC IN MODERN ULTRA HIGH }}$}

STRENGTH STAINLESS STEELS

$\begin{array}{lll}4.1 & \text { Problem Statement } & 121\end{array}$

$\begin{array}{llr}4.2 & \text { RESULTS } & 122\end{array}$

4.2.1 EBSD MEASUREMENT OF MARTENSite Microstructure 122

4.2.2 EBSD Measurement PreCipttated Austenite in UHSSS 136

$\begin{array}{lll}\text { 4.2.3 } & \text { FRACTAL ANALYSIS OF MARTENSITE INTERFACES IN UHSSS } & 139\end{array}$

4.2.4 Measurement of K K ANd Ductile Fracture Morphology 145

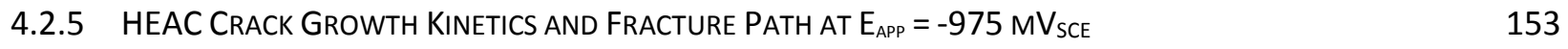

4.2.6 EFFECT OF $E_{\text {APP }}$ ON HEAC KINETICS AND FRACTURE MORPHOLOGY IN FERRIUM PH48S - H900 161

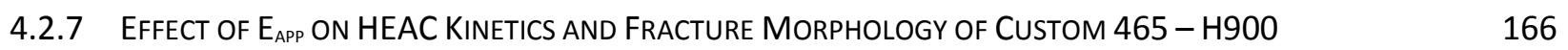

4.2.8 EFFECT OF $E_{\text {App }}$ ON HEAC PROPERTIES OF CUSTOM 465 AND FERRIUM PH48S 171

4.2.9 HydRogen SOLUBiLITY In FERRIUM PH48S AND CUSTOM 465

4.2.10 TDS ANAlysis Of HydRogen TRAP StATES In FERRIUM PH48S AND CUSTOM 465

$\begin{array}{lll}4.3 & \text { DisCUSSION } & 188\end{array}$

$\begin{array}{lll}\text { 4.3.1 HydRogen TRAPPING AND SOLUBILITY UHSSS Alloys } & 188\end{array}$

4.3.2 MeChanism OF HEAC In Modern UHSSS 198

4.3.3 Measurement Of PreCipitated Austenite in UHSSS alloys 211

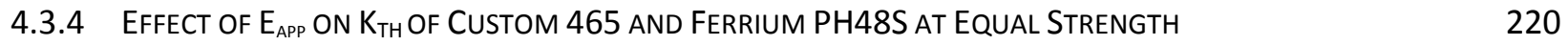

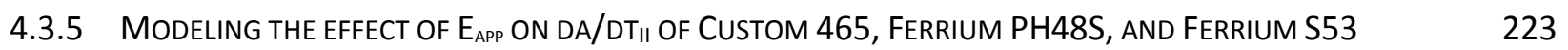

4.3.6 Metallurgical Design of HeAC-Resistant UHSS and UHSSS Alloys 232

$\begin{array}{lll}4.4 & \text { Conclusions } & 235\end{array}$

$\underline{5}$ REFERENCES $\quad 237$ 


\section{List of Figures}

Figure 1 Generic example of an RBN (black + red) with a highlighted MRBC (red). A $25 \mu \mathrm{m}$ grid $(\eta=25 \mu \mathrm{m})$ is overlaid for scale and fractal analysis of MRBC connectivity. . 23

Figure 2 Idealized crack growth kinetics delineating SCC-HEAC parameters $\left(\mathrm{K}_{\mathrm{TH}}, \mathrm{da} / \mathrm{dt}_{\mathrm{II}}\right)$ which are characteristic of UHSS behavior.

Figure 3 Typical crack growth kinetics of Ferrium S53 stressed under slow rising displacement (Initial $\mathrm{dK} / \mathrm{dt}=1 \mathrm{MPa} \sqrt{\mathrm{m}} / \mathrm{h}$ ) in $3.5 \% \mathrm{NaCl}$, exhibiting strong SCC-HEAC resistance and deviation from the ideal form of HEAC kinetics. 29

Figure 4 Construction of da/dt resolution limit for full immersion of UHSS alloys. Regression of false da/dt rise is shown as the central line (red); with the bounds of a 95\% CI around the regression shown above and below (green). The upper bound of this confidence interval is employed as the resolution limit in all further analyses. 30

Figure 5 Variation in the form of typical HEAC kinetics for 300M (•), AerMet $100(\mathbf{\square})$, and Ferrium S53 ( $\mathbf{\Delta})$ stressed under slow rising displacement in $3.5 \% \mathrm{NaCl}$ at $\mathrm{E}_{\text {app }}=-$ $550 \mathrm{mV}_{\mathrm{SCE}}$ 32

Figure 6 OIM map of 300M utilizing IPF-Z coloring. A single prior austenite grain is highlighted (white line); 4 individual martensite packets are denoted with white arrows separated by dotted white lines. 37

Figure 7 OIM map of AerMet 100 utilizing IPF-Z coloring. A single prior austenite grain is highlighted (white line); 3 individual packets are denoted with white arrows, separated by dotted white lines. 38

Figure 8 OIM map of Ferrium M54 utilizing IPF-Z coloring. A single prior austenite grain is highlighted (white line); 4 individual packets are denoted with white arrows, separated by dotted white lines. 39

Figure 9 OIM map of CrNiMoWV utilizing IPF-Z coloring. A single prior austenite grain is highlighted (white line); individual packets are denoted with white arrows, separated by dotted white lines. 40

Figure 10 OIM map of Ferrium S53 utilizing IPF-Z coloring. A single prior austenite grain is highlighted (white line); individual packets are denoted with white arrows, separated by dotted white lines. Parallel martensite block features are shown with black arrows. 41

Figure 11 Distribution of EBSD-measured precipitated austenite (red) in AerMet 100 shown against a trace of all martensite interfaces with $>2^{\circ}$ misorientation. 42 
Figure 12 (a) Classical hierarchy of martensite structure of UHSS alloys, adapted from [52], (b) Isolated 300M prior austenite grain. Martensite packet boundaries are highlighted in white dotted lines and individual packet orientations are numbered. 43

Figure 13 (a) Orientation map of in an isolated AerMet 100 martensite packet colored using IPF$\mathrm{Z}$ coloring. (b) Map of martensite boundaries showing the distribution of high $\left(>15^{\circ}\right.$, green) and low $\left(2^{\circ} \leq \theta<15^{\circ}\right.$, black) angle interfaces. (c) Identical misorientation map as (b) with high angle $\sum 3$ CSL boundaries highlighted in red. 45

Figure 14 Point to point misorientation profile from (1) to (2) in Figure 13b. 47

Figure 15 Isolated packets of 5 UHSS alloys: (Column 1) Orientation map of an isolated martensite packet colored using IPF-Z coloring. (Column 2) Map of martensite boundaries showing the distribution of high $\left(15^{\circ} \leq \theta\right.$, green $)$ and low angle $\left(2^{\circ} \leq \theta<\right.$ $15^{\circ}$, black) interfaces. (Column 3) Identical misorientation coloring with $\sum 3 \mathrm{CSL}$ boundaries highlighted (red). 50

Figure 16 (a) Orientation map of an isolated AerMet 100 martensite packet colored using IPF-Z coloring. (b) Map of martensite boundaries showing the distribution of high angle $\left(15^{\circ}\right.$ $\leq \theta$, green), low angle $\left(2^{\circ} \leq \theta<15^{\circ}\right.$, black) and dominant $\sum 3$ CSL boundaries (red) (c) Identical map to (b) with the distribution of $\sum 11$ (teal), $\sum 13 \mathrm{~b}$ (maroon), and $\sum 25 \mathrm{~b}$ (fuchsia) boundaries highlighted. 52

Figure 17 Subcritical crack growth rate versus $\mathrm{K}$ for five ultra-high strength steels stressed at $550 \mathrm{mV}_{\mathrm{SCE}}$ in $0.6 \mathrm{M} \mathrm{NaCl}$.

Figure 18 Subcritical crack growth rate versus $\mathrm{K}$ for five ultra-high strength alloys stressed at $1,000 \mathrm{mV}_{\mathrm{SCE}}$ in $0.6 \mathrm{M} \mathrm{NaCl}$ 57

Figure 19 TG HEAC of AerMet $100(\mathrm{~K}=9 \mathrm{MPa} \sqrt{\mathrm{m}})$ stressed in $3.5 \% \mathrm{NaCl}$ at $\mathrm{E}_{\text {app }}=-1,000$ $\mathrm{mV}_{\text {SCE. }}$ Large white and black arrows indicate regions of QC and 'flat' morphologies, respectively. 58

Figure 20 TG HEAC morphology of Ferrium $\mathrm{S} 53(\mathrm{~K} \approx 7 \mathrm{MPa} \sqrt{\mathrm{m}})$ stressed in $3.5 \% \mathrm{NaCl}$ at $\mathrm{E}_{\text {app }}$ $=-1,000 \mathrm{mV}_{\mathrm{SCE}}$. White and black arrows indicate regions of $\mathrm{QC}$ and flat facets, respectively. 60

Figure 21 TG HEAC morphology of Ferrium M54 (K $\approx 9 \mathrm{MPa} \sqrt{ })$ stressed in $3.5 \% \mathrm{NaCl}$ at $\mathrm{E}_{\text {app }}$ $=-1,000 \mathrm{mV}$ SCE. White and black arrows indicate regions of $\mathrm{QC}$ and 'flat', respectively. 62

Figure 22 IG HEAC morphology $300 \mathrm{M}(\mathrm{K} \approx 8 \mathrm{MPa} \sqrt{\mathrm{m}})$ stressed in $3.5 \% \mathrm{NaCl}$ at $\mathrm{E}_{\text {app }}=-1,000$ $\mathrm{mV}_{\mathrm{SCE}}$. White and black arrows indicate regions of QC and 'flat', respectively.... 63

Figure 23 (a) Mixed IG and TG HEAC of CrNiMoWV $(\mathrm{K} \approx 13 \mathrm{MPa} \sqrt{\mathrm{m}})$ stressed in $3.5 \% \mathrm{NaCl}$ at $\mathrm{E}_{\mathrm{app}}=-1,000 \mathrm{mV}_{\mathrm{SCE}}$, (b) Multiple IG HEAC facets (c) Detail of TG quasi-cleavage from the white arrow in (a), and (d) detail of an IG facet from (b). 66 
Figure 24 Threshold stress intensity $\left(\mathrm{K}_{\mathrm{TH}}\right)$ vs. applied potential $\left(\mathrm{E}_{\mathrm{app}}\right)$ for five ultra-high strength steels stressed in $0.6 \mathrm{M} \mathrm{NaCl}$. Closed squares ( $\mathbf{a}$ ) represent previously reported data [1] while open squares ( $\square$ ) represent data generated in the present study for AerMet 100. The trend line for CrNiMoWV is extrapolated from trends observed in similar alloys Ferrium M54 and AerMet 100. Three replicate experiments $\left(E_{\text {app }}=-550 \mathrm{mV}_{\mathrm{SCE}}\right.$, $\left.\mathrm{K}_{\mathrm{TH}}=12.0,13.8,14.4 \mathrm{MPa} \sqrt{\mathrm{m}}\right)$ with $\mathrm{CrNiMoWV}$ are spread in applied potential for clarity. 68

Figure 25 Stage II crack growth rate (da/dt $\left.{ }_{I I}\right)$ versus applied potential ( $\left.\mathrm{E}_{\text {app }}\right)$ for five ultra-high strength steels stressed in $0.6 \mathrm{M} \mathrm{NaCl}$. Closed squares (a) represent previously reported data [1], while open squares $(\square)$ represent data generated in the present study for AerMet 100. Three replicate experiments $\left(\right.$ Eapp $=-550 \mathrm{mV}_{\mathrm{SCE}}, \mathrm{da} / \mathrm{dt}_{\mathrm{II}}=20,20,28$ $\mathrm{nm} / \mathrm{s}$ ) of CrNiMoWV are spread in potential for clarity. 70

Figure 26 RBN map of 300M (a) Map of random interfaces corresponding to the microstructure of 300M shown in Figure 2. (b) Detail image showing the small interruptions (red arrows) that are present at regular intervals on random boundaries from (a). 72

Figure 27 Quasi-cleavage facets and representative areas of martensite block structure in OIM maps (Figure 6-9) for AerMet 100 (a,d), Ferrium S53 (b,e) and Ferrium M54 (c,f).

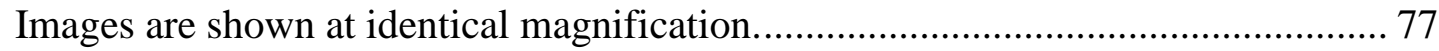

Figure 28 Comparison of lath martensitic structure observed by Nagao (a) [11] and the microstructure of AerMet 100 from the present study (b) both utilizing IPF coloring. 78

Figure 29 The bold-surface applied potential dependence of crack tip diffusible H concentration established for AerMet 100 using the measurement and modeling approach of Kehler and Scully [20]. Points were estimated from their Figure 14, and the OCP of AerMet 100 declines from about -0.50 to $-0.60 \mathrm{~V}_{\mathrm{SCE}}$ for a $20 \mathrm{~h}$ exposure in $0.6 \mathrm{M} \mathrm{NaCl}$.... 93

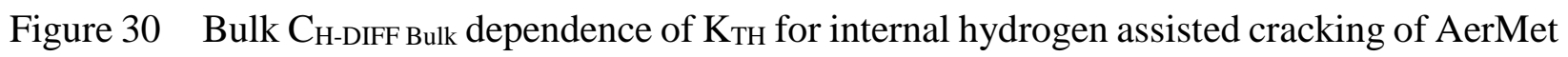
$100[145]$. 94

Figure 31 Measured ( $\square \boldsymbol{\Delta}$ ) and model predicted (--) applied potential dependence of $\mathrm{K}_{\mathrm{TH}}$ for AerMet 100 and Ferrium M54, using the measured H concentration dependence of $\mathrm{K}_{\mathrm{TH}}$ determined for $\mathrm{H}$ precharged AerMet 100, as given by Figure 30. 96

Figure 32 Measured ( $\square \mathbf{\Delta}$ ) and model predicted (--) $E_{\text {app }}$ dependence of $K_{\mathrm{TH}}$ for AerMet 100 and Ferrium M54, using a H-decohesion based model (Equation 1) with $\beta$ ', $\alpha$,', kIG, and $\alpha$ calibrated by fit to IHAC data for H precharged AerMet 100 (Figure 30) and lower bound $\sigma_{\mathrm{H}}=4.2 \sigma_{\mathrm{Ys}}$. 99

Figure 33 Comparison of $\mathrm{K}_{\mathrm{TH}}$ measured for IHAC by Thomas et al [144] and model predicted $\mathrm{K}_{\mathrm{TH}}$ for the conditions shown in Figure 32 ................................................... 100 
Figure 34 Measured ( $\square \mathbf{\Delta}$ ) and model predicted (--) $\mathrm{E}_{\text {app }}$ dependence of $\mathrm{K}_{\mathrm{TH}}$ for AerMet 100 and Ferrium M54, using a H-decohesion based model (Equation 1) with $\beta$ ', $\alpha$ ", kIG, and $\alpha$ calibrated by fit to IHAC data for $\mathrm{H}$ precharged AerMet 100 (Figure 30) and lower bound $\sigma_{\mathrm{H}}=6.8 \sigma_{\mathrm{Ys}}$. 101

Figure 35 Comparison of $\mathrm{K}_{\mathrm{TH}}$ measured for IHAC by Thomas et al [144] and model predicted $\mathrm{K}_{\mathrm{TH}}$ for the conditions shown in Figure 34 . 102

Figure 36 Measured ( $\square \mathbf{A}$ ) and model predicted (-) applied potential dependence of da/dt II for AerMet 100 and Ferrium M54, using the H-diffusion limited model (Equation 1) with measured $\mathrm{D}_{\mathrm{H}-\mathrm{EFF}}[2]$, correlated $\chi_{\text {crit }}[2,3]$, and best fit $\mathrm{C}_{\text {Hocrit }}$ for lower (top) and upper (bottom) bounds of $\sigma_{\mathrm{H}}=4.2 \sigma_{\mathrm{YS}}$ and $6.8 \sigma_{\mathrm{YS}}$. 105

Figure 37 Measured ( $\boldsymbol{\Delta}$ ) and model predicted (--) applied potential dependence of $\mathrm{K}_{\mathrm{TH}}$ for $300 \mathrm{M}$, using the H-decohesion based model (Equation 1) for lower and upper bounds of $\sigma_{\mathrm{H}}$ $=4.2 \sigma_{\mathrm{YS}}$ (upper) and 6.8 $\sigma_{\mathrm{YS}}$ (lower). 110

Figure 38 Measured ( $\square \mathbf{\Delta} \bullet$ ) and model predicted (--) applied potential dependence of da/dt II for AerMet 100, Ferrium M54 and 300M, using the H diffusion limited model (Equation 2) with measured $D_{H-E F F}[1]$, correlated $\chi_{\text {crit }}[2]$, and best fit $C_{H \sigma-c r i t}$ for: the lower bound of $\sigma_{H}=4.2 \sigma_{Y S}$ (top plot) and upper bound of $\sigma_{H}=6.8 \sigma_{Y S}$ (bottom plot)... 113

Figure 39 OIM Map of Custom 465 - H900 utilizing IPF-Z coloring. Three prior austenite grains are highlighted (white line); martensite packets within a prior austenite grain are separated by dotted white lines. This coloring scheme is identical for all EBSD-OIM images in the present work. 123

Figure 40 OIM Map of Ferrium PH48S - H900 utilizing IPF-Z coloring. A single prior austenite grain is highlighted (white line); martensite packets within a prior austenite grain are separated by dotted white lines. 124

Figure 41 High resolution OIM Map of Custom 465 - H900 utilizing IPF-Z coloring. Two martensite packets are isolated by dashed white lines. 125

Figure 42 High resolution OIM Map of Ferrium PH48S - H900 utilizing IPF-Z coloring. A prior austenite grain boundary is shown by the dashed white line. 126

Figure 43 Determination of prior austenite grain boundaries in Custom 465 - H900: a) IPF-Z OIM map, b) map of random martensite interfaces with a misorientation of $20-40^{\circ}$, c) IGSCC of Custom $465-\mathrm{H} 900$ stressed in $3.5 \% \mathrm{NaCl}$ at $\mathrm{E}_{\mathrm{app}}=-975 \mathrm{mV}_{\mathrm{SCE}}$, and d) Polished section of Custom 465 - H900 etched via full immersion Beraha's No. 4 sulfamic acid etch for $240 \mathrm{~s}$. 128

Figure 44 (a) Classical hierarchy of martensite structure of UHSS alloys, adapted from [52], and (b) Isolated 300M prior austenite grain. Martensite packet boundaries are highlighted in white dotted lines, and individual packet orientations are numbered. 131 
Figure 45 (a) Orientation map of an isolated Custom 465 - H900 martensite packet colored using IPF-Z coloring. (b) Map of martensite boundaries showing the distribution of high (> $15^{\circ}$, green) and low angle $\left(2^{\circ} \leq \theta<15^{\circ}\right.$, black) interfaces. (c) Identical misorientation map as (b) with high angle $\sum 3$ CSL boundaries highlighted in red, and (d) Identical misorientation map as (c) with minor fractions of $\sum 11$ (teal) and $\sum 25 \mathrm{~b}$ (magenta) highlighted. 133

Figure 46 (a) Orientation map of an isolated Ferrium PH48S - H900 martensite packet colored using IPF-Z coloring. (b) Map of martensite boundaries showing the distribution of high $\left(>15^{\circ}\right.$, green) and low angle $\left(2^{\circ} \leq \theta<15^{\circ}\right.$, black) interfaces. (c) Identical misorientation map as (b) with high angle $\sum 3$ CSL boundaries highlighted in red, and (d) Identical misorientation map as (c) with minor fractions of $\sum 11$ (Teal) and $\sum 25 \mathrm{~b}$ (Magenta) highlighted. 135

Figure 47 Detail of Ferrium PH48S - H900 martensite block structure presented in Figure 42, acquired with at a very fine EBSD pixel step size $(13 \mathrm{~nm})$. The prior austenite grain boundary is indicated by the white dashed line. 136

Figure 48 Distribution of EBSD-Measured Austenite in Custom 465 - H900. 137

Figure 49 Distribution of EBSD-Measured Austenite in Ferrium PH48S - H900............... 138

Figure 50 MRBC of Custom $465(\mathrm{a}, \mathrm{c})$ and Ferrium PH48S (b,d), using a pixel gap tolerance of zero $(\mathrm{a}, \mathrm{b})$ and 2 pixels $(\mathrm{c}, \mathrm{d})$. Two pixels represent 488 and $380 \mathrm{~nm}$ of physical distance for Custom 465 and Ferrium PH48S, respectively. 142

Figure 51 Random boundary network of Ferrium PH48S - H900: (a) High resolution random boundary trace corresponding to the OIM map presented in Figure 42 and (b) Detail of the region indicated by the red dashed line in (a). Representative gaps in the random boundary network are indicated by red arrows and correspond to small segments of CSL or low angle boundaries. 144

Figure 52 Crack growth resistance curves of Ferrium PH48S and Custom 465 constructed using a dcPD-measured crack growth after $\mathrm{K}_{\mathrm{JICi}}$. Initial crack extension is determined by $1 / 2$ of the crack tip opening displacement (CTOD), shown as the intersection of the RCurve with the blunting line from [104]. 147

Figure 53 Fracture morphology of (a) Custom 465 - H900 and (b) Ferrium PH48S - H900 tested under slow rising displacement in moist air. 150

Figure 54 Typical morphology of microvoid nucleants in Ferrium PH48S - H900: (a) spheroidal rare earth oxy-sulfides and (2) cuboidal Ti-rich precipitates. 151

Figure 55 Typical morphology of microvoid nucleants in Custom 465 - H900: (a) spheroidal impurity rich particles (2) cuboidal Ti-rich precipitates. 152

Figure 56 HEAC Kinetics for UHSSS stressed under slow-rising $\mathrm{K}$ in $3.5 \% \mathrm{NaCl}$ at $\mathrm{E}_{\mathrm{app}}=-975$ $\mathrm{mV}$ SCE. 154 
Figure 57 HEAC fracture morphology of Custom $465-\mathrm{H} 900(\mathrm{~K} \approx 10 \mathrm{MPa} \sqrt{\mathrm{m}})$ stressed in $3.5 \%$ $\mathrm{NaCl}$ at $\mathrm{E}_{\mathrm{app}}=-975 \mathrm{mV}$ SCE. The white arrow indicates a region of transgranular cracking among predominantly IG fracture. 155

Figure 58 HEAC fracture morphology of Ferrium PH48S - H860 (K $\approx 9 \mathrm{MPa} \sqrt{\mathrm{m}})$ stressed in $3.5 \% \mathrm{NaCl}$ at $\mathrm{E}_{\text {app }}=-975 \mathrm{mV}_{\text {SCE}}$. White and black arrows indicate regions of QC and flat fracture, respectively.

Figure 59 HEAC fracture morphology of Ferrium PH48S - $900(\mathrm{~K} \approx 9 \mathrm{MPa} \sqrt{\mathrm{m}})$ stressed in 3.5\% $\mathrm{NaCl}$ at $\mathrm{E}_{\mathrm{app}}=-975 \mathrm{mV}_{\text {SCE}}$. White and black arrows indicate regions of QC and flat fracture, respectively. White arrowheads denote minor secondary cracking, possibly at prior austenite grain boundaries. 158

Figure $60 \mathrm{HEAC}$ fracture morphology of Ferrium PH48S - $968(\mathrm{~K} \approx 9 \mathrm{MPa} \sqrt{\mathrm{m}})$ stressed in 3.5\% $\mathrm{NaCl}$ at $\mathrm{E}_{\mathrm{app}}=-975 \mathrm{mV}_{\mathrm{SCE}}$. White and black arrows indicate regions of $\mathrm{QC}$ and flat fracture, respectively. 159

Figure 61 Comparison of QC fracture in: (a) AerMet 100 at $\mathrm{E}_{\mathrm{app}}=-1,000 \mathrm{~m} \mathrm{~V}_{\mathrm{SCE}}$ and (b) Ferrium PH48S H900 at $\mathrm{E}_{\mathrm{app}}=-975 \mathrm{mV}_{\text {SCE. }}$ 160

Figure 62 Effect of $\mathrm{E}_{\text {app }}$ on subcritical crack growth kinetics of Ferrium PH48S - H900 stressed under slow rising displacement in $3.5 \% \mathrm{NaCl}$. The specimen tested at $\mathrm{E}_{\text {app }}=-250$ $\mathrm{mV}_{\mathrm{SCE}}$ reached the load limit of the testing frame and the test was terminated. ..... 162

Figure 63 HEAC fracture morphology of Ferrium PH48S - H900 stressed under slow rising displacement in $3.5 \% \mathrm{NaCl}$ at $\mathrm{E}_{\mathrm{app}}=-250$ (a), -575 (b), -775 (c), -975 (d), and -1,200 (e) $\mathrm{mV}$ SCE. 165

Figure 64 Effect of $E_{\text {app }}$ on the subcritical crack growth kinetics of Custom $465-\mathrm{H} 900$ stressed under slow rising displacement in $3.5 \% \mathrm{NaCl}$ solution. The specimen tested at $\mathrm{E}_{\mathrm{app}}=$ $-575 \mathrm{mV}$ SCE was conducted by Yongwon Lee without the use of a reference probe and analyzed using 52 point polynomial averaging, rather than 7 point polynomial averaging [173]. 167

Figure 65 HEAC fracture morphology of Custom 465 - H900 stressed under slow rising displacement in $3.5 \% \mathrm{NaCl}$ at $\mathrm{E}_{\mathrm{app}}=-575$ (a), -975 (b) and $-1,300$ (c) $\mathrm{mV}_{\mathrm{SCE}} \ldots \ldots .169$

Figure 66 Detail of a single IG facet from (a) Figure $65 b\left(E_{a p p}=-975 m V_{\text {SCE }}\right)$ and (b) Figure $65 \mathrm{c}\left(\mathrm{E}_{\mathrm{app}}=-1,300 \mathrm{mV} \mathrm{SCE}\right)$ showing the presence of lines (white arrows).............. 170

Figure 67 Threshold stress intensity $\left(\mathrm{K}_{\mathrm{TH}}\right)$ vs. $\mathrm{E}_{\text {app }}$ for Custom 465 - H900 and Ferrium PH48S - H860. Specimens which reached the load limit of the testing frame are denoted with an upward arrow; for these specimens $\mathrm{K}_{\mathrm{TH}}$ is taken at maximum load. 172

Figure 68 Effect of $E_{\text {app }}$ on Stage II crack growth rate at $\mathrm{K}=40 \mathrm{MPa} \sqrt{\mathrm{m}}$ in Custom $465-\mathrm{H} 900$ and Ferrium PH48S - H860. The trend line for PH48S - H860 is modeled after the more expansive set of kinetics for Ferrium PH48S - H900, which are presented in Figure 70 . 173

\section{XVIII}


Figure 69 Effect of $E_{a p p}$ and aging condition on the threshold stress intensity for HEAC in Ferrium PH48S. Upward arrows denote specimens which reached the load limit of the test frame. For these specimens, $\mathrm{K}_{\mathrm{TH}}$ is denoted as the stress intensity at maximum load. 175

Figure 70 Effect of Eapp and aging condition on Stage II crack growth rate $(\mathrm{K}=40 \mathrm{MPa} \sqrt{\mathrm{m}})$ in UHSSS. Plotted trend line for PH48S - H860 was extrapolated from PH48S - H900. The data for Custom 465 - H900 are shown for reference. 176

Figure 71 Hydrogen content of Ferrium PH48S and Custom 465 coupons charged for variable time $(609 \mathrm{~h} \max )$ at $\mathrm{E}_{\mathrm{app}}=-1,350 \mathrm{mV}_{\mathrm{SCE}}\left(\eta_{\mathrm{H} / \mathrm{H}+}=-520 \mathrm{mV}\right)$ in $0.001 \mathrm{M} \mathrm{NaOH}(\mathrm{pH}=$ 10) and subsequently outgassed at room temperature for up to $888 \mathrm{~h}$. 179

Figure 72 Regression fitting of $\mathrm{D}_{\mathrm{H}-\mathrm{EFF}}$ to the H-concentration data of Ferrium PH48S-H950 shown in Figure 71. 182

Figure 73 Hydrogen desorption rate $\left(\mathrm{dC}_{\mathrm{H}} / \mathrm{dt}\right)$ as a function of temperature for Ferrium PH48S H900and Custom $465-\mathrm{H} 900$. Specimens were charged at $\mathrm{E}_{\mathrm{app}}=-1,350 \mathrm{mV} \mathrm{SCE}_{\mathrm{S}}\left(\eta_{\mathrm{H} / \mathrm{H}+}\right.$ $=-520 \mathrm{mV}$ ) for $609 \mathrm{~h}$. 183

Figure 74 Determination of the activation energy for $\mathrm{H}$ desorption energy associated with the desorption peak of Ferrium PH48S and Custom 465 presented in Figure 73. The data are compared to an identical analysis conducted on the primary strengthening precipitate desorption peak in AerMet 100 [130]. 186

Figure $75 \mathrm{H}$-desorption rate $\left(\mathrm{dC}_{\mathrm{H}} / \mathrm{dt}\right)$ versus temperature for as-quenched (dashed line) and aged (solid line) AerMet 100, H-charged at $\eta_{\text {chg }}=-0.62 \mathrm{~V}$ and TDS tested at a heating rate (dT/dt) of $5^{\circ} \mathrm{C} / \mathrm{min}$, taken from [130] 187

Figure 76 Comparison of QC fracture features for AerMet 100 (a, $\mathrm{E}_{\mathrm{app}}=-1,000 \mathrm{mV} \mathrm{V}_{\mathrm{SCE}}$ ) and Ferrium PH48S-H900 (b and c, $\mathrm{E}_{\mathrm{app}}=-975 \mathrm{mV}$ SCE) with EBSD-measured martensite microstructure. OIM maps of AerMet 100 (d) and Ferrium PH48S (e) are shown at equal magnification to their respective fractographs. Black arrows indicate regions of martensite block cleavage across a small number of block features. 203

Figure 77 Detail of flat fracture in Ferrium PH48S $-\mathrm{H} 860$ stressed in $3.5 \% \mathrm{NaCl}$ at $\mathrm{E}_{\text {app }}=-975$ $\mathrm{mV}_{\mathrm{SCE}}$. Black arrows indicate fine river marks on the flat cleavage surface. 204

Figure 78 Fracture morphology of UHSS(S) stressed under slow rising displacement in 3.5\% $\mathrm{NaCl}$, (a) IGSCC of $300 \mathrm{M}$ at $\mathrm{E}_{\mathrm{app}}=1,000 \mathrm{mV}_{\mathrm{SCE}}$ and, (b) IGSCC of Custom $465-$ $\mathrm{H} 900$ at $\mathrm{E}_{\mathrm{app}}=1,000 \mathrm{mV}_{\mathrm{SCE}}$. Images (c) and (d) show detail regions of (a) and (b), respectively. 209

Figure 79 High magnification SEM-BSE imaging of a prior austenite grain boundary in Custom 465. The boxed region is shown magnified in the lower right inserted image........ 214 
Figure 80 High magnification SEM-BSE imaging of a prior austenite grain boundary in Custom 465. A prior austenite grain or packet boundary is indicated by a yellow dashed line. The boxed region is shown magnified in the lower right image. 215

Figure 81 Low magnification TEM micrograph martensite block structure in Ferrium PH48S H900. 217

Figure 82 Transmission electron micrographs of Ferrium PH48S - H900: (a) Bright field image of martensite block structure of Ferrium PH48S - H900 and (b) High magnification bright field image of the boxed region in (a). A single, identical block boundary is indicated by white arrows in each image. Both images were taken in collaboration with Matt Schneider at The University of Virginia. 218

Figure $83 \mathrm{C}_{\mathrm{H}-\mathrm{DIFF}}$ as a function of $\mathrm{E}_{\text {app }}$ for five ultra-high strength stainless steel-aging temperature combinations. Values of $\mathrm{C}_{\mathrm{H}-\mathrm{DIFF}}$ are calculated from $\mathrm{K}_{\mathrm{TH}}$ versus $\mathrm{E}_{\text {app }}$ measurements (Figures 67 and 69) through Equation 1 and Equation 12.............. 226

Figure 84 Measured da/dtII at $\mathrm{K}=40 \mathrm{MPa} \sqrt{\mathrm{m}}(\triangleleft)$ and the model predicted trend line of the applied potential dependence of $\mathrm{da} / \mathrm{dt}_{\text {II }}$ for Ferrium PH48S - H860, using the Hdiffusion limited model (Equation 2) with inputs of $\mathrm{C}_{\mathrm{H}-\mathrm{DIFF}}$ calculated from Equation 15 , average $\mathrm{D}_{\text {H-EFF }}$ as described in the text, correlated $\chi_{\text {crit }}[2,3]$, best fit $\mathrm{C}_{\text {Ho-crit }}$, and the upper bound of $\sigma_{\mathrm{H}}=6.8 \sigma \mathrm{YS}$.

Figure 85 Measured da/dtII at $\mathrm{K}=40 \mathrm{MPa} \sqrt{\mathrm{m}}(\bullet)$ and the model predicted trend line of the applied potential dependence of da/dt II for Ferrium PH48S - H900, using the H-diffusion limited model (Equation 2) with inputs of $\mathrm{C}_{\mathrm{H}-\mathrm{DIFF}}$ calculated from Equation 16, measured $\mathrm{D}_{\mathrm{H}-\mathrm{EFF}}$, correlated $\chi_{\text {crit }}[2,3]$, best fit $\mathrm{C}_{\mathrm{H \sigma}-\text { crit }}$, and the upper bound of $\sigma_{\mathrm{H}}=$ $6.8 \sigma_{\mathrm{YS}}$. 228

Figure 86 Measured $\mathrm{da} / \mathrm{dt}_{\mathrm{II}}$ at $\mathrm{K}=40 \mathrm{MPa} \sqrt{\mathrm{m}}(\mathbf{m})$ and the model predicted trend line of the applied potential dependence of da/dtII for Ferrium PH48S - H968, using the Hdiffusion limited model (Equation 2) with inputs of $\mathrm{C}_{\mathrm{H} \text {-DIFF }}$ calculated from Equation 17, measured $\mathrm{D}_{\mathrm{H}-\mathrm{EFF}}$, correlated $\chi_{\text {crit }}[2,3]$, best fit $\mathrm{C}_{\mathrm{H} \sigma-\mathrm{crit}}$, and the upper bound of $\sigma_{\mathrm{H}}$ $=6.8 \sigma_{\mathrm{YS}}$. 229

Figure 87 Measured da/dt II at $\mathrm{K}=40 \mathrm{MPa} \vee \mathrm{m}$ ( $\mathbf{0})$ and the model predicted trend line of the applied potential dependence of da/dt II for Ferrium S53, using the H-diffusion limited model (Equation 2) with inputs of $\mathrm{C}_{\mathrm{H}-\mathrm{DIFF}}$ calculated from Equation 18, average $\mathrm{D}_{\mathrm{H}}$ EFF for AerMet 100, correlated $\chi_{\text {crit }}[2,3]$, best fit $\mathrm{C}_{\mathrm{H \sigma} \text {-crit }}$, and the upper bound of $\sigma_{\mathrm{H}}=$ $6.8 \sigma \mathrm{Ys}$. 230 


\section{$\underline{\text { List of Tables }}$}

Table 1 Conventional Heat Treatment and Reported Mechanical Properties of UHSS and

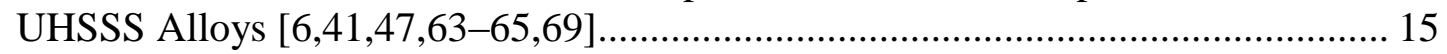

Table 2 Measured Compressive Flow Properties of Selected Alloys......................................... 16

Table 3 Measured Bulk Composition of UHSS Alloys ......................................................... 16

Table 4 Measured Impurity and Rare Earth Element Contents of UHSS Alloys ..................... 17

Table 5 Measured OCP of UHSS and UHSSS with Respect to the Saturated Calomel Electrode 24

Table 6 Interfacial area and fraction of total boundary area of selected martensite boundaries 51

Table 7 Calculated $\mathrm{D}_{\mathrm{R}}$ of UHSS alloys, using a variable pixel gap tolerance. 73

Table 8 Calculated maximum stress distances for UHSS at $\mathrm{K}=40 \mathrm{MPa} \sqrt{\mathrm{m}}$ using Equation 8 . 116

Table 9 Interfacial area and fraction of total boundary area of selected martensite boundaries 134

Table 10 The effect of increasing pixel gap tolerance on calculated $D_{R}$ of all UHSS(S) alloys in the present study. The physical distance for each pixel gap tolerance is indicated for each value of $D_{R}$ in $n m$. 145

Table 11 Measured values of $\mathrm{K}_{\mathrm{JICi}}$ and $0.2 \mathrm{~mm}$ offset $\mathrm{K}_{\mathrm{JIC}}$ for the R-curve data of selected alloys presented in Figure 52. (*) The value of K KIC for Ferrium PH48S - H968 was extrapolated from a linear regression of the first $0.1 \mathrm{~mm}$ of crack extension after $\mathrm{K}_{\text {JICi. }}$. 148

Table 12 Regression fit $\mathrm{D}_{\mathrm{H}-\mathrm{EFF}}$ for the H-solubility data represented in Figure 71 using an assumed $\mathrm{C}_{\mathrm{H}-\mathrm{TOTAL}} / \mathrm{C}_{\mathrm{H}-\mathrm{EQ}}=0.99$ at $609 \mathrm{~h}$ of charging time 180

Table 13 ThermoCalc predictions of the equilibrium volume fraction (\%) of phases in Ferrium PH48S under different aging conditions [43]..... 205

Table 14 Measured and Calculated Total Austenite Content of Custom 465 and Ferrium PH48S. 


\section{Introduction}

\subsection{Problem Statement}

Modern martensitic ultra-high strength steels (UHSS) exhibit exceptionally high combinations of tensile strength and fracture toughness, ideal for structural aerospace applications such as a landing gear, rotor shaft, and arresting hook [4,5]. However, high-strength martensitic microstructures are susceptible to severe stress corrosion cracking (SCC) via a hydrogen environment assisted cracking (HEAC) mechanism during extended exposure to service environments that produce atomic hydrogen $(\mathrm{H})[6,7]$. Hydrogen-driven degradation of mechanical properties is severe and significantly limits operating stress levels and component life [7]. Significant advances have been made to the measurement and modeling of alloy-environment dependent SCC-HEAC cracking kinetics, paving the way for damage tolerant SCC management. However, the present literature database of SCC-HEAC kinetics for modern alloys is severely limited. Further, the microstructural dependences of key variables in models of SCC-HEAC properties have not been sufficiently investigated.

The overarching goal of this research is to quantitatively measure high-resolution subcritical crack growth kinetics for modern UHSS, to further the fundamental understanding of the electrochemical and microstructural basis for key variables in micro-mechanical models of SCC-HEAC properties. Towards this goal, a suite of UHSS was selected to encompass a wide range of metallurgical variables and quantitatively characterized for SCC resistance in a range of environmental severities. Alloy microstructure, HEAC fracture path, and hydrogen trapping phenomena were characterized to gain a comprehensive view of the variables which can affect SCC resistance. Fundamental knowledge gained from the present work can be used to inform SCC management, lifetime prediction, and future SCC-resistant alloy development.

This dissertation consists of four Chapters. The present chapter provides the background material for HEAC of UHSS alloys. Due to the similarity of characterization techniques used, experimental methods are collected in Chapter 2. Chapters 3 and 4 form the investigative body of this dissertation, examining the origins of SCC resistance in martensitic UHSS and ultra-high strength stainless steels (UHSSS), respectively. 


\subsection{Mechanism of HEAC}

In the broadest sense, SCC propagation in many alloys occurs by the simultaneous action of mechanical loading and (electro) chemical reaction(s) which produce atomic hydrogen at the crack tip. Above a fracture mechanics based threshold ( $\mathrm{K}_{\mathrm{TH}}$ or $\mathrm{K}_{\mathrm{ISCC}}$ ) of mechanical driving force, crack growth occurs at a quantifiable-subcritical rate (da/dt) which depends on stress intensity (K) [7]. Atomic hydrogen produced on the crack tip surface diffuses as an interstitial solute to the fracture process zone (FPZ) ahead of the crack tip and initiates hydrogen damage through bonddecohesion, H-enhanced plasticity, or the interaction of these prominent mechanisms [8]. Maximum crack growth rate is often limited by H-diffusion to the FPZ, and asymptotically approaches a characteristic K-independent Stage II crack growth rate (da/dtII) [7].

\subsubsection{Micromechanical Models of SCC-HEAC}

Three hydrogen embrittlement (HE) mechanisms have significant experimental or theoretical support in non-hydride forming structural metals: hydrogen enhanced decohesion (HEDE), hydrogen enhanced localized plasticity (HELP), and adsorption induced dislocation emission (AIDE) [7,9]. The contribution of each mechanism in HEAC is controversial, and the conjoint action of multiple mechanisms is likely [10]. However, there is evidence that both HELP and HEDE mechanisms are active in UHSS alloys [7,11,12]. Apart from the mechanistic controversy, it is critically important for a given mechanism to provide means to predict SCC-HEAC properties. As detailed elsewhere $[3,7,13,14]$, HEDE provides this capability. However, predictive capability is hindered by unknown-adjustable parameters integral to the models. In the remainder of this section, micromechanical models for $\mathrm{K}_{\mathrm{TH}}$ and da/dtII will be presented.

Hydrogen embrittlement by interface decohesion above a threshold stress intensity [15-17], and $\mathrm{H}$ diffusion limited crack growth [2], provide a mechanism for models to predict the dependencies of $\mathrm{K}_{\mathrm{TH}}$ and da/dt $\mathrm{dI}_{\text {II }}$ on crack tip hydrogen concentration. As developed by Gerberich et al. [13,14]:

$$
\text { (1) } K_{T H}=\frac{1}{\beta^{\prime}} \exp \frac{\left(k_{I G}-\alpha C_{H \sigma}\right)^{2}}{\alpha^{\prime \prime} \sigma_{Y S}}
$$


where $\beta^{\prime}\left((\mathrm{MPa} \sqrt{ })^{-1}\right)$ and $\alpha^{\prime \prime}(\mathrm{MPa} \cdot \mathrm{m})$ are constants in a dislocation-shielding model of crack tip elastic stress, $\mathrm{C}_{\mathrm{H \sigma}}$ (atom fraction $\mathrm{H}$ ) is the crack tip stress enhanced diffusible-H concentration adjacent to the crack path in the FPZ, and $\alpha(\mathrm{MPa} \sqrt{ } \mathrm{m}$ per atom fraction $\mathrm{H})$ is a weighting factor which defines lowering of the Griffith toughness $\left(\mathrm{k}_{\mathrm{IG}}, \mathrm{MPa} \sqrt{\mathrm{m}}\right)$ per unit $\mathrm{H}$ concentration. To briefly summarize the model mechanism: cohesion of microstructural interfaces, such as prior austenite grain boundaries, is reduced by the accumulation of $\mathrm{H}$. Cracking occurs when the crack tip/microstructure-scale tensile stress exceeds the cohesive strength of local interfaces. $\mathrm{H}$ concentration is elevated at the crack tip $\left(\mathrm{C}_{\mathrm{H \sigma}}\right)$ due to the interaction of hydrostatically stressed lattice dilation and H-trapping at specific microstructure features $[18,19]$. The Gerberich model successfully predicted $\mathrm{K}_{\mathrm{TH}}$ for a $\mathrm{Ni}-\mathrm{Cu}$ superalloy (Monel $\mathrm{K}-500$ ) and to a limited extent for AerMet 100 [3,6,20].

Considering diffusion limited SCC growth, one dimensional modeling of H-diffusion ahead of a discontinuously growing crack front yielded $[2,6,7]$ :

$$
\text { (2) }\left(\frac{d a}{d t}\right)_{I I}=\frac{4 D_{H-E F F}}{\chi_{c r i t}}\left\{\operatorname{erf}^{-1}\left(1-\frac{C_{H \sigma-c r i t}}{C_{H \sigma}}\right)\right\}^{2}
$$

where $\mathrm{D}_{\text {H-EFF }}$ is the reversible-trap affected diffusivity of $\mathrm{H}, \chi_{\text {crit }}$ is the critical distance ahead of the crack tip where $\mathrm{H}$ cracking nucleates for an increment of discontinuous crack advance, and $\mathrm{C}_{\mathrm{H \sigma} \text {-crit }}$ is the critical concentration of $\mathrm{H}$ necessary for decohesion at $\chi_{\text {crit. }} \mathrm{C}_{\mathrm{H \sigma} \text {-crit }}$ represents a critical concentration of local hydrogen, in the context of stress filed enhancement; it is a property of the interface and inversely depends on a function of local tensile stress. Regression fitting of Equation 2 to fastest observed da/dt $/$ II versus $D_{H-E F F}$ data yielded a constant $\chi_{\text {crit }}$ of $900 \mathrm{~nm}$ for high strength alloys [2,3]. Proportionality between fastest observed da/dt $\mathrm{dI}_{\mathrm{I}}$ and $\mathrm{D}_{\mathrm{H}, \mathrm{EFF}}$ across multiple structural material systems (Ni superalloy, UHSS, $\beta / \alpha \mathrm{Ti}, \mathrm{Al}$ ) supports the validity of the model [2].

Quantitative predictions of $\mathrm{K}_{\mathrm{TH}}$ and da/dt $\mathrm{II}$ models require determination of $\mathrm{C}_{\mathrm{H \sigma}}$ given by [21,22]:

$$
\text { (3) } C_{H \sigma}=\left[C_{L} \frac{\left(1-C_{L \sigma}\right)}{\left(1-C_{L}\right)} \exp \left(\frac{\sigma_{H} V_{H}}{R T}\right)\right]\left[1+\frac{\left(1-C_{R T}\right)}{\left(1-C_{L}\right)} \exp \left(\frac{E_{B}}{R T}\right)\right]
$$


where $\sigma_{\mathrm{H}}$ is crack tip hydrostatic stress (a multiple of $\sigma_{\mathrm{YS}}$ ), $\mathrm{V}_{\mathrm{H}}$ is the partial molar volume of $\mathrm{H}$ in the lattice $\left(2.0 \mathrm{~cm}^{3} / \mathrm{mol} \mathrm{Fe}\right), \mathrm{E}_{\mathrm{B}}$ is the binding energy of $\mathrm{H}$ to the dominant-reversible trap site in the FPZ, $C_{L \sigma}$ is the concentration of stress enhanced lattice $H, C_{R T}$ is the concentration of reversibly trapped $\mathrm{H}$ governed by $\mathrm{E}_{\mathrm{B}}, \mathrm{T}$ is temperature and $\mathrm{R}$ is the ideal gas constant. $\mathrm{C}_{\mathrm{L}}$ and $\mathrm{C}_{\mathrm{RT}}$ are certainly much less than 1 atom fraction $\mathrm{H}$, but $\mathrm{C}_{\mathrm{L} \sigma}$ may be more significant and simplification of $\left(1-C_{L \sigma}\right)$ to 1 must be verified if crack tip stress $\left(\sigma_{H} V_{H}\right)$ is large. In Equation 2 the stress affected crack tip hydrogen concentration is in equilibrium with H-production on the crack surface and is directly influenced by alloy-specific microstructure, phase composition, and mechanical properties. $\mathrm{C}_{\mathrm{H \sigma}}$ is the key-unifying variable in modeling of $\mathrm{HEAC}$ kinetics for alloy performance or mechanism validation.

The models in Equations 1 and 2 provide a basis for interpreting the effects of metallurgical and environmental variables on observed trends in $\mathrm{K}_{\mathrm{TH}}$ and $\mathrm{da} / \mathrm{dt}_{\mathrm{II}}$. Variables affecting HEAC properties fall into 4 categories including those that affect: (a) H-production and uptake $\left(\mathrm{C}_{\mathrm{L}}\right)$, (b) trap sensitive $\mathrm{H}$ mobility approximated by $\mathrm{D}_{\mathrm{H}-\mathrm{EFF}}$, (c) H-localization at crack tip FPZ damage sites $\left(\mathrm{C}_{\mathrm{H \sigma}}, \sigma_{\mathrm{YS}}\right)$, and (d) susceptibility of the microstructure to atomic $\mathrm{H}\left(\alpha, \mathrm{C}_{\mathrm{H}-\mathrm{crit}}, \mathrm{k}_{\mathrm{IG}}\right)$. Critically, many of the model variables have not been conclusively linked to specific microstructural or metallurgical features. For example, $\chi_{\text {crit }}$ has been solely interpreted as a fitting parameter with some justification through continuum fracture mechanics location of maximum crack tip $\sigma_{\mathrm{H}}$; however, it is reasonable to suggest that local microstructure (e.g, martensite interfaces) could affect $\chi_{\text {crit }}$. This point has not been investigated by comparison of careful microstructure and fractographic findings.

\subsection{Metallurgical Design of UHSS}

\subsubsection{Key Developments of Modern UHSS}

Ultra-high strength steels have benefited from five decades of composition and microstructure refinement. From this dedicated study, three key developments are fundamental to current modern and next-generation UHSS alloys, particularly in the context of SCC/HEAC: (1) precipitation of coherent, nano-scale carbides or intermetallics which trap hydrogen, reducing the effective diffusivity of hydrogen $\left(\mathrm{D}_{\mathrm{H}, \mathrm{EFF}}\right)$ and diffusion-limited da/dt $\mathrm{II}_{\mathrm{II}},(2)$ reduced impurity content for 
increased intergranular cohesion and improved $\mathrm{K}_{\mathrm{TH}}$ in hydrogen environments, and (3) rare earth additions to further reduce boundary segregation and increase impurity particle spacing and improve $\mathrm{K}_{\mathrm{IC}}$.

The efficacy of $\mathrm{D}_{\mathrm{H}-\mathrm{EFF}}-$ limiting carbide precipitation is illustrated by comparing legacy $300 \mathrm{M}$ $(0.4 \mathrm{C}-1.8 \mathrm{Ni}-0.8 \mathrm{Cr}-0.4 \mathrm{Mo})$ to modern, secondary hardening AerMet $100(0.2 \mathrm{C}-13.4 \mathrm{Co}-11.1 \mathrm{Ni}-$ 3.0Cr-1.2Mo). Both alloys exhibit ultra-high strength and a similar tempered martensite matrix. However, a dispersion of nano-scale coherent $\mathrm{M}_{2} \mathrm{C}(\mathrm{M}=\mathrm{Fe}, \mathrm{Mo}, \mathrm{Cr})$ carbides reduces $\mathrm{D}_{\mathrm{H}-\mathrm{EFF}} 80 \mathrm{x}$ in AerMet 100 compared to $300 \mathrm{M}$ at the same strength, and decreases diffusion-limited da/dtII by 1 to 2 orders of magnitude when stressed in $3.5 \% \mathrm{NaCl}$ under cathodic applied potential $\left(\mathrm{E}_{\mathrm{app}}\right)$ $[1,6,23,24]$. Nano-scale $\mathrm{M}_{2} \mathrm{C}$ precipitation in AerMet 100 is promoted by significant additions of cobalt which increase the driving force for precipitation $[25,26]$. Further, cobalt retards recovery of martensite dislocation structure, which acts as nucleation sites for precipitates during tempering $[25,27]$. Characteristics of the primary strengthening precipitate, while not directly probed, are discussed for the selected UHSS alloys in ensuing sections.

It has been well established that mobile-atomic impurities ( $\mathrm{S}, \mathrm{P}, \mathrm{Mn}, \mathrm{Si}, \mathrm{As}, \mathrm{Sn}, \mathrm{Sb})$ segregate to prior austenite grain boundaries and act synergistically with environmental $\mathrm{H}$ to promote intergranular decohesion, drastically reducing $\mathrm{K}_{\mathrm{TH}}$ [28]. For example, high purity 4340-type steels exhibit $5 \mathrm{x}$ greater $\mathrm{K}_{\mathrm{TH}}$ in gaseous $\mathrm{H}_{2}$ when impurities $(\mathrm{P}+\mathrm{S})$ are reduced $4 \mathrm{x}(0.025$ to $0.006 \mathrm{wt} \%)$ [29]. The adverse effect of impurities is exacerbated by elevated $\mathrm{Mn}$ and $\mathrm{Si}$, which act to promote segregation to the grain boundaries [29]. As such, the purity of modern UHSS is carefully controlled through vacuum induction melting and vacuum arc remelting (VIM/VAR) techniques and feed stock selection [30]. The presence of mobile impurities is further controlled through the addition of rare earth elements such as lanthanum or cerium [31]. Rare earth elements preferentially form stable impurity-oxide particles with increased mean spacing compared to deleterious impurity phases such as $\mathrm{MnS}$ or $\mathrm{CrS}$ [32]. Widely spaced impurity precipitates have been conclusively linked to improved toughness in UHSS AF1410 [32,33], but are not likely (as particles) to affect HEAC. The origin of this fracture toughness improvement has not been mechanistically proven, but appears to be tied to the plastic strain required for void generation which is relatively low in small, closely spaced $\mathrm{MnS}$ and $\mathrm{CrS}$ precipitates [34]. 


\subsubsection{Development of Next Generation $\mathrm{M}_{2} \mathrm{C}$ Strengthened UHSS}

Targeted compositional advancement for next-generation $\mathrm{M}_{2} \mathrm{C}$ carbide strengthened UHSS is threefold: (1) reduce cobalt content for cost savings, (2) improve corrosion resistance through chromium addition so as to eliminate the need for corrosion resistant coatings, and (3) produce an additional strengthening dispersion of nano-scale MC carbides to further reduce $\mathrm{D}_{\mathrm{H}-\mathrm{EFF}}$. Critically, alloy advancement must be achieved with consistent-high mechanical properties and strong SCC resistance compared to an AerMet 100 baseline. The next generation UHSS selected for the present study maintain mechanical properties similar to AerMet 100, but have not been quantitatively evaluated for HEAC resistance.

Commercial UHSS Ferrium ${ }^{\circledR} \mathrm{M} 54{ }^{\circledR 1}(0.3 \mathrm{C}-10.1 \mathrm{Ni}-7.0 \mathrm{Co}-2.1 \mathrm{Mo}-1.0 \mathrm{Cr}-1.3 \mathrm{~W}-0.1 \mathrm{~V}$ wt $\%)$ and an experimental low alloy steel CMU-CrNiMoWV ${ }^{2}(0.4 \mathrm{C}-4.5 \mathrm{Cr}-3.0 \mathrm{Ni}-2.0 \mathrm{Mo}-0.5 \mathrm{~W}-0.5 \mathrm{~V}$ wt\%) have achieved significant alloy element reductions compared to AerMet 100 while maintaining equivalent mechanical properties $[23,35]$. When compared, the triplet of alloys provides a means to qualitatively evaluate the effect of decreasing $\mathrm{Ni}$ and Co on the SCC-HEAC resistance of UHSS from an AerMet 100 baseline composition. Stainless UHSS Ferrium ${ }^{\circledR}$ S53 ${ }^{\circledR 3}(0.21 \mathrm{C}-14.1 \mathrm{Co}-$ $10.0 \mathrm{Cr}-5.5 \mathrm{Ni}-2.0 \mathrm{Mo}-1.0 \mathrm{~W}-0.3 \mathrm{~V} \mathrm{wt} \%$ ) fulfills the need for increased corrosion resistance in UHSS, but suffers from reduced $\sigma_{\mathrm{ys}}$ and $\mathrm{K}_{\mathrm{IC}}$ relative to AerMet 100 [36]. When compared to AerMet 100, it elucidates the effect of chromium on HEAC resistance. Minor additions of $\mathrm{W}$ and $\mathrm{V}$ (1.0-1.4 wt\%) in all next generation $\mathrm{M}_{2} \mathrm{C}$ strengthened alloys (Ferrium M54, Ferrium S53, and CrNiMoWV) promote precipitation of fine $\mathrm{MC}(\mathrm{M}=\mathrm{W}, \mathrm{V})$ carbides known to reduce HEAC by acting as strong reversible H-traps [37-40]. The HEAC resistance of next-generation lower alloy UHSS compositions is reportedly strong, but has not been quantitatively assessed in the context of microstructural or compositional variables [35]. The origins of SCC-HEAC resistance in next generation UHSS will be probed in Chapter 3 though a systematic examination of HEAC behavior using modern characterization techniques and micromechanical models of HEAC properties.

\footnotetext{
$1 \quad$ Ferrium and M54 are registered trademarks of QuesTek Innovations LCC, Evanston, IL

2 CMU-CrNiMoWV is an experimental low alloy UHSS developed at Carnegie Mellon University. For brevity it will be referenced as CrNiMoWV.

3 S53 is a registered trademark of QuesTek Innovations LLC, Evanston, IL
} 


\subsubsection{Development of Next Generation Ni $i_{3}$ Ti Strengthened UHSSS}

Ultra-high strength stainless steel (UHSSS) fills the technical need for UHSS in structural applications where increased corrosion resistance is required. Corrosion resistance is conferred through the formation of a stable surface oxide film, eliminating the need for a corrosion resistant coating. Basic microstructure is nearly identical between UHSS and UHSSS, with the exception of strengthening precipitates. UHSSS are strengthened with nano-sized intermetallic particles such as hexagonal $\mathrm{Ni}_{3} \mathrm{Ti}, \mathrm{B} 2 \mathrm{NiAl}$, or bcc $\mathrm{Cu}$ rather than $\mathrm{M}_{2} \mathrm{C}$ typical to modern UHSS. Current alloy development of UHSSS is primarily centered on improving SCC resistance and achieving ultrastrength levels.

Modern martensitic UHSSS Custom ${ }^{\circledR} 465^{4}$ (0.01C-11.4Cr-10.9Ni-0.9Mo-0.7W-1.6Ti wt\%) was developed to replace $300 \mathrm{M}$ or 4340-type steels in aerospace applications where increased corrosion resistance is required. Custom 465 utilizes intermetallic $\mathrm{Ni}_{3} \mathrm{Ti}(\eta)$ as the primary strengthening precipitate within a tempered martensitic matrix. When compared to AerMet 100 on an equal strength basis, Custom 465 in the ultra-high strength temper (H900) exhibits dramatically reduced $\mathrm{K}_{\mathrm{IC}}(80 \mathrm{vs} 130 \mathrm{MPa} \sqrt{\mathrm{m}})$ and $\mathrm{K}_{\mathrm{TH}}(10 \mathrm{vs} 15 \mathrm{MPa} \sqrt{\mathrm{m}}$ at free corrosion in $3.5 \% \mathrm{NaCl})$ [6,41]. The origins of reduced $\mathrm{K}_{\mathrm{IC}}$ and $\mathrm{K}_{\mathrm{TH}}$ in Custom 465 at ultra-high strength have not been investigated. However, the technical need remains high for a stainless UHSS which maintains AerMet 100 level mechanical properties and strong SCC resistance.

Next-generation Ferrium PH48S ${ }^{5}$ (0.02C-10.9Cr-8.6Ni-7.3Co-1.6Mo-0.1W-0.6Ti-0.4Al wt\%) was originally developed to replace high strength cast titanium components in marine applications by maximizing strength to weight ratio, fracture toughness, and resistance to SCC. Three metallurgical factors distinguish Ferrium PH48S from modern Custom 465. First, precipitation strengthening is accomplished through an alloyed $\eta$ phase $\left(\mathrm{Ni}_{3}(\mathrm{Ti}, \mathrm{Al})\right)$ and ordered $\mathrm{NiAl}[42,43]$. Second, $\mathrm{Ti}$ and $\mathrm{C}$ contents are limited to allow single phase austenite homogenization, and subsequent precipitation of fine grain boundary pinning MC carbides during forging [42]. Finally, the addition of $\mathrm{Co}$ is designed to reduce strengthening precipitates size and maximize $\mathrm{Cr}$

4 Custom 465 is a registered trademark of CRS Holdings Inc., Reading, PA

5 Ferrium and PH48S are registered trademarks of QuesTek Innovations LLC, Evanston, IL 
partitioning to the chromium oxide passive film. Literature support of these effects is limited and confined to similar alloy systems $[36,44,45]$. However, compared to Custom 465-H900, fracture toughness $(\sim 100 \mathrm{MPa} \sqrt{\mathrm{m}})$ and $\mathrm{SCC}$ resistance $\left(\mathrm{K}_{\mathrm{ISCC}}>80 \mathrm{MPa} \sqrt{\mathrm{m}}\right.$ at free corrosion in $\left.3.5 \% \mathrm{NaCl}\right)$ are dramatically improved in Ferrium PH48S, at the cost of tensile strength ( $\left.\sigma_{\mathrm{YS}} \sim 1500 \mathrm{MPa}\right)$ $[46,47]$. It is unknown at this time if improved SCC resistance in PH48S is due to reduced strength (decreasing $\mathrm{C}_{\mathrm{H \sigma}}$ ) sourced as an inherent metallurgical variable, or the result of inaccurate SCC characterization. The origins of this behavior will be probed in Chapter 4 though systematically decreasing the aging temperature (and increasing $\sigma_{Y S}$ ) of Ferrium PH48S until the strength is identical to Custom 465.

\subsection{The Role of Grain Boundary Character}

It has been established in the literature that the misorientation, frequency, and connectivity of microstructural interfaces can influence the macroscopic fracture and embrittlement resistances of polycrystalline metals [48-52]. Established by Watanabe, the grain boundary character distribution (GBCD) is used to describe the nature of boundaries within an alloy [51,53]. The GBCD distribution is an empirical description of the relative area of various boundaries within a microstructure. Boundaries are classified according to misorientation: (1) low angle boundaries with small misorientation $\left(\theta<15^{\circ}\right)$, (2) boundaries with a sufficiently low coincident site lattice (CSL) orientation relationship $(3 \leq \Sigma \leq 29)$, and (3) high angle random boundaries without a special orientation relationship $\left(\theta>15^{\circ}\right)$. Low angle and CSL boundaries are considered resistant to fracture and impurity driven embrittlement when compared to high angle random boundaries [52], but the connections have not been quantitative nor detailed from the micromechanics and damage mechanism perspectives. The fracture stress for CSL boundaries in a variety of simple metals (Mo, $\mathrm{Zn}$, and $\mathrm{Al}$ ) was found to be 2-18 times higher than random boundaries; strongly dependent on the CSL boundary type, material system, and the presence of embrittling species [48]. When impurities are present, random high angle boundaries serve as a weak connected crack path though polycrystalline microstructure [48,49]. Further, there is evidence that random boundaries provide a preferential site for metalloid impurity and hydrogen segregation [52,54-57]. Preferential impurity segregation originates due to the relatively high interfacial energy and poor atomic fit of random high angle boundaries [52]. For these reasons, it is critically important to limit the 
connectivity of random high energy interfaces as they provide a weak, interconnected crack path through the microstructure.

Recent work has examined the effect of increasing CSL fraction to disrupt the connectivity of random interfaces in polycrystalline nickel [49,50]. Results establish that susceptibility to hydrogen and sulfur induced intergranular embrittlement is significantly reduced by increasing the fraction of CSL boundaries [49,50]. Specifically, Bechtle et al. found that increasing the length fraction of CSL (primarily $\Sigma 3$ ) boundaries by 1.6 times in polycrystalline nickel resulted in 20$30 \%$ higher fracture toughness, 2-fold increase in ductility, and a shift from brittle IG to more ductile failure during IHAC.

Two hypotheses were put forth by Bechtle et al. to explain increased resistance to IG IHAC, centered on the inherent susceptibility of random boundaries. First, the critical hydrogen concentration for decohesion of a random boundary is lower than a CSL boundary, implying that random boundaries will fracture at significantly lower local hydrogen concentrations. Second, random boundaries are hypothesized to have a higher trap-binding energy $\left(E_{B}\right)$ for hydrogen compared to CSL boundaries [52,58]. This effect would increase the hydrogen concentration local to the boundary and reduce grain boundary cohesion. Critically, the spatial connectivity of random interfaces with increasing CSL area fraction was not directly probed in this study despite literature which indicates this is a critical factor [49,51,59]. Regardless of the mechanistic origin, increasing CSL fraction strongly benefits IHAC resistance in polycrystalline Ni [50].

In a similar study, Kobayashi et al. examined the effect of random boundary connectivity on sulfurinduced IG fracture of polycrystalline nickel [49]. Kobayashi demonstrated that decreasing connectivity of random boundaries dramatically reduces the susceptibility to sulfur segregation induced IG fracture; emphasizing the importance of understanding the connectivity of random interfaces [49]. The positive effect of reducing random boundary connectivity is attributed to segments of CSL boundaries interrupting the random boundary network (RBN) and forcing the crack to deflect or branch for continued travel along random boundaries. Taken together, the results of Kobayashi et al. and Bechtle et al. underscore the importance of understanding the fractional distribution and connectivity of interfaces for impurity or hydrogen driven IG fracture. It is notable, however, that such studies have not been reported for more complex microstructures with 
a high density and variable characteristics of interfaces, as encountered for martensitic UHSS and UHSSS.

The effect of GBCD on hydrogen or impurity driven fracture, established in polycrystalline nickel should, in principle, apply to martensite interfaces in modern UHSS and UHSSS. The CSL boundary description is not affected by the crystal system; CSL boundaries in martensite should be low energy relative to random high angle boundaries [52]. Similarly, hydrogen is likely to preferentially segregate to high angle interfaces as modeled by Jothi et al. for a simple nickel bicrystal [57]. The fundamental challenges in applying the principles of grain boundary engineering to HEAC of martensitic UHSS is two-fold: (1) general length scales of the microstructure are significantly reduced, and (2) the strength of the alloys is massively increased over polycrystalline nickel (1600 MPa versus $50 \mathrm{MPa}$, [50]). In the case of the latter, the deleterious effect of increased yield strength on susceptibility to hydrogen assisted cracking (HAC) has been well established [7]. Increasing yield strength allows greater crack tip hydrostatic stress to be supported, subsequently increasing $\mathrm{C}_{\mathrm{H} \sigma}$ during hydrogen assisted cracking. Increased crack tip $\mathrm{H}$ concentration may dominate the role of beneficial CSL boundaries. However, a limited beneficial effect of CSL boundaries on IGSCC has been previously reported in ferrite/pearlite pipeline steels $\left(\sigma_{\mathrm{YS}} \sim 500\right.$ MPa) [60]. Low angle and low $\Sigma$ CSL boundaries $(\Sigma 11, \Sigma 13 b)$ were observed to resist crack propagation, while random high angle boundaries formed a preferential crack path [60]. Recently, Seita et al. studied the effect of the GBCD on hydrogen assisted cracking of a high strength Nibased superalloy, Inconel 725 , and confirmed that $\sum 3$ boundaries strongly resist hydrogen cracking [61]. This extension of previous work with low strength polycrystalline nickel suggests that the GBCD may play a role in HEAC of ultra-high strength alloys.

\subsection{Objectives}

The objective of this research is to further the understanding of the metallurgical and electrochemical mechanisms which dictate the SCC resistance of modern UHSS. Cracking kinetics gathered from high-resolution subcritical crack growth experiments quantitatively establish the SCC resistance of modern UHSS and provide a basis for future fracture mechanics based life time prediction. SCC fracture path is established through correlation of sub-micrometer martensite microstructure with fine scale fracture features. Using the expansive database of collected SCC 
kinetics, micromechanical models of HEAC properties are evaluated and the physical basis for adjustable-fitted parameters is investigated.

This dissertation investigates the metallurgical origins of SCC resistance in three chapters. Experimental procedures are identical for the investigative chapters (Chapters 3, 4) and, therefore, collated in Chapter 2 for brevity. The fundamental objectives for each chapter are outlined below, and specific objectives will be given at the beginning of each chapter.

\subsubsection{Chapter 2: Experimental Procedures}

The goal of Chapter 2 is to document the detailed experimental procedures for the remaining chapters and discuss the inherent resolution limit of SCC-HEAC characterization. Towards this goal, the present work answers three questions:

1) What is the current state of literature characterization of complex, multi-scale microstructures present in modern UHSS?

2) Can the influence of dcPD testing artifacts be effectively eliminated through a plasticity based resolution limit?

3) In the context of variable cracking kinetics, how are the fundamental HEAC properties $\left(K_{T H}, d a / d t_{I I}\right)$ best defined?

\subsubsection{Chapter 3: Measurement and Modeling of HEAC in Modern UHSS}

The goal of Chapter 3 is to develop the HEAC kinetics database and understanding of structureproperty relationships necessary to develop martensitic UHSS with improved performance in SCC-HEAC situations, in the context of a modern damage tolerance approach $[14,62]$, and building on baseline AerMet 100 behavior. First, the resistance of modern UHSS to SCC across a wide range of applied polarization is probed using subcritical crack growth experiments. Second, the fracture path is established through conjoint fine-scale fractographic and EBSD studies of microstructure. Finally, the efficacy of micromechanical models in predicting HEAC properties 
$\left(\mathrm{K}_{\mathrm{TH}}, \mathrm{da} / \mathrm{dt}_{\mathrm{II}}\right)$ is evaluated using the established database of HEAC kinetics. Three fundamental questions are addressed:

1) Can susceptibility to SCC-HEAC be managed in modern UHSS with applied polarization?

2) What compositional, microstructural, and electrochemical variables promote susceptibility to SCC?

3) Do the present models for HEAC properties effectively predict the behavior of modern UHSS in variable electrochemical conditions?

\subsubsection{Chapter 4: Origins of HEAC Resistance in Modern UHSSS}

The goal of Chapter 3 is to develop the HEAC kinetics database and understanding of structureproperty relationships necessary to further develop stainless UHSS with improved performance in SCC-HEAC situations, in the context of a modern damage tolerant approach [14,62]. Emphasis is placed upon the origins of variable SCC-HEAC resistance in compositionally similar UHSSS alloys. First, the resistance of modern UHSSS to SCC across a large range of applied polarization is probed using subcritical crack growth experiments. Second, the fracture path is established through conjoint fine-scale fractographic and EBSD studies. Finally, the hydrogen trapping behavior of each UHSSS is quantified using $\mathrm{H}$ uptake experiments and limited thermal desorption spectroscopy (TDS). Two fundamental questions are addressed:

1) What is the mechanistic origin of low SCC resistance in UHSSS at ultra-high strength?

2) Between similar UHSSS, is improved SCC resistance sourced at compositional, metallurgical, or H-trapping variables? 


\section{Experimental Methods}

The following chapter will discuss the experimental methods used to characterize the SCC-HEAC resistance, microstructure, and hydrogen trapping phenomena of the selected UHSS alloys. As discussed in Chapter 1, mechanistic understanding of SCC-HEAC in UHSS lies at the intersection of metallurgical, electrochemical, and hydrogen trapping variables. Potential-dependent HEAC kinetics must therefore be viewed in the context of alloy composition, fine scale microstructure, fracture path, and hydrogen trapping phenomena.

Toward this end, each alloy is subjected to a suite of characterization techniques aimed at quantifying HEAC-relevant variables. The process consists of five characterization steps:

(1) Bulk alloy and impurity compositions are measured via ICP-AES (inductively coupled plasma - atomic emission spectroscopy) and GDMS (glow discharge mass spectroscopy), respectively.

(2) The martensite microstructure is probed using EBSD (electron back scatter diffraction), and statistical methods are employed to quantify the GBCD.

(3) High resolution HEAC kinetics are quantified across a range of applied polarizations.

(4) High resolution SEM fractographs of HEAC fracture are compared to sub-micrometer martensite structure to establish the HEAC fracture path.

(5) For a limited set of alloys, hydrogen solubility and trapping were quantified using long term H-charging experiments and TDS.

Finally, the inherent variability in the measurement of high resolution HEAC kinetics in modern UHSS is addressed. The addition of a plasticity based resolution limit is experimentally supported and determination of HEAC properties is defined for variable forms of HEAC kinetics. 


\subsection{Material Composition and Heat Treatment}

Seven ultra-high strength steels were heat treated to optimize strength and fracture toughness. Original stock format, heat treatment procedures, and typical mechanical properties are shown in Table 1. Mechanical property data were obtained from literature studies and technical datasheets $[6,23,41,47,63-65]$. The selected heat treatments are representative of commercial procedures and denoted as the 'conventional' heat treatment for each alloy in the remainder of the text. Two exceptions to this convention are notable. First, Custom $465-\mathrm{H} 900$ is aged at a significantly lower temperature $\left(482^{\circ} \mathrm{C}\right)$ than is typically recommended by the manufacturer. The $482^{\circ} \mathrm{C}$ aging temperature was chosen for increased yield strength compared to commercial tempers (510$565^{\circ} \mathrm{C}$ ), allowing for a direct comparison to UHSS AerMet 100. Second, specimens of nextgeneration Ferrium PH48S were subjected to four different aging temperatures: 460, 482, 510, $520^{\circ} \mathrm{C}$. The goal of this series was to systematically examine the effect of increasing yield strength on HEAC properties and provide an equal strength comparison to Custom 465. Mechanical property data for the commercial aging temperature $\left(520^{\circ} \mathrm{C}\right)$ of Ferrium PH48S are shown in Table $1[47]$.

To verify reported mechanical properties and assess the efficacy of ultra-high strength aging temperatures in PH48S, a limited number of alloys were selected for compression testing. The work hardening behavior was determined by calculation of Ramberg-Osgood (RO) parameters from measured load versus displacement data for each alloy. Flow curves measured from compression loading were regression fit with the Ramberg-Osgood equation [66]:

$$
\text { (4) } \varepsilon=\frac{\sigma}{E}+\alpha \frac{\sigma_{0}}{E}\left(\frac{\sigma}{\sigma_{0}}\right)^{n}
$$

where $\varepsilon$ is the total elastic-plastic strain, $\sigma$ is the tensile stress, $\mathrm{E}$ is the elastic modulus, $\sigma_{0}$ is a reference stress taken as the material yield strength ( $\left.\sigma_{Y S}\right)$, and $\alpha$ and $n$ are Ramberg-Osgood fitting parameters. Measured compressive mechanical properties and RO parameters are shown in Table 2. Compressive yield strength values for the UHSS in Table 2 are universally elevated compared to tensile values (Table 1). This effect has been previously observed in a wide range of UHSS and does not constitute experimental error [67]. Further, RO parameters shown in Table 2 are close to literature values for compressive stress strain testing of AerMet 100 (13 vs 16), supporting the 
efficacy of compression testing and RO analysis in the present work [67,68]. Bulk composition was measured by a vendor using inductively coupled plasma optical emission spectroscopy (ICPOES) and infrared combustion (IC). The results of ICP-OES and IC measurements are shown in Table 3. Trace impurity and rare earth content were measured using glow discharge mass spectroscopy (GDMS). The results of the GDMS analysis are presented in Table 4.

\begin{tabular}{|c|c|c|c|c|c|c|c|}
\hline$\cdot$ & $\begin{array}{l}\text { Stock } \\
\text { Format }\end{array}$ & Austenitization & Temper & $\begin{array}{c}\text { OYS } \\
(\mathrm{MPa})\end{array}$ & $\begin{array}{l}\text { UTS } \\
\text { (MPa) }\end{array}$ & $\mathrm{RH}_{\mathrm{C}}$ & $\begin{array}{c}\mathrm{K}_{\mathrm{IC}} \\
(\mathrm{MPa} \sqrt{ } \mathrm{m})\end{array}$ \\
\hline $300 \mathrm{M}$ & $\begin{array}{l}1.4 \mathrm{~cm} \\
\varnothing \mathrm{Bar}\end{array}$ & $871^{\circ} \mathrm{C} 1 \mathrm{~h} / \mathrm{OQ}$ & $\begin{array}{c}2 \times 300^{\circ} \mathrm{C} 3 \mathrm{~h} / \\
\mathrm{AC}\end{array}$ & 1580 & 1850 & 58 & 65 \\
\hline AerMet 100 & $\begin{array}{l}15 \mathrm{~cm} \varnothing \\
\text { Forging }\end{array}$ & $\begin{array}{c}885^{\circ} \mathrm{C} 1 \mathrm{~h} / \mathrm{OQ} \\
/ \mathrm{LN}_{2}\end{array}$ & $482^{\circ} \mathrm{C} 5 \mathrm{~h}$ & 1725 & 1965 & 54 & 130 \\
\hline CrNiMoWV & - & $\begin{array}{c}1050^{\circ} \mathrm{C} 1 \mathrm{~h} / \\
\mathrm{OQ} / \mathrm{LN}_{2}\end{array}$ & $\begin{array}{c}3 \times 575^{\circ} \mathrm{C} 1 \mathrm{~h} / \\
W Q / \mathrm{LN}_{2}\end{array}$ & 1580 & 1880 & 48 & 145 \\
\hline Ferrium M54 & $\begin{array}{l}1.4 \mathrm{~cm} \\
\varnothing \mathrm{Bar}\end{array}$ & $\begin{array}{c}1060^{\circ} \mathrm{C} 1 \mathrm{~h} / \\
\mathrm{OQ} / \mathrm{DI}\end{array}$ & $516^{\circ} \mathrm{C} 10 \mathrm{~h}$ & 1720 & 2020 & 54 & 126 \\
\hline Ferrium S53 & $\begin{array}{l}1.4 \mathrm{~cm} \\
\varnothing \mathrm{Bar}\end{array}$ & $\begin{array}{c}1085^{\circ} \mathrm{C} 1 \mathrm{~h} / \\
\mathrm{OQ} / \mathrm{DI}\end{array}$ & $\begin{array}{c}501^{\circ} \mathrm{C} 3 \mathrm{~h} / \\
\mathrm{WQ} / \mathrm{DI} 482^{\circ} \mathrm{C} \\
12 \mathrm{~h} / \mathrm{AC}\end{array}$ & 1550 & 1985 & 54 & $71-77$ \\
\hline Custom 465 & $\begin{array}{l}1.9 \mathrm{~cm} \\
\varnothing \mathrm{Bar}\end{array}$ & $\begin{array}{c}982^{\circ} \mathrm{C} 1 \mathrm{~h} / \\
\mathrm{OQ} / \mathrm{DI}\end{array}$ & $482^{\circ} \mathrm{C} 4 \mathrm{~h} / \mathrm{AC}$ & 1700 & 1810 & 51 & $78-89$ \\
\hline $\begin{array}{l}\text { Ferrium } \\
\text { PH48S }\end{array}$ & $\begin{array}{l}10 \mathrm{~cm} \varnothing \\
\text { Forging }\end{array}$ & $\begin{array}{c}825^{\circ} \mathrm{C} 1 \mathrm{~h} / \\
\mathrm{OQ} / \mathrm{LN}_{2}\end{array}$ & $520^{\circ} \mathrm{C} 2 \mathrm{~h} / \mathrm{AC}$ & $\begin{array}{l}1450- \\
1515\end{array}$ & 1585 & 49 & 100 \\
\hline
\end{tabular}

Table 1 Conventional Heat Treatment and Reported Mechanical Properties of UHSS and UHSSS Alloys [6,41,47,63-65,69] 


\begin{tabular}{|c|c|c|c|c|}
\hline & Temper & Comp. OYs (MPa) & E (GPa) & RO Parameter (n) \\
\hline AerMet 100 & $482^{\circ} \mathrm{C}$ 5 h & 1985 & 194 & 13 \\
\hline Ferrium M54 & $516^{\circ} \mathrm{C} 10 \mathrm{~h}$ & 1951 & 198 & 14 \\
\hline Ferrium S53 & $\begin{array}{c}501^{\circ} \mathrm{C} 3 \mathrm{~h} / \mathrm{WQ} / \mathrm{DI} \\
482^{\circ} \mathrm{C} 12 \mathrm{~h} / \mathrm{AC}\end{array}$ & 1820 & 209 & 10 \\
\hline Custom 465 & $482^{\circ} \mathrm{C} 4 \mathrm{~h} / \mathrm{AC}$ & 1827 & 201 & $23-31$ \\
\hline Ferrium PH48S & $460^{\circ} \mathrm{C} 4 \mathrm{~h} / \mathrm{AC}$ & 1806 & 222 & $41-43$ \\
\hline Ferrium PH48S & $482^{\circ} \mathrm{C} 4 \mathrm{~h} / \mathrm{AC}$ & 1696 & 202 & $26-38$ \\
\hline Ferrium PH48S & $510^{\circ} \mathrm{C} 4 \mathrm{~h} / \mathrm{AC}$ & 1689 & 203 & $40-50$ \\
\hline Ferrium PH48S & $520^{\circ} \mathrm{C} 2 \mathrm{~h} / \mathrm{AC}$ & 1640 & 203 & $63-72$ \\
\hline OQ - Oil Quench & AC - Air Cool / LN - Liquid Nitrogen Quench | DI - Dry Ice Quench \\
\hline Austenitization/quench/chill conditions are given in Table 1 & \\
\hline
\end{tabular}

Table 2 Measured Compressive Flow Properties of Selected Alloys

\begin{tabular}{|c|c|c|c|c|c|c|c|c|c|}
\hline & $\mathrm{C}^{1}$ & $\mathrm{Cr}^{2}$ & $\mathrm{Ni}^{2}$ & $\mathrm{Co}^{2}$ & $\mathrm{Mo}^{2}$ & $\mathrm{~W}^{2}$ & $\mathrm{~V}^{2}$ & $\mathrm{Ti}^{2}$ & $\mathrm{Al}^{2}$ \\
\hline 300M & 0.42 & 0.8 & 1.8 & - & 0.4 & - & 0.1 & - & - \\
\hline AerMet 100 & 0.23 & 3.0 & 11.1 & 13.4 & 1.2 & - & - & - & - \\
\hline CrNiMoWV & 0.38 & 4.5 & 3.0 & - & 2.0 & 0.5 & 0.5 & - & - \\
\hline Ferrium M54 & 0.30 & 1.0 & 10.1 & 7.0 & 2.1 & 1.3 & 0.1 & - & - \\
\hline Ferrium S53 & 0.21 & 10.0 & 5.5 & 14.1 & 2.0 & 1.0 & 0.3 & - & - \\
\hline Custom 465 & 0.01 & 11.4 & 10.9 & 0.1 & 0.9 & 0.7 & - & 1.6 & 0.1 \\
\hline Ferrium PH48S & 0.02 & 10.9 & 8.6 & 7.3 & 1.6 & 0.1 & - & 0.6 & 0.4 \\
\hline${ }^{1}$ Combustion Infrared Absorption ${ }^{2} \mathrm{ICP}-\mathrm{AES}$ & & & $\mathrm{Wt} \%$ & & \\
\hline
\end{tabular}

Table 3 Measured Bulk Composition of UHSS Alloys 


\begin{tabular}{|c|c|c|c|c|c|c|c|c|c|}
\hline & $\mathrm{Si}$ & $\mathrm{Mn}$ & $\mathrm{S}$ & $\mathrm{P}$ & As & $\mathrm{Sn}$ & $\mathrm{Sb}$ & $\mathrm{La}$ & $\mathrm{Ce}$ \\
\hline 300M & 18,000 & 9,000 & 3 & 71 & 44 & 65 & 10 & $<1$ & $<1$ \\
\hline AerMet 100 & 62 & 150 & 5 & 10 & 8 & 1 & 1 & 16 & 39 \\
\hline CrNiMoWV & $>1,000^{6}$ & 2 & 2 & 6 & $<1$ & $<1$ & $<1$ & 25 & 49 \\
\hline Ferrium M54 & 82 & 17 & 3 & 32 & 2 & $<1$ & $<1$ & 41 & $<1$ \\
\hline Ferrium S53 & 170 & 88 & 7 & 13 & 7 & 2 & 1 & 25 & $<1$ \\
\hline Custom 465 & 65 & 150 & 5 & 18 & 19 & 9 & 6 & $<1$ & $<1$ \\
\hline Ferrium PH48S & 39 & 3 & 2 & 5 & $<1$ & 1 & $<1$ & 35 & $<1$ \\
\hline & & & & \multicolumn{7}{|c|}{ wppm } \\
\hline
\end{tabular}

\section{Table 4 Measured Impurity and Rare Earth Element Contents of UHSS Alloys}

\subsubsection{UHSS Microstructure in Literature}

Current literature microstructural data are summarized for each UHSS alloy. The microstructure of legacy $300 \mathrm{M}$ and AerMet 100 has been extensively characterized through high resolution TEM in previous studies [24,26,63,70,71]. However, relatively little published data exist for the majority of alloys in the present work. Logical assumptions on the structure of these alloys will be made based on manufacturer information and studies of similar alloys. Unless otherwise noted, the microstructural descriptions below are for the conventional heat treatment for each alloy.

\subsubsection{M}

Low alloy 300M exhibits a typical martensitic matrix containing significant amounts of retained austenite and coarse carbide precipitates. The matrix is comprised of highly dislocated martensite within an average prior austenite grain size of $20 \pm 2 \mu \mathrm{m}$ for conventionally heat treated 300M [72]. Individual laths $(0.3 \mu \mathrm{m}$ wide $)$ are often separated by a twin boundary with a $\{112\}$ boundary plane [72]. Multiple studies show 300M tempered in the range of $280-315^{\circ} \mathrm{C}$ exhibits

\footnotetext{
6 Silicon content of CrNiMoWV was unexpectedly high and above the characterization limit of GDMS.
} 
discontinuous thin film retained austenite (10-20 nm thick, 2-5 vol\%) present at martensite lath and prior austenite interfaces [63,72], but precipitated austenite is not expected due to the low tempering temperature. Carbide precipitation is dominated by large ( $\sim 1 \mathrm{x} 0.1 \mu \mathrm{m})$ incoherent cementite present along all microstructural interfaces and inside of martensite laths [71]. Large irregular regions of auto-tempered martensite (1-10 $\mu \mathrm{m}$ in diameter) along prior austenite grain boundaries were observed in a wide range of heat treatment conditions[72]. Such regions often exhibited extensive internal and boundary carbide precipitation.

\subsubsection{AerMet 100}

Secondary hardening AerMet 100 has a lath martensite matrix with a nano-scale dispersion of strengthening carbides and a small amount of precipitated austenite. Conventionally heat treated AerMet 100 has an average prior austenite grain size of $\sim 11 \mu \mathrm{m}$ [73], but no retained austenite due to cryogenic processing. Lath width measured from limited TEM images is $0.1-0.25 \mu \mathrm{m}$, indicating a relatively fine scale structure compared to 300M [24]. Strengthening of the lath martensite matrix is primarily accomplished by a dispersion of needle shaped $\mathrm{M}_{2} \mathrm{C}(\mathrm{M}=\mathrm{Fe}, \mathrm{Mo}, \mathrm{Cr})$ carbides typically $10 \mathrm{~nm}$ long and $5 \mathrm{~nm}$ in diameter [24,74]. Cementite precipitation is absent and lath interfaces are covered in a continuous thin film of precipitated austenite $3 \mathrm{~nm}$ thick with a small total volume fraction ( 2 vol\%) [24,74]; each of these features is traced to the relatively high aging temperature. The methodology for determining the small-total austenite content in UHSS is controversial and discussed in Chapter 3.

\subsubsection{Ferrium M54}

TEM characterization by Wang et al. indicates that the general microstructure of Ferrium M54 is near-identical to AerMet 100. The matrix is comprised of lath martensite with an average width of $0.1-0.25 \mu \mathrm{m}[75]$. Lath boundaries are covered in a continuous thin film of precipitated austenite 5-10 $\mathrm{nm}$ thick and devoid of extensive cementite precipitation [75]. No information on prior austenite grain size is available. Strengthening carbides are coherent, needle shaped, and reduced in size $(10 \times 2 \mathrm{~nm})$ relative to those in AerMet 100 [24,75]. In addition to $\mathrm{M}_{2} \mathrm{C}$ (perhaps with reduced Cr content), these carbides should include WC, VC and perhaps other crystal structures tied to the presence of $\mathrm{W}$ and $\mathrm{V}$, not included in AerMet 100 or $300 \mathrm{M}$. 


\subsubsection{CrNiMoWV}

No published microstructural data exist for CrNiMoWV. A tempered martensite microstructure, similar to $300 \mathrm{M}$ is expected based on composition and heat treatment schedule. Two notable microstructural differences are expected compared to AerMet 100: (1) extensive precipitation of nano-scale coherent $(\mathrm{W}, \mathrm{V}) \mathrm{C}+\mathrm{M}_{2} \mathrm{C}$ (needle shaped) strengthening carbide dispersions, and (2) removal of thin film austenite through a triple temper plus cryogenic quench heat treatment (Table 1) [69].

\subsubsection{Ferrium S53}

Literature microstructure characterization of Ferrium S53 is limited. This corrosion resistant steel is designed to utilize the $\mathrm{M}_{2} \mathrm{C}$ carbide as the primary strengthening precipitate in a tempered-lath martensite matrix [44,45,76]. As such, a basic martensitic microstructure similar to AerMet 100 or Ferrium M54 is expected. Elevated chromium in Ferrium S53, relative to AerMet 100, should preferentially replace $\mathrm{Mo}$ in $\mathrm{M}_{2} \mathrm{C}$ carbides, possibly reducing precipitate size and coherency [70,77]. Small amounts of retained austenite are expected, even after cryogenic treatment due to depressed martensite finish temperature due to $\mathrm{Cr}[64,65]$. Austenite content may be elevated compared to other UHSS, sourced at austenite precipitation during tempering, which is promoted by high chromium concentration in Ferrium S53 [74,78]. However, this hypothesis is speculative and the presence of interlath precipitated austenite has not been investigated in Ferrium S53.

\subsubsection{Custom 465}

Microstructure data for the ultra-high strength $\mathrm{H} 900\left(482^{\circ} \mathrm{C}, 4 \mathrm{~h}\right)$ aging condition of Custom 465 is not available in published literature. Three sources of microstructure data are presented for Custom 465: (1) literature publications on Custom 465 with a different thermo-mechanical processing route, (2) detailed TEM of precipitation reactions in similar maraging steels, and (3) limited Thermo-Calc predictions of equilibrium phase composition. The matrix of as-quenched Custom 465 is primarily lath martensite with no major element grain boundary segregation or precipitation [79]. Martensite lath width for a cold worked and aged $\left(482^{\circ} \mathrm{C}\right)$ specimen is $\sim 300$ $900 \mathrm{~nm}$ [79]. Strengthening of the matrix is accomplished by intermetallic $\mathrm{Ni}_{3} \mathrm{Ti}$ precipitates rather than $\mathrm{M}_{2} \mathrm{C}$ carbides. The precipitation sequence, composition, and morphology of intermetallic 
particles in precipitation strengthened stainless steels is controversial and strongly dependent on composition [80]. As reported, but not demonstrated, by Grujicic et al. intermetallic precipitation in Custom 465 at short aging times is dominated by needle shaped coherent hexagonal $\omega$-phase which gradually transforms into equilibrium orthorhombic $\eta$-phase $\mathrm{Ni}_{3}(\mathrm{Ti}, \mathrm{Mo})$ [81,82]. However, in a Co-free maraging steel $(18.5 \mathrm{Ni}-3.0 \mathrm{Mo}-1.5 \mathrm{Ti}$ by wt $\%)$, $\eta$-phase nucleates within intralath islands of reverted austenite [83]. Recent work by Schober et al. demonstrates that the dominant precipitates in a stainless maraging steel $(13.0 \mathrm{Cr}-8.3 \mathrm{Ni}-1.0 \mathrm{Si}-1.0 \mathrm{Ti}-1.1 \mathrm{Al}-0.5 \mathrm{Mo}-0.1$ Mn by wt $\%$ ) are rod shaped $\eta$-phase $\mathrm{Ni}_{3}(\mathrm{Ti}, \mathrm{Mo})$ and spherical G-phase $\left(\mathrm{Ni}_{16} \mathrm{Si}_{7} \mathrm{Ti}_{6}\right)$. The formation

of the latter is precluded due to elevated silicon not present in Custom 465 [84]. Nanometer-scale characterization of precipitates in Custom 465 is beyond the scope of the present study. However, there is a general consensus that $\mathrm{Ni}_{3} \mathrm{Ti}$ is the equilibrium precipitate in similar maraging steels and in the absence of significant silicon, aluminum, and cobalt [78,85-87]. Molybdenum may be incorporated into the $\eta$-phase at sufficiently high aging temperatures or times [83].

Thermo-Calc predictions of phase composition in Custom 465 show that a single equilibrium precipitate exists across a wide range of aging temperatures [43]. When aged at $482^{\circ} \mathrm{C} \mathrm{Custom} 465$ is expected to have an equilibrium $\eta$-phase $\left(\mathrm{Ni}_{3} \mathrm{Ti}\right)$ concentration of $8.5 \mathrm{vol} \%$. A small amount of $\mathrm{Fe}$ and $\mathrm{Mo}$ are predicted to substitute for $\mathrm{Ni}$ and $\mathrm{Ti}$, respectively [43]. Based on atom probe measurements by Schober et al. [84], these precipitates are expected to be rod shaped, but literature does not support assumptions of their dimensions or coherency; both are heavily dependent on time-temperature heat treatment conditions.

\subsubsection{Ferrium PH48S}

In the simplest sense, Ferrium PH48S is Custom 465 with Co, Mo, and Al additions. While no microstructure data for Ferrium PH48S exist in the literature, three key microstructural changes are suggested by the governing patent [42]: (1) decreased prior austenite grain and martensite feature size, (2) reduced precipitate size and, (3) increased passivity of the surface oxide film. Ferrium PH48S is homogenized as single phase austenite and grain boundary pinning MC-type carbides are precipitated during subsequent hot working, yielding a refined prior austenite grain size. Martensite packet and block size is expected to be reduced proportionately [88]. Further, cobalt additions to secondary hardening high strength steels retard dislocation recovery during 
tempering [25,27]. As such, the lath martensite matrix may have increased dislocation density and reduced martensite lath size when compared to identically tempered, Co-poor UHSS alloys.

Thermo-Calc predictions of conventionally tempered Ferrium PH48S indicate precipitation of both $\eta$-phase, $\mathrm{Ni}_{3} \mathrm{Ti}_{0.8} \mathrm{Al}_{0.2}(3.0 \mathrm{vol} \%)$ and $\mathrm{B} 2 \mathrm{NiAl}(0.6 \mathrm{vol} \%)$ [43]. In both strengthening precipitates a small amount $(<0.2 \mathrm{wt} \%)$ of $\mathrm{Fe}$ and $\mathrm{Co}$ will substitute for $\mathrm{Ni}$. As aging temperature is decreased from the conventional $520^{\circ} \mathrm{C}$ to $460^{\circ} \mathrm{C}$ the volume fractions of $\eta$-phase and $\mathrm{NiAl}$ each increase, reaching 4.0 and $1.0 \mathrm{vol} \%$ respectively at $460^{\circ} \mathrm{C}$. No significant change is predicted in precipitate composition. Limited 3-dimensional atom probe measurements show that three precipitates are present in the PH48S martensitic matrix: (1) Rod shaped $\eta$-phase with a composition of $\left(\mathrm{Ni}_{0.9} \mathrm{Co}_{0.1}\right)_{3}\left(\mathrm{Ti}_{0.81} \mathrm{Al}_{0.19}\right)$, (2) spherical B2 $\left(\mathrm{Ni}_{0.75} \mathrm{Fe}_{0.16} \mathrm{Co}_{0.09}\right)\left(\mathrm{Ti}_{36} \mathrm{Al}_{64}\right)$, and (3) BCC Cr-rich clusters $\left(\mathrm{Cr}_{.57} \mathrm{Fe}_{0.34} \mathrm{Ni}_{0.05} \mathrm{Mo}_{0.03} \mathrm{Co}_{0.02}\right)$ [43]. When compared to Thermo-Calc predictions, atom probe measurements indicate that the equilibrium phase distribution is not reached, even at the highest tempering temperature. When compared to Custom 465, Ferrium PH48S has a lesser amount of strengthening precipitates ( $\eta$ and B2 NiAl).

\subsection{EBSD Measurement of Microstructure}

\subsubsection{Sample Preparation}

Specimens were mechanically polished through 1200 grit SiC papers and glycerol polycrystalline diamond suspensions $(3,1,0.5,0.25,0.1 \mu \mathrm{m})$. Final vibratory polishing with $0.05 \mu \mathrm{m}$ alumina suspension in propylene glycol reduced surface deformation and improved EBSD pattern quality [89]. Grain boundary/martensite interface misorientation and morphology information was collected in an FEI Quanta 650 SEM operating at $20 \mathrm{kV}$. Orientation imaging maps (OIM) were acquired over a representative area for each alloy using a constant pixel step size selected to ensure adequate pixels inside of the features of interest. Non-indexed zero solutions were nearly exclusively located at heavily dislocated martensite interfaces. Standard image reconstruction techniques were employed to fill the zero solutions with the dominant adjacent orientation [90,91]. 


\subsubsection{Characterization of Grain Boundary Character}

Quantitative stereological methods were applied to EBSD maps to yield the surface area to volume ratio $\left(S_{\mathrm{v}}\right)$ of each-identified martensite interface misorientation type [92]. The distribution of low energy coincident site lattice (CSL) boundaries, which have a specific plane-angle misorientation relationship, was measured. Boundaries are referred to as 'random' if they do not hold a CSL orientation relationship, as they may have a wide range of misorientations. Four boundary types were analyzed: randomly oriented low-misorientation angle boundaries (2-15 $)$, randomly oriented high angle boundaries $\left(>15^{\circ}\right), \sum 3^{7}$ symmetrical twin boundaries, and all other CSL boundaries. Boundaries were classified as 'special' CSL boundaries via Brandon's Criterion $\left(\Delta \theta=15 / \sum^{0.5}\right)$ for sufficiently low $\sum\left(1<\sum<29\right)[93,94]$.

\subsubsection{Fractal Analysis of Random Boundary Connectivity}

The connectivity of random high angle boundaries was measured using the box-counting-method to determine the fractal dimension $\left(\mathrm{D}_{\mathrm{R}}\right)$ of the maximum random boundary connectivity (MRBC) $[49,95]$. The MRBC is the longest set of contiguous random boundaries present in a 2D map of random boundary network $(\mathrm{RBN})$ and classically represents an interconnected-weak crack path through the microstructure. For clarity, an RBN, with highlighted MRBC and sampling grid is shown in Figure 1. An outline of all random high angle boundaries was obtained from OIM maps. The MRBC was determined using an algorithm that finds the longest connected set of black pixels. Successive square grids of size $\eta(\eta=50,25,12.5,5 \mu \mathrm{m})$ are scribed over the MRBC map, and the number of grid squares $(\mathrm{N})$ containing a portion of the MRBC was counted. The fractal dimension of the MRBC $\left(D_{R}\right)$, a proxy for connectivity, is defined as [49]:

$$
D_{R}=\frac{\log [N(\eta)]}{\log [\eta]}
$$

\footnotetext{
7 CSL boundaries are described by the reciprocal density of common lattice points of the two crystal orientations sharing a boundary plane with a given misorientation. For an ideal bi-crystal, misorientation would be $0^{\circ}$ and $\sum=1$; all sites in one crystal are present in the second. Increasing $\sum$ corresponds to rising disorder at a grain boundary. Higher $\sum$ are typically associated with higher grain boundary energy, lower excess free volume, and lower resistance to intergranular corrosion $[93,94]$
} 
In principle as the connectivity of random boundaries in a microstructure decreases, the measured $D_{R}$ will decrease. Falling connectivity in polycrystalline metals is attributed to an increasing fraction of CSL or low angle boundaries which disrupt the interconnected-weak RBN crack path. Critically, this method is commonly applied to relatively simplistic microstructures such as polycrystalline Ni or Monel K-500 [96,97]. Within coarse polycrystalline microstructures, the number of random boundary pathways through the microstructure is relatively small compared to fine scale martensitic structures. Increased fraction of embrittlement-resistant CSL boundaries are therefore more likely to significantly disrupt the network and reduce $D_{R}$ in a coarse grain structure as opposed to fine microstructures.

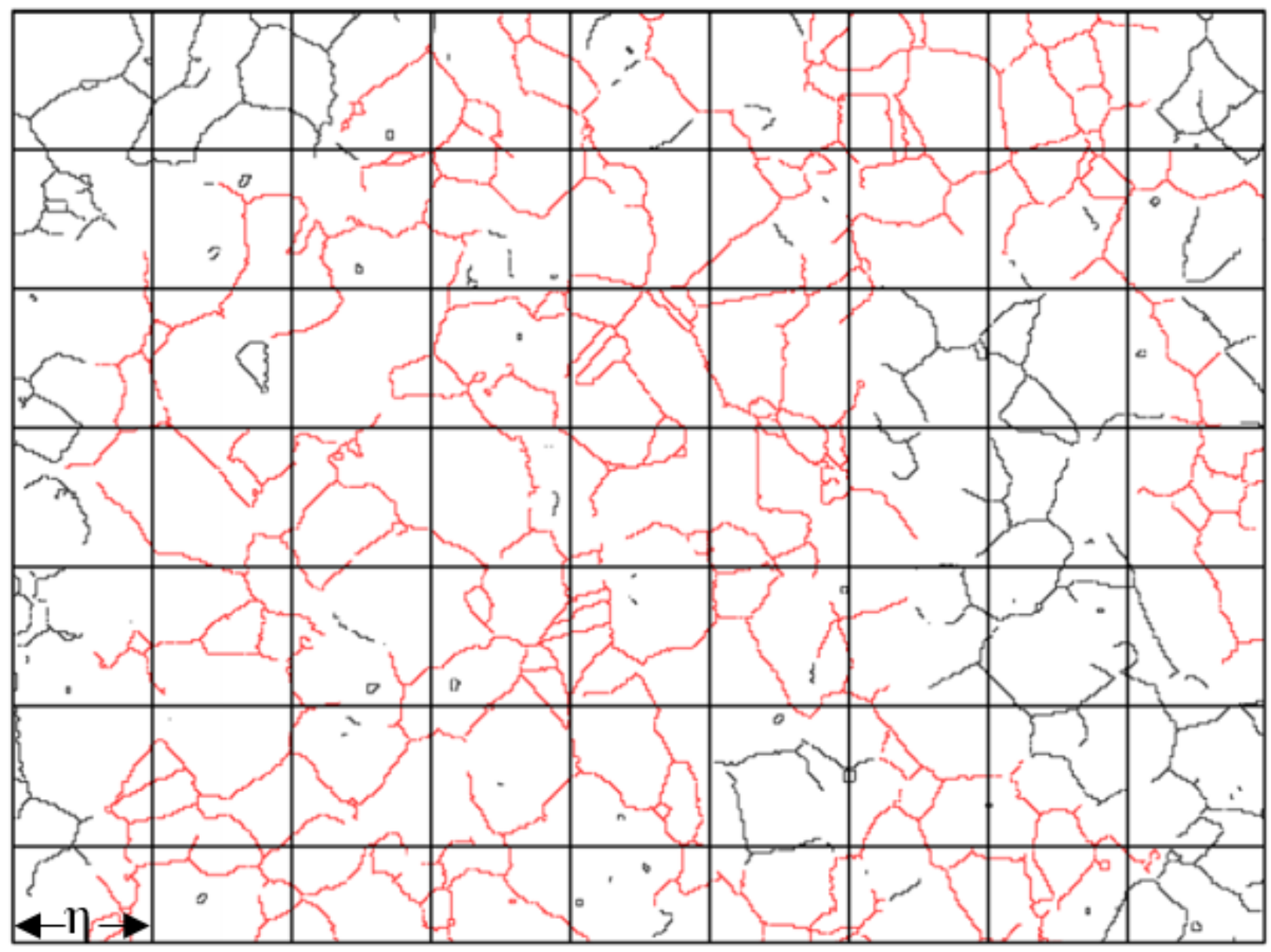

Figure 1 Generic example of an RBN (black + red) with a highlighted MRBC (red). A $25 \mu \mathrm{m}$ grid $(\eta=25 \mu \mathrm{m})$ is overlaid for scale and fractal analysis of MRBC connectivity. 


\subsection{Characterization of HEAC Kinetics}

\subsubsection{SENT Specimen Design and Precracking}

Heat treated blanks were machined to the single edge notch tensile (SENT) geometry with a flat gage section $35.6 \mathrm{~mm}$ long, $10.7 \mathrm{~mm}$ (W) wide and 2.54-mm (B) thick. Electrical discharge machining (EDM) was employed to cut the through-thickness edge notch at the center of the gage section to a depth of $200 \pm 25 \mu \mathrm{m}$, with a mouth opening of $230 \pm 30 \mu \mathrm{m}$. All steel microstructures were assumed to be isotropic and crack orientation varied among the product forms. SENT specimens were fatigue precracked using a $\mathrm{K}_{\mathrm{MAX}}$ gradient protocol ( $\mathrm{K}_{\mathrm{MAX}}$ of 18-15 MPa $\sqrt{\mathrm{m}}$ shed to $10-9 \mathrm{MPa} \sqrt{\mathrm{m}}, \mathrm{R}=0.1, \mathrm{f}=5-20 \mathrm{~Hz}$ ) in lab air to a total crack plus notch depth of 0.5 to $1.0 \mathrm{~mm}$. This protocol uniformly initiated a fatigue crack front, with minimal through-thickness depth difference, and minimized final crack tip plastic zone size.

\subsubsection{Electrochemical Control and Loading}

A precracked specimen was contained in an o-ring sealed $400 \mathrm{~mL}$ cylindrical acrylic cell filled with circulating $(20 \mathrm{ml} /$ minute), non-deaerated $0.6 \mathrm{M} \mathrm{NaCl}$ solution. The gage section of the specimen was fully immersed in solution for the duration of the experiment, and gripped outside of the cell to preclude galvanic coupling. Open circuit potential (OCP) for each steel, after 1 hour of immersion in $0.6 \mathrm{M} \mathrm{NaCl}$ solution, is shown in Table 5. Four aging conditions of Ferrium PH48S (H860, H900, H950, and H968) exhibited identical (-225 mV SCE) OCP.

\begin{tabular}{|c|c|c|c|c|c|c|c|}
\hline & $300 \mathrm{M}$ & $\begin{array}{c}\text { AerMet } \\
100\end{array}$ & $\begin{array}{c}\text { Ferrium } \\
\text { M54 }\end{array}$ & CrNiMoWV & $\begin{array}{c}\text { Ferrium } \\
\text { S53 }\end{array}$ & $\begin{array}{c}\text { Custom } \\
465\end{array}$ & $\begin{array}{c}\text { Ferrium } \\
\text { PH48S }\end{array}$ \\
\hline & UHSS & \multicolumn{2}{|c|}{ Secondary Hardening UHSS } & \multicolumn{2}{|c|}{ Secondary Hardening UHSSS } \\
\hline $\begin{array}{c}\text { OCP } \\
\left(\mathrm{mV}_{\text {sce }}\right)\end{array}$ & -650 & -525 & -510 & -560 & -370 & -225 & -225 \\
\hline
\end{tabular}

Table 5 Measured OCP of UHSS and UHSSS with Respect to the Saturated Calomel Electrode 
Crack growth experiments were conducted at constant applied potential ( $\left.\mathrm{E}_{\text {app }}\right)$ ranging from -150 to $-1,100 \mathrm{mV}_{\text {sce. }}$. The grounded SENT specimen was the working electrode, while a floating potentiostat controlled $\mathrm{E}_{\mathrm{app}}$. Solution $\mathrm{pH}$ was periodically measured and did not significantly vary $(\mathrm{pH}=6.5 \pm 0.5)$ during a cracking experiment. Pinned-clevis gripping ensured the validity of the freely rotating SENT elastic stress intensity solution [98]. The specimen was loaded in a servoelectric or hydraulic, feedback controlled, tensile machine operating in grip displacement control. A constant-applied displacement rate of 0.0003 to $0.0008 \mathrm{~mm} / \mathrm{min}$ yielded elastic stress intensity rates $(\mathrm{dK} / \mathrm{dt}$ ) of $1.0-1.5 \mathrm{MPa} \sqrt{\mathrm{m}} / \mathrm{h}$ until the onset of crack growth; where after, $\mathrm{dK} / \mathrm{dt}$ increased with crack growth.

\subsubsection{Crack Length Measurement}

The direct current potential difference (dcPD) method was employed to measure crack length in situ [99-101]. Alumel or platinum wires were spot welded $500 \pm 200 \mu \mathrm{m}$ above and below the notch centerline, across the specimen thickness, prior to fatigue precracking. A constant current of 4.000 A was applied through the grips. Voltage measurements from the dcPD probes were amplified through a DC amplifier $\left(10^{3}-10^{5}\right.$ times) and converted to a crack length through Johnson's equation [100]. Initial dcPD voltage (following $2 \mathrm{~h}$ of stabilization first in air then in solution at $\mathrm{K}=2 \mathrm{MPa} \sqrt{\mathrm{m}}$ ) was referenced to the measured fatigue crack depth. $60 \mathrm{~Hz}$ AC voltage noise was eliminated through block averaging; 56 voltage readings are taken in $1 / 60^{\text {th }}$ of a second [101]. Thermal voltage contributions were eliminated through current reversals, where 1,120 (20 x 56 reading blocks) active potentials were measured at $+4.000 \mathrm{~A}$, then current polarity was changed and 1,120 potentials were measured after a $0.5 \mathrm{~s}$ delay for amplifier response. Absolute dcPD values at each polarity were averaged to yield a single dcPD value without thermal contribution. Crack length was determined from an average of 10 such voltages for a total measurement time of $8.6 \mathrm{~s}$. A secondary set of Alumel or Pt wires was spot welded to the specimen $15 \pm 0.5 \mathrm{~mm}$ from the notch centerline [101]. Due to their position, voltage readings taken from the reference probes experience identical changes to the active probe pair due to temperature, net section elasticity, and input current, with limited influence of crack tip plasticity. The measured active voltage was linearly corrected for the change in the reference probe voltage [101]. Specimen load, actuator displacement, crack length, dcPD voltage, and applied current were recorded at 30$120 \mathrm{~s}$ intervals until SENT failure. Crack growth rate was calculated at a given time, $t$, (thus K) 
through the slope of a second order polynomial fit to sets of successive $a$ versus $t$ over $t \pm 3$ data points, as described for fatigue crack propagation rate determination [102].

\subsubsection{HEAC Test Analysis}

Crack growth kinetics obtained from subcritical crack growth experiments must be further analyzed to determine $\mathrm{K}_{\mathrm{TH}}$ and Stage II da/dt. In the ideal case, dcPD measurement of da/dt is nil until crack growth initiates at $\mathrm{K}_{\mathrm{TH}}$ and rapidly transitions to Stage II growth through a steeply rising Stage I. An example of this behavior is shown in Figure 2. $\mathrm{K}_{\mathrm{TH}}$ is delineated as the stress intensity where $\mathrm{da} / \mathrm{dt}$ rapidly rises, typically several orders of magnitude. The $\mathrm{da} / \mathrm{dt}_{\mathrm{II}}$ is defined as rate limited, $\mathrm{K}$-independent da/dt for all $\mathrm{K}$ larger than $\mathrm{K}_{\mathrm{TH}}$, less a small range of transition values.

Measured SCC-HEAC kinetics in UHSS do not typically display this simplified behavior. Four significant deviations from idealized behavior are typical of UHSS HEAC kinetics: (1) All specimens display rising dcPD in the absence of physical crack extension as $\mathrm{K}$ rises from a very low level, (2) Stage I crack growth exhibits weaker K-dependence, making $\mathrm{K}_{\mathrm{TH}}$ determination difficult, (3) Stage II da/dt is often only constant over a small K interval and/or rises mildly with increasing $\mathrm{K}$, and (4) specimens tested at heavily cathodic $\mathrm{E}_{\text {app }}$ exhibit elevated-falling da/dt as $\mathrm{K}$ rises at very low stress intensities $(\mathrm{K}<5 \mathrm{MPa} \sqrt{\mathrm{m}})$. In the following sub-sections, each issue will be addressed for SCC-HEAC kinetics typical of UHSS alloys. 


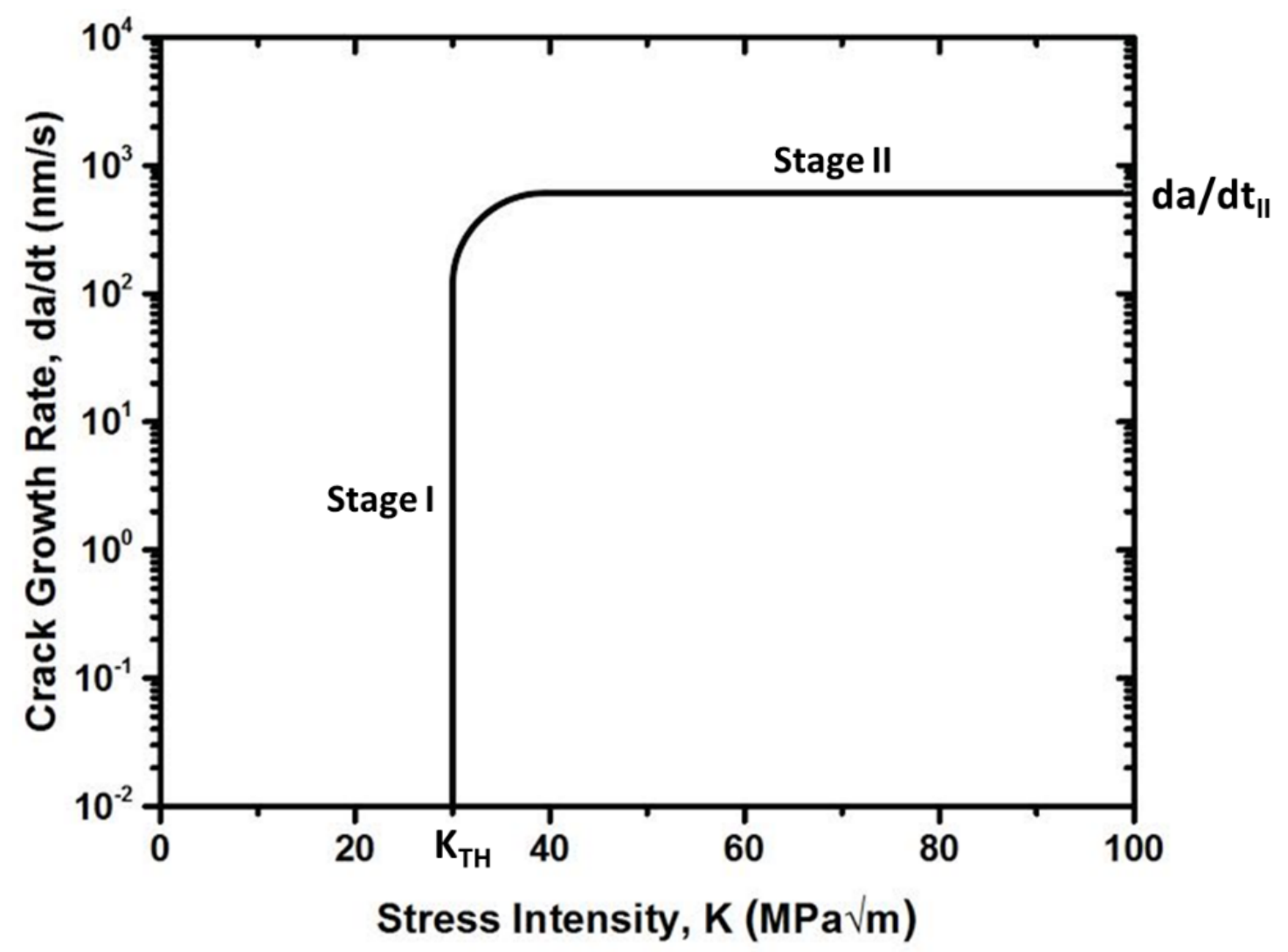

Figure 2 Idealized crack growth kinetics delineating SCC-HEAC parameters (KTH, da/dt $\left.{ }_{I I}\right)$ which are characteristic of UHSS behavior.

\subsubsection{Resolution Limit of the dcPD Method}

As shown in Figure 3, typical SCC-HEAC kinetics exhibit rising-false da/dt at stress intensities well below $\mathrm{K}_{\mathrm{TH}}$. Specimens tested in lab air show similar false da/dt rise at stress intensities well below $\mathrm{K}_{\mathrm{IC}}$. False-rising dcPD voltage is attributed to increasing crack tip plasticity with rising K; deformation both increases metal resistivity and causes physical crack extension due to geometric blunting $[99,103]$. The expected contribution of geometric crack extension to total false da/dt rise is small. At $\mathrm{K}=100 \mathrm{MPa} \sqrt{\mathrm{m}}, 18-22 \mu \mathrm{m}$ of crack extension is expected from crack tip blunting for the steels presented in Table 1 [104]. At a constant K-rate of $1 \mathrm{MPa} \sqrt{\mathrm{m}} / \mathrm{h}$ this would yield an average crack extension rate of $0.05-0.06 \mathrm{~nm} / \mathrm{s}$. Typical false da/dt (Figure 3) is significantly 
faster than this predicted average rate, indicating that crack tip resistivity increase may be the dominant contribution.

Compared to typical HEAC kinetics, false da/dt rise is small and only significant in the case of HEAC-resistant alloys that exhibit small $d a / d t$ at high K. For this set of alloys a plasticity based resolution limit was determined to help accurately measure $\mathrm{K}_{\mathrm{TH}}$. The basis for an operationally defined resolution limit for dcPD detection of subcritical crack propagation is shown in Figure 4. Crack growth rate for Custom 465-H900 and Ferrium PH48S SENT specimens, calculated from dcPD voltage, rises steadily with increasing K for situations where SCC crack growth did not occur as confirmed via SEM fractography. Average da/dt rise was calculated by least squares regression of all false da/dt versus $\mathrm{K}$ data. Maximum expected false da/dt is the upper bound of a $95 \%$ confidence interval constructed around the regression line. The efficacy of the resolution limit in capturing false da/dt rise is readily observed in the SCC-resistant alloy presented in Figure 5.

While the magnitude of false-da/dt is highly variable, the slope of the rise is largely consistent across alloys, and the constructed resolution limit effectively captures the slope of this rise. In isolated instances, an alloy exhibited false-da/dt consistently above the resolution limit with an identical slope. In these cases, the resolution limit is shifted upwards for $\mathrm{K}_{\mathrm{TH}}$ determination. 


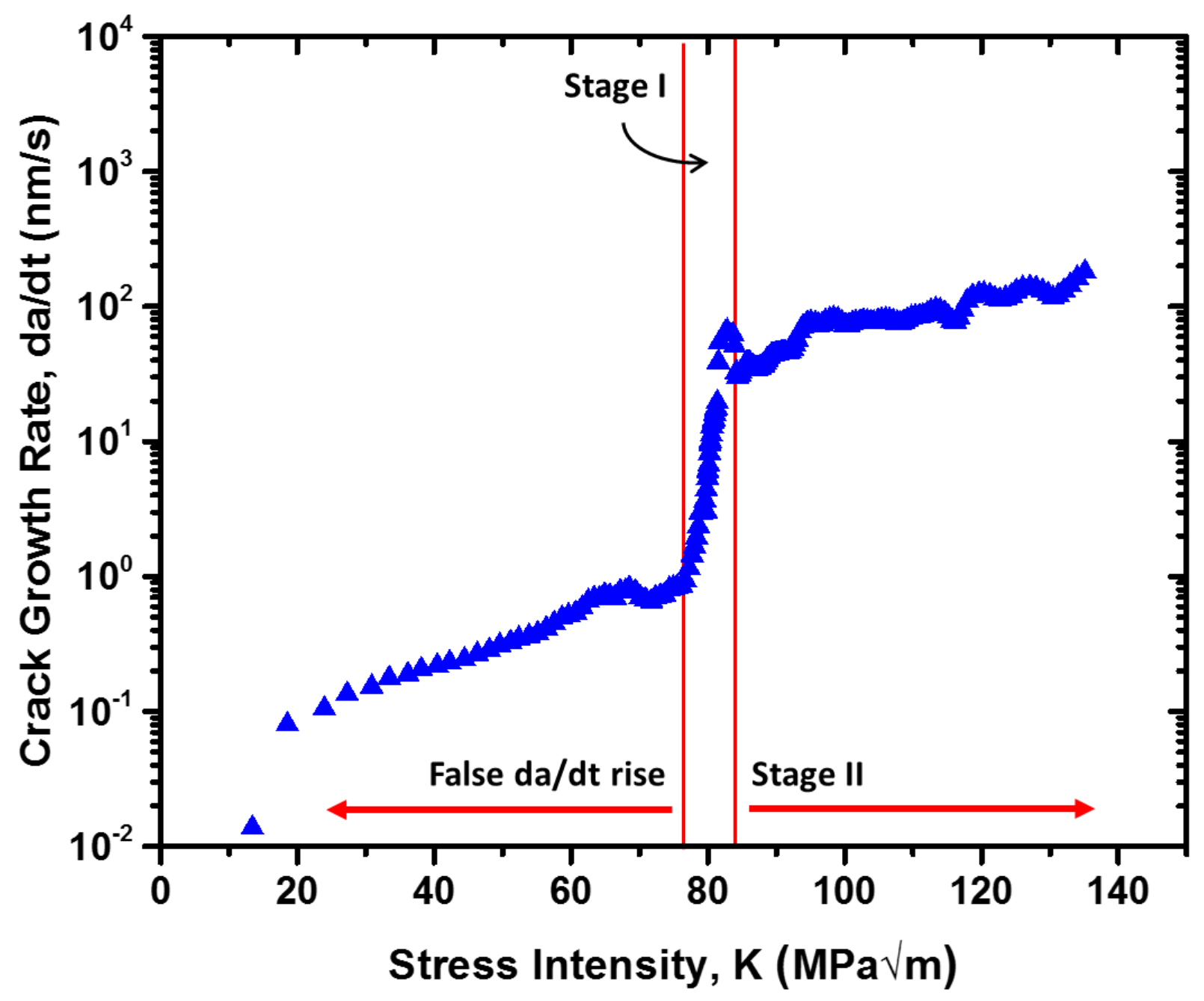

Figure 3 Typical crack growth kinetics of Ferrium S53 stressed under slow rising displacement (Initial $d \mathrm{~K} / \mathrm{dt}=1 \mathrm{MPa} \sqrt{\mathrm{m} / \mathrm{h}}$ ) in $3.5 \% \mathrm{NaCl}$, exhibiting strong $\mathrm{SCC}$-HEAC resistance and deviation from the ideal form of HEAC kinetics. 


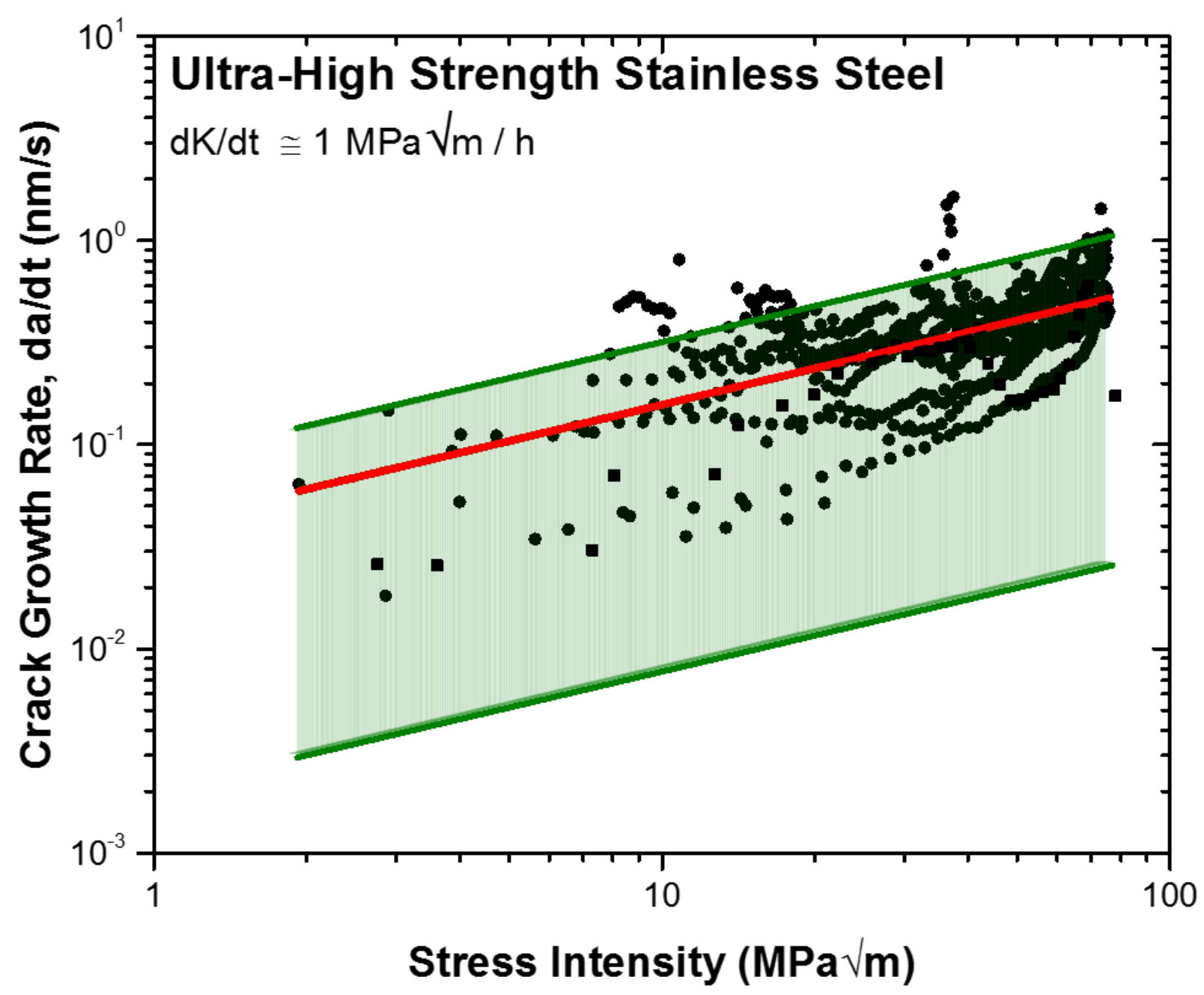

Figure 4 Construction of da/dt resolution limit for full immersion of UHSS alloys. Regression of false da/dt rise is shown as the central line (red); with the bounds of a 95\% CI around the regression shown above and below (green). The upper bound of this confidence interval is employed as the resolution limit in all further analyses. 


\subsubsection{Anomalous da/dt at Low $K$}

In addition to false crack growth from plasticity, the aqueous environment for HEAC testing provides another mechanism which obscures da/dt resolution and $\mathrm{K}_{\mathrm{TH}}$ determination. Specimens tested at $\mathrm{E}_{\text {app }}$ levels heavily cathodic or anodic to OCP often exhibited significantly elevated da/dt $(\sim 10 \mathrm{~nm} / \mathrm{s})$ at very low $\mathrm{K}(\mathrm{K}<5 \mathrm{MPa} \sqrt{\mathrm{m}})$ where HEAC was highly unlikely, and which declined rapidly with increasing $\mathrm{K}$. Falling da/dt reached a minimum $(\mathrm{K} \sim 5 \mathrm{MPa} \sqrt{\mathrm{m}})$ and increased with rising $\mathrm{K}$ to resume the false da/dt rise associated with crack tip plasticity effects (Figure 4). An example of this behavior is shown by the shifted-susceptible case in Figure $5(\bullet)$. Beyond the minima, Stage I / Stage II kinetics typical of HEAC are observed. Falling da/dt at low K has been observed for a Ni-based superalloy, Monel K-500, stressed under similar mechanical and electrochemical conditions [3]. Falling-elevated da/dt at low $\mathrm{K}$ was attributed to crack surface electrical contact, which decreases with rising $\mathrm{K}$ and crack opening displacement [3]. Electrical contact of the crack surfaces is enabled by the reduction of an insulating surface oxide film at cathodic or anodic $\mathrm{E}_{\mathrm{app}}$, relative to OCP. Falling-elevated da/dt in the present study is false and attributed to an identical crack surface electrical contact mechanism. As such, initial da/dt $(\mathrm{K}<8$ $\mathrm{MPa} \sqrt{\mathrm{m}}$ ) is not associated with physical crack extension and is subtracted from measured da/dt prior to measurement of $\mathrm{K}_{\mathrm{TH}}$ or $\mathrm{da} / \mathrm{dt}_{\mathrm{II}}$.

\subsubsection{Determination of $K_{T H}$}

In the majority of cases, $\mathrm{K}_{\mathrm{TH}}$ is readily determined by da/dt rising rapidly over a small stress intensity range. For the case of an alloy susceptible to SCC-HEAC (Figure 5, 匹), kinetics are similar to the idealized case presented in Figure $2 . \mathrm{K}_{\mathrm{TH}}$ is determined by the onset of rapidly rising $\mathrm{da} / \mathrm{dt}(\mathrm{K}=10 \mathrm{MPa} \sqrt{\mathrm{m}})$. Since $\mathrm{K}_{\mathrm{TH}}$ is sufficiently low, plasticity based da/dt rise is not significant, and the operationally defined resolution limit provides confidence that rising da/dt represents SCC crack growth. Any small ambiguity in the precise value of $\mathrm{K}_{\mathrm{TH}}$ by this method is sufficiently low so as to not affect subsequent analyses. 


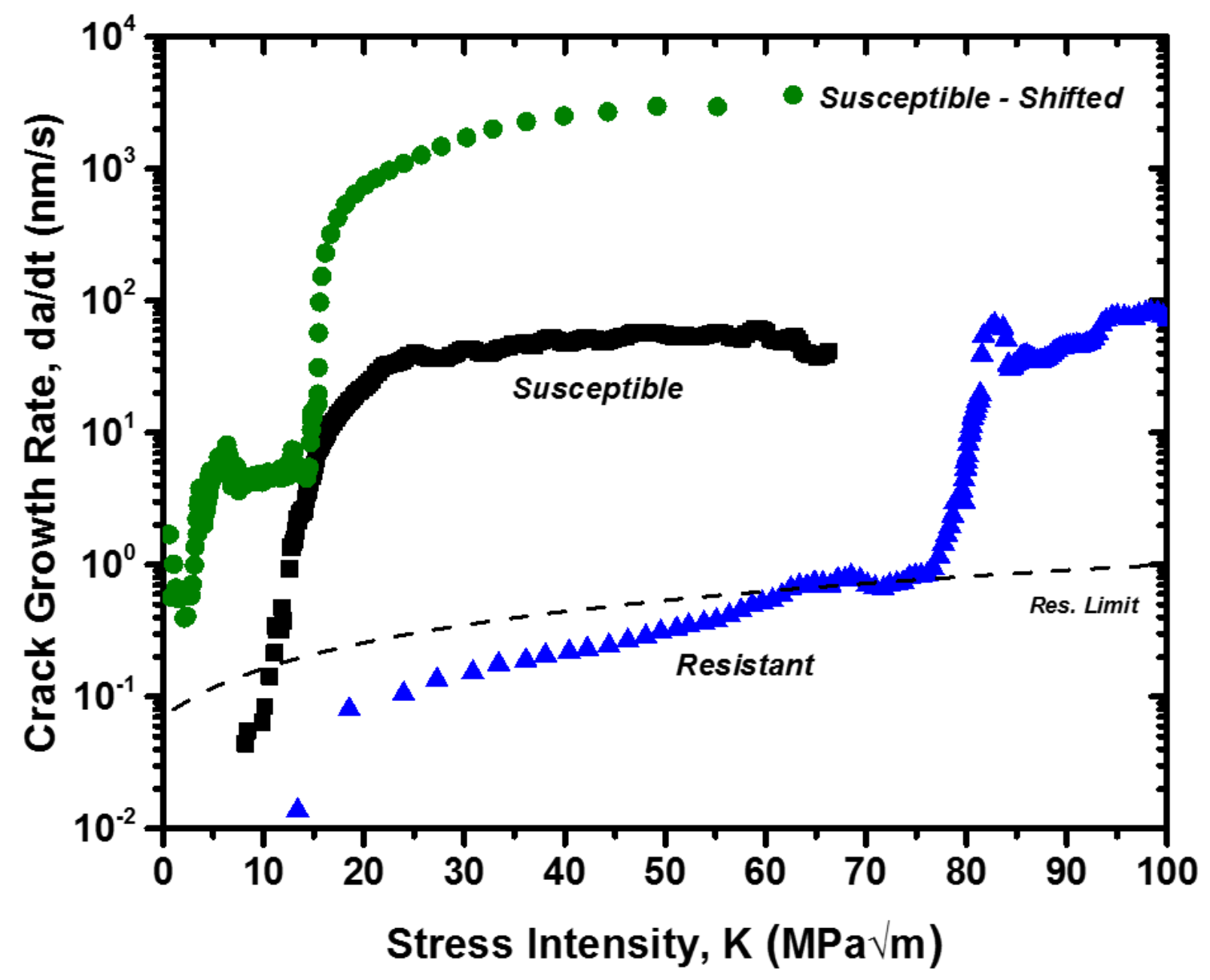

Figure 5 Variation in the form of typical HEAC kinetics for $300 M(\bullet)$, AerMet $100(\square)$, and Ferrium S53 ( $\triangle$ ) stressed under slow rising displacement in $3.5 \% \mathrm{NaCl}$ at $E_{\text {app }}=-550 \mathrm{~m} V_{S C E}$.

In SCC-resistant alloy-environment pairs (Figure 5, $\Delta$ ) $\mathrm{K}_{\mathrm{TH}}$ is determined by the first sustainedsignificant deviation above the resolution limit $(\mathrm{K}=79 \mathrm{MPa} \sqrt{\mathrm{m}})$. Isolated instances of da/dt above the resolution limit (Figure $5(\Delta) \mathrm{K}=60-70 \mathrm{MPa} \vee \mathrm{m}$ ) are typical and do not represent $\mathrm{K}_{\mathrm{TH}}$. Finally, a small minority of specimens exhibit a large shift of typical da/dt at low $\mathrm{K}$ (Figure $5(\bullet), \mathrm{K}=8$ $15 \mathrm{MPa} \sqrt{\mathrm{m}})$. In this region, the slope of da/dt vs. $\mathrm{K}$ is typical of the plasticity based resolution limit, but increased by 2 orders of magnitude. The precise origins of this shift are unknown, but attributed to an experimental rather than material variable. Near-ideal Stage I / Stage II kinetics are observed above $\mathrm{K}=15 \mathrm{MPa} \sqrt{\mathrm{m}}$ supporting the quality of the measurement in the face of anomalous low-K behavior. To determine $\mathrm{K}_{\mathrm{TH}}$, the resolution limit is shifted to capture the slow- 
rising da/dt $(\mathrm{K}=8-15 \mathrm{MPa} \sqrt{\mathrm{m}})$, and the first sustained-significant deviation is measured (15.4 $\mathrm{MPa} \sqrt{\mathrm{m}})$.

\subsubsection{Determination of $d a / d t_{I I}$}

As shown in Figure 5, the form of HEAC cracking kinetics is variable among alloys, even when stressed under identical conditions. Further, diffusion limited $\mathrm{da} / \mathrm{dt}_{\mathrm{II}}$ varies over several orders of magnitude and rises mildly with increasing K. Due to the breadth of the gathered SCC-HEAC kinetics, it is logical to define a single measure of $\mathrm{da} / \mathrm{dt}_{\text {II }}$ for comparing the HEAC resistance of similar alloys at constant $\mathrm{E}_{\mathrm{app}}$. For the present work, $\mathrm{da} / \mathrm{dt}_{\mathrm{II}}$ is taken at $\mathrm{K}=40 \mathrm{MPa} \sqrt{\mathrm{m}}$, comparing crack growth rates at a single mechanical driving force across all alloys.

While this methodology works extremely well for SCC-susceptible alloy-environment pairs $\left(\mathrm{K}_{\mathrm{TH}}\right.$ 10-40 MPa $\sqrt{\mathrm{m}})$, it does not effectively capture da/dt fI for SCC-resistant $\left(\mathrm{K}_{\mathrm{TH}}>40 \mathrm{MPa} \sqrt{\mathrm{m}}\right)$ conditions. In this case, $\mathrm{da} / \mathrm{dt}$ at $\mathrm{K}=40 \mathrm{MPa} \sqrt{\mathrm{m}}$ is essentially equal to the resolution limit, as no crack growth has occurred. Specimens exhibiting strong SCC resistance are typically limited to a small range of $E_{a p p}$ slightly cathodic to OCP and do not represent the majority of specimens. Moreover, many of these specimens have $\mathrm{K}_{\mathrm{TH}}$ sufficiently close to $\mathrm{K}_{\mathrm{IC}}$ that a contribution of stableductile crack growth to da/dt II cannot be ruled out. Despite the inherent inaccuracy of portraying $\mathrm{da} / \mathrm{dt}_{\mathrm{II}}$ in SCC-resistant alloys, the utility of easy comparisons between the wide-ranging SCCHEAC kinetics in the present research clearly justifies the use of a single definition of da/dt $\mathrm{II}$.

\subsection{Fracture Surface Analysis}

SENT specimen fracture surfaces were ultrasonically cleaned in acetone for 10 minutes and stored under vacuum. Fracture surfaces were examined in an FEI Quanta 650 SEM equipped with a field emission gun operating at 5-10 kV accelerating voltage and $10 \mathrm{~mm}$ working distance. Crack growth occurred from the bottom to top of each fractograph.

\subsection{Hydrogen Uptake / Outgassing Behavior of UHSSS}

Thin plate specimens of Custom 465 and Ferrium PH48S were heat treated according to Table 1 (H900 and H900/H950/H968, respectively) and ground to a constant thickness of $0.7 \mathrm{~mm}$. Specimens were fully immersed in non-deaerated 0.001M NaOH $(\mathrm{pH}=10)$ and hydrogen charged 
at a constant applied polarization $\left(\mathrm{E}_{\mathrm{app}}=-1350 \mathrm{mV}\right.$ SCE $)$. Electrochemical conditions for hydrogen charging were chosen for congruency with previous hydrogen solubility experiments conducted on Custom 465 [105]. Specimens were charged for 0, 25, 72, 168, 333, and $610 \mathrm{~h}$. Maximum charging time corresponds to $99 \%$ of the steady state $\mathrm{H}$ concentration, as calculated using an assumed hydrogen diffusivity of $10^{-9} \mathrm{~cm}^{2} / \mathrm{s}$ and a semi-infinite diffusion solution [106].

Following hydrogen charging, specimens were rinsed in deionized water and immediately stored in liquid nitrogen to prevent room temperature hydrogen outgassing. A limited number of fully charged $(610 \mathrm{~h})$ specimens were then allowed to outgas at room temperature for 144 or $888 \mathrm{~h}$. Total-dissolved hydrogen concentration for all specimens was measured using the carrier gas extraction method on a Bruker G4 Hydrogen gas analyzer. Chamber temperature was ramped using an infrared heating element to a maximum temperature of $550^{\circ} \mathrm{C}$. A hydrogen concentration versus charging-then-outgas time curve was constructed for each alloy, and $\mathrm{D}_{\mathrm{H}-\mathrm{EFF}}$ was fit to the data using a semi-infinite diffusion solution [106]. Comparison of these results will provide an estimate of the effect of steel composition/microstructure on reversibly trapped and very strongly trapped $\mathrm{H}$, which should inform modeling of metallurgical effects on HEAC.

\subsection{Thermal Desorption Spectroscopy (TDS) of H-Trap States in UHSSS}

Hydrogen uptake measurements were determined through thermal desorption spectroscopy (TDS). The TDS system was configured on an ultra-high vacuum (UHV) detection chamber (maintained at $\sim 13 \mu \mathrm{Pa}$ ) connected to a high vacuum load lock chamber which allows for sample insertion without disturbing the chamber pressure of the detection area. Sample heating is by a programmable resistive furnace. During analysis, while heating the sample, the mass to charge signals versus time of desorbed gasses were collected by a quadrupole mass spectrometer. The partial pressure of hydrogen was then compared to a time temperature profile collected simultaneously. Three different temperature ramp rates; $2.5,5$, and $10^{\circ} \mathrm{C} / \mathrm{min}$, were used for analysis over a range of 25 to $550^{\circ} \mathrm{C}$. All scans were corrected by the background partial pressure and the mass of each sample. 


\section{Measurement and Modeling of HEAC in Modern UHSS}

\subsection{Problem Statement}

The exceptionally high combination of yield strength and fracture toughness characteristic of modern, secondary hardening ultra-high strength steels make then ideal candidates for highly stressed structural components in aerospace or marine applications. Due to their extraordinarily high strength, martensitic UHSS are susceptible to SCC via a HEAC mechanism in environments which electrochemically produce hydrogen. The degradation of mechanical properties in components experiencing SCC is severe and safe component life could be substantially reduced. Critically, component life is currently based upon overly conservative go or no-go threshold stress intensity for crack growth, which does not account for slow-safe subcritical crack growth in modern SCC resistant UHSS. Significant advances have been made to the measurement and modeling of alloy-environment dependent SCC-HEAC kinetics; paving the way for a transition in SCC management to a damage tolerance approach. However, the present literature database of alloy-environment dependent SCC-HEAC kinetics is severely limited. Further the microstructure dependences of key variables in modern models of SCC-HEAC properties have not been sufficiently investigated. The overarching goal of this chapter is to quantitatively measure highresolution subcritical crack growth kinetics for a suite of modern UHSS, to further the fundamental understanding of the electrochemical and microstructural basis of key variables in micromechanical models of SCC-HEAC properties.

The goal of Chapter 3 is to develop the HEAC kinetics database and understanding of microstructure necessary to further develop UHSS with improved performance in SCC-HEAC situations, in the context of a modern damage tolerant approach $[14,62]$. Towards this goal, 4 primary objectives are identified:

1) Measure SCC-HEAC kinetics across a wide range of electrochemical environments for a systematically chosen set of UHSS.

2) Correlate HEAC fracture path to alloy dependent, fine scale martensite microstructure

3) Explain observed trends in SCC-HEAC resistance using microstructural, H-trapping, and fractographic data in the context of modern micromechanical models of HEAC properties 
4) Link micromechanical model parameters to specific microstructural or metallurgical variables to improve the efficacy of HEAC modeling in high strength alloys

The experimental approach taken to achieve these objectives is given in Chapter 2 .

\subsection{Results}

\subsubsection{EBSD Measured Microstructure}

Martensite microstructure for each alloy was characterized via EBSD as shown in Figures 6-10. Measured orientation imaging maps (OIM) utilize inverse pole figure coloring (IPF-Z $)^{8}$. This color assignment is identically used for all OIM maps in the present work. All steels exhibit a fully martensitic matrix with small amounts of precipitated austenite consistent with previous studies of 300M, AerMet 100, and CrNiMoWV [23,24,107].

Total EBSD-measured austenite for AerMet 100, CrNiMoWV, Ferrium S53, Ferrium M54, and $300 \mathrm{M}$ is $1.5,0.9,1.9,0.8$, and 1.6 volume percent, respectively. Isolated islands of austenite, 75 $250 \mathrm{~nm}$ in diameter, are predominantly located along martensite interfaces and triple points in AerMet 100 (Figure 11, red islands). This morphology of austenite is characteristic of precipitation during aging, rather than retention during the cryogenic quench, and is consistent among alloys. Thin films of precipitated austenite, 3-10 nm in thickness have been previously observed in tempered AerMet 100 and Ferrium M54 based on TEM [24,75]. Detection of such films in the present study is precluded by the comparatively large EBSD step size and interaction volume. The measurement of total austenite content in UHSS will be discussed in later sections using AerMet 100 as a model alloy.

8 In IPF-Z visualization, color is assigned to a pixel based on the crystal axis parallel to the normal of the polished plane, projected on the stereographic triangle. For example, in Figure 6 features with the $\langle 111\rangle$ direction parallel to the polished plane normal would be colored pure blue. This color assignment is identical for all OIM maps in the present work. 


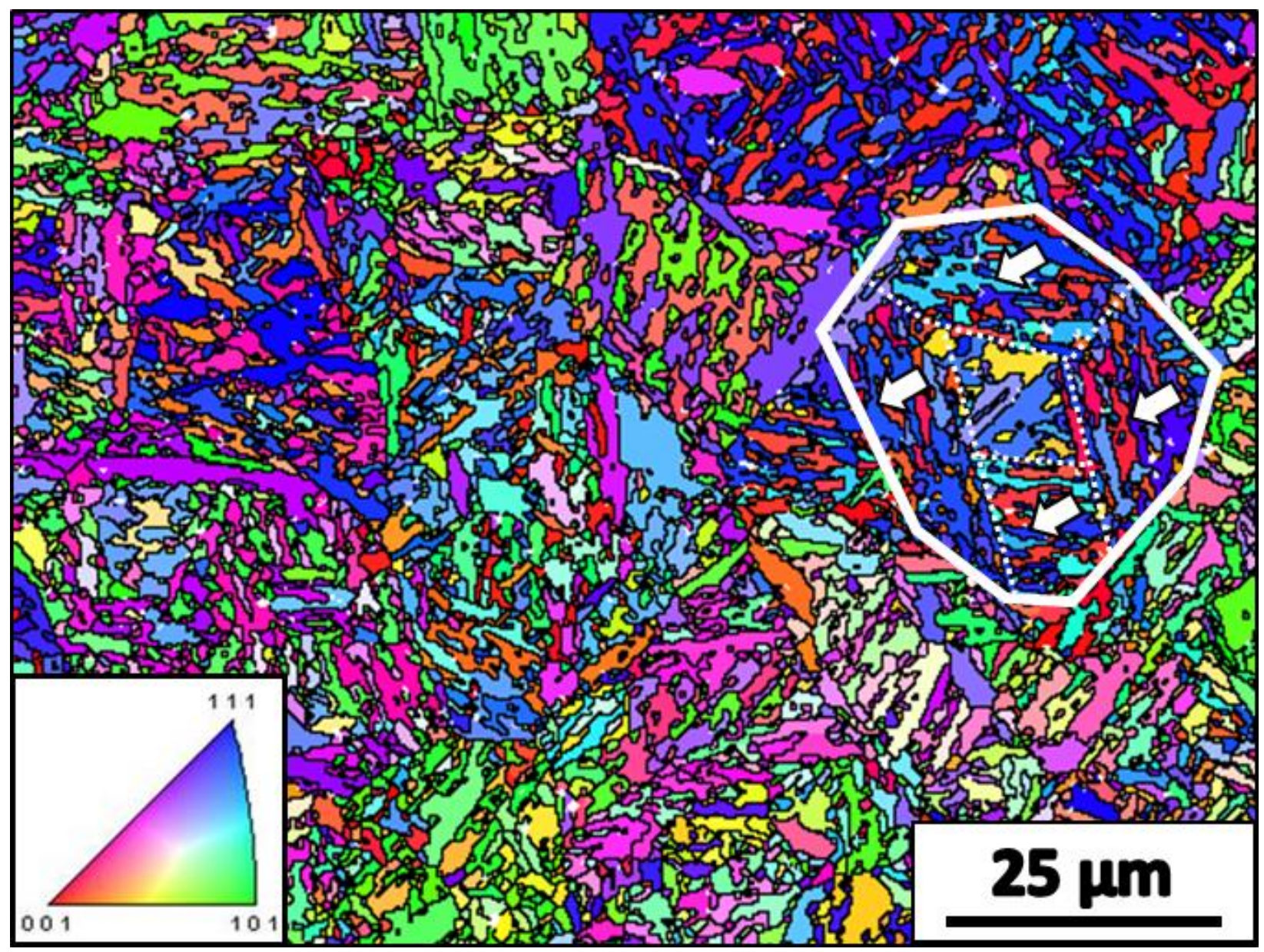

Figure 6 OIM map of 300M utilizing IPF-Z coloring. A single prior austenite grain is highlighted (white line); 4 individual martensite packets are denoted with white arrows separated by dotted white lines. 


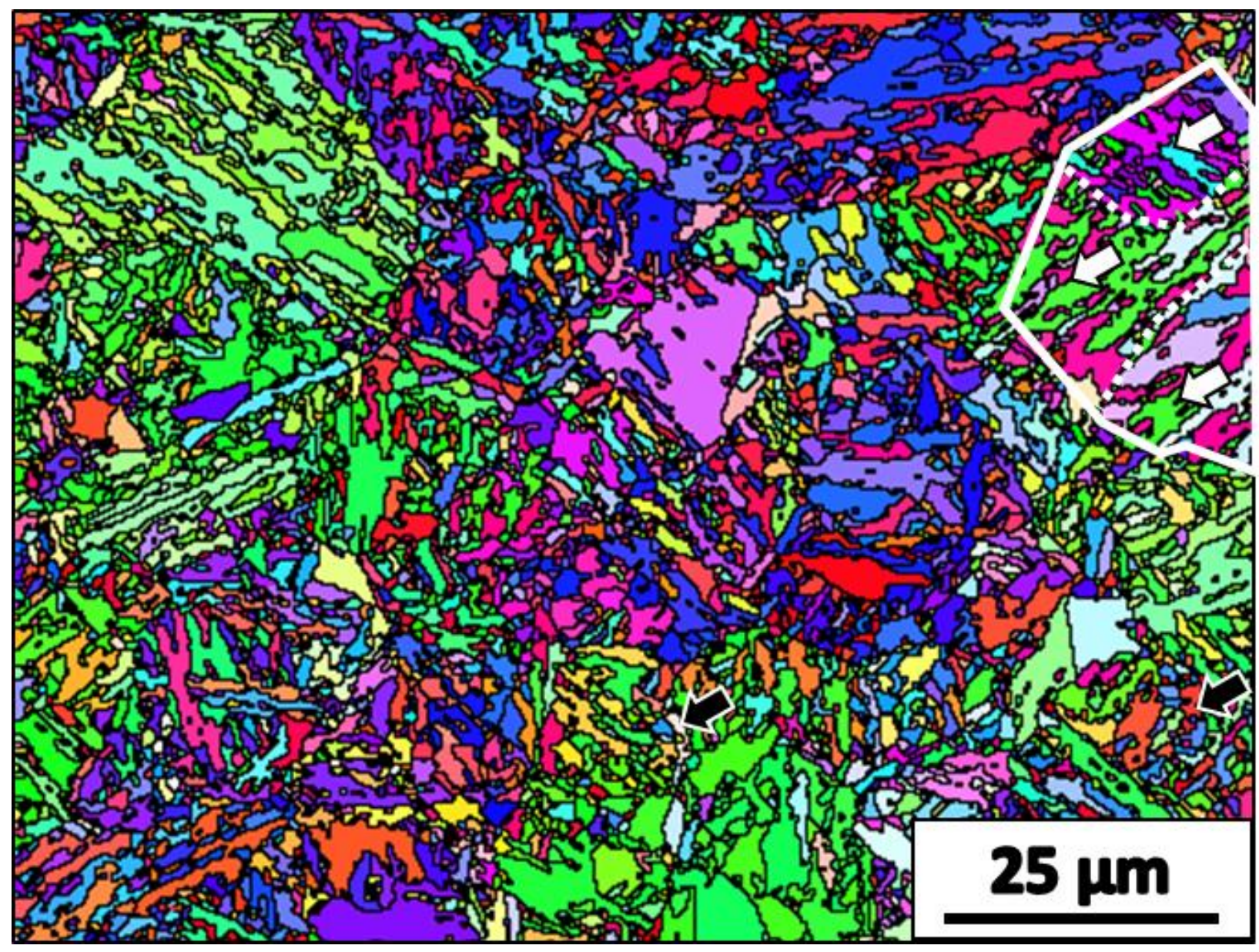

Figure 7 OIM map of AerMet 100 utilizing IPF-Z coloring. A single prior austenite grain is highlighted (white line); 3 individual packets are denoted with white arrows, separated by dotted white lines. 


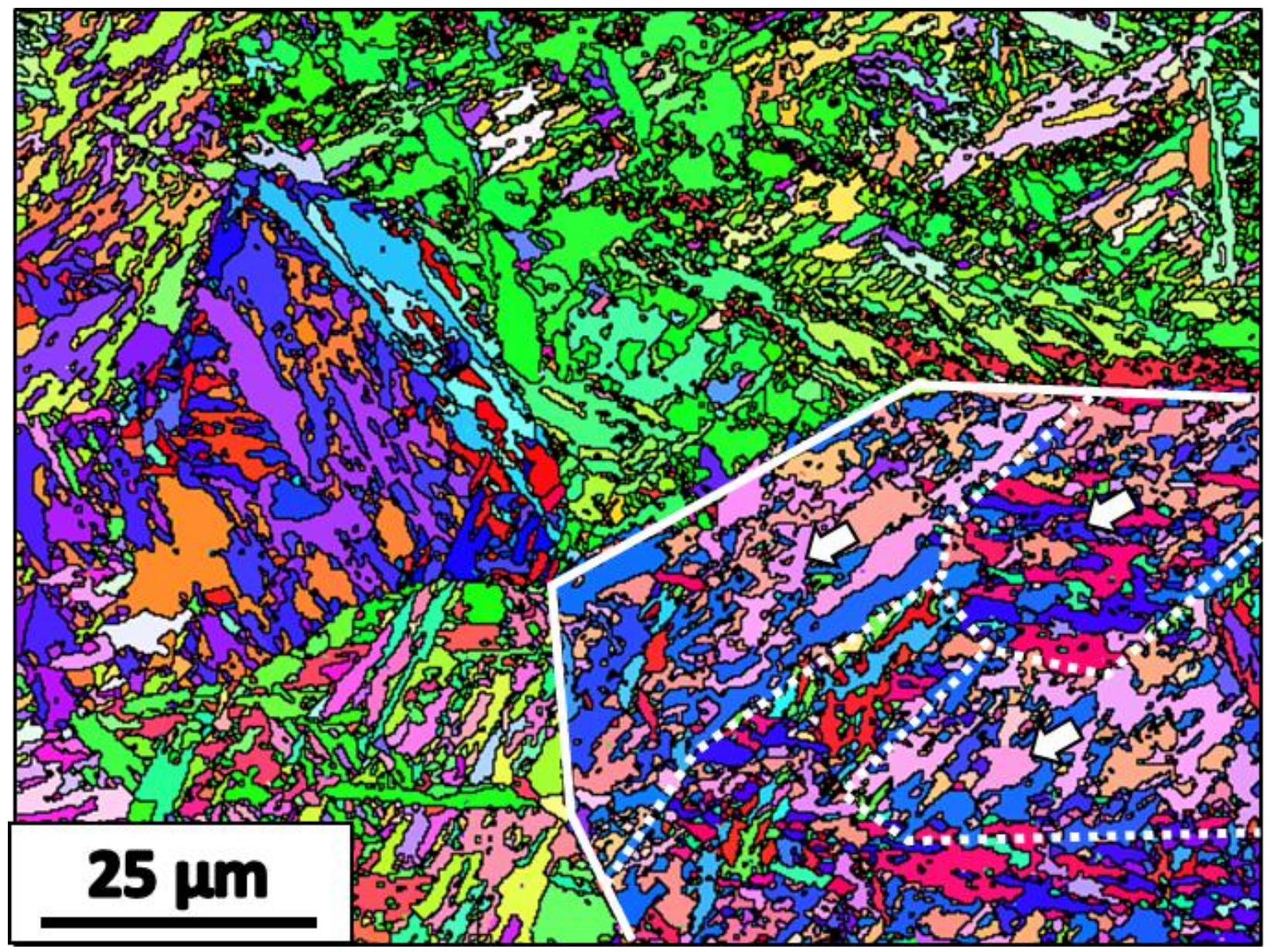

Figure $8 \quad$ OIM map of Ferrium M54 utilizing IPF-Z coloring. A single prior austenite grain is highlighted (white line); 4 individual packets are denoted with white arrows, separated by dotted white lines. 


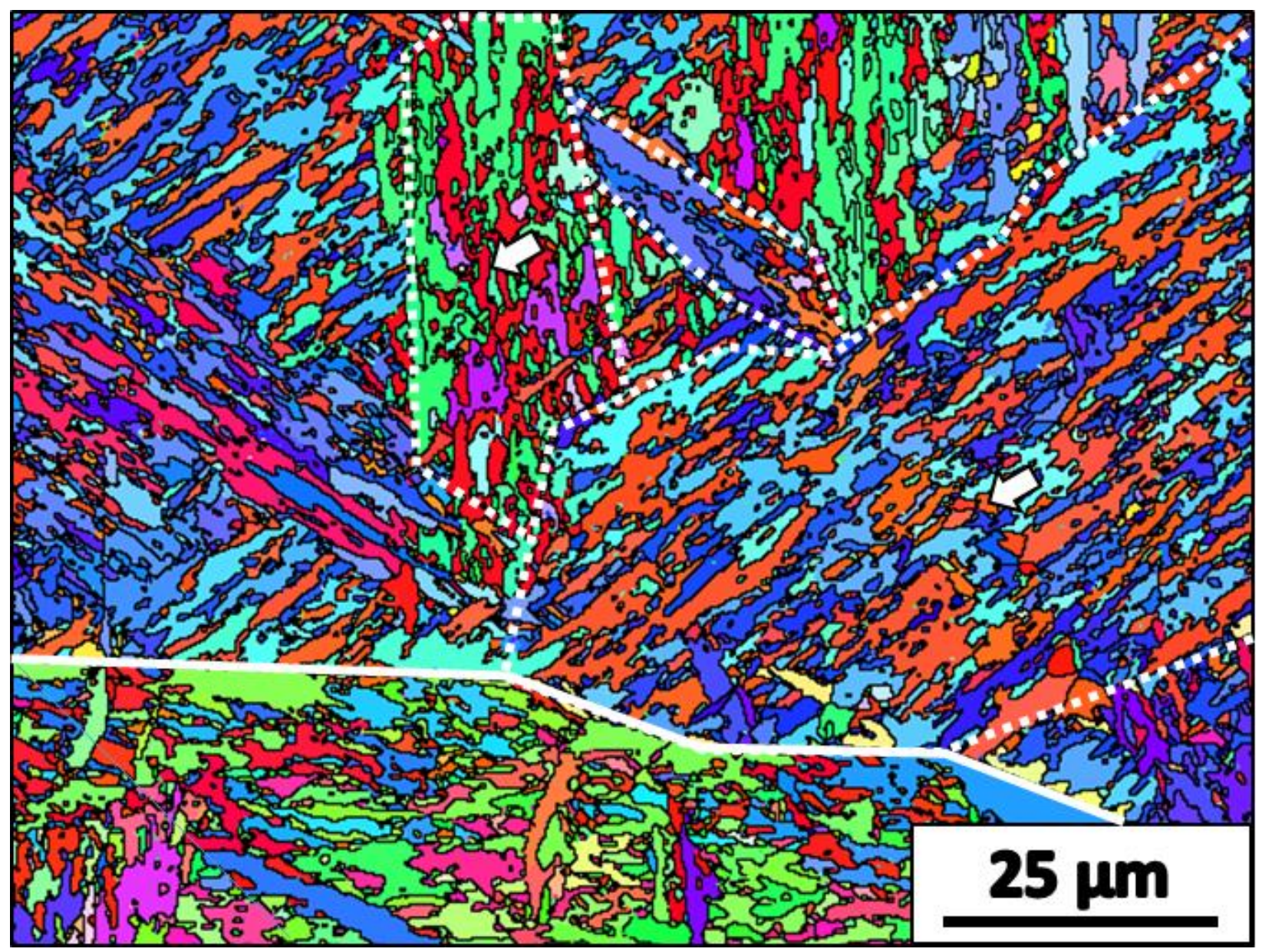

Figure 9 OIM map of CrNiMoWV utilizing IPF-Z coloring. A single prior austenite grain is highlighted (white line); individual packets are denoted with white arrows, separated by dotted white lines. 


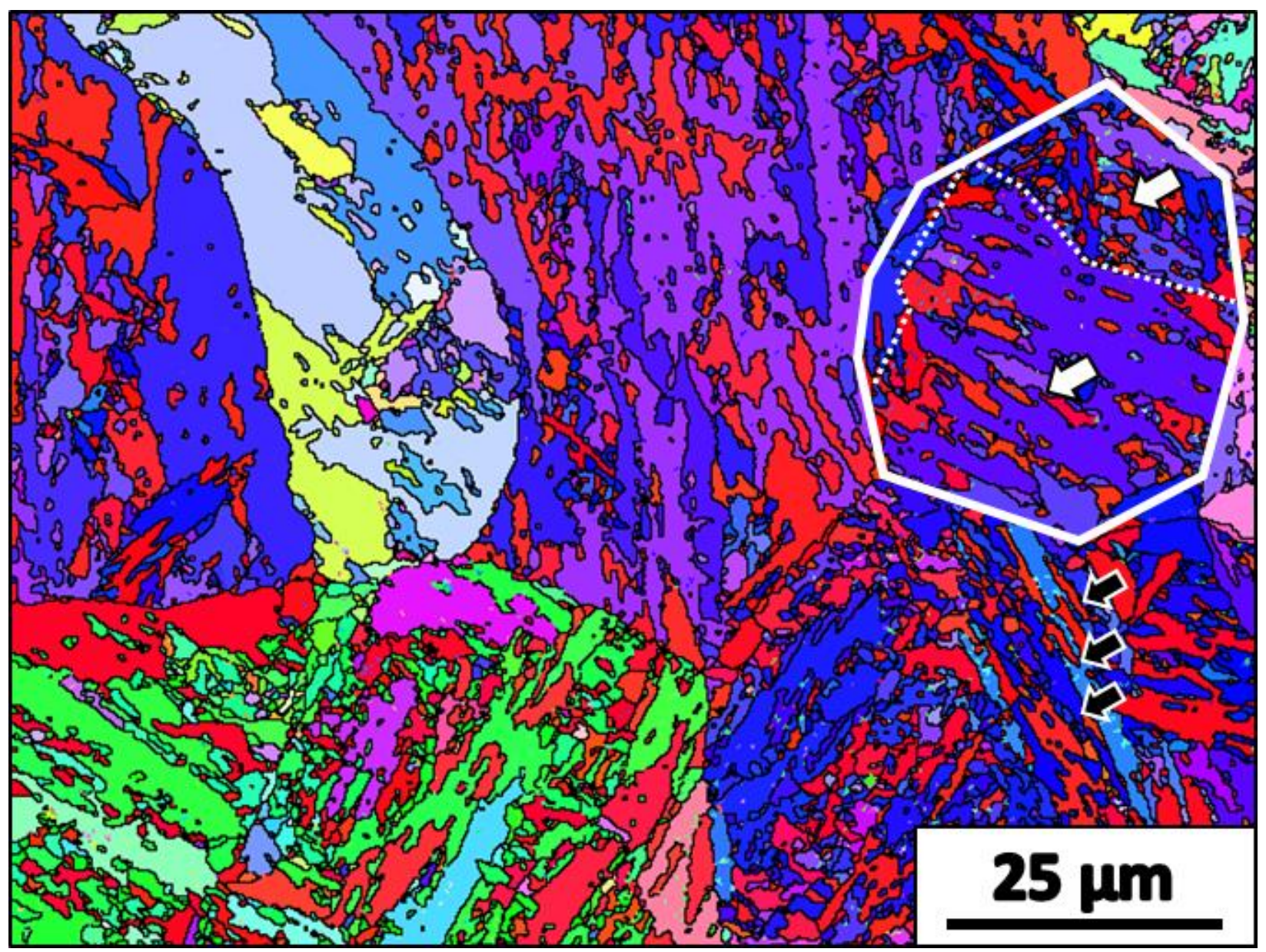

Figure 10 OIM map of Ferrium S53 utilizing IPF-Z coloring. A single prior austenite grain is highlighted (white line); individual packets are denoted with white arrows, separated by dotted white lines. Parallel martensite block features are shown with black arrows.

Prior austenite grain boundaries in a lath martensite microstructure are randomly oriented high angle interfaces, with typical misorientations of $20-40^{\circ}$ [108]. Conversely packet and block interfaces typically exhibit a bimodal distribution of misorientation, consisting of a low angle peak $\left(10-15^{\circ}\right)$ and high angle peak $\left(50-60^{\circ}\right)$. Therefore, to determine the location of prior austenite grains, a map of all randomly boundaries with a misorientation of $20-40^{\circ}$ was compared to the IPF-Z OIM maps presented in Figures 6-10. An example of this comparison is shown for Custom 465 in Chapter 4 (Figure 43a-d), including approach validation. While an insufficient number of prior austenite grains was sampled to meet the ASTM requirement for statistically rigorous grain 
size measurement [109], average prior austenite grain size from this EBSD images is consistent with previous studies: 11 and 16 - $22 \mu \mathrm{m}$ for AerMet 100 and 300M, respectively (Figures 6-7, white lines) $[63,71,73]$. Grain size is increased 2-5x in Ferrium M54 and Ferrium S53 compared to AerMet 100 (Figure 8 and Figure 10). The scale of Ferrium M54 and Ferrium S53 grains is similar to that observed using optical etchant microscopy (OEM) [64,65]. No published microstructure data exist for the experimental CrNiMoWV steel; however, average prior austenite grain diameter is $\sim 100 \mu \mathrm{m}$ based on grains observed on intergranular fracture surfaces.

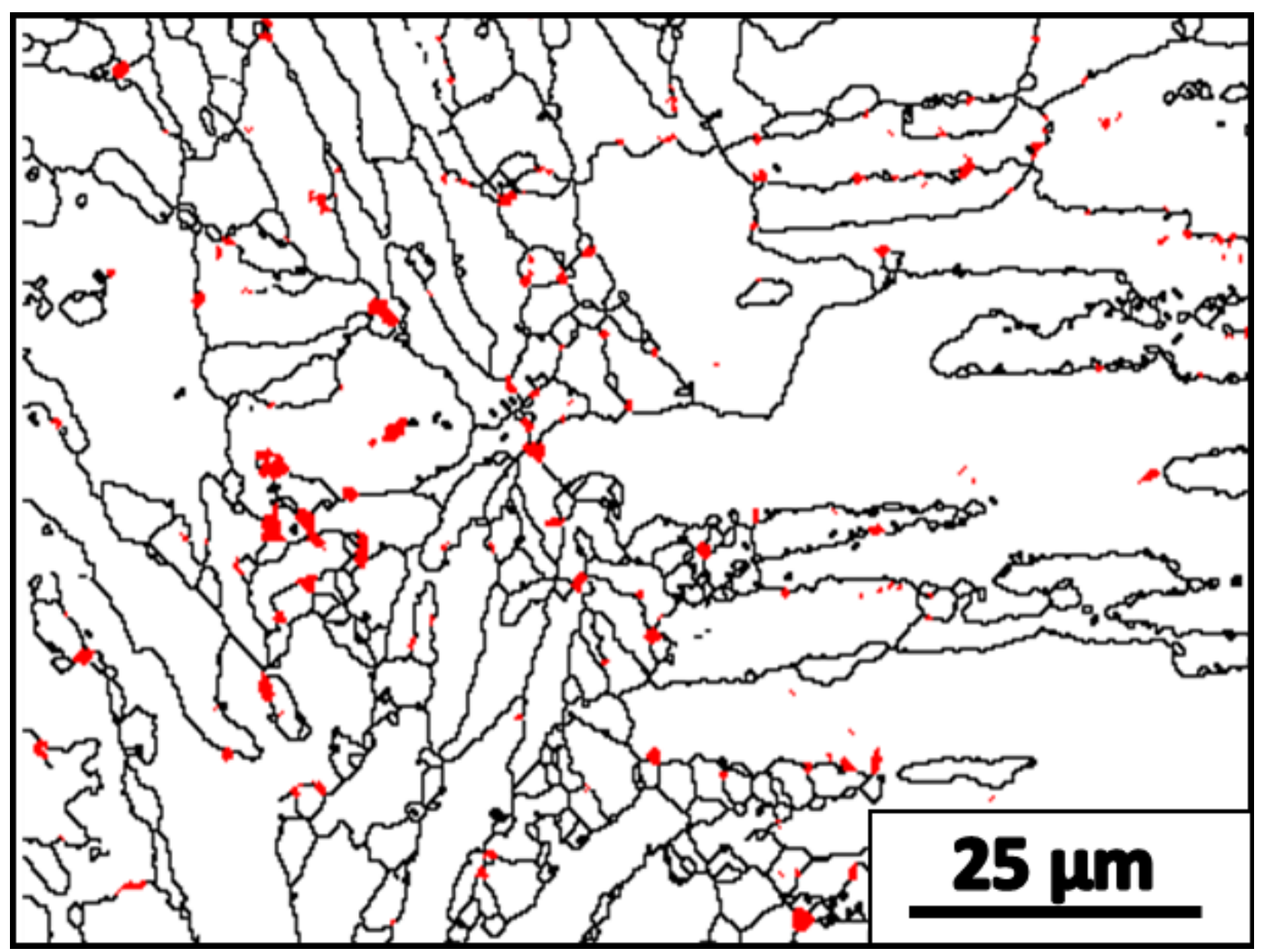

Figure 11 Distribution of EBSD-measured precipitated austenite (red) in AerMet 100 shown against a trace of all martensite interfaces with $>2^{\circ}$ misorientation.

The martensite structures shown in Figures 6-10 are complex, but consistent with detailed literature studies on martensite morphology and crystallography reported for model Fe-C-Mn microstructures. As background based on modern work, classic low-carbon lath martensite 
microstructure exhibits a three level hierarchical structure comprised of laths, blocks, and packets within a prior austenite grain, as schematically shown in Figure 12a [110-113]. Crystallographic orientations of martensite laths classically obey the Kurdjumov-Sachs (K-S) orientation relationship, which defines the crystallographic orientation of newly formed martensite with respect the parent austenite [113]. Close packed $\{111\}_{\gamma}$ FCC planes are parallel to $\{110\}_{\alpha}$, in transformed body centered tetragonal martensite and the $\langle 101\rangle_{\gamma}$ direction is parallel to $<111>_{\alpha \prime}$ [113]; where $\gamma$ and $\alpha^{\prime}$ denote parent austenite and newly formed martensite, respectively. Cubic symmetry of both phases dictates 24 possible martensite lath orientations following K-S: 6 uniquely oriented directional variants $<101>_{\gamma} / /<111>_{\alpha}$, within each of 4 possible planes $\{111\}_{\gamma} / /\{110\}_{\alpha},[114]$.
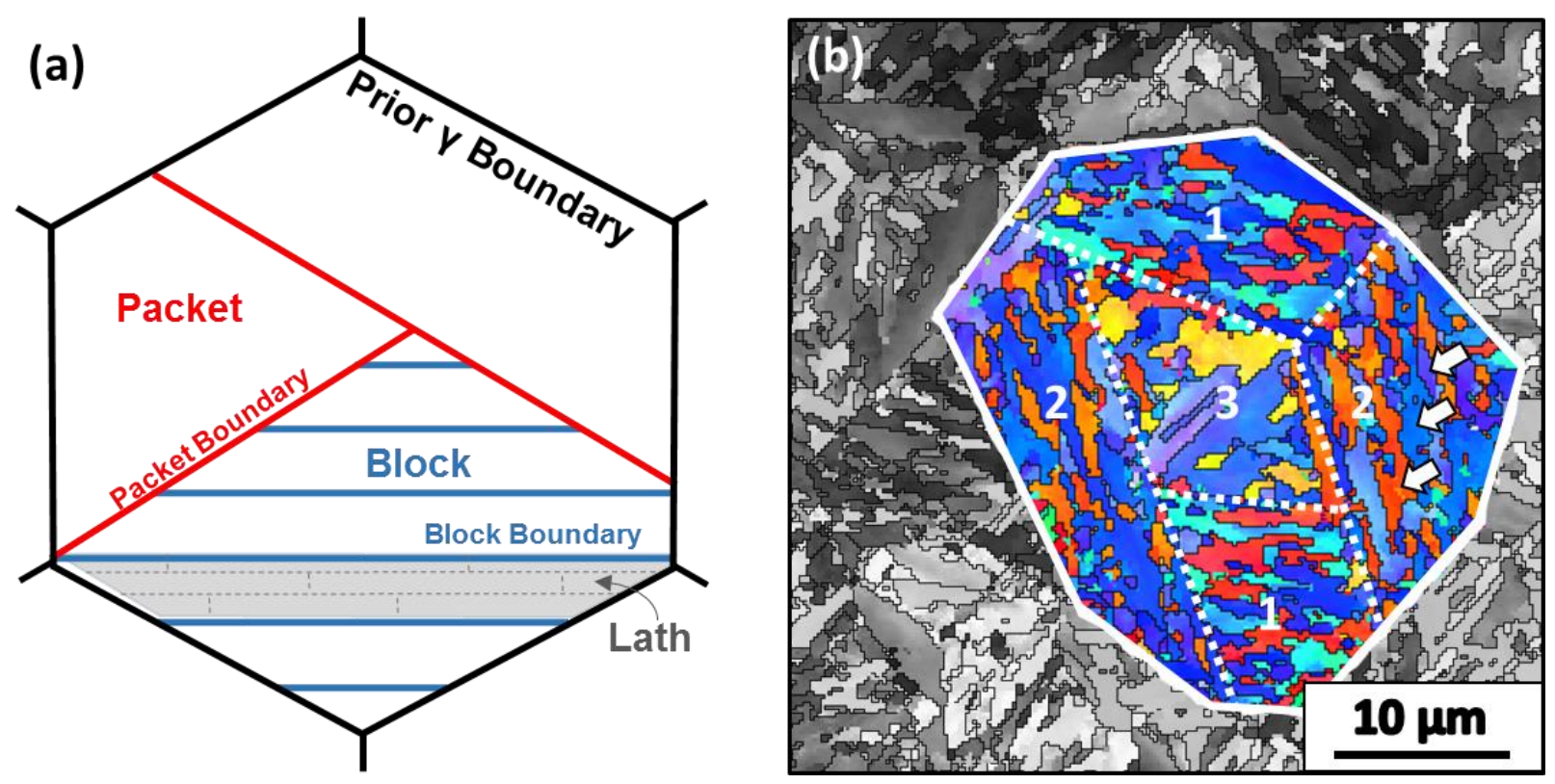

Figure 12 (a) Classical hierarchy of martensite structure of UHSS alloys, adapted from [52], (b) Isolated 300M prior austenite grain. Martensite packet boundaries are highlighted in white dotted lines and individual packet orientations are numbered.

Laths in low-carbon steels are acicular, heavily dislocated single-crystal, each with a single K-S crystallographic orientation. Blocks are comprised of either two interleaved lath variants, which are misoriented by less than $10^{\circ}$, or multiple parallel single variant laths separated by similar low angle boundaries. A martensite packet is comprised of a set of parallel blocks sharing a single habit 
plane from the parent austenite. The martensite habit plane is the plane of the parent austenite crystal where martensite laths and blocks form and grow during transformation. The habit plane for low carbon lath martensitic steels, $\{557\}_{\gamma}$, is irrational and lies relatively close to $\{111\}_{\gamma}$ $[112,113]$. Due to this similarity, it is common practice to refer to $\{111\}_{\gamma}$ as the habit plane in the parent austenite. However, the corresponding parallel plane of lath martensite is highly dependent upon alloy composition and has been measured to be $\{110\}_{\alpha},\{225\}_{\alpha},\{335\}_{\alpha}[113,115,116]$.

Determination of the exact orientation relationship in multiple UHSS alloys is beyond the scope of this study. EBSD measured orientation relationships have not been reported for highly alloyed UHSS. For the purposes of discussion, it will be assumed that the alloys obey classical K-S: the habit plane of martensite in austenite is $\{111\}_{\gamma}$ and packet structures are sets of parallel block/lath features sharing a common $\{110\}_{\alpha^{\prime}}$ boundary plane, as recently observed by Morris et al. [111]. In structures obeying K-S, no more than 4 unique packet orientations, corresponding to 4 independent $\{111\}_{\gamma}$ habit planes, can be present within a single prior austenite grain. An ideal K-S orientation relationship dictates three dominant block orientations per packet, each with 2 lath variants $[111,112]$. The classical view of martensite morphology will serve as a basis for the interpretation of the present UHSS results. However, significant alloy additions increase the complexity of martensite features, which may require crystallographic relationships other than K-S $[111,117]$.

Martensite microstructural hierarchy for the five observed UHSS alloys is varied in morphological appearance and scale, but is consistent with the K-S dominance and symmetry. In general prior austenite grains are subdivided into a small number of packets, highlighted by dotted lines in Figure 12b and Figures 6-10, which contain features largely aligned along the same austenite habit plane. Three unique packet orientations (numbered 1, 2 and 3 in Figure 12b), each for a single $\{111\}_{\gamma}$ variant are represented, among 5 distinct packets. Within each martensite packet there are multiple irregular but parallel features (denoted by white arrows) containing shades of a single coloration. Three orientations (3 IPF-Z colors) of features dominate in a packet, e.g. redorange/blue/purple in packet 2 of Figure 12b (white arrows). In an ideal K-S relationship, packets contain equal fractions of three block features to minimize transformational strain [111]. Single color parallel features observed in Figures 6-10 and labeled with white arrows in Figure 12b are therefore assumed to be martensite blocks within the classical martensite definition. No finer-scale structure was detected within these features, suggesting three possibilities: (1) fine scale parallel 
lath structure is below the resolution limit of EBSD analysis, (2) single IPF-Z color features represent laths rather than blocks, or (3) single IPF-Z features represent blocks which contain a single lath variant. To investigate these possibilities, a single AerMet 100 martensite packet was characterized by high resolution EBSD analysis, with reduced pixel size ( $24 \mathrm{~nm})$ and prolonged machine time. The result shows the typical packet/block structure in Figure 13a-c.

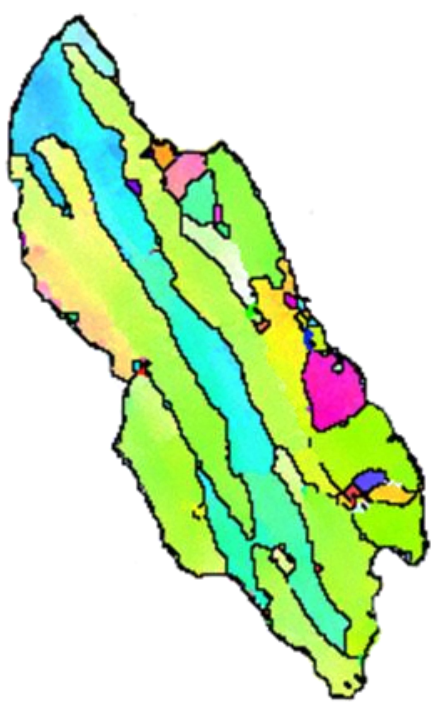

(a)

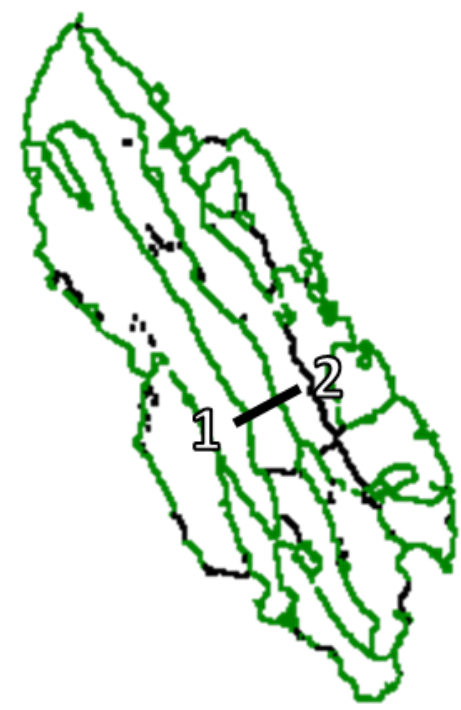

(b)

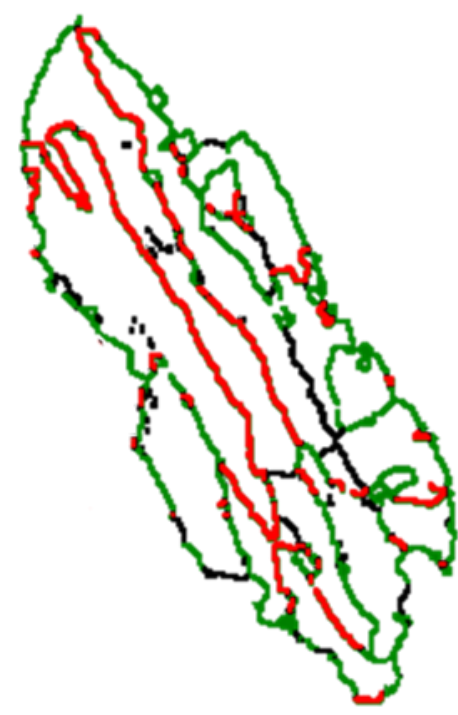

(c)

\section{$2 \mu \mathrm{m}$}

Figure 13 (a) Orientation map of in an isolated AerMet 100 martensite packet colored using IPF-Z coloring. (b) Map of martensite boundaries showing the distribution of high $\left(>15^{\circ}\right.$, green) and low $\left(2^{\circ} \leq \theta<15^{\circ}\right.$, black) angle interfaces. (c) Identical misorientation map as (b) with high angle $\sum 3$ CSL boundaries highlighted in red. 
Three visualizations of martensite packet structure are presented: (a) standard IPF-Z coloring, (b) boundary traces by misorientation, and (c) a trace of the dominant CSL special boundaries ( $\Sigma 3)$. An acicular-needle block shape is diminished compared to classical martensite structure; for AerMet 100, block boundaries are often nonlinear and rounded. Block dimensions are highly variable within a packet: $0.25-6 \mu \mathrm{m}$ in length and $0.25-1.25 \mu \mathrm{m}$ in width. Martensite blocks are separated by highly misoriented boundaries with misorientations $>15^{\circ}$ (Figure 13b), and typically $50^{\circ}<\theta$ (Figure 14). This result is consistent with previous misorientation analyses of block interfaces in carbon and low alloy steels [112,118]. Morito et al observed that block interfaces in Fe-C-Mn martensitic steel are, on average, misoriented by $\sim 60^{\circ}$ with a $\{110\}_{\alpha^{\prime}}$ interface plane. Further, lath interfaces were observed to be exclusively low angle (2-4 $)$, as predicted by a K-S orientation relationship [112]. Based upon these observations, green/red lines internal to the packet shown in Figure 13c represent highly misoriented martensite block interfaces. It is therefore logical to assume that the single IPF-Z color features (Figure 12b, white arrows) are martensite blocks. As shown in Figure 14, point-to-point misorientation within blocks is less than $1^{\circ}$. If lath misorientations in UHSS follow observations by Morito et al. [112], then this would suggest that each martensite block contains a single lath variant. Transmission electron microscopy of identically aged AerMet 100 by Ayer et al. reveals that the finest martensite features, which these authors denoted as laths, have widths typically 0.3-0.5 $\mu \mathrm{m}$ [24]. This spacing is on the same order as martensite blocks observed in the present study and the distinction between laths and blocks is not made by Ayer. Speculatively, the features described by Ayer et al. as laths are martensite blocks which contain a single lath variant.

Characterization of the martensite boundaries in AerMet 100, via Brandon's criterion, establishes that a significant portion of inter-block boundaries have a high angle special $\Sigma 3$ CSL relationship (Figure 13c, red lines). Martensite packet boundaries are highly misoriented (exclusively $>50^{\circ}$ ), but as expected, do not have a special orientation relationship. The observations of martensite boundary morphology in fine probe EBSD of AerMet 100 (Figure 13) are mirrored in the results of identical analyses conducted on 300M, Ferrium M54, CrNiMoWV, and Ferrium S53 UHSS, as shown in Figure 15. These martensite structure maps will be compared to SEM fractographic results which show the operative HEAC path(s) in these UHSS. 


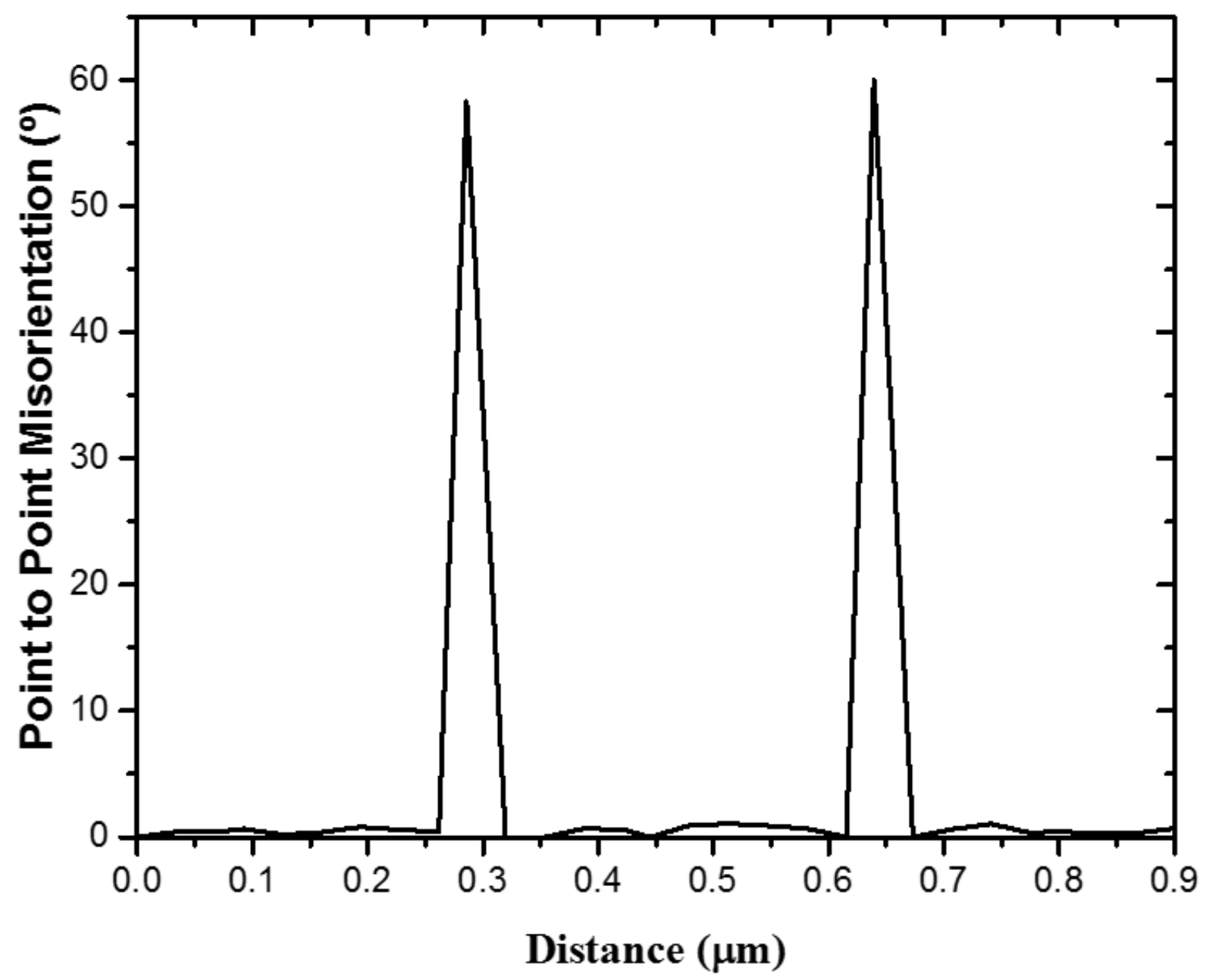

Figure 14 Point to point misorientation profile from (1) to (2) in Figure $13 b$. 


\section{M}

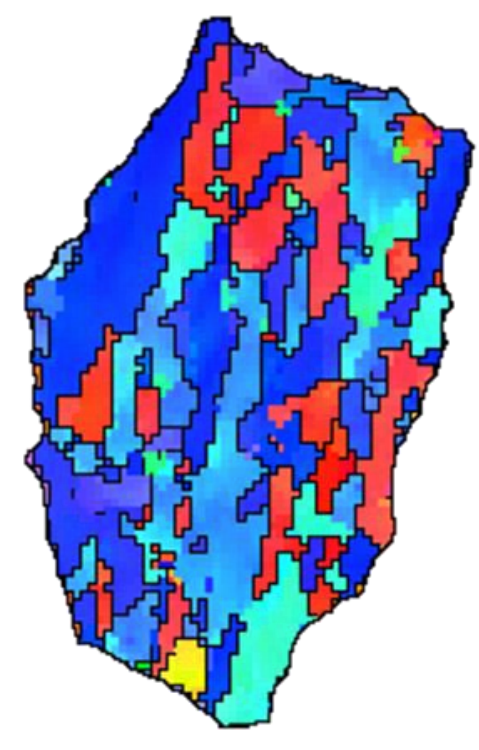

(a)

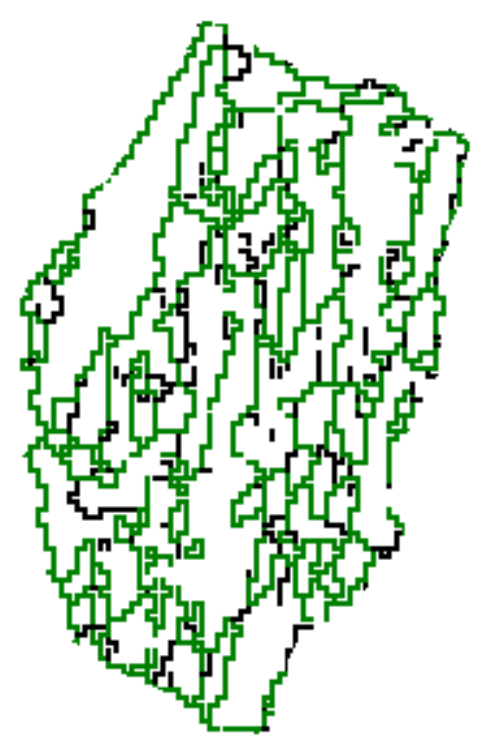

(b)

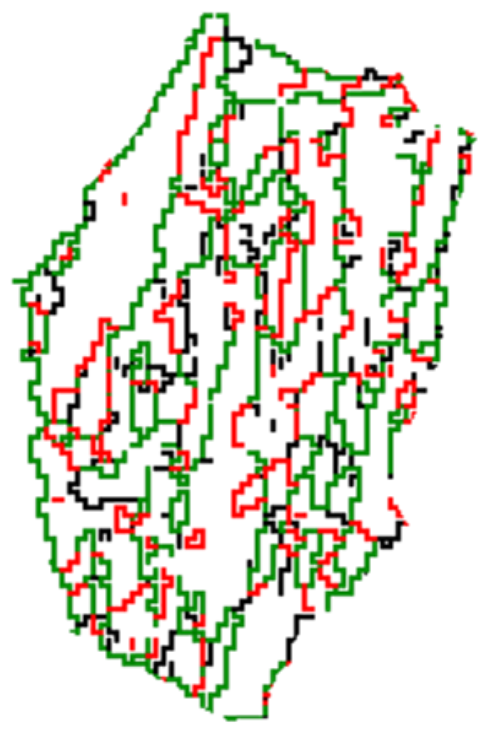

(c)

$6 \mu \mathrm{m}$

\section{AerMet 100}

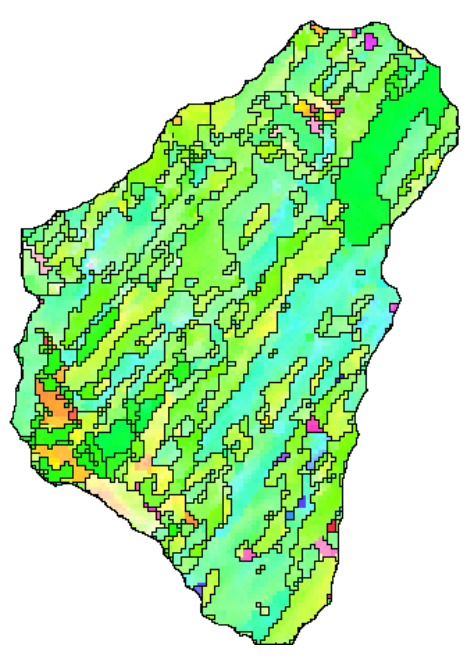

(d)

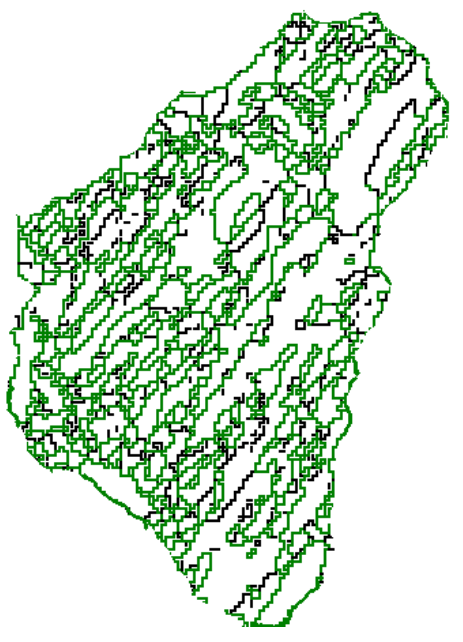

(e)

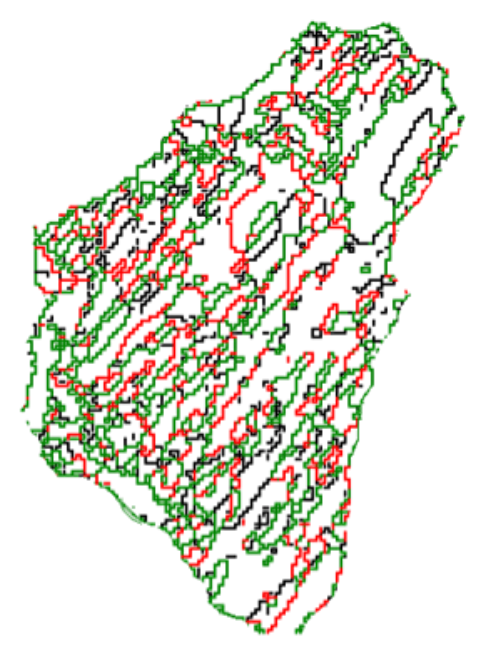

(e)

$9 \mu \mathrm{m}$ 


\section{CrNiMoWV}

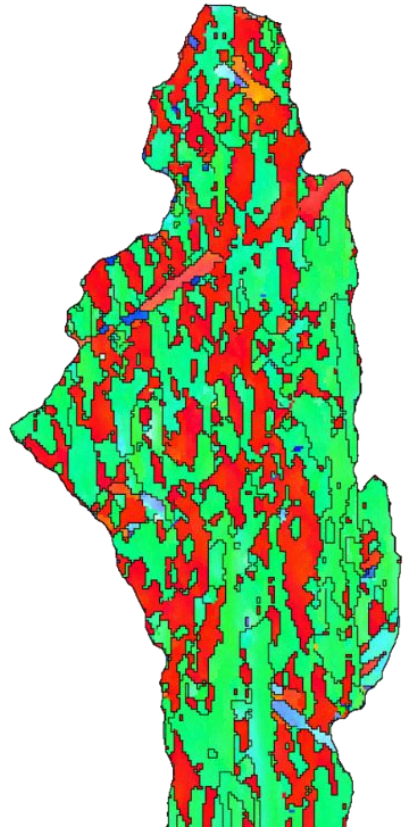

(g)

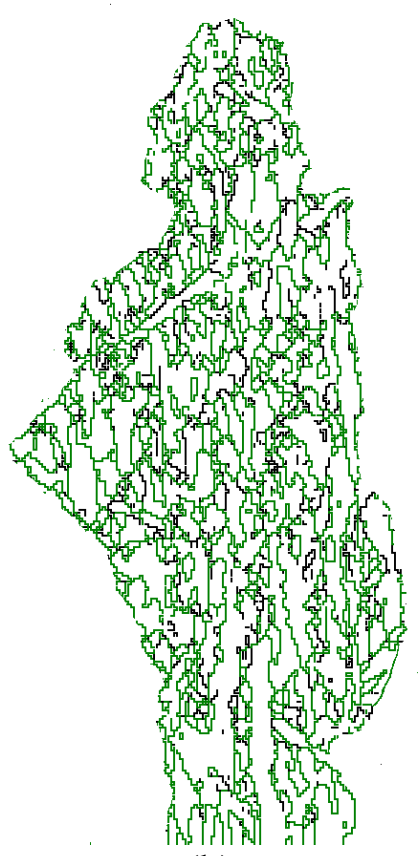

(h)

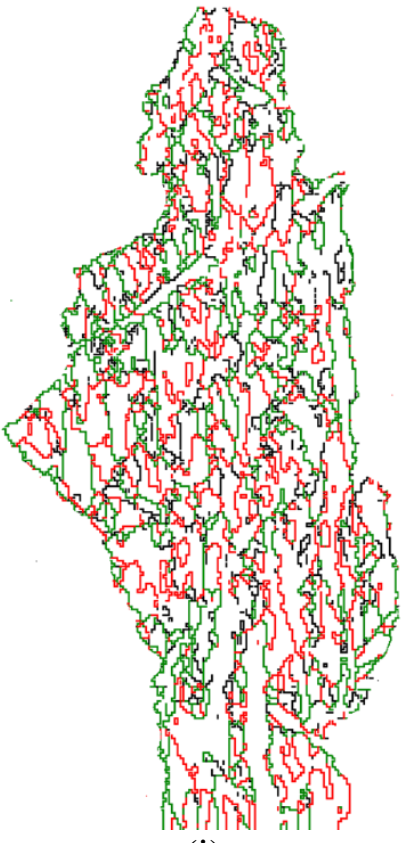

(i)

\section{$7 \mu \mathrm{m}$}

\section{Ferrium S53}

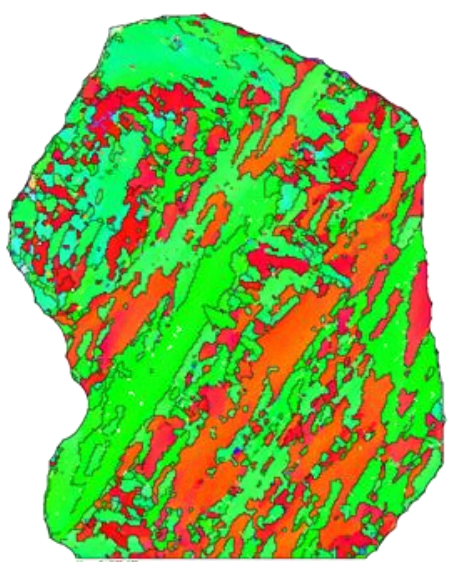

(j)

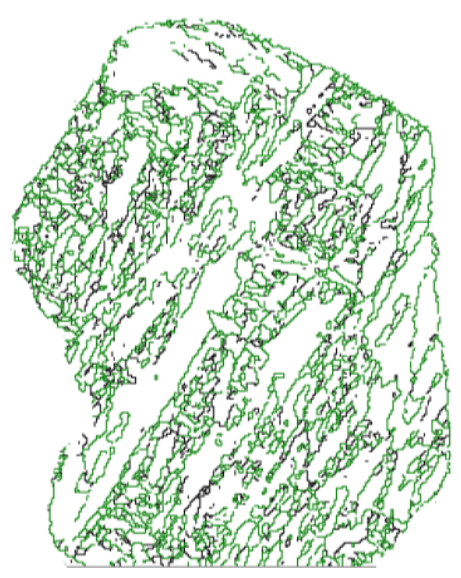

(k)

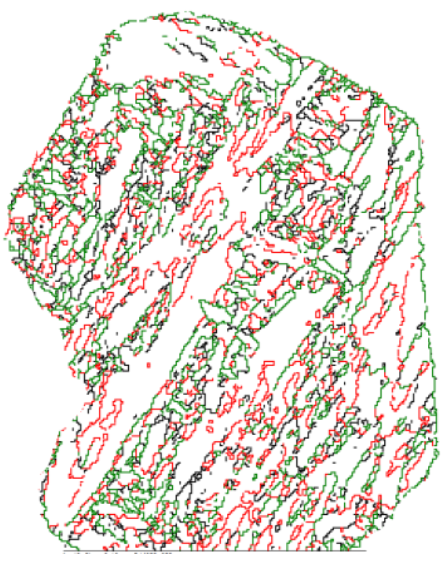

(1)

\section{$14 \mu \mathrm{m}$}




\section{Ferrium M54}

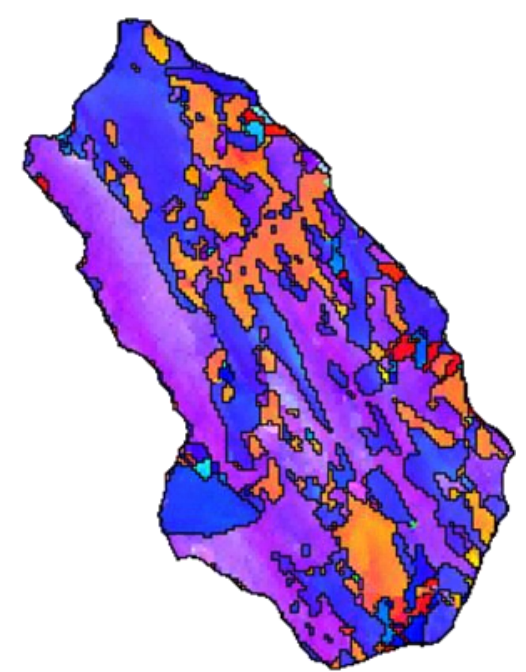

(m)

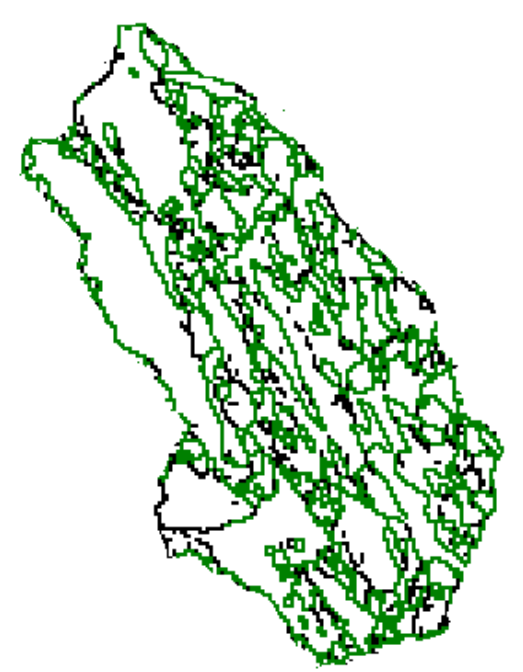

(n)

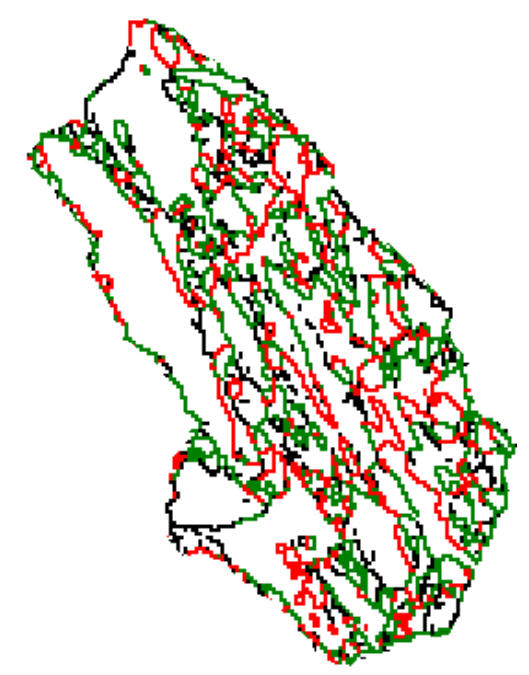

(o)

\section{$7 \mu \mathrm{m}$}

Figure 15 Isolated packets of 5 UHSS alloys: (Column 1) Orientation map of an isolated martensite packet colored using IPF-Z coloring. (Column 2) Map of martensite boundaries showing the distribution of high $\left(15^{\circ} \leq \theta\right.$, green) and low angle $\left(2^{\circ} \leq \theta<15^{\circ}\right.$, black) interfaces. (Column 3) Identical misorientation coloring with $\sum 3$ CSL boundaries highlighted (red).

Figures 6-10, 13 and 15 illustrate that martensite structure in UHSS alloys is highly variable compared to the classic description presented in Figure 12a. Martensite structure in 300M, AerMet 100, and Ferrium M54 exhibit similar, fine-scale features, and difficult-to-distinguish austenite grain boundaries. Regions of extremely fine block size (Figure 7, black arrows), which do not fit the classic martensite definition, are observed in all alloys. Similar morphology has been previously observed for a Fe-9Ni martensitic steel, though its origin was not addressed by the authors [111]. Such fine scale disordered regions may be attributed to the short direction(s) of a martensite packet lying nearly parallel to the $2 \mathrm{D}$-sectioning plane. Isolated, well-defined grains (Figures 6-10, white lines) indicate that 1-5 martensite packets fill a prior austenite grain. Packet size is determined by the prior austenite grain size and is accordingly variable. An insufficient area 
was mapped to collect statistically significant packet size data. However, average block interface spacing is consistently small: $0.96 \pm 0.03,0.81 \pm 0.05$, and $0.95 \pm 0.06 \mu \mathrm{m}$ for $300 \mathrm{M}$, AerMet 100, and Ferrium M54, respectively. Near-classic martensite structure is clearly distinguishable in Ferrium S53 and CrNiMoWV (Figures 9-10). Martensite packet length is qualitatively proportional to the increase in prior austenite grain size. This effect is most evident in CrNiMoWV where a single packet is $>75 \mu \mathrm{m}$ in length and $35 \mu \mathrm{m}$ wide (Figure 9, dotted line). Average spacing between martensite block interfaces is increased: $1.16 \pm 0.09$ and $1.27 \pm 0.08 \mu \mathrm{m}$ for Ferrium S53 and CrNiMoWV, indicating a coarsening of the martensite block structure which is more pronounced in CrNiMoWV.

The surface area and fractional occurrence of randomly oriented, high energy martensite interfaces, and low energy coincident site lattice (CSL) interfaces, are shown in Table 6. The distribution of interfacial area is consistent across UHSS: 59-67\% of the total interfacial area per volume is randomly oriented high angle boundaries; total CSL fraction varies from 20-30\%, and $12-17 \%$ of the boundaries are randomly oriented low angle. There is no distinguishing characteristic, in the context of martensite interface characteristics, for any of the UHSS cases examined.

\begin{tabular}{|c|c|c|c|c|c|}
\hline $\begin{array}{c}\text { Interfacial Area } \\
\left(\mathrm{S}_{\mathrm{V}}, \mathrm{mm}^{2} / \mathrm{mm}^{3}\right)\end{array}$ & $\begin{array}{c}\text { Random High } \\
\text { Angle }\end{array}$ & $\begin{array}{c}\text { Random } \\
\text { Low Angle }\end{array}$ & $\sum 3$ & Other CSL & Total \\
\hline $300 \mathrm{M}$ & $1,312(64 \%)$ & $304(15 \%)$ & $192(9 \%)$ & $258(12 \%)$ & 2,066 \\
\hline AerMet 100 & $1,456(59 \%)$ & $290(12 \%)$ & $314(13 \%)$ & $412(17 \%)$ & 2,472 \\
\hline CrNiMoWV & $972(62 \%)$ & $202(13 \%)$ & $203(13 \%)$ & $201(13 \%)$ & 1,578 \\
\hline Ferrium M54 & $1,280(61 \%)$ & $350(17 \%)$ & $178(8 \%)$ & $301(14 \%)$ & 2,110 \\
\hline Ferrium S53 & $1,154(67 \%)$ & $230(13 \%)$ & $223(13 \%)$ & $117(7 \%)$ & 1,724 \\
\hline
\end{tabular}

Table 6 Interfacial area and fraction of total boundary area of selected martensite boundaries 


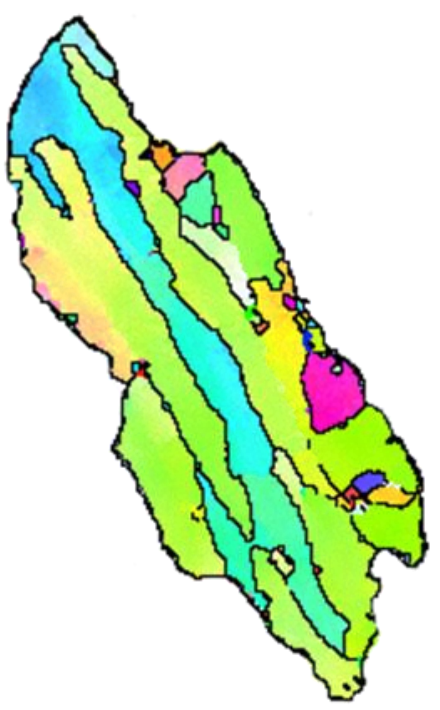

(a)

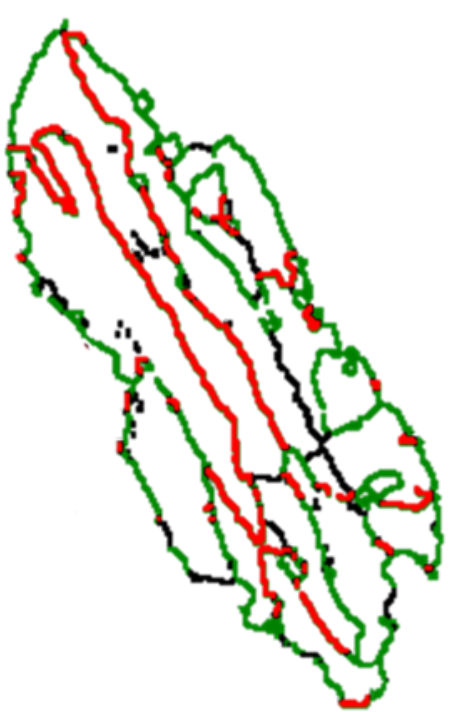

(b)

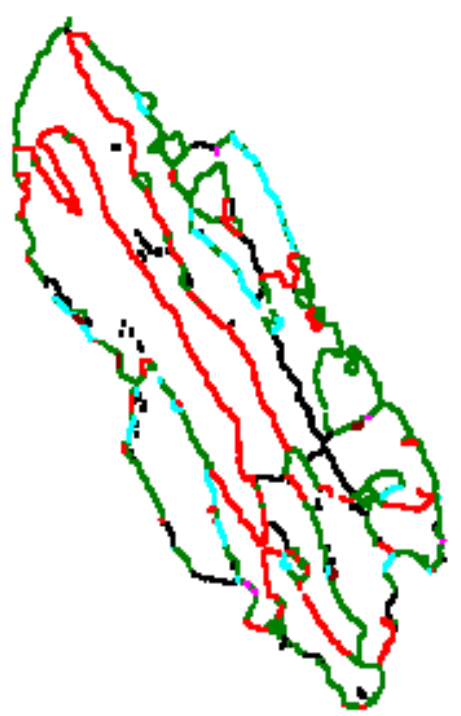

(c)

\section{$2 \mu \mathrm{m}$}

Figure 16 (a) Orientation map of an isolated AerMet 100 martensite packet colored using IPF-Z coloring. (b) Map of martensite boundaries showing the distribution of high angle $\left(15^{\circ}\right.$ $\leq \theta$, green), low angle $\left(2^{\circ} \leq \theta<15^{\circ}\right.$, black) and dominant $\sum 3$ CSL boundaries (red) (c) Identical map to (b) with the distribution of $\sum 11$ (teal), $\sum 13 b$ (maroon), and $\sum 25 b$ (fuchsia) boundaries highlighted.

Contiguous, high-angle $\left(15^{\circ} \leq \theta\right)$ interfaces with no CSL relationships are found along packet interfaces and along block interfaces (Figures 15 and 16, green lines). In all alloys, $\sum 3$ boundaries comprised the largest fraction of CSL boundaries; specifically, 37-66\% of the total CSL interfacial area per volume. Remaining CSL interfacial area was approximately equally distributed among $\sum 11, \sum 13 \mathrm{~b}, \sum 25 \mathrm{~b}$, and a small fraction of other CSL orientations. As shown in Figures 15 and 16 (red lines), $\sum 3$ boundaries are primarily found along martensite block interfaces, following the lengthwise direction and often running the entire length of a block boundary. Conversely, the $\sum 11$, $\sum 13 \mathrm{~b}$, and $\sum 25 \mathrm{~b}$ boundaries are present in short $(0.25-1.5 \mu \mathrm{m})$ disconnected segments, as shown in Figure 16c for an isolated packet of AerMet 100. Notably, all of the CSL boundary types observed have high angles of misorientation: $\sum 3\left(60^{\circ}\right), \sum 11\left(50.5^{\circ}\right), \sum 13 \mathrm{~b}\left(27.8^{\circ}\right)$, and $\sum 25 \mathrm{~b}$ $\left(43.6^{\circ}\right)$ and correspond to the boundary between specific martensite lath pairs [110]. The 
occurrence and distribution of these various packet and block interfaces are correlated in the Discussion with HEAC fractographic observations. This comparison is motivated by the observations that such interfaces provide a preferential HEAC path dominated by $\mathrm{H}$ decohesion $[49,50,57]$.

\subsubsection{HEAC Crack Growth Kinetics}

\subsubsection{HEAC Kinetics at $E_{a p p}=-550 m V_{S C E}$}

AerMet 100, Ferrium M54, Ferrium S53, CrNiMoWV and 300M tempered for ultra-high strength are susceptible to severe HEAC when stressed in $0.6 \mathrm{M} \mathrm{NaCl}$ at a fixed potential $\left(\mathrm{E}_{\text {app }}=-550\right.$ $\mathrm{mV}_{\mathrm{SCE}}$ ) near to OCP. Subcritical crack growth kinetics for each alloy are represented in Figure 17 as da/dt versus elastic $\mathrm{K}$ for $\mathrm{E}_{\mathrm{app}}=-550 \mathrm{mV}_{\text {SCE}}$. Relative to $1 \mathrm{~h}$ OCP measurements, $300 \mathrm{M}$ and CrNiMoWV are anodically polarized (by 100 and $10 \mathrm{mV}_{\text {SCE}}$, respectively), while AerMet 100, Ferrium M54, and Ferrium S53 are cathodically polarized by - $25-40$ and - $180 \mathrm{mV}_{\mathrm{SCE}}$, respectively. The operational $\mathrm{K}_{\mathrm{TH}}$ is clearly delineated for AerMet 100, Ferrium M54 and Ferrium S53 by rapidly increasing $\mathrm{K}$-dependent $\mathrm{da} / \mathrm{dt}$ rising above the plasticity defined resolution limit (dotted line). In contrast classic Stage I-Stage II HEAC kinetics are exhibited by 300M and CrNiMoWV, but preceded by significantly elevated initial $\mathrm{da} / \mathrm{dt}$ at very low $\mathrm{K}$. This initial da/dt is attributed to an unknown testing artifact rather than being representative of real crack growth. $\mathrm{K}_{\mathrm{TH}}$ for the minor number of specimens exhibiting this behavior is established by shifting the resolution limit upwards until it aligns with rising da/dt prior to Stage I. Stage II crack growth rate (da/dtII) is shown for each alloy as nearly $\mathrm{K}$-independent da/dt after $\mathrm{K}_{\mathrm{TH}}$.

Three HEAC behavioral classes are noted: (1) Severe SCC/HEAC exhibited by legacy 300M as characterized by low $\mathrm{K}_{\mathrm{TH}}(15.4 \mathrm{MPa} \sqrt{\mathrm{m}})$ and dramatically high da/dt $/ \mathrm{II}(2,540 \mathrm{~nm} / \mathrm{s})$, (2) Moderate susceptibility of modern, non-stainless UHSS AerMet 100, Ferrium M54, and CrNiMoWV to SCC/HEAC at similar $\mathrm{K}_{\mathrm{TH}}(12-18 \mathrm{MPa} \sqrt{\mathrm{m}})$, but with significantly reduced da/dtII $(20-60 \mathrm{~nm} / \mathrm{s})$ compared to $300 \mathrm{M}$, and (3) strong SCC resistance of stainless Ferrium S53 exhibiting $\mathrm{K}_{\mathrm{TH}}=77$ $\mathrm{MPa} \sqrt{\mathrm{m}}$ and $\mathrm{da} / \mathrm{dt}_{\mathrm{II}}=80 \mathrm{~nm} / \mathrm{s} . \mathrm{K}_{\mathrm{TH}}$ of Ferrium $\mathrm{S} 53$ at $\mathrm{E}_{\text {app }}=-550 \mathrm{mV}_{\mathrm{SCE}}$ is identical to the manufacturer-reported $\mathrm{K}_{\mathrm{IC}}(71-77 \mathrm{MPa} \sqrt{\mathrm{m}})[36,65]$. However, crack growth above $\mathrm{K}=77 \mathrm{MPa} \sqrt{\mathrm{m}}$ displays near $\mathrm{K}$-independent da/dtII at $\sim 80 \mathrm{~nm} / \mathrm{s}$, indicative of diffusion limited HEAC. Due to this high $\mathrm{K}$, a portion of the da/dt above $\mathrm{K}=77 \mathrm{MPa} \sqrt{ } \mathrm{m}$ may be due to stable-ductile microvoid 


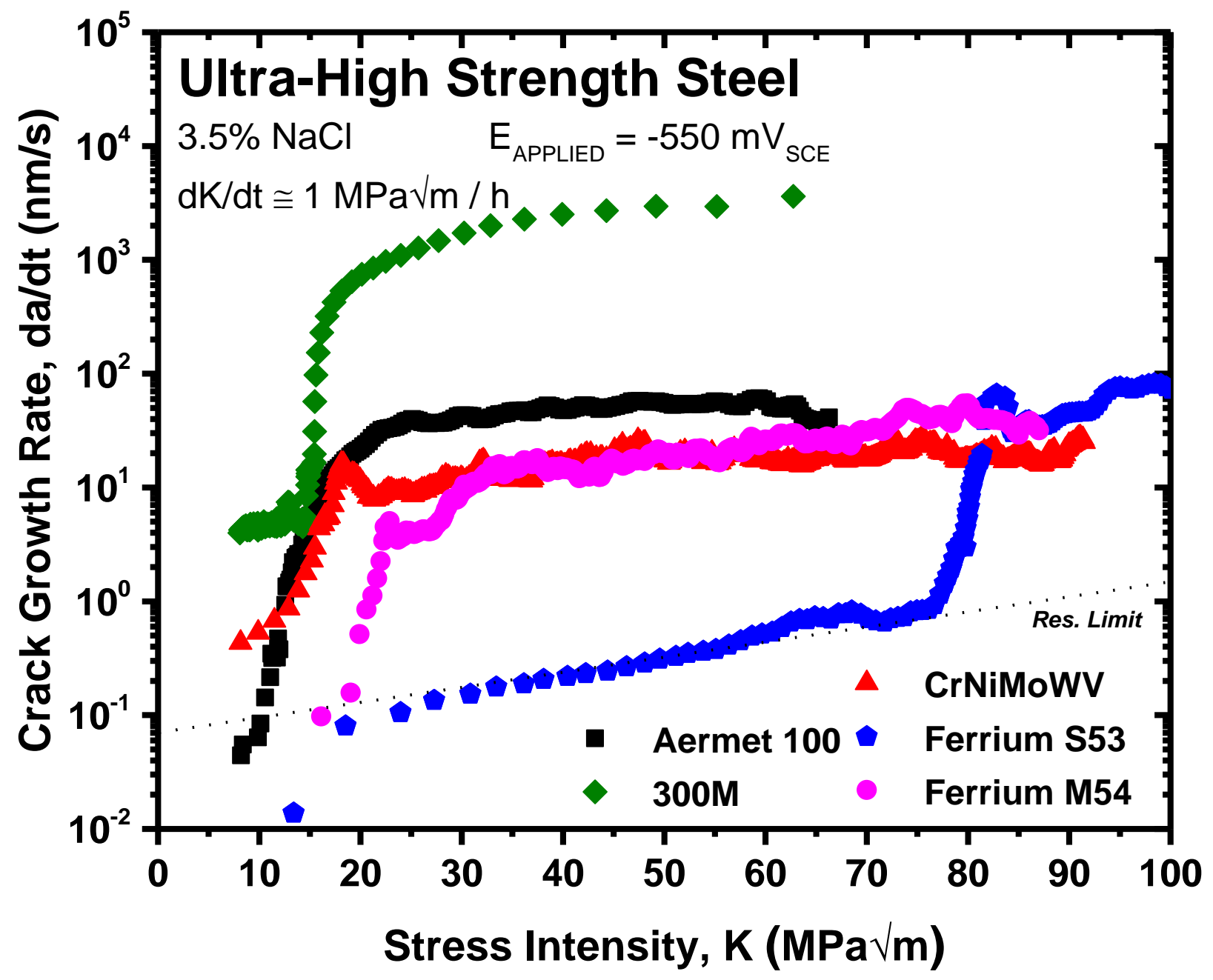

Figure 17 Subcritical crack growth rate versus $\mathrm{K}$ for five ultra-high strength steels stressed at $-550 \mathrm{~m} V_{S C E}$ in $0.6 \mathrm{M} \mathrm{NaCl}$.

cracking (MVC). SEM fractography confirms an equal mixture of TG-HEAC and ductile MVC fracture morphologies, indicating that stable-ductile crack growth significantly contributed to da/dt for Ferrium $\mathrm{S} 53$ at $\mathrm{K}>77 \mathrm{MPa} \sqrt{\mathrm{m}}$.

Observed differences in $\mathrm{K}_{\mathrm{TH}}$ and da/dt ${ }_{\mathrm{II}}$ for modern, non-stainless UHSS are comparable to the variability shown for six replicate experiments with AerMet 100 heat treated according to Table 1 and stressed at $-550 \mathrm{mV}_{\mathrm{SCE}}\left(7<\mathrm{K}_{\mathrm{TH}}<13 \mathrm{MPa} \sqrt{\mathrm{m}}\right.$ and $40<\mathrm{da} / \mathrm{dt}_{\mathrm{II}}<75 \mathrm{~nm} / \mathrm{s}$ at $\left.\mathrm{K}=40 \mathrm{MPa} \sqrt{\mathrm{m}}\right)$ 
[62,119]. Accordingly, the HEAC behavior of AerMet 100 and Ferrium M54 can be assumed identical, as would be exhibited by each alloy with sufficient sample size. Three replicate experiments with CrNiMoWV at $-550 \mathrm{mV}_{\mathrm{SCE}}$ show low variability: $\mathrm{K}_{\mathrm{TH}}$ ranges from 13-14 $\mathrm{MPa} \sqrt{\mathrm{m}}$ and $\mathrm{da} / \mathrm{dt}_{\mathrm{II}}$ is low at $20-28 \mathrm{~nm} / \mathrm{s}$. The strong SCC/HEAC resistance of Ferrium S53 is unique at $\mathrm{E}_{\mathrm{app}}=-550 \mathrm{mV} \mathrm{SCE}$ and illustrates the plasticity-based resolution limit. The different HEAC resistances shown in Figure 17 are specific to a fixed $E_{\text {app }}$ of $-550 \mathrm{mV}_{\mathrm{SCE}}$, which was either somewhat cathodic $\left(\sim 180 \mathrm{mV}_{\mathrm{SCE}}\right)$ to OCP for Ferrium S53 that exhibited the highest $\mathrm{K}_{\mathrm{TH}}$, somewhat anodic $\left(\sim 100 \mathrm{mV}_{\mathrm{SCE}}\right)$ to OCP for $300 \mathrm{M}$ that was most susceptible, or about equal to OCP for AerMet 100, Ferrium M54 and CrNiMoWV. Prior work identified that HEAC resistance was improved by mild cathodic polarization and degraded by anodic polarization for AerMet 100 in $\mathrm{NaCl}$ solution [1]. Clearly, it is important to establish the $\mathrm{E}_{\mathrm{app}}$ dependence of $\mathrm{K}_{\mathrm{TH}}$ and da/dt $\mathrm{dI}$ for each of these UHSS in order to understand metallurgical effects on HEAC.

\subsubsection{HEAC Kinetics and Fracture Path at $E_{a p p}=-1,000 m V_{S C E}$}

AerMet 100, Ferrium M54, Ferrium S53, CrNiMoWV and 300M tempered for ultra-high strength are susceptible to severe $\mathrm{HEAC}$ when stressed in $0.6 \mathrm{M} \mathrm{NaCl}$ with strong-cathodic polarization $\left(E_{\text {app }}=-1,000 m V_{S C E}\right)$. Subcritical crack growth kinetics for each alloy are represented in Figure 18. For all alloys, $\mathrm{K}_{\mathrm{TH}}$ is similarly low, 7-12 MPa $\sqrt{\mathrm{m}}$, compared to the variable-high values of $\mathrm{K}_{\mathrm{IC}}$ (Table 1). Variability in $\mathrm{K}_{\mathrm{TH}}$ between alloys is identical to previously reported replicate tests of AerMet 100 at $\mathrm{E}_{\mathrm{app}}=-550 \mathrm{mV}_{\mathrm{SCE}}[62,119]$. Following $\mathrm{K}_{\mathrm{TH}}$, crack growth is nearly $\mathrm{K}$-independent indicating a valid da/dt II measurement. Stage II crack growth rates are universally low for modern UHSS, and reduced by 2 orders of magnitude compared to legacy 300M. Stage II crack growth rates for Ferrium S53, Ferrium M54, and AerMet 100 are similar; 22-50 nm/s at K = $40 \mathrm{MPa} \sqrt{\mathrm{m}}$. Crack growth rate for CrNiMoWV is noticeably reduced; da/dt $/$ at $\mathrm{K}$ of $40 \mathrm{MPa} \sqrt{\mathrm{m}}$ is $9 \mathrm{~nm} / \mathrm{s}$. A replicate specimen of CrNiMoWV tested at $\mathrm{E}_{\mathrm{app}}=-1,000 \mathrm{mV}_{\mathrm{SCE}}$ exhibited similar-low crack growth rates and da/dt $/$ II $(13 \mathrm{~nm} / \mathrm{s}$ at $40 \mathrm{MPa} \sqrt{\mathrm{m}})$. Due to the small number of specimens tested, it is difficult to determine if low da/dt exhibited by CrNiMoWV represents a statistically significant departure from that for the other similar modern UHSS. However, six replicate experiments of AerMet 100 tested at $\mathrm{E}_{\mathrm{app}}=-550 \mathrm{mV}$ SCE showed $\sim 2$ times variability from low to high da/dtII. If

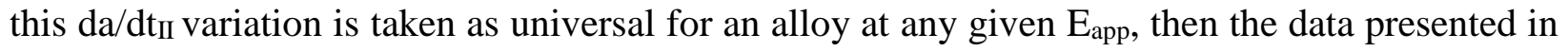
Figure 18 show that AerMet 100, Ferrium M54, and Ferrium S53 are within the expected single 
range of da/dt II. Stage II da/dt for CrNiMoWV is reduced 2.6 to 3.7 times from the mean da/dt $/$ of other modern UHSS at $\mathrm{E}_{\mathrm{app}}=-1,000 \mathrm{mV}_{\mathrm{SCE}}$ and likely represents cracking at a significantly lower rate.

Fracture path for SCC/HEAC is classified as intergranular (IG) along prior austenite grain boundaries, or transgranular (TG) with respect to prior austenite grains. Such TG cracking could reasonably involve the various martensite packet, block and/or lath interfaces. Previous studies of HEAC fracture in martensitic steels established 'quasi-cleavage' (QC) fracture along atypical cleavage planes $\left(\{110\}_{\alpha}\right.$, vs $\{100\}_{\alpha}$, for low temperature cleavage) as the dominant failure morphology $[116,120]$. Close agreement of $\{110\}_{\alpha^{\prime}}$ QC cleavage planes to $\{110\}_{\alpha^{\prime}}$ block/lath boundary planes indicates that $\mathrm{QC}$ fracture is correlated to cracking along block or lath interfaces [120]. A largely featureless 'flat' morphology is often observed intermixed with TG QC, and is associated with limited cracking along prior austenite boundaries [12]. Scanning electron fractographs in Figures 19-23 indicate the HEAC morphology for crack growth in each UHSS, at a $\mathrm{K}$ level immediately above $\mathrm{K}_{\mathrm{TH}}$ and adjacent to the precrack tip.

AerMet 100, Ferrium S53, and Ferrium M54 exhibit predominantly transgranular (TG) HEAC (Figures 19-21) with minor secondary (out-of-plane) cracking, speculatively along prior austenite or high angle packet boundaries (Figure 19a, small-black arrows). TG cracking correlates with low $\mathrm{K}_{\mathrm{TH}}$ of 7, 9, and $11 \mathrm{MPa} \sqrt{\mathrm{m}}$, respectively (Figure 18). TG HEAC in each alloy is dominated by QC (white arrows) intermixed with a few larger 'flat' facets (white/black arrows) $[11,12,116,120]$. The typical morphology of QC fracture features is shown for AerMet in Figure 19b (regions surrounding white arrows). QC facets are decorated with fine lath-like features and are near identical to previously observed SCC-HEAC fracture of AerMet 100 and similar lath martensite UHSS $[6,11,116]$. 


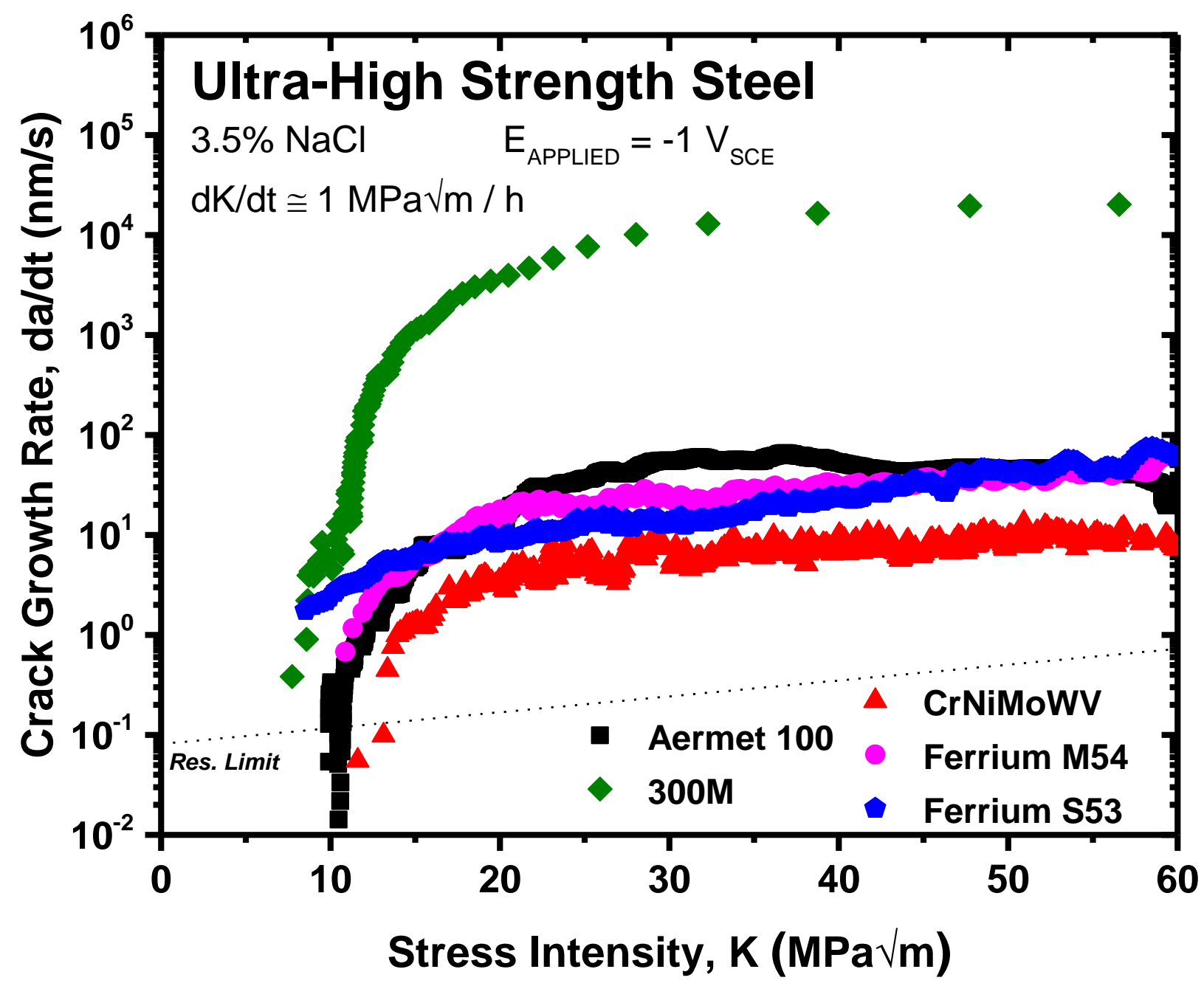

Figure 18 Subcritical crack growth rate versus $K$ for five ultra-high strength alloys stressed at $-1,000 \mathrm{~m} V_{S C E}$ in $0.6 \mathrm{M} \mathrm{NaCl}$ 

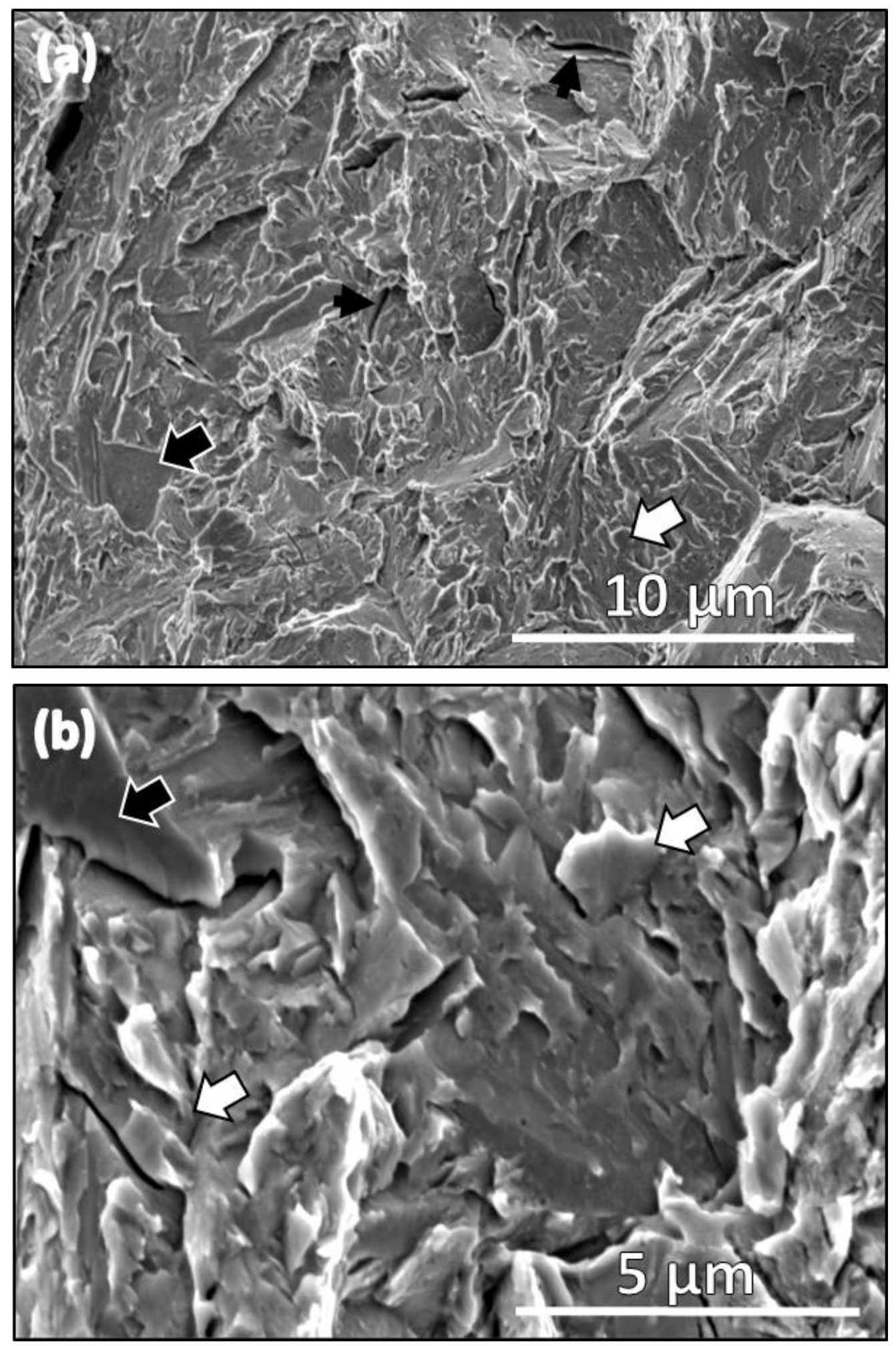

Figure 19 TG HEAC of AerMet $100\left(\mathrm{~K}=9 \mathrm{MPa}{ }^{\mathrm{m}}\right)$ stressed in $3.5 \% \mathrm{NaCl}$ at $\mathrm{E}_{\text {app }}=-1,000$ $m V_{S C E}$. Large white and black arrows indicate regions of $Q C$ and 'flat' morphologies, respectively. 

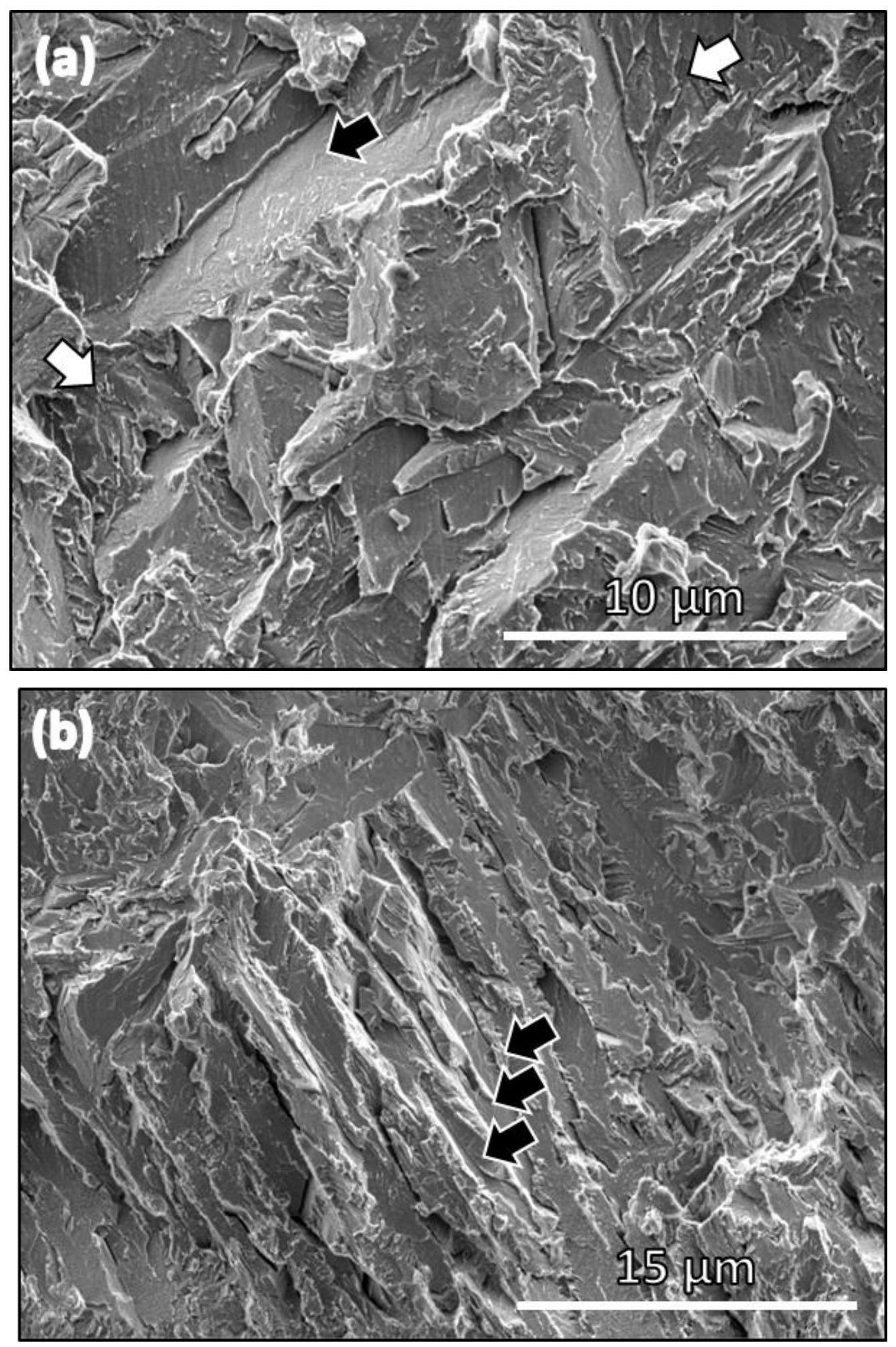


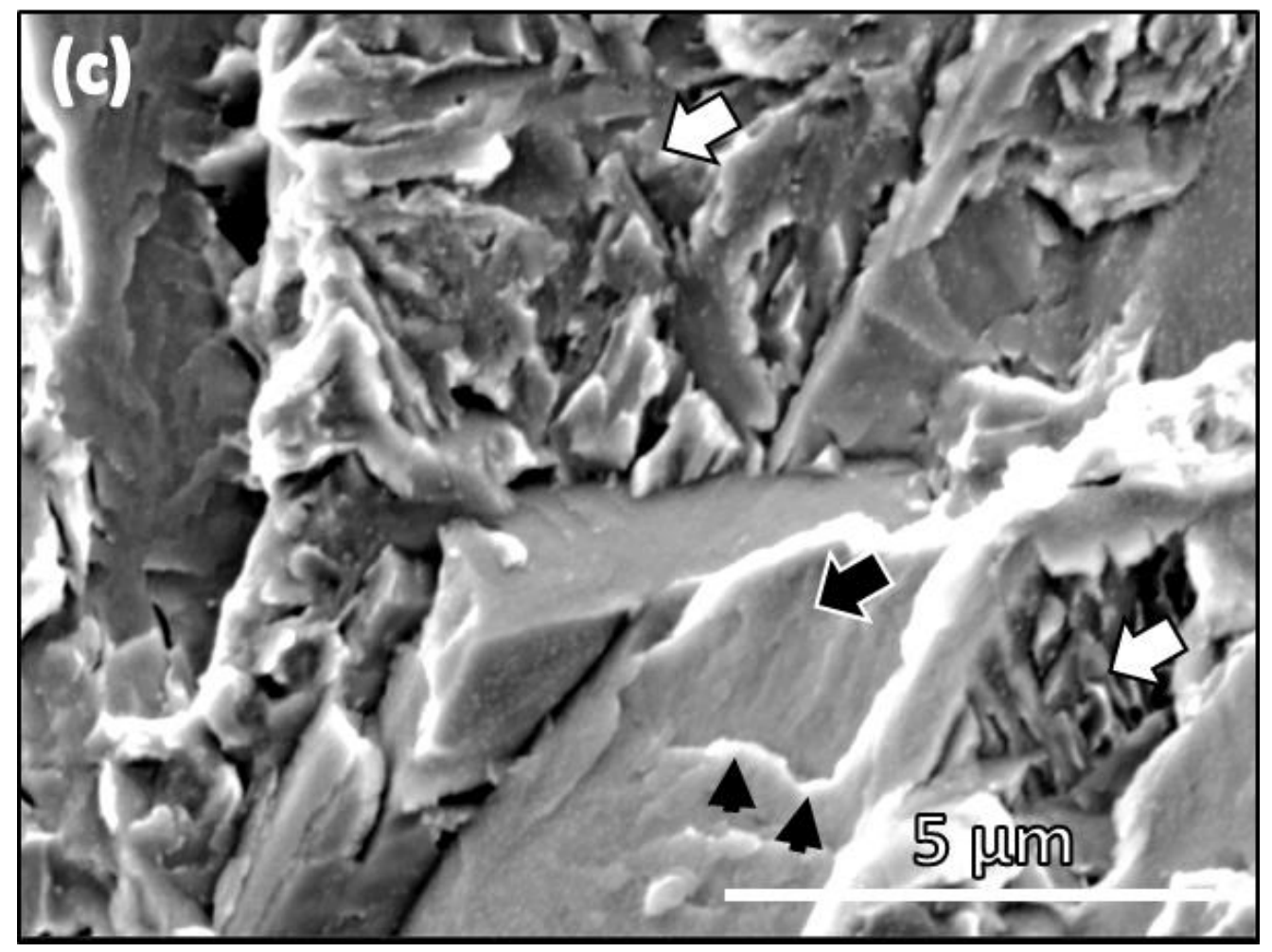

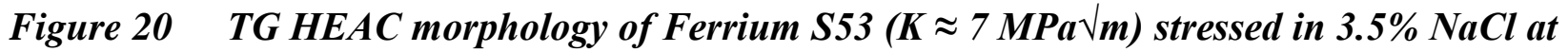
$E_{a p p}=-1,000 \mathrm{~m} V_{S C E}$. White and black arrows indicate regions of $Q C$ and flat facets, respectively.

HEAC in Ferrium S53 is TG with an equal mixture of QC identical to AerMet 100 (Figure 20, white arrows) and flat cleavage (Figure 20, black arrows). Three morphologies of 'flat' features are observed: (1) broad-flat clearly associated with large scale cracking along prior austenite grain or packet boundaries (Figure 20a, black arrow), (2) regular blocky steps of flat morphology 1-3 $\mu \mathrm{m}$ wide (Figure 20b, black arrows) which match the average spacing and shape of single martensite blocks shown in Figure 10 (black arrows), and (3) fine scale isolated regions of flat facets intermixed with QC fracture on TG facets (Figure 20c, black arrow). All morphologies are decorated with fine cleavage-like river marks (Figure 20c, black arrowheads), but represent different crack paths through the microstructure.

TG HEAC in Ferrium M54 exhibits unique large martensite packet-shaped features (Figure 21a, dotted line) often separated by out of plane cracking along prior austenite or martensite packet boundaries (Figure $21 \mathrm{a}$ and b, black arrows). The surface of larger packet features is decorated with a fine scale mixture of QC and 'flat' features (Figure 21c, white and black arrows, 
respectively). Identical to Ferrium S53, many 'flat' regions are intermixed with TG QC features and cannot be associated with intergranular cracking along prior austenite grain boundaries.
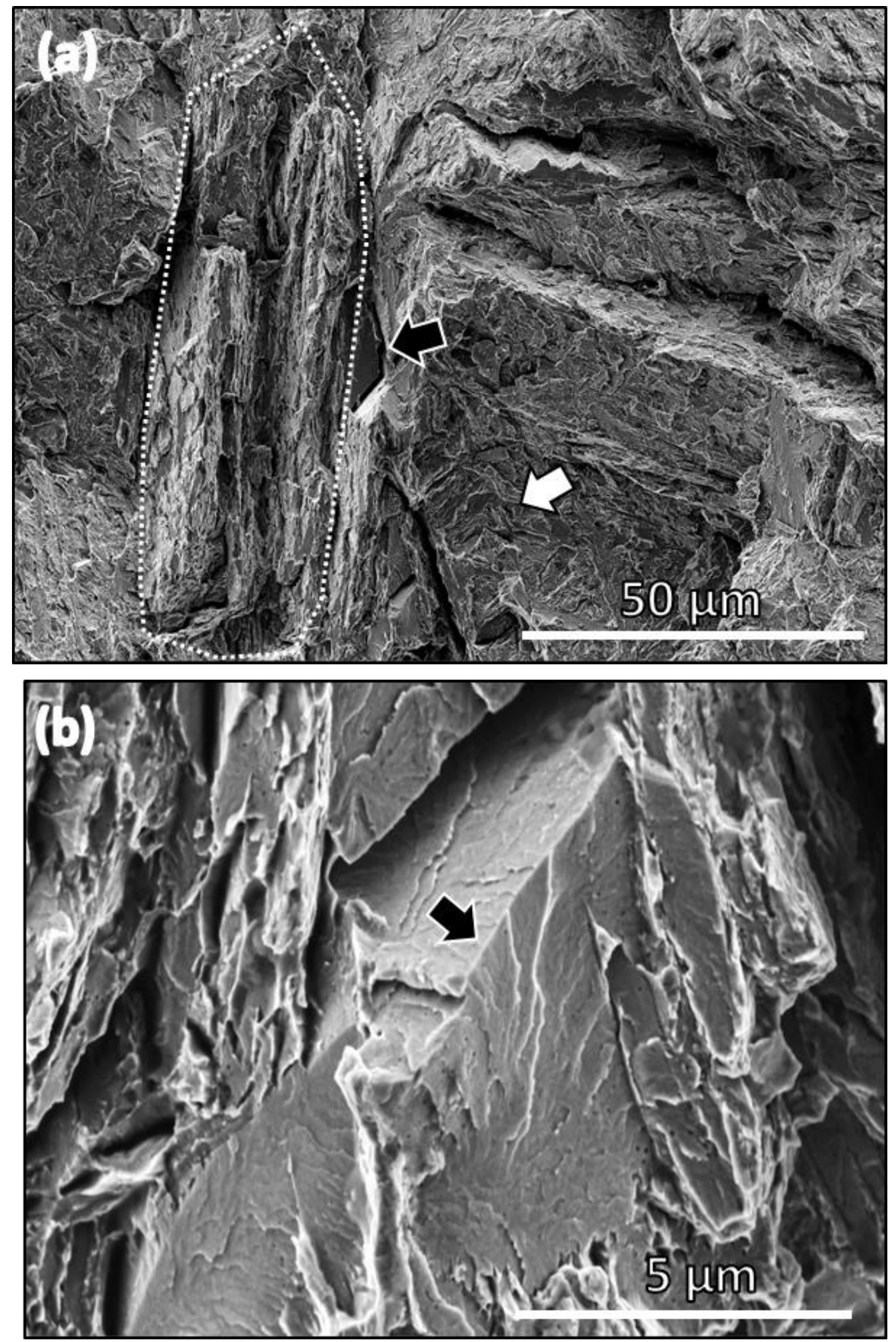


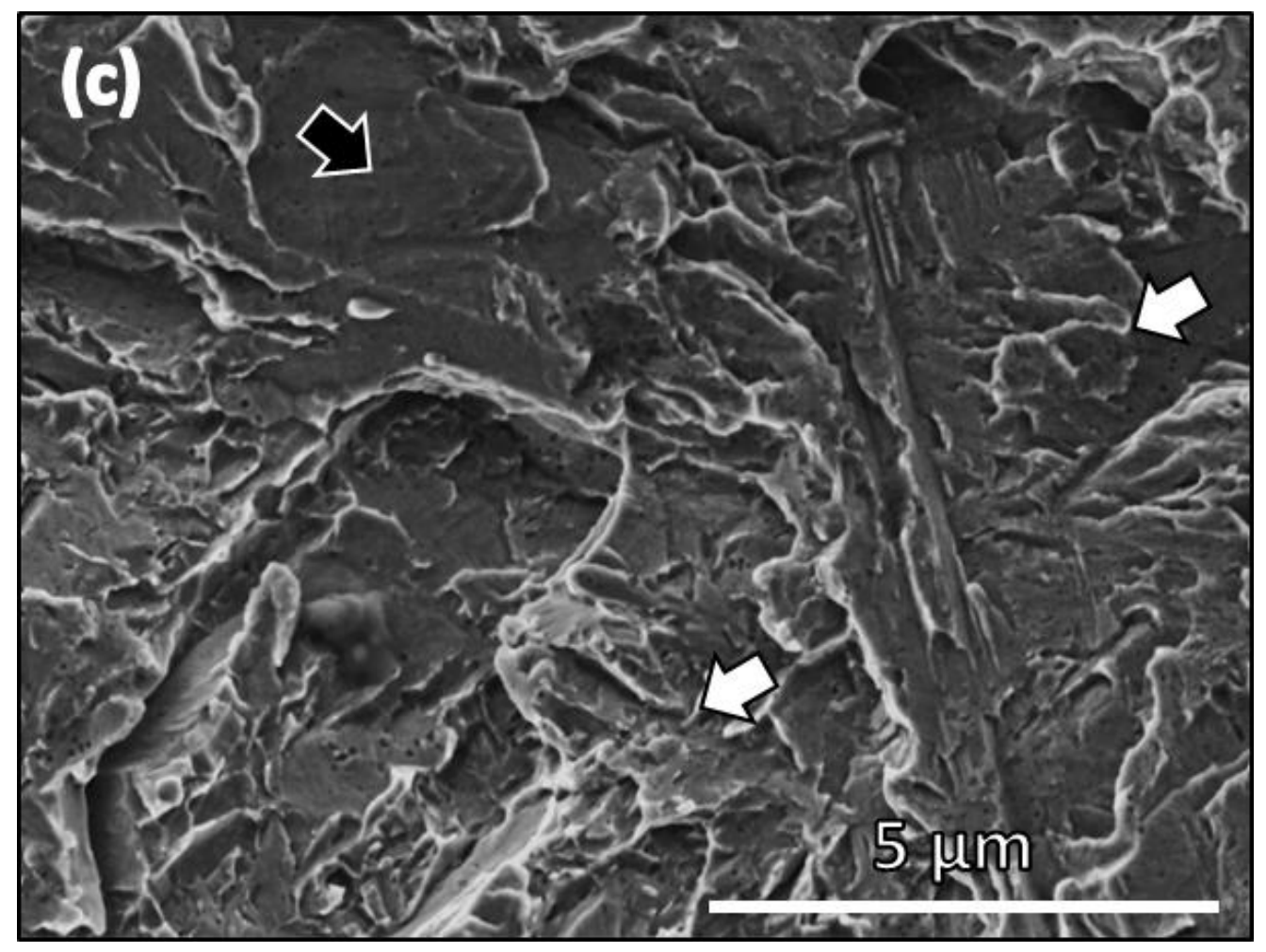

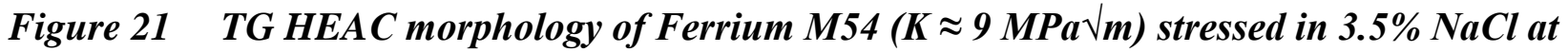
$E_{a p p}=-1,000 \mathrm{~m} V_{S C E}$. White and black arrows indicate regions of $Q C$ and 'flat', respectively.

Predominantly IG SCC-HEAC along prior austenite grain boundaries is exhibited by 300M and CrNiMoWV (Figure 22-23), correlated with low $\mathrm{K}_{\mathrm{TH}}$ of 8 and $13 \mathrm{MPa} \sqrt{\mathrm{m}}_{\text {, respectively (Figure }}$ 18). Dominant IG HEAC is intermixed with TG features (Figure 22a and Figure 23a, white arrows), often bisecting prior austenite grains and decorated with quasi-cleavage features. Intergranular facets in $300 \mathrm{M}$ are largely featureless, showing limited amounts of small ridges (Figure 22b, black arrow heads). Shallow, parallel crevices are present on a large fraction of the 300M IG facets (Figure 22b). It is unknown if these are associated with H-cracking, as they do not appear to match any EBSD-measured microstructural feature. The linear nature and spacing of the crevices suggests that they could represent slip steps or ridges from discontinuous crack advance. Speculatively, such features experienced localized corrosion during the short time during HEAC test unloading where the specimen was not protected under cathodic polarization. $300 \mathrm{M}$ is extremely susceptible to corrosion in chloride containing environments and small slip band features could be rapidly dissolved [8,121]. 

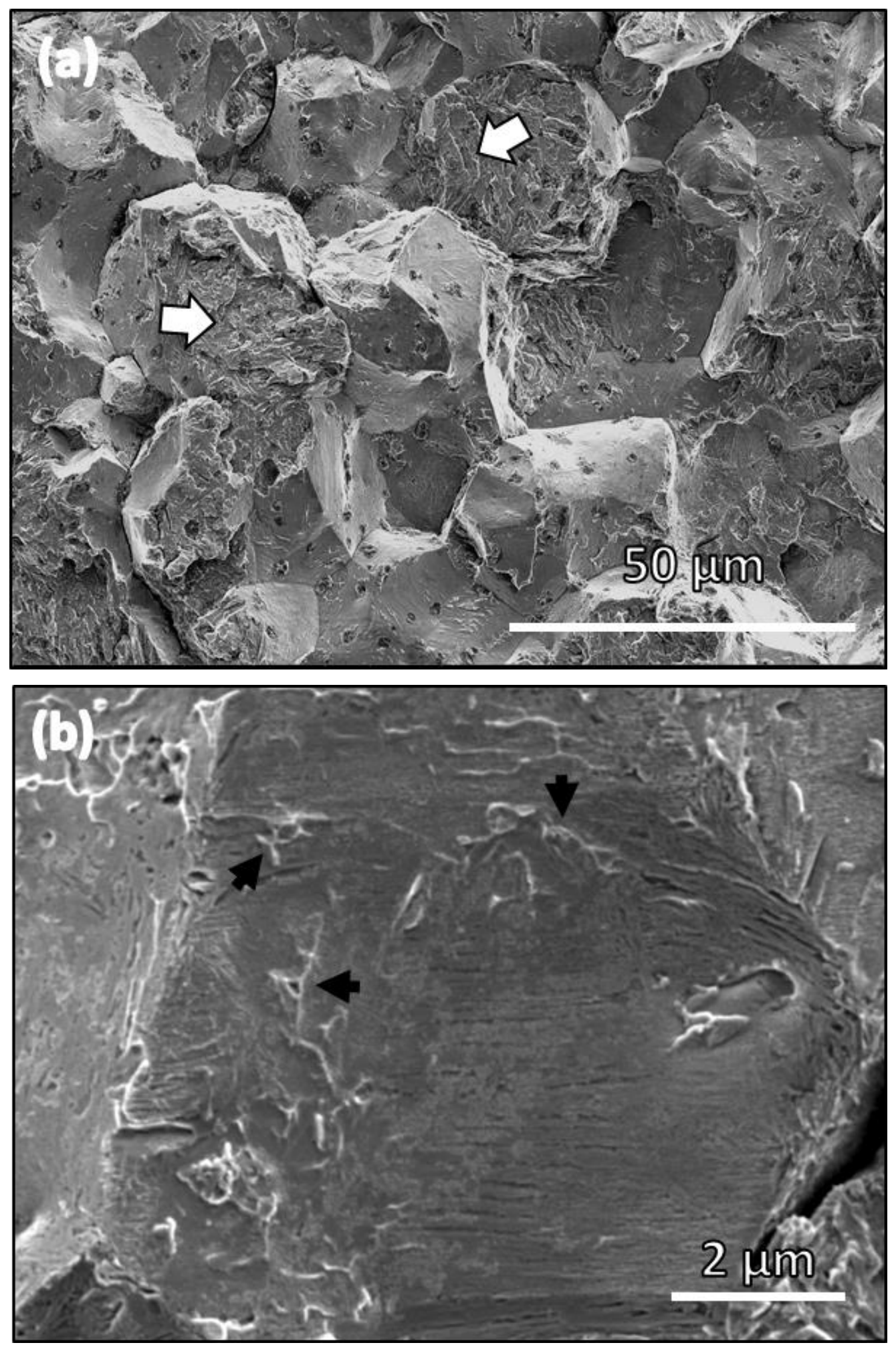

Figure 22 IG HEAC morphology 300M $(\mathrm{K} \approx 8 \mathrm{MPa} \sqrt{\mathrm{m}})$ stressed in $3.5 \% \mathrm{NaCl}$ at $E_{a p p}=$ $-1,000 \mathrm{~m} V_{S C E}$. White and black arrows indicate regions of $Q C$ and 'flat', respectively. 
Intergranular HEAC facets in CrNiMoWV show a substantially increased density of fine ridges compared to 300M, and some evidence of fine scale microvoid coalescence (Figure 23d, black and white arrowheads, respectively). Such ridges and small microvoids are strong evidence that IGHEAC is associated with significant small-scale plasticity at the prior austenite grain boundaries. This result stands in contrast to IG-HEAC in 300M which exhibits flat, largely featureless IG facets typical of brittle fracture. This TG HEAC in CrNiMoWV exhibits a similar QC morphology as Ferrium M54 (Figure 21c and Figure 23c). However, the size of the intermixed intragranular 'flat' regions is substantially reduced in CrNiMoWV. 

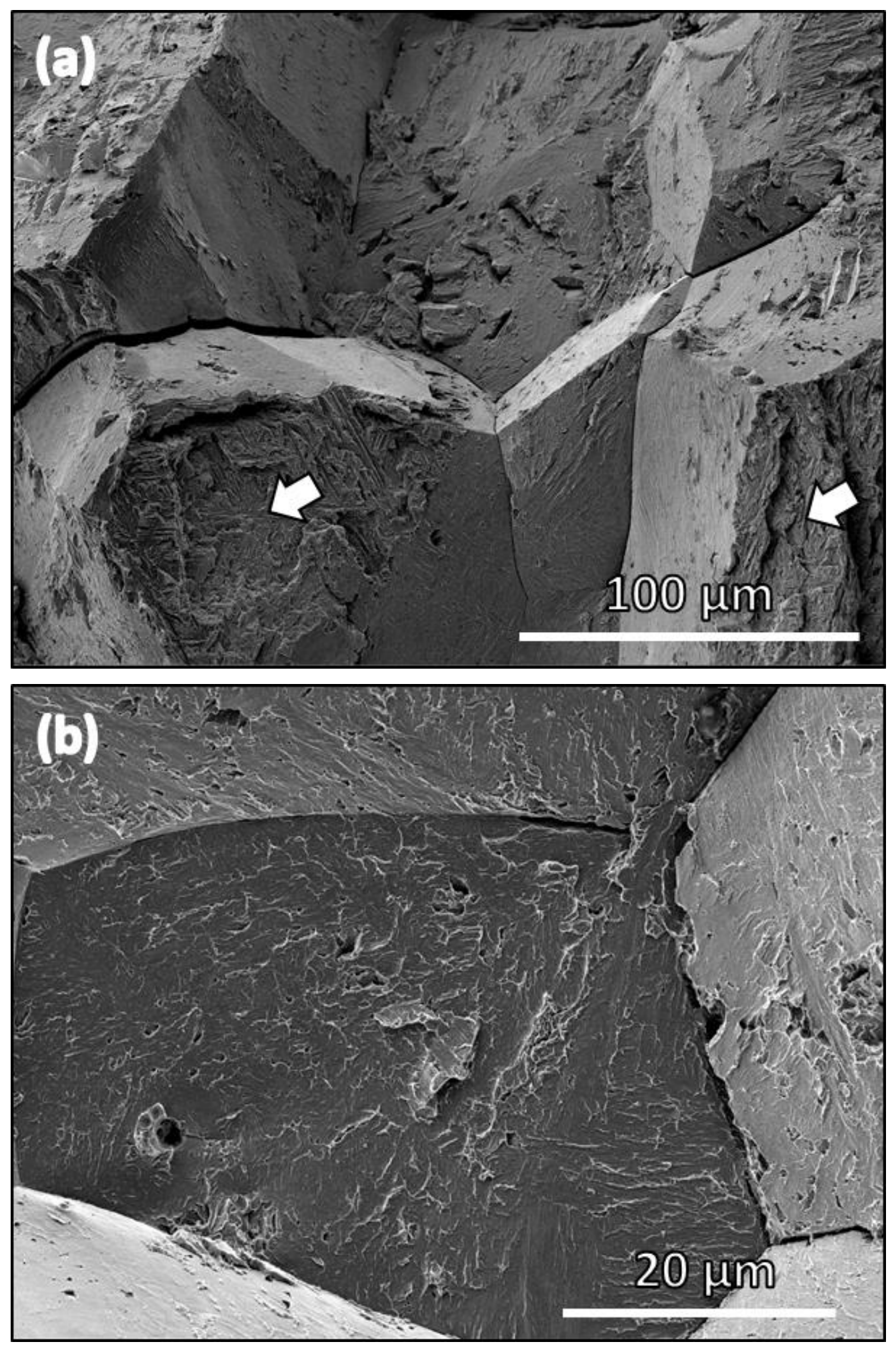

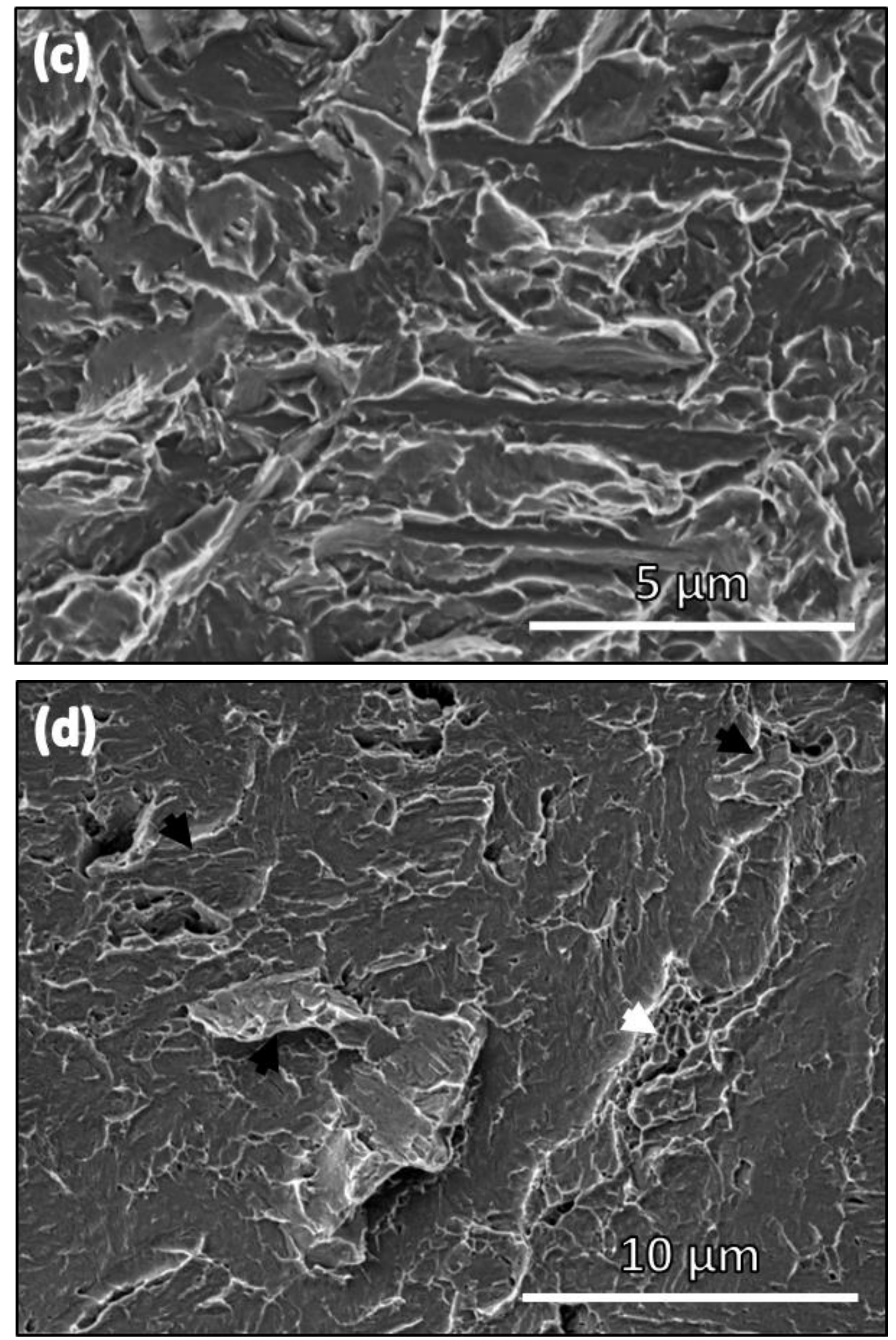

Figure 23 (a) Mixed IG and TG HEAC of CrNiMoWV $(K \approx 13 \mathrm{MPa} \vee \mathrm{m})$ stressed in 3.5\% $\mathrm{NaCl}$ at $\mathrm{E}_{\text {app }}=-1,000 \mathrm{mV} \mathrm{V}_{\mathrm{SCE}}$, (b) Multiple IG HEAC facets (c) Detail of TG quasi-cleavage from the white arrow in (a), and (d) detail of an IG facet from (b). 


\subsubsection{Effect of $E_{\text {app }}$ on HEAC Kinetics}

The effect of applied potential on $\mathrm{K}_{\mathrm{TH}}$ for five UHSS alloys is shown in Figure 24. With the exception of $300 \mathrm{M}$, all alloys exhibit unambiguous evidence of a window of mild-to-moderate cathodic polarization that confers strong HEAC resistance. The precise shape of the threshold dependence on $E_{\text {app }}$ in this regime is uncertain due to the necessarily limited number of experiments, and approximate trend lines are logically positioned. As $\mathrm{E}_{\text {app }}$ is decreased from OCP, $\mathrm{K}_{\mathrm{TH}}$ rises sharply reaching an observed peak at 57, 76, 76, and $79 \mathrm{MPa} \sqrt{\mathrm{m}}$ for Ferrium M54, AerMet 100, CrNiMoWV, and Ferrium S53, respectively. Significant $\mathrm{K}_{\mathrm{TH}}$ variability is observed in replicate experiments of AerMet 100 at $\mathrm{E}_{\mathrm{APP}}=-625 \mathrm{mV}_{\mathrm{SCE}}$; SEM-confirmed HEAC growth occurred in $50 \%$ of four replicate tests $\left(\mathrm{K}_{\mathrm{TH}}=40\right.$ and $\left.44 \mathrm{MPa} \sqrt{\mathrm{m}}\right)$, indicating that the remaining AerMet 100 specimens tested at $-625 \mathrm{mV}_{\mathrm{SCE}}$ show HEAC immunity to $>70 \mathrm{MPa} \sqrt{ } \mathrm{m}$. Bimodal $\mathrm{K}_{\mathrm{TH}}$ variability at $\mathrm{E}_{\text {app }}$ slightly cathodic to OCP has been previously observed in austenitized, cryogenically chilled (5 h in liquid nitrogen) and optimally aged AerMet 100 [119]. It is unknown if replicate experiments of CrNiMoWV, Ferrium M54, and Ferrium S53 would exhibit similar variation in this regime where $\mathrm{E}_{\mathrm{app}}$ confers near-maximum $\mathrm{K}_{\mathrm{TH}}$.

Strong HEAC resistance is stable over a range of applied potentials (e.g. $-750 \leq \mathrm{E}_{\text {app }} \leq-550 \mathrm{mV} \mathrm{V}_{\mathrm{SCE}}$ for Ferrium S53 and $-725 \leq \mathrm{E}_{\text {app }} \leq-575 \mathrm{mV}_{\mathrm{SCE}}$ for Ferrium M54). Limited samples of CrNiMoWV were tested; expected trends (Figure 24, red dash) in $\mathrm{K}_{\mathrm{TH}} \mathrm{vs} \mathrm{E}_{\text {app }}$ are extrapolated based on the behavior observed in Ferrium M54 and AerMet 100. The data for Ferrium M54 are limited between $E_{\text {app }}=-550 \mathrm{mV}_{\mathrm{SCE}}$ and $\mathrm{E}_{\mathrm{app}}=-650 \mathrm{mV} \mathrm{V}_{\mathrm{SCE}}$. A sharp spike in $\mathrm{K}_{\mathrm{TH}}$, identical to AerMet 100, in this range of mildly cathodic $\mathrm{E}_{\mathrm{app}}$ cannot be ruled out. Given the comparatively high maximum $\mathrm{K}_{\mathrm{TH}}$ in similar UHSS AerMet 100, Ferrium S53, and CrNiMoWV, it is logical to assume that such a peak would be revealed by additional testing.

A window of protective polarization conferring elevated $\mathrm{K}_{\mathrm{TH}}$ is not observed in $300 \mathrm{M}$; measured $\mathrm{K}_{\mathrm{TH}}$ monotonically decreases from 15.4 MPa $\sqrt{\mathrm{m}}$ at $\mathrm{E}_{\text {app }}=-550 \mathrm{mV}_{\mathrm{SCE}}$ to $8.2 \mathrm{MPa} \sqrt{\mathrm{m}}$ at $-1,000$ $\mathrm{mV}_{\mathrm{SCE}}$. Due to the limited number of samples tested, improved HEAC resistance at $\mathrm{E}_{\mathrm{app}}$ which is 100 to $200 \mathrm{mV}_{\text {SCE }}$ cathodic to the OCP (-650 mV $\mathrm{mCE}_{\mathrm{SC}}$ cannot be ruled out. However, previous work did not observe cathodic prevention of HEAC in identically tempered 300M stressed in 3.5\% $\mathrm{NaCl}$; $\mathrm{K}_{\mathrm{ISCC}}$ measured via rising step-load is constant (14.5 to $\left.17.7 \mathrm{MPa} \sqrt{\mathrm{m}}\right)$ in the examined 
potential range $\left(E_{\text {app }}=-700,-800,-1000\right.$, and $\left.-1200 \mathrm{mV}_{\mathrm{SCE}}\right)[122,123]$. Logically, this would imply two possibilities: (1) 300M does not exhibit a window of $\mathrm{E}_{\text {app }}$ conferring elevated $\mathrm{K}_{\mathrm{TH}}$, or (2) $\mathrm{K}_{\mathrm{TH}}$ is locally elevated between either $-800 \leq \mathrm{E}_{\text {app }} \leq-800 \mathrm{mV}_{\mathrm{SCE}}$ or $-1000 \leq \mathrm{E}_{\text {app }} \leq-800 \mathrm{mV} \mathrm{V}_{\text {SCE}}$. Mechanism-based modeling in the Discussion will examine reasons for the lack and the degree of cathodic prevention of HEAC in 300M.

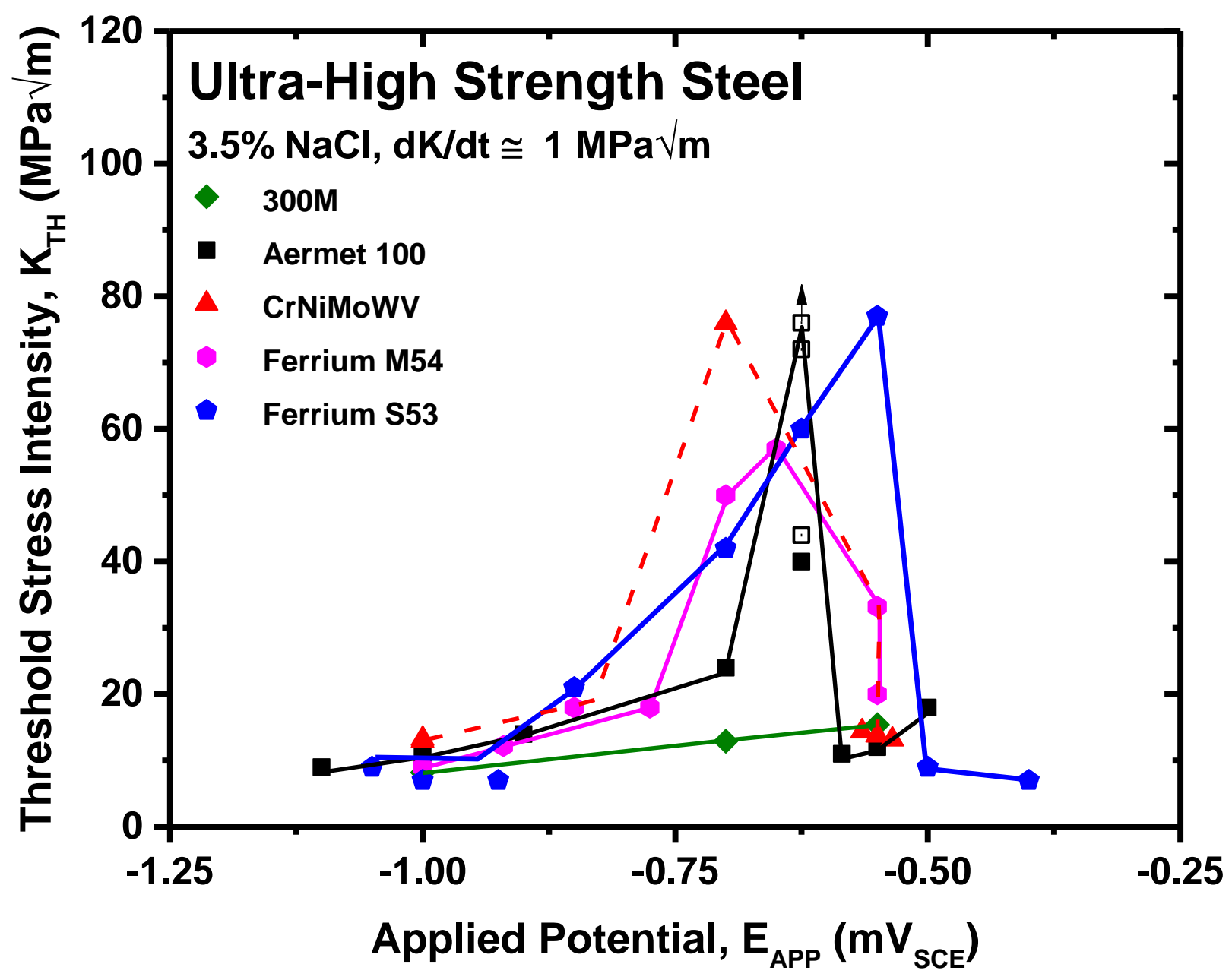

Figure 24 Threshold stress intensity $\left(K_{T H}\right)$ vs. applied potential $\left(E_{\text {app }}\right)$ for five ultra-high strength steels stressed in $0.6 \mathrm{M} \mathrm{NaCl}$. Closed squares (ロ) represent previously reported data [1] while open squares ( $\square$ ) represent data generated in the present study for AerMet 100. The trend line for CrNiMoWV is extrapolated from trends observed in similar alloys Ferrium M54 and AerMet 100. Three replicate experiments $\left(E_{a p p}=-550 \mathrm{~m} V_{S C E}, K_{T H}=12.0,13.8,14.4 \mathrm{MPa}{ }^{\mathrm{m}}\right)$ with CrNiMoWV are spread in applied potential for clarity. 
Observed maximum values of $\mathrm{K}_{\mathrm{TH}}$ for $\mathrm{CrNiMoWV}$ and AerMet $100(76 \mathrm{MPa} \sqrt{\mathrm{m}})$ are significantly greater than those for Ferrium M54 (57 MPa $\sqrt{m})$. However, the threshold variability observed in AerMet 100 may be present in Ferrium M54 at $\mathrm{E}_{\text {app }}$ slightly cathodic to OCP, raising $\mathrm{K}_{\mathrm{TH}}$ at these unique $\mathrm{E}_{\mathrm{app}}$. At $\mathrm{E}_{\mathrm{app}}$ outside of the protective window for AerMet 100, Ferrium M54, Ferrium S53, and CrNiMoWV, $\mathrm{K}_{\mathrm{TH}}$ rapidly reduces to a common value of 6-12 $\mathrm{MPa} \sqrt{\mathrm{m}}$. This degradation is much sharper in the anodic direction compared to the cathodic direction. For example, in AerMet $100 \mathrm{~K}_{\mathrm{TH}}$ falls by 4-7 times from $40-76 \mathrm{MPa} \sqrt{\mathrm{m}}$ to $11 \mathrm{MPa} \sqrt{\mathrm{m}}$ over an anodic increase in $\mathrm{E}_{\mathrm{app}}$ of $40 \mathrm{mV}_{\mathrm{SCE}}\left(-625\right.$ to $\left.-585 \mathrm{mV}_{\mathrm{SCE}}\right)$. Conversely, a cathodic shift of $75 \mathrm{mV}_{\mathrm{SCE}}$ reduces $\mathrm{K}_{\mathrm{TH}}$ by a comparatively small $1.6-3$ times $\quad\left(40-76 \mathrm{MPa} \sqrt{\mathrm{m}}\right.$ at $\mathrm{E}_{\mathrm{app}}=-625 \mathrm{mV}_{\mathrm{SCE}}$ to $24 \mathrm{MPa} \sqrt{\mathrm{m}}_{\text {at }} \mathrm{E}_{\mathrm{app}}$ $=-700 \mathrm{mV}_{\text {sce}}$ ). Anodic-direction $\mathrm{K}_{\mathrm{TH}}$ degradation is similarly sharp in Ferrium S53; specifically, 77 to $9 \mathrm{MPa} \sqrt{\mathrm{m}}$ over an anodic increase in $\mathrm{E}_{\text {app }}$ of $50 \mathrm{mV}_{\mathrm{SCE}}\left(-550\right.$ to $\left.-500 \mathrm{mV}_{\mathrm{SCE}}\right)$. Reduction in $\mathrm{K}_{\mathrm{TH}}$ due to cathodic polarization of Ferrium S53 is less sharp than in AerMet 100 or Ferrium M54; a cathodic shift of $225 \mathrm{mV}_{\mathrm{SCE}}\left(-700\right.$ to $-925 \mathrm{mV}_{\mathrm{SCE}}$ ) is required to reduce $\mathrm{K}_{\mathrm{TH}}$ from 42 to $7 \mathrm{MPa} \sqrt{\mathrm{m}}_{\text {. }}$ However, it must be noted that these trends are based upon relatively few data points in the nearOCP and anodic regimes of $E_{\text {app. }}$ Reduction in $\mathrm{K}_{\mathrm{TH}}$ with increasing anodic or cathodic polarization is unknown for CrNiMoWV without further experiments at moderate cathodic $\mathrm{E}_{\text {app. }}$.

In all alloys, reduced $\mathrm{K}_{\mathrm{TH}}$ with increasing cathodic and anodic-direction polarization is accompanied by a rise in $\mathrm{da} / \mathrm{dt}_{\mathrm{II}}$, as shown in Figure $25 \mathrm{using}$ the $\mathrm{da} / \mathrm{dt}$ measured at $\mathrm{K}=40 \mathrm{MPa} \sqrt{\mathrm{m}}$. The rate of $\mathrm{da} / \mathrm{dt}_{\mathrm{II}}$ increase with increasingly cathodic $\mathrm{E}_{\text {app }}$ below the protection regime is nearly identical for the secondary hardening alloys (AerMet 100, Ferrium M54, and Ferrium S53), rising on average by two orders of magnitude as $E_{\text {app }}$ is decreased to $-1,000 \mathrm{mV}_{\mathrm{SCE}}$. Crack growth rates for legacy alloy $300 \mathrm{M}$ are significantly elevated at all investigated potentials, and increase modestly (6.5 times) as $\mathrm{E}_{\mathrm{app}}$ is decreased from $-550 \mathrm{mV}$ SCE to $-1,000 \mathrm{mV}_{\mathrm{SCE}}$. 


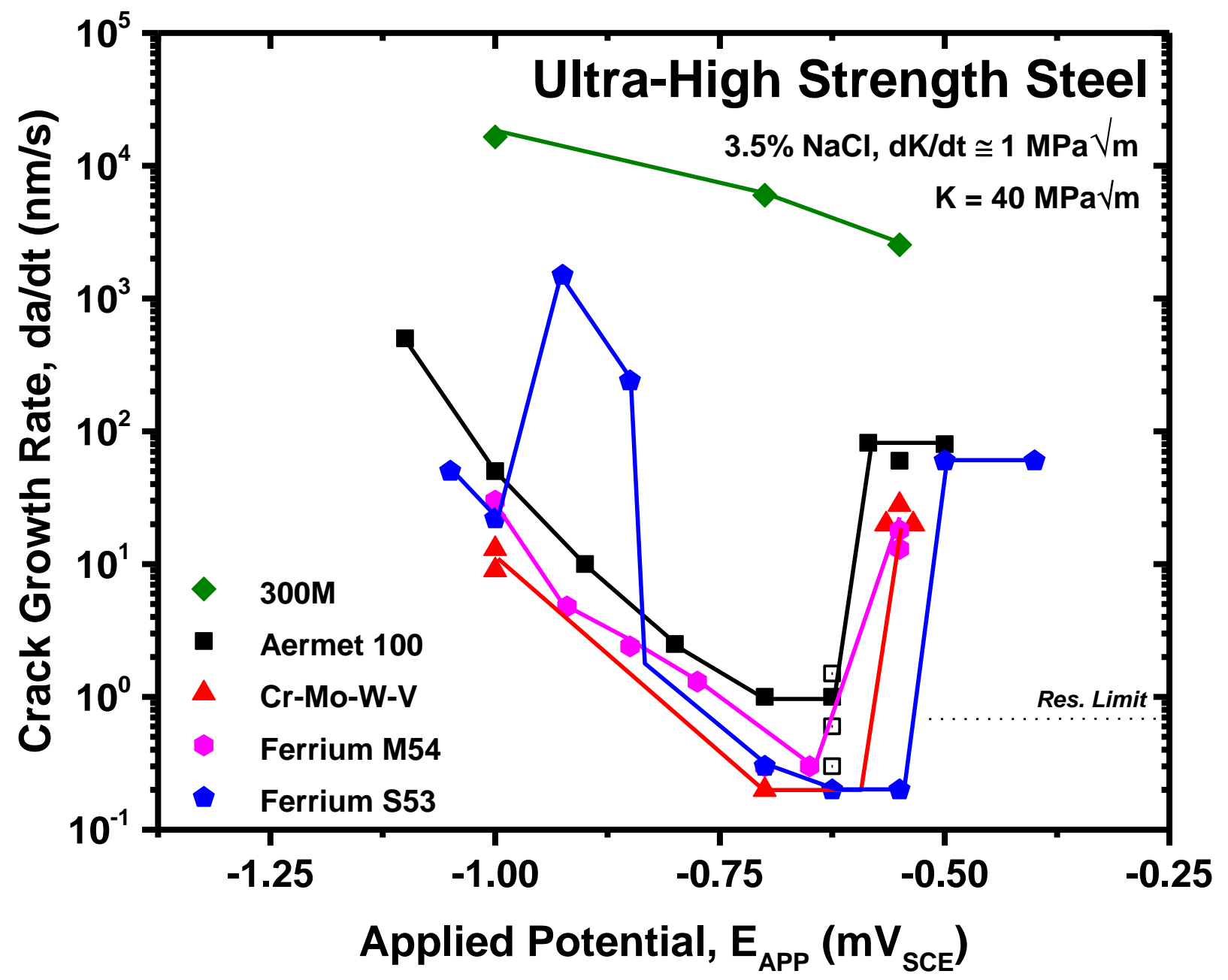

Figure 25 Stage II crack growth rate (da/dt $\left.t_{I I}\right)$ versus applied potential $\left(E_{a p p}\right)$ for five ultrahigh strength steels stressed in $0.6 \mathrm{M} \mathrm{NaCl}$. Closed squares (a) represent previously reported data [1], while open squares () represent data generated in the present study for AerMet 100. Three replicate experiments (Eapp $\left.=-550 \mathrm{~m} V_{S C E}, d a / d t_{I I}=20,20,28 \mathrm{~nm} / \mathrm{s}\right)$ of CrNiMoWV are spread in potential for clarity.

At $E_{\text {app }}=-925$ and $-850 \mathrm{mV}_{\mathrm{SCE}}$, corrosion resistant Ferrium S53 with significant $\mathrm{Cr}$ addition exhibits da/dtII values that are 1-2 orders of magnitude greater than the average trend for the other modern UHSS. Additional cathodic polarization by $75 \mathrm{mV}_{\text {SCE }}$ reduces da/dt II to $22 \mathrm{~nm} / \mathrm{s}$, in line with the general trend of modern UHSS in Figure 22. Acceleration of $\mathrm{da} / \mathrm{dt}_{\mathrm{II}}$ is also observed in Ferrium S53 tested at $E_{\text {app }}=-700 \mathrm{mV}_{\mathrm{SCE}}$, but is not captured in da/dt II measured at $\mathrm{K}=40 \mathrm{MPa} \sqrt{\mathrm{m}}$ because $\mathrm{K}_{\mathrm{TH}}$ equals $42 \mathrm{MPa} \sqrt{\mathrm{m}}$. This behavior has been previously observed in $\mathrm{Ni}_{3} \mathrm{Ti}$ strengthened, stainless UHSS Ferrium PH48S at similar Eapp [124], as discussed in Chapter 4. The source of 
$\mathrm{da} / \mathrm{dt}$ acceleration at moderate cathodic polarization in modern stainless UHSS is considered in the Discussion.

\subsubsection{Fractal Analysis of Martensite Interface Connectivity}

Previous work has established that the connectivity of randomly arranged interface boundaries can directly influence the susceptibility of polycrystalline metals to segregated impurity and hydrogen assisted cracking $[49,50]$. Based on these observations, the connectivity of the random boundary network (RBN) was hypothesized to contribute to the observed differences in HEAC susceptibility of UHSS (Figure 24). As shown in Table 6 the fractional distribution of low-misorientation angle CSL, and high-misorientation angle boundaries is largely similar, implying the same qualitative makeup of the boundaries in each UHSS. Total interfacial area $\left(\mathrm{S}_{\mathrm{V}}\right)$ of random high-angle interfaces varies from 972 (CrNiMoWV) to 1,456 (AerMet 100) $\mathrm{mm}^{2} / \mathrm{mm}^{3}$. Significantly increased $\mathrm{S}_{\mathrm{V}}$ of random boundaries suggests that the connectivity of random high-angle interfaces varies among UHSS. Connectivity of the RBN was assessed via the box counting method, as characterized by the fractal dimension of the MRBC, $D_{R}$.

Analysis established that the RBN network in each martensitic UHSS microstructure is near 0, without differentiation among the steels investigated. As shown in Figure 13c and Figure 16c, small segments of CSL $\left(\sum 11, \sum 13 \mathrm{~b}\right.$ and $\left.\sum 25\right)$ boundaries of 0.25 to $1.5 \mu \mathrm{m}$ length were often observed to interrupt otherwise continuous high angle random boundaries. Such boundaries disrupt the connectivity of the RBN, as they constitute discrete gaps in the network. This effect is visualized in Figure 26 and severely limits the length of the MRBC for all UHSS alloys. Subsequently, $D_{R}$ is reduced to unity for all alloys, implying near zero connectivity of the RBN.

Critically, the box counting method for calculating $D_{R}$ has only been previously applied to coarse grained polycrystalline nickel where the main concern is control of IG cracking [49]. This calculation is extremely sensitive to interruptions in random boundary connectivity, which reduce the length of the MRBC. The size scale of the CSL interruptions $(0.25$ to $1.5 \mu \mathrm{m})$ is reduced $\sim 100$ times in modern UHSS compared to previous work in polycrystalline nickel. Many fine scale CSL boundaries are $<1 \mu \mathrm{m}$ in length compared to 20-100 $\mu \mathrm{m}$ in nickel. Similar fractal connectivity analyses in lath martensite required the use of computational methods to 'reconstruct' uneven grain boundaries with a gap of up to $0.3 \mu \mathrm{m}$ [108]. Further, hydrogen damage during HEAC initiates 

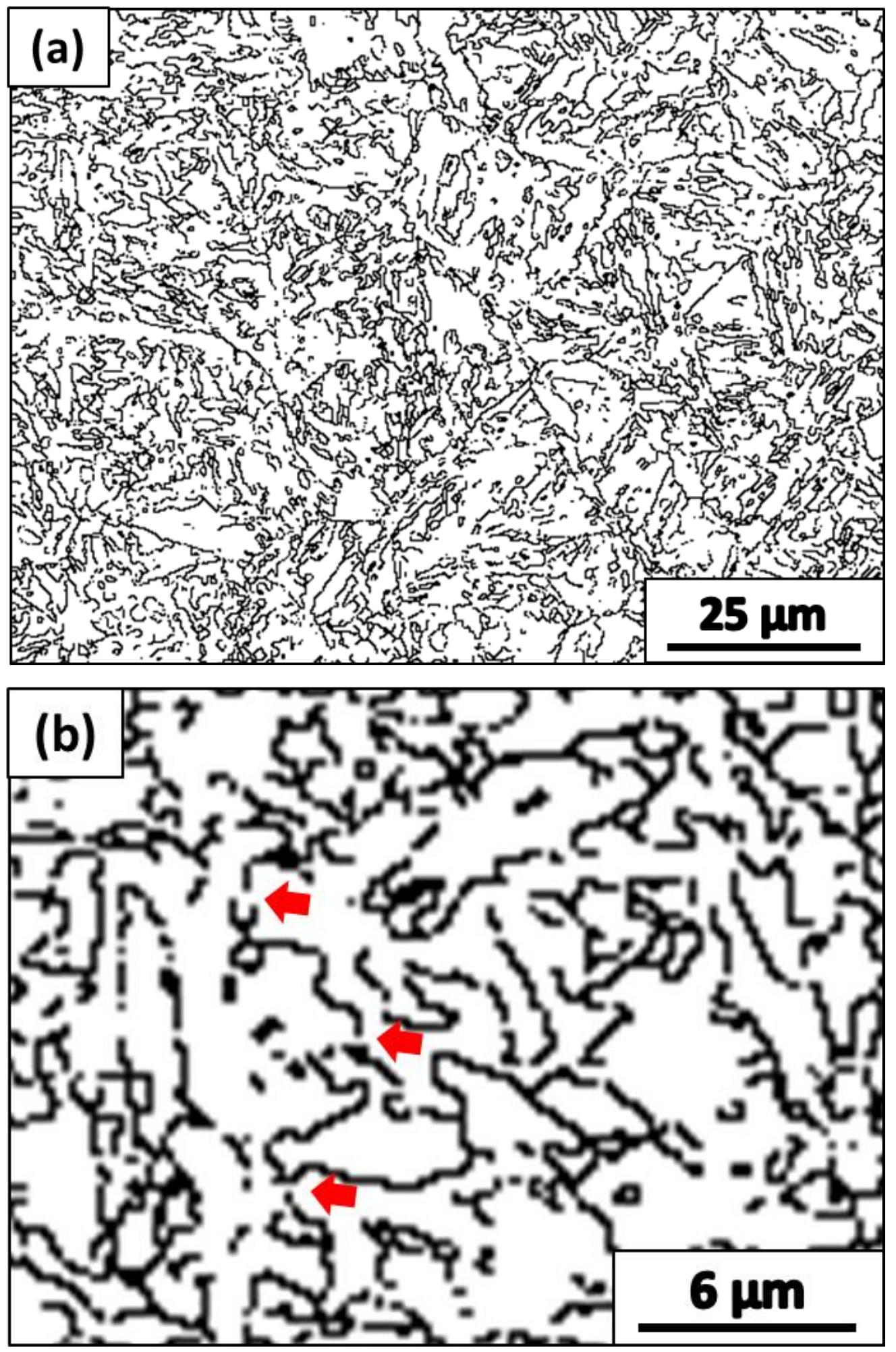

Figure $26 \quad R B N$ map of 300M (a) Map of random interfaces corresponding to the microstructure of 300M shown in Figure 2. (b) Detail image showing the small interruptions (red arrows) that are present at regular intervals on random boundaries from (a). 
ahead of the crack tip at $\chi_{\text {crit }}(1 \mu \mathrm{m})$, and may completely bypass relatively small segments of CSL boundary in an otherwise continuous RBN, particularly when the 3-dimensional character of the crack tip FPZ is considered.

Based upon these observations, the fractal dimension of the MRBC for the five UHSS alloys was reassessed, allowing variable-small (1-5 pixel) discrete gaps in the network to be considered as connected. The result is a dramatic increase in measured connectivity. The fractal dimension of the MRBC exhibits a step function with increasing gap tolerance in all alloys. Allowing a pixel gap tolerance of $\geq 2$ pixels (320-400 nm of physical distance) resulted in measured $D_{R}$ of 1.8-1.9, indicating near-full connectivity of the RBN through the microstructure [49].

\begin{tabular}{|c|c|c|c|c|}
\hline & \multicolumn{4}{|c|}{ Pixel Gap Allowed } \\
\cline { 2 - 5 } & $\mathbf{0}$ & $\mathbf{1}$ & $\mathbf{2}$ & $\mathbf{5}$ \\
\hline $\mathbf{3 0 0 M}$ & 1.01 & 1.01 & 1.82 & 1.85 \\
\hline AM100 & 1.03 & 1.04 & 1.87 & 1.89 \\
\hline CrNiMoWV & 1.02 & 1.02 & 1.91 & 1.93 \\
\hline Ferrium M54 & 1.01 & 1.04 & 1.86 & 1.88 \\
\hline Ferrium S53 & 1.03 & 1.04 & 1.90 & 1.95 \\
\hline
\end{tabular}

Table 7 Calculated $D_{R}$ of UHSS alloys, using a variable pixel gap tolerance

The physical basis for a pixel gap tolerance in boundary connectivity must be carefully considered before any quantitative conclusions can be reached. Regardless of the chosen tolerance, it can be conclusively stated that the connectivity of high angle boundaries in UHSS is invariant between the alloys investigated. The implications of RBN connectivity to fine scale transgranular HEAC in ultra-high strength alloys are addressed in ensuing Discussion sections.

\subsection{Discussion}

The slow-rising $\mathrm{K}$ method, with precision dcPD monitoring of crack growth, effectively characterized the SCC resistance of a suite of ultra-high strength steels across a wide range of $\mathrm{E}_{\mathrm{app}}$. The results establish that modern secondary hardening UHSS alloys, developed for improved fracture toughness at ultra-high strength, exhibit good resistance to SCC and the causal HEAC 
mechanism. Compared to the baseline $300 \mathrm{M}$, crack growth is commonly TG rather than IG and Stage II crack growth rates are remarkably reduced. However, $\mathrm{K}_{\mathrm{TH}}$ for the onset of HEAC under severe cathodic polarization is unchanged and is similarly low relative to the increased $\mathrm{K}_{\mathrm{IC}}$ for each steel. The present results demonstrate a window of mild-cathodic polarization that confers very high resistance to SCC, which could be used to control SCC via galvanically coupled coatings. The data in Figures 17, 18, 24, and 25 are relevant to the simulation of component SCC behavior $[14,62]$.

HEAC kinetics, EBSD-measured microstructure and fractographic observations are interpreted and integrated to improve mechanistic understanding regarding three key issues: (a) the microstructural origins of TG SCC-HEAC for modern UHSS, (b) the impact of steel composition and purity on SCC of UHSS, and (c) interaction of steel composition and microstructure on the observed-strong potential dependence of SCC-HEAC. Each issue is, in turn, examined in the ensuing sections. Emphasis is placed upon the $\mathrm{E}_{\mathrm{app}}$ dependence of HEAC properties, which is assessed based on quantitative micromechanical models of $\mathrm{K}_{\mathrm{TH}}$ and da/dt $\mathrm{dI}_{\text {II }}$.

\subsubsection{TG HEAC Mechanism in UHSS}

Transgranular HEAC of modern UHSS exhibited a predominance of quasi-cleavage intermixed with several morphologies of near-featureless regions, denoted 'flat'. Such morphologies have been characterized in previous work reviewed below, which shows a definitive link between the HEAC fracture plane and fine-scale martensite interfaces. However, the transgranular crack path has not been previously established for modern secondary hardening UHSS.

Literature studies of low alloy martensitic steels collectively show that TG HEAC occurs primarily along martensite lath interfaces. TG HEAC fracture has been unambiguously shown to occur along $\{110\}_{\alpha}$, planes in 4340 steel $\left(\sigma_{Y S}=1,344 \mathrm{MPa}\right)$ [116]. This plane is both a slip plane in the BCT martensite and is parallel the interface plane which is the $\{111\}_{\gamma}$ habit plane in austenite and $\{110\}_{\alpha}$, in martensite. Notably, quasi-cleavage HEAC fracture along $\{110\}_{\alpha}$, planes was confirmed to be an inter-lath interfacial cracking process, rather than a trans-lath cleavage process in Ni-added martensitic steel [120]. Fracture was hypothesized to initiate with nucleation of interlath microcracks at microstructural irregularities, such as boundary intersections or precipitates, on lath boundaries ahead of the crack tip. Microcracks then propagate parallel to or 
along $\{110\}_{\alpha}$, lath boundary planes via lath boundary decohesion. Cracking along or parallel to $\{110\}_{\alpha}$, lath/block interfaces was confirmed by Shibata et al. in H-embrittled carbon steel $\left(\sigma_{\mathrm{YS}}=\right.$ $844 \mathrm{MPa}$ ) [125]. Building on this scenario, Nagao et al. recently attributed internal hydrogen assisted cracking (IHAC) fracture of lath martensitic steel $(\mathrm{Fe}-0.4 \mathrm{C}-1.9 \mathrm{Mn}-0.26 \mathrm{Si}-0.23 \mathrm{Mo}$ by wt $\%, \sigma_{Y S}=1,290 \mathrm{MPa}$ ) to a conjoint HELP/HEDE mechanism [11,12]: (1) H-enhanced dislocation movement along $\{110\}_{\alpha}$, slip planes redistributes $\mathrm{H}$ to martensite boundaries, (2) local plasticity severely work hardens the microstructure near the slip system-boundary intersection to support locally high tensile stress, and (3) a microcrack initiates and propagates along the lath/block interface via decohesion of the H-embrittled boundary [12]. Within this mechanism, the site of fracture initiation is determined by the combination of highest local stress and hydrogen accumulation, each on the sub-micrometer length scale.

It is logical to assume that multiple microcracks initiate ahead of a growing crack, in the 3dimensional FPZ, and fracture proceeds along the interface with the highest local stress and lowest $\mathrm{H}$-affected cohesive strength. This hypothesis is supported by observations that a large number of microcracks initiate away from the crack path when a critical plastic strain is reached $[125,126]$. Nagao et al. observed that the orientation of activated slip systems relative to martensite lath/block boundaries correlates to the $\mathrm{H}$-fracture morphology [12]. Beneath the fracture surface of a flat IG fracture facet, activated slip systems lie parallel to the length direction of laths (consistent with the fact that the martensite interface plane and slip plane are both $\{110\}_{\alpha}$, in martensite), and such slip planes intersect prior austenite boundaries at a glancing angle. Conversely, activated slip systems beneath QC fracture features are inclined relative to and thus intersect lath boundaries at relatively larger angles. These observations demonstrate that the appearance of $\mathrm{H}$-assisted fracture in lath martensite is dictated by the specific interface (lath/block/packet/prior $\gamma$ ) that decohesion proceeds along. The present results provide an opportunity to compare the relative contributions of lath/block/packet interfacial cracking in HEAC of modern ultra-high strength UHSS. For this analysis, the HEAC mechanism proposed by Nagao et al. will be used as the basis for interpretation of HEAC fracture morphology.

Transgranular quasi-cleavage facets presented in Figures 19 - 21, and 23 for 4 UHSS alloys bear a striking resemblance to the previously observed QC driven by $\mathrm{H}$-assisted martensite interface decohesion [11,12,116,120]. Further, QC facets surfaces presented in Figures 19-21, and 23 
display strong similarity to one another, supporting the hypothesis that a similar damage mechanism is active in multiple alloys. Close agreement between UHSS alloys is particularly evident in the comparison of AerMet 100 (Figure 19b, lower left), Ferrium S53 (Figure 20, upper portion), and Ferrium M54 (Figure 21, lower right), shown collated in Figure 27a-c. Fine lath features and ridges in Figure 27a-c are near identical to observations of QC by Nagao et al. for IHAC by interlath/interblock fracture following the conjoint HELP/HEDE mechanism in high strength martensitic steel. Congruency of QC facet morphology with observations by Nagao et al indicates the HELP/HEDE mechanism likely controls HEAC of UHSS alloys.

The spacing of fine scale surface features on QC facets in Figure 27a-c closely matches the width of block interfaces shown by the EBSD-OIM images in Figure 27d-f. Notably, no sub-block structure of parallel laths was observed in the present study, as indicated by the OIM map and misorientation profile presented in Figures 13-14. In contrast to the classical description of martensite morphology, martensite blocks contain a single crystallographic variant and are separated from adjacent blocks by a high angle boundary. Previous characterization of lath martensite obeying K-S demonstrates that block boundaries typically have a near- $\{110\}_{\alpha}$, crystallographic plane. In the context of the present results, this implies that transgranular quasicleavage in modern UHSS, governed by the HELP/HEDE mechanism, likely nucleates and propagates along high angle $\{110\}_{\alpha}$, block boundaries. Highly localized plasticity and rupture are required to accommodate $\{110\}_{\alpha}$, cracks occurring in the distribution of relatively fine scale blocks. The larger blocks apparent in Figure 27 (f) for the Ferrium M54, appear to correlate with larger trans-block crack surface features and reduced plasticity/rupture. Notably, this subtle difference in martensite structure and TG QC features does not correlate with a resolvable difference in either $\mathrm{K}_{\mathrm{TH}}$ or da/dt versus $\mathrm{K}$ for this UHSS compared to, for example, AerMet 100 at any $E_{\text {app. }}$. 


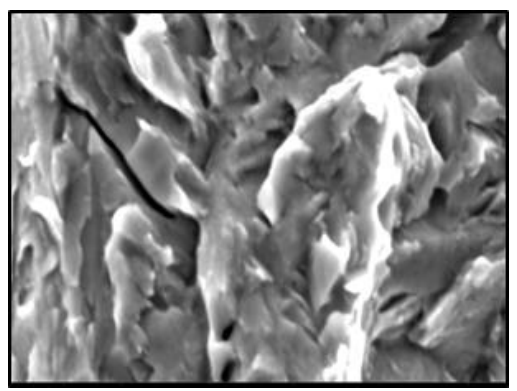

(a)

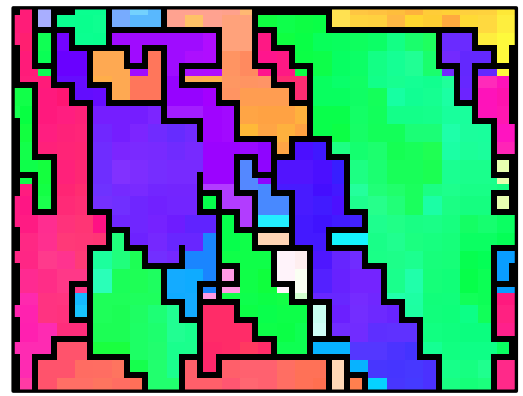

(d)

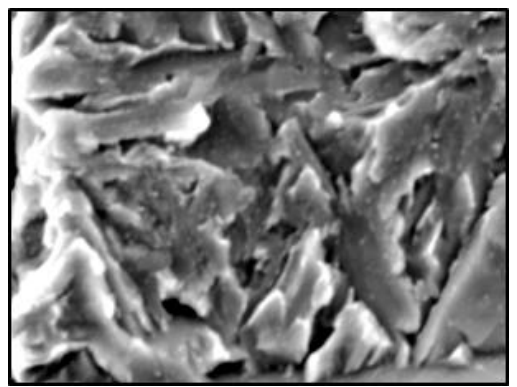

(b)

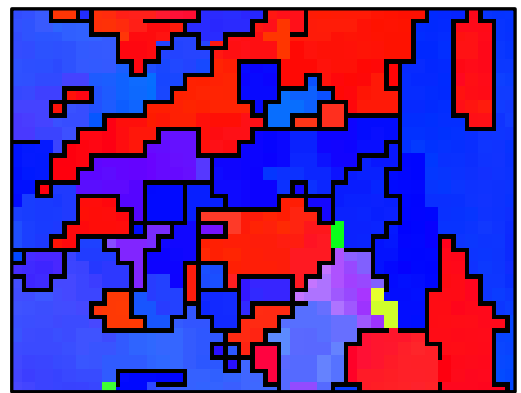

(e)

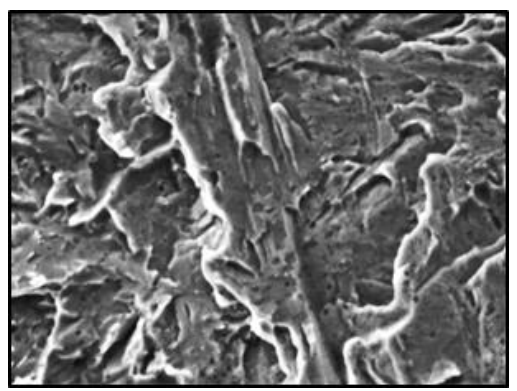

(c)

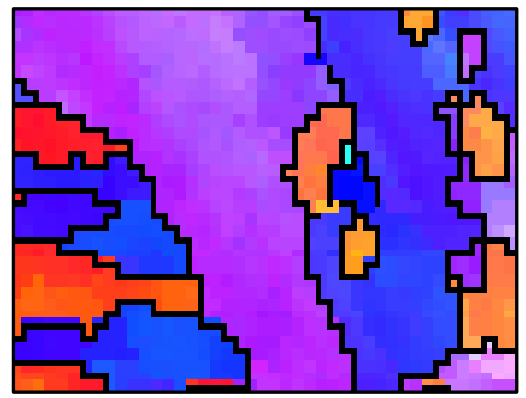

(f)

\section{$2 \mu \mathrm{m}$}

Figure 27 Quasi-cleavage facets and representative areas of martensite block structure in OIM maps (Figure 6-9) for AerMet $100($ a,d), Ferrium S53 (b,e) and Ferrium M54 (c,f). Images are shown at identical magnification.

As shown in Figures 19-23, both intergranular and intragranular (with respect to a prior austenite grain) flat features are evidenced on the HEAC fracture surface of UHSS alloys. The origins of intergranular HEAC will be discussed in a later section for the unique cases of $300 \mathrm{M}$ and CrNiMoWV. Intragranular 'flat' regions were not observed in development of the HELP/HEDE mechanism $[11,12]$. Critically, the ultra-high strength martensitic steel studied by Nagao et al. has a relatively well-defined, ordered structure which matches the classical definition of martensite morphology presented in Figure 12. Martensite packet/block features of UHSS are reduced in length and widened compared to classic morphologies. Further, the UHSS microstructures contain heterogeneity, which is not classically observed in lath martensite, as illustrated by Figure 28. 

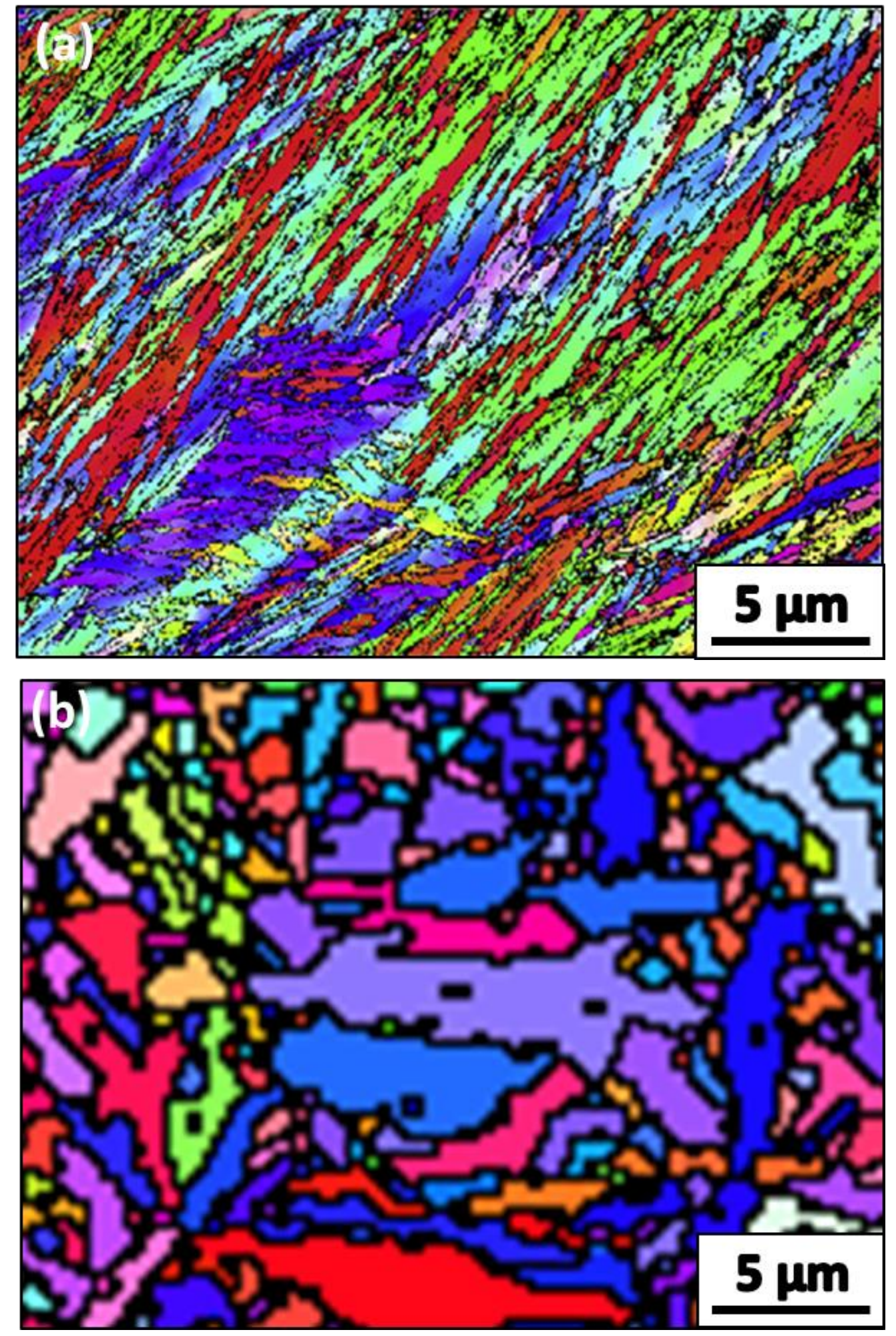

Figure $28 \quad$ Comparison of lath martensitic structure observed by Nagao (a) [11] and the microstructure of AerMet 100 from the present study (b) both utilizing IPF coloring. 
The source of the intragranular 'flat' fracture morphology can be logically traced to this relative coarseness and heterogeneity of the block structure, which can be observed in all alloys (Figures 6-10). TEM of the martensite microstructure beneath IHAC QC fracture has shown that intralath decohesion is accompanied by trans-lath steps [11,12]. Many of the intragranular 'flat' features observed to be intermixed with QC fracture (Figure 19-21, 23, black arrows) match the block widths observed in the OIM maps of the alloy microstructure. This agreement is illustrated by comparing the width of successive parallel blocks in Figure 10 (black arrows) to regular blocky steps of 'flat' 1-3 $\mu \mathrm{m}$ wide features on the HEAC fracture surface of Ferrium S53 (Figure 20b, black arrows). Such features are hypothetically traced to trans-block cleavage steps in modern UHSS. Regions of 'flat' morphology which do not have a regular spacing close to the martensite block width are occasionally observed in AerMet 100 (Figure 19b, black arrow), Ferrium S53 (Figure 20c, black arrow), and Ferrium M54 (Figure 21c, black arrow). Two possible origins for isolated flat features logically follow from the HELP/HEDE mechanism: (1) flat features represent trans-block cleavage steps across unusually large single variant blocks (Figure 7, large blue block in the center), and (2) flat features represent limited decohesion-driven cracking along packet interfaces in the interior of a prior austenite grain. In the case of the latter, Figure 21 clearly shows that cracking along packet interfaces within a prior austenite grain occurs during HEAC of Ferrium M54. Further, martensite packet interfaces are exclusively high angle boundaries without a special CSL relationship. In this regard, packet boundaries are similar to prior austenite grain boundaries and may display identical 'flat' fracture on a size scale consistent with packet size. In the case of Ferrium M54, the isolated packet shown in Figure $15 \mathrm{~m}$ is about $10 \times 30 \mu \mathrm{m}$ in size, significantly larger than the regions of flat fracture observed intermixed with QC ( 1 - $4 \mu \mathrm{m}$ in diameter). This does not preclude packet boundary decohesion, as the features are not planar and often interrupted by block protrusions from neighboring packets. As such, packet boundary decohesion could be limited to a small portion of the boundary surrounded by typical QC block decohesion and cleavage.

All fractographic evidence (Figures 19-23) points to a similar-universal H-damage mechanism for transgranular quasi-cleavage fracture of modern martensitic UHSS alloys. The conjoint HELP/HEDE mechanism, proposed by Nagao, provides a reasonable set of mechanistic steps for nucleation and propagation of microcracks ahead of the crack tip and on the sub-micrometer length 
scale, which link to form the macroscopic crack path. Microstructural heterogeneity of modern UHSS provides a logical explanation for the occurrence of intragranular flat fracture morphology. The mechanism elucidates the importance of the relative susceptibility of the various martensite interfaces to hydrogen and impurity driven reduction in cohesive strength. In the next section, the effect of martensite interface character and connectivity on HEAC is investigated for UHSS.

\subsubsection{Effect of Grain Boundary Character Distribution on HEAC on UHSS}

In the present study of the five UHSS alloys, EBSD characterization of fine scale martensite microstructure (Figures 6-10) provides a unique opportunity to probe the effect of the GBCD on measured HEAC properties. A bimodal split of random boundary connectivity, as characterized by $D_{R}$, was observed for all alloys. Including 300-400 nm long gaps in random boundaries, $D_{R}$ is near zero for all steels, indicating no connectivity of the RBN, consistent with the extensive local interruptions. Conversely, allowing a 'gap tolerance' of $\sim 2$ pixels (320-400 nm of physical distance) yielded $D_{R}=1.8$ to 1.9 for all alloys, indicating near-complete connectivity of the RBN consistent with the high density of random interfaces in the prior austenite grain boundarymartensite packet-block/lath microstructures. Given the stark contrast in the two bounding cases of RBN connectivity results, it is important to justify or dismiss the use of a 'gap tolerance' when analyzing connectivity in microstructurally complex $\operatorname{UHSS}(\mathrm{S})$ alloys.

The use of the gap tolerance in calculating the connectivity of random high angle boundaries is supported by a rational-assumed morphology for small CSL boundaries in a 3D microstructure and curve fit values for $\chi_{\text {crit. }}$ Small CSL disruptions represented in Figure 16 must have a physical origin on an otherwise continuous random block interface. Speculatively, they may represent ultrafine regions of recrystallized ferrite grains formed during the tempering process. Such recrystallization is greatly limited at typical aging temperatures for modern UHSS due to the

presence of solute Co as well as nano-scale precipitates that immobilize the dislocation and recrystallized boundary structures. Higher secondary hardening temperature would drive the recrystallization of locally-high dislocation density, but this is not typically employed due to strength and toughness losses [26]. Newly formed ferrite would be small in size and possibly have an orientation relationship with the parent martensite crystal, yielding a CSL boundary in some cases [127]. In this scenario, the fine CSL disruptions would be represented as isolated islands of 
good atomic fit on a 2D high angle boundary plane. This scenario is speculative since such measured CSL segments relate to ferrite precipitation in austenite and not ferrite formed by recovery and recrystallization of highly dislocated BCC/BCT martensite [127].

As established in previous sections, TG quasi-cleavage fracture of modern UHSS is likely governed by a coupled HELP/HEDE mechanism, and decohesion driven fracture proceeds along martensite block interfaces with occasional transblock steps. Critically, during hydrogen assisted QC, microcracks are hypothesized to initiate ahead of the macro-crack tip at microstructural features which enhance local tensile/hydrostatic stress (precipitates, block/lath boundary intersections) [120]. Such microcracks should logically initiate in the region of highest hydrostatic stress some distance ahead of the crack tip. In tempered martensitic steel ( $\left.\sigma_{\mathrm{YS}} \sim 1,600 \mathrm{MPa}\right)$ stressed at a $\mathrm{K}=40 \mathrm{MPa} \sqrt{\mathrm{m}}$, this distance was calculated to be $6 \mu \mathrm{m}$ [7]. If the stress intensity is reduced to $10 \mathrm{MPa} \sqrt{\mathrm{m}}$ the maximum stress is located $0.3 \mu \mathrm{m}$ ahead of the crack tip. Alternate models of crack tip stresses justify a substantially smaller location for the maximum stress, on the order of 0.5 to $1 \mu \mathrm{m}$. Similarly, reasonable literature values of $\chi_{\text {crit }}$ obtained from regression fitting of fastest reported da/dt II Vs $\mathrm{D}_{\mathrm{H}-\mathrm{EFF}}$ (Equation 2) are 0.7-1.0 $\mu \mathrm{m}$ and represent the interaction of the crack tip stress field and falling H-concentration away from the crack tip [2,3]. The applicability of a universal, alloy-independent $\chi_{\text {crit }}$ will be discussed in an ensuing section. However, it is clear that $\chi_{\text {crit }}$ is small and on the order of average martensite block spacing $(0.8-1.3 \mu \mathrm{m})$ for martensitic UHSS. Compared to the minimum pixel gap tolerance required for high $\mathrm{D}_{\mathrm{R}}$ (2 pixels, 320-400 nm of physical distance), these $\chi_{\text {crit }}$ are relatively large for HEAC-relevant stress intensities. In the case of crack propagation along a 2D high angle boundary plane, (represented by the 1D trace in Figure 26) small islands of CSL boundary may limit crack propagation through that highly localized island. However, the crack front could advance between islands of CSL boundary on the 2D plane, leaving behind 'pillars' of connected CSL boundary. Such pillars would fail under increased stress as the surrounding boundary cracks and local stress increases. The mechanism would be enhanced by microcracks initiating ahead of any CSL disruption and cracking back to the advancing crack front, as suggested by curve fit values of $\chi_{\text {crit. }}$ The $3 \mathrm{D}$ island morphology for small CSL boundary segments, and $\chi_{\text {crit }}$ on the order of the martensite block spacing, both imply that the effect of CSL disruptions in three dimensions is likely minimal. As such, the use of a gap tolerance in determination of $D_{R}$ is justified and consistent with previous work [108] EBSD measurements 
therefore establish that the random boundaries of all UHSS are fully connected, as characterized by the high fractal dimension of the MRBC.

The preceding analysis did not consider the effect of extremely high crack tip $\mathrm{H}$ concentrations present during HEAC of UHSS at potentials either at/just anodic to OCP as well as modest/strong cathodic polarization relative to OCP (Figure 29). Hydrogen segregation to CSL boundaries may be limited by good atomic matching [52]. However, there should be a local lattice $\mathrm{H}$ concentration in an open system HEAC situation [21] where such a beneficial effect of low $\mathrm{H}$ interfaces is overwhelmed and the interface fails via decohesion. Elevated $\mathrm{C}_{\mathrm{H} \text {-DIFF }}$ may sufficiently decrease CSL cohesive strength to allow crack propagation through these interfaces. The effect on RBN connectivity would be identical to using a high pixel gap tolerance; with high-local $\mathrm{H}$ concentration, CSL boundaries are susceptible to $\mathrm{H}$ cracking similar to random boundaries and the RBN would be considered fully connected.

\subsubsection{Measurement of Precipitated Austenite in UHSS}

Austenite precipitation during aging is hypothesized to be detrimental to HEAC resistance in UHSS alloys $[119,128]$. Thin film austenite, present along martensite packet and block interfaces in the FPZ is saturated with $\mathrm{H}$ during HEAC. In principle, high crack tip stress and plastic strain should drive transformation of $\mathrm{H}$-saturated precipitated austenite into H-supersaturated, untempered martensite. Untempered martensite from precipitated austenite, hardened by dislocations and carbon, would provide a H-laden, interconnected crack path through the martensite microstructure along martensite block interfaces. High resolution EBSD measurements in the present study of UHSS alloys provide the opportunity to examine the applicability of this hypothesis.

The presence, distribution, and morphology of precipitated austenite in AerMet 100 has been extensively characterized via XRD and TEM [24,26,69,74,129], and is a factor to consider when interpreting microstructure effects on IHAC and HEAC. Retained austenite is expected to be reduced or eliminated by air/oil cooling following austenitization then cryogenic processing [24]. TEM of commercially aged AerMet 100 specimens conclusively shows that precipitated austenite is present in a continuous $3 \mathrm{~nm}$ thick film along martensite lath interfaces [24,74]. X-ray diffraction (XRD) measurements of identically aged specimens indicate that the summation of retained and 
thin film austenite volume fraction is universally small but highly variable between studies, $0.8-$ $4.5 \mathrm{vol} \%$, for identically tempered specimens [23,24,26,74,129]. Some variability may be attributed to varying methods of XRD specimen-surface preparation represented in the separate studies. However, seven replicate measurements conducted at a single laboratory, with identical surface preparation, show a large variance in austenite content for AerMet 100: $1.97 \pm 0.66$ vol $\%$ (95\% confidence interval) [69]. The fine scale of the AerMet 100 microstructure relative to XRD specimen size precludes statistical sampling errors of a sufficient thin film volume. As such, the variability in volume fracture of austenite measured via XRD is likely attributed to the difficulty of distinguishing a small amount of austenite above XRD background scatter.

EBSD measurements indicate that precipitated austenite is present in a small island morphology along martensite interfaces. Total EBSD-measured austenite for AerMet 100, CrNiMoWV, Ferrium S53, Ferrium M54, and 300M is 1.5, 0.9, 1.9, 0.8, and 1.6 vol\%, respectively. Isolated islands of austenite, $75-250 \mathrm{~nm}$ in diameter, are predominantly located along martensite interfaces and triple points (Figure 11, red islands). Thin film austenite is likely present in each steel, but precluded from measurement by the relatively large EBSD step size. The expected volume fraction of continuous thin film austenite on all martensite interfaces can be estimated from the total $\mathrm{S}_{\mathrm{V}}$ (Table 4) of martensite interfaces and the film thickness. For AerMet 100, a $3 \mathrm{~nm}$ film of precipitated austenite over all interfaces would yield 0.7 vol $\%$, which is below the confidence interval of XRD measurements. The volume fraction of precipitated austenite held in small island morphology ( 1.5 vol \% for AerMet 100$)$ is likely additive to the estimated thin film contribution, yielding an expected total precipitated austenite content of $2.2 \mathrm{vol} \%$ for AerMet 100 . This result is in close agreement with the XRD-measured average austenite content of $1.97 \mathrm{vol} \%$ noted above.

Significantly thicker (5-10 nm) precipitated austenite films in Ferrium M54 [75] suggest 1 - 2 vol\% of continuous thin film austenite, yielding a total austenite content of $1.8-2.8$ vol \%. Interlath films of austenite in $300 \mathrm{M}$ are relatively thin $(0.1-0.2 \mathrm{~nm})$, discontinuous, and do not contribute significantly to the total austenite fraction $(<0.04$ vol \% total calculated compared to 1.6 vol \% EBSD measured for the precipitate morphology) [72]. No information on the presence and morphology of precipitated thin film austenite is available for Ferrium S53 and CrNiMoWV. Thin films of precipitated austenite are expected in these alloys, so the total precipitated austenite content should be significantly higher than measured via EBSD. The results of the present study 
indicate that the XRD measured austenite content in UHSS is a summation of austenite held in thin films and small island morphologies. Further, the thickness of thin film intra lath austenite in UHSS can be hypothetically calculated from the difference of average-total XRD and EBSDmeasured small island morphologies.

In the context of the conjoint HELP/HEDE mechanism, thin film austenite could synergistically act with high local hydrogen concentrations to reduce HEAC resistance. Thin film austenite along martensite block boundaries should increase the hydrogen concentration local to the block interface relative to an austenite free structure. This effect is due to both the higher solubility of $\mathrm{H}$ in FCC austenite and strong-reversible H-trapping at the austenite-martensite interface $[9,128,130]$. During HEAC, high plastic strain should transform H-saturated precipitated austenite into H-supersaturated, untempered martensite along martensite interfaces [128]. Kim et al. hypothesized that the volume change associated with martensite transformation will impose a tensile stress across the newly formed H-laden martensite boundary and initiate a microcrack [128]. Critically, precipitated austenite examined by Kim et al. in 5.5Ni steel was in discrete islands rather than the continuous thin film morphology likely present in UHSS alloys. Strain transformation of continuous thin film precipitated austenite in UHSS would produce a tensile stress across the entire lath/block boundary. The tensile stress would reduce the local stress necessary to initiate fracture at the block boundary in the HELP/HEDE mechanism for HEAC. Microcracks would logically initiate at the comparatively large islands of austenite observed along block boundaries (Figure 11, red islands) where the transformational strain is the largest. Simultaneously, microcracks could initiate at the intersection of slip bands and the newly transformed H-saturated martensite interface due to the high local stress. In either case, fracture would proceed via decohesion along the martensite interface, possibly at an increased-average rate due to the high local hydrogen concentration in newly transformed martensite.

Continuous thin film austenite has been observed along all block boundaries in AerMet 100 and Ferrium M54, with larger $75-250 \mathrm{~nm}$ islands at triple points or other boundary irregularities [24,75]. Notably, the HEAC resistance of AerMet 100 and Ferrium M54 is near identical (Figure 24) despite a relatively 2 to 3 fold difference in thin film thickness ( 3 versus 5-10 nm, respectively) $[24,75]$ and EBSD-interface area estimated thin film volume fraction (0.7 vs 1-2 vol \%, respectively). This result indicates that in the context of the HELP/HEDE mechanism, the 
thickness of precipitated austenite films is not driving differences in HEAC resistance. Speculatively, a continuous thin film plus island precipitated austenite morphology is present in CrNiMoWV and Ferrium S53, comprising a similar-small total volume fraction. If a similar morphology of precipitated austenite is present in all modern UHSS, then the effect on HEAC resistance should be universally detrimental. For small differences in the film thickness and island size, the effect on HEAC kinetics is likely minimal, but cannot be assessed without quantitative measurement of precipitated austenite morphology in Ferrium S53 and CrNiMoWV.

\subsubsection{Effect of Purity on Composition on SCC-HEAC Fracture Path}

The group of precipitation strengthened martensitic steels, which were investigated provide for a systematic comparison of the effects of potentially important composition variables on susceptibility to SCC by the HEAC mechanism.

\subsubsection{Steel Purity and IG HEAC}

The dominant HEAC path is intergranular along prior austenite boundaries in $300 \mathrm{M}$ and CrNiMoWV. McMahon and coworkers extensively characterized the conjoint action of steel purity and environmental $\mathrm{H}$ on cracking in high strength ( $\sigma_{\mathrm{YS}} \sim$ 1,400 MPa) 4340 type steels $[28,29,131]$. Prior austenite boundary cohesion is degraded by the contributions of impurities and elemental hydrogen, as empirically characterized though the parameter $\Psi(\Psi=[\mathrm{Mn}+0.5 \mathrm{Si}+\mathrm{S}$ $+\mathrm{P}]+\left[10^{4} \mathrm{C}_{\mathrm{H}}\right]$ in at\%); where $\mathrm{C}_{\mathrm{H}}$ is the bulk $\mathrm{H}$ concentration in the lattice [131]. Falling $\Psi$ correlates with decreased fraction of brittle IG cracking and increased $\mathrm{K}_{\mathrm{TH}}$ for HEAC in a gaseous $\mathrm{H}_{2}$ environment [131]. IG HEAC is essentially eliminated when the impurity component of $\Psi$ is less than $0.05 \mathrm{wt} \%$ for 4340 steel $\left(\sigma_{\mathrm{YS}}=1,450 \mathrm{MPa}\right)$ stressed in low pressure $\mathrm{H}_{2}\left(\mathrm{p}_{\mathrm{H} 2}=0.1 \mathrm{MPa}\right)$ [131]. However, recent work by Liu et al has shown that ultra-high purity $(\mathrm{Mn}+\mathrm{Si}+\mathrm{P}+\mathrm{S}<$ $0.024 \mathrm{wt} \%) 4340$ steel $\left(\sigma_{\mathrm{ys}}=1,860 \mathrm{MPa}\right)$ exhibits IG cracking when stressed under very slow rising displacement in an identical $0.1 \mathrm{MPa}_{2}$ atmosphere [132]. This result indicates that sufficiently high hydrogen concentrations can drive IG HEAC in the absence of a high impurity content, as expected if either hydrostatic stress or strong-adverse $\mathrm{H}$ trapping enhance the level of crack tip $\mathrm{H}$ available for HEAC. 
For the current study, the $\Psi$ parameter correlates with the minor fraction of IG cracking in AerMet 100, Ferrium S53 and Ferrium M54. AerMet 100, Ferrium M54 and Ferrium S53 exhibit very low $\Psi_{\text {Impurity }}{ }^{9}$ of $0.02,0.01,0.02 \mathrm{wt} \%$, respectively (Table 4). Additionally, each of these modern UHSS alloys contains rare earth elements to reduce free impurity content through stable oxide precipitation, implying a substantial reduction in $\Psi_{\text {Impurity }}[5,7,133]$. Each of these steels exhibits a negligible amount of IG HEAC in chloride solution, as shown in Figures 19-21 and 23. Notably, this correlation holds for higher $\mathrm{C}_{\mathrm{H}}\left(\mathrm{C}_{\mathrm{H \sigma}}\right)$ levels for severe cathodic as well as mild anodic $\mathrm{E}_{\mathrm{app}}$. In sharp contrast, 300M exhibits essentially exclusive IG HEAC (Figure 22). Impurity content in $300 \mathrm{M}$ is significantly elevated $\left(\Psi_{\text {Impurity }}=1.8 \mathrm{wt} \%\right)$ compared to modern UHSS. Moreover, no

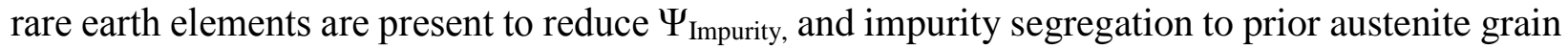
boundaries is promoted by high $\mathrm{Mn}$ and $\mathrm{Si}[29,134]$. IG HEAC in $300 \mathrm{M}$ is, therefore most likely caused by the embrittlement of prior austenite grain boundaries by environmental $\mathrm{H}$ and significant free impurity concentration.

CrNiMoWV steel exhibits anomalous behavior with regard to the dominant role of metalloid impurity concentration in IG HEAC. As shown in Figure 23, the HEAC fracture mode for CrNiMoWV is a predominant IG morphology with minor TG components. IG fracture of CrNiMoWV is predicted by $\Psi_{\text {Impurity }}>0.05 \mathrm{wt} \%$; however, with the exception of Si, impurity concentrations are extremely low and likely reduced compared to Table 4 due to substantial rare earth treatment. Literature work has not conclusively isolated an adverse effect of Si on IG HEAC in the absence of $\mathrm{Mn}, \mathrm{P}$, and $\mathrm{S}$. Two pieces of evidence point towards a microstructural origin of IG fracture in CrNiMoWV. First, Bandyopadhyay et al. observed that Si does not segregate to prior austenite grain boundaries, as confirmed by auger electron spectroscopy of IG facets [131,135]. Second, IG facets in CrNiMoWV show evidence of small scale plasticity, as opposed to largely featureless IG surfaces driven by impurity segregation in low purity 300M. Near identical IG morphology was observed in hydrogen assisted fracture of an ultra-high strength ( $\sigma_{Y S}$ $=1,860 \mathrm{MPa})$, ultra-high purity $\left(\Psi_{\text {Impurity }}=0.024 \mathrm{wt} \%\right)$ 4340-type steel by Liu et al, who noted that IG facets showed signs of 'ductile rupture' [132]. Similar strength 4340 steels with significant impurity concentration $(\mathrm{Mn}+\mathrm{Si}+\mathrm{P}+\mathrm{S})$ display typical featureless, glassy IG facets identical to

$9 \quad \Psi_{\text {Impurity }}$ denotes the measured $\Psi$ parameter without the $\mathrm{C}_{\mathrm{H}}$ contribution. 
300M [29]. Based on this evidence, it is logical to assume that IG HEAC in CrNiMoWV is not

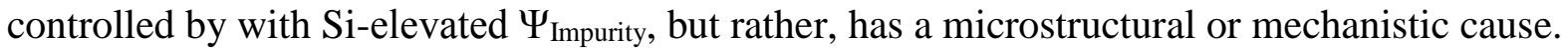

The HELP/HEDE mechanism for HEAC developed by Nagao et al. proposes that the morphology of fracture is determined by the orientation of martensite lath interfaces relative to activated slip systems. Slip bands beneath an intergranular fracture surface were oriented approximately parallel to lath/block interfaces $[11,12]$. In the classical view of martensite morphology (Figure 12), slip bands parallel to the $\{110\}_{\alpha}$, martensite lath/block interface plane would travel the length of a packet and intersect a packet or prior austenite interface prior to a block boundary. In this scenario, hydrogen enhanced dislocation movement both work hardens the local microstructure and redistributes significant amounts of $\mathrm{H}$ to the slip band / boundary intersection. Prior austenite grain and martensite packet size are increased dramatically in CrNiMoWV compared to similar UHSS (Figures 6-10). As a hypothesis, IG fracture of CrNiMoWV stems from simultaneous factors: (1) due to increased grain size, slip bands running parallel to $\{110\}_{\alpha}$, block interfaces are able to pile up significantly more dislocations at the slip band / boundary intersection, (2) the boundary $\mathrm{H}$ concentration is nominally elevated compared to the QC case due to increased slip band length, (3) boundary disruptions (and perhaps nano-voiding) are elevated due to increased slip band activity and could provide strong-deleterious sites for $\mathrm{H}$ trapping, and (4) prior austenite grain boundaries are exclusively high angle random boundaries which will likely trap H. Logical interpretation of the generic HELP/HEDE mechanism dictates that decohesion-based microcrack propagation will proceed with a weakest link criterion. Crack propagation is favored along the interface with lowest $\mathrm{H}$-affected cohesive strength and highest local stress state. In the hypothesized scenario for CrNiMoWV this almost certainly is prior austenite grain boundaries.

The present study establishes that IG HEAC is largely eliminated in modern UHSS by control of prior austenite grain boundary segregation through steel purity and rare earth treatment. This result is significant because previous work showing the benefit of reduced boundary segregation was established for somewhat lower strength levels and substantially lower crack tip H concentrations [131,136]. At the higher strengths investigated (Table 1), high $\mathrm{C}_{\mathrm{H}}$ from crack tip reaction is not sufficient to produce IG HEAC under slow-rising displacement in the majority of modern UHSS alloys examined. However, modern UHSS alloys are broadly prone to significant TG HEAC, which is not driven by impurity effects. 


\subsubsection{Steel Composition and TG HEAC}

For severe cathodic and mild anodic $\mathrm{E}_{\mathrm{app}}, \mathrm{K}_{\mathrm{TH}}$ values are similar for IG and TG cracking in the examined alloys (Figure 24). However, da/dtII is dramatically reduced in modern steels compared to baseline legacy $300 \mathrm{M}$ (Figure 25). The dependencies of $\mathrm{K}_{\mathrm{TH}}$ and da/dtII on $\mathrm{E}_{\text {app }}$ are discussed in an ensuing section. As illustrated by Table 3-4 and Figure 24, $\mathrm{K}_{\mathrm{TH}}$ is insensitive to alloy purity at the extremes of polarization. As such, observed differences in da/dt II cannot be attributed to differing intrinsic susceptibilities to IG versus TG H damage. This conclusion is validated by the discussion of the EBSD characterizations of the martensitic microstructures, particularly the similarities in special boundary characteristics and random boundary connectivity, in these UHSS alloys. Rather, observed variation in $\mathrm{da} / \mathrm{dt}_{\text {II }}$ is sourced at differences in trap sensitive $\mathrm{H}$ diffusivity, as represented by Equation 2, which shows that da/dt ${ }_{I I}$ is directly proportional to $\mathrm{D}_{\mathrm{H}-\mathrm{EFF}}$ [2].

Legacy $300 \mathrm{M}$ provides an ideal baseline control for investigating the effect of composition on measured Stage II crack growth in modern secondary hardening UHSS. Figueroa and Robinson reported that $\mathrm{D}_{\mathrm{H}, \mathrm{EFF}}$ for optimally aged AerMet 100 is 80 times less than $300 \mathrm{M}$, as determined by the first-rise transient in $\mathrm{H}$ permeation experiments [1,137]. The da/dt II values for AerMet 100 are 36-56-times and 330-times less than 300M when stressed in $\mathrm{NaCl}$ solution at $-550 \mathrm{mV}_{\mathrm{SCE}}$ and $1,000 \mathrm{mV}_{\mathrm{SCE}}$, respectively (Figures 17-18). Growth rate reduction at $-550 \mathrm{mV}_{\mathrm{SCE}}$ is quantitatively consistent with the $\mathrm{H}$ diffusivity reduction given by permeation experiments, if all other terms in Equation 2 are equal for $300 \mathrm{M}$ and AerMet 100 . If $\chi_{\text {crit }}$ is dictated by the location of the maximum crack tip hydrostatic stress [2,7], then it is reasonable to assume that $\chi_{\text {crit }}$ is equal for each UHSS examined and should not change with $E_{\text {app. }}$. The squared error function term in Equation 2 is dampened to rise from 1 to 5 as the ratio of $\mathrm{C}_{\mathrm{H \sigma}} / \mathrm{C}_{\text {Crit }}$ rises from 6 to 500 . In order to explain the 330x faster da/dtII in the context of Equation 2, it is necessary for the squared error function term to increase 4-fold for $300 \mathrm{M}$ compared to AerMet 100 with each polarized to $-1000 \mathrm{mV}_{\mathrm{SCE}}$, but not so at $-550 \mathrm{mV}_{\text {SCE. }}$. Whether this is possible depends on the precise value of $\mathrm{C}_{\text {Crit }}$ for each steel and relative to $\mathrm{C}_{\mathrm{H}}$. Kehler and Scully reported that crack tip $\mathrm{C}_{\mathrm{H}}$ for AerMet $100 \mathrm{in} \mathrm{NaCl}$ solution is 0.7 wppm at $-550 \mathrm{mV}_{\text {SCE }}$ and $4.2 \mathrm{wppm}$ at $-1,000 \mathrm{mV}_{\text {SCE }}$ (Figure 26) [20]. There is no basis to conclude that $\mathrm{C}_{\mathrm{H} \sigma}$ is different for $300 \mathrm{M}$, due to equal strength levels. However, the $\mathrm{C}_{\text {Crit }}$ can be assumed lower for impurity-driven IG cracking in $300 \mathrm{M}$ versus TG $\mathrm{H}$ cracking. As such, $\mathrm{C}_{\mathrm{H} \sigma} / \mathrm{C}_{\mathrm{Crit}}$ may be higher for $300 \mathrm{M}$ compared to AerMet 100 at fixed $\mathrm{E}_{\mathrm{app}}$, and the impact on the squared 
inverse error function term in Equation 2 could rise with increasing cathodic polarization. This sort of comparison is amplified in an ensuing section of this Discussion, which considers quantitative predictions of $\mathrm{K}_{\mathrm{TH}}$ and $\mathrm{da} / \mathrm{dt}_{\mathrm{II}}$ based on occluded crack electrochemistry.

Detailed thermal desorption spectroscopy (TDS) experiments established that reduced $\mathrm{D}_{\mathrm{H}-\mathrm{EFF}}$ in AerMet 100 is due to reversible $\mathrm{H}$-trapping at needle-shaped, nano-scale ( $\mathrm{Mo}, \mathrm{Cr})_{2} \mathrm{C}$ precipitates $[1,24,130,137]$. (Mo,Cr) $2 \mathrm{C}$ precipitation is due to $\mathrm{Cr}$ and Mo content in AerMet 100, enhanced by significant additions of cobalt [4,74]. Recent TEM characterization of Ferrium M54 has indicated a near-identical distribution of needle shaped $\mathrm{M}_{2} \mathrm{C}$ carbides [75]. Based upon these TEM observations and the compositions presented in Table 3-4, similar nano-scale $\mathrm{M}_{2} \mathrm{C}$ carbides are likely present in Ferrium S53 and CrNiMoWV, but the precise sizes and volume fractions have not been established. Nano-scale (Mo, Cr) $)_{2} \mathrm{C}$ are not present in $300 \mathrm{M}$, which precipitates large $(\sim 1$ x $0.1 \mu \mathrm{m}$ ) intralath, incoherent $(\mathrm{Fe}, \mathrm{Cr})_{3} \mathrm{C}$ during tempering [71,72]. Further, CrNiMoWV, Ferrium M54, and Ferrium S53 contain significant additions of tungsten and vanadium, to promote precipitation of fine $\mathrm{MC}(\mathrm{M}=\mathrm{W}, \mathrm{V})$ carbides which are likely to further reduce $\mathrm{D}_{\mathrm{H}-\mathrm{EFF}}$ [3740,138]. As such, similar low values of $\mathrm{D}_{\mathrm{H}-\mathrm{EFF}}$ are expected for each of these modern secondary hardening steels, explaining the similar-lower $\mathrm{da} / \mathrm{dt}_{\mathrm{II}}$ measured for each compared to $300 \mathrm{M}$ under high cathodic polarization. The dependence of $\mathrm{da} / \mathrm{dt}_{\text {II }}$ on the composition of the modern high purity steels, as observed for moderate cathodic $\mathrm{E}_{\mathrm{app}}$, is explained by possible-strong reductions in $\mathrm{C}_{\mathrm{H \sigma}} / \mathrm{C}_{\mathrm{H}-\mathrm{Crit}}$ amplified by the inverse error function (Equation 2). This ratio may not significantly decrease for low purity $300 \mathrm{M}$ due to impurity driven, intrinsically low $\mathrm{C}_{\mathrm{H}-\mathrm{Crit}}$. The quantitative crack tip electrochemical basis for this conclusion is amplified in an ensuing section. As a general conclusion, the primary factor which governs reduced da/dt II of AerMet 100, Ferrium M54, Ferrium S53 and CrNiMoWV is reduced DH-EFF driven by the presence of a high density of nanoscale carbide $\left(\mathrm{M}_{2} \mathrm{C}, \mathrm{MC}\right)$ precipitates which act as sites for strong-reversible $\mathrm{H}$ trapping [1,137].

The selected alloy steels provide a basis to qualitatively assess the individual effects of $\mathrm{Ni}$, Co and Cr on HEAC kinetics. The alloy sequence of AerMet 100 to Ferrium M54 to CrNiMoWV provides stepwise decreasing $\mathrm{Co}$ and $\mathrm{Ni}$ from 13 to 0 and from 11 to $3 \mathrm{wt} \%$, respectively. As Ni addition promotes reduced ductile-to-brittle transition temperature, Co was historically added to increase $\mathrm{K}_{\mathrm{IC}}$ at the ultra-high strength level, offsetting the negative effect of Ni reduction. Increased $\mathrm{K}_{\mathrm{IC}}$ due to Co addition is attributed to 4 factors: (1) reduced martensite recovery at high secondary 
hardening temperatures $\left(400-600^{\circ} \mathrm{C}\right),(2)$ solid solution strengthening of the lattice, (3) elimination of large, incoherent $(\mathrm{Fe}, \mathrm{Cr})_{3} \mathrm{C}$, and $(4)$ reduced $(\mathrm{Mo}, \mathrm{Cr})_{2} \mathrm{C}$ size $[25,26]$.

As outlined in Chapter 1, detailed TEM is only available for AerMet 100 and to a limited extent for Ferrium M54, demonstrating the complex microstructure of each UHSS [24,75]. The present work conclusively shows that reduction in $\mathrm{Co}, \mathrm{Cr}$, and $\mathrm{Ni}$ did not adversely impact SCC-HEAC resistance, which is essentially equal for AerMet 100 and Ferrium M54 at identical $\sigma_{\text {Ys }}(1,720$ $\mathrm{MPa}$, Table 1). Moreover, Figures 17-18, and 25 suggest that da/dt $\mathrm{II}$ values are lower for Ferrium M54, CrNiMoWV, and Ferrium S53 compared to AerMet 100, though a statistically significant conclusion cannot be made. The lowest crack growth rates at $\mathrm{E}_{\mathrm{app}}=-1,000 \mathrm{mV}_{\mathrm{SCE}}$ were measured for CrNiMoWV, with the lowest Ni of modern UHSS and no Co. This result shows that Co is not necessary for high SCC-HEAC resistance, as characterized by da/dtII. The comparison further suggests that a sufficient volume fraction of nano-scale carbides $\left((\mathrm{Mo}, \mathrm{Cr})_{2} \mathrm{C}, \mathrm{WC}\right.$, and $\left.\mathrm{VC}\right)$ formed to reduce $\mathrm{H}$ mobility. The strength of this comparison is reduced by lower yield and ultimate strengths exhibited by CrNiMoWV (Table 1). Detailed TEM and $\mathrm{D}_{\mathrm{H}-\mathrm{EFF}}$ measurements have not been reported to affirm these speculations for Ferrium M54 and CrNiMoWV. The measuredstrong SCC resistance and significantly lower cost of low alloy UHSS justify further investigation.

The comparison between Ferrium M54, AerMet 100 and Ferrium S53 provides for assessment of the impact of increasing $\mathrm{Cr}(1,3,10 \mathrm{wt} \%$, respectively) on TG SCC-HEAC. The high Cr content in Ferrium S53 was added for corrosion resistance via passive film formation which eliminates the need for Cd electroplating [36,139]. Passivity is logarithmically dependent on the matrix $\mathrm{Cr}$ content [140]. Moreover, Co additions to Ferrium S53 were hypothesized to significantly increase passivity by raising the activity of $\mathrm{Cr}$ in the $\mathrm{Fe}$ matrix and thus promoting partitioning of $\mathrm{Cr}$ to the oxide film [36,44]. Consideration of the crack tip H production which governs SCC, detailed in an ensuing section, leads to the hypothesis that $\mathrm{Cr}$ (and $\mathrm{Co}$ ) could improve HEAC resistance for low-to-moderate cathodic polarization [141]. Alternately, Cr may exacerbate SCC for Eapp near to OCP and somewhat anodic to OCP where significant crack tip dissolution and cation acidification may occur. The relative balance of beneficial versus harmful effects is controlled by the sensitivity of crack tip $\mathrm{H}$ production attributed to $\mathrm{Cr}$ and $\mathrm{Co}$ additions. Detailed crack chemistry measurement and modeling is outside the scope of the present study. However, the data summarized in Figures 17-18 and 24-25 do not provide any evidence of either a strong-beneficial 
or resolvable-deleterious effect of $\mathrm{Cr}$ content on SCC. Each of these 3 steels exhibits a similar window of HEAC-protective potentials cathodic to OCP which confers elevated $\mathrm{K}_{\mathrm{TH}}$. Compared to Ferrium M54 or AerMet 100, high Cr Ferrium S53 exhibits equal da/dt ${ }_{\text {II }}$ and somewhat lower $\mathrm{K}_{\mathrm{TH}}$ at potentials, which are both cathodic as well as anodic versus this window. The SCC results do not evidence either a detrimental or beneficial effect. For cathodic polarization, lack of a benefit from $\mathrm{Cr}$ is not likely to change if the steel yield strength is increased to the $1,720 \mathrm{MPa}$ level. However, lower $\mathrm{K}_{\mathrm{TH}}$ and higher da/dt $\mathrm{dI}_{\mathrm{I}}$ could be produced for a higher strength of steel near OCP, but this is questionable given the high tensile strength for Ferrium S53, similar to AerMet 100 and Ferrium M54. Notably, da/dt II significantly elevated in Ferrium S53 for a range of moderate to severe $\mathrm{E}_{\mathrm{app}}$. The origins of this trend are considered in Chapter 4, with a comparison to similar UHSSS. The impact of $\mathrm{Cr}$ on the width of the window of cathodic prevention of TG SCC is also considered in an ensuing section. Suffice to conclude at this point that the present results do not evidence a dramatic-consistent effect of high $\mathrm{Cr}$ on SCC by HEAC for UHSS in NaCl solution.

\subsubsection{Modeling the Effect of $E_{a p p}$ on HEAC Kinetics}

Applied potential affects the kinetics of SCC by a HEAC mechanism in modern AerMet 100, CrNiMoWV, Ferrium M54, and Ferrium S53, but much less so for legacy 300M. Figure 24 and Figure 25 show elevated $\mathrm{K}_{\mathrm{TH}}$ and reduced da/dt at polarizations mildly cathodic to the OCP, with decreasing SCC resistance at potentials anodic or cathodic with respect to this window. Such behavior was examined in detail for AerMet 100 in order to identify the governing occluded crack electrochemistry for hydrogen production/uptake and micromechanics of crack tip HAC $[6,7,20,142,143]$. The extensive data base in Figures 17-18, and 24-25, plus recent modeling developments, provide for a quantitative reassessment and improvement of models that capture the $\mathrm{E}_{\text {app }}$ dependence of HEAC. The result provides a framework for additional assessment of the impact of alloy $\mathrm{Cr}, \mathrm{Co}$, and martensite interface microstructure on HEAC in UHSS.

The $E_{a p p}$ dependencies shown in Figures 17 and 18 were explained for AerMet 100 based on the dissolved lattice concentration of hydrogen $\left(C_{L}\right)$ in the FPZ at equilibrium with the crack tip environment. Increasing severity of HEAC at anodic or cathodic $\mathrm{E}_{\text {app }}$ was governed by increasing crack tip overpotential for hydrogen production $(\eta)$, which dictates $C_{L}[20]$. Anodic polarization

promotes crack tip dissolution and hydrolytic acidification of $\mathrm{Fe}^{+2}$ or +3 and $\mathrm{Cr}^{+3}$ for lower local $\mathrm{pH}$ 
and, thus increases $\eta$ and $C_{L}$ [20,142]. Cathodic polarization promotes an alkaline crack solution, but $\eta$ is large, promoting water reduction to form $\mathrm{H}[6,20,144]$. HEAC resistance is maximized at mild cathodic polarizations where both crack tip dissolution/acidification and $\eta$ are sufficiently reduced, limiting $\mathrm{C}_{\mathrm{L}}$ in the FPZ. These explanations, and the important effect of FPZ stress state, are quantified as follows.

Kehler and Scully established the $\mathrm{E}_{\text {app }}$ dependence of occluded crack tip hydrogen concentration $\left(\mathrm{C}_{\mathrm{H}-\mathrm{Diff}}\right)$ for AerMet 100 based on measured local $\mathrm{pH}$ and potential in an artificial crevice, combined with a geometric scaling relationship to relate crevice chemistry to a real crack tip [20]. The result is presented in Figure 29, and least squares regression yields the potential dependence of $\mathrm{C}_{\mathrm{H}, \mathrm{Diff}}$ :

$$
C_{H, D i f f}=19.125 E_{a p p}^{3}+78.568 E_{a p p}^{2}+80.026 E_{a p p}+24.560 \quad\left(R^{2}=0.99\right)
$$

where $\mathrm{E}_{\mathrm{app}}$ is $\mathrm{V}_{\mathrm{SCE}}$ and $\mathrm{C}_{\mathrm{H} \text {,Diff }}$ is wppm as closely approximated by $\mathrm{C}_{\mathrm{L}} \exp \left[\mathrm{E}_{\mathrm{B}} / \mathrm{RT}\right.$ ] consistent with Equation 3. This Equation 5 potential dependence of $\mathrm{C}_{\mathrm{H} \text {,Diff }}$ was experimentally established with five conditions and assumptions: (1) a single crevice scaling parameter $\left(\mathrm{x}^{2} / \mathrm{G}\right.$, where $(\mathrm{x})$ is crevice depth and (G) is mouth opening) of $100 \mathrm{~mm}$ was used for the typical SCC crack depth and crack opening distances at HEAC-relevant $\mathrm{K}$ levels, (2) $\mathrm{C}_{\mathrm{L}}$ was computed from a $\mathrm{pH}$ dependent uptake law governed by $\eta$ and using crack tip $\mathrm{pH}$ of 1 for each $E_{\text {app }}$ up to $-450 \mathrm{mV}$ SCE with $\mathrm{pH}$ set to 2 for more cathodic $E_{\text {app, }}$ overall simulating severe hydrolysis, (3) flat-electrode surfaces were polished rather than passivated for $\mathrm{H}$ uptake, (4) $\mathrm{C}_{\mathrm{H} \text {,Diff }}$ was measured by Barnacle Cell extraction, as a function of known $\eta$, and (5) several points were interpolated between crack chemistry measurements [20]. The well-established potential dependence of $\mathrm{C}_{\mathrm{H}, \text { Diff, }}$, summarized in Equation 5, enables quantitative assessment of the model predictions of HEAC kinetics (Equations 1 and 2) with regard to their $\mathrm{E}_{\mathrm{app}}$ dependencies. Previous work suggested that these models are qualitatively reasonable $[6,20]$, but the available $\mathrm{K}_{\mathrm{TH}}$ and da/dt data were not sufficient for quantitative analysis. 


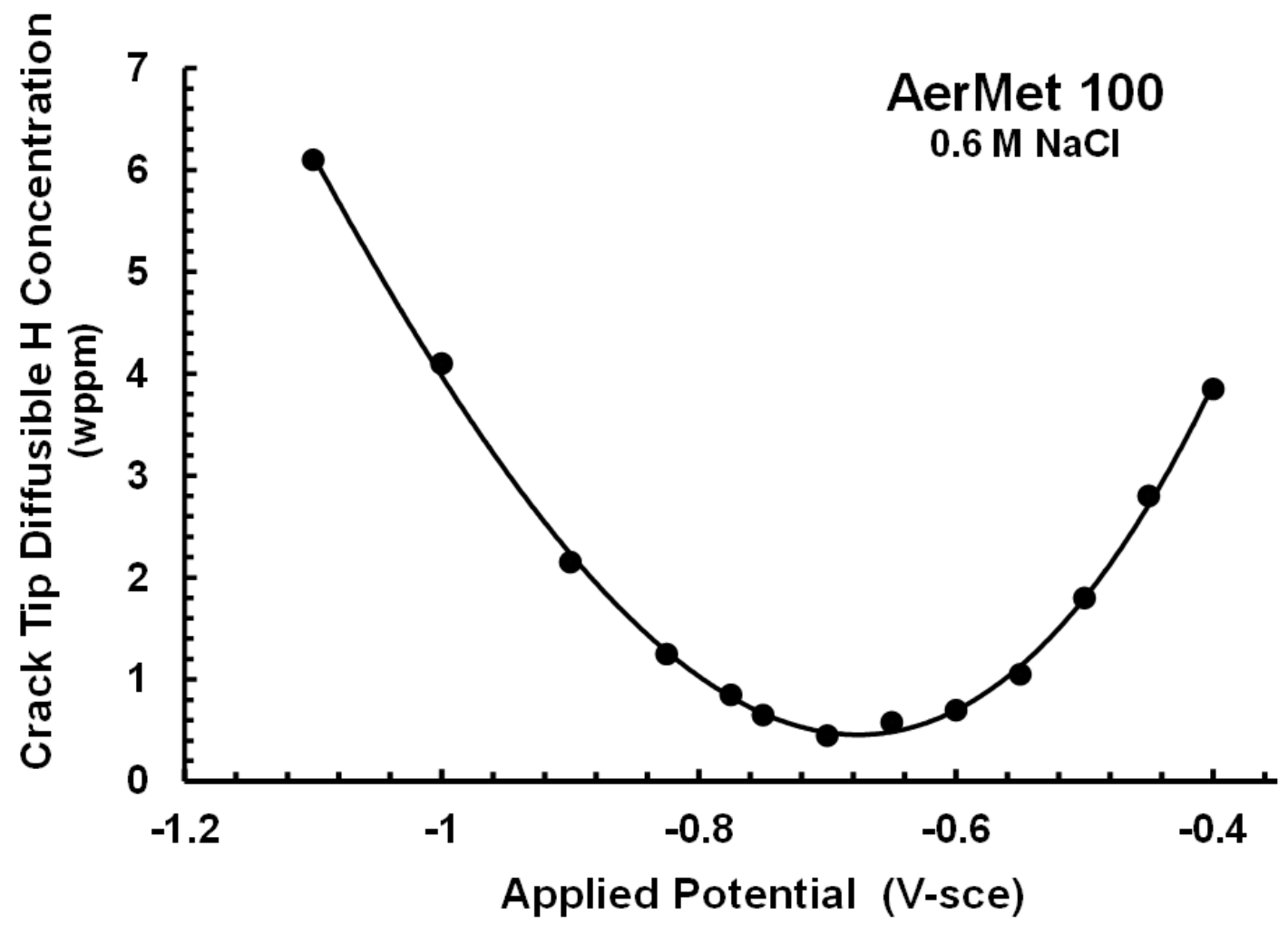

Figure 29 The bold-surface applied potential dependence of crack tip diffusible $H$ concentration established for AerMet 100 using the measurement and modeling approach of Kehler and Scully [20]. Points were estimated from their Figure 14, and the OCP of AerMet 100 declines from about -0.50 to $-0.60 \mathrm{VSCE}$ for a $20 \mathrm{~h}$ exposure in $0.6 \mathrm{M} \mathrm{NaCl}$.

\subsubsection{Modeling of $K_{T H}$ for Similar UHSS Aermet 100 and Ferrium M54}

The fundamental challenge in predicting $\mathrm{K}_{\mathrm{TH}}$ using crack tip $\mathrm{C}_{\mathrm{H} \text {,Diff }}$ is to independently establish values of the model parameters in Equation 1 [3,6,14]. Towards this goal, the independently measured dependence of $\mathrm{K}_{\mathrm{TH}}$ on the level of bulk-precharged diffusible $\mathrm{H}$ content $\left(\mathrm{C}_{\mathrm{H}-\mathrm{DIFF}}\right.$ Bulk $)$ is shown in Figure 30 for the same lot of AerMet 100 used in the present study [73,145]. 


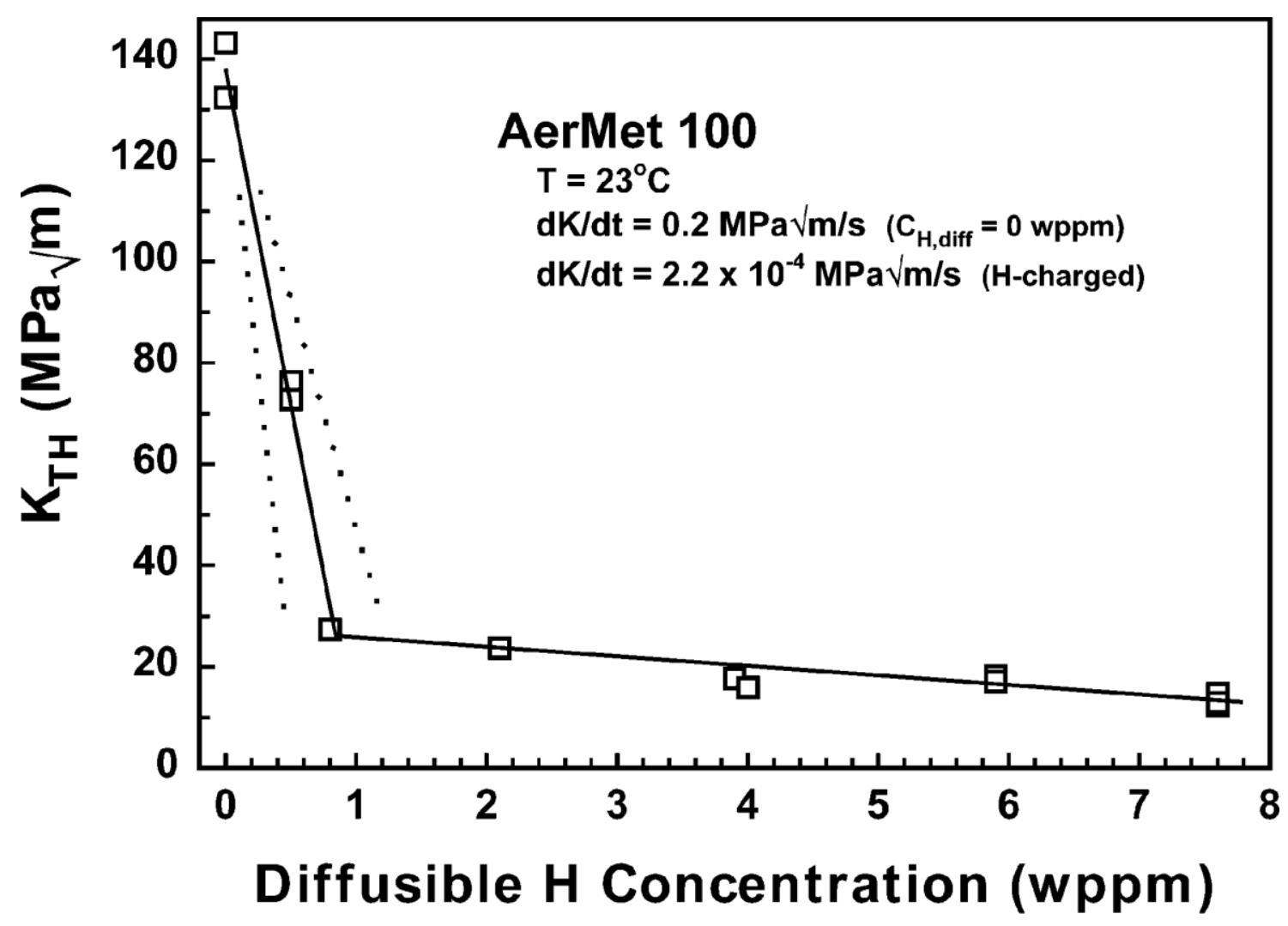

Figure 30 Bulk $C_{H-D I F F}$ Bulk dependence of $K_{T H}$ for internal hydrogen assisted cracking of AerMet 100 [145].

For these internal hydrogen assisted cracking (IHAC) experiments, $\mathrm{K}_{\mathrm{TH}}$ was measured by the same slow-rising $\mathrm{K}$ method used in the present study, applied to H-precharged compact tension (CT) specimens stressed in moist air [145]. Crack length was measured with a near-identical dcPD method. $\mathrm{C}_{\mathrm{H}-\mathrm{DIFF}}$ Bulk was measured by Barnacle Cell extraction, identical to the method used by Kehler and Scully to establish Equation 5 [20]. Severe IHAC cracking is demonstrated by rapidly falling $\mathrm{K}_{\mathrm{TH}}$ as bulk diffusible $\mathrm{H}$ concentration rises from near 0 to 8 wppm. The data in Figure 30 are described by two linear segments:

$$
K_{T H}=a-b C_{H-D i f f B u l k}
$$

where $\mathrm{K}_{\mathrm{TH}}$ is $\mathrm{MPa} \sqrt{\mathrm{m}}$ and $\mathrm{C}_{\mathrm{H} \text {-Diff-Bulk }}$ is in wppm. Fitting constants for the two behavioral regions are $a=28.87$ and $b=1.77$ for $\mathrm{C}_{\mathrm{H}-\text {-Diff-Bulk }} \geq 0.82 \mathrm{wppm}$, and $a=147.5$ and $b=146.04$ for $\mathrm{C}_{\mathrm{H}-\mathrm{Diff}-\mathrm{Bulk}} \leq 0.82 \mathrm{wppm}$. The dramatic change in $\mathrm{K}_{\mathrm{TH}}$ versus $\mathrm{C}_{\mathrm{H} \text {-DIFF Bulk }}$ correlates with a fracture path transition from $100 \%$ transgranular QC (identical to Figure 19) for all $\mathrm{C}_{\mathrm{H}-\mathrm{DIF} \text { BulkF }}$ above 0.82 
wppm to a mixed fracture morphology with increasing fraction of microvoid-based cracking as $\mathrm{C}_{\mathrm{H}-\mathrm{DIFF} \text { Bulk }}$ is reduced from $0.82 \mathrm{wppm}$ to near zero.

As a first step in determining the constants for use in Equation 1, Equation 6 is used to empirically predict the dependence of $\mathrm{K}_{\mathrm{TH}}$ on $\mathrm{E}_{\mathrm{app}}$, where $\mathrm{C}_{\mathrm{H} \text {,Diff }}$ relevant to the crack tip is related to $\mathrm{E}_{\mathrm{app}}$ through Equation 5. This connection is based on the assumption that uniformly precharged $\mathrm{H}$ ( $\mathrm{C}_{\mathrm{H} \text {-Diff Bulk }}$ ) for IHAC and crack tip $\mathrm{H}$ solubility $\left(\mathrm{C}_{\mathrm{H} \text {,Diff }}\right)$ for $\mathrm{HEAC}$ will produce identical $\mathrm{K}_{\mathrm{TH}}$ if loading is sufficiently slow to permit redistribution of $\mathrm{H}$ from either source to the crack tip FPZ. For this analysis it is assumed that AerMet 100 and Ferrium M54 have identical crack tip H production/uptake behavior, and the data shown in Figures 29-30 are representative of Ferrium M54 if similar measurements were conducted. This point has not been experimentally verified. The result of this analysis is represented by the upper-dashed line in Figure 31 (Model 1.0).

Three points are notable. First, this approach using the IHAC input from Equation 5 captures the $\mathrm{E}_{\text {app }}$ dependence of $\mathrm{K}_{\mathrm{TH}}$ reasonably well without any adjustable-fitting parameters. Second, the window of mildly cathodic $\mathrm{E}_{\mathrm{app}}$ with increased $\mathrm{K}_{\mathrm{TH}}$ correlates well with the sharp change in the $\mathrm{K}_{\mathrm{TH}}$ dependence of precharged $\mathrm{H}$ seen in Figure 30. The HEAC fracture surfaces of AerMet 100 and Ferrium M54 tested at $\mathrm{E}_{\mathrm{app}}=-625$ to $650 \mathrm{mV}$ SCE each exhibit a mixed TG-microvoid fracture morphology, identical to observations of IHAC fracture in the region of rapidly rising $\mathrm{K}_{\mathrm{TH}}$ (shown in Figure 30). This result supports the hypothesis that an identical $\mathrm{C}_{\mathrm{H} \text {,Diff }}$ will yield identical $\mathrm{K}_{\mathrm{TH}}$ regardless of the origin of the diffusible $\mathrm{H}$, and that the hydrogen uptake behavior of Aermet 100 and Ferrium M54 is similar. Finally, predicted $\mathrm{K}_{\mathrm{TH}}$ is systematically higher compared to measured $\mathrm{K}_{\mathrm{TH}}$. For example, the lowest $\mathrm{K}_{\mathrm{TH}}$ reported for IHAC of H-precharged AerMet 100 is $15 \mathrm{MPa} \sqrt{\mathrm{m}}$ at high $\mathrm{C}_{\mathrm{H}-\mathrm{DIFF}}$ Bulk of 8 wppm [145]. The lowest $\mathrm{K}_{\mathrm{TH}}$ measured for HEAC in AerMet 100 and Ferrium M54 is 9-10 MPa $\sqrt{\mathrm{m}}$ for strong-cathodic as well as mild anodic polarizations. It is unlikely that differences in measured $\mathrm{C}_{\mathrm{H} \text {,Diff }}$ and $\mathrm{C}_{\mathrm{H}-\mathrm{DIFF} \text { Bulk }}$ explain this difference $[20,145]$. 


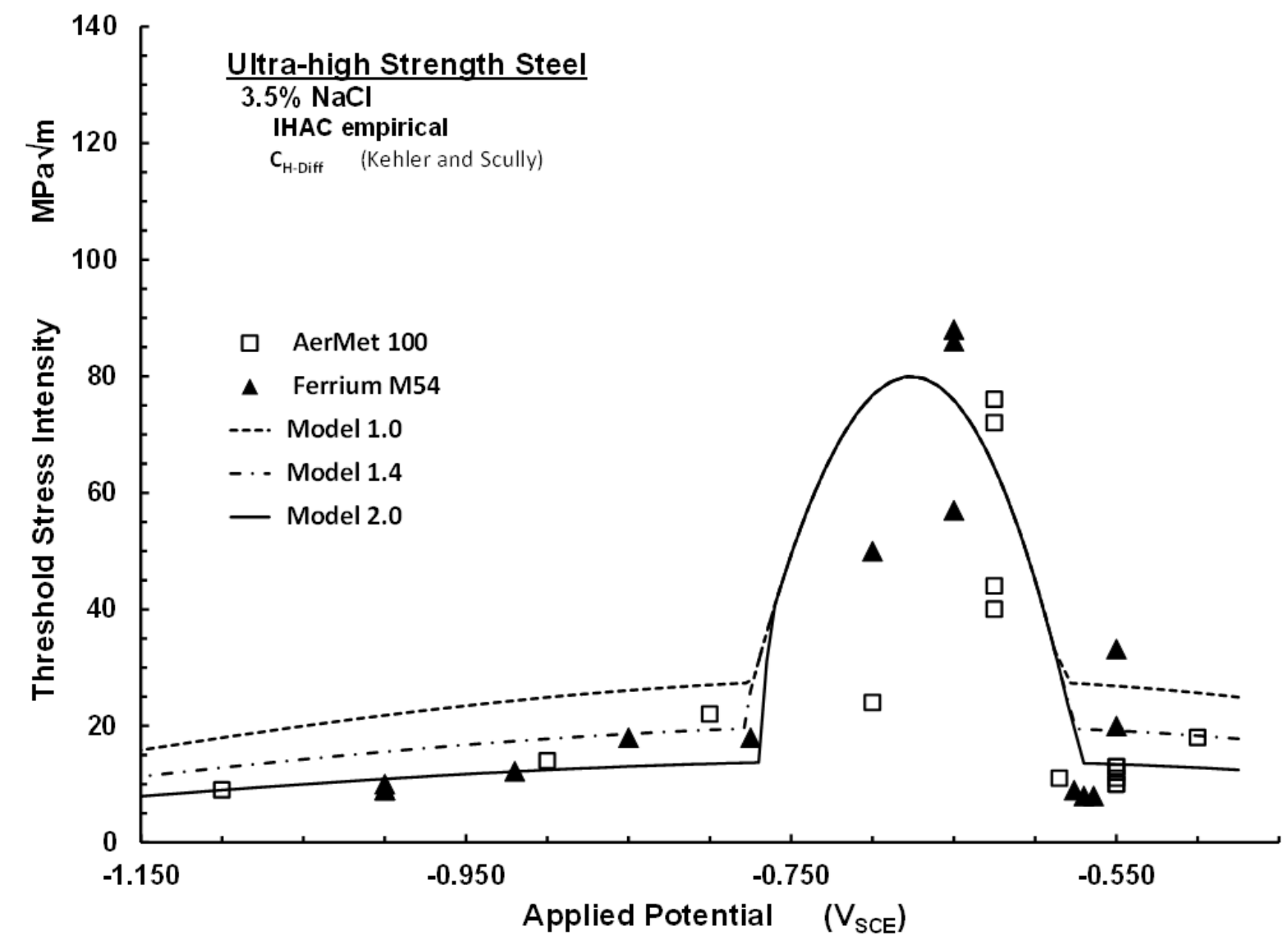

Figure 31 Measured $(\square \Delta)$ and model predicted (--) applied potential dependence of $K_{T H}$ for AerMet 100 and Ferrium M54, using the measured $H$ concentration dependence of $K_{T H}$ determined for H precharged AerMet 100, as given by Figure 30.

Speculatively, the difference between measurements and model predictions shown in Figure 31 is traced to the different experimental measurements of $\mathrm{K}_{\mathrm{TH}}$. For HEAC measurements, the high precision dcPD method applied to the SENT specimen with a small fatigue precrack likely detected small amounts of $\mathrm{H}$-enhanced microcracking at a lower bound $\mathrm{K}_{\mathrm{TH}}(\square$ and $\boldsymbol{\Delta}$ in Figure 31). For IHAC measurement (Figure 30), a CT specimen with long-fatigue precrack was monitored by dcPD and upper bound $\mathrm{K}_{\mathrm{TH}}$ was established based on an operational definition of a threshold potential change that would likely not capture very small amounts of microcracking [145-147]. Published dcPD voltage versus crack mouth opening displacement data (Figure 2 in [145]) suggest that $\mathrm{H}$ cracking may have initiated at a $\mathrm{K}$ level $40 \%$ lower than the values shown in Figure 30. 
This reduction was assumed to apply for all $\mathrm{E}_{\mathrm{app}}$ where $\mathrm{C}_{\mathrm{H}-\mathrm{DIFF}}$ Bulk (Equation 5) exceeded 0.8 wppm (The threshold between $\mathrm{K}_{\mathrm{TH}}$ vs $\mathrm{C}_{\mathrm{H}-\mathrm{DIFF}-\mathrm{Bulk}}$ behavioral regions), but not for lower $\mathrm{H}$ concentrations where H-enhanced microcracking is less likely. In summary, there is some evidence that the data analysis methods used to determine $\mathrm{K}_{\mathrm{TH}}$ during IHAC of AerMet 100 may yield substantial over estimates of the threshold for the onset of slow crack growth. To improve model predictions, $a$ and $b$ in Equation 6 were reduced by a factor of 1.4, but only for the high$\mathrm{C}_{\mathrm{H}-\mathrm{DIFF}-\mathrm{Bulk}}$ segment of the IHAC data. The revised prediction of $\mathrm{K}_{\mathrm{TH}}$ versus $\mathrm{E}_{\mathrm{app}}$ for HEAC is shown by the dot-dashed line in Figure 31 (Model 1.4). Further, the improved resolution of the dcPD method applied to a small crack could produce an additional lowering of the upper bound $\mathrm{K}_{\mathrm{TH}}$ detected by the long crack IHAC experiments [99]. The magnitude of this $\mathrm{K}_{\mathrm{TH}}$ difference was estimated by forcing model predicted $\mathrm{K}_{\mathrm{TH}}$ to agree with the HEAC $\mathrm{K}_{\mathrm{TH}}$ for the most severe level of cathodic polarization (-1.100 $\left.\mathrm{V}_{\mathrm{SCE}}\right)$ in Figure 31. The resulting $\mathrm{K}_{\mathrm{TH}}$ reduction of 2.0 was applied to $a$ and $b$ in Equation 6, and the resulting empirical prediction is given by the solid line (Model 2.0) in Figure 31. Notably, predicted $\mathrm{K}_{\mathrm{TH}}$ for this model show the best agreement to measured HEAC $\mathrm{K}_{\mathrm{TH}}$ over a wide range of $\mathrm{E}_{\mathrm{app}}$.

Given the good agreement of Model 2.0 in Figure 31, resolution-corrected values of $\mathrm{K}_{\mathrm{TH}}$ versus $\mathrm{C}_{\mathrm{H} \text {-DIFF Bulk }}$ for IHAC were fit using Equations 1 and 3 to establish the parameters for the decohesion mechanism-based model of $\mathrm{K}_{\mathrm{TH}}$ put forth by Gerberich [13]. Prior analysis has suggested that the $\mathrm{K}_{\mathrm{TH}}$ values shown in Figure 30 were well predicted versus $\mathrm{C}_{\mathrm{H}-\mathrm{DIFF}}$ Bulk above 0.8 wppm using Equation 1 with: $\alpha "=0.0002 \mathrm{MPa}^{-1}, \beta^{\prime}=0.13(\mathrm{MPa} \sqrt{\mathrm{m}})^{-1}, \mathrm{k}_{\mathrm{IG}}=0.85 \mathrm{MPa} \sqrt{\mathrm{m}}, \alpha=0.5$ $\mathrm{MPa} \sqrt{ } \mathrm{m} /$ atom fraction $\mathrm{H}$, and $\sigma_{\mathrm{H}}=5.2 \sigma_{\mathrm{YS}}$ in Equation 3 (Figure 31) [145], in good agreement with work by Gerberich and coworkers [148]. However, recent work suggests alternate values for these parameters, which are more physically justified [3]. Modification of these parameters for modeling in the present work is two-fold. First, the set of $\mathrm{k}_{\mathrm{IG}}, \alpha^{\prime}$ ' and $\beta^{\prime}$ must be constrained to yield a physically reasonable value of the plasticity-shielded H-free toughness of the HEAC crack path. For transgranular martensite interface cracking, this toughness must logically exceed the measured $\mathrm{K}_{\mathrm{IC}}$ of AerMet 100 and Ferrium M54 (126-130 MPa $\sqrt{\mathrm{m}}$, Table 1), which are dictated by microvoidbased fracture. Simply put, the Gerberich model must predict a $\mathrm{K}_{\mathrm{TH}}$ similar to $\mathrm{K}_{\mathrm{IC}}$ in the absence of hydrogen. With $\mathrm{C}_{\mathrm{H}-\mathrm{DIFF} \text { Bulk }}=0$, assuming a martensite interface toughness of $140 \mathrm{MPa} \sqrt{\mathrm{m}}$, and solving for the remaining parameters yielded $\alpha^{\prime \prime}=0.00022 \mathrm{MPa}^{-1}, \beta^{\prime}=0.20(\mathrm{MPa} \sqrt{\mathrm{m}})^{-1}$, and $\mathrm{k}_{\mathrm{IG}}$ 
$=1.125 \mathrm{MPa} \sqrt{\mathrm{m}}$. Second, the value of crack tip $\sigma_{\mathrm{H}}$ in Equation 3 is controversial [3]. Original modeling by Gerberich and coworkers suggested very high crack tip normal stresses of 16-25 GPa depending on the details of the dislocation simulation and level of stress relaxation due to $\mathrm{H}$ localization. This result corresponds to crack tip hydrostatic stress $\left(\sigma_{H}\right)$ of $11,000 \mathrm{MPa}\left(6.6 \sigma_{\mathrm{Ys}}\right.$ for AerMet 100) to $18,000 \mathrm{MPa}\left(10.4 \sigma_{Y S}\right)[13,149]$. Such high crack tip stresses are supported by a small deformation strain gradient plasticity model $[150,151]$. A large-strain blunt-crack model applied to AerMet 100 type UHSS yields a lower bound for crack tip $\sigma_{H}$ of $2.1 \sigma_{Y S}$, which is increased by strain gradient plasticity to $4.2 \sigma_{Y S}$ or higher depending on the length scale used in the strain gradient model [152]. This latter SGP modeling is presently in progress. Based on these considerations, two levels of $\sigma_{\mathrm{H}}$ are examined, $4.2 \sigma_{\mathrm{YS}}$ and $6.8 \sigma_{\mathrm{Ys}}$.

To complete prediction of the $\mathrm{E}_{\mathrm{app}}$ dependence of $\mathrm{K}_{\mathrm{TH}}$ for HEAC for AerMet 100 and Ferrium M54, measured $\mathrm{K}_{\mathrm{TH}}$ on the lower segment of the IHAC data for $\mathrm{C}_{\mathrm{H} \text {-DIFF Bulk }}(\geq 0.9$ wppm $\mathrm{H}$, Figure 30) was reduced by a factor of 2.0 to account for measurement resolution discussed in the previous section, and fit to Equations 1 and 3 to calculate the 'best' independent prediction of $\alpha$. This $\alpha$ from IHAC (plus the adjusted $\beta$ ', $\alpha$ ", $\sigma_{\mathrm{H}}$, and $\mathrm{k}_{\mathrm{IG}}$ ) were then used in conjunction with Equations 1 and 3 to predict the $\mathrm{E}_{\text {app }}$ dependence of $\mathrm{K}_{\mathrm{TH}}$ with $\mathrm{C}_{\mathrm{H} \text {,Diff }}$ related to $\mathrm{E}_{\mathrm{app}}$ through Equation 5. The results are shown for $\sigma_{H} / \sigma_{Y S}=4.2$ (Figure 32) and $\sigma_{H} / \sigma_{Y S}=6.8$ (Figure 34), where Figure 33 and Figure 35 each show the quality of the IHAC fit that yielded the adjustable $\alpha$ values of 8.0 and 0.9 $\mathrm{MPa} \sqrt{ } \mathrm{m} /$ atom fraction $\mathrm{H}$, respectively, for these two crack tip hydrostatic stress levels. The dashed portion of each HEAC model prediction represents the regime where IHAC $\mathrm{K}_{\mathrm{TH}}$ transitions to a steeply rising dependence on $\mathrm{C}_{\mathrm{H}-\mathrm{DIFF} \text { Bulk }}$ (Figure 30), associated with progressively increasing of amounts microvoid fracture in IHAC of AerMet 100. Such MVC is present in HEAC of AerMet 100 and Ferrium M54 for the low values of $\mathrm{C}_{\mathrm{H} \text {,Diff }}$ in this dotted-line regime of $\mathrm{E}_{\text {app }}$. As such, this portion of the Equation 1 model in Figure 32 and 34 provides a lower bound of $\mathrm{K}_{\mathrm{TH}}$ relevant to HEAC; modeling of a fractional contribution from $\mathrm{H}$-independent MVC fracture in this potential regime would result in higher-predicted levels of $\mathrm{K}_{\mathrm{TH}}$ compared to Equation 1. 


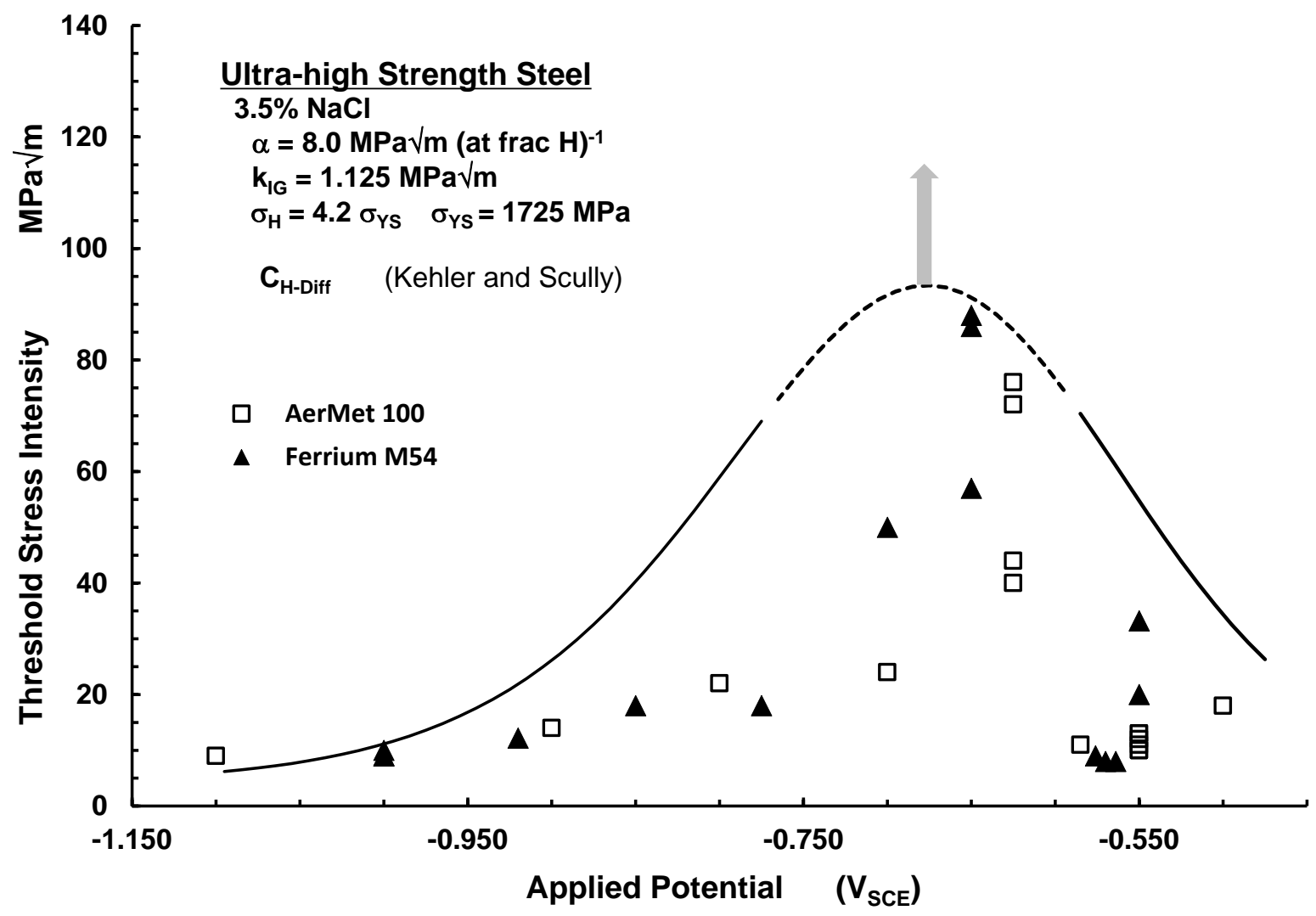

Figure 32 Measured $\left(\square \Delta\right.$ ) and model predicted (--) $E_{\text {app }}$ dependence of $K_{T H}$ for AerMet 100 and Ferrium M54, using a H-decohesion based model (Equation 1) with $\beta$ ', $\alpha$ ", kIG, and $\alpha$ calibrated by fit to IHAC data for $H$ precharged AerMet 100 (Figure 30) and lower bound $\sigma_{H}=$ 4.2 $\sigma_{Y S}$. 


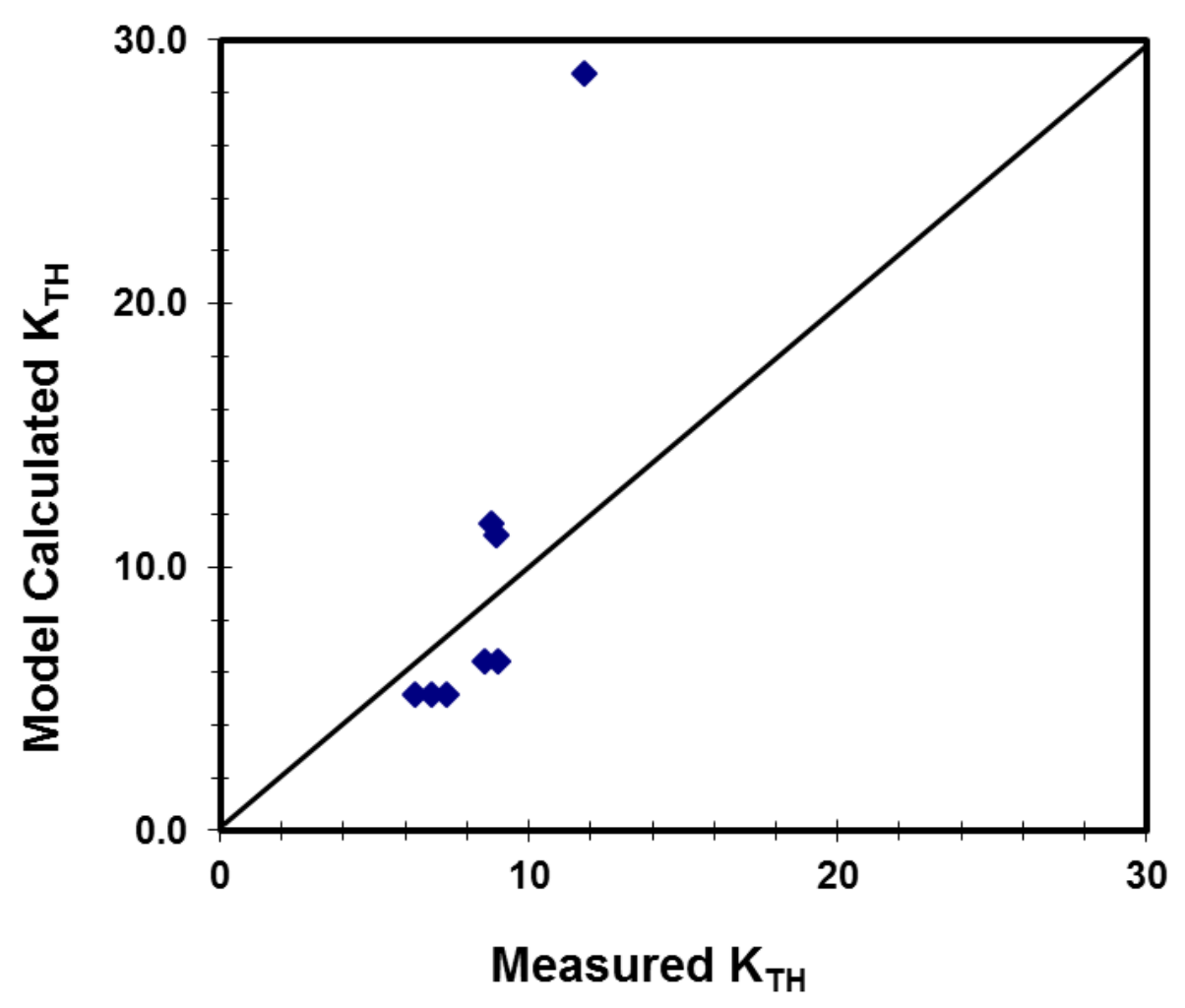

Figure 33 Comparison of $K_{T H}$ measured for IHAC by Thomas et al [144] and model predicted $K_{T H}$ for the conditions shown in Figure 32.

In summary, the results in Figure 32 and 34 validate the $\mathrm{H}$ decohesion based threshold model (Equation 1) coupled with the independent measurements of crack tip $\mathrm{C}_{\mathrm{H} \text {,Diff }}$ versus bold-surface $\mathrm{E}_{\text {app }}$ in AerMet 100 (Equation 5) [71] and independent model calibration of the $\mathrm{K}_{\mathrm{TH}}$ model parameters using IHAC data (Figure 33 and Figure 35) [145]. In this sense, there are no adjustable parameters apart from the uncertain crack tip hydrostatic tensile stress. A similar-strong validation was reported for IHAC in a Ni-Cu superalloy using values of the model input parameters similar to those selected for UHSS [9]. Two points are noteworthy. First, Figure 33 and Figure 35 each show good agreement between measured and predicted $\mathrm{K}_{\mathrm{IH}}$ for IHAC of AerMet 100, similar to that previously reported [145]. The exception is the case of $\mathrm{C}_{\mathrm{H} \text {-DIFF Bulk }}=0.85 \mathrm{wppm}$. This data point is at the diffusible $\mathrm{H}$ concentration where MVC begins to contribute to $\mathrm{H}$ fracture, but the cause of over-predicted $\mathrm{K}_{\mathrm{TH}}$ is not known. Second, the best agreement between measured and

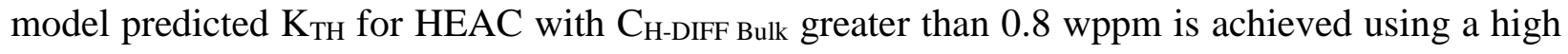
level of $\sigma_{H} / \sigma_{Y S}=6.8$, consistent with dislocation-based simulation [149], small-deformation SGP 
modeling [150], and experience with the Ni-Cu superalloy [3]. The agreement between measured and modeled $\mathrm{K}_{\mathrm{TH}}$ systematically improves as $\sigma_{\mathrm{H}} / \sigma_{\mathrm{YS}}$ increases from 4.2 to 8.0 , with the associated $\alpha$ decreasing from 8.0 to 0.70 . Additional modeling of crack tip stresses is required, including refined SGP formulation and length scale as well as large-deformation theory [152], before concluding that the results in Figure 34 validate the threshold model represented in Equation 1.

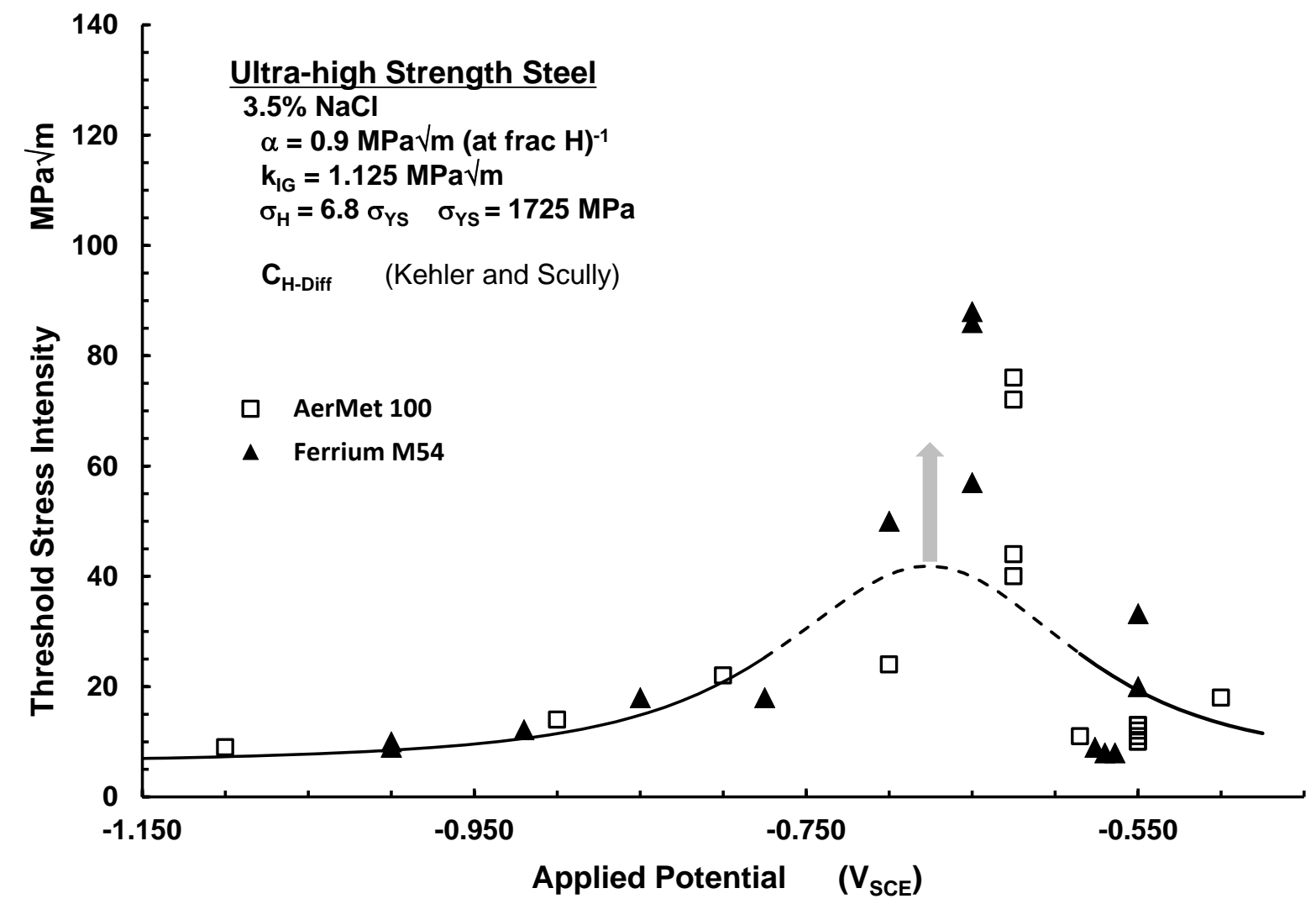

Figure 34 Measured ( $\square \Delta$ ) and model predicted (--) $E_{\text {app }}$ dependence of $K_{T H}$ for AerMet 100 and Ferrium M54, using a H-decohesion based model (Equation 1) with $\beta$ ', $\alpha$ ", kIG, and $\alpha$ calibrated by fit to IHAC data for H precharged AerMet 100 (Figure 30) and lower bound $\sigma_{H}=$ 6.8$\sigma_{Y S}$. 


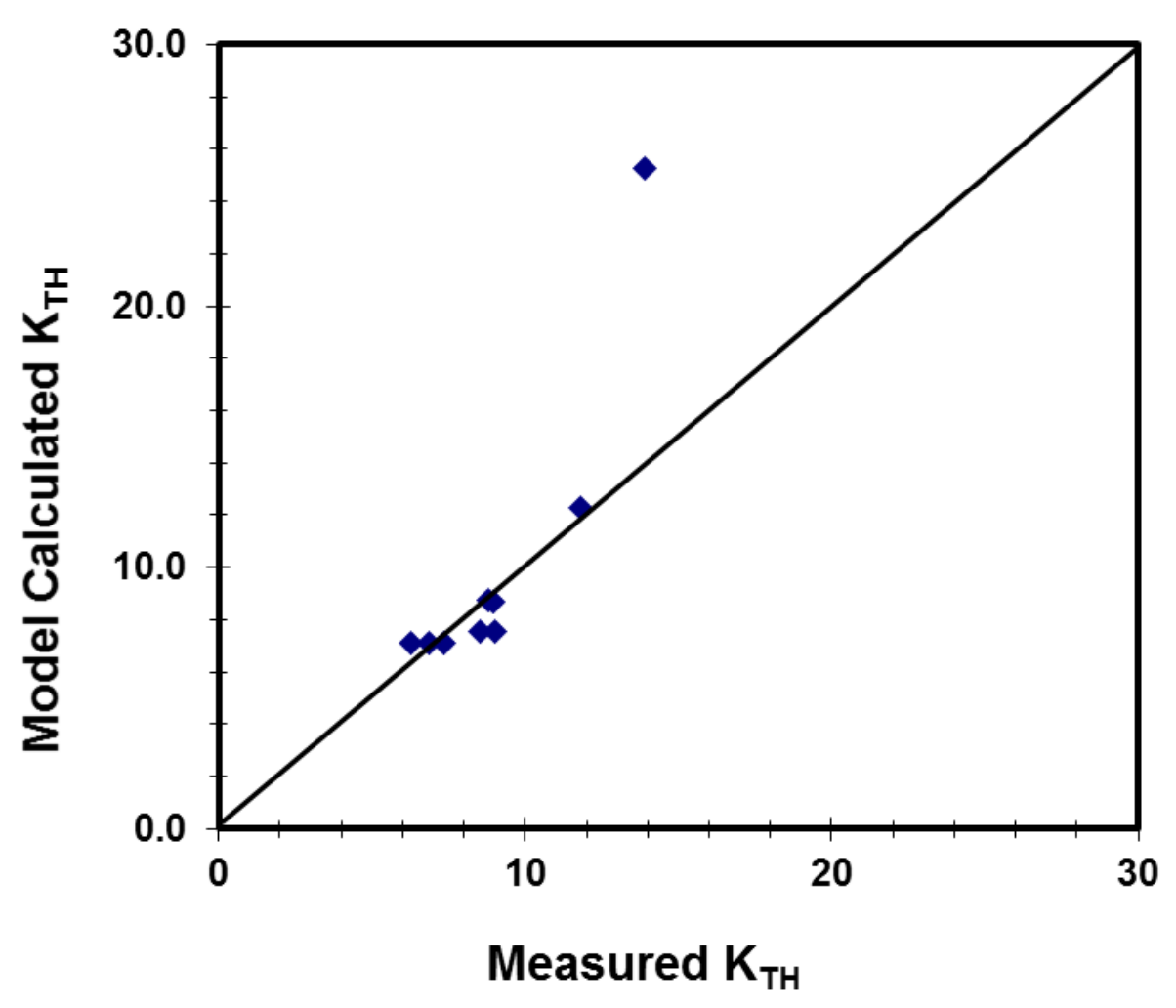

Figure 35 Comparison of $K_{T H}$ measured for IHAC by Thomas et al [144] and model predicted $K_{T H}$ for the conditions shown in Figure 34.

\subsubsection{Modeling of da/dtII for AerMet 100 and Ferrium M54}

The dependence of da/dt II on $E_{\text {app }}$ is predicted through Equation 2, where the important parameters of $\mathrm{D}_{\mathrm{H}-\mathrm{EFF}}$ and $\chi_{\text {crit }}$ are taken from independent literature, and $\mathrm{C}_{\mathrm{H \sigma}-\mathrm{crit}} / \mathrm{C}_{\mathrm{H} \sigma}$ is established to be consistent with the results of $\mathrm{C}_{\mathrm{H}, \mathrm{Diff}}$ determination (Equations 5 and 6). A broad correlation between fastest measured da/ $\mathrm{dt}_{\text {II }}$ and reported $\mathrm{D}_{\text {H-EFF }}$ demonstrated that $\chi_{\text {crit }}=900 \mathrm{~nm}$ for a wide range of materials and orders of magnitude change in $\mathrm{H}$ diffusivity [2]. The effect of $\sigma_{\mathrm{H}}$ on crack tip $\mathrm{C}_{\mathrm{H \sigma}}$ is described by the bounding values of $\sigma_{\mathrm{H}} / \sigma_{\mathrm{YS}}(4.2$ and 6.8$)$ used and justified in previous sections of $\mathrm{K}_{\mathrm{TH}}$ modeling.

The challenge for crack tip $\mathrm{H}$ diffusion modeling is to capture the effect of $\mathrm{H}$ trapping, which dictates $\mathrm{H}$-concentration dependent $\mathrm{D}_{\mathrm{H} \text {-EFF }}$ and thus the experimental method dependent $\mathrm{H}$ concentration profile at the crack tip $[153,154]$. The $\mathrm{D}_{\mathrm{H}-\mathrm{EFF}}$ for AerMet 100 at $23^{\circ} \mathrm{C}$ was previously measured by two methods: galvanostatic H-uptake yielded $D_{H-E F F}=1 \times 10^{-9} \mathrm{~cm}^{2} / \mathrm{s}$ [17] and TDS 
extraction yielded $D_{\mathrm{H}-\mathrm{EFF}}=1 \times 10^{-8} \mathrm{~cm}^{2} / \mathrm{s}$ to $6 \times 10^{-9} \mathrm{~cm}^{2} / \mathrm{s}$ as $C_{\mathrm{H}-\mathrm{DIFF}}$ fell from 6.0 wppm to 0.8 wppm [16]. D D-EFF from an $\mathrm{H}$ uptake experiment (e.g., permeation or galvanostatic charging) should reasonably estimate $\mathrm{D}_{\mathrm{H}-\mathrm{EFF}}$ for $\mathrm{da} / \mathrm{dt}_{\mathrm{II}}$ prediction due to the close agreement of experimental conditions with HEAC testing. The galvanostatic $\mathrm{H}$ charging result of $1 \times 10^{-9} \mathrm{~cm}^{2} / \mathrm{s}$ is used in the present $\mathrm{HEAC}$ modeling for high $\mathrm{C}_{\mathrm{H} \text {-diff }}$ conditions, closely approximated by the high hydrogen fugacity charging condition used by Figueroa and Robinson $\left(-1 \mathrm{~mA} / \mathrm{cm}^{2} 0.2 \mathrm{M} \mathrm{NaOH}\right.$, hydrogen recombination was poisoned with thiourea) [145]. This absolute $\mathrm{D}_{\mathrm{H}-\mathrm{EFF}}$ may be reduced to reflect the effect of reversible trapping, which increasingly slows $\mathrm{H}$ diffusion as $\mathrm{C}_{\mathrm{H}, \mathrm{Diff}}$ falls [145].

The da/dt $/$ dependence on $\mathrm{E}_{\mathrm{app}}$ is modeled using $\mathrm{C}_{\mathrm{H} \text {,Diff }}$ from Equation 5, $\mathrm{C}_{\mathrm{H \sigma}}$ from Equation 3 and an adjustable value of $\mathrm{C}_{\mathrm{H \sigma} \text {-crit }}$ for best fit of Equation 2 to da/dt $\mathrm{dI}_{\text {II }}$ measurements for AerMet 100 and Ferrium M54 (Figure 25). The results are shown in Figure 36 for the two bounds of $\sigma_{H} / \sigma_{Y S}$, 4.2 and 6.8 as previously justified for $\mathrm{K}_{\mathrm{TH}}$ modeling. Figure 36 demonstrates good overall agreement between the measured and predicted $E_{\text {app }}$ dependencies of da/dt at $\mathrm{K}=40 \mathrm{MPa} \sqrt{\mathrm{m}}$ with adjustable-fit values of $\mathrm{C}_{\mathrm{H \sigma} \text {-crit; }} 180 \mathrm{wppm} \mathrm{H}$ for $\sigma_{\mathrm{H}} / \sigma_{\mathrm{YS}}=4.2$ and 6,500 wppm $\mathrm{H}$ for $\sigma_{\mathrm{H}} / \sigma_{\mathrm{YS}}=6.8$. Four important points are established. First, the strong reduction then increase in da/dt $\mathrm{II}_{\mathrm{I}}$ with increasing-cathodic polarization below OCP is governed by crack tip $\mathrm{H}$ solubility through Equation 5. Compare Figure 29 and Figure 36: the minima in crack tip diffusible H concentration at $\mathrm{E}_{\mathrm{app}}$ of $-700 \mathrm{mV}_{\mathrm{SCE}}$ correlates with the predicted and observed minima in $\mathrm{da} / \mathrm{dt}$ at $\mathrm{K}=40$ $\mathrm{MPa} \sqrt{ } \mathrm{m}$. Second, the absolute value of the highest $\mathrm{da} / \mathrm{dt}_{\mathrm{II}}$ at the most severe level of cathodic polarization is accurately predicted for each crack tip stress state using the selected value of $\mathrm{D}_{\mathrm{H}-\mathrm{EFF}}$. In this regime, $\mathrm{C}_{\mathrm{H} \text {,Diff }}$ is large (6.2 wppm), dictating very high $\mathrm{C}_{\mathrm{H} \sigma} ; 2,175$ wppm and 82,000 wppm for the two bounds of crack tip $\sigma_{\mathrm{H}}$. Accordingly, the inverse error function term in Equation 2 is 1.4 . As such, da/dt $\mathrm{dI}_{\text {II }}$ essentially equals $4 \mathrm{D}_{\mathrm{H}-\mathrm{EFF}} / \chi_{\text {crit }}$ and the good agreement with measured $\mathrm{da} / \mathrm{dt}_{\mathrm{II}}$ supports this $\mathrm{H}$ diffusion model since $\mathrm{D}_{\mathrm{H}-\mathrm{EFF}}$ and $\chi_{\text {crit }}$ are independently established in previous work $[2,20,145]$. Third, the sharp decline in $\mathrm{da} / \mathrm{dt}_{\mathrm{II}}$ at $\mathrm{E}_{\mathrm{app}}$ within the protective window is leveraged by the inverse error function term in Equation 2, which falls from 2.9 to 1.0 to 0.0003

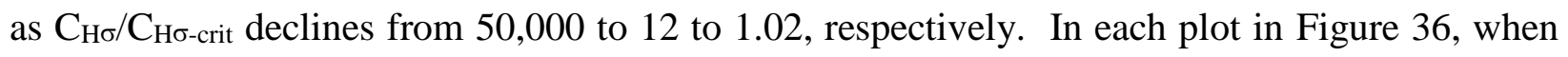
the argument for the inverse error function term is negative due to $\mathrm{C}_{\mathrm{H \sigma}} \leq \mathrm{C}_{\mathrm{H \sigma} \text {-crit, }}$, the predicted $\mathrm{da} / \mathrm{dt}_{\text {II }}$ was equated to $4 \times 10^{-5} \mu \mathrm{m} / \mathrm{s}$; essentially the best possible resolution limit of $\mathrm{dcPD}$ monitoring of crack growth. As such, this model predicts immunity to HEAC in this protective 
$\mathrm{E}_{\text {app }}$ regime. Forth, absolute values of $\mathrm{da} / \mathrm{dt}_{\text {II }}$ are over-predicted for $\mathrm{E}_{\text {app }}$ below the $\mathrm{da} / \mathrm{dt}_{\mathrm{II}}$ minimum for each stress condition. This effect will be considered in an ensuing section.

Knowledge of the $\mathrm{E}_{\mathrm{app}}$ dependence of $\mathrm{K}_{\mathrm{TH}}$ provides an independent means to establish $\mathrm{C}_{\mathrm{H \sigma}-\mathrm{crit}}$, which was previously used as an adjustable parameter to establish the predictions in Figure 36. The $\mathrm{H}$ embrittlement models for $\mathrm{K}_{\mathrm{TH}}$ (Equation 1) and $\mathrm{H}$ diffusion limited da/dt $\mathrm{dt}_{\mathrm{II}}$ (Equation 2) each depend on $\mathrm{C}_{\mathrm{H \sigma}}$ (Equation 3). Solving Equations 1 and 2 to eliminate $\mathrm{C}_{\mathrm{H \sigma}}$ yields:

$$
\mathrm{C}_{\mathrm{H} \sigma-\text { crit }}=\frac{1}{\alpha}\left(\mathrm{k}_{\mathrm{IG}}-\sqrt{\alpha^{\prime \prime} \sigma_{\mathrm{YS}} \ln \left(\beta^{\prime} \mathrm{K}_{\mathrm{TH}}\right)}\right)\left(1-\operatorname{erf}\left(\sqrt{\mathrm{X}_{\mathrm{CRIT}} \frac{\mathrm{da}}{\mathrm{dt}_{\mathrm{II}} / 4 \mathrm{D}_{\mathrm{H}-\mathrm{EFF}}}}\right)\right)
$$

This result enables an absolute prediction of $\mathrm{da} / \mathrm{dt}_{\text {II }}$ versus $\mathrm{E}_{\text {app }}$ using the independently established values of $\mathrm{D}_{\mathrm{H}-\mathrm{EFF}}$, crack tip $\mathrm{H}$ solubility (Equation 5) and literature-based $\chi_{\text {crit }}$ coupled with $\beta$ ', $\alpha$ ", $\sigma_{H}, \alpha$, and $k_{I G}$ established by $K_{T H}$ modeling for the bounding stress conditions $\left(\sigma_{H} / \sigma_{Y S}=4.2\right.$, Figure 32 and $\sigma_{H} / \sigma_{Y S}=6.8$, Figure 34 ). Any set of measured $\mathrm{K}_{\mathrm{TH}}$ and da/dt $\left(\right.$ II constant $\mathrm{E}_{\mathrm{app}}$ ) can be used in Equation 7 to solve for $\mathrm{C}_{\mathrm{H \sigma}-\text { crit. }}$. The values of $\mathrm{K}_{\mathrm{TH}}$ and da/dt $\mathrm{dt}_{\mathrm{II}}$ at $\mathrm{E}_{\mathrm{app}}$ of $-1,100 \mathrm{mV}_{\mathrm{SCE}}$ are readily measured. To test model consistency, Equation 7 was used with these parameters to predict the $\mathrm{C}_{\mathrm{H \sigma} \text {-crit }}$ of Ferrium M54 and AerMet 100 for comparison with the values given from previous curve fits in Figure 36. The ratio of curve fit to predicted (Equation 7) $\mathrm{C}_{\mathrm{H} \sigma \text {-crit }}$ is 3.4 for $\sigma_{\mathrm{H}} / \sigma_{\mathrm{YS}}=6.8,1.7$ for $\sigma_{\mathrm{H}} / \sigma_{\mathrm{YS}}=5.7$, and 0.91 for $\sigma_{\mathrm{H}} / \sigma_{\mathrm{YS}}=4.2$. These comparisons suggest that the independently developed models for $\mathrm{K}_{\mathrm{TH}}$ and $\mathrm{da} / \mathrm{dt}_{\mathrm{II}}$ are consistent, which supports this approach to HEAC predictions. The agreement is near perfect for $\sigma_{H} / \sigma_{Y S}$ of 4.2 , and is less so for the higher $\sigma_{H} / \sigma_{Y S}$. This result is reversed compared to modeling the $\mathrm{E}_{\text {app }}$ dependence of $\mathrm{K}_{\mathrm{TH}}$, where the best fit was achieved for the higher level of $\sigma_{H} / \sigma_{Y S}$ (compare Figure 32 with Figure 34). 

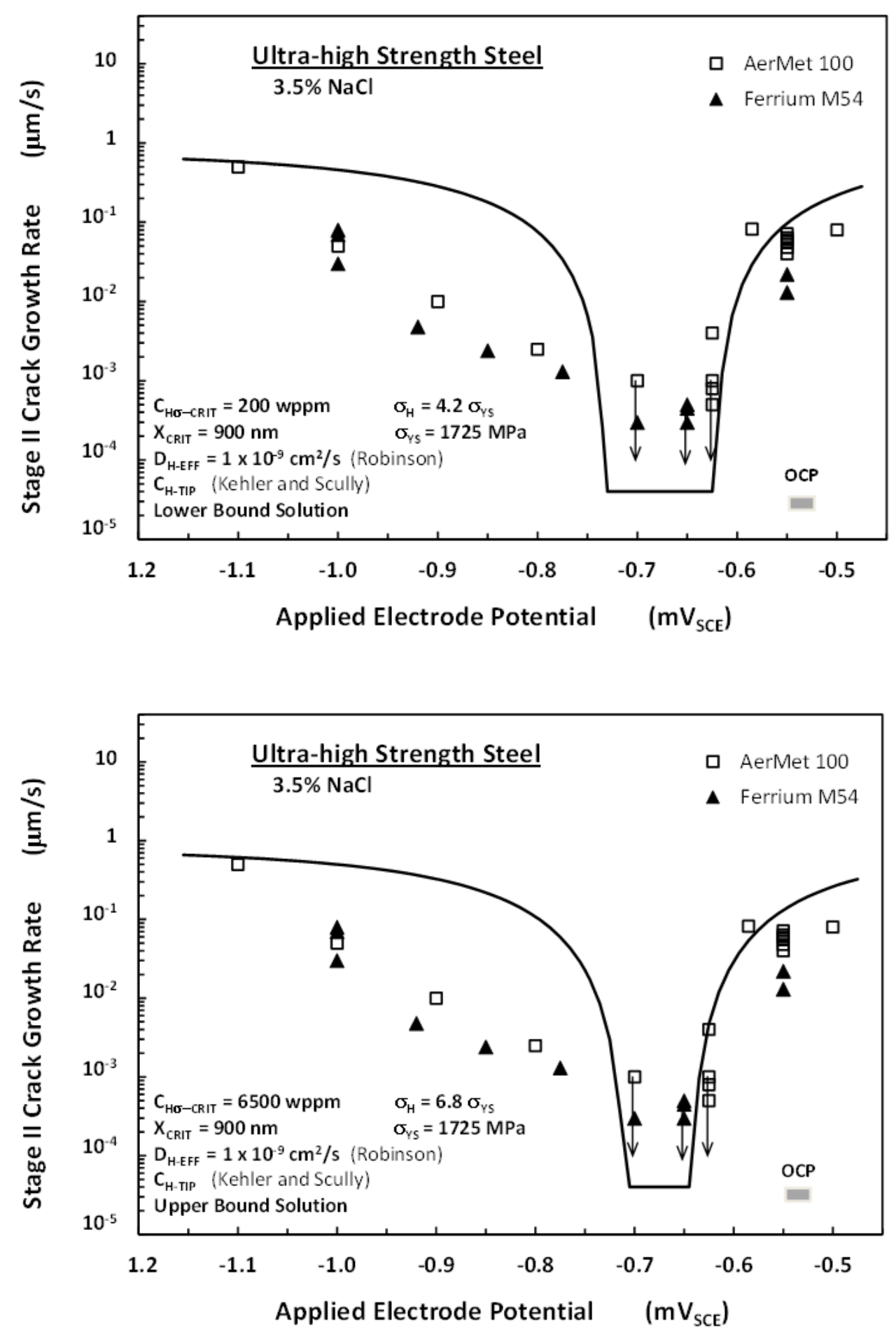

Figure $36 \quad$ Measured ( $\square \Delta$ ) and model predicted (-) applied potential dependence of da/dt $t_{I I}$ for AerMet 100 and Ferrium M54, using the H-diffusion limited model (Equation 1) with

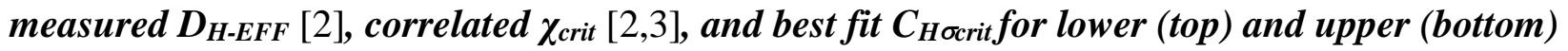
bounds of $\sigma_{H}=4.2 \sigma_{Y S}$ and $6.8 \sigma_{Y S}$. 


\subsubsection{Variability of measured and predicted da/dtII and $K_{T H}$}

Measured values of da/dtII are systematically over-predicted at moderate levels of cathodic polarization, as shown in Figure 36 for AerMet 100 and Ferrium M54. For example, predicted $\mathrm{da} / \mathrm{dt}$ is 50 -times higher than the measured value at $\mathrm{E}_{\mathrm{app}}$ of $-850 \mathrm{mV} \mathrm{V}_{\mathrm{SCE}}$. This difference is not due to the measured da/dt values being within Stage I for these applied potentials; while the da/dt at K of $40 \mathrm{MPa} \sqrt{\mathrm{m}}$ increased by 1.5 to 2 times as $\mathrm{K}$ rose to $60 \mathrm{MPa} \sqrt{\mathrm{m}}$ for AerMet 100 and Ferrium M54 in the $\mathrm{E}_{\text {app }}$ range from -850 to $-1,000 \mathrm{mV}_{\mathrm{SCE}}$, this change only explains a very small part of the difference in measured versus predicted da/dtII in Figure 36. Moreover, application of this model to HEAC in Ni-Cu Monel K-500 stressed in $\mathrm{NaCl}$ solution revealed a similar reduction in

measured Stage II da/dt, when compared to model predictions, with rising $E_{\text {app }}$ from highly cathodic polarization, transitioning to a very sharp near-identical decline in HEAC growth at a critical $E_{\text {app }}$ [3]. However, the error in model predictions is noticeably reduced in Monel K-500, e.g. predicted da/dt exceeded measured values by only 7 -times in this potential regime.

Based on Equation 2, there are at least 3-possible reasons for the divergence between measured and modeled da/dtII at moderate to severe cathodic $\mathrm{E}_{\text {app }}$ for UHSS. First, the value of $\mathrm{D}_{\mathrm{H}-\mathrm{EFF}}$ used

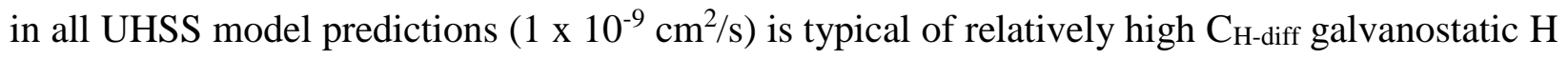
charging [1]. Due to H-trapping, $\mathrm{D}_{\mathrm{H}-\mathrm{EFF}}$ is strongly dependent on total hydrogen concentration and falls $50 \%$ as $\mathrm{C}_{\mathrm{H}-\mathrm{DIFF}}$ declines from 6 to $0.06 \mathrm{wppm}$ [137]. An identical $\mathrm{D}_{\mathrm{H}-\mathrm{EFF}}$ reduction would only explain a small part of the over-prediction of da/dt in Figure 36, which varies from 15\% at $E_{\text {app }}$ of $-1,100 \mathrm{mV}_{\text {SCE }}$ to 100-times at $E_{\text {app }}$ of $-900 \mathrm{mV}_{\text {SCE. }}$ Second, the diffusion model represented in Equation 2 is based on a 1-D simplification of 3-D crack tip diffusion, which is driven by the $\mathrm{H}$ concentration gradient but not stress gradient term in Fick's laws [154-156].

Third, H-diffusion limited da/dt is based on the requirement that surface reaction rates are sufficiently rapid to maintain crack tip $C_{L}$ as essentially constant. Scully and Moran experimentally demonstrated that two factors elevate the importance of surface reaction controlled HEAC rates for 4340 UHSS in $\mathrm{NaCl}$ solution: (1) mild cathodic polarization which promotes an alkaline crack solution and, (2) small values of $\mathrm{x}_{\mathrm{CRIT}} / \mathrm{D}_{\mathrm{H}-\mathrm{EFF}}[157,158]$. In essence for point (1) applied to the current situation, a surface film is stable in this regime of $\mathrm{pH} /$ potential and reduces the surface coverage of adsorbed hydrogen relative to a film-free surface (for $\mathrm{E}_{\mathrm{app}}$ at and cathodic 
to the minimum in Figure 29). The effect of such films during HEAC is a hypothetical reduction in $\mathrm{C}_{\mathrm{H \sigma}}$, due to $\mathrm{C}_{\mathrm{L}}$ limited by film-mitigated $\mathrm{H}$-uptake. This film is likely eliminated at heavy cathodic and mild anodic potentials or by sufficient crack tip strain rate. A coupled surface reaction and H-diffusion model has not been reported in literature for HEAC. However, this effect could be approximated by reduced $\mathrm{C}_{\mathrm{H} \text {,Diff }}$ and thus $\mathrm{C}_{\mathrm{H \sigma}}$ in Equation 2. For example, for UHSS at $\mathrm{E}_{\mathrm{app}}$ $=-900 \mathrm{mV}_{\mathrm{SCE}}$ and $\sigma_{\mathrm{H}} / \sigma_{\mathrm{YS}}=6.8$ (Figure 36), $\mathrm{C}_{\mathrm{H}, \text { Diff }}$ from Figure 29 must be reduced by 3.8 times (from $2.3 \mathrm{wppm}$ to $0.6 \mathrm{wppm}$ ) in order for predicted da/dt to equal measured values. This reduction factor produces good agreement between measured and predicted da/dt for $\mathrm{E}_{\text {app }}$ below $-650 \mathrm{mV} \mathrm{V}_{\mathrm{SCE}}$, and should gradually decrease in importance as $\mathrm{E}_{\mathrm{app}}$ is reduced from -700 to $-1,100 \mathrm{mV}_{\mathrm{SCE}}$.

Considering point (2) Scully and Moran argued that surface reaction is relevant for 4340 steel stressed in alkaline $\mathrm{NaCl}$ under mild cathodic polarization, when the ratio of $\chi_{\text {crit }} / \mathrm{D}_{\mathrm{H}-\mathrm{EFF}}$ is less than $1.6 \times 10^{5}$ to $4.4 \times 10^{5} \mathrm{~s} / \mathrm{cm}$ [157]. For the conditions in Figure 36 and considering the 50\%reduced $D_{H-E F F}$ for lower $C_{H-D i f f}$, this ratio is $(900 \mathrm{~nm}) /\left(5 \times 10^{-10}\right) \mathrm{cm}^{2} / \mathrm{s}=1.8 \times 10^{5} \mathrm{~s} / \mathrm{cm}$. This comparison validates the possibility for surface reaction and $\mathrm{H}$ diffusion to interactively govern $\mathrm{da} / \mathrm{dt}$ in a way not captured by Equation 2. This conclusion is not definitive because the critical ratio of $\chi_{\text {crit }} / \mathrm{D}_{\mathrm{H}}$ has not been broadly established with permeation experiments applied to a range of alloy steels. Moreover, slow-rising K loading may destabilize such surface films by active crack tip plastic deformation, at an increasingly rapid rate during crack growth (also impacting point (1)). However, the $\mathrm{dK} / \mathrm{dt}$ (and da/dt) dependence of crack tip film rupture/passivation is not known for these UHSS alloys so a definitive conclusion is not possible. Given these considerations, the precise cause of the over-prediction of da/dt in Figure 36 is not established, but surface reaction rate limitation appears to be the most reasonable-possible cause among those considered.

Notably, measured values of $\mathrm{K}_{\mathrm{TH}}$ for HEAC during slow-rising $\mathrm{K}$ loading are variable in the regime of protective-mild cathodic polarization, as shown in Figures 31, 32 and 34 for AerMet 100 and Ferrium M54. For replicate experiments of AerMet 100 polarized at $-625 \mathrm{mV}_{\mathrm{SCE}}$, measured $\mathrm{K}_{\mathrm{TH}}$ varied from 40 to $76 \mathrm{MPa} \sqrt{\mathrm{m}}$ and from 57 to $88 \mathrm{MPa} \sqrt{\mathrm{m}}$ for Ferrium M54 polarized at $-650 \mathrm{mV}_{\mathrm{SCE}}$. In contrast, replicate experiments produced measured $\mathrm{K}_{\mathrm{TH}}$ values from 10 to 13 MPa $\sqrt{ } \mathrm{m}$ for AerMet 100 at $-550 \mathrm{mV}$ SCE and from 8 to $9 \mathrm{MPa} \sqrt{m}_{\mathrm{m}}$ at $-570 \mathrm{mV}$ SCE for Ferrium M54 (but from 20 to $33 \mathrm{MPa} \sqrt{\mathrm{m}}$ at $-550 \mathrm{mV}_{\text {SCE. }}$ ) This variability is strongly dependent on $\mathrm{E}_{\text {app }}$ and is 
speculatively ascribed to an effect of crack surface microstructure in reducing local $\mathrm{H}$ uptake, coupled with the hydrogen embrittlement regime where $\mathrm{K}_{\mathrm{TH}}$ strongly depends on $\mathrm{C}_{\mathrm{H} \text {,Diff }}$ (Figure 30). Two possible microstructural origins for this phenomena are discussed in the remainder of this section. Further, as addressed in Chapter 2, reproducible measurement of $\mathrm{K}_{\mathrm{TH}}$ in the lower $\mathrm{H}$ concentration regime is challenging because $\mathrm{da} / \mathrm{dt}$ for HEAC is very low, on the order of the false da/dt from crack tip plasticity/blunting (Figure 4) and other transient-unknown causes. Small false changes in da/dt could be incorrectly interpreted as a $\mathrm{K}_{\mathrm{TH}}$.

At issue is the physical basis for variable-low $\mathrm{H}$ uptake accentuated in a window of $\mathrm{E}_{\text {app }}$ just cathodic to OCP and where HEAC susceptibility is low. Kehler and Scully demonstrated that measured crevice $\mathrm{pH}$ fell below bulk solution neutral $\mathrm{pH}$ when AerMet 100 crevice surfaces were polished and polarized above $-700 \mathrm{mV}_{\mathrm{SCE}}$. However, passive surfaces did not promote acidification because of limited cation formation [20]. This behavior resulted in a demonstrated surface-condition dependence of crevice-tip $\eta$. Specifically, local $\eta$ was less than 0 for the polished/acidified crevice at essentially all $\mathrm{E}_{\text {app }}$ and above 0 for a passivated/neutral-alkaline crevice coupled with $\mathrm{E}_{\text {app }}$ above $-700 \mathrm{mV}_{\mathrm{SCE}}$. A surface passive film could also hinder $\mathrm{H}$ production and absorption for a given $\eta$, exacerbated by the slow loading used for $\mathrm{K}_{\mathrm{TH}}$ determination $[157,158]$. In essence, crack tip $C_{H, D i f f}$ in the vicinity of the minimum in Figure 29 is strongly sensitive to the extent of crack surface passivity. Notably, dcPD measurements suggested the presence of a crack surface film, but only for those values of $E_{\text {app }}$ where HEAC was generally mitigated. The presence of a passive film was evidenced by a lack of a crack-surface separation dependence of the measured dcPD signal at very low $\mathrm{K}$ (1-8 MPa $\sqrt{\mathrm{m}})$, as discussed in Chapter 2. When the film was eliminated for more cathodic or more anodic values of $E_{a p p}$, the dcPD increased notably with initial rising $\mathrm{K}$ in the low $\mathrm{K}$ regime, suggesting separation of electrically conducting surfaces.

As a second hypothesis, microstructural heterogeneity such as large oxy-sulfide inclusions, could cause specimen-to-specimen variability in $\mathrm{K}_{\mathrm{TH}}$. Dissolution of sulfides to form sulfur-based anions could accelerate crack solution hydrolysis and/or $\mathrm{H}$ production/uptake to thus reduce $\mathrm{K}_{\mathrm{TH}}$. The presence and distribution of such particles in UHSS alloys has not been probed in the present study. However, previous work has confirmed the presence of rare-earth impurity particles in a similar UHSS (AF1410) [32]. The effect of impurity particle dissolution may be reduced under strongly 
anodic or cathodic polarization where the crack tip chemistry is likely dominated by $\mathrm{E}_{\mathrm{app}}$ In this scenario strong-anodic polarization should dominate dissolution to reduce $\mathrm{pH}$, and strongly limit

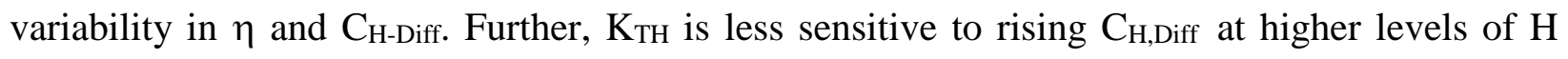
concentration. In the cathodic direction, crack solution is alkaline at all $\mathrm{E}_{\text {app }}$ below about -700 $\mathrm{mV}_{\mathrm{SCE}}$ and $\mathrm{K}_{\mathrm{TH}}$ variability from a microstructural-dissolution source is less likely. Microstructural variability may be captured by the artificial crevice experiment, but replicate measurements were not presented to quantify the variability of crevice $\eta$ [20]. While correct in theory, the presence of a substantial rare earth treatment in AerMet 100 and Ferrium M54 indicates that most impurities will be bound in rare-earth impurity particles. The applicability of this hypothesis is strongly dependent upon the solubility of such particles in the acidic chloride environment. Quantification of rare earth impurity particle solubility in occluded crack tip environments is beyond on the scope of the present work. However, the possibility remains that the presence and distribution of impurity containing phases could significantly alter the crack tip chemistry and subsequent $\mathrm{H}$-uptake in the regime of mildly cathodic $\mathrm{E}_{\mathrm{app}}$ which confer enhance HEAC resistance.

\subsubsection{Modeling of $K_{T H}$ in $300 M$ vs Modern UHSS}

The dependency of $\mathrm{K}_{\mathrm{TH}}$ on $\mathrm{E}_{\mathrm{app}}$ for legacy 300M HEAC (Figure 24) represents a large departure from the trends observed for modern secondary hardening UHSS. Accordingly, significant modifications to the micromechanical model of $\mathrm{K}_{\mathrm{TH}}$ (Equation 1) were made to account for increased total impurity content (Table 4) and reduced KIC (Table 1) compared to modern UHSS.

Two logical parameter modifications are relevant: (1) increased impurity content on prior austenite grain boundaries may increase $\alpha$ through iteraction with high local $\mathrm{H}$ concentration, as supported by studies in 4340-types steels by Bandyopadhyay et al. [131], and (2) a constant reduction in kIG due to temper embrittlement of prior austenite grain boundaries in the absence of $\mathrm{H}$. Identical to previous analyses, $\mathrm{K}_{\mathrm{TH}}$ at $\mathrm{C}_{\mathrm{H}-\mathrm{DIFF}}=0 \mathrm{wppm}$ is constrained to be the plane strain fracture toughness of $300 \mathrm{M}(65 \mathrm{MPa} \sqrt{\mathrm{m}})$, yielding a reduction in $\mathrm{k}_{\mathrm{IG}}$ : $\mathrm{k}_{\mathrm{IG}}=0.99 \mathrm{MPa} \sqrt{\mathrm{m}}$ when $\alpha "=0.00022 \mathrm{MPa}^{-1}$ and $\beta^{\prime}=0.20(\mathrm{MPa} \sqrt{ })^{-1}$. There is no reason to suspect that hydrogen uptake is altered in $300 \mathrm{M}$ compared to AerMet 100 or Ferrium M54, so an identical $\mathrm{E}_{\text {app }}$ dependency of $\mathrm{C}_{\mathrm{H} \text {,Diff }}$ (Equation 5 and Figure 29) was used to calculate best-fit $\alpha$ for the two standard values of $\sigma_{H}$. The result of this modeling is shown for $\sigma_{\mathrm{H}}=4.2 \sigma_{\mathrm{YS}}$ and $\sigma_{\mathrm{H}}=6.8 \sigma_{\mathrm{YS}}$ in Figure 37. 

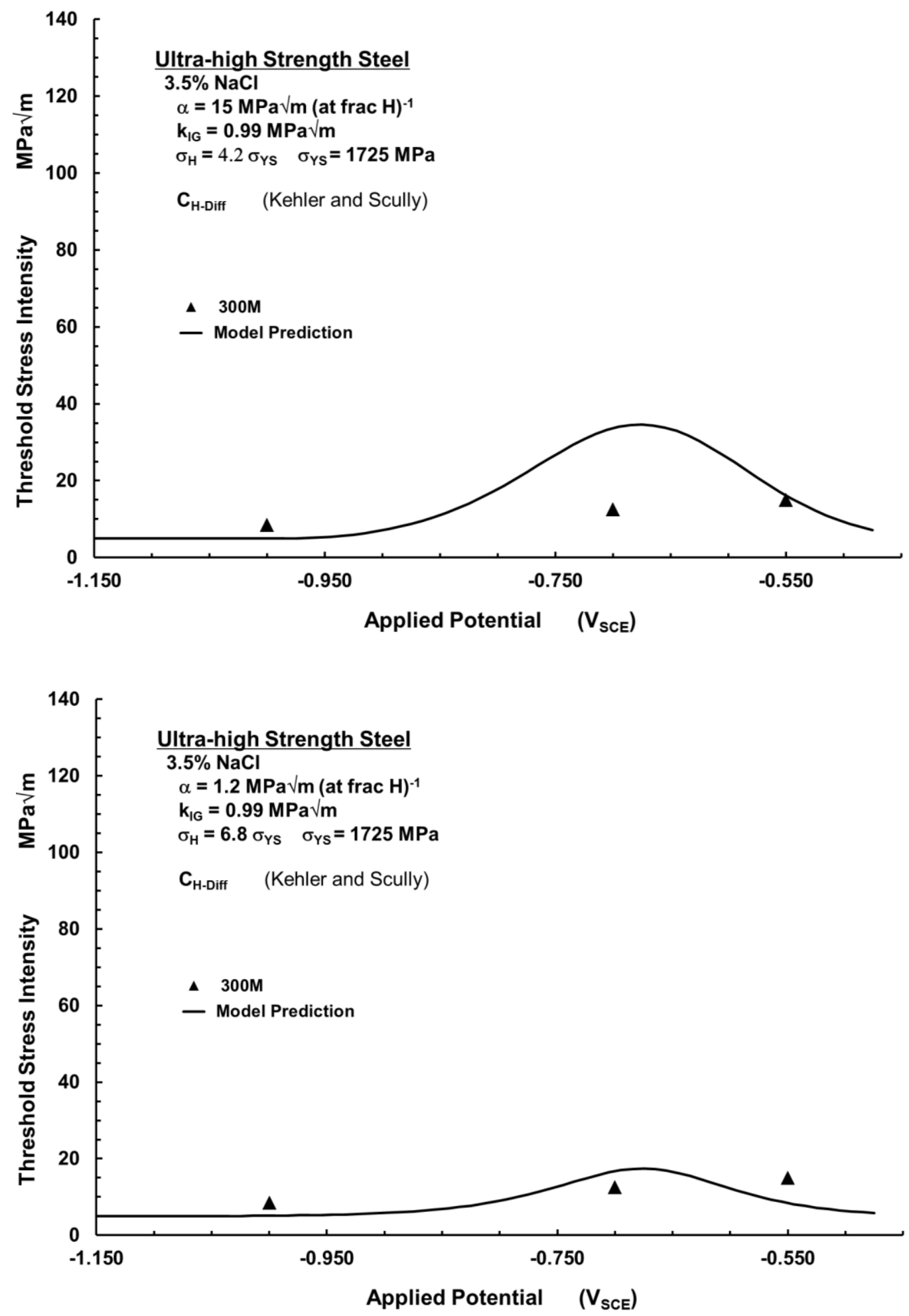

Figure 37 Measured ( $\Delta$ ) and model predicted (--) applied potential dependence of $\boldsymbol{K}_{T H}$ for 300M, using the H-decohesion based model (Equation 1) for lower and upper bounds of $\sigma_{H}=$ $4.2 \sigma_{Y S}$ (upper) and 6.8 $\sigma_{Y S}$ (lower). 
As expected, good fit of measured $\mathrm{K}_{\mathrm{TH}}$ by the decohesion-based model (Equation 1) requires that $\alpha$ be significantly increased. For rational crack tip hydrostatic stress levels of $\sigma_{H} / \sigma_{Y S}$ (4.2 and 6.8), the best fit $\alpha$ is 15.0 and $1.2 \mathrm{MPa} \sqrt{\mathrm{m}}$ (atom frac $\mathrm{H}^{-1}$, respectively and compared to 8.0 and 0.9 $\mathrm{MPa} \sqrt{\mathrm{m}}\left(\right.$ atom frac $\mathrm{H}^{-1}$ for AerMet 100 (Figures 31 and 33). No independent $\mathrm{K}_{\mathrm{TH}}$ IHAC data are available for $300 \mathrm{M}$ to quantitatively judge the fit of predicted $\mathrm{K}_{\text {TH }}$ as was done for AerMet 100 .

Identical to previous modeling of $\mathrm{K}_{\mathrm{TH}}$ AerMet 100 and Ferrium M54, the qualitative fit of Equation 1 to experimental data increases as the ratio of $\sigma_{\mathrm{H}} / \sigma_{\mathrm{YS}}$ is increased from 4.2 to 6.8 . Similarly, bestfit $\alpha$ falls from 15.0 to $1.2 \mathrm{MPa} \sqrt{\mathrm{m}}$ (atom fraction $\mathrm{H}$ ) ${ }^{-1}$ as the crack tip stress is increased. The significance of the results presented in Figure 37 is two-fold. First, identical to $K_{T H}$ modeling in previous sections, the best qualitative fit is achieved at $\sigma_{H} / \sigma_{\mathrm{YS}}=6.8$, supporting the use of dislocation-based simulation [149] and small-deformation SGP modeling [150] for prediction of crack tip hydrostatic stress. Second, $\alpha$ is elevated in 300M compared to AerMet 100 and Ferrium M54 for both levels of $\sigma_{H} / \sigma_{Y S}$. Best fit $\alpha$ rises from 0.9 to 1.2 in as the embrittlement parameter $\Psi$ rises from 0.02 to $1.8 \mathrm{wt} \%$ in legacy $300 \mathrm{M}$ compared to modern UHSS (AerMet 100). This observation supports previous work by Bandyopadhyay et al. in establishing the combined effect of hydrogen and elevated impurity content in reducing grain boundary cohesion [131]. Further, the results in Figure 37 quantitatively link improved alloy purity and rare earth treatment with the parameter $\alpha$. Future work in the area should be targeted at understanding the effects of each impurity element (Table 4) and specific combinations of impurity elements which may synergistically act to promote segregation [159]. Based on previous work by Bandyopadhyay et al a logical link exists between the impurity parameter $\Psi$ and $\alpha$. If such a parameter could be quantified for hydrogen embrittlement high strength martensitic steels, it would allow for more accurate predictions of SCC-HEAC resistance in modern ICME-designed alloys such as Ferrium S53 or Ferrium M54. 


\subsubsection{Modeling of da/dt $t_{I I}$ in 300M vs. Modern UHSS}

Identical to previous analysis, the $\mathrm{da} / \mathrm{dt}_{\mathrm{II}}$ dependence on $\mathrm{E}_{\mathrm{app}}$ for legacy $300 \mathrm{M}$ is modeled using $\mathrm{C}_{\mathrm{H \sigma}}$ from Equations 3 and 5, and an adjustable value of $\mathrm{C}_{\mathrm{H \sigma} \text {-crit }}$ for best fit of Equation 2 to da/dt measurements of 300M (Figure 25). Hydrogen diffusivity for 300M was obtained from previously reported galvanostatic $\mathrm{H}$-uptake measurements and assumed constant, $\mathrm{D}_{\mathrm{H}-\mathrm{EFF}}=8.0 \times 10^{-8} \mathrm{~cm}^{2} / \mathrm{s}$ [1]. The results of this modeling are shown in Figure 38 for the two reasonable bounds of $\sigma_{H} / \sigma_{Y S}$, 4.2 and 6.8 .

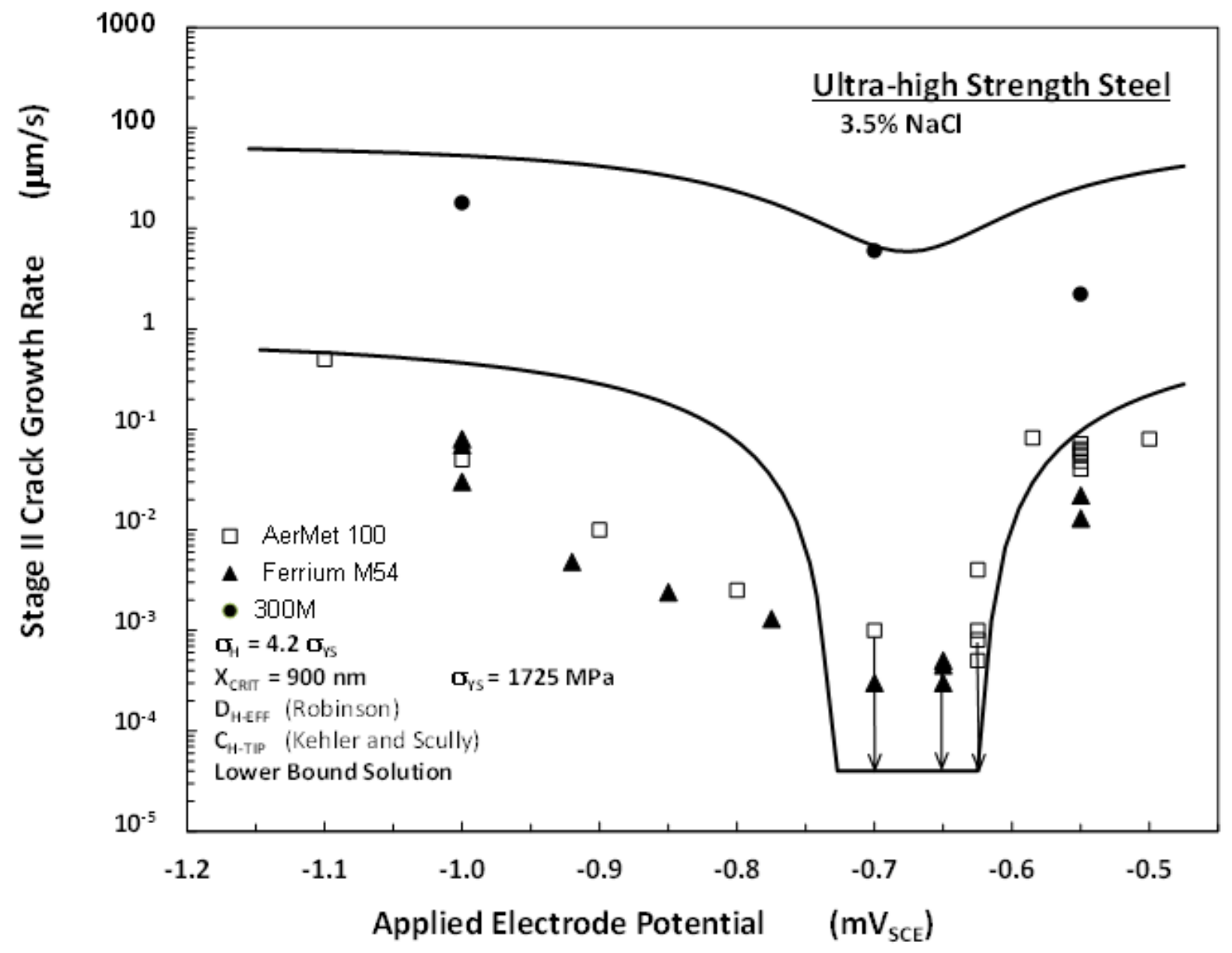




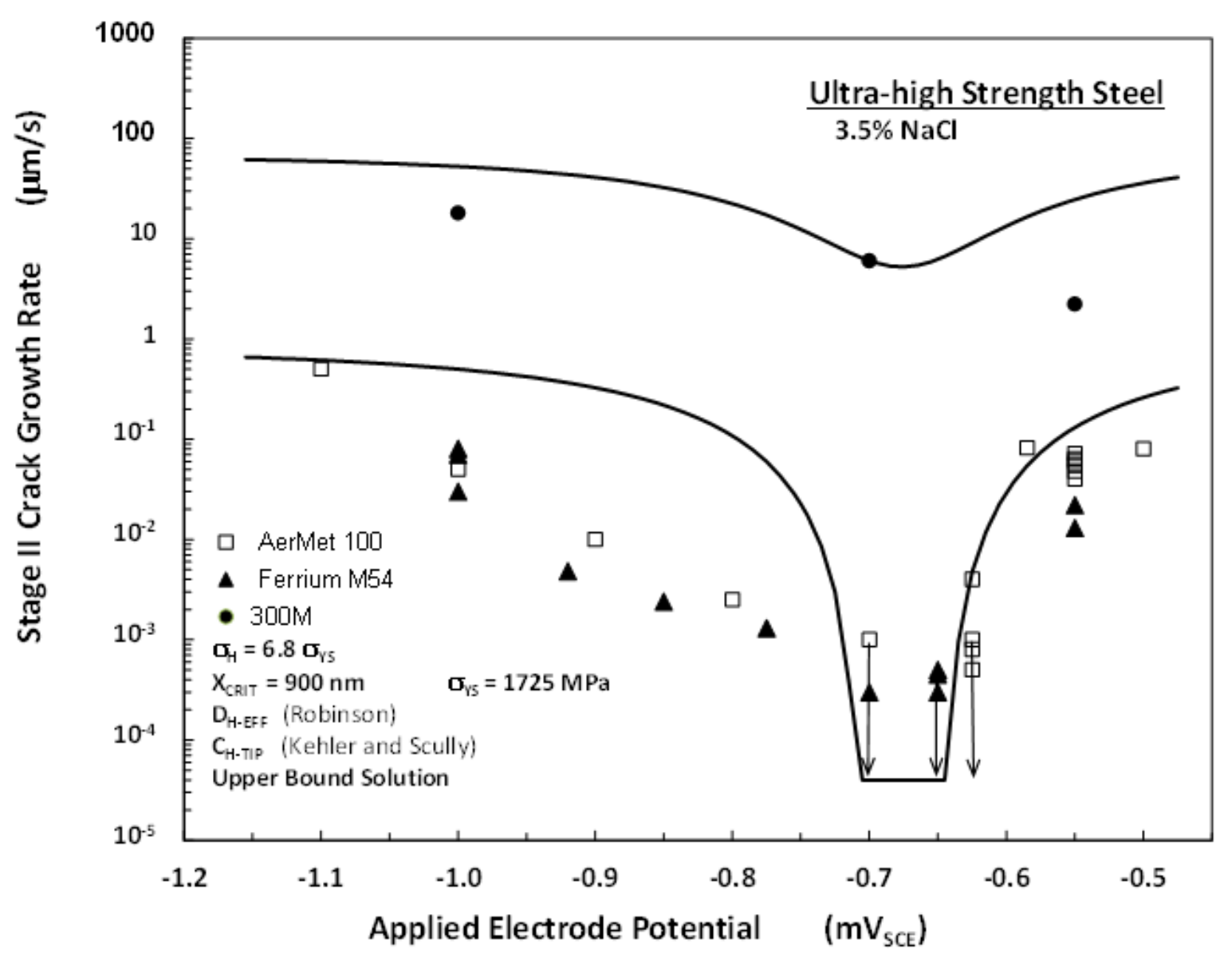

Figure 38 Measured ( $\square \Delta \bullet$ ) and model predicted (--) applied potential dependence of da/dt $t_{I I}$ for AerMet 100, Ferrium M54 and 300M, using the H diffusion limited model (Equation 2) with measured $D_{H-E F F}[1]$, correlated $\chi_{\text {crit }}$ [2], and best fit $C_{H \sigma-c r i t}$ for: the lower bound of $\sigma_{H}=4.2 \sigma_{Y S}$ (top plot) and upper bound of $\sigma_{H}=6.8 \sigma_{Y S}$ (bottom plot).

The results shown in Figure 38 establish that the potential dependence of da/dtII is reasonably modeled for both the upper and lower bound crack tip hydrostatic stress levels using the $\mathrm{H}$ diffusion limited model in Equation 2. The single adjustable parameter in the model, $\mathrm{C}_{\mathrm{H \sigma}-\mathrm{crit}}$, rises from $90 \mathrm{wppm}$ to $3,500 \mathrm{wppm}$ as $\sigma_{\mathrm{H}} / \sigma_{\mathrm{YS}}$ is increased from 4.2 to 6.8 , as expected since this critical concentration is referenced to the magnitude of the crack tip stress field. This trend is similar but reduced from fitted $\mathrm{C}_{\mathrm{H \sigma}-\mathrm{crit}}$ for AerMet 100 and Ferrium M54, which rises from 180 wppm to 6500 wppm as $\sigma_{H} / \sigma_{Y S}$ is identically increased. The reduced $\mathrm{C}_{\mathrm{H} \sigma \text {-crit }}$ for $300 \mathrm{M}$ parallels the increased $\alpha$, as predicted by Equation 7, and associated with impurity-H interaction. 
Two points are noteworthy in comparing the potential dependences of 300M, AerMet 100, and Ferrium M54. First, substantially reduced $\mathrm{C}_{\mathrm{H \sigma} \text {-crit }}$ in $300 \mathrm{M}$ appears to eliminate the beneficial effect of moderate-cathodic potentials observed for all modern UHSS. This loss of cathodic prevention is understood based on the small values of $\mathrm{C}_{\mathrm{H \sigma}}$ exceeding the $\mathrm{C}_{\mathrm{H \sigma} \text {-crit }}$ at mild cathodic $\mathrm{E}_{\mathrm{app}}$, causing a rapid increase in the inverse error function term in Equation 2. Reduced $\mathrm{C}_{\mathrm{H \sigma} \text {-crit }}$ in $300 \mathrm{M}$ compared to modern UHSS is a result of the impurity dependence of $\alpha$ and kIG. Metalloid impurities present at the grain boundaries work with local hydrogen to reduce the cohesive strength of the boundary [131]. The result is a net reduction in the amount of hydrogen needed to initiate a microcrack compared to an unembrittled boundary, under identical loading.

Second, the dominating effect of $\mathrm{D}_{\mathrm{H}-\mathrm{EFF}}$ on da/dt II in high strength alloys is confirmed at all $\mathrm{E}_{\mathrm{app}}$. As outlined in a previous section, reduced $\mathrm{D}_{\mathrm{H}-\mathrm{EFF}}$ in AerMet 100 compared to $300 \mathrm{M}$ due to the precipitation of nano scale $\mathrm{M}_{2} \mathrm{C}$ carbides, which effectively trap $\mathrm{H}[130,137]$. Microstructural characterization in the present work strongly supports this conclusion. The tempered martensite microstructure of AerMet 100 and $300 \mathrm{M}$ is quantitatively identical with regard to average martensite block spacing ( $0.81 \pm 0.05$ versus $0.96 \pm 0.03$, respectively), EBSD measured austenite content (1.5 versus $1.6 \mathrm{vol} \%)$, and the connectivity of random high angle boundaries $\left(\mathrm{D}_{\mathrm{R}}=1.8\right.$ 1.9). In the context of quantitative microstructure data and a strong-established literature, the results in Figure 38 demonstrate reduced da/dt $\mathrm{dI}_{\mathrm{I}}$ in modern UHSS is attributed to a distribution of nano-scale carbides which are not present in 300M. This result underscores the importance of controlling precipitate morphology in modern ultra-high strength alloys and in the design of next generation HEAC-resistant alloys. Such microstructure control affects crack growth rates, but not the threshold stress intensity for HEAC, which is an equilibrium property. This behavior is well illustrated in Figure 18 for high H contents, and to an extent in Figure 17 where H uptake is limited for the stainless alloy.

\subsubsection{Microstructural Aspect of Micromechanical Modeling and $\chi_{\text {crit }}$}

Collectively, the crack tip models of HEAC provide consistent and reasonably accurate predictions of the $\mathrm{E}_{\mathrm{app}}$ dependencies of $\mathrm{K}_{\mathrm{TH}}$ and da/dt II. The present effort provides the broadest application of such models to date, using reasoned-independent estimates of important and difficult to measure/model input terms taken from the literature, particularly: (1) occluded crack H solubility 
( $\mathrm{C}_{\mathrm{H} \text {,Diff }}$ ) versus $\mathrm{E}_{\mathrm{app}}$, (2) trap sensitive $\mathrm{H}$ diffusivity ( $\mathrm{D}_{\mathrm{H}-\mathrm{EFF}}$ ) versus $\mathrm{C}_{\mathrm{H} \text {,Diff, and }}$ (3) crack tip stress hydrostatic stress $\left(\mathrm{C}_{\mathrm{H \sigma}}\right)$. Further improvements in the rigor of this modeling are contingent on future developments in each of these areas. The good agreement between measured and predicted HEAC kinetics in Figures 31, 33, 35, and 36 justifies this fundamental effort. From the materials science perspective, these models provide a framework to consider the effects of UHSS composition and microstructure on HEAC. Further, integration of the present results in ICMEbased alloy development schemas could produce next generation UHSS with significantly improved HEAC resistance.

Work to date has not focused on a microstructural origin and definition of $\chi_{\text {crit, }}$ framed in the context of a H-damage mechanism such as the interacting HELP-HEDE, and integrated with continuum fracture mechanics be it classic or length/gradient scale based.

\subsubsection{Microstructural basis for $\chi_{\text {crit }}$ in TG quasi-cleavage of modern UHSS}

As relevant to the diffusion limited model of HEAC da/dt fractographic characterization put forth in previous sections presents the opportunity to probe $\chi_{\text {crit }}$ in UHSS. At issue is the question of the physical origin(s) for $\chi_{\text {crit, }}$ which at the coarse-correlation level is shown to be identical $(900 \mathrm{~nm})$ for a wide range of high strength alloys (Ni-superalloy, Al, $\mathrm{Fe}, \beta / \alpha \mathrm{Ti}$ ), microstructures, and HEAC fracture paths [2,6]. As reviewed elsewhere [2], literature points towards four possible origins of $\chi_{\text {crit: }}$ (1) location of highest tensile/hydrostatic stress ahead of the crack tip, (2) spacing of microstructure features such as precipitates or grain boundaries, (3) strain generated H-trap sites, and (4) the crack tip surface. The high resolution microstructural characterization shown in Figures 6-10 and the mechanical properties presented in Table 1-2 are relevant to evaluating points (1) and (2) for the physical origin of $\chi_{\text {crit. }}$.

Within the HELP/HEDE mechanism for HEAC of UHSS, the location of highest tensile stress ahead of the crack tip provides the largest driving force for $\mathrm{H}$ accumulation and microcrack nucleation. Fracture mechanics based elastic-plastic modeling of the stress and strain fields around a crack tip has yielded the location of highest crack opening stress $[2,7,160]$ :

$$
\delta_{T}=\frac{K^{2}}{2 \sigma_{Y S} E}
$$


where $\mathrm{K}$ is the elastic-plastic stress intensity, nearly identical to $\mathrm{K}_{\text {elastic }}$ for the ultra-high strength alloys in the present study. The location of maximum stress is $\sim 2 \delta_{\mathrm{T}}$ but may range from $1-4 \delta_{\mathrm{T}}$ $[2,7]$. As discussed previously, if the effect of strain gradient plasticity is considered the maximum crack tip stress will rise substantially. However, the approximate location of stress maximum should be similar and certainly no larger in strain gradient plasticity compared to a conventional elastic plastic prediction [7]. Calculated maximum stress distance for UHSS is shown in Table 8, at $\mathrm{K}=40 \mathrm{MPa} \sqrt{\mathrm{m}}$ and using Equation 8.

\begin{tabular}{|c|c|c|c|c|}
\hline Alloy & бYs $(\mathbf{M P a})$ & $\mathbf{K}(\mathbf{M P a} \sqrt{\mathbf{m}})$ & $\mathbf{2 \delta}_{\mathbf{T}}(\boldsymbol{\mu m})$ & $\mathbf{1}-\mathbf{4} \boldsymbol{\delta}_{\mathbf{T}}(\boldsymbol{\mu m})$ \\
\hline 300M & 1,580 & 40 & 5.0 & $2.5-10.0$ \\
\hline AerMet 100 & 1,725 & 40 & 4.6 & $2.3-9.2$ \\
\hline CrNiMoWV & 1,580 & 40 & 5.0 & $2.5-10.0$ \\
\hline Ferrium M54 & 1,720 & 40 & 4.6 & $2.3-9.2$ \\
\hline Ferrium PH48S & 1,550 & 40 & 5.2 & $2.6-10.4$ \\
\hline
\end{tabular}

Table 8 Calculated maximum stress distances for UHSS at $\mathrm{K}=40 \mathrm{MPa} \bigvee_{\mathrm{m}}$ using Equation 8.

At a moderate stress intensity the predicted maximum stress distance is increased 2.5 to 11.5 times larger than to the regression fit value of $\chi_{\text {crit }}$ in UHSS. The choice of $\mathrm{K}$ for this calculation is arbitrary above $\mathrm{K}_{\mathrm{TH}}$, as da/dt in UHSS is stable across a wide range of stress intensities (Figure 17). Further, an identical calculation performed on a broad range of materials (Ni-superalloy, precipitation hardened aluminum, 18Ni Maraging Steel, $\beta / \alpha$ Ti) indicates that UHSS alloys exhibit the minimum ratio of $\delta_{\mathrm{T}} / \chi_{\text {crit }}$ for a constant stress intensity. Due to the discrepancy in magnitude between $\delta_{\mathrm{T}}$ and $\chi_{\text {crit }}$ it is reasonable to assume that $\chi_{\text {crit }}$ is not directly dictated by the location of maximum-continuum crack tip stress. In the remainder of this section the basis for $\chi_{\text {crit }}$ in the present suite of martensitic UHSS alloys will be explored in the context of the high resolution characterization of martensite microstructures presented in Figures 6-10.

A microstructurally based $\chi_{\text {crit., }}$ in martensitic UHSS would logically be linked to the spacing of a microstructural feature such as grain boundaries, martensite interfaces, or precipitates. As shown in Figure 6-10, the spacing of prior austenite grain boundaries and packet boundaries are large compared to $\chi_{\text {crit }}$ and can be eliminated from the possibilities. Conversely, the average spacing of 
strengthening precipitates in UHSS is very small, likely less $50 \mathrm{~nm}$, and can be eliminated as a direct source of $\chi_{\text {crit. }}$ Section 3.3.2 establishes that the UHSS alloys studied exhibit TG HEAC, but involving fracture of martensite block interfaces. The HELP/HEDE mechanism proposed by Nagao et al. and previous literature indicates that HELP-nucleated microcracks likely initiate by martensite block interface decohesion a distance ahead of the crack tip $[11,12,120]$. As such, it is logical to hypothesize that $\chi_{\text {crit }}$ in UHSS may be dictated by the martensite block spacing. Average spacing of block interfaces is consistently small among UHSS: $0.96 \mu \mathrm{m} \pm 0.03 \mu \mathrm{m}, 0.81 \mu \mathrm{m} \pm$ $0.05 \mu \mathrm{m}, 0.95 \mu \mathrm{m} \pm 0.06 \mu \mathrm{m}, 1.16 \mu \mathrm{m} \pm 0.09 \mu \mathrm{m}$ and $1.27 \mu \mathrm{m} \pm 0.08 \mu \mathrm{m}$ for 300M, AerMet 100, Ferrium M54, Ferrium S53, and CrNiMoWV, respectively. The curve fitted value of $\chi_{\text {crit }}$ predicted from a wide range of fastest-observed da/dt $\mathrm{dI}_{\mathrm{II}} \mathrm{D}_{\mathrm{H}-\mathrm{EFF}}$ is $0.9 \mu \mathrm{m}$, falling directly within the distribution of average martensite block spacing (0.81-1.27) [2]. In the context of the accuratereasonable modeling results when using $\chi_{\text {crit }}=0.9 \mu \mathrm{m}$ as a fundamental parameter (Figure 36), close agreement of the block spacing and this $\chi_{\text {crit }}$ would imply that the microstructural origin of $\chi_{\text {crit }}$ is the martensite block spacing. However, the quality of modeling fit for the $\mathrm{E}_{\text {app }}$ dependency of $\mathrm{da} / \mathrm{dt}_{\mathrm{II}}$ is not degraded when the $\chi_{\text {crit }}=0.9 \mu \mathrm{m}$ is used for predictions of IG-HEAC in legacy $300 \mathrm{M}$ (Figure 38 ). Further, the regression fit value of $\chi_{\text {crit }}$ was obtained from a very wide range of alloys and microstructures, many of which do not have microstructural features on the order of $900 \mathrm{~nm}$. Any microstructural or mechanistic basis for $\chi_{\text {crit }}$ must be able to address these two points.

Speculatively, an identical critical distance for IG and TG HEAC of UHSS is supported by IG fracture in which microcracks nucleate at the intersection of high angle block boundaries and prior austenite grain boundaries. Bulk average spacing of martensite block interfaces will roughly translate into the spacing of the block boundaries intersecting a prior austenite grain boundary. The intersection point would be an exclusively high angle, poor atomic fit triple point; ideal for trapping $\mathrm{H}$ and nucleating a microcrack. As observed by Nagao, slip bands beneath an IG fracture surface are oriented approximately parallel to martensite lath and block boundaries. The possibility exists for a $\{110\}_{\alpha}$, slip plane to encompass a $\{110\}_{\alpha}$, block boundary plane, transmitting slip and dislocations directly along the boundary and to the hypothesized-weak intersection with the prior austenite grain boundary. The result of this scenario in an idealized, classic martensite structure (Figure 12) would be a series of high $\mathrm{H}$, high stress regions, spaced on the order of the martensite block width, across the surface of a prior austenite grain. 
This preceding analysis provides a reasonable hypothesis for the interaction of HELP and martensite microstructure to produce a constant $\chi_{\text {crit }}$ for both IG and TG HEAC of martensitic alloys. However, this set of mechanistic steps does not explain the fundamental reason for constant $\chi_{\text {crit }}$ in simple precipitation strengthened Ni-base superalloys (IN 718, IN 903, and Monel K-500) and austenitic stainless steel (JBK-75) [2]. These microstructures are remarkably coarse compared to tempered martensite, and contain predominantly FCC austenite grain boundaries and fine scale strengthening phase. Austenite grain size will be large and strengthening precipitate spacing will be small, relative to $\chi_{\text {crit }}$; yielding no direct basis for a microstructural feature origin of $\chi_{\text {crit. }}$

Recent characterization of the microstructure directly beneath the H-induced fracture surface of polycrystalline $\mathrm{Ni}$ and $\mathrm{Fe}$ revealed a high density of organized dislocations formed as a result of $\mathrm{H}$ interaction with dislocations activated by strain [161-163]. When H-precharged to a high hydrogen concentration, high purity polycrystalline Ni exhibits a regular dislocation cell structure $600-1,200 \mathrm{~nm}$ in diameter, up to $3 \mathrm{~mm}$ beneath the IG fracture surface [162,164,165]. Similarly, IG fracture of $\mathrm{H}$-precharged pure Fe shows the beginnings of dislocation organization into a dense cell structure, but full organization of the structure was limited due to the severity of $\mathrm{H}$ embrittlement [163]. In both cases, it appears that hydrogen promotes the evolution of a dislocation cell structure beneath the fracture surface, which would not be present in the absence of $\mathrm{H}$ [161]. Speculatively, dense dislocation structure is developed ahead of the straining crack tip during HEAC and IHAC of microstructurally simple high strength alloys and provides the microstructural origin for $\chi_{\text {crit }}$ in these alloys. Hydrogen damage will nucleate in the highly stressed region ahead of the crack tip on the highly dislocated wall of a dislocation cell. Hydrogen trapping at cells walls is likely extensive, due to the high density of strong-reversible dislocations H-traps $[130,166]$. In essence the dislocation cell walls function identically to high angle martensite block boundaries in the generic HELP/HEDE mechanism. This hypothesis requires the primary strengthening precipitate in alloys such as JBK-75 or IN-718 does not significantly hinder the H-enhanced development of dislocation cells. Probing the efficacy of this hypothesis across high strength material systems is beyond the scope of the present work. However, future work could be targeted at verifying the presence of dislocation cell structure beneath the HEAC fracture surface of microstructurally coarse high strength alloys (Ni-base superalloys, PH austenitic stainless steels). If the hypothesized dislocation structures can be confirmed for multiple alloys, then the 
microstructural basis for $\chi_{\text {crit }}$ would be directly established. The critical importance of micromechanical model based lifetime prediction for HEAC-susceptible high strength components strongly supports this future effort.

\subsection{Conclusions}

The stress corrosion cracking resistance of a systematically selected range of ultra-high strength martensitic steels was characterized for slow-rising $\mathrm{K}$ loading in aqueous chloride solution for a wide range of $\mathrm{E}_{\mathrm{app}}$. Precisely measured crack growth kinetics and crack surface morphologies are correlated with a high resolution EBSD-based analysis of martensitic microstructure, including feature size/morphology and interface crystallographic characteristics. Results are interpreted in the context of quantitative models of crack tip hydrogen environment embrittlement, with predictions of associated threshold stress intensity and Stage II crack growth rates.

- 300M, experimental CrNiMoWV, AerMet 100, Ferrium M54, and Ferrium S53 martensitic steels aged to ultra-high strength are susceptible to severe HEAC when polarized near to OCP or substantially cathodically.

- Experimental CrMoWV, AerMet 100, Ferrium M54, and Ferrium S53 steels aged to ultrahigh strength are highly resistant to HEAC when stressed within a limited range of $E_{a p p}$, mildly cathodic to OCP, which confers elevated $\mathrm{K}_{\mathrm{TH}}$ and reduced da/dt $\mathrm{dI}_{\mathrm{II}}$. The breath of this protective window is correlated to low $\mathrm{C}_{\mathrm{H} \text {,Diff }}$ owed to a minimum in overpotential for hydrogen production and hydrolytic crack tip acidification. Legacy 300M does not exhibit this behavior, but experimental results are limited.

- The suite of modern UHSS alloys resist SCC-HEAC by virtue of crack growth rates which are reduced 2.5 - 3 orders of magnitude compared to legacy $300 \mathrm{M}$; as attributed to a nanoscale carbide distribution $\left((\mathrm{Mo}, \mathrm{Cr})_{2} \mathrm{C}, \mathrm{WC}\right.$, and/or $\left.\mathrm{VC}\right)$ which reduces the mobility of deleterious $\mathrm{H}$ due to strong-reversible trapping at strengthening carbide interfaces.

- Variable additions of $\mathrm{Cr}$ and Co do not impact the absolute values and applied potential dependencies of the threshold stress intensity factor for the growth kinetics of HEAC in 
spite of the potentially strong impact of these elements on the hydrolytic acidification and surface passivation elements of occluded crack electrochemistry and $\mathrm{H}$ uptake which govern crack tip H-damage.

- Intergranular SCC of UHSS is well predicted by the embrittlement parameter ( $\Psi)$ threshold of $0.5 \mathrm{wt} \%$, notably for the ultra-high strength level and elevated crack tip H concentrations achieved here and compared to prior studies. Transgranular quasi-cleavage cracking dominates HEAC for AerMet 100, Ferrium M54, and Ferrium S53 ( $\Psi=0.02,0.01$, and 0.02 wt $\%$, respectively, likely further reduced due to rare earth treatment), while predominately intergranular fracture is exhibited by $300 \mathrm{M}(\Psi=1.8 \mathrm{wt} \%)$, which did not contain rare earth elements.

- Close matching of fracture surface features with EBSD measured martensite microstructure indicates that HEAC is likely governed by a coupled HELP/HEDE mechanism; H cracking primarily proceeds along $\{110\}_{\alpha}$, martensite block boundary planes.

- The GBCD does not significantly vary between the present suite of UHSS alloys. Randomly oriented high energy martensite interfaces form the majority of boundaries (59$67 \%)$ in each alloy and exhibit high connectivity $\left(\mathrm{D}_{\mathrm{R}}=1.8-1.9\right)$. High energy interfaces speculatively trap significantly more hydrogen during HEAC providing an interconnected crack path through the microstructure. However, no alloy-specific effect of boundary structure on HEAC kinetics can be determined.

- Crack tip models of HEAC provide consistent and reasonably accurate predictions of the $\mathrm{E}_{\mathrm{app}}$ dependence of $\mathrm{K}_{\mathrm{TH}}$ and da/dt $\mathrm{II}$ in legacy 300M, Aermet 100, and Ferrium M54. Comparison of predicted and measured HEAC kinetics among these alloys demonstrates the dominating-beneficial effect of increased alloy purity and precipitation of nano scale carbides in reducing HEAC susceptibility of UHSS. 


\section{Characterization of HEAC in Modern Ultra High Strength $\underline{\text { Stainless Steels }}$}

\subsection{Problem Statement}

The combination of high mechanical properties and general corrosion resistance characteristic of modern, secondary hardening ultra-high strength stainless steels make them ideal candidates for structural aerospace components which require additional corrosion resistance. However, martensitic UHSSS are susceptible to SCC via a HEAC mechanism in environments which electrochemically produce hydrogen. Hydrogen-driven degradation of mechanical properties is severe and limits both operating stress levels and component life [7]. Critically, the susceptibility to SCC-HEAC is severe in UHSSS, such as Custom 465, at the tensile strength level required to replace modern UHSS $(\sim 1,700 \mathrm{MPa})$ in corrosion-limited applications [6,7,41]. The mechanistic origins of reduced SCC-HEAC resistance in UHSSS compared to UHSS near the 1,700 $\mathrm{MPa}$ strength level have not been investigated. Further, the present literature database of alloyenvironment dependent SCC-HEAC kinetics of UHSSS is severely limited.

The goal of Chapter 4 is to develop the HEAC kinetics database and understanding of microstructure necessary to further develop stainless UHSS with improved performance in SCCHEAC situations, in the context of a modern damage tolerant approach [14,62]. Emphasis is placed upon the origins of strong differences in SCC-HEAC resistance of compositionally similar UHSSS alloys. Towards this goal, four primary objectives are identified:

1) Measure SCC-HEAC kinetics of a systematically chosen set of UHSSS across a wide range of electrochemical potential for full immersion in a chloride solution environment.

2) Characterize the complex martensite microstructure across multiple lengths scales and determine the HEAC crack path for each alloy.

3) Measure hydrogen solubility, estimate $\mathrm{H}$ diffusivity, and qualitatively assess trap states in a limited set of UHSSS; link observed phenomena to specific microstructural features 
4) Explain observed trends in SCC-HEAC resistance using microstructural, H-trapping, and fractographic data.

The experimental approach taken to achieve these objectives is given in Chapter 2 .

\subsection{Results}

\subsubsection{EBSD Measurement of Martensite Microstructure}

The martensite microstructures of Ferrium PH48S - H900 and Custom 465 - H900 were characterized via EBSD, and the results are shown in Figures 39 to 40 using IPF-Z coloring to show the crystallographic orientation of individual martensite features. The microstructure of each alloy is examined at low magnification (500x) in Figures 39 to 40 and at high magnification (5000x) in Figures 41 to 42. Representative prior austenite grains are highlighted in Figures 39 to 40 by heavy white lines and intragranular packet boundaries are delineated by dotted white lines. OIM maps at 5000x were acquired using a $20 \mathrm{~nm}$ pixel step size for improved resolution of fine scale martensite block structure. Prior austenite grain boundaries in lath martensite microstructures are randomly oriented high angle boundaries, with typical misorientations of 20 to $40^{\circ}$ [108]. Conversely, packet and block interfaces typically exhibit a bimodal distribution of misorientation, consisting of a low angle peak (10-15 $)$ and high angle peak (50-60 $)$ [108].

The location of prior austenite grain boundaries was determined by comparison of a map of all random boundaries with misorientations of 20 to $40^{\circ}$ to the IPF-Z OIM maps. An example of this comparison is shown in Figure 43a,b for the low magnification EBSD-OIM map of Custom 465 H900 in Figure 39. A relatively complete set of prior austenite boundaries is clearly revealed in Figure $43 \mathrm{~b}$ using a misorientation criterion of 20 to $40^{\circ}$. Prior austenite grains highlighted by white lines in Figure 43a are indicated in Figure 43b by black arrows. Many of the prior austenite grain boundaries in Figure 43b show irregularities apart from classic grain structure such as concavity or nonlinearity. However, this structure closely matches the size/shape of prior austenite grains observed in IGSSC fractographs (Figure 43c and detailed in an ensuing section) and opticaletchant microscopy (Figure 43d). For example, nonlinear grain boundaries are clearly visible in Custom 465 - H900 when etched using Beraha's No. 4 sulfamic acid etch and viewed under 
polarized light (Figure 43d, white arrows). While atypical in the classic description of prior austenite grains as space filling polyhedra, non-linearity and concavity of grain boundaries has been previously observed in similar lath martensitic steels $[108,112,167]$. As such, the boundaries defined by the misorientation criterion (Figure 39 and 40, white lines) represent the physical reality of prior austenite grains in Custom 465 - H900 and Ferrium PH48S - H900.

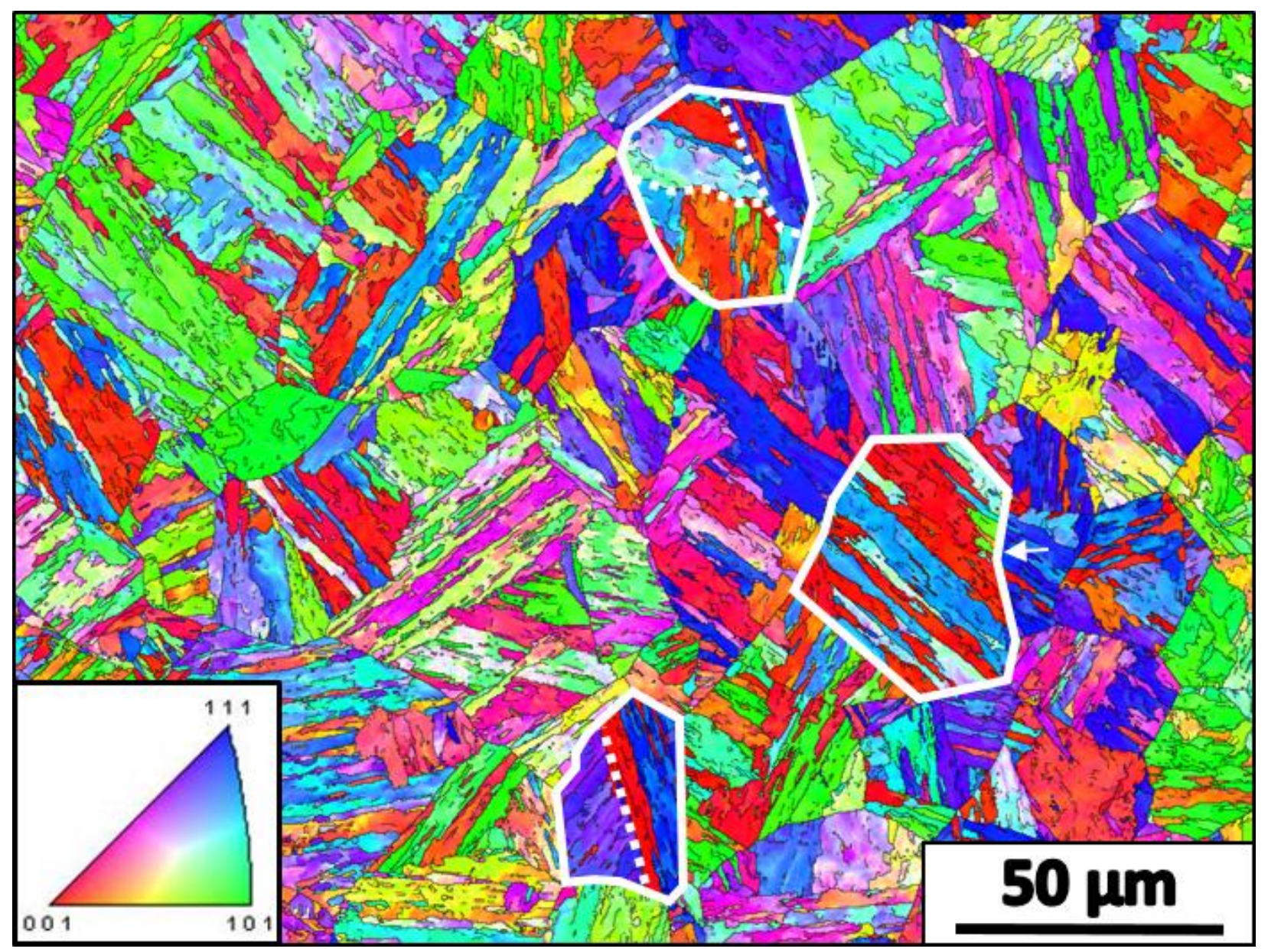

Figure 39 OIM Map of Custom 465 - H900 utilizing IPF-Z coloring. Three prior austenite grains are highlighted (white line); martensite packets within a prior austenite grain are separated by dotted white lines. This coloring scheme is identical for all EBSD-OIM images in the present work. 


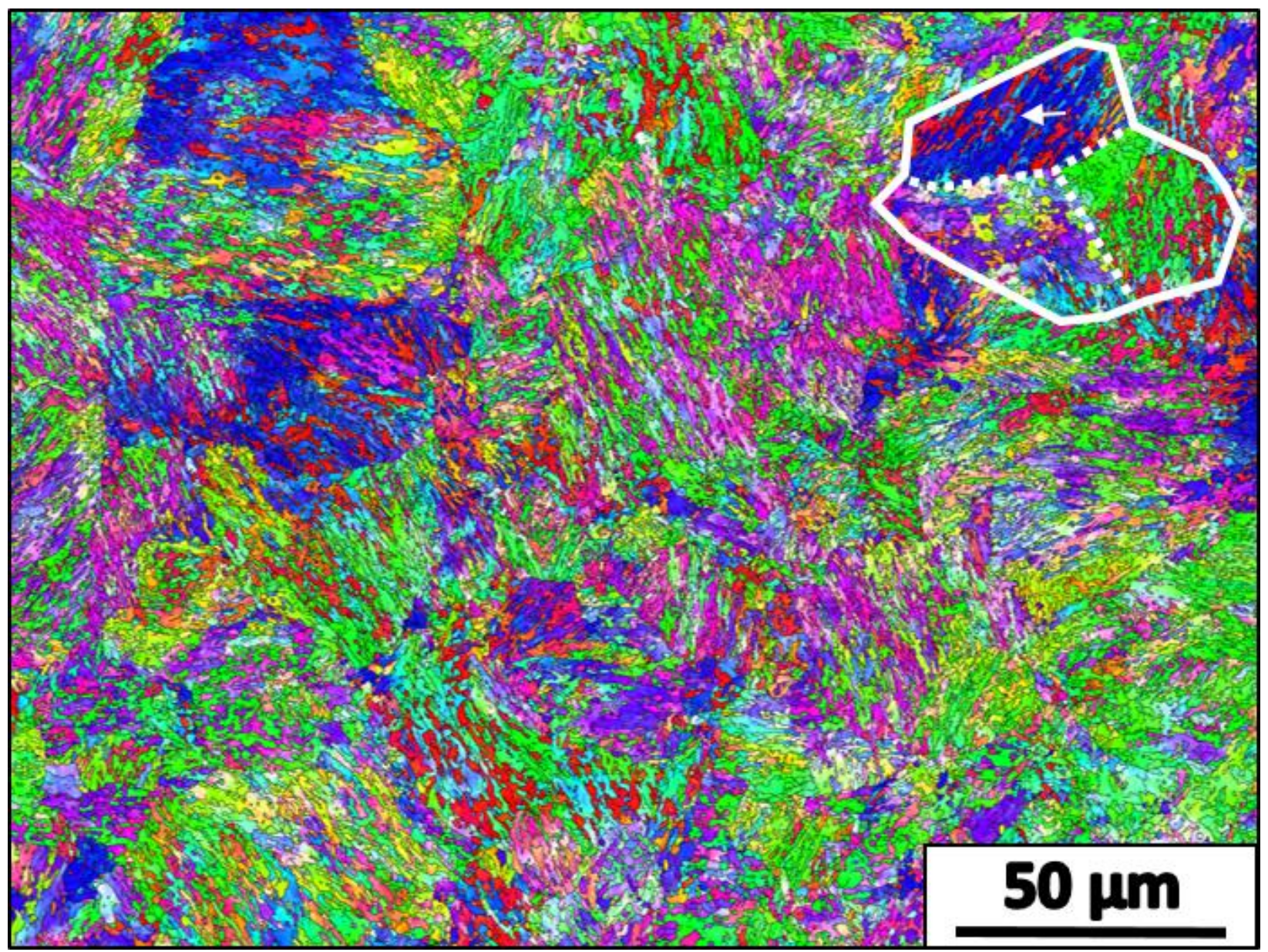

Figure $40 \quad$ OIM Map of Ferrium PH48S - H900 utilizing IPF-Z coloring. A single prior austenite grain is highlighted (white line); martensite packets within a prior austenite grain are separated by dotted white lines. 


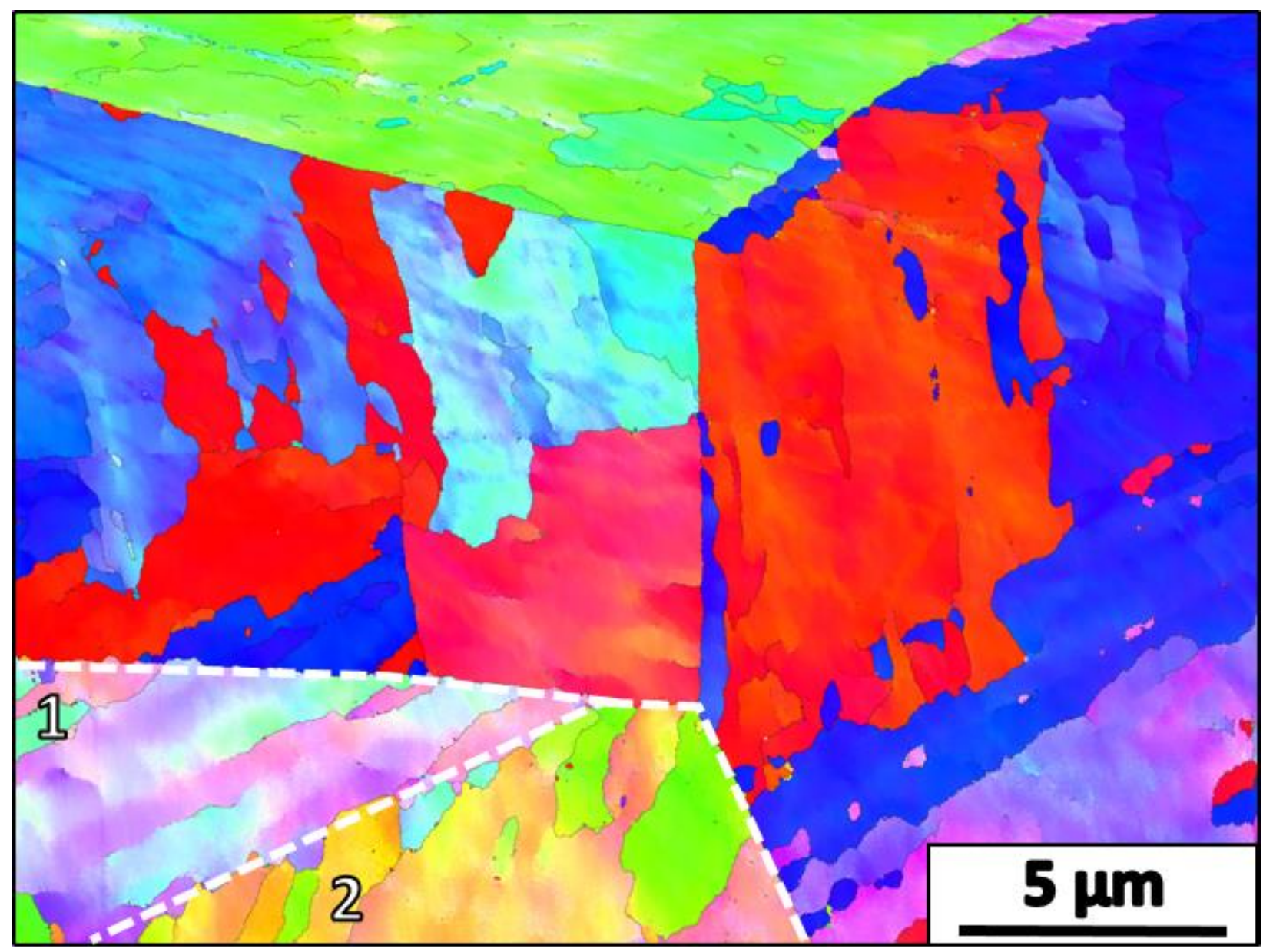

Figure 41 High resolution OIM Map of Custom $465-H 900$ utilizing IPF-Z coloring. Two martensite packets are isolated by dashed white lines. 


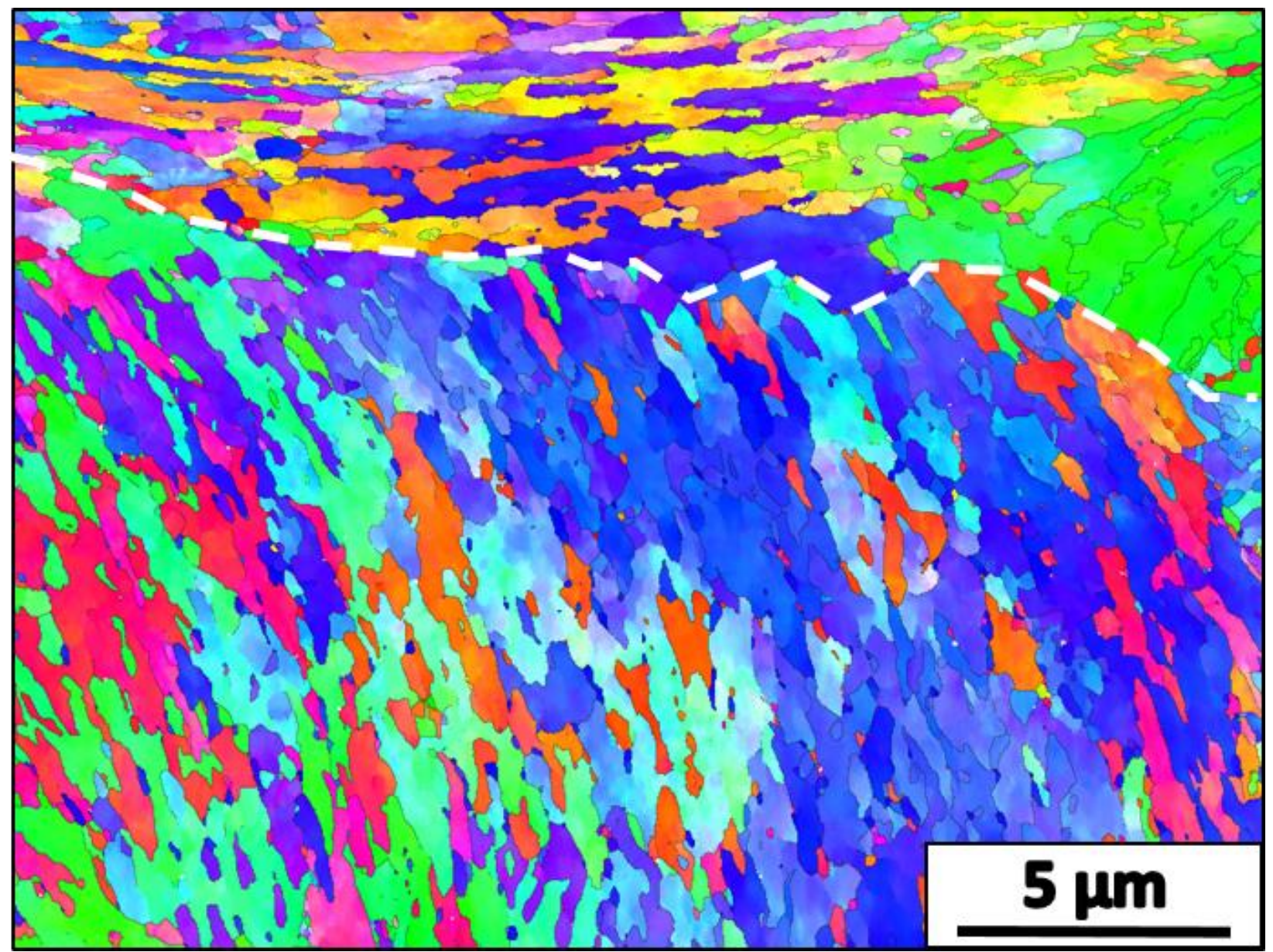

Figure 42 High resolution OIM Map of Ferrium PH48S-H900 utilizing IPF-Z coloring. A prior austenite grain boundary is shown by the dashed white line. 

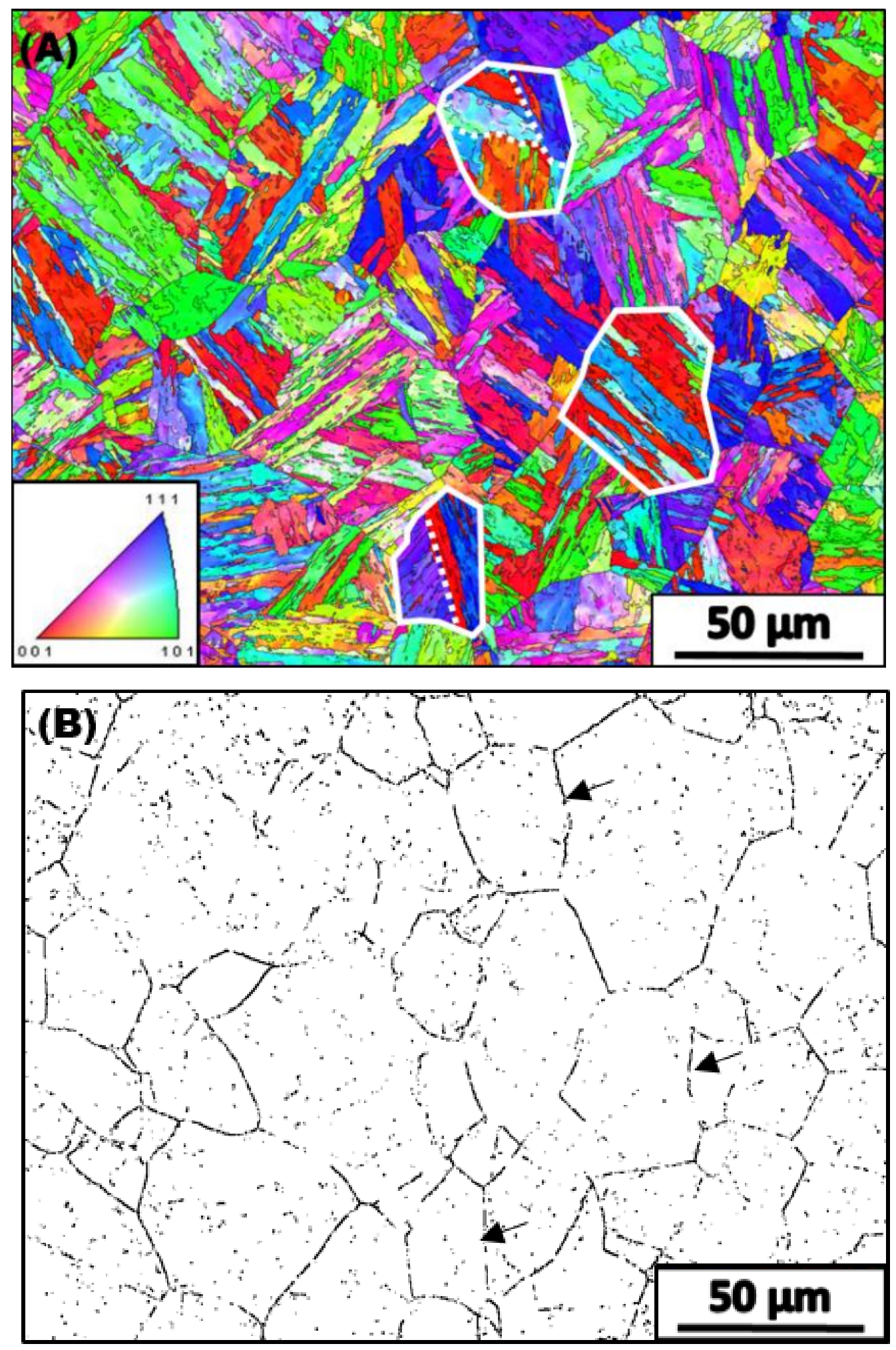

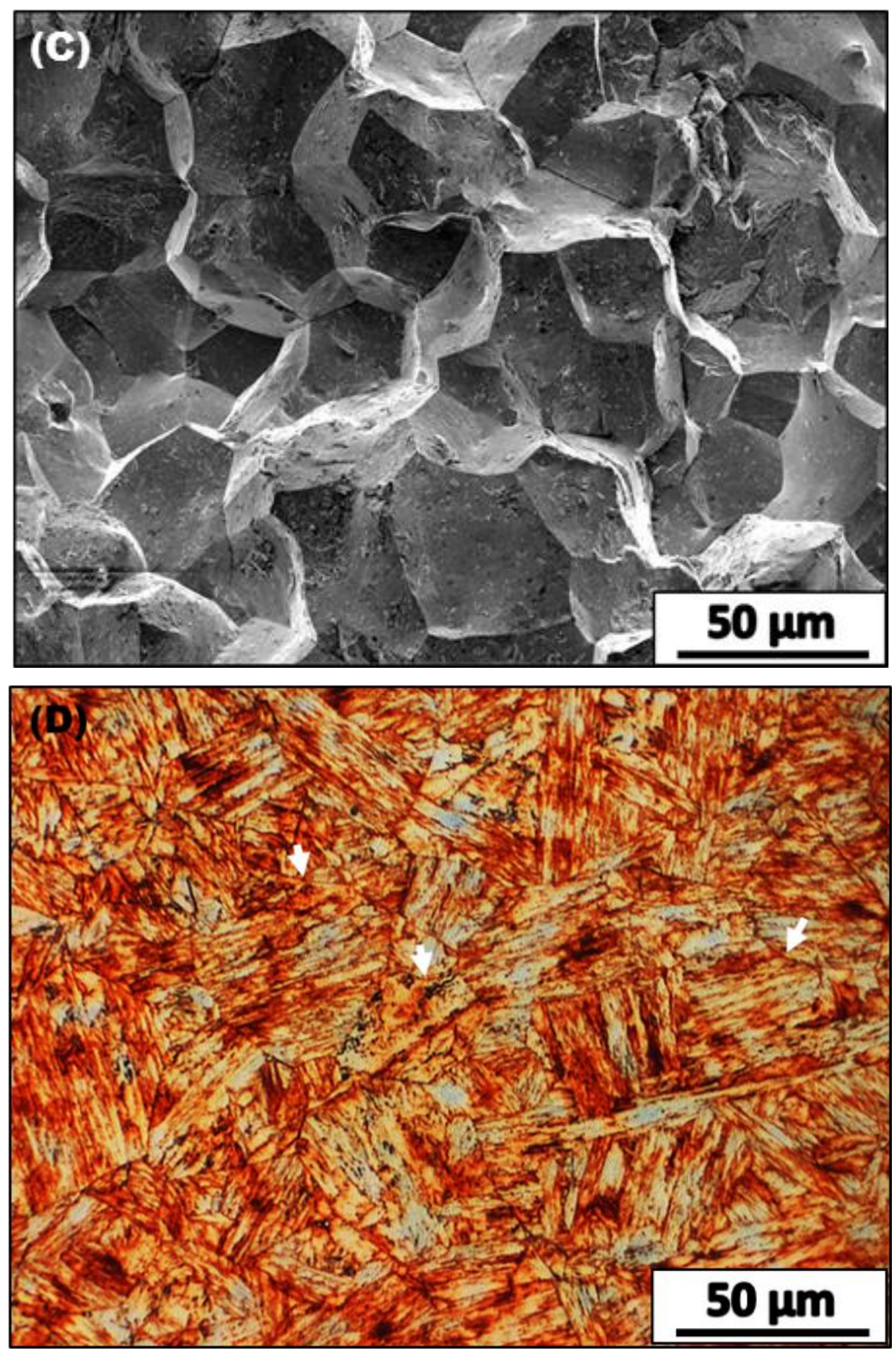

Figure 43 Determination of prior austenite grain boundaries in Custom 465 - H900: a) IPF$Z$ OIM map, b) map of random martensite interfaces with a misorientation of $\left.20-40^{\circ}, c\right)$ IGSCC of Custom 465-H900 stressed in $3.5 \% \mathrm{NaCl}$ at $\mathrm{E}_{\text {app }}=-975 \mathrm{mV} \mathrm{V}_{\mathrm{SCE}}$, and d) Polished section of Custom 465 - H900 etched via full immersion Beraha's No. 4 sulfamic acid etch for 240 s. 
Custom 465 exhibits a classical microstructure with well-defined prior austenite grain boundaries and martensite packet features (Figure 39 solid white and dotted lines, respectively). Ferrium PH48S has a comparatively fine-scaled martensite structure that does not exhibit well-formed prior austenite grains (Figure 40). An insufficient area was sampled to establish the prior austenite grain size in accordance with ASTM E112-13 [109]. However, prior austenite grain size in Custom 465 - H900 is measured to be $20-100 \mu \mathrm{m}$ with an average of $40 \mu \mathrm{m}$, based upon the EBSD-OIM map shown in Figure 39. In contrast prior austenite grain boundaries are not readily observed in OIM maps of Ferrium PH48S, but are distinguishable using the misorientation criterion for random boundaries. Isolated well-formed prior austenite grains (Figure 40, white lines) indicate the grain size of Ferrium PH48S - H900 is close to that of Custom 465 - H900: 20-100 $\mu$ m with a nominal average of $50 \mu \mathrm{m}$. Grain size of Ferrium PH48S is designed to be small relative to conventional alloys $(5-50 \mu \mathrm{m})$; controlled by a fine scale dispersion of grain boundary carbides which are stable during hot working and austenitization. However, no published microstructural data exist to support this design goal, and the OIM map in Figure 40 gives no indication of prior austenite grain size refinement compared to conventional Custom 465 - H900.

To further analyze the size of prior austenite grains, optical etchant microscopy was conducted on Custom 465 - H900 and Ferrium PH48S - H900. Standard solutions of four common reagents for martensitic stainless steels were prepared: Ralphs reagent, Viella's reagent, waterless Kalling's reagent, and Beraha's sulfamic acid etchant [168]. Polished sections of UHSSS were fully immersed or swabbed with each etchant in $15 \mathrm{~s}$ intervals for up to 10 minutes of total time. At each interval, the surface was examined under white and polarized light in an optical microscope. Immersion of Custom 465 - H900 in Beraha's sulfamic acid etch for $240 \mathrm{~s}$ (Figure 43d) effectively reveals prior austenite grain boundaries and general martensite microstructure when viewed under polarized light. However, the etching response is spatially heterogeneous, and a large number of prior austenite grains were unable to be sampled. The remaining etchants did not effectively reveal the martensite microstructure of Custom 465 - H900 and a similar spatially sensitive grain boundary attack was observed. Prior austenite grain boundaries in Ferrium PH48S were not revealed by any of the selected etchants. Fine scale martensite structure was observed by immersion in Kalling's (15 s) and Viella's (3 min) reagents, but the size of the smallest martensite features is near the resolution limit of optical microscopy, and quantitative characterization was 
not possible. However, this result does indicate that prior austenite grain boundaries in Ferrium PH48S - H900 are more resistant to chemical attack than those of Custom $465-\mathrm{H} 900$.

The martensite structures represented in Figures 39 to 43 represent the extremes of martensite feature size when compared to the UHSS alloys examined in Chapter 3 (Figures 6-10). Identical to the microstructure analysis of UHSS alloys, a classic martensite hierarchy of martensite packets, blocks and laths within prior austenite grains is used to interpret the EBSD results. The classical martensite hierarchy of structure, originally shown in Figure 12 is reproduced in Figure 44 for ease of comparison. As was previously discussed in Chapter 3, the crystallographic orientations of martensite laths typically obey the Kurdjumov-Sachs (K-S) orientation relationship, which defines the crystallographic orientation of newly formed martensite (BCC) with respect the parent austenite (FCC) [113]. Close packed $\{111\}_{\gamma}$ FCC habit planes are parallel to $\{110\}_{\alpha}$, in transformed body centered tetragonal martensite, and the $\langle 101\rangle_{\gamma}$ direction is parallel to $<111>_{\alpha \prime}$ [113]; where $\gamma$ and $\alpha^{\prime}$ denote parent austenite and newly formed martensite, respectively. Cubic symmetry of both phases dictates 24 possible martensite lath orientations following K-S: 6 uniquely oriented directional variants $<101>_{\gamma} / /<111>_{\alpha}$, within each of 4 possible planes $\{111\}_{\gamma} / /\{110\}_{\alpha}$, [114]. In structures obeying K-S, no more than 4 unique packet orientations, corresponding to 4 independent $\{111\}_{\gamma}$ habit planes, can be present within a single prior austenite grain. Further, an ideal K-S orientation relationship dictates three dominant block orientations per packet, each with 2 lath variants [111,112]. This orientation relationship was indicated for microstructurally similar UHSS (Chapter 3) and will be employed to determine the morphology of packets, blocks and laths in UHSSS. 

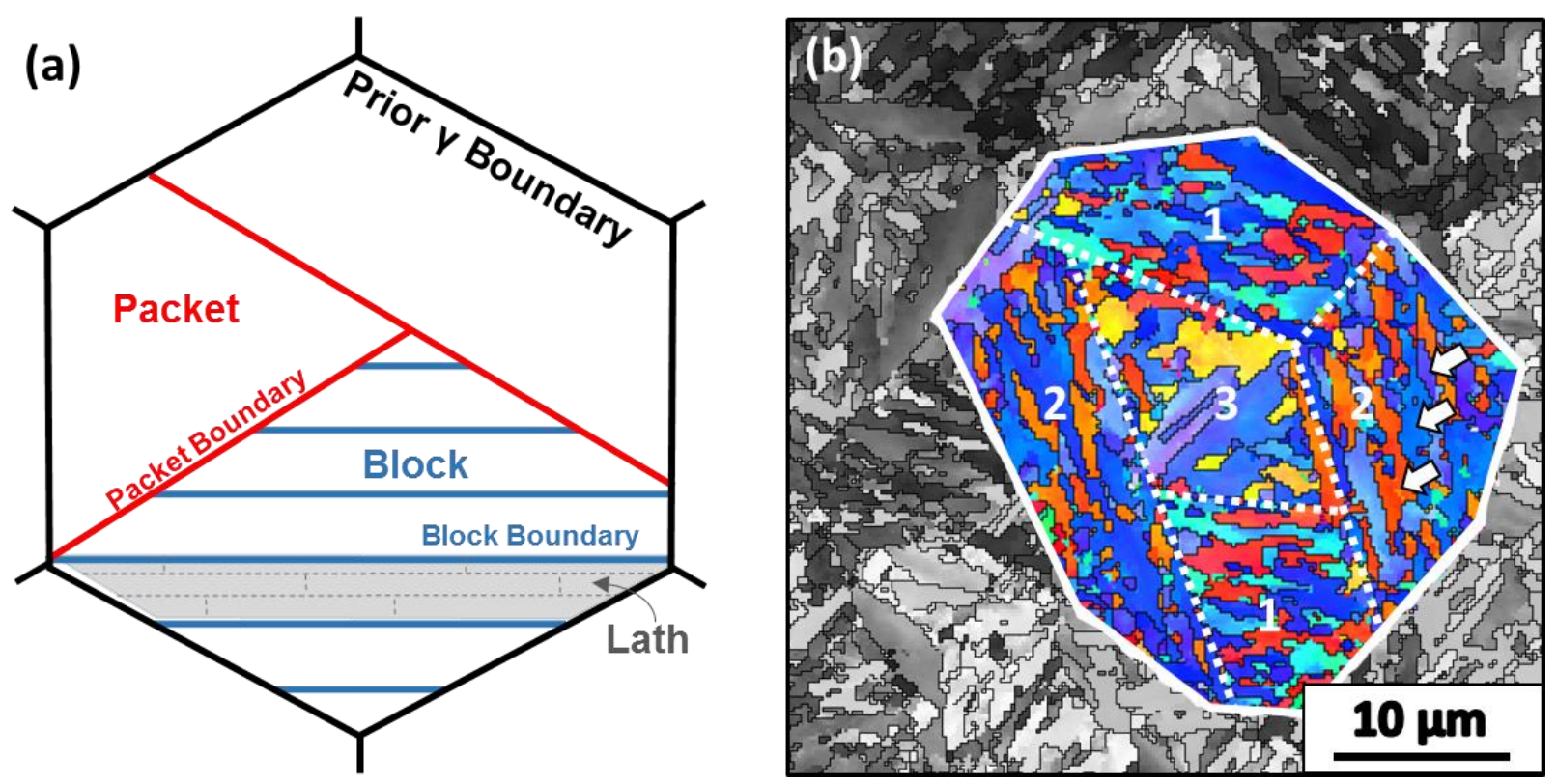

Figure 44 (a) Classical hierarchy of martensite structure of UHSS alloys, adapted from [52], and (b) Isolated 300M prior austenite grain. Martensite packet boundaries are highlighted in white dotted lines, and individual packet orientations are numbered.

While massively different in size scale, the martensite structure of both alloys is consistent with UHSS martensite structure and the classical Kurdjumov-Sachs orientation relationship. Prior austenite grains (Figures 39-40, white lines) are divided into 1 to 4 crystallographically distinct martensite packets (Figures 39-40, separated by white dotted lines). Packets are subdivided into multiple irregular, but parallel features containing a single orientation (IPF-Z color). Three orientations of single-color features typically dominate within a packet, indicating that they are martensite blocks, as established for all UHSS in Chapter 3. For example, the central Custom 465 - H900 prior austenite grain in Figure 39 (white arrow) contains a single martensite packet which is dominated by three block orientations indicated by three IPF-Z colors (red-blue-cyan). This morphology is identical for typical packets of Ferrium PH48S - H900 (Figure 40, white arrow, red-blue-cyan block features). A small fraction of martensite packets in Custom 465 - H900 exhibit two dominant block orientations. Detail of this dual block packet morphology is shown in Figure 41 (outlined packets: (1) cyan - purple IPF-Z colors, and (2) yellow - green IPF-Z colors) and further examples are visible via inspection of Figure 39. The predominance of three martensite blocks in K-S lath martensite is understood based on a minimization of the shape strain during transformation from austenite to martensite [111]. Two block packets may therefore have a higher 
transformational strain. However, transformation strain calculations are based upon simulations martensite transformation in pure iron [112,114]. Significant alloy additions in Custom 465 may cause a deviation from this ideal packet-block structure by altering the lattice parameter of transformed martensite [169]. To aid in examination of fine scale martensite packet and block structure features, a single packet is isolated for each alloy in Figures 45 and 46 . Four visualizations of martensite packet structure are presented: (a) standard IPF-Z coloring, (b) boundary traces by misorientation, (c) a trace of the dominant $\Sigma 3$ CSL special boundary, and (d) a trace of the three dominant CSL special boundary types $\left(\sum 3, \sum 11\right.$, and $\left.\sum 25 b\right)$.

The martensite block structure of Custom 465 - H900 is well ordered and coarse compared to all other examined alloys. As shown in Figure 45a, parallel block features are elongated along one of four variants of K-S $\{111\}_{\gamma}$ habit planes in austenite that define the packet and are separated by relatively straight $\{110\}_{\alpha}$, block boundaries. Block length is dictated by the prior austenite grain size and number of packets inside the grain [170]. Average spacing between block interfaces is 2.4 $\pm 0.1 \mu \mathrm{m}$, substantially larger than all other UHSS $(0.51-1.27 \mu \mathrm{m})$. Martensite blocks are separated by highly misoriented boundaries with misorientations $>15^{\circ}$ (Figure 45b), consistent with previous misorientation analyses of K-S block interfaces in carbon and low alloy steels [112,118]. Packet boundaries in Custom 465 - H900 are exclusively high angle (Figure 45b), identical to packet structure in UHSS (Figure 15) and result from the intersection of K-S packet variants across an irregular interface. A minor fraction of high angle boundaries along block and packet interfaces obey the plane-angle orientation relationship to be a CSL boundary, as defined by Brandon's Criterion [93]. CSL boundaries do not have a preferential location and are randomly located along martensite packet and block interfaces in short 1-5 $\mu \mathrm{m}$ segments typically bordered by random high angle boundaries (Figure $45 \mathrm{~b}$ and c). The fractional distribution of interfacial area for each boundary type is shown in Table 9. 


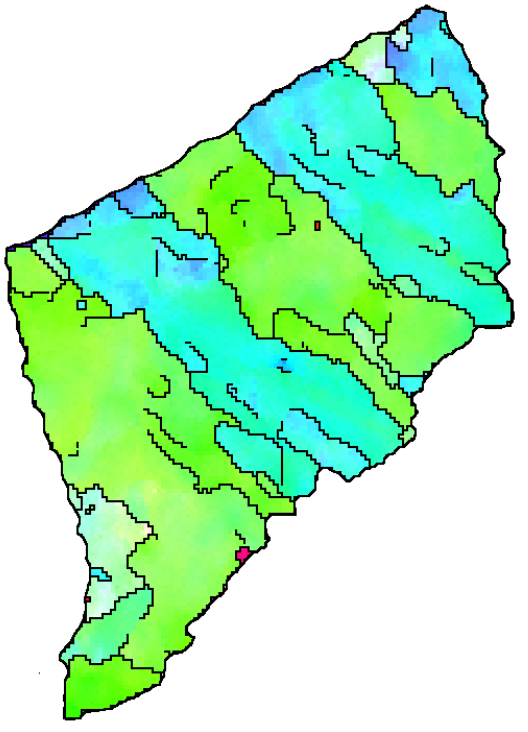

(a)

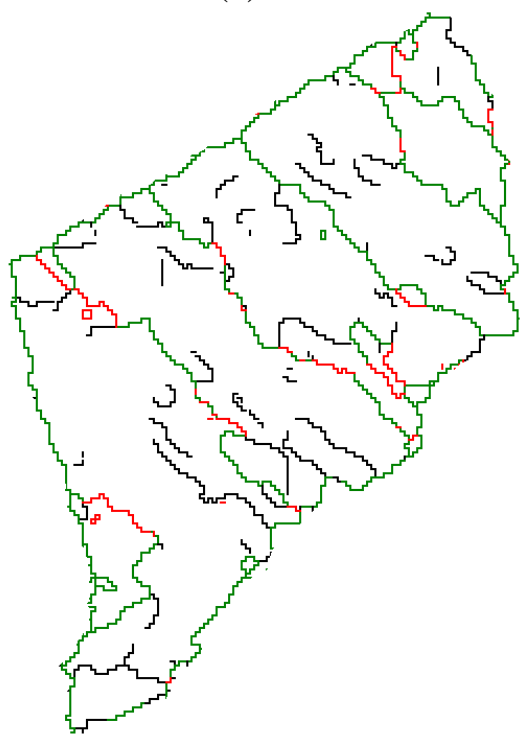

(c)

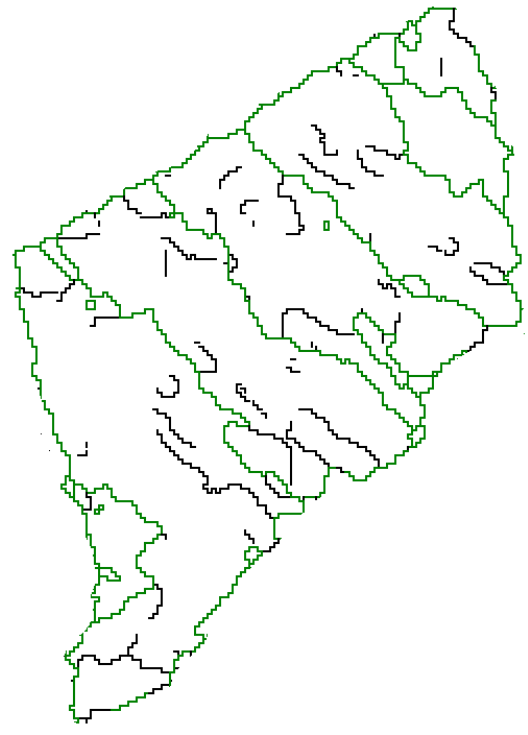

(b)

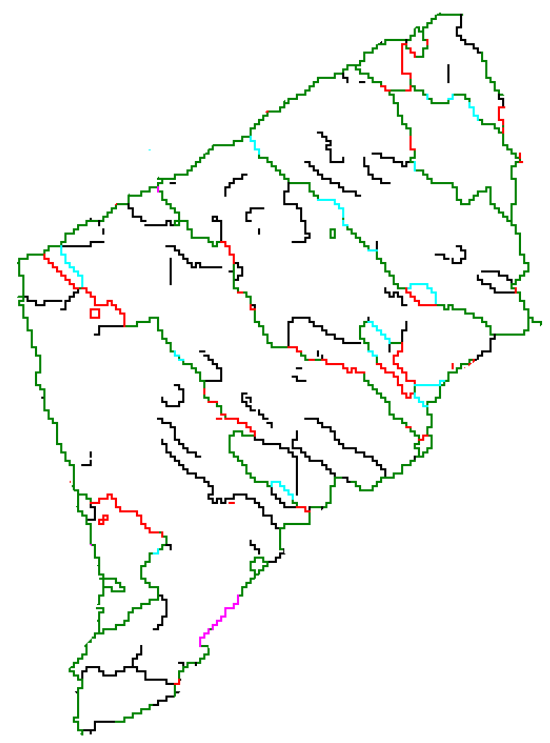

(d)

\section{$10 \mu \mathrm{m}$}

Figure 45 (a) Orientation map of an isolated Custom 465 - H900 martensite packet colored using IPF-Z coloring. (b) Map of martensite boundaries showing the distribution of high (> $15^{\circ}$, green) and low angle $\left(2^{\circ} \leq \theta<15^{\circ}\right.$, black) interfaces. (c) Identical misorientation map as (b) with high angle $\sum 3$ CSL boundaries highlighted in red, and (d) Identical misorientation map as (c) with minor fractions of $\sum 11$ (teal) and $\sum 25 b$ (magenta) highlighted. 


\begin{tabular}{|c|c|c|c|c|c|}
\hline $\begin{array}{c}\text { Interfacial Area } \\
\left(\mathrm{S}_{\mathrm{V}}, \mathrm{mm}^{2} / \mathrm{mm}^{3}\right)\end{array}$ & $\begin{array}{c}\text { Random High } \\
\text { Angle }\end{array}$ & $\begin{array}{c}\text { Random } \\
\text { Low Angle }\end{array}$ & $\sum 3$ & Other CSL & Total \\
\hline $300 \mathrm{M}$ & $1,312(64 \%)$ & $304(15 \%)$ & $192(9 \%)$ & $258(12 \%)$ & 2,066 \\
\hline AerMet 100 & $1,456(59 \%)$ & $290(12 \%)$ & $314(13 \%)$ & $412(17 \%)$ & 2,472 \\
\hline CrNiMoWV & $972(62 \%)$ & $202(13 \%)$ & $203(13 \%)$ & $201(13 \%)$ & 1,578 \\
\hline Ferrium M54 & $1,280(61 \%)$ & $350(17 \%)$ & $178(8 \%)$ & $301(14 \%)$ & 2,109 \\
\hline Ferrium S53 & $1,154(67 \%)$ & $230(13 \%)$ & $223(13 \%)$ & $117(7 \%)$ & 1,724 \\
\hline PH48S-H900 & $1,761(46 \%)$ & $248(7 \%)$ & $1,303(34 \%)$ & $483(13 \%)$ & 3,795 \\
\hline C465-H900 & $695(80 \%)$ & $11(1 \%)$ & $41(6 \%)$ & $116(13 \%)$ & 863 \\
\hline
\end{tabular}

Table 9 Interfacial area and fraction of total boundary area of selected martensite boundaries

As shown in Figure 46, the martensite packet and block structure of Ferrium PH48S is extremely fine scale, but obeys the hierarchy and boundary characteristics of a classic K-S relationship, identical to Custom 465 (Figure 45), as well as AerMet 100 (Figure 13) and similar UHSS (Figure 15). Martensite blocks are roughly parallel and separated by irregular, non-linear boundaries. The non-linearity of block boundaries is especially evident at high magnification (Figure 47); martensite blocks are routinely intruded by the orientation of the adjacent block by up to $50 \%$ of the block width. Average spacing of block interfaces is reduced compared to all other UHSS alloys: $0.52 \pm 0.03 \mu \mathrm{m}$. Similar to AerMet 100 (Figure 13), martensite blocks lack the classical acicularneedle shape and are often non-linear and rounded. Three martensite block orientations dominate within a given packet (Figure 46, red-cyan-blue block features) in close agreement with previous observations of K-S lath martensite [110,112]. Further, block and packet boundaries are nearly exclusively high angle (Figure 46b), as would be predicted by a K-S orientation relationship.

However, a significant portion of block and packet boundaries in Ferrium PH48S - H900 meet Brandon's Criterion for a CSL boundary. CSL interfaces are preferentially located on block boundaries and are predominantly $\sum 3$ (Figure 46c), with a minor fraction of $\sum 11$ and $\Sigma 25 \mathrm{~b}$ (Figure 46d). Notably, Ferrium PH48S has a substantially higher fraction $\sum 3$ boundaries (34\%, Table 9) compared to Custom 465 (6\%) and all other UHSS alloys (6-13\%). Further, due to the fine scale 
of martensite features in Ferrium PH48S, there is greater interfacial area of $\Sigma 3$ boundaries compared to other UHSS $\left(1,303 \mathrm{vs} 41-314 \mathrm{~mm}^{2} / \mathrm{mm}^{3}\right)$. Similarly from Table 9 , the total martensite interface area is largest for PH48S $\left(3,795 \mathrm{~mm}^{2} / \mathrm{mm}^{3}\right)$ compared to Custom $465\left(863 \mathrm{~mm}^{2} / \mathrm{mm}^{3}\right)$ and the other UHSS $\left(1,578-2,472 \mathrm{~mm}^{2} / \mathrm{mm}^{3}\right.$, Table 8$)$.

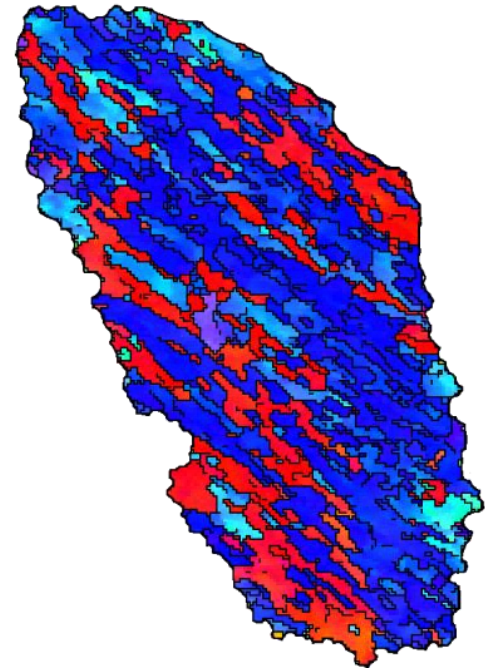

(a)

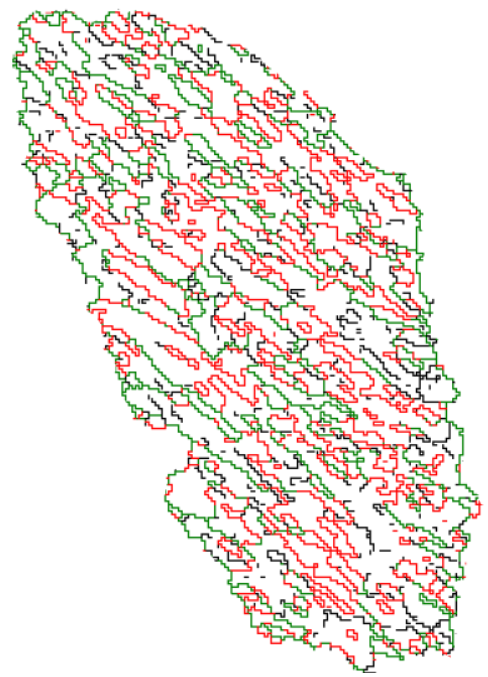

(c)

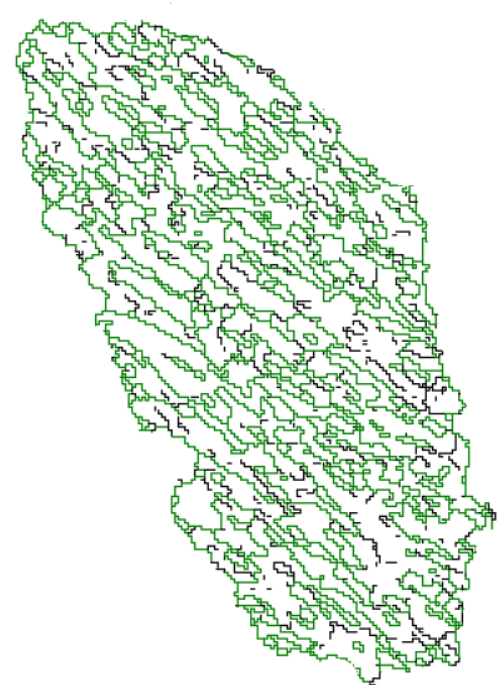

(b)

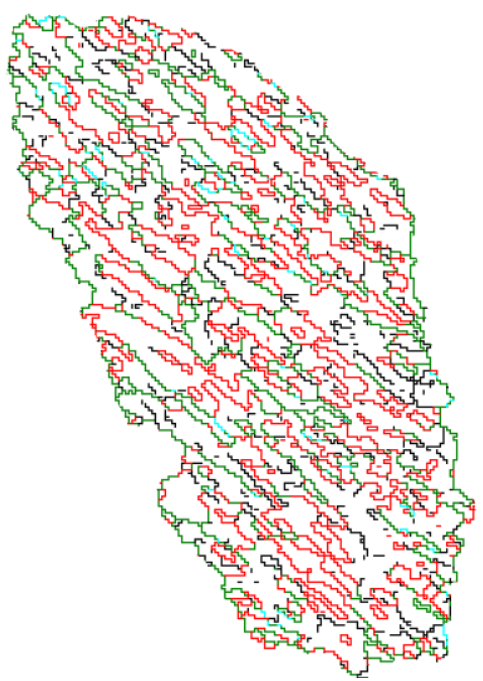

(d)

\section{$10 \mu \mathrm{m}$}

Figure 46 (a) Orientation map of an isolated Ferrium PH48S-H900 martensite packet colored using IPF-Z coloring. (b) Map of martensite boundaries showing the distribution of high $\left(>15^{\circ}\right.$, green) and low angle $\left(2^{\circ} \leq \theta<15^{\circ}\right.$, black) interfaces. (c) Identical misorientation map as (b) with high angle $\sum 3$ CSL boundaries highlighted in red, and (d) Identical misorientation map as (c) with minor fractions of $\sum 11$ (Teal) and $\sum 25 b$ (Magenta) highlighted. 


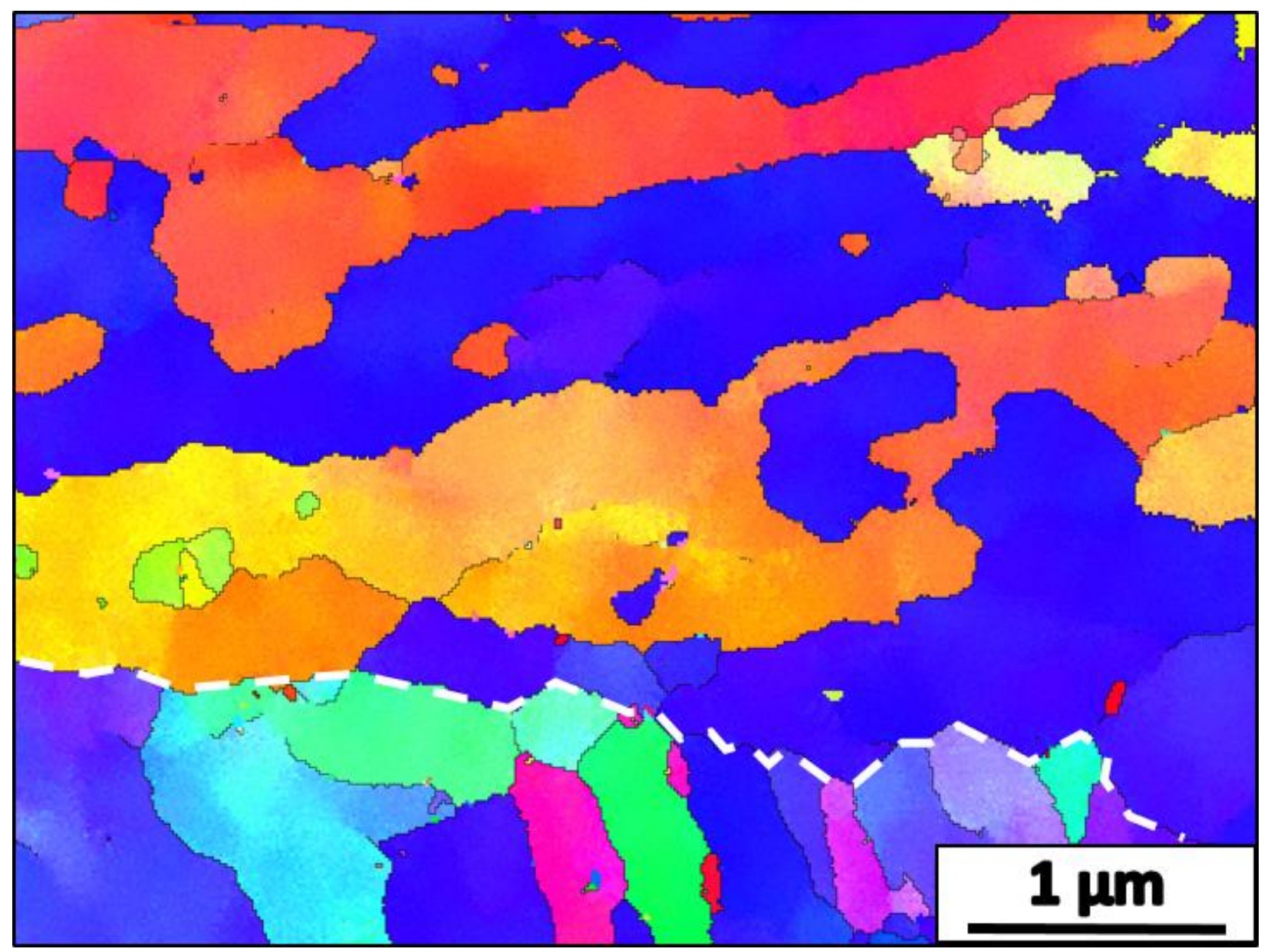

Figure 47 Detail of Ferrium PH48S - H900 martensite block structure presented in Figure 42, acquired with at a very fine EBSD pixel step size $(13 \mathrm{~nm})$. The prior austenite grain boundary is indicated by the white dashed line.

\subsubsection{EBSD Measurement Precipitated Austenite in UHSSS}

After austenitization, quench, and aging, both Custom 465 - H900 and Ferrium PH48S - H900 exhibit a martensitic matrix, which contains a small fraction of austenite phase. Average EBSD measured austenite content is 0.1 and 0.3 vol \% for Custom 465 - H900 and Ferrium PH48S H900, respectively. Isolated islands of austenite 20-200 nm in size are exclusively located on martensite interfaces in both alloys, as shown in Figure 48 and Figure 49. The island morphology is identical to austenite observed in AerMet 100 (Figure 11) and strongly suggests it represents austenite precipitated during aging at high temperature. Substantially higher amounts of total austenite are predicted by Thermo-Calc modeling for the H900 aging condition (15 and 18 vol \% 
for Ferrium PH48S and Custom 465, respectively) [43]. There is no evidence to suggest that an experimental error in the EBSD could account for this discrepancy. Austenite islands are spatially well defined by a statistically significant number of pixels. Further, mechanical polishing conducted on EBSD specimens ended with a vibratory final polishing designed to reduce surface deformation which may convert austenite to martensite.

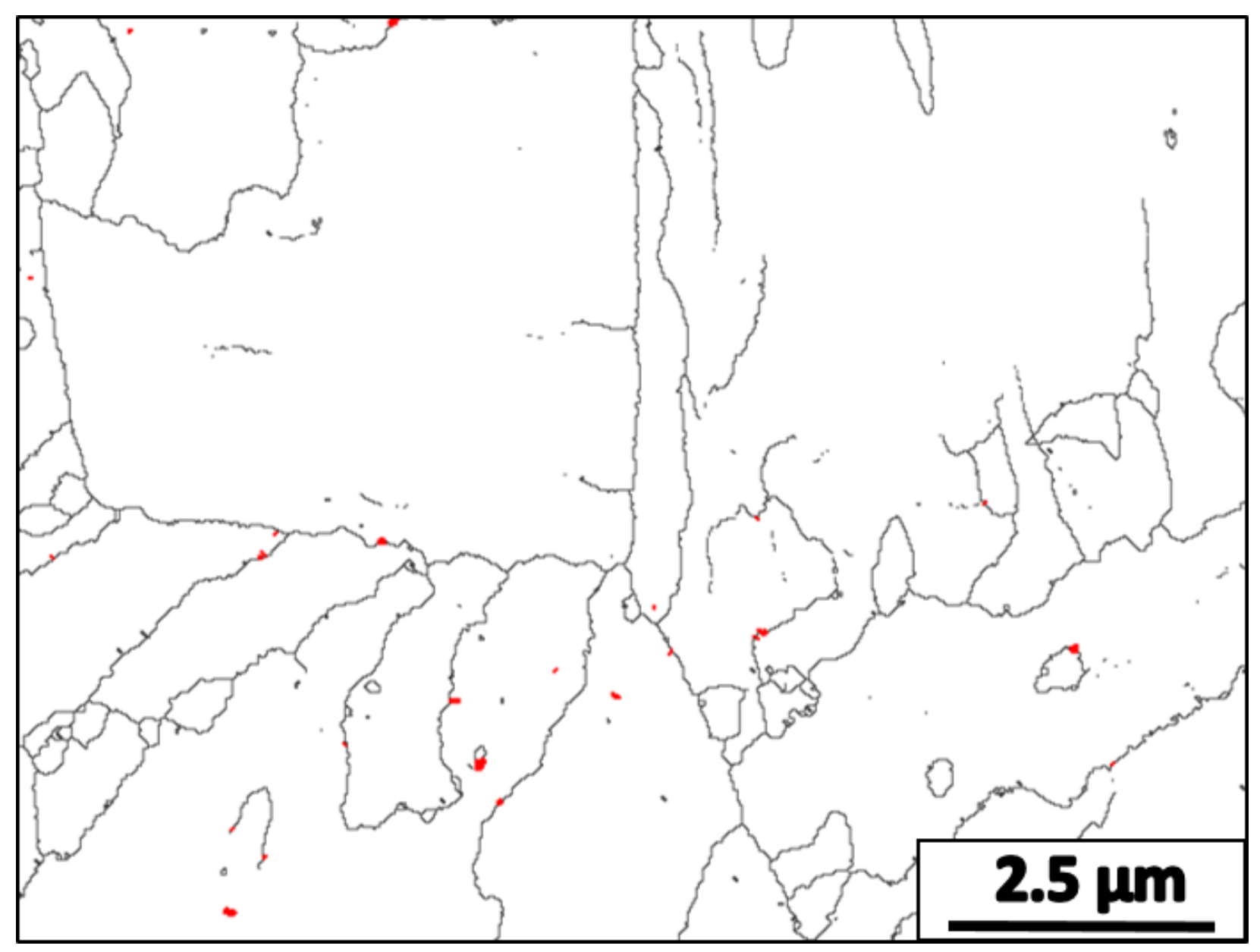

Figure 48 Distribution of EBSD-Measured Austenite in Custom $465-H 900$. 


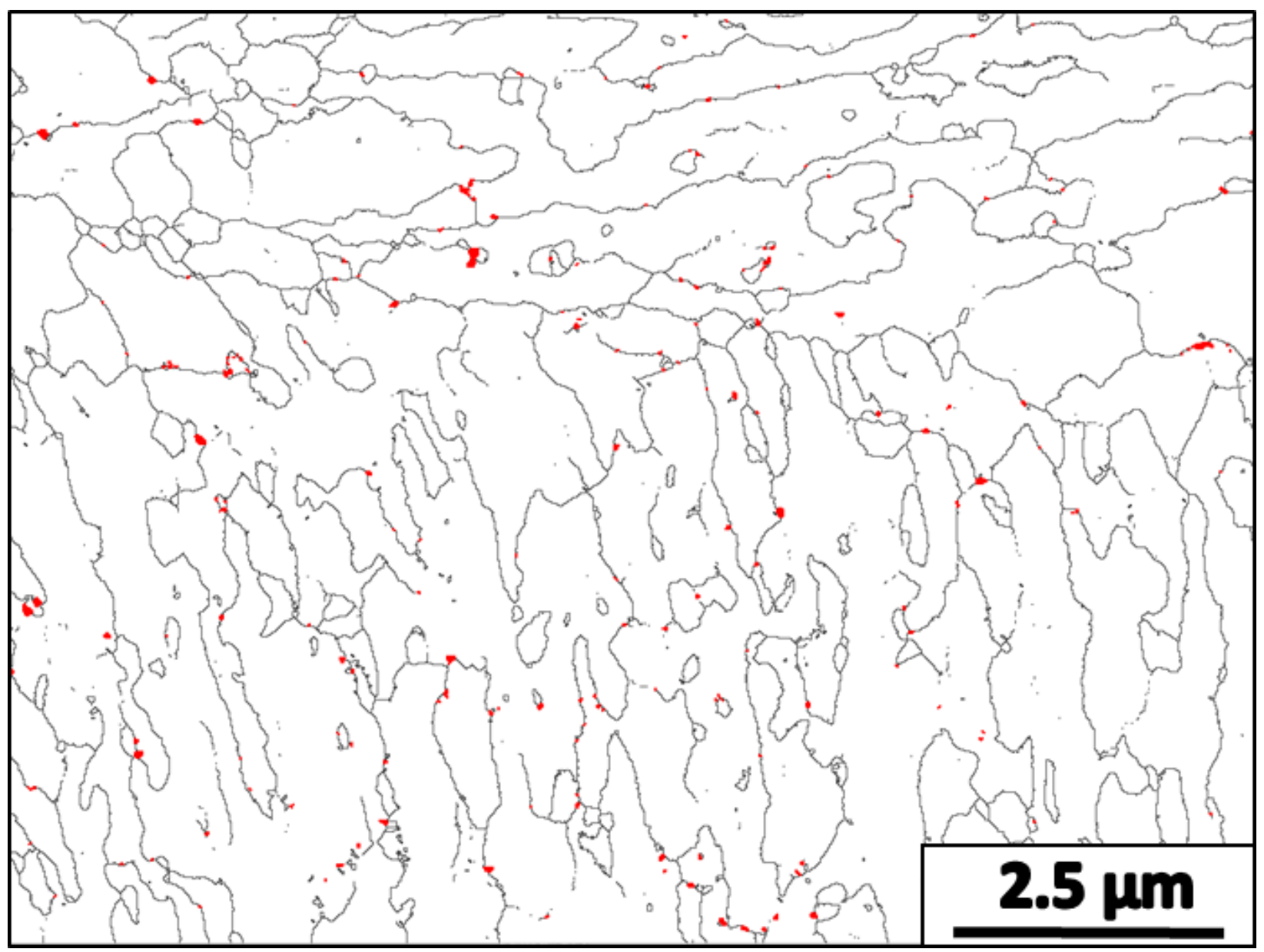

Figure 49 Distribution of EBSD-Measured Austenite in Ferrium PH48S - H900.

The analysis of martensite structure presented in Figures 13, 15, 43 and 44, has revealed a universal hierarchy for martensite structure in UHSS and UHSSS alloys. Prior austenite grains are divided into a small number (1-5) of packets of parallel martensite block features, on up to 4 possible variants of $\{111\}_{\gamma}$ habit plane, with three dominant block orientations per packet as associated with the variants of possible directions in the KS relationships. No structure below the martensite block has been observed in any alloy, even when EBSD pixel size is reduced to an order magnitude below the expected lath width (13 nm versus 0.1-0.25 $\mu \mathrm{m}$ ). Martensite block and packet interfaces are near exclusively high angle. Only a very small amount of precipitated austenite is observed by EBSD along packet and block interfaces; however, the EBSD method would not resolve thin layer (1-5 nm thick) austenite along block interfaces, as discussed in Chapter 3 for UHSS such as 
AerMet 100. Measurement of thin film austenite in UHSSS alloys will be discussed in an ensuing section. A fraction of high angle boundaries are CSL special boundaries, as defined by Brandon's Criterion. The size scale of martensite features varies significantly between alloys; however, the preferential location of low, high, and CSL boundaries does not vary significantly between $\operatorname{UHSS}(\mathrm{S})$.

\subsubsection{Fractal Analysis of Martensite Interfaces in UHSSS}

As detailed in Chapters 1 and 3, the connectivity of random boundaries has been shown to influence the susceptibility of polycrystalline metals to segregated impurity and hydrogen assisted cracking [49,50]. Bechtle et al. established that resistance to IG hydrogen embrittlement is significantly reduced in polycrystalline $\mathrm{Ni}$ when CSL fraction is increased from 46 to 75 length percent of all boundaries. This result is predicated upon the hypothesis that increasing fraction of CSL special boundaries will reduce the connectivity of the random boundary network (RBN), which is inherently susceptible to fracture and provides a weak crack path through the microstructure $[48,49]$. CSL special boundaries are hypothesized to be inherently more resistant to fracture and hydrogen embrittlement compared to random boundaries [48,57]. Critically, the connectivity of such boundaries was not probed by Betchtle et al., despite literature which indicates connectivity is an important factor in determining fracture resistance [49,51,59]. Moreover, these studies focused on simple polycrystalline microstructures; similar studies have not been reported for martensitic steels with complex microstructures and a high density of interfaces.

Results presented in Chapter 3 establish all UHSS exhibit small, discrete segments of CSL boundary in along otherwise continuous random boundaries. The presence of discrete-small gaps in the RBN necessitates the use of a pixel gap tolerance, which considers small gaps in a continuous random boundary to be connected in calculation of the maximum random boundary connectivity (MRBC). The physical basis for employing a CSL gap tolerance is discussed in Chapter 3 and is fundamentally based on the hypothesis that a relevant gap tolerance is small relative to HEAC critical length scales. Compared to the minimum pixel gap tolerance required for high $\mathrm{D}_{\mathrm{R}}$ in UHSS (2 pixels, 320-400 nm of physical distance), $\chi_{\text {crit }}$ for HEAC is relatively large. In the case of crack propagation along a 2D high angle boundary plane, small segments of CSL boundary may limit crack propagation. However, if the small gaps in the measured RBN are relatively localized in 
three dimensions, a crack front could advance between islands of CSL boundary on the martensite block interface, leaving behind pillars of connected CSL boundary. Such pillars would fail under increased stress as the boundary around them cracks and local stress increases. The mechanism would be enhanced by microcracks initiating ahead of any CSL disruption and cracking back to the advancing crack front, as suggested by curve fit values of $\chi_{\text {crit }}$ and literature $[2,11,12,120]$. The hypothesized 3D island morphology for small CSL boundary segments and relatively large $\chi_{\text {crit, }}$ both imply that the effect of CSL disruptions in three dimensions is likely minimal. As such, the use of a gap tolerance in determination of $\mathrm{D}_{\mathrm{R}}$ is logical and consistent with previous work [108].

Random boundary network connectivity is strongly dependent upon the use of a pixel gap tolerance during the fractal analysis. When the small-justified gap ( $\geq 2$ pixels, $190-500 \mathrm{~nm}$ of physical distance, dependent on the EBSD pixel size) in the random boundaries is considered to be connected, all UHSS alloys exhibit full connectivity of the RBN $\left(D_{R}=1.8-1.9\right)$. Without the use of a gap tolerance, there is essentially zero calculated connectivity of the RBN ( $\left.D_{R} \sim 1.0\right)$. This result cannot be physically justified in the context of the fine scale martensite interfaces. Due to identical grain boundary character distribution (GBCD) of UHSS characterized in Chapter 3, no specific effect on HEAC behavior could be discerned from the data. However, the extremely high CSL fraction in UHSSS Ferrium PH48S - H900, relative to Custom 465 and other UHSS (Table 6) offers the opportunity to assess the effect of increasing CSL fraction on RBN connectivity and HEAC properties.

Fractal analysis of the MRBC in Ferrium PH48S and Custom 465 establishes that the connectivity of the random boundaries in the absence of a gap tolerance filter is near zero, identical to other UHSS. As shown in Figure 50 ( $\mathrm{a}$ and $\mathrm{b}$ ), where black boundaries are not connected and red shows the extent of connected-random boundaries), the MRBC is extremely short for both UHSSS alloys when no pixel gap tolerance is employed in the fractal analysis. That is, the black-line paths are all disrupted by very small gaps, and the fractal dimension of this MRBC is near unity, indicating zero connectivity of the random boundaries. If the pixel gap tolerance is increased to greater than 2 pixels (greater than 190 - $500 \mathrm{~nm}$ of physical distance), then the MRBC encompasses the entire RBN (red boundaries in Figure 50c and d), and the $D_{R}$ increases to 1.89-1.92, indicating full connectivity of the random boundaries within the microstructure for each steel. 

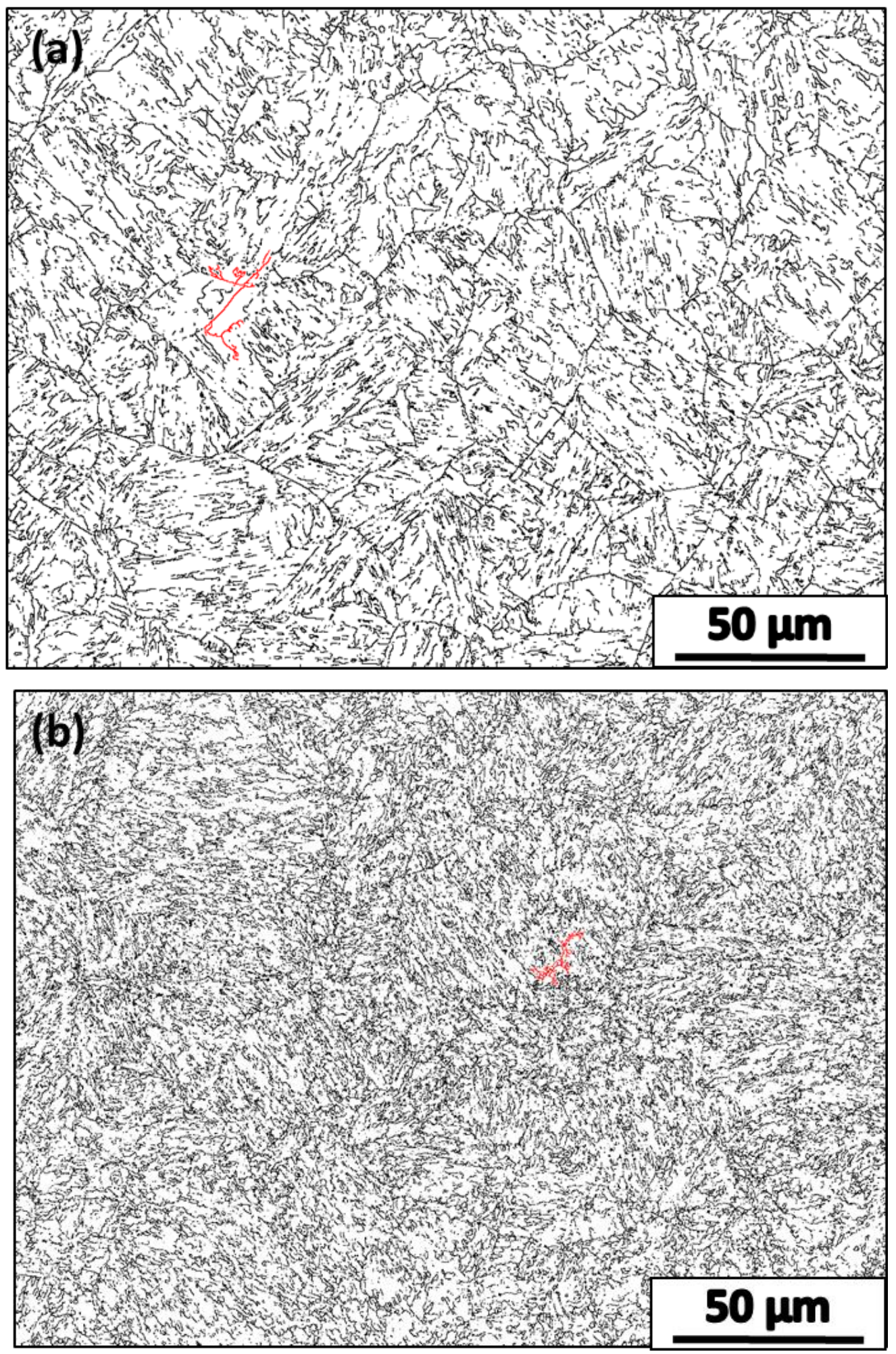

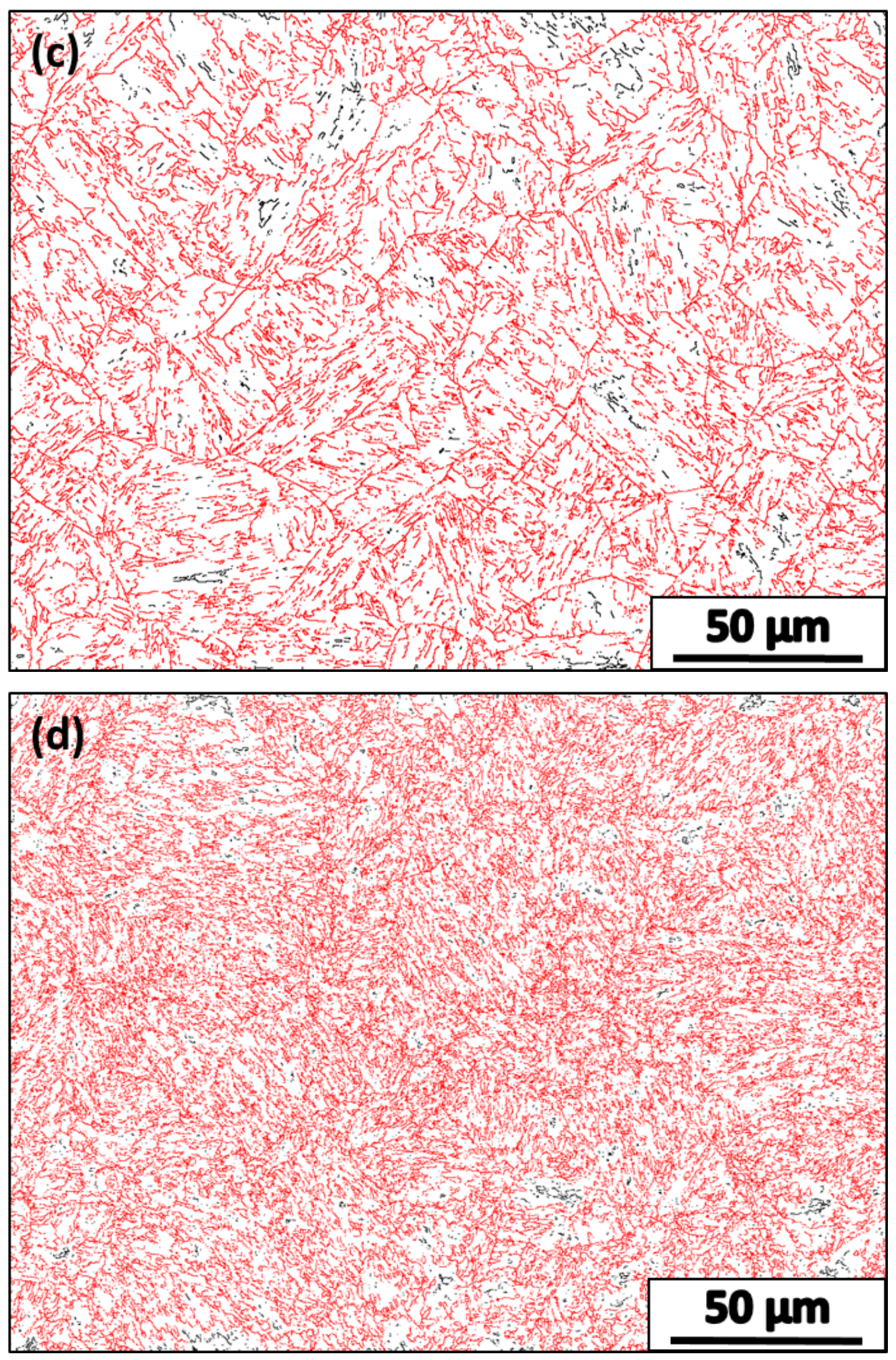

Figure 50 MRBC of Custom 465 (a,c) and Ferrium PH48S (b,d), using a pixel gap tolerance of zero $(a, b)$ and 2 pixels $(c, d)$. Two pixels represent 488 and $380 \mathrm{~nm}$ of physical distance for Custom 465 and Ferrium PH48S, respectively. 
Identical to UHSS in Chapter 3, the source of this bimodal behavior is the interruptions of the RBN provided by short segments of CSL or low angle boundaries. Small segments of CSL ( $\sum 3, \sum 11$ and $\sum 25 \mathrm{~b}$ ) boundaries of 0.2 to $3.0 \mu \mathrm{m}$ length were observed to interrupt otherwise continuous high angle random boundaries. As shown in Figure 51, such boundaries disrupt the connectivity of the RBN, as they constitute discrete gaps in the network. It must be noted that the connectivity analysis of Ferrium PH48S and Custom 465 was conducted over a broad area using the EBSD pixel size shown in Table 10 (160 - $244 \mathrm{~nm}$ ), while high resolution boundary traces were measured using a significantly lower pixel size (Figure 51, 28 and $13 \mathrm{~nm}$ for (a) and (b), respectively). As such a two pixel gap in the low magnification boundary traces (Figure 50) will appear as multiple pixels in high magnification maps, but will represent the same physical distance.

Increasing the pixel gap tolerance allows the $\mathrm{RBN}$ to be considered connected even in the presence of a small CSL gap. The effect of increasing pixel gap tolerance on the fractal dimension of the MRBC is shown in Table 10 for all alloys in the present study. Notably, increased CSL fraction in Ferrium PH48S - H900 did not significantly affect the connectivity of MRBC at any level of pixel gap tolerance. This result clearly indicates that random boundaries in Custom 465 - H900 and Ferrium PH48S - H900 can be considered fully connected, identical to previous observations in UHSS alloys (Chapter 3). 

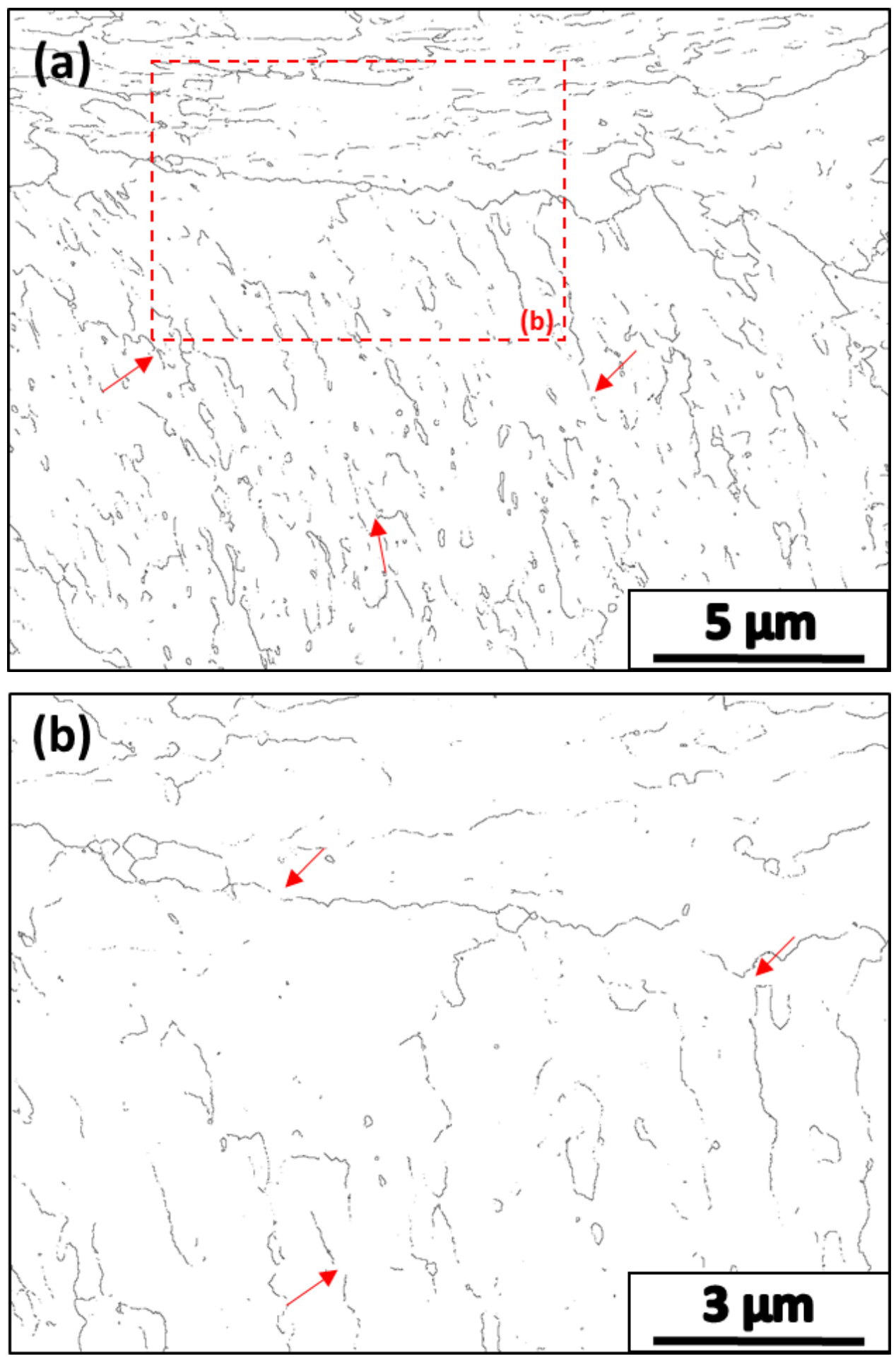

Figure 51 Random boundary network of Ferrium PH48S - H900: (a) High resolution random boundary trace corresponding to the OIM map presented in Figure 42 and (b) Detail of the region indicated by the red dashed line in (a). Representative gaps in the random boundary network are indicated by red arrows and correspond to small segments of CSL or low angle boundaries. 


\begin{tabular}{|c|c|c|c|c|c|}
\hline & \multicolumn{4}{|c|}{ Pixel Gap Tolerance } & \multirow[b]{2}{*}{$\begin{array}{l}\text { Total CSL } \\
\text { Sv Fraction }\end{array}$} \\
\hline DR (Phys. Dist.) & $\mathbf{0}$ & 1 & 2 & 5 & \\
\hline 300M & 1.01 & $1.01(205 \mathrm{~nm})$ & $1.82(410 \mathrm{~nm})$ & $1.85(1025 \mathrm{~nm})$ & 0.36 \\
\hline AM100 & 1.03 & $1.04(220 \mathrm{~nm})$ & $1.87(440 \mathrm{~nm})$ & $1.89(1100 \mathrm{~nm})$ & 0.42 \\
\hline CrNiMoWV & 1.02 & $1.02(180 \mathrm{~nm})$ & $1.91(360 \mathrm{~nm})$ & $1.93(900 \mathrm{~nm})$ & 0.39 \\
\hline Ferrium M54 & 1.01 & $1.04(172 \mathrm{~nm})$ & $1.86(344 \mathrm{~nm})$ & $1.88(860 \mathrm{~nm})$ & 0.39 \\
\hline Ferrium S53 & 1.03 & $1.04(160 \mathrm{~nm})$ & $1.90(320 \mathrm{~nm})$ & $1.95(800 \mathrm{~nm})$ & 0.33 \\
\hline Custom 465 & 1.02 & $1.10(244 \mathrm{~nm})$ & $1.92(488 \mathrm{~nm})$ & $1.94(1220 \mathrm{~nm})$ & 0.54 \\
\hline Ferrium PH48S & 1.01 & $1.08(190 \mathrm{~nm})$ & $1.89(380 \mathrm{~nm})$ & $1.91(950 \mathrm{~nm})$ & 0.20 \\
\hline
\end{tabular}

Table 10 The effect of increasing pixel gap tolerance on calculated $D_{R}$ of all UHSS(S) alloys in the present study. The physical distance for each pixel gap tolerance is indicated for each value of $D_{R}$ in $\mathrm{nm}$.

The present results establish that the connectivity of high angle boundaries is invariant among the seven UHSS(S) in the present study. Each steel contains relatively small CSL segments that are accurately resolved by EBSD and would, if included, result in each steel showing no boundary connectivity. These small CSL segments are justifiably eliminated from the analysis of connectivity. The implications of RBN connectivity to fine scale transgranular HEAC in ultrahigh strength alloys was addressed in the Discussion of Chapter 3. There is no evidence to suggest that the connectivity of martensite interfaces in Custom 465 or Ferrium PH48S is significantly different from other UHSS and is, therefore, unlikely to drive any differences in SCC-HEAC resistance.

\subsubsection{Measurement of $K_{I C}$ and Ductile Fracture Morphology}

To provide a ductile fracture baseline for HEAC properties, the cracking resistance of Custom 465 - H900 and three aging conditions (H860, H900, H968) of Ferrium PH48S was assessed in moist air rather than full aqueous-chloride immersion. The small SENT specimens were stressed under slow-rising displacement identical to that applied during HEAC testing. A J-integral analysis approximately following ASTM E-1820 and prior work on Monel K-500 was conducted on crack 
growth data obtained from open air tests using Ramberg-Osgood flow hardening constants (Table 2) to determine the total elastic-plastic stress intensity $\left(K_{J}\right)[3,171]$. For all $K$, the plastic component of $\mathrm{K}_{\mathrm{J}}$ was sufficiently small to be excluded from the analysis without significantly altering the value of measured fracture toughness. As such, $\mathrm{K}_{\text {elastic }}$ is considered identical to $\mathrm{K}_{\mathrm{J}}$, and $\mathrm{K}_{\mathrm{IC}}$ is considered identical to $\mathrm{K}_{\mathrm{JIC}}$, for the crack growth resistance data presented in this section. For each alloy, the stress intensity for the onset of physical crack growth $\left(\mathrm{K}_{\mathrm{JICi}}\right)$ was determined using the first sustained deviation of da/dt above the plasticity based resolution limit (Figure 4). All dcPD-measured false crack extension below $\mathrm{K}_{\mathrm{JICi}}$ were neglected and replaced by the expected amount of crack tip blunting according to [104]:

$$
\Delta a_{\text {blunt }}=\frac{2}{\pi} \frac{K^{2}}{\sigma_{Y S} E}
$$

This procedure eliminates any small plasticity-based dcPD artifacts and replaces false $\Delta$ a prior to $\mathrm{K}_{\mathrm{JICi}}$ with a well-known analytical expression for the amount of geometric crack extension by blunting at a known $\mathrm{K}$. The constructed crack growth resistance curves (R-curves) are shown in Figure 52 for Ferrium PH48S (H860, H900, H968) and Custom 465 (H900).

Previous work has established that the dcPD method effectively characterizes the onset of ductile microvoid cracking in aluminum alloys $\left(\mathrm{K}_{\mathrm{JICi}}\right)$ [147]. Such cracking initiates at the midline of a fracture mechanics specimen in the region of high plane strain constraint. The elastic-plastic stress intensity at which dcPD cracking initiates $\left(\mathrm{K}_{\mathrm{JIC}}\right)$ is shown to be a conservative estimate of plane strain fracture toughness $\left(\mathrm{K}_{\mathrm{IC}}\right)$ when compared to $\mathrm{K}_{\mathrm{JIC}}$, determined according to ASTM E813 which uses the intersection of the K- $\Delta$ a curve with a $0.2 \mathrm{~mm}$ offset crack tip blunting line [147]. A similar methodology for determining $\mathrm{K}_{\mathrm{JICi}}$ is applied to the specimens represented in Figure 52: the stress intensity corresponding to the first dcPD crack growth above the resolution limit is shown by the intersection of each R-curve with the blunting line. Based on the work of Haynes et al. this value represents a conservative estimate of $\mathrm{K}_{\mathrm{IC}}$ for each alloy [147]. However, $\mathrm{K}_{\mathrm{JIC}}$ determined from the intersection of the R-curve with a $0.2 \mathrm{~mm}$ offset blunting line, approximately following ASTM 1820, provides a non-conservative upper bound of $\mathrm{K}_{\mathrm{JIC}}$ that would be expected from a relatively thin specimen. Measured values of $\mathrm{K}_{\mathrm{JIC}}$ and $\mathrm{K}_{\mathrm{JIC}}$ (offset) are shown in Table 11. 


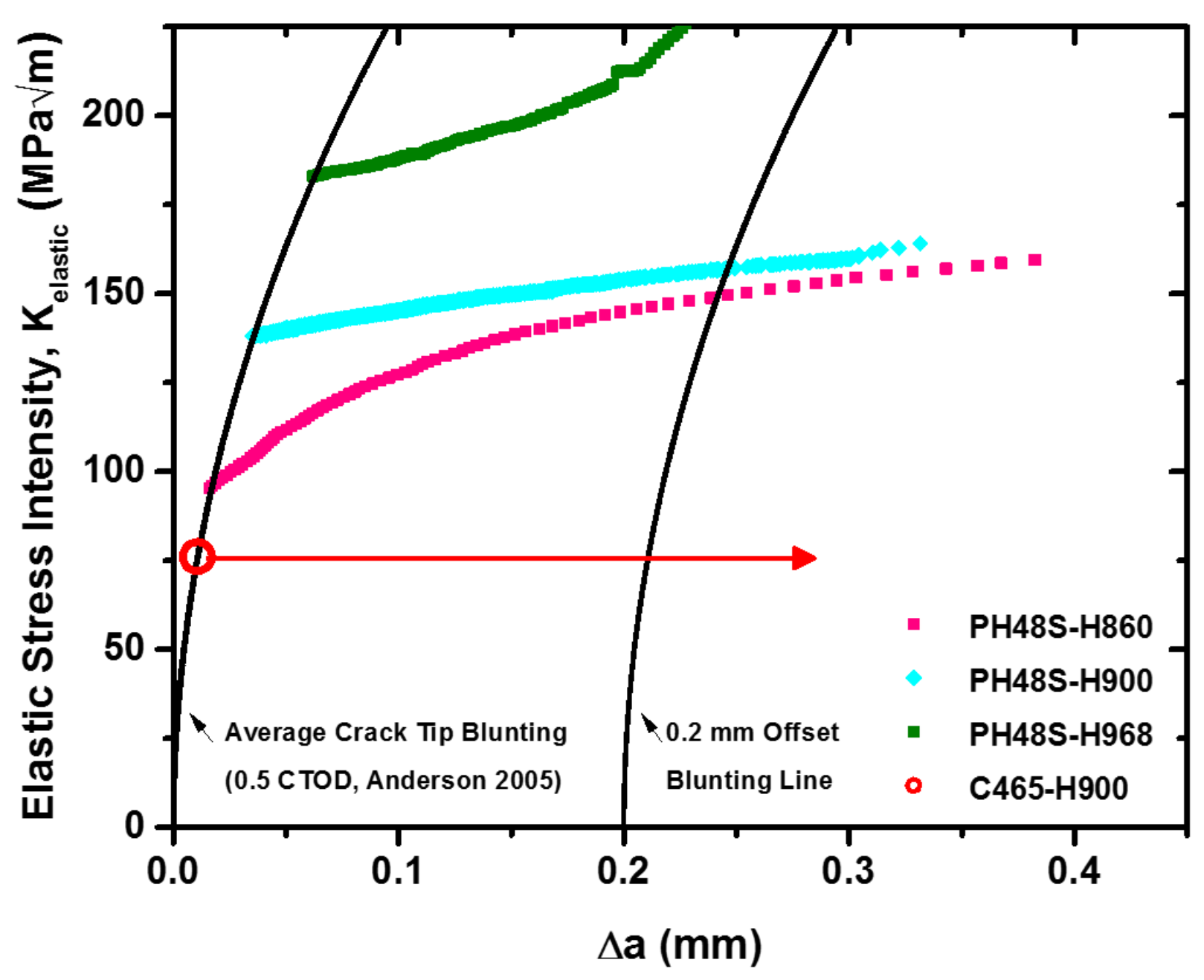

Figure 52 Crack growth resistance curves of Ferrium PH48S and Custom 465 constructed using a dcPD-measured crack growth after $K_{J I C i}$. Initial crack extension is determined by $1 / 2$ of the crack tip opening displacement (CTOD), shown as the intersection of the R-Curve with the blunting line from [104]. 


\begin{tabular}{|l|c|c|}
\hline & K $_{\text {JICi }}(\mathbf{d c P D})$ & $\mathbf{K}_{\text {JIC }}$ (Offset) \\
\hline Ferrium PH48S - H860 & $95 \mathrm{MPa} \sqrt{\mathrm{m}}$ & $149 \mathrm{MPa} \sqrt{\mathrm{m}}$ \\
\hline Ferrium PH48S - H900 & $139 \mathrm{MPa} \sqrt{\mathrm{m}}$ & $158 \mathrm{MPa} \sqrt{\mathrm{m}}$ \\
\hline Ferrium PH48S - H968 & $182 \mathrm{MPa} \sqrt{\mathrm{m}}$ & $212 \mathrm{MPa} \sqrt{\mathrm{m}}^{*}$ \\
\hline Custom 465 - H900 & $79 \mathrm{MPa} \sqrt{\mathrm{m}}$ & $79 \mathrm{MPa} \sqrt{\mathrm{m}}$ \\
\hline
\end{tabular}

Table 11 Measured values of $K_{\text {IICi }}$ and $0.2 \mathrm{~mm}$ offset $K_{\text {JIC }}$ for the $R$-curve data of selected alloys presented in Figure 52. (*) The value of $K_{\text {IIC }}$ for Ferrium PH48S - H968 was extrapolated from a linear regression of the first $0.1 \mathrm{~mm}$ of crack extension after $K_{\text {JICi. }}$

As shown in Figure 52, Ferrium PH48S - H860 (magenta) and H900 (cyan) exhibit a rising Rcurve; classically representative of ductile fracture in metals [104]. Two significant deviations from this behavior are noted for Ferrium PH48S - H968 (green) and Custom 465 - H900 (red). First, no stable crack extension was observed in Custom 465 stressed under slow rising displacement in lab air: physical crack extension initiated at $79 \mathrm{MPa} \sqrt{ } \mathrm{m}$ and propagated through the specimen in the time between dcPD data points $(1 \mathrm{~min})$. The result is a flat $\mathrm{R}$-curve indicating unstable-ductile fracture. Second, the R-curve of Ferrium PH48S - H968 rises at an increasing rate with rising $\Delta \mathrm{a}$, indicating a significant toughening of the microstructure with crack extension. The origins of this behavior are unknown and may include a contribution from plane stress relaxation of crack tip stresses. The data in Figure 52 indicate microstructure-scale discontinuous cracking in the early stage of ductile fracture evolution, or an experimental error as the only logical sources of the observed deviation from classic rising R-curve (Figure 52, PH48S - H860, H900).

Three key pieces of evidence support the accuracy of dcPD based KIICi in UHSSS: (1) the measured $\mathrm{K}_{\text {IICi }}$ of Custom $465-\mathrm{H} 900(79 \mathrm{MPa} \sqrt{\mathrm{m}})$ is identical to reported values of $\mathrm{K}_{\mathrm{IC}}(78-89 \mathrm{MPa} \sqrt{\mathrm{m}})$ [41], (2) Ferrium PH48S $-\mathrm{H} 900$ exhibits a $\mathrm{K}_{\mathrm{JICi}}=139 \mathrm{MPa} \sqrt{\mathrm{m}}$, closely matching the reported fracture toughness of similar-modern UHSS AerMet 100 (130 MPa $\sqrt{\mathrm{m}}$, Table 1), and (3) $\mathrm{K}_{\mathrm{JICi}}$ rationally decreases as aging temperature is decreased and tensile strength rises. However, measured $\mathrm{K}_{\mathrm{IIC}}$ is elevated in Ferrium PH48S - H968 (182 MPa $\left.\sqrt{\mathrm{m}}\right)$ relative to the literature 
reported value of $\mathrm{K}_{\mathrm{IC}}$ for the $\mathrm{H} 968$ condition (100 MPa $\sqrt{\mathrm{m}}$ ) [46]. The methodology for determining reported this $\mathrm{K}_{\mathrm{IC}}$ of conventionally tempered Ferrium PH48S has not been published beyond the specimen thickness $(25.4 \mathrm{~mm})$. Due to the experimental nature of Ferrium PH48S, some variation in the composition and thermal processing of the alloy is expected. It is possible that this is the source of increased toughness in the present lot of PH48S material compared to reported values.

The fracture morphologies of Custom 465 - H900 and Ferrium PH48 - H900 stressed in moist air are shown in Figure 53. The fracture mode in both alloys is exclusively microvoid failure, indicating ductile fracture. Microvoids are observed in a wide range of sizes, often containing the nucleating particle at the bottom of the void. High resolution images of the microvoid nucleating particles are shown in Figure 54 (Ferrium PH48S) and Figure 55 (Custom 465). Particle composition was analyzed using energy dispersive $\mathrm{x}$-ray spectroscopy, operating at $15 \mathrm{kV}$. Due to the size of the EDS interaction volume, it is not possible to quantitatively assess the composition of the observed particles. However, the basic elemental makeup can be qualitatively probed.

Two primary particle morphologies are observed in each alloy: a spherical impurity-rich particle and a cuboidal, titanium-rich precipitate. In Ferrium-PH48S, large (Figure 54a, 1-2 $\mu \mathrm{m}$ ) spherical particles with high concentrations of lanthanum, oxygen and sulfur are found exclusively in large voids $\sim 20 \mu \mathrm{m}$ in diameter. Smaller cuboidal precipitates (Figure 54a, 0.5-1.5 $\mu \mathrm{m}$ ) are rich in titanium and carbon, and found within $\sim 10 \mu \mathrm{m}$ diameter microvoids. In contrast spherical impurity (P,S, and As) rich particles in Custom 465 (Figure 55a) are small (200-400 nm), devoid of resolvable rare earth elements, and found at the bottom of small voids $(<5 \mu \mathrm{m})$. Extremely large Ti-C rich precipitates (Figure 55b), 5-15 $\mu \mathrm{m}$ in diameter, are found in moderately sized microvoids and are often cracked into several pieces. These large Ti-C rich cracked particles were not observed for Ferrium PH48S. No precipitates were found at the bottom of fine scale voids, though it is likely that they represent classic void sheeting of the smaller strengthening precipitate phase between large voids [172]. 

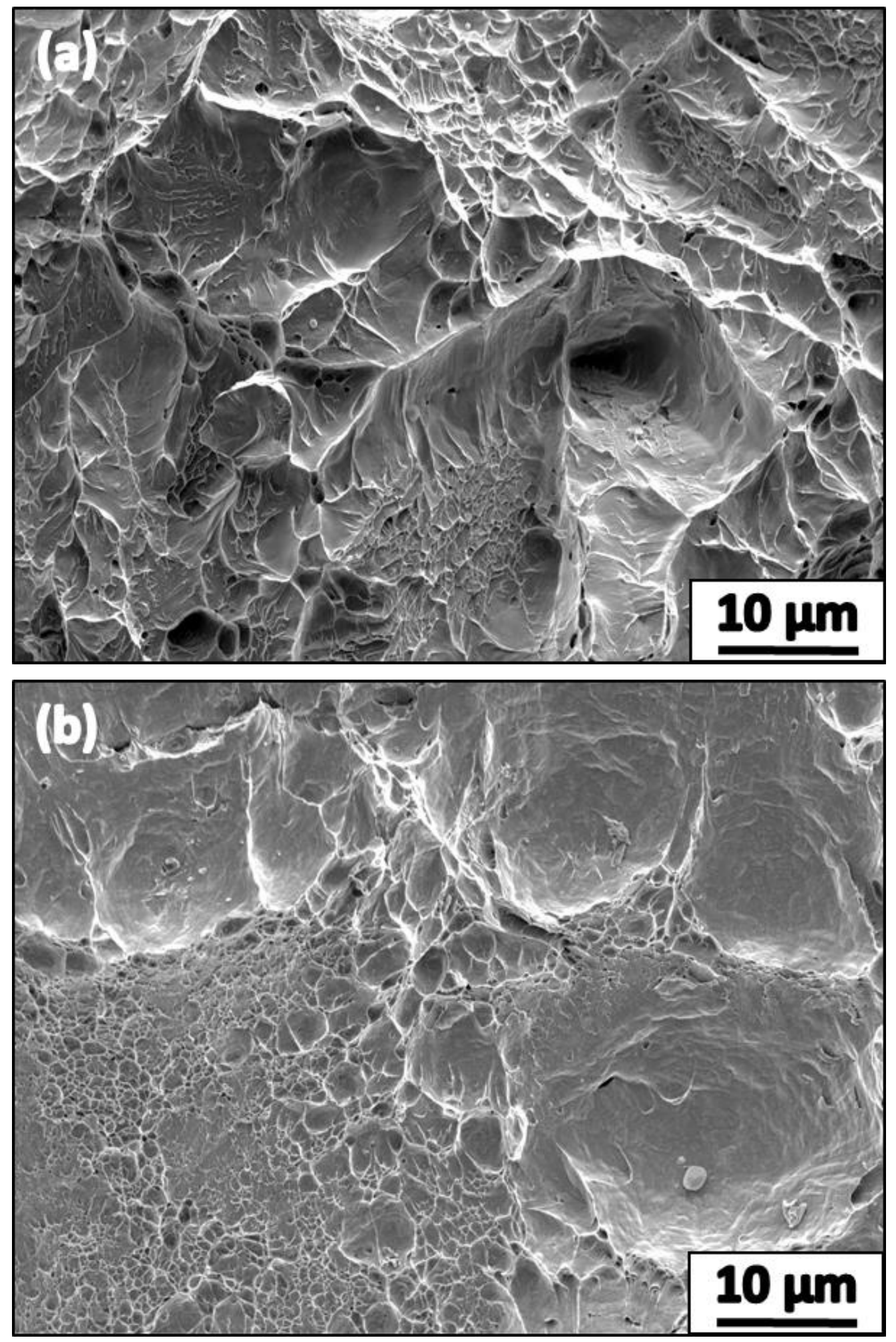

Figure 53 Fracture morphology of (a) Custom 465 - H900 and (b) Ferrium PH48S - H900 tested under slow rising displacement in moist air. 

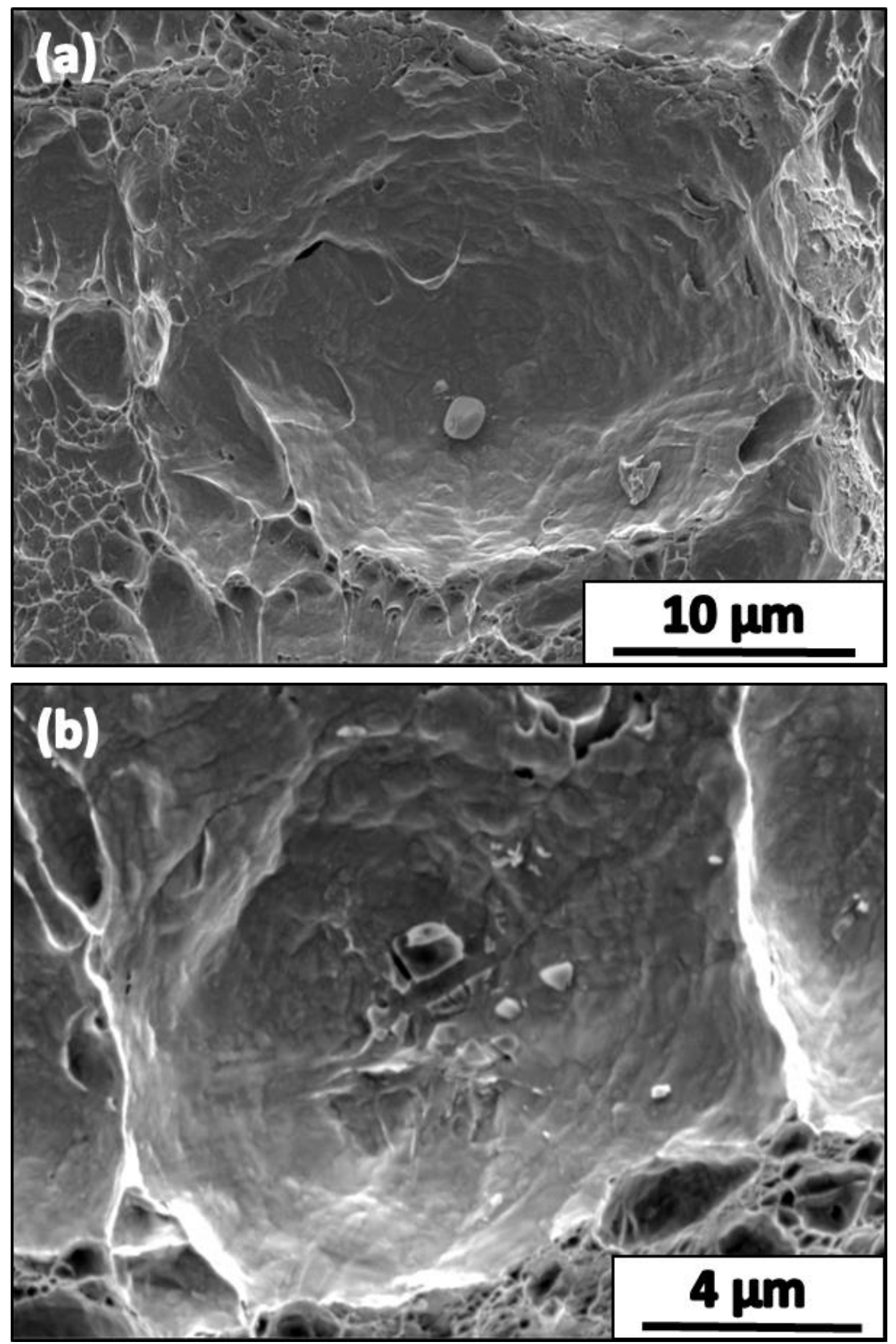

Figure $54 \quad$ Typical morphology of microvoid nucleants in Ferrium PH48S - H900: (a) spheroidal rare earth oxy-sulfides and (2) cuboidal Ti-rich precipitates. 

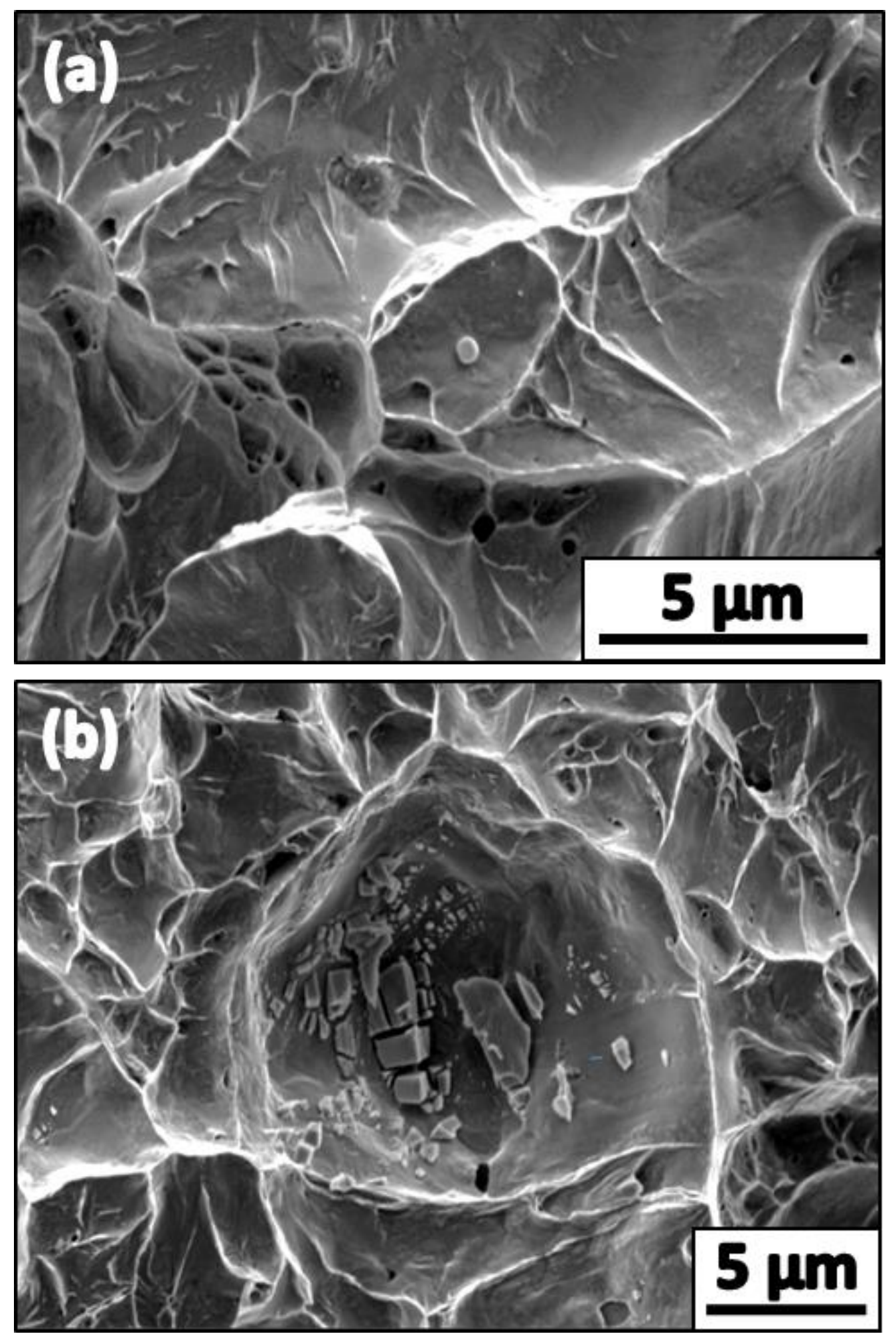

Figure 55 Typical morphology of microvoid nucleants in Custom 465 - H900: (a) spheroidal impurity rich particles (2) cuboidal Ti-rich precipitates. 


\subsubsection{HEAC Crack Growth Kinetics and Fracture Path at $E_{a p p}=-975 m V_{S C E}$}

Custom 465 - H900 and Ferrium PH48S (H860, H900, H950, and H968) are each susceptible to severe HEAC when stressed in neutral $0.6 \mathrm{M} \mathrm{NaCl}$ at fixed $\mathrm{E}_{\text {app }}$ of $-975 \mathrm{mV}_{\text {SCE. }}$ Subcritical crack growth kinetics for each alloy and aging condition are presented in Figure 56. For all conditions, $\mathrm{K}_{\mathrm{TH}}$ is similarly low (8-15 MPa $\sqrt{\mathrm{m}}$ ), and near identical to the range of $\mathrm{K}_{\mathrm{TH}}$ observed in UHSS tested at $E_{\text {app }}=-1,000 \mathrm{mVsce}$ (Figure 18, 7-12 MPa $\sqrt{\mathrm{m}}$ ). Threshold stress intensity is clearly discernable by da/dt rapidly rising two orders of magnitude above the plasticity based resolution limit. Stage II crack growth rates taken at $\mathrm{K}=40 \mathrm{MPa} \sqrt{\mathrm{m}}$ are 1-2 orders of magnitude lower for each aging condition of Ferrium PH48S $(10-200 \mathrm{~nm} / \mathrm{s})$ versus Custom $465-\mathrm{H} 900(2,500 \mathrm{~nm} / \mathrm{s})$. The subcritical crack growth experiment for PH48S $-\mathrm{H} 860$ at $\mathrm{E}_{\text {app }}=-975 \mathrm{mV} \mathrm{V}_{\mathrm{SCE}}$ was terminated at $\mathrm{K}=24 \mathrm{MPa} \sqrt{\mathrm{m}}$ due to a power failure. Stage II da/dt for this specimen is extrapolated to be 200 $\mathrm{nm} / \mathrm{s}$ based upon the crack growth kinetics in PH48S - H900. Notably, as compressive yield strength is decreased from Custom $465-\mathrm{H} 900(1,827 \mathrm{MPa})$ through the aging conditions of Ferrium PH48S (1806 to $1640 \mathrm{MPa}$, from H860 to H968), da/dt similarly decreased from 2,500 $\mathrm{nm} / \mathrm{s}$ to $18 \mathrm{~nm} / \mathrm{s}$. However, the sharp drop in da/dt, from Custom 465 - H900 to PH48S - H860 where yield strength is near equal, suggests that strength alone is not the root cause of reduced da/dt shown in Figure 56. The effect of decreasing strength on HEAC in UHSS alloys will be discussed in an ensuing section.

SEM fractography of the specimens represented in Figure 56 reveals a fundamental change in the microscopic path of HEAC for Custom 465 versus Ferrium PH48S. The fracture morphologies of Custom 465 - H900, Ferrium PH48S - H860, Ferrium PH48S - H900, and Ferrium PH48S H968 are shown in Figures 57 and 58, respectively. Each is clearly transformed from the microvoid cracking typical of ductile fracture in moist air (Figure 53). Intergranular cracking dominates the fracture surface of Custom 465 - H900 (Figure 58a). Close agreement of the intergranular feature size to prior austenite boundary spacing revealed in EBSD measurements (Figure 41, white outline) establishes that IG fracture in Custom 465 - H900 occurs along prior austenite grain boundaries. Intergranular facets are exceptionally flat, featureless and nearly void of any smallscale ductile processes that were observed in IG fracture of 300M (Figure 22b, black arrows) or CrNiMoWV (Figure 23c, white arrows). Identical to IG HEAC in 300M, isolated regions of transgranular HEAC are present on the fracture surface of Custom 465 - H900 (Figure 57a, white 
arrow). IG fracture in all three alloys occurred at similar-low $\mathrm{K}_{\mathrm{TH}}(8,9$, and $13 \mathrm{MPa} \sqrt{\mathrm{m}}$ for $300 \mathrm{M}$, Custom 465, and CrNiMoWV; respectively), at near identical $\mathrm{E}_{\mathrm{app}}$ (-975 to -1,000 mV $\mathrm{mCE}$ ).

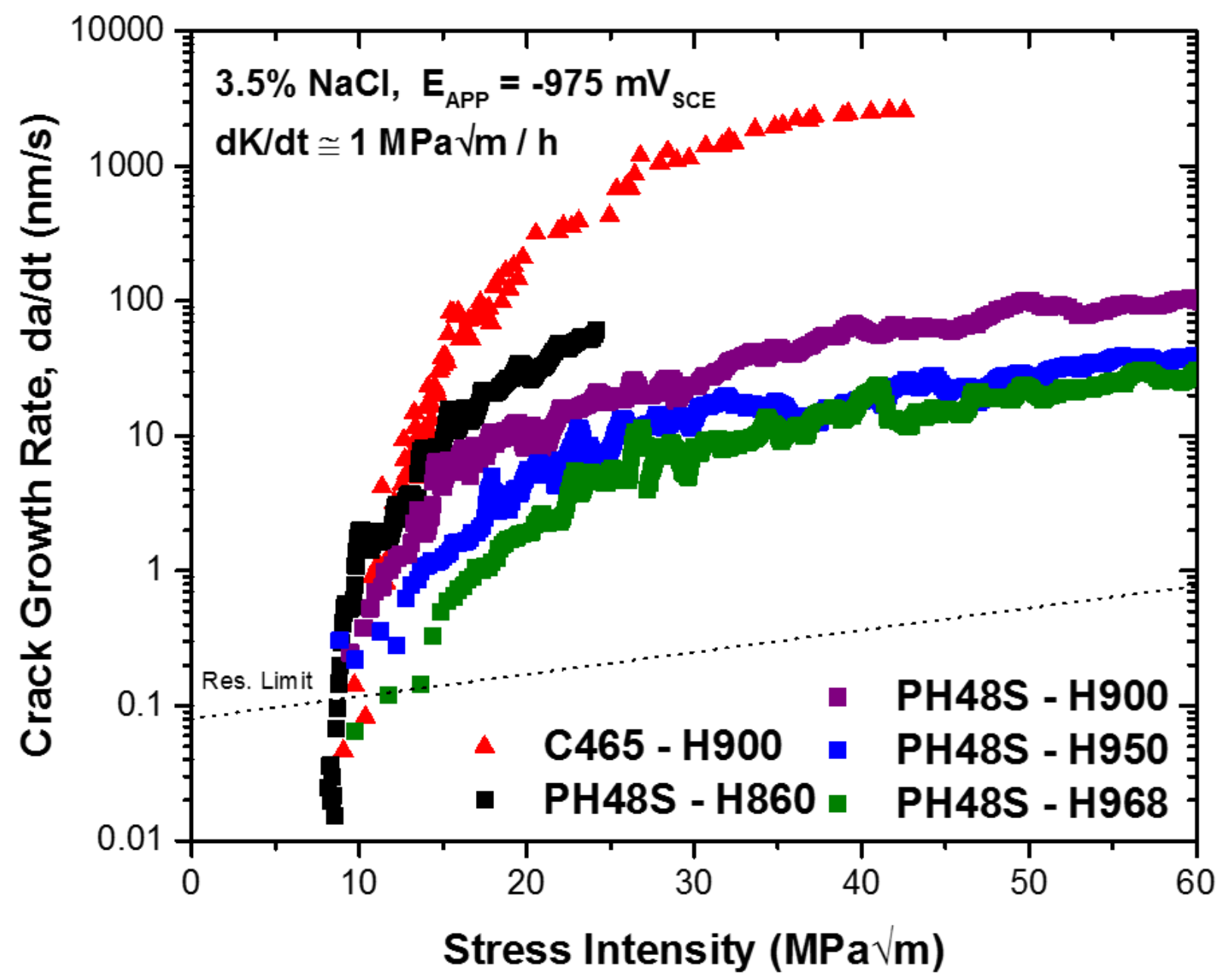

Figure 56 HEAC Kinetics for UHSSS stressed under slow-rising $\mathrm{K}$ in $3.5 \% \mathrm{NaCl}$ at $\mathrm{E}_{\text {app }}=$ -975 $m V_{\text {SCE. }}$ 

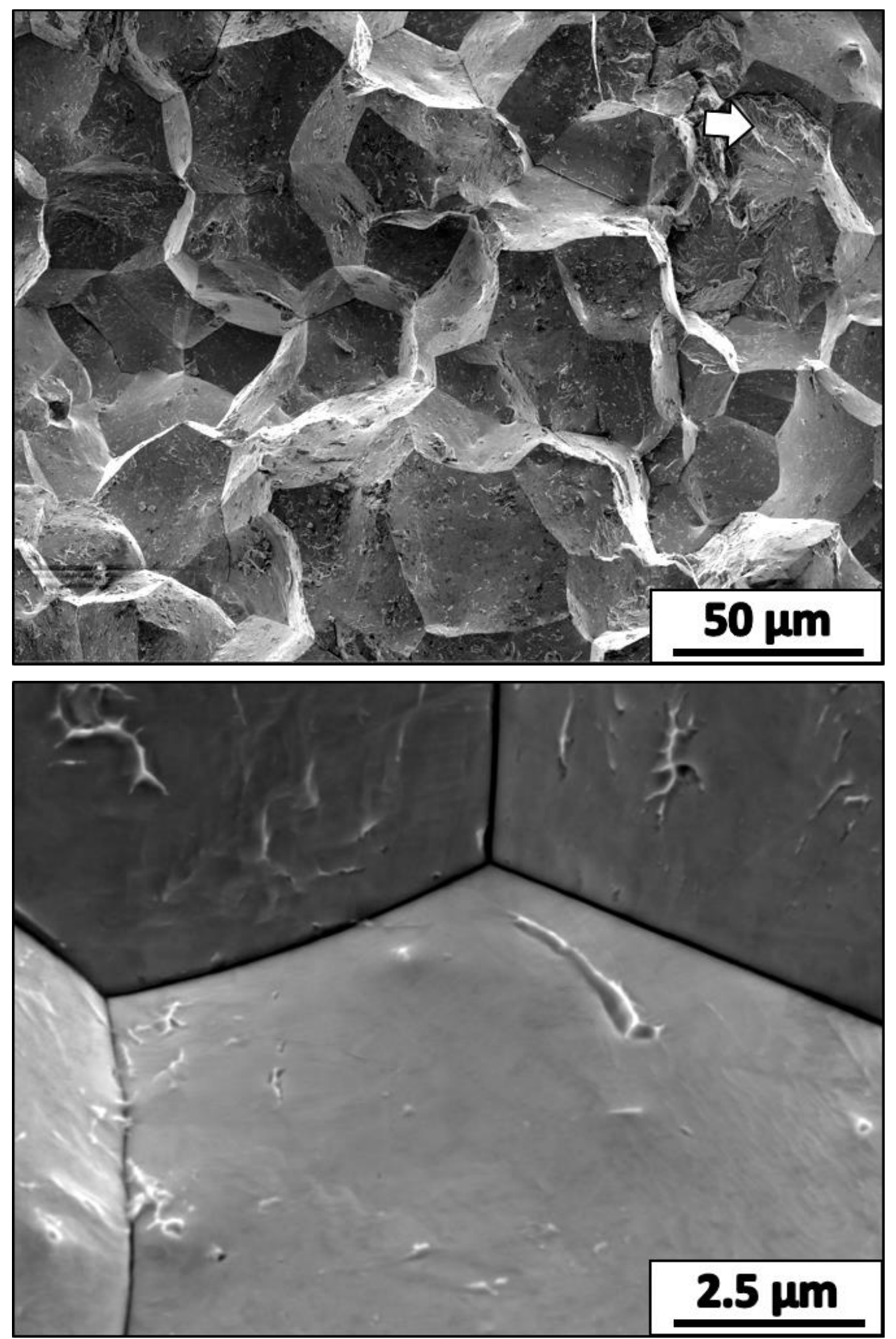

Figure 57 HEAC fracture morphology of Custom $465-H 900(K \approx 10 \mathrm{MPa} \sqrt{\mathrm{m}})$ stressed in $3.5 \% \mathrm{NaCl}$ at $E_{a p p}=-975 \mathrm{~m} V_{S C E}$. The white arrow indicates a region of transgranular cracking among predominantly $I G$ fracture. 
In sharp contrast to Custom 465 - H900, Ferrium PH48S exhibits exclusively transgranular HEAC when stressed at $E_{a p p}=-975 \mathrm{mV}_{\mathrm{SCE}}$. The transgranular fracture morphology for the H860, H900, and H968 aging conditions (Figure 58, Figure 59, and Figure 60, respectively) is identical and composed of broad quasi-cleavage (QC, white arrows), small scale regions of flat fracture (black arrows), and minor instances of secondary cracking (white arrow heads). As discussed in Chapter 3 , transgranular QC along atypical $\{110\}_{\alpha^{\prime}}$ cleavage planes (compared to $\{100\}_{\alpha^{\prime}}$ for low temperature cleavage) is the dominant HEAC fracture morphology for high strength martensitic steels $[116,120]$. Close agreement of $\{110\}_{\alpha^{\prime}}$ planes to $\{110\}_{\alpha^{\prime}}$ lath boundary planes indicates that QC fracture in lath martensite is correlated to cracking along lath/block interfaces [120]. The fractional distribution of quasi-cleavage and flat fracture varies slightly between adjacent regions on a single specimen, but the average distribution of these HEAC fracture morphologies is constant between aging conditions.

As discussed in Chapter 3, QC features in UHSS (Figures 19-18 and 20) bear a striking resemblance to literature observations of $\mathrm{QC}$ in lath martensite driven by $\mathrm{H}$-assisted interface decohesion [11,12,116,120]. Similarity of QC in UHSS (Figures 19-18, 20) supports the hypothesis that a similar damage mechanism is active in multiple alloys. Further, congruency of QC facet morphology with observations by Nagao et al. indicates the generic HELP/HEDE mechanism likely controls HEAC of UHSS alloys [11,12]. The HEAC fracture morphology of Ferrium PH48S closely matches HEAC QC fracture facets in UHSS alloys (Chapter 3) and provides additional experimental support of this hypothesis. For example, HEAC fracture of AerMet 100 (Figure 61a) displays an identical QC morphology with slightly increased spacing of fine lath-like features compared to Ferrium PH48S - H900 (Figure 61b). Increased density of lathlike features and ridges is expected if HEAC fracture primarily proceeds along $\{110\}_{\alpha}{ }^{\prime}$ martensite block interfaces as was observed in UHSS alloys. Average spacing of block interfaces is increased from $0.51 \mu \mathrm{m}$ in Ferrium PH48S to $0.81 \mu \mathrm{m}$ in AerMet 100, supporting the hypothesis of a generic, microstructure sensitive mechanism. The effect of subtle changes in the martensite block structure on QC HEAC morphology of Ferrium PH48S - H900 and AerMet 100 will be discussed in an ensuing section. 

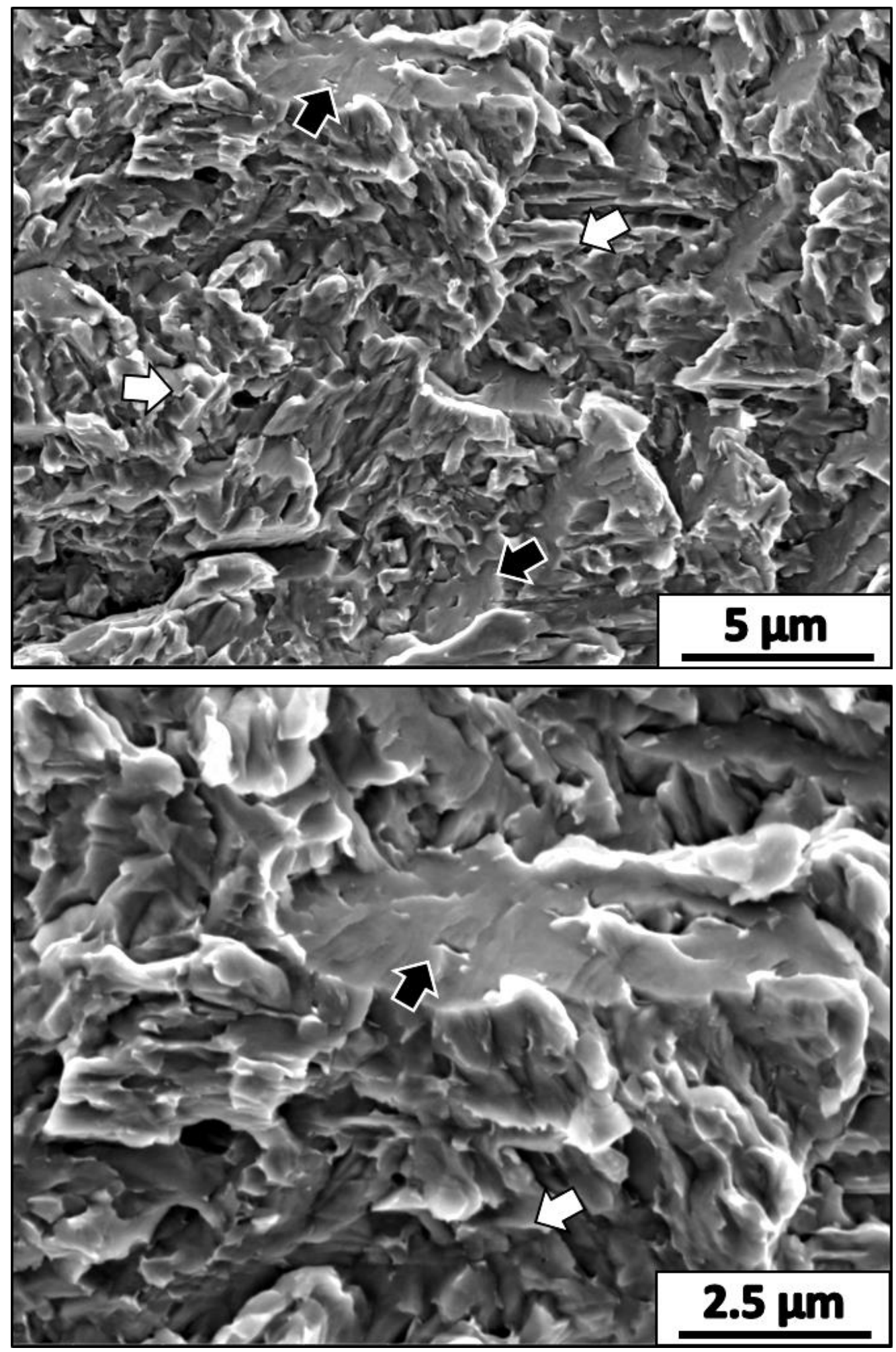

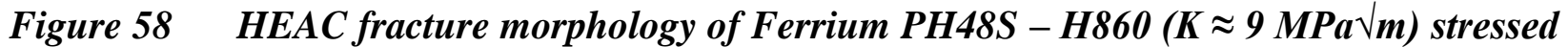
in 3.5\% $\mathrm{NaCl}$ at $\mathrm{E}_{a p p}=-975 \mathrm{mVSCE}$. White and black arrows indicate regions of $Q \mathrm{C}$ and flat fracture, respectively. 


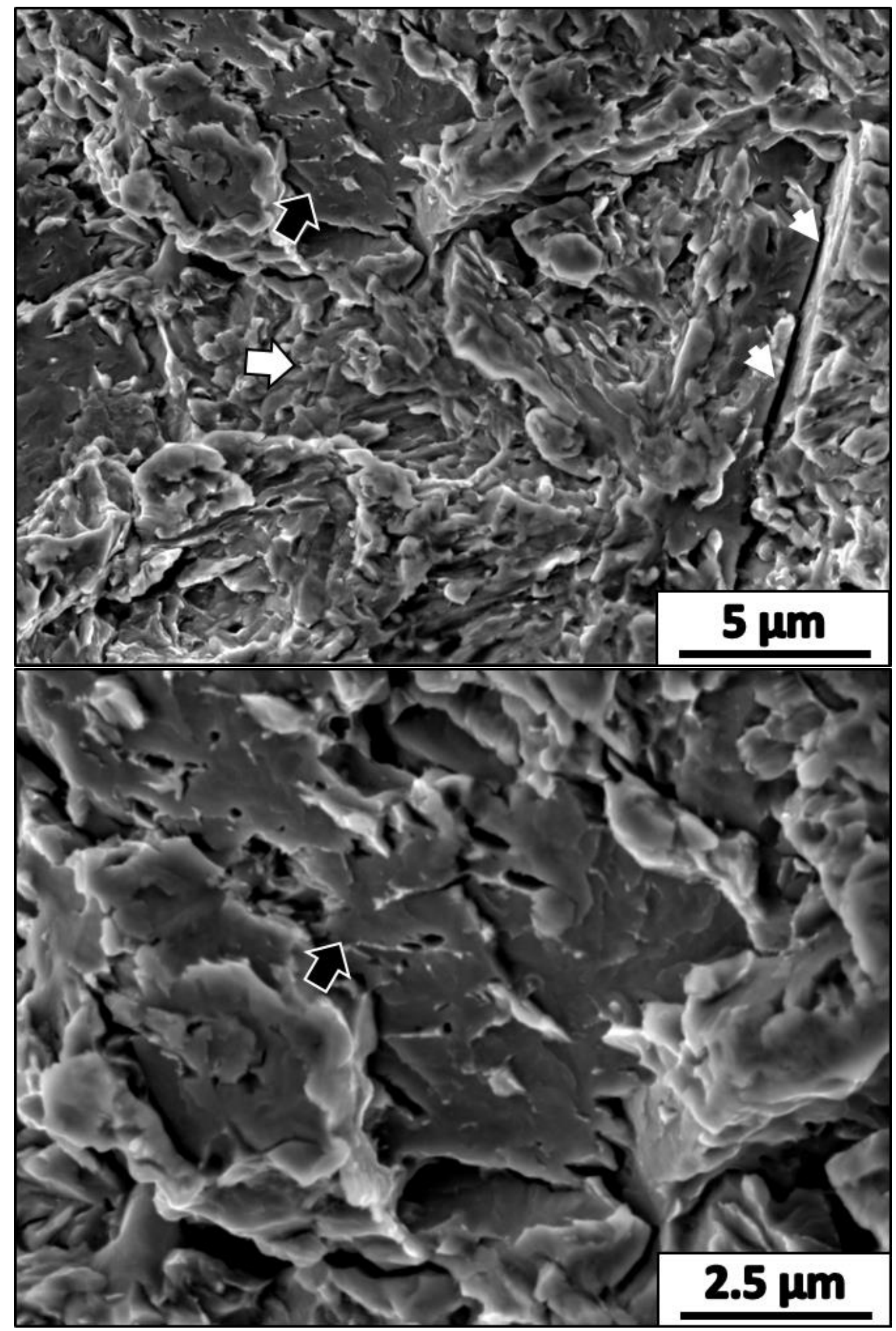

Figure 59 HEAC fracture morphology of Ferrium PH48S - 900 (K $\approx 9 \mathrm{MPa} \sqrt{\mathrm{m}})$ stressed in $3.5 \% \mathrm{NaCl}$ at $E_{a p p}=-975 \mathrm{mV}$ SCE. White and black arrows indicate regions of $Q C$ and flat fracture, respectively. White arrowheads denote minor secondary cracking, possibly at prior austenite grain boundaries. 

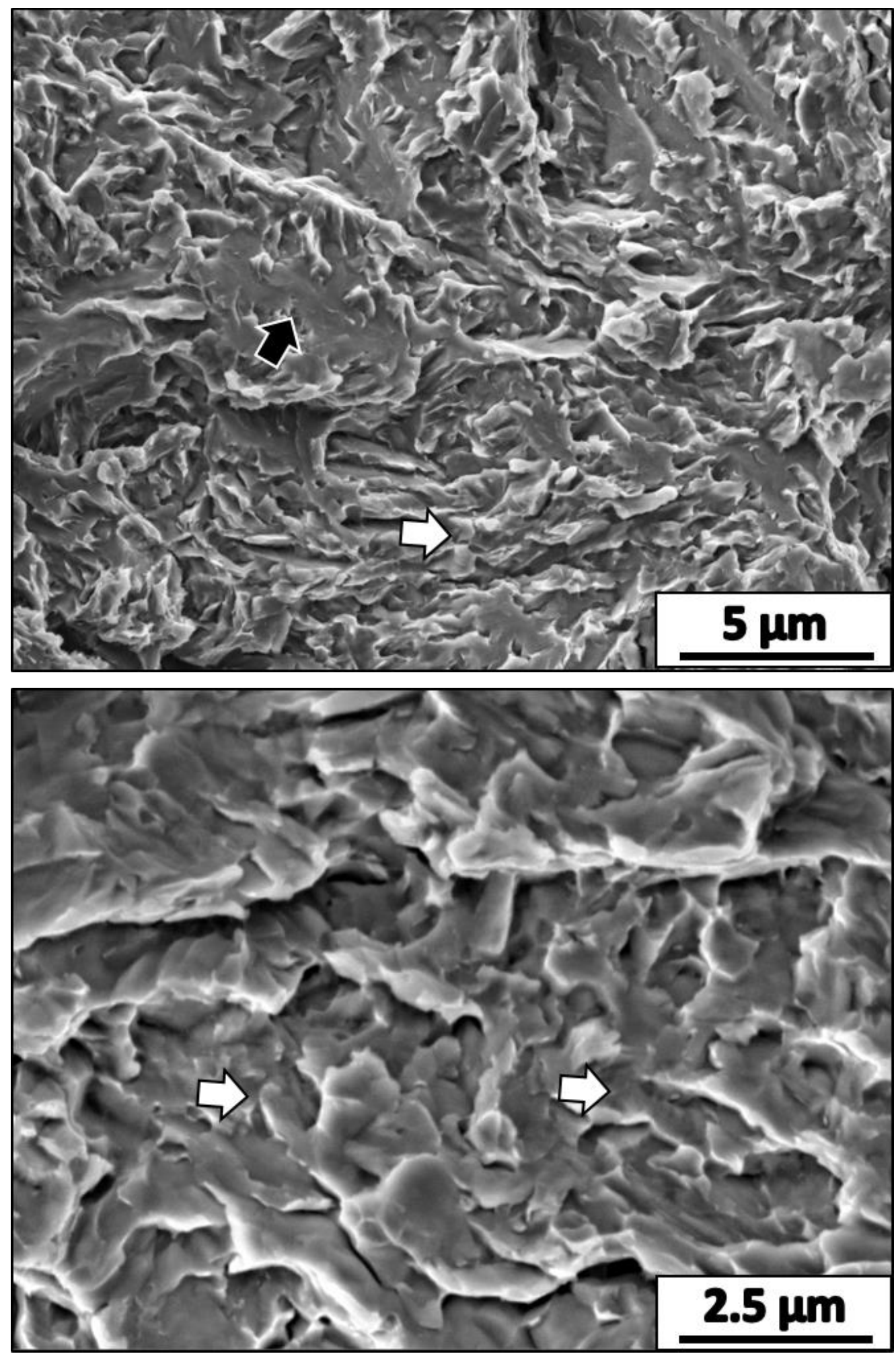

Figure 60 HEAC fracture morphology of Ferrium PH48S - 968 (K $\approx 9 \mathrm{MPa} \sqrt{\mathrm{m}})$ stressed in $3.5 \% \mathrm{NaCl}$ at $E_{\text {app }}=-975 \mathrm{mV}$ SCE. White and black arrows indicate regions of $Q \mathrm{C}$ and flat fracture, respectively. 

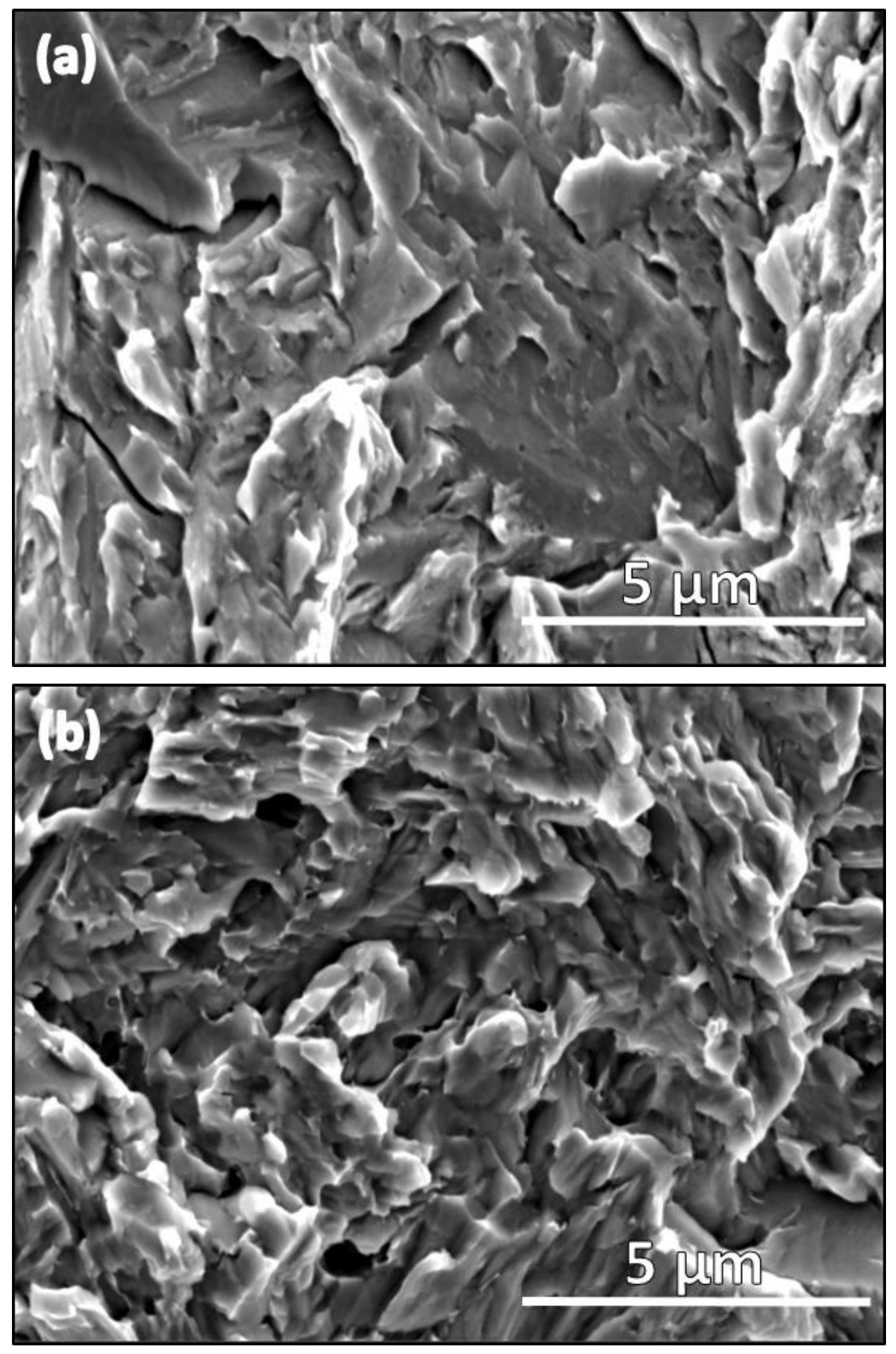

Figure 61 Comparison of QC fracture in: (a) AerMet 100 at $E_{a p p}=-1,000 \mathrm{~m} V_{S C E}$ and (b) Ferrium PH48S H900 at $E_{a p p}=-975 m V_{S C E}$. 


\subsubsection{Effect of $E_{a p p}$ on HEAC kinetics and fracture morphology in Ferrium PH48S - H900}

Ferrium PH48S - H900 is susceptible to HEAC when stressed under slow rising displacement in $3.5 \% \mathrm{NaCl}$ across a wide range of $\mathrm{E}_{\text {app. }}$ Subcritical crack growth kinetics of PH48S - H900 specimens loaded at fixed $E_{\text {app }}$, ranging from slightly cathodic to OCP (-250 mV $\left.\mathrm{VCE}_{\mathrm{SC}}\right)$ to heavy cathodic polarization (-1,200 mV $\mathrm{SCE})$, are shown in Figure 62. Three specimens were tested at $\mathrm{E}_{\text {app }}$ $=-150 \mathrm{mV}_{\mathrm{SCE}}$, but each test was terminated prematurely due to corrosion of the dcPD wire specimen interface. As such, no HEAC kinetics are presented for $\mathrm{E}_{\text {app }}$ anodic to OCP. However, the replicate experiments suggest that $\mathrm{K}_{\mathrm{TH}}$ for Ferrium PH48S - H900 is $\sim 25 \mathrm{MPa} \sqrt{\mathrm{m}}$ when tested at $E_{\text {app }}=-150 \mathrm{mV}_{\mathrm{SCE}}$. This result indicates that $\mathrm{K}_{\mathrm{TH}}$ for HEAC of Ferrium PH48S $-\mathrm{H} 900$ rapidly declines as $\mathrm{E}_{\text {app }}$ is increased anodic to OCP, identical to Custom 465 - H900.

Five key results are notable from this data set. First, $\mathrm{K}_{\mathrm{TH}}$ for each set of crack growth kinetics is readily measured based on the rapid increase in da/dt. In several cases $\left(E_{\text {app }}=-640,-710\right.$, and -775 $\left.\mathrm{mV}_{\mathrm{SCE}}\right) \mathrm{da} / \mathrm{dt}$ increase is near discontinuous, rapidly rising several orders of magnitude with a small increase in K. Second, the plasticity based resolution limit accurately captures the general trend of false da/dt rise, but does not encompass the broad range of slow-rising da/dt observed prior to $\mathrm{K}_{\mathrm{TH}}$. For example, the plasticity based resolution limit accurately predicts the false $\mathrm{da} / \mathrm{dt}$ rise in PH48S - H900 at $E_{\text {app }}=-575 \mathrm{mV}_{\mathrm{SCE}}$ up to $97 \mathrm{MPa} \sqrt{\mathrm{m}}$, indicating the efficacy of the method. However, in specimens tested at $\mathrm{E}_{\mathrm{app}}=-640$ or $-710 \mathrm{mV} \mathrm{SCE}_{\mathrm{SC}}$, the general slope of $\mathrm{da} / \mathrm{dt}$ rise is identical, but shifted significantly upwards. Consistency in the slope of da/dt rise prior to a welldefined $\mathrm{K}_{\mathrm{TH}}$ supports shifting the resolution limit upwards for $\mathrm{K}_{\mathrm{TH}}$ determination in these cases. Third, $\mathrm{K}_{\mathrm{TH}}$ is very high for the two-least cathodic potentials examined, near to OCP, and this threshold declines with increasing cathodic polarization from near OCP. Fourth, Stage II crack growth rate measured at $\mathrm{K}=40 \mathrm{MPa} \sqrt{\mathrm{m}}$ is nil for the least cathodic potentials examined, and increases as the level of cathodic polarization increases. For example, as $E_{\text {app }}$ decreases from -250 to -975 to $-1200 \mathrm{mV}$ SCE, da/dtII increases from 0.2 to 61 to $413 \mathrm{~nm} / \mathrm{s}$. Finally and notably, the specimens tested at $E_{\text {app }}=-775$ and $-875 \mathrm{mV}_{\mathrm{SCE}}$ do not follow this trend and exhibit unexpectedly rapid da/dtII equal to the specimen tested at $\mathrm{E}_{\mathrm{app}}=-1,200 \mathrm{mV} \mathrm{V}_{\mathrm{SCE}}$. Acceleration of $\mathrm{da} / \mathrm{dt}_{\text {II }}$ in a similar potential range was previously observed in chrome-rich UHSS Ferrium S53 at $E_{\text {app }}=-850$ and $-925 \mathrm{mV}_{\mathrm{SCE}}$; da/dtII increased nearly two orders of magnitude above the average trend in $\mathrm{da} / \mathrm{dt}_{\mathrm{II}}$ versus $\mathrm{E}_{\mathrm{app}}$ for this limited range of cathodic polarizations (Figure 25). The origin of 
accelerated da/dt II in a limited range of moderate cathodic polarization will be discussed in an ensuring section.

Fracture morphology for SCC-susceptible specimens of Ferrium PH48S - H900 is invariant with increasing cathodic polarization below the OCP range. SEM fractographs for specimens tested at $E_{\text {app }}=-250,-575,-775,-975$, and $-1,200 \mathrm{mV}_{\mathrm{SCE}}$ (Figure 62) are shown in Figure 63a-e. All images

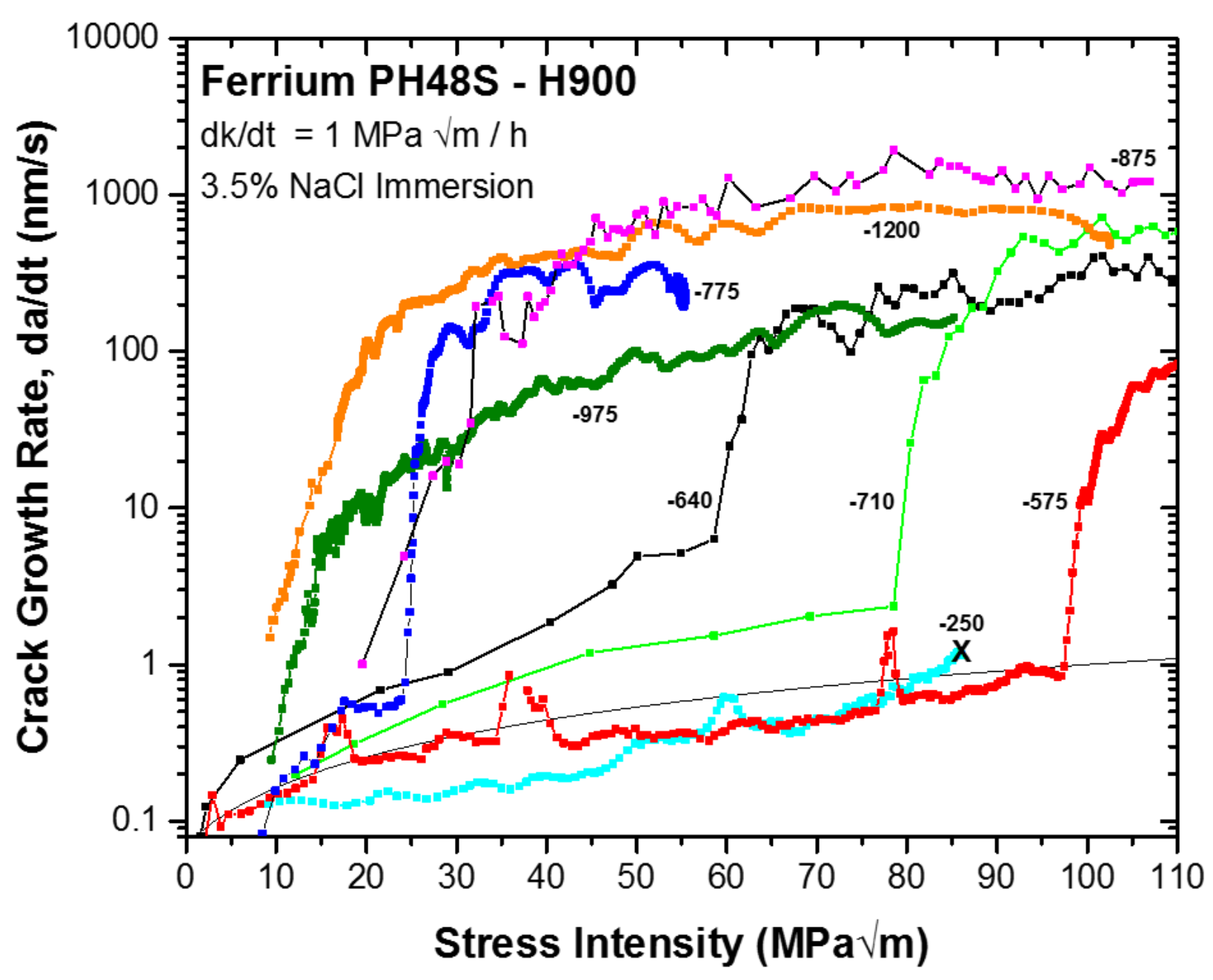

Figure 62 Effect of $E_{a p p}$ on subcritical crack growth kinetics of Ferrium PH48S-H900 stressed under slow rising displacement in $3.5 \% \mathrm{NaCl}$. The specimen tested at $\mathrm{E}_{\text {app }}=-250$ $m V_{S C E}$ reached the load limit of the testing frame and the test was terminated. 

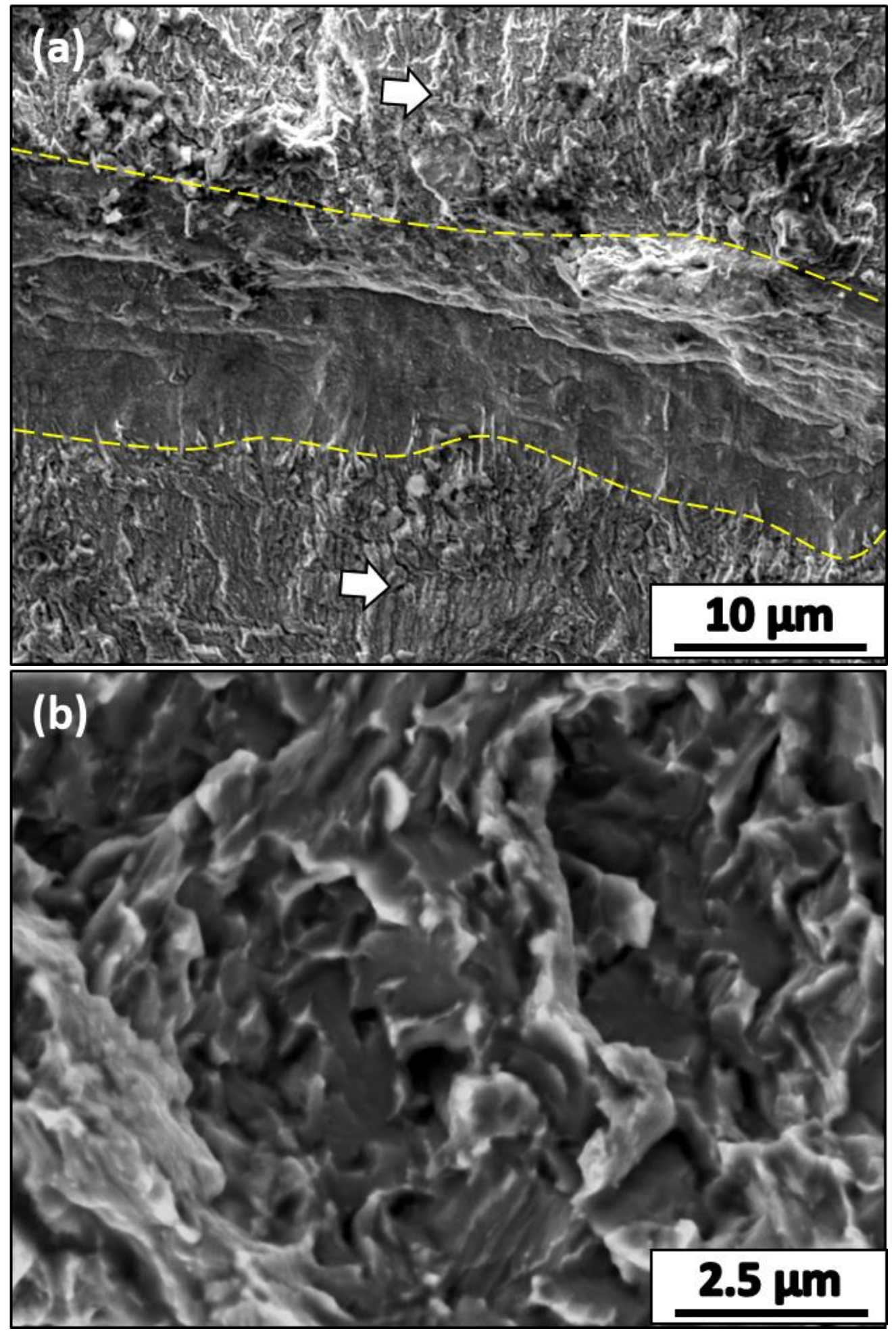

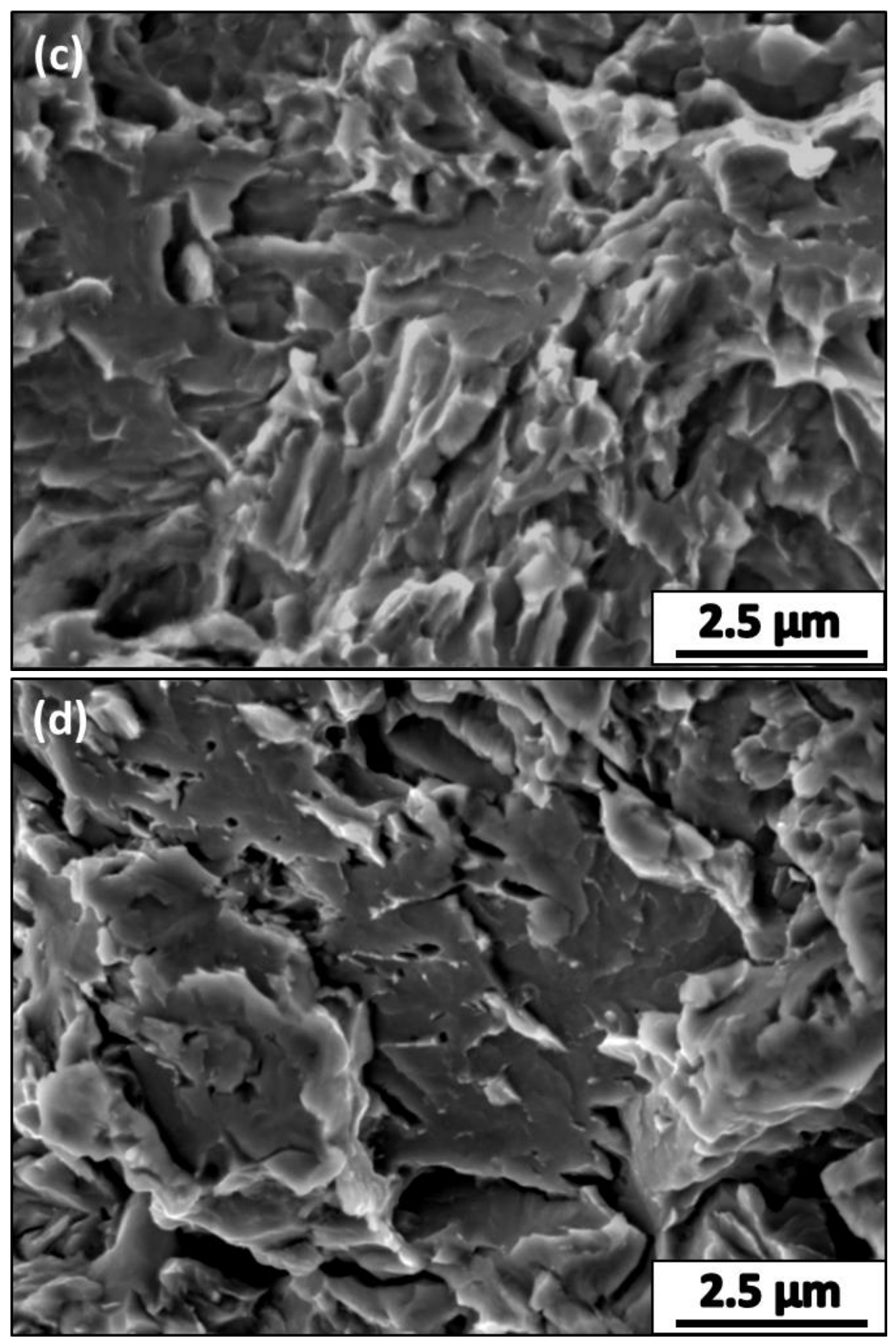


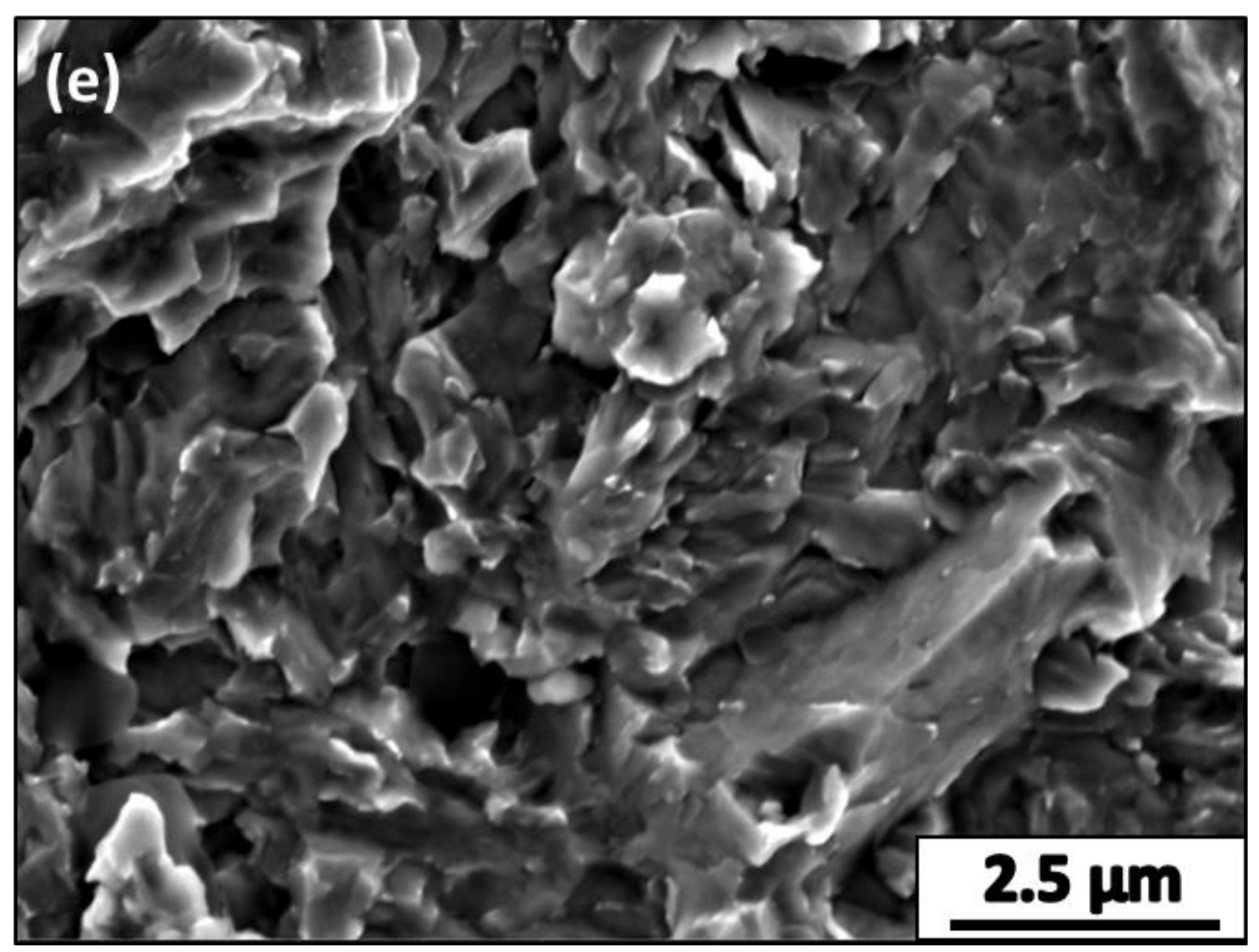

Figure 63 HEAC fracture morphology of Ferrium PH48S-H900 stressed under slow rising displacement in 3.5\% $\mathrm{NaCl}$ at $E_{a p p}=-250(a),-575(b),-775(c),-975(d)$, and -1,200 (e) $m V_{S C E}$.

in Figure 62 were taken in the region directly adjacent to the end of the fatigue precrack where stress intensity was approximately $\mathrm{K}_{\mathrm{TH}}$ for each alloy- $\mathrm{E}_{\text {app }}$ pair. As shown in Figure 63b-e, HEAC fracture at all SCC-susceptible $E_{a p p}$ is dominated by transgranular quasi-cleavage with a small fraction of transgranular flat fracture. The spacing of fine lath-like features on QC facets is consistent across $\mathrm{E}_{\mathrm{app}}$, as is the relative fractions of QC and flat fracture. This result suggests that a single HEAC damage mechanism is active at all SCC-susceptible levels of $\mathrm{E}_{\text {app }}$ applied to Ferrium PH48S $-\mathrm{H} 900$. Notably, the specimen tested at $\mathrm{E}_{\mathrm{app}}=-250 \mathrm{mV}_{\mathrm{SCE}}$ (Figure 63a) reached the load limit of the test frame (at $\mathrm{K}$ of $86 \mathrm{MPa} \sqrt{\mathrm{m}}$ ) and did not experience HEAC. For this specimen, the fracture surface is dominated by pre and post-test fatigue features (white arrows) that bound a large featureless blunted zone (yellow dash) at the root of the precrack. A geometric crack extension of $15.2 \mu \mathrm{m}$ is expected based upon the crack tip blunting equation presented in Equation 9 (Assuming $\sigma_{Y S}=1,550 \mathrm{MPa}$ and $\mathrm{E}=200 \mathrm{GPa}$ ) [104]. An average blunted zone width of $13.3 \mu \mathrm{m}$ is observed in Figure 63a, closely matching the geometric prediction and supporting the use of this blunting equation. Moreover, this lack of TG HEAC features affirms the use of the 
plasticity-based resolution limit for $\mathrm{K}_{\mathrm{TH}}$ definition and demonstates that HEAC did not occur in PH48S-H900 stressed in $\mathrm{NaCl}$ solution at $\mathrm{E}_{\text {app }}$ of $-250 \mathrm{mV}$ SCE.

\subsubsection{Effect of $E_{a p p}$ on HEAC Kinetics and Fracture Morphology of Custom 465 - H900}

Custom 465 - H900 is susceptible to severe HEAC when stressed under slow rising displacement in $3.5 \% \mathrm{NaCl}$ at $\mathrm{E}_{\mathrm{app}}$ more cathodic than $-450 \mathrm{mV}_{\mathrm{SCE}}$. The subcritical crack growth kinetics of Custom 465 - H900 specimens stressed at fixed $E_{\text {app }}$ are shown in Figure 64. For all SCCsusceptible specimens $\left(-1,300 \leq \mathrm{E}_{\mathrm{app}}<-450 \mathrm{mV}_{\mathrm{SCE}}\right), \mathrm{K}_{\mathrm{TH}}$ is low $(8-11 \mathrm{MPa} \sqrt{\mathrm{m}})$ and da/dt $\mathrm{dI}_{\mathrm{II}}$ is very rapid (900 to $3200 \mathrm{~nm} / \mathrm{s}$ ). The fracture morphology for these specimens is exclusively intergranular along prior austenite grain boundaries, as shown in Figure 65a-c. IG facets are featureless, identical to Figure 57b, and show little evidence of localized plasticity or microvoid fracture. As shown in Figure 66 (white arrows), a minor fraction of IG facets exhibited closely spaced, parallel markings. No correlation to $\mathrm{E}_{\mathrm{app}}$ or location on a fracture surface was observed for this feature. Similar features observed on IG IHAC facets of pure nickel were conclusively identified to be slip traces [165]. In pure nickel slip traces on IG IHAC facets were abundant and observed to readily transmit across grain boundaries [165]. Further, multiple orientations of identical slip features were commonly observed on a single IG facet. Although there is no evidence of multiple orientations of the parallel markings in Figure 66 and the frequency of such features is reduced in IG fracture of Custom 465 - H900, it is assumed that parallel markings represent slip traces. Relative to OCP $(-225 \mathrm{mV}$ SCE $)$, small amounts of cathodic polarization $\left(-450 \leq \mathrm{E}_{\mathrm{app}} \leq-250\right.$ $\left.\mathrm{mV}_{\mathrm{SCE}}\right)$ conferred immunity to SCC-HEAC. Specimens tested at $\mathrm{E}_{\mathrm{app}}=-250 \mathrm{mV}_{\mathrm{SCE}}$ and -450 $\mathrm{mV}_{\mathrm{SCE}}($ Figure $64, \mathrm{O}, \Delta)$ were loaded to near $\mathrm{K}_{\mathrm{JICi}}(79 \mathrm{MPa} \sqrt{\mathrm{m}})$ and reached the load limit of the test frame, without any HEAC growth. False da/dt rise from $\mathrm{K}=8$ to $70 \mathrm{MPa} \sqrt{\mathrm{m}}$ is well described by the plasticity based resolution limit for these specimens, supporting its use in determining $\mathrm{K}_{\mathrm{TH}}$ for specimens exhibiting strong SCC resistance. 


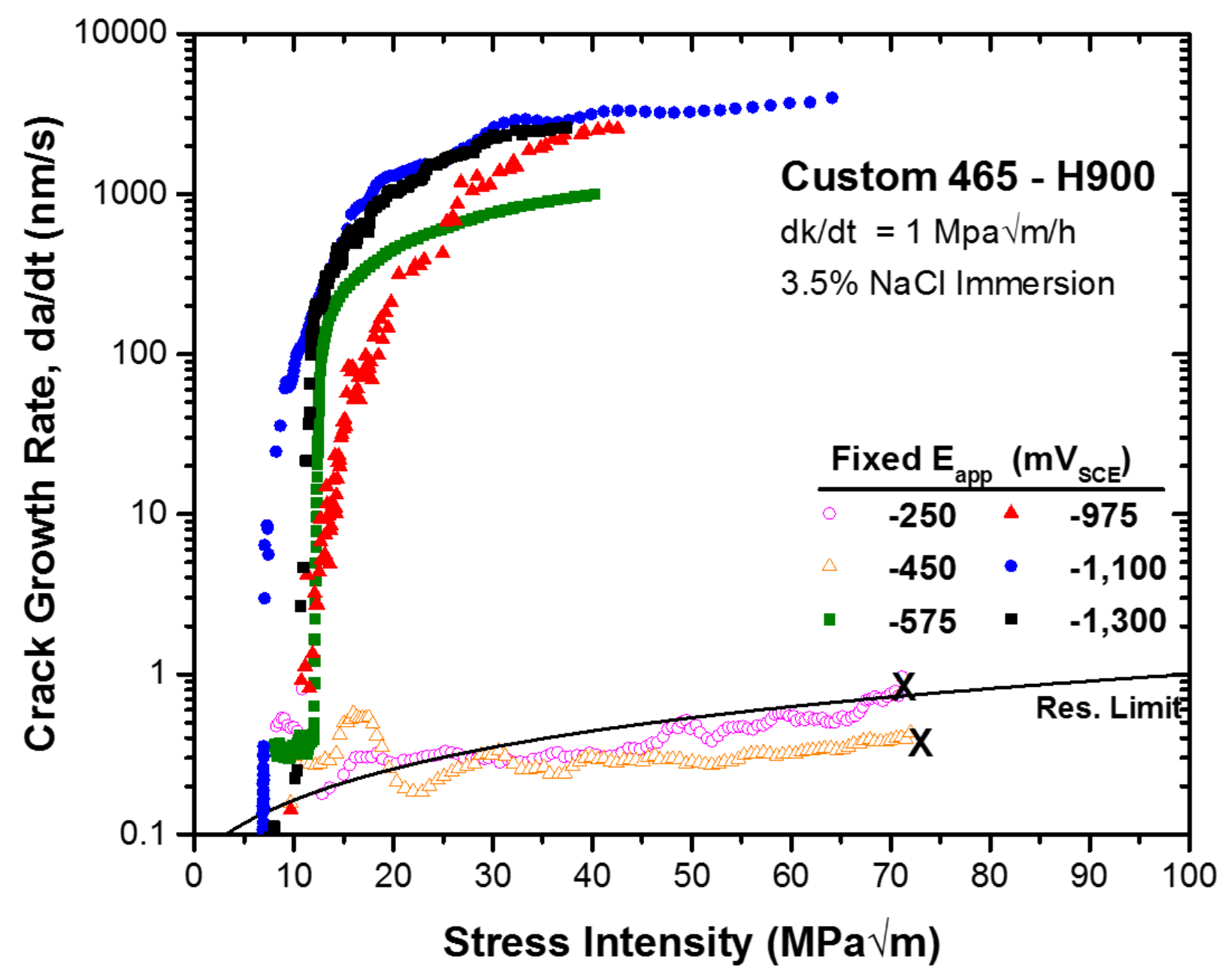

Figure 64 Effect of $E_{a p p}$ on the subcritical crack growth kinetics of Custom $465-H 900$ stressed under slow rising displacement in $3.5 \% \mathrm{NaCl}$ solution. The specimen tested at $\mathrm{E}_{\text {app }}=$ $-575 \mathrm{mV} V_{S C E}$ was conducted by Yongwon Lee without the use of a reference probe and analyzed using 52 point polynomial averaging, rather than 7 point polynomial averaging [173]. 

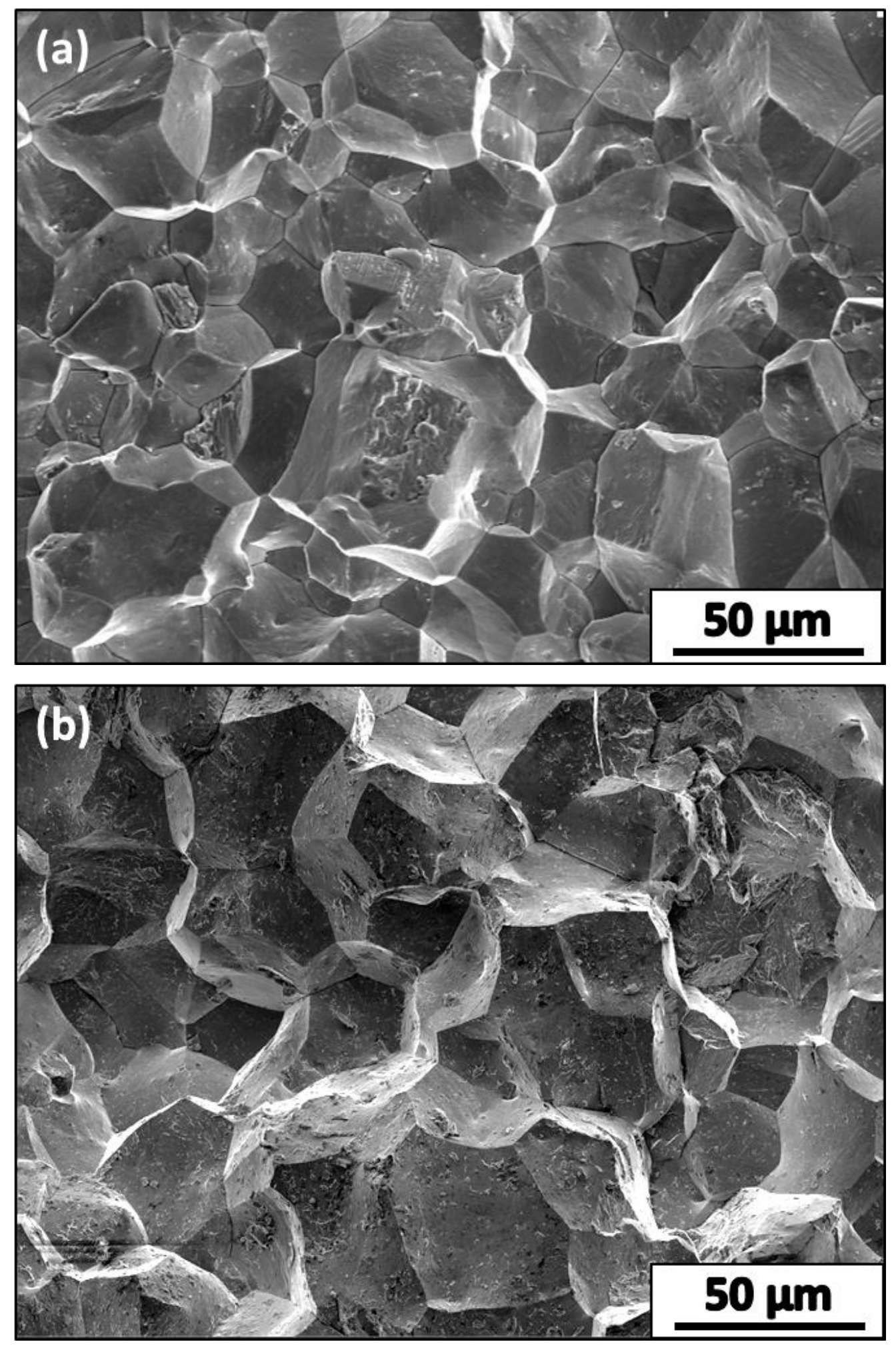


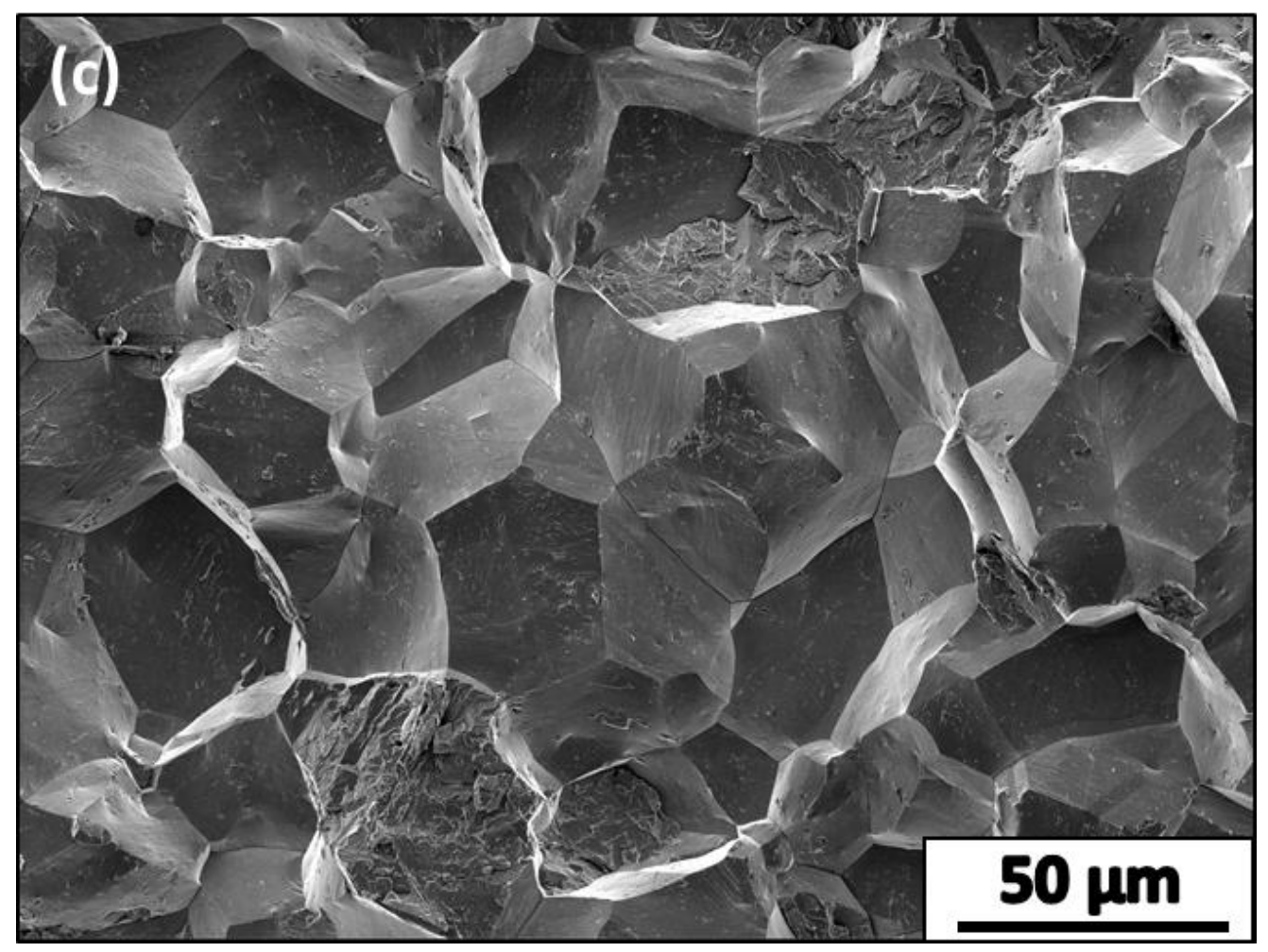

Figure 65 HEAC fracture morphology of Custom 465 - H900 stressed under slow rising displacement in $3.5 \% \mathrm{NaCl}$ at $E_{a p p}=-575(a),-975(b)$ and $-1,300(\mathrm{c}) \mathrm{mV}$ SCE. 

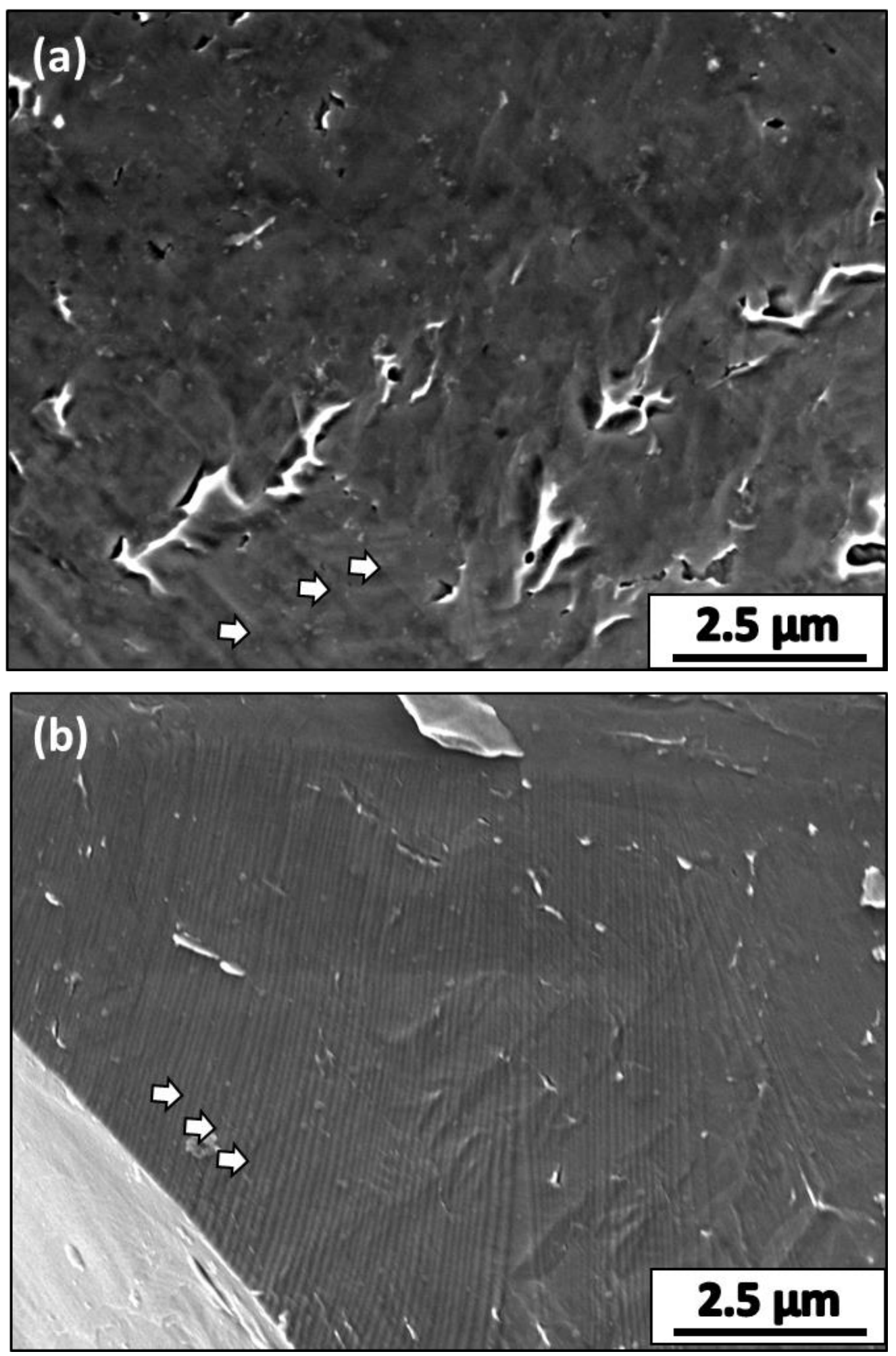

Figure 66 Detail of a single IG facet from (a) Figure $65 b\left(E_{a p p}=-975 \mathrm{~m} V_{S C E}\right)$ and $(b)$ Figure $65 c\left(E_{a p p}=-1,300 m V_{S C E}\right)$ showing the presence of lines (white arrows). 


\subsubsection{Effect of $E_{\text {app }}$ on HEAC Properties of Custom 465 and Ferrium PH48S}

\subsubsection{Equal Strength Comparison of C465 - H900 and Ferrium PH48S - H860}

The effect of applied potential on $\mathrm{K}_{\mathrm{TH}}$ for Custom 465 - H900 and Ferrium PH48S - H860, aged to identical strength (Table 2), is shown in Figure 67. Both alloys exhibit a similar window of mild cathodic polarization which confers strong resistance to HEAC. This conclusion assumes that this temper of PH48S behaves analogous to Ferrium S53 (Figures 24 and 25), and also as indicated by the reduced threshold evidenced in unsuccessful experiments with PH48S H900 at $E_{\text {app }}=-150$ $\mathrm{mV}_{\text {SCE. }}$ As $\mathrm{E}_{\text {app }}$ is decreased from OCP $\left(\sim-225 \mathrm{mV}_{\mathrm{SCE}}\right)$ to $-450 \mathrm{mV}_{\mathrm{SCE}}$, average $\mathrm{K}_{\mathrm{TH}}$ is high and constant, near $\mathrm{K}_{\mathrm{JICi}}$ for both Custom 465 (79 MPa $\sqrt{\mathrm{m}}$ ) and Ferrium PH48S - H860 (95 MPa $\sqrt{\mathrm{m}}$ ). The fracture surfaces for PH48S-H860 in this regime were heavily corroded. However, classic TG QC identical to HEAC of Ferrium PH48S - H900 (Figure 59) with a minor fraction of fine scale microvoid fracture was observed. This result indicates that Ferrium PH48S is not immune to HEAC under mild-cathodic polarization. A bimodal behavior is exhibited by Custom $465-\mathrm{H} 900$ at moderate cathodic polarization. Three specimens of Custom $465-\mathrm{H} 900\left(\mathrm{E}_{\mathrm{app}}=-250,-250\right.$, and $-450 \mathrm{mV}_{\mathrm{SCE}}$ ) exhibit immunity to HEAC and reached the load limit of the test frame, as indicated by the upward arrows in Figure 62. Two specimens of Custom $465-\mathrm{H} 900\left(\mathrm{E}_{\mathrm{app}}=-250\right.$ and -350 $\mathrm{mV}_{\mathrm{SCE}}$ ) exhibited significantly lower $\mathrm{K}_{\mathrm{TH}}, 47$ and $53 \mathrm{MPa}{ }^{\mathrm{m}}$. Crack growth for these specimens was rapid ( $>5,000 \mathrm{~nm} / \mathrm{s}$ ) and exclusively IG along prior austenite grain boundaries, identical to Figure 65. The origin of this $\mathrm{K}_{\mathrm{TH}}$ variability in Custom 465 - H900 stressed under moderate cathodic polarization is unknown. The presence of IG fracture features indicates that specimen to specimen variation in $\mathrm{K}_{\mathrm{JICi}}$ is not the source, as ductile fracture in Custom $465-\mathrm{H} 900$ is dominated by microvoid coalescence (Figure 53). Near identical threshold variability is observed in replicate specimens of AerMet 100 tested at $E_{a p p}=-625 \mathrm{mV}_{\mathrm{SCE}}\left(100 \mathrm{mV}_{\mathrm{SCE}}\right.$ cathodic to OCP). In Chapter 3 , two hypotheses were put forth to explain this variability: (1) H production/absorption limited by a passive film, the strength of which could be microstructurally sensitive [157,158], or (2) heterogeneous dissolution of sulfides could accelerate crack solution hydrolysis and $\mathrm{H}$ production and/or uptake. The efficacy of each hypothesis in explaining the $\mathrm{K}_{\mathrm{TH}}$ variability in Custom 465H900 will be discussed in an ensuing section. 


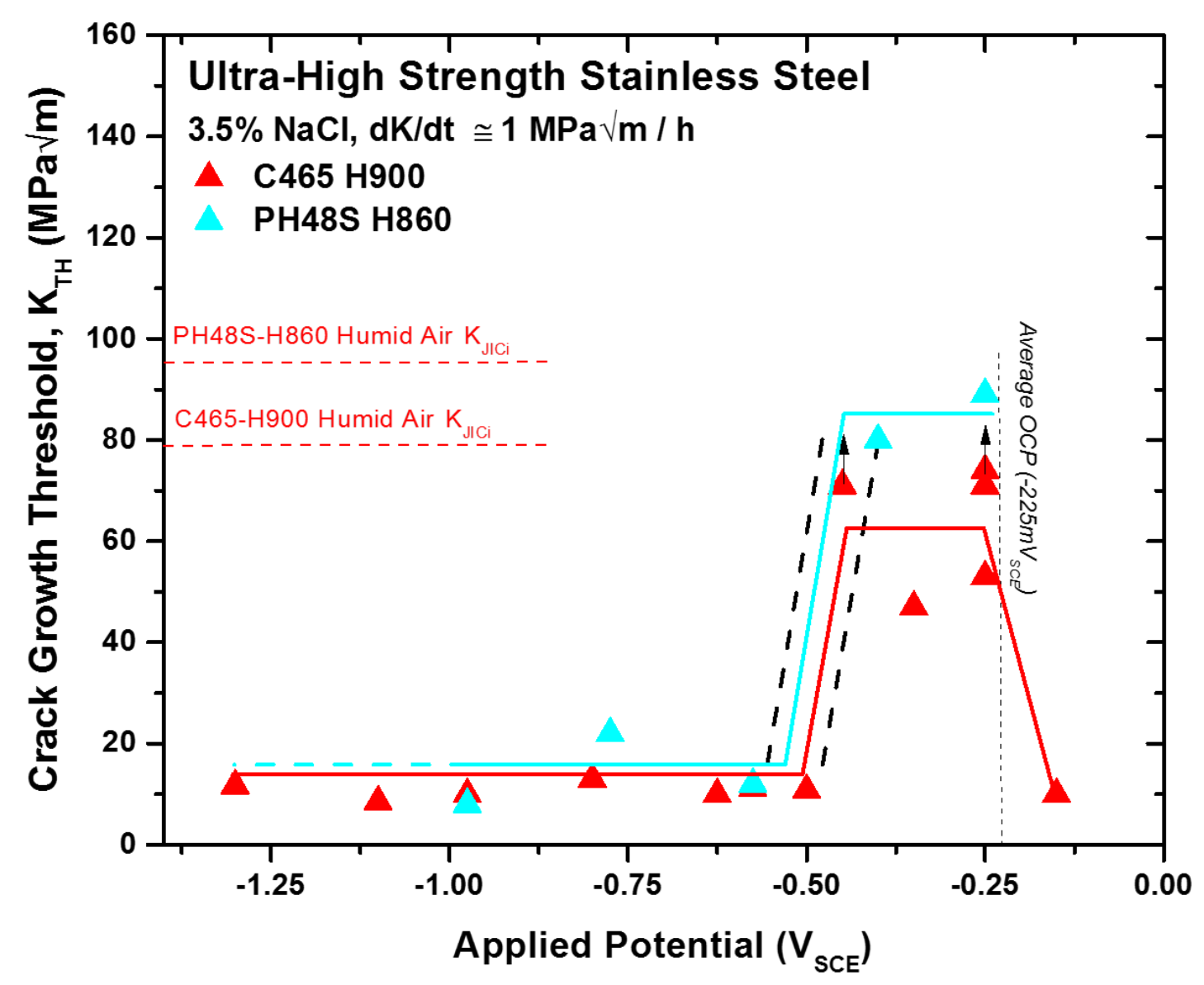

Figure 67 Threshold stress intensity $\left(K_{T H}\right)$ vs. $E_{\text {app }}$ for Custom $465-H 900$ and Ferrium PH48S - H860. Specimens which reached the load limit of the testing frame are denoted with an upward arrow; for these specimens $K_{T H}$ is taken at maximum load.

At $\mathrm{E}_{\text {app }}<-450 \mathrm{mV}_{\mathrm{SCE}}$, HEAC is severe, as characterized by low $\mathrm{K}_{\mathrm{TH}}(8$ to $22 \mathrm{MPa} \sqrt{ } \mathrm{m})$, which approaches a common value of 8 to $12 \mathrm{MPa} \sqrt{\mathrm{m}}$ in both alloys (Figure 67). Degradation from SCC immunity ( $\mathrm{E}_{\mathrm{app}} \geq-450 \mathrm{mV} \mathrm{VCE}_{\mathrm{SC}}$ ) to SCC-susceptibility is sharp; $\mathrm{K}_{\mathrm{TH}}$ for Custom $465-\mathrm{H} 900$ falls from 71 to $11 \mathrm{MPa} \sqrt{\mathrm{m}}$ over $50 \mathrm{mV}$ of increasingly cathodic polarization from -450 to $-500 \mathrm{mV} \mathrm{V}_{\mathrm{SEE}}$. The $\mathrm{K}_{\mathrm{TH}}$ drop with increasing cathodic polarization appears to be equally sharp in Ferrium PH48S - H860. Limited specimens of Ferrium PH48S - H860 were tested and the first SCC-susceptible $\mathrm{E}_{\text {app }}$ could fall anywhere between -400 and $-575 \mathrm{mV}_{\mathrm{SCE}}$. The range of possible trend lines for Ferrium PH48S-H860 in the transition regime is bounded in Figure 67 by black dashed lines, assuming an identical rate of $\mathrm{K}_{\mathrm{TH}}$ decline as observed for Custom $465-\mathrm{H} 900$. 
In both alloys, reduced $\mathrm{K}_{\mathrm{TH}}$ with increasing cathodic polarization is accompanied by a rise in da/dtII, as shown in Figure 68. In Custom 465- H900, da/dtII sharply rises from 0.4 to $500 \mathrm{~nm} / \mathrm{s}$ over $50 \mathrm{mV}$ of increasingly cathodic polarization from -450 to $-500 \mathrm{mV}_{\text {SCE. }}$. Following this initial rise, da/dtII approaches a stable value of 2,500 to $3,000 \mathrm{~nm} / \mathrm{s}$ for $\mathrm{E}_{\text {app }} \leq-800 \mathrm{mV} \mathrm{V}_{\text {SCE}}$. Rise in da/dtII is similarly sharp in Ferrium PH48S - H860, but Stage II da/dt is on average an order of magnitude slower compared to Custom 465. For instance, da/dtII at $\mathrm{E}_{\text {app }}=-775 \mathrm{mV}$ SCE is $365 \mathrm{~nm} / \mathrm{s}$ for PH48S - H860, compared to $3,000 \mathrm{~nm} / \mathrm{s}$ for Custom $465-\mathrm{H} 900$ stressed at $\mathrm{E}_{\text {app }}=-800 \mathrm{mV}_{\text {SCE}}$. Notably, PH48S - H860 exhibits a peak in da/dt $/ \mathrm{dI}_{\mathrm{II}}(365 \mathrm{~nm} / \mathrm{s})$ at $\mathrm{E}_{\text {app }}=-775 \mathrm{mV} \mathrm{V}_{\mathrm{SCE}}$, and decreases to $60-80$ $\mathrm{nm} / \mathrm{s}$ over $200 \mathrm{mV}$ of polarization in the anodic or cathodic directions. Due to the limited number of samples tested, the exact shape of the peak in $\mathrm{da} / \mathrm{dt}_{\mathrm{II}}$ is unknown. Similar elevated da/dt at moderate $\mathrm{E}_{\text {app }}$ is observed for PH48S - H900 (Figure 62).

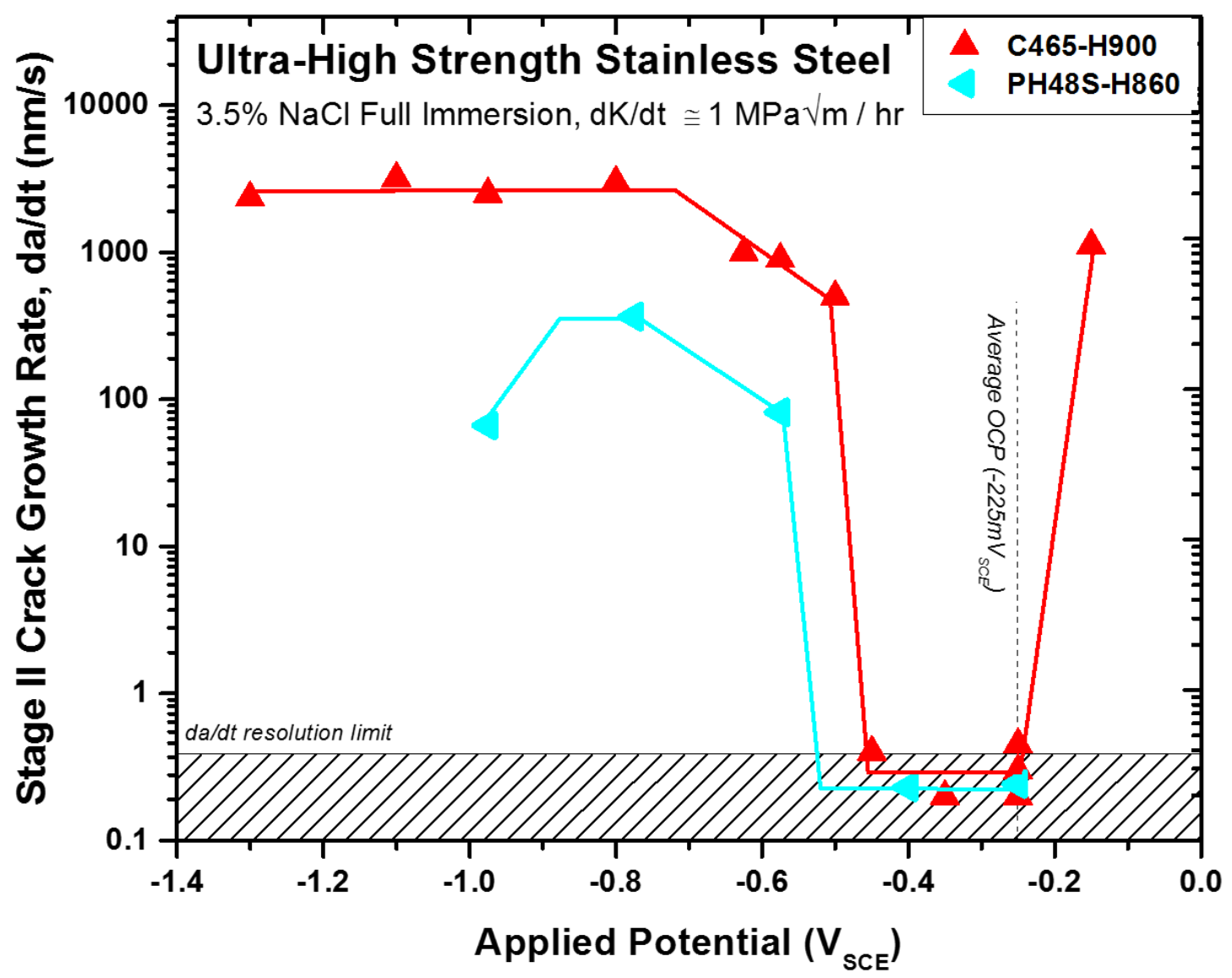

Figure 68 Effect of $E_{\text {app }}$ on Stage II crack growth rate at $K=40 \mathrm{MPa} \backslash \mathrm{m}$ in Custom $465-$ H900 and Ferrium PH48S - H860. The trend line for PH48S-H860 is modeled after the more expansive set of kinetics for Ferrium PH48S - H900, which are presented in Figure 70. 


\subsubsection{Effect of $E_{\text {app }}$ and Aging Condition on HEAC of Ferrium PH48S}

The effect of applied potential on $\mathrm{K}_{\mathrm{TH}}$ for four ultra-high strength aging conditions of Ferrium PH48S is quantified in Figure 69. All heat treatments exhibit a window of mild cathodic polarization which confers strong resistance to HEAC. Examination of the anodic HEAC properties in Ferrium PH48S was attempted, but yielded limited results due to corrosion of the dcPD wire - specimen interface. It is assumed that all aging conditions and Ferrium PH48S will exhibit a dramatic fall off in $\mathrm{K}_{\mathrm{TH}}$ under mild anodic polarization, relative to OCP ( $\mathrm{E}_{\text {app }}>-225$ $\mathrm{mV}_{\mathrm{SCE}}$ ). This assumption is justified from two results: (1) the sharp reduction in $\mathrm{K}_{\mathrm{TH}}$ observed in both Custom $465-\mathrm{H} 900$ at $\mathrm{E}_{\text {app }}=-150 \mathrm{mV}_{\mathrm{SCE}}$ (Figure 67) and in stainless Ferrium S53 at $\mathrm{E}_{\text {app }}=$ -500 to $-550 \mathrm{mV}_{\mathrm{SCE}}$ (Figures 24 and 25), and (2) replicate experiments of Ferrium PH48S - H900 tested at $\mathrm{E}_{\mathrm{app}}=-150 \mathrm{mV}_{\mathrm{SCE}}$ indicate that $\mathrm{K}_{\mathrm{TH}}=\sim 25 \mathrm{MPa} \sqrt{ } \mathrm{m}$. Further experiments are needed to confirm the anodic HEAC properties of Ferrium PH48S across the range of aging conditions.

The breadth of cathodic polarization conferring elevated $\mathrm{K}_{\mathrm{TH}}$ is strongly dependent on the aging condition. In general, as the aging temperature decreases (H968 $\rightarrow \mathrm{H} 950 \rightarrow \mathrm{H} 900 \rightarrow \mathrm{H} 860$ ), the breadth of cathodic polarizations conferring elevated $\mathrm{K}_{\mathrm{TH}}$ is reduced. For instance, $\mathrm{PH} 48 \mathrm{~S}-\mathrm{H} 968$ is essentially immune to $\mathrm{HEAC}\left(\mathrm{K}_{\mathrm{TH}}>86 \mathrm{MPa} \sqrt{ } \mathrm{m}\right)$ from $\mathrm{E}_{\text {app }}$ of -250 to $-875 \mathrm{mV} \mathrm{V}_{\mathrm{SCE}}$, while higher strength PH48S - H900 exhibits elevated $\mathrm{K}_{\mathrm{TH}}$ from -250 to $-575 \mathrm{mV}_{\mathrm{SCE}}$. As strength is further increased in the H860 condition, the range of protective potentials further declines, such that -400 $\leq \mathrm{E}_{\text {app }} \leq-250 \mathrm{mV} \mathrm{SCE}_{\mathrm{SE}}$ confers $\mathrm{K}_{\mathrm{TH}}>80 \mathrm{MPa} \sqrt{\mathrm{m}}$. Due to the relatively low number of samples tested, it is not possible to know the precise range of protective $\mathrm{E}_{\text {app }}$ for PH48S $-\mathrm{H} 860$. However, the specimen tested at $E_{\text {app }}=-400 \mathrm{mV}_{\mathrm{SCE}}$ shows a decline in $\mathrm{K}_{\mathrm{TH}}$ from $\mathrm{E}_{\mathrm{app}}=-250 \mathrm{mV} \mathrm{V}_{\mathrm{SCE}}$, possibly indicating the onset of declining $\mathrm{K}_{\mathrm{TH}}$ with increasing cathodic polarization. For the remainder of analyses, it is assumed that applied potential in the range of $-400 \leq \mathrm{E}_{\text {app }} \leq 250 \mathrm{mV}_{\text {SCE }}$ confers elevated $\mathrm{K}_{\mathrm{TH}}$ in PH48S - H860; further testing is needed to firm up this behavior. Notably, the H950 and H968 aging conditions exhibit identical behavior across all tested $\mathrm{E}_{\text {app }}$, despite a $50 \mathrm{MPa}$ difference in compressive yield strength (Table 2) compared to the H950 and H900 aging conditions (6 MPa, Table 2). 


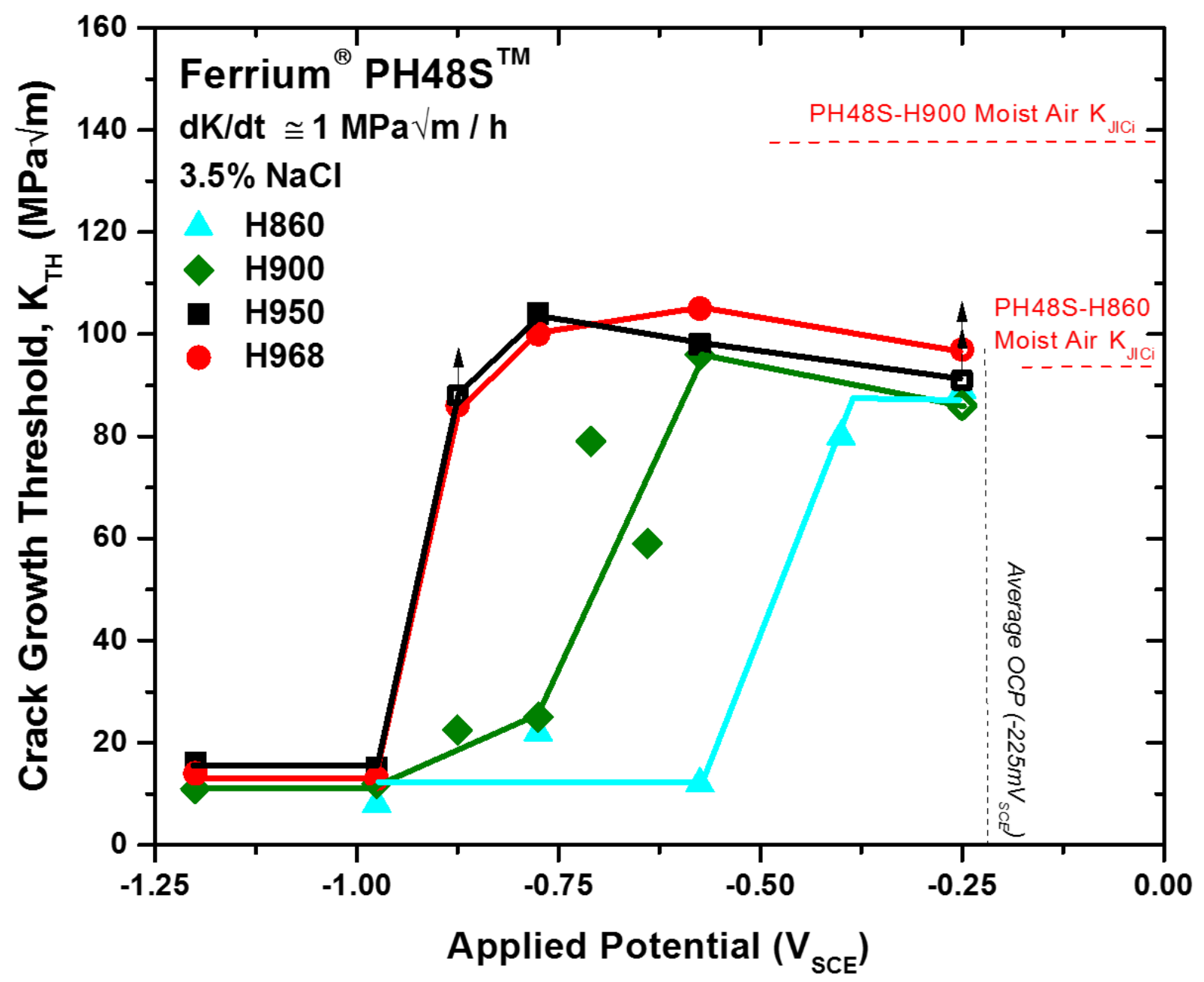

Figure 69 Effect of $E_{a p p}$ and aging condition on the threshold stress intensity for HEAC in Ferrium PH48S. Upward arrows denote specimens which reached the load limit of the test frame. For these specimens, $K_{T H}$ is denoted as the stress intensity at maximum load.

For all aging conditions, $\mathrm{K}_{\mathrm{TH}}$ sharply declines at $\mathrm{E}_{\text {app }}$ cathodic to the protective window and approaches a stable value of 10 to $16 \mathrm{MPa} \sqrt{\mathrm{m}}$ at $\mathrm{E}_{\text {app }} \leq-975 \mathrm{mV}_{\mathrm{SCE}}$. The rate of decline in $\mathrm{K}_{\mathrm{TH}}$ is strongly aging temperature dependent. $\mathrm{K}_{\mathrm{TH}}$ for HEAC of Ferrium PH48S - H968 and 950 decreases sharply from $>86 \mathrm{MPa} \sqrt{ } \mathrm{m}$ to $13-15 \mathrm{MPa} \sqrt{\mathrm{m}}$ over only $100 \mathrm{mV}$ of additional cathodic polarization. The rate of decline with increasing cathodic polarization is much less sharp in PH48S - H900; $\mathrm{K}_{\text {TH }}$ decreases from 96 to $13 \mathrm{MPa} \sqrt{\mathrm{m}}$ over $400 \mathrm{mV}$ of cathodic polarization ( $\mathrm{E}_{\text {app }}=-575$ to $-975 \mathrm{mV}$ SCE). 
As shown in Figure 70, falling $\mathrm{K}_{\mathrm{TH}}$ with increasing cathodic polarization is accompanied by a rise in da/daII for each aging temperature of Ferrium PH48S. The magnitude of $\mathrm{da} / \mathrm{dt}_{\text {II }}$ in the SCCsusceptible $E_{\mathrm{app}}$ regime correlates to aging condition. For example, at $\mathrm{E}_{\mathrm{app}}=-975 \mathrm{mV} \mathrm{VCE}_{\mathrm{SC}} \mathrm{da} / \mathrm{dt}_{\mathrm{II}}$ increases from 18 to 61 to $200 \mathrm{~nm} / \mathrm{s}$ as aging temperature is decreased from H968 to H900 to

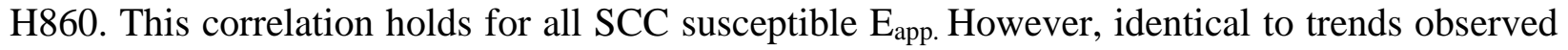
in $\mathrm{K}_{\mathrm{TH}}$ versus $\mathrm{E}_{\mathrm{app}}$, the behavior of the H968 and H950 aging conditions is identical across all $E_{\text {app. }}$ Stage II crack growth rate is elevated at moderate cathodic $E_{\text {app }}$ in the H860/H900 aging conditions, but not for the H950/H968 conditions. Both the H900 and H860 aging conditions of Ferrium PH48S exhibit increased da/dt $\mathrm{dI}_{\text {II }}$ at moderate cathodic $\mathrm{E}_{\mathrm{app}}$. Notably, da/dt $\mathrm{dI}_{\text {II }}$ falls in the

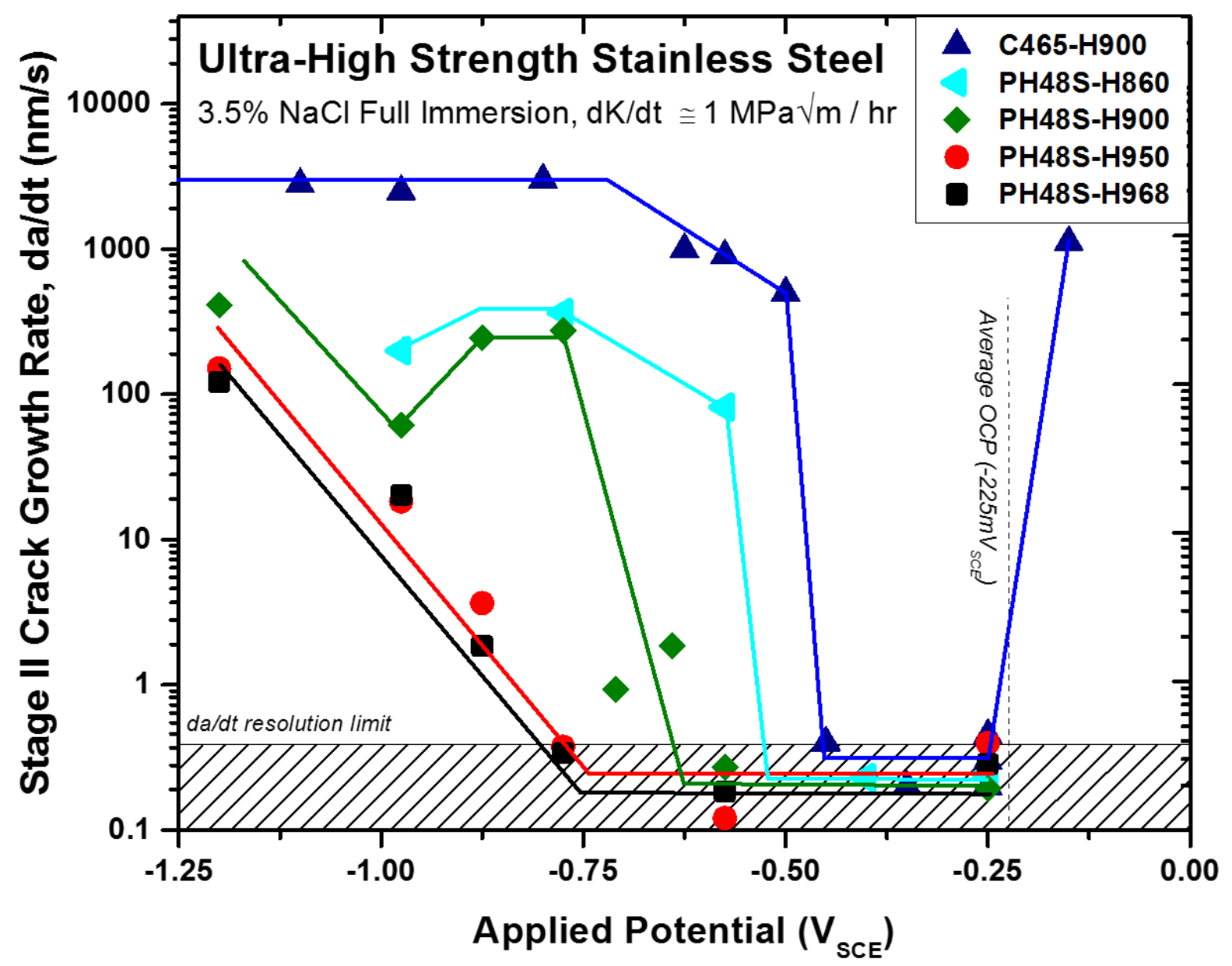

Figure 70 Effect of $E_{\text {app }}$ and aging condition on Stage II crack growth rate $(K=40 \mathrm{MPa} \vee \mathrm{m})$ in UHSSS. Plotted trend line for PH48S - H860 was extrapolated from PH48S - H900. The data for Custom 465 - H900 are shown for reference. 
PH48S-H900 at $\mathrm{E}_{\mathrm{app}}<-875 \mathrm{mV}_{\mathrm{SCE}}$ and returns to the general trend of rising $\mathrm{da} / \mathrm{dt}_{\mathrm{II}}$ versus decreasing $E_{\text {app }}$ that is observed for PH48S - H968/H950 from $E_{\text {app }}=-775$ to $-1,200 \mathrm{mV}_{\mathrm{SCE}}$. There is strong evidence of an identical return to a general trend of slow-rising da/dt $\mathrm{II}_{\mathrm{I}}$ with decreasing $\mathrm{E}_{\text {app }}$ in PH48S - H860, but insufficient samples were tested to validate this hypothesis. Regardless, it is clear from the data presented in Figure 70 that elevated da/dt II at moderate $\mathrm{E}_{\text {app }}$ is affected by aging conditions and electrochemical polarization.

\subsubsection{Hydrogen Solubility in Ferrium PH48S and Custom 465}

The solubility of hydrogen in Custom 465 (as quenched, AQ, and H900) and Ferrium PH48S (AQ, $\mathrm{H} 900, \mathrm{H} 950$, and $\mathrm{H} 968$ ), charged for up to $609 \mathrm{~h}$ in $0.001 \mathrm{M} \mathrm{NaOH}$ at $\mathrm{E}_{\mathrm{app}}=-1,350 \mathrm{mV}_{\mathrm{SCE}}$, is quantified in Figure 71. Maximum charging time for the thin plate specimens was calculated to be $99 \%$ of the steady state $\mathrm{H}$ concentration, using an assumed hydrogen diffusivity of $10^{-9} \mathrm{~cm}^{2} / \mathrm{s}$ and a semi-infinite plate diffusion solution [106]. Total hydrogen content $\left(\mathrm{C}_{\mathrm{H}-\mathrm{TOTAL}}\right)$ increases with charging time and approaches a steady state value in all alloys. Experimental error either in

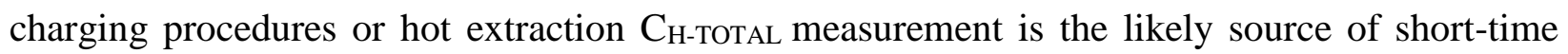
variations in $\mathrm{H}$ concentration. For all conditions except Ferrium PH48S - H950, steady state

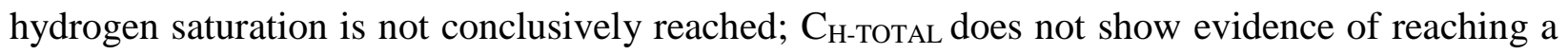
constant value with increasing time. As such, the maximum hydrogen concentration for each aging condition is likely higher than what is shown for $609 \mathrm{~h}$ in Figure 71. Ferrium PH48S - H900

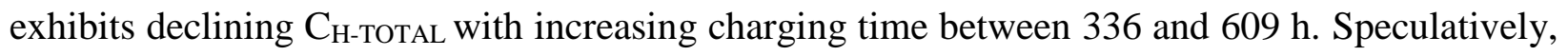

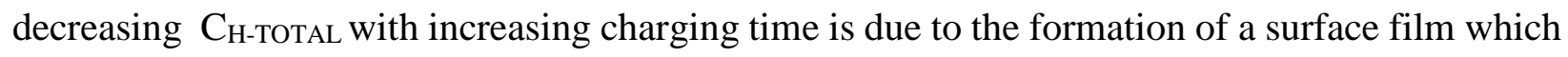
hinders $\mathrm{H}$-uptake and results in reduced $\mathrm{H}$-solubility at fixed $\mathrm{E}_{\mathrm{app}}$.

Hydrogen solubilities for Custom 465 and Ferrium PH48S in the as-quenched condition are remarkably similar (10.0 and 11.9 wppm, respectively), but strongly diverge after aging. For three aging conditions of Ferrium PH48S, the highest measured hydrogen content is low but variable: 2.3, 4.0, and 8.4 wppm for the H900, H968, and H950 heat treatments. Maximum hydrogen concentration is highest in the H950 condition (3.6 times H900 and 2.1 times H968), and decreases as the aging temperature is increased (H968) or decreased (H900). The observed trend in maximum $\mathrm{C}_{\mathrm{H}-\mathrm{TOTAL}}$ with increasing aging temperature does not match the trends in equilibrium phase concentrations of precipitated austenite, $\mathrm{BCC}-\mathrm{Cr}, \mathrm{NiAl}$, or $\eta$, as predicted by ThermoCalc 
modeling. These features could be trap states, which would increase $\mathrm{C}_{\mathrm{H} \text {-TOTAL. }}$ All three heat

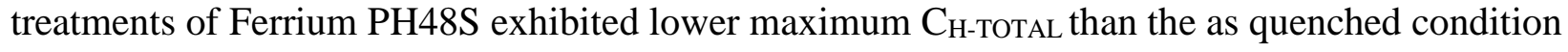
(12.0 wppm). Decreased $\mathrm{C}_{\mathrm{H}-\text { TOTAL }}$ after aging in Ferrium PH48S suggests the loss of a significant $\mathrm{H}$-trap state in the microstructure, despite the known precipitation of $\eta, \mathrm{BCC}-\mathrm{Cr}$, and ordered $\mathrm{NiAl}$ strengthening phases upon aging. Austenite retained after quenching from solution treatment would be a strong-reversible H-trap state, and the high solubility of $\mathrm{H}$ in FCC austenite would allow for a large volume fraction of $\mathrm{H}$ to be trapped $[9,128,130]$. Decomposition of retained austenite at elevated aging temperature would eliminate the hypothetical trap state and reduce the solubility of $\mathrm{H}$ in the microstructure. At all charging times, hydrogen solubility is dramatically increased in Custom 465 - H900, compared to both the C465-AQ case and each condition of Ferrium PH48; Custom 465 - H900 exhibits a maximum $\mathrm{C}_{\mathrm{H}-\mathrm{TOTAL}}$ of 72.5 wppm (compared to 2.3 - 8.4 wppm in PH48S - H900/H950/H968) at 609 h of charging time.

Following H-charging, a portion of the H-charged specimens were allowed to outgas at room

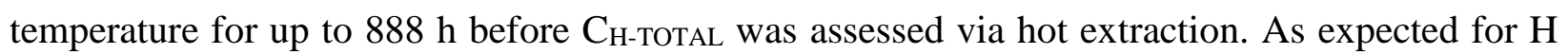
diffusion governed by lattice and reversibly trapped $\mathrm{H}, \mathrm{C}_{\mathrm{H}-\mathrm{TOTAL}}$ falls as outgassing time increases at $25^{\circ} \mathrm{C}$. For example, the hydrogen content of Custom 465 - H900 falls from 72.5 to 23.3 to 0.3 wppm as outgassing time increases from 0 to 144 to $888 \mathrm{~h}$ (total times of 609, 753, and $1497 \mathrm{~h}$ in Figure 71, respectively). For each alloy and all aging conditions, the hydrogen content of outgassed specimens approaches the value of uncharged specimens. This result indicates that hydrogen in Ferrium PH48S and Custom 465 is exclusively trapped at low to moderate binding energy sites, rather than high energy sites which would vastly slow $\mathrm{H}$ outgassing. Further, there is no observable effect of aging temperature on this outgassing behavior, indicating that this distribution of high and low energy $\mathrm{H}$-trap states is constant with aging temperature. 


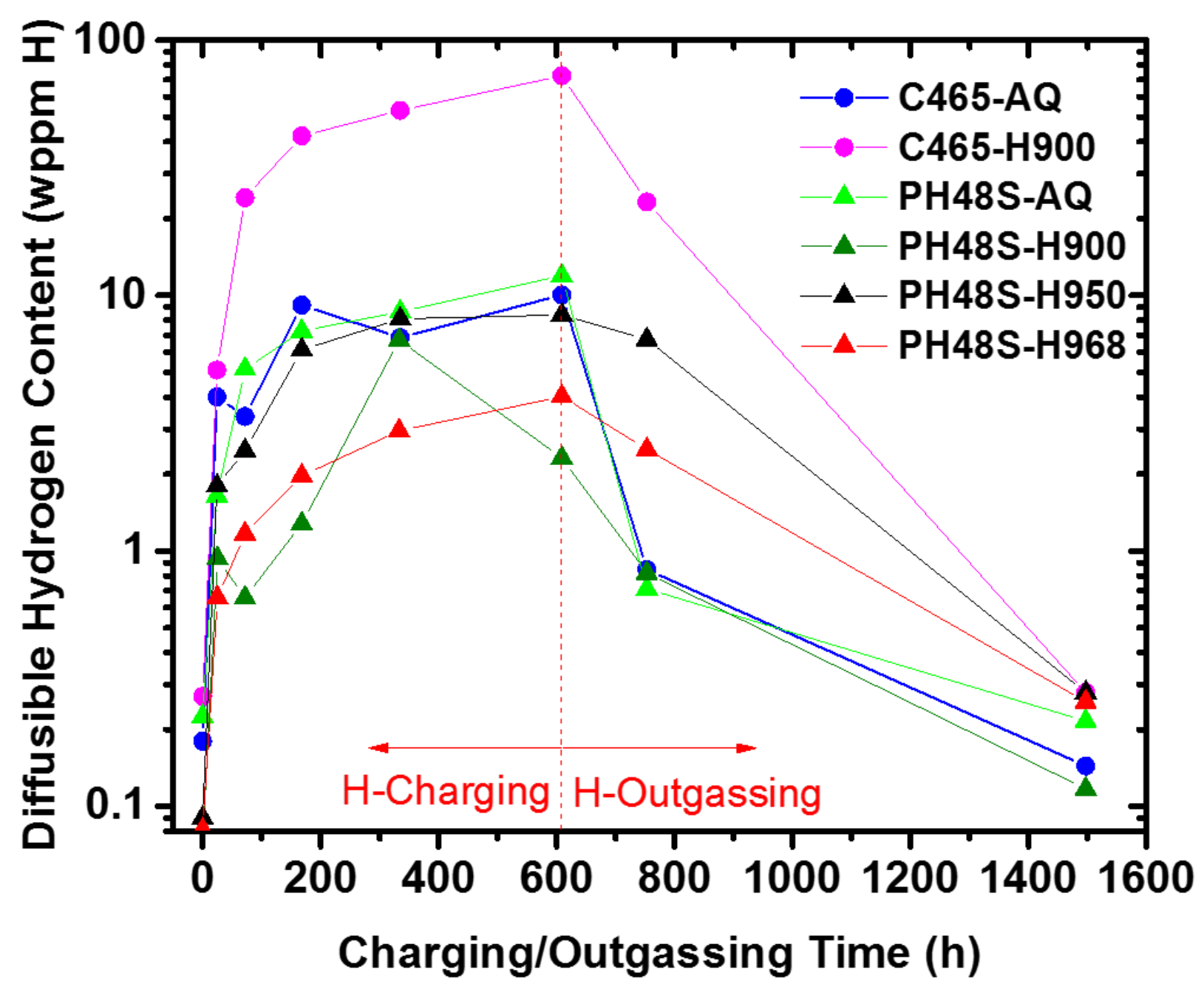

Figure 71 Hydrogen content of Ferrium PH48S and Custom 465 coupons charged for variable time (609 h max) at $E_{a p p}=-1,350 \mathrm{~m} V_{S C E}\left(\eta_{H / H+}=-520 \mathrm{mV}\right)$ in $0.001 \mathrm{M} \mathrm{NaOH}(p H=10)$ and subsequently outgassed at room temperature for up to $888 \mathrm{~h}$.

Measurement of hydrogen solubility as a function of charging time allows for an estimate of $\mathrm{D}_{\mathrm{H}}$ EFF to be made for Ferrium PH48S and Custom 465. DH-EFF may be regression fit to the $\mathrm{H}$ uptake solubility data (Figure 71) using a semi-infinite diffusion solution for a thin plate specimen. However, this analysis requires knowledge of the steady state $\mathrm{H}$-concentration $\left(\mathrm{C}_{\mathrm{H}-\mathrm{EQ}}\right)$, and subsequent $\mathrm{C}_{\text {H-TOTAL }} / \mathrm{C}_{\mathrm{H}-\mathrm{EQ}}$ (percentage of steady state) for all data points. In the ideal case, the

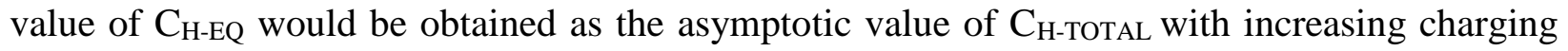
time. However, as shown in Figure 71, with the exception of Ferrium PH48S - H950, a steady

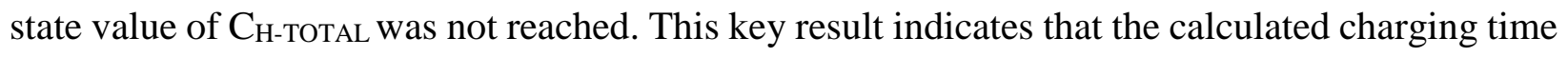


for $99 \%$ of equilibrium $\mathrm{H}$-concentration was not sufficiently long, and implies that $\mathrm{D}_{\mathrm{H}-\mathrm{EFF}}$ is lower than $10^{-9} \mathrm{~cm}^{2} / \mathrm{s}$ for all conditions except Ferrium PH48S - H950.

The H-concentration data of Ferrium PH48S - H950 (Figure 71, $\boldsymbol{\Delta}$ ) are unique among aging

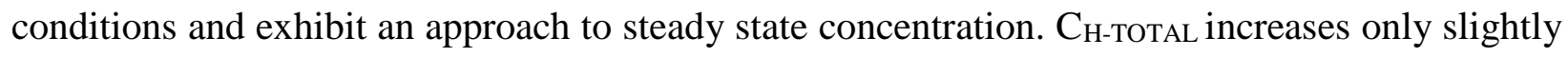
from $8.1 \mathrm{wppm}$ to $8.4 \mathrm{wppm}$ between 335 to $609 \mathrm{~h}$ of charging time. For this unique data, $\mathrm{C}_{\mathrm{H}-\mathrm{TOTAL}}$ at $609 \mathrm{~h}$ of charging time was assumed to be $99 \%$ of $\mathrm{C}_{\mathrm{H}-\mathrm{EQ}}\left(\mathrm{C}_{\mathrm{H}-\mathrm{EQ}}=8.48 \mathrm{wppm}\right)$, and $\mathrm{D}_{\mathrm{H}-\mathrm{EFF}}$ was regression fit to the $\mathrm{H}$-concentration data. As shown in Figure 72, the semi-infinite plate diffusion model effectively fits the data set. From regression fitting, $\mathrm{D}_{\mathrm{H}-\mathrm{EFF}}$ for Ferrium PH48S - H950 is $6.9 \times 10^{-10} \mathrm{~cm}^{2} / \mathrm{s}$, significantly slower than the assumed-conservative value of $10^{-9} \mathrm{~cm}^{2} / \mathrm{s}$. Without knowledge of $\mathrm{C}_{\mathrm{H}-\mathrm{EQ}}$ for each of the remaining aging conditions, regression fitting the remaining $\mathrm{H}$-solubility data can provide only a reasonable estimation of $\mathrm{D}_{\mathrm{H}-\mathrm{EFF}}$. For this analysis, the fraction of steady state $\mathrm{C}_{\mathrm{H}-\mathrm{EQ}}$ represented by the data in Figure 71 at $609 \mathrm{~h}$ was assumed to be 0.99 , and $\mathrm{D}_{\mathrm{H}-\mathrm{EFF}}$ was fit for each alloy. The results of this analysis for both $\mathrm{H}$-uptake and H-outgas are shown in Table 12. For all conditions, calculated H-uptake $D_{H-E F F}$ is low $\left(3.7-6.9 \times 10^{-10} \mathrm{~cm}^{2} / \mathrm{s}\right)$, and reduced in the heat treated conditions compared to the as-quenched conditions. Further, all alloys exhibit slower $\mathrm{D}_{\mathrm{H}-\mathrm{EFF}}$ than Ferrium PH48S - H968, as indicated by the H-charging curves in Figure 71.

\begin{tabular}{|c|c|c|c|c|}
\hline Alloy & Heat Treatment & $\begin{array}{c}\text { 609 h Solubility } \\
\text { (CH-TOTAL wppm) }\end{array}$ & $\begin{array}{c}\text { DH-EFF }\left(\mathbf{c m}^{2} / \mathbf{s}\right) \\
\text { Uptake }\end{array}$ & $\begin{array}{c}\text { DH-EFF }\left(\mathbf{c m}^{2} / \mathbf{s}\right) \\
\text { Outgas }\end{array}$ \\
\hline C465 & As-Quenched & 10.0 & $6.3 \times 10^{-10}$ & $1.6 \times 10^{-9}$ \\
\hline C465 & H900 & 72 & $3.7 \times 10^{-10}$ & $2.6 \times 10^{-9}$ \\
\hline PH48S & As-Quenched & 11.9 & $5.9 \times 10^{-10}$ & $2.4 \times 10^{-10}$ \\
\hline PH48S & H900 & 2.31 & $5.4 \times 10^{-10}$ & $7.0 \times 10^{-10}$ \\
\hline PH48S & H950 & 8.4 & $6.9 \times 10^{-10}$ & $2.3 \times 10^{-10}$ \\
\hline PH48S & H968 & 4.0 & $4.4 \times 10^{-10}$ & $3.1 \times 10^{-10}$ \\
\hline
\end{tabular}

Table 12 Regression fit $D_{H-E F F}$ for the $H$-solubility data represented in Figure 71 using an assumed $C_{H-T O T A L} / C_{H-E Q}=0.99$ at $609 \mathrm{~h}$ of charging time 
Literature characterization of $\mathrm{D}_{\mathrm{H}-\mathrm{EFF}}$ in Ferrium PH48S is nonexistent; however, $\mathrm{D}_{\text {H-EFF }}$ has been reported for similar UHSS(S) alloys such as Custom 465, PH 17-4, and AerMet 100. For example, scanning kelvin probe (SKP) measurements indicate that $D_{H-E F F}$ is $2.5 \times 10^{-8} \mathrm{~cm}^{2} / \mathrm{s}$ for Custom $465-\mathrm{H} 900$ specimens precharged in $0.6 \mathrm{M} \mathrm{NaCl}$ at $\mathrm{E}_{\text {app }}=-1,032 \mathrm{mV}_{\mathrm{SCE}}$ [174]. Further, extensive isothermal TDS experiments have measured $\mathrm{D}_{\text {H-EFF }}$ of Custom 465-AQ and Custom 465 - H900 charged in $0.6 \mathrm{M} \mathrm{NaCl}(\mathrm{pH}=10)$ to be $2.4-3.4 \times 10^{-9}$ and $6.2 \times 10^{-9} \mathrm{~cm}^{2} / \mathrm{s}$, respectively [175]. A similar UHSSS, PH 17-4, has a DH-EFF in the range of $2.8-9.7 \times 10^{-8} \mathrm{~cm}^{2} / \mathrm{s}$ depending on aging condition, as characterized by the electrochemical permeation method [176]. As discussed in Chapter 3, the $\mathrm{D}_{\mathrm{H}-\mathrm{EFF}}$ for AerMet 100 was previously measured by two methods: galvanostatic $\mathrm{H}$ uptake yielded $D_{\mathrm{H}-\mathrm{EFF}}=1 \times 10^{-9} \mathrm{~cm}^{2} / \mathrm{s}$ [17], and TDS extraction yielded $D_{\mathrm{H}-\mathrm{EFF}}=1 \times 10^{-8} \mathrm{~cm}^{2} / \mathrm{s}$ to $6 \times 10^{-9} \mathrm{~cm}^{2} / \mathrm{s}$ as $\mathrm{C}_{\mathrm{H}-\mathrm{DIFF}}$ fell from $6.0 \mathrm{wppm}$ to $0.8 \mathrm{wppm} \mathrm{[16].} \mathrm{DH-EFF} \mathrm{values} \mathrm{for} \mathrm{Ferrium} \mathrm{PH48S}$ and Custom 465 (Table 12) are substantially reduced compared to literature characterization of $\mathrm{D}_{\mathrm{H}-\mathrm{EFF}}$ in AerMet 100 and PH 17-4. However, close agreement of TDS-measured $\mathrm{D}_{\mathrm{H}-\mathrm{EFF}}$ by Schaller at UVa, using an outgassing boundary condition, to regression fit $\mathrm{D}_{\text {H-EFF }}$ for $\mathrm{H}$-outgassing (Table 12) supports the accuracy of the current measurements and support their use in modeling efforts of diffusion limited da/dtII. For example, note that both studies show that $\mathrm{D}_{\text {H-EFF }}$ rises by a factor of 2 due to H900 aging of as-quenched Custom 465. Comparison of the regression fit

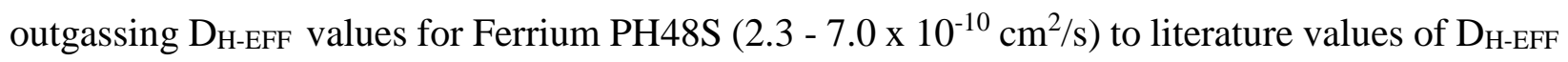
in UHSS, and to the data for $\mathrm{D}_{\mathrm{H}-\mathrm{EFF}}$ in Custom $465\left(10^{-8}\right.$ to $\left.10^{-9}\right)$, indicates that the diffusivity of Ferrium PH48S is substantially reduced from conventional UHSS as well as compared to Custom 465 in both the as-quenched and high strength conditions. In the absence of significant changes in $\mathrm{C}_{\mathrm{H \sigma}} / \mathrm{C}_{\mathrm{H \sigma}-\mathrm{CRIT}}$, diffusion limited da/dtII would be proportionately reduced for Ferrium PH48S, as examined in an ensuing section of the Discussion.

\subsubsection{TDS Analysis of Hydrogen Trap States in Ferrium PH48S and Custom $465^{10}$}

Hydrogen desorption results $\left(\mathrm{dC}_{\mathrm{H}} / \mathrm{dt}\right.$. temperature), measured during temperature-ramp rate controlled TDS, are shown in Figure 73 for two H-precharged UHSSS alloys. Specimens were charged for up to $609 \mathrm{~h}$ in $0.001 \mathrm{M} \mathrm{NaOH}$ at $\mathrm{E}_{\text {app }}=-1350 \mathrm{mV}_{\mathrm{SCE}}$ (Figure 71). Three fixed

10 TDS experiments were conducted by Rebecca Schaller under the advisement of Professor John R. Scully. 


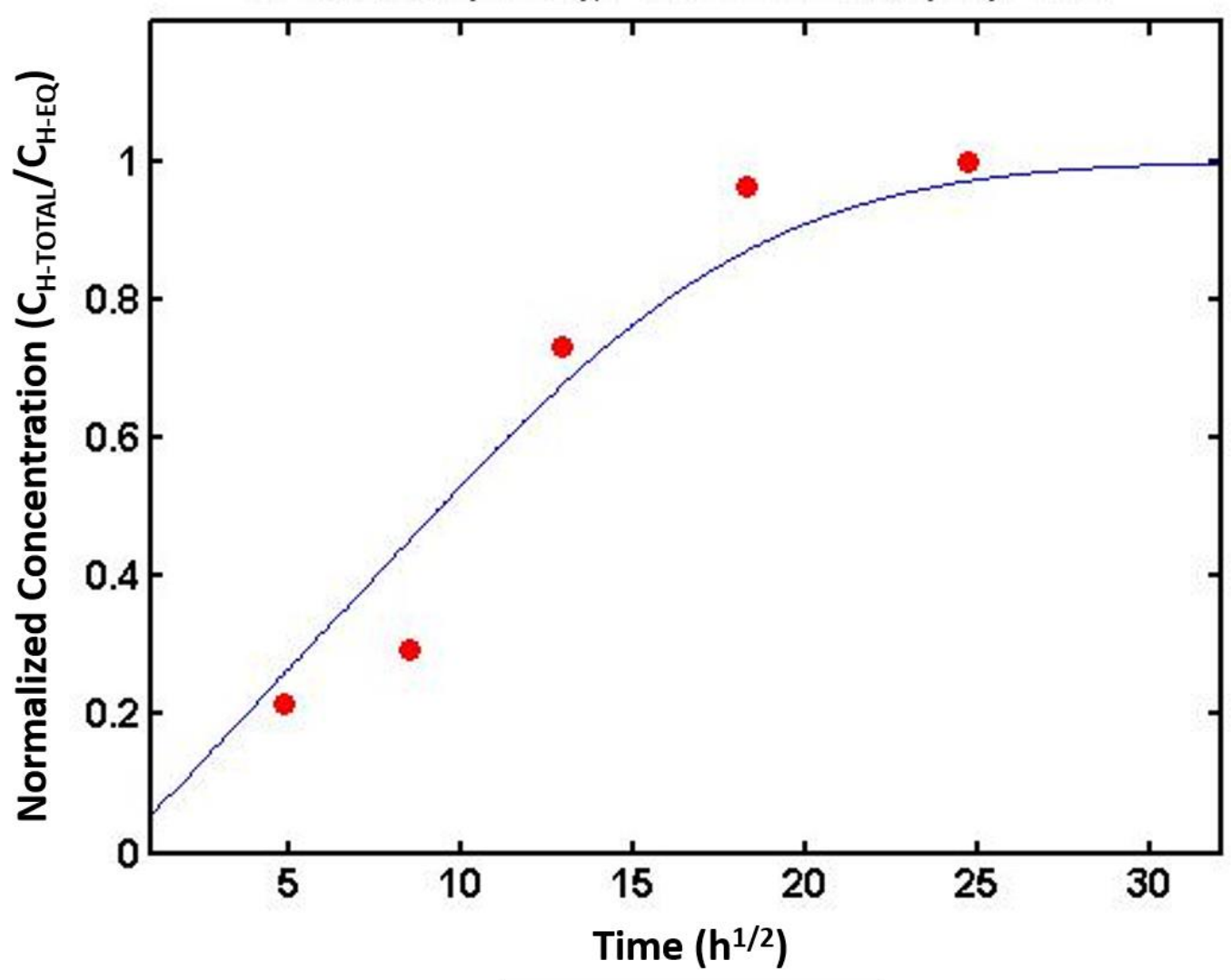

Figure 72 Regression fitting of $D_{H-E F F}$ to the H-concentration data of Ferrium PH48S-H950 shown in Figure 71.

temperature-ramp rates are shown $\left(2.5,5.0,10.0^{\circ} \mathrm{C} / \mathrm{min}\right)$ for Ferrium PH48S and Custom 465, each in the high strength $\mathrm{H} 900$ aging condition. For the temperature range of 23 to $550{ }^{\circ} \mathrm{C}$, each specimen exhibits a single Gaussian desorption peak, which shifts to higher temperatures as the ramp rate is increased. Maximum desorption rate, and area enclosed by the outgassing peak, are significantly increased in Custom 465 - H900 compared to Ferrium PH48S - H900. Following this single desorption peak, $\mathrm{dC}_{\mathrm{H}} / \mathrm{dt}$ rapidly approaches a constant background level without resolvable evidence of higher temperature peaks for either steel. The background level in Ferrium $\mathrm{PH} 48 \mathrm{~S}-\mathrm{H} 900$ is noticeably elevated at $0.8-5 \times 10^{-5} \mathrm{wppm} / \mathrm{s}$ compared to $0.5-5 \times 10^{-6}$ for Custom 465 - H900. No material variable would logically cause a change in the background level, and this difference is attributed to experimental differences. However, both background levels are near the 
estimated resolution limit of the TDS system $\left(\sim 10^{-5} \mathrm{wppm} / \mathrm{s}\right)$, and no effect on the $\mathrm{dT} / \mathrm{dt}$ analysis is expected.

Increased total hydrogen content in Custom 465 - H900, relative to Ferrium PH48S - H900, is clearly visible in Figure 73 through comparison of the peak areas. In the absence of multiple peaks, the ratio of total hydrogen content can be qualitatively assessed based upon the total area under the peak, less the TDS background. For all heating rates, desorption peaks are significantly larger in Custom 465 - H900 compared to Ferrium PH48S - H900. This result, while not quantitative, is consistent with and affirms the $\mathrm{H}$ solubility data in Figure 69 for identically charged samples.

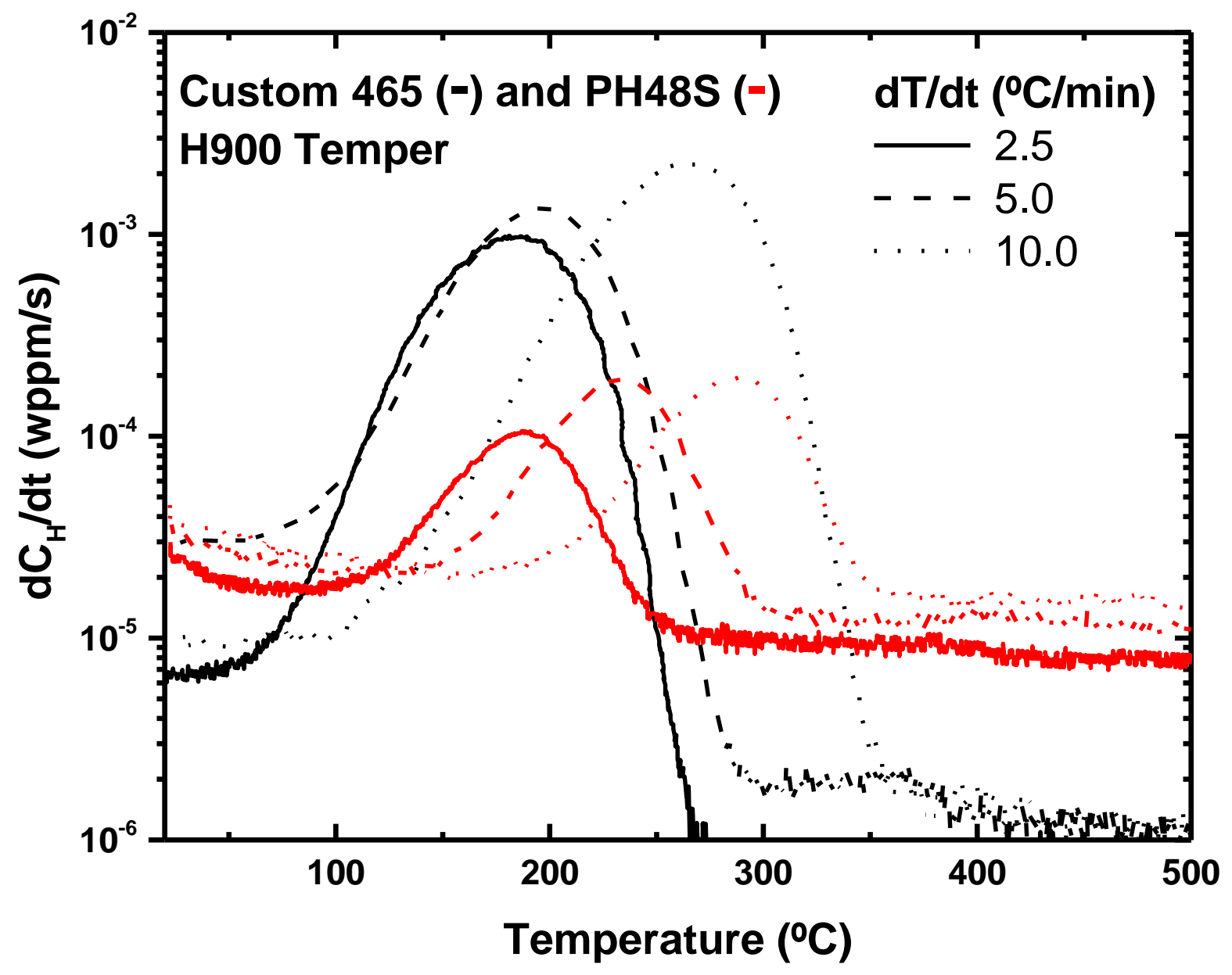

Figure $73 \quad$ Hydrogen desorption rate $\left(d C_{H} / d t\right)$ as a function of temperature for Ferrium PH48S - H900 and Custom $465-H 900 . \quad$ Specimens were charged at $E_{a p p}=-1,350 m V_{S C E}$ $\left(\eta_{H / H+}=-520 \mathrm{mV}\right)$ for $609 \mathrm{~h}$. 
For each specimen and ramp rate, the temperature associated with the peak maximum $\left(\mathrm{T}_{\mathrm{m}}\right)$ was measured, and the data presented in Figure 73 were analyzed to produce a plot of $\ln \left[(d T / d t) / T_{m}^{2}\right]$ against reciprocal temperature (Figure 74). The activation energy for H-desorption $\left(\mathrm{E}_{\mathrm{d}}\right)$ can be determined from the variation in $\mathrm{T}_{\mathrm{M}}$ with increasing $\mathrm{dT} / \mathrm{dt}$, according to Equation 10 [130]:

$$
\frac{d\left(\ln \left((d T / d t) / T_{m}^{2}\right)\right)}{d\left(T_{m}\right)}=-\frac{E_{d}}{R}
$$

The value of $E_{d}$ determined from this analysis provides a basis for determining the binding energy $\left(E_{B}\right)$ for the hydrogen trap state associated with the peak, assuming that only one such state is stable for the temperature range of the measured peak. Following a TDS analysis conducted on AerMet 100, $E_{d}$ is assumed to equal $E_{B}+E_{M}$, where $E_{M}$ is the activation energy for H-diffusion in a trap free lattice [130]. For the present study, $\mathrm{E}_{\mathrm{M}}$ is assumed to be $10.0 \mathrm{~kJ} / \mathrm{mol}$, identical to the previous analysis for AerMet 100 [130]. Based on this relationship, $E_{B}$ was determined for the observed hydrogen desorption peak, shown in the inset of Figure 74. Calculated $E_{B}$ is identical (12.1-12.2 kJ/mol) for the single H-desorption peak of Custom 465 - H900 and Ferrium PH48S presented in Figure 73. As shown in Figure 74, the magnitude of this binding energy is close to the $\mathrm{E}_{\mathrm{B}}$ of coherent $\mathrm{M}_{2} \mathrm{C}$ carbide traps in AerMet 100 [130]. Based upon this similarity, it is reasonable to hypothesize that the single desorption peak in Custom 465 and Ferrium PH48S corresponds to a single trap state, and that this trap state is the primary strengthening precipitate. This assignment was justified for AerMet 100 in that the primary peak shown in Figure 71 was not observed for an as-quenched specimen, which did not contain strengthening precipitates [124].

While experimentally calculated values of $\mathrm{E}_{\mathrm{B}}$ are identical for PH48S and AerMet 100, the data presented for this UHSSS in Figure 74 are shifted relative to prior work on AerMet 100 [130]. This shift is a result of a consistent increase in the $T_{m}$ value for desorption peaks in UHSSS compared to AerMet 100. Critically, the effect of increasing TDS ramp rate (dT/dt) on $\mathrm{T}_{\mathrm{m}}$ must be identical between UHSSS and Aermet 100 to measure the same H-trap binding energy. Previous work has analytically modeled the effect of material and experimental parameters on theoretical hydrogen desorption profiles [177]. Based upon this fundamental work, the consistent $\mathrm{T}_{\mathrm{m}}$ shift in Ferrium PH48S and Custom 465 is attributed to the TDS specimen thickness, which is increased compared to that used for AerMet 100 (0.7 vs $0.5 \mathrm{~mm})$ [130]. Increased specimen thickness 
increases the amount of time required for hydrogen to diffuse from the center of the specimen and be measured by the TDS system. At a constant temperature ramp increased diffusion time for outgassing hydrogen directly shifts the peak heights to higher temperatures, producing the shift in $\mathrm{T}_{\mathrm{m}}$ observed in Figure 74.

Notably, previous TDS measurements of H-trapping in AerMet 100 revealed two additional high energy desorption peaks (Figure 75) [124]. The moderate energy desorption peak (Figure 75, Peak 2 at about $325^{\circ} \mathrm{C}$ ) was associated with martensite interfaces, prior austenite grain boundaries, and mixed dislocation cores $\left(E_{B}=61.3-62.2 \mathrm{~kJ} / \mathrm{mol}\right.$ ). The high energy desorption peak (Figure 75, Peak 3) was associated with undissolved-large metal carbides and highly misoriented grain boundaries $\left(\mathrm{E}_{\mathrm{B}}=89.1-89.9 \mathrm{~kJ} / \mathrm{mol}\right)$ [130]. Both of these peaks are absent from the UHSSS desorption spectra presented in Figure 73 despite a similar martensite microstructure (Figure 7, Figure 39, and Figure 40), heat treatment (Table 1), and H-charging conditions [130]. The implications of this result on SCC-HEAC of UHSSS will be discussed in an ensuing section. 


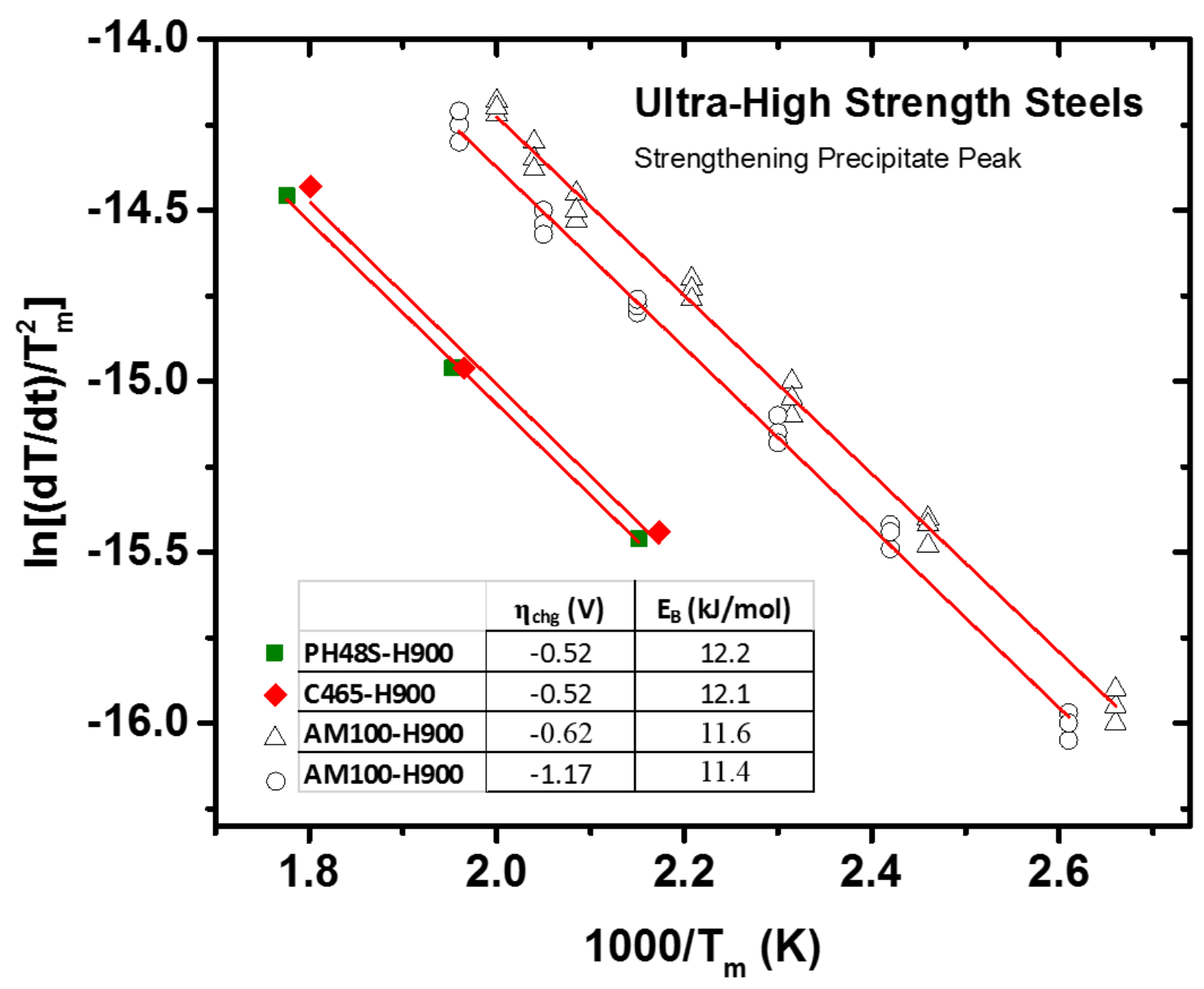

Figure 74 Determination of the activation energy for $H$ desorption energy associated with the desorption peak of Ferrium PH48S and Custom 465 presented in Figure 73. The data are compared to an identical analysis conducted on the primary strengthening precipitate desorption peak in AerMet 100 [130]. 


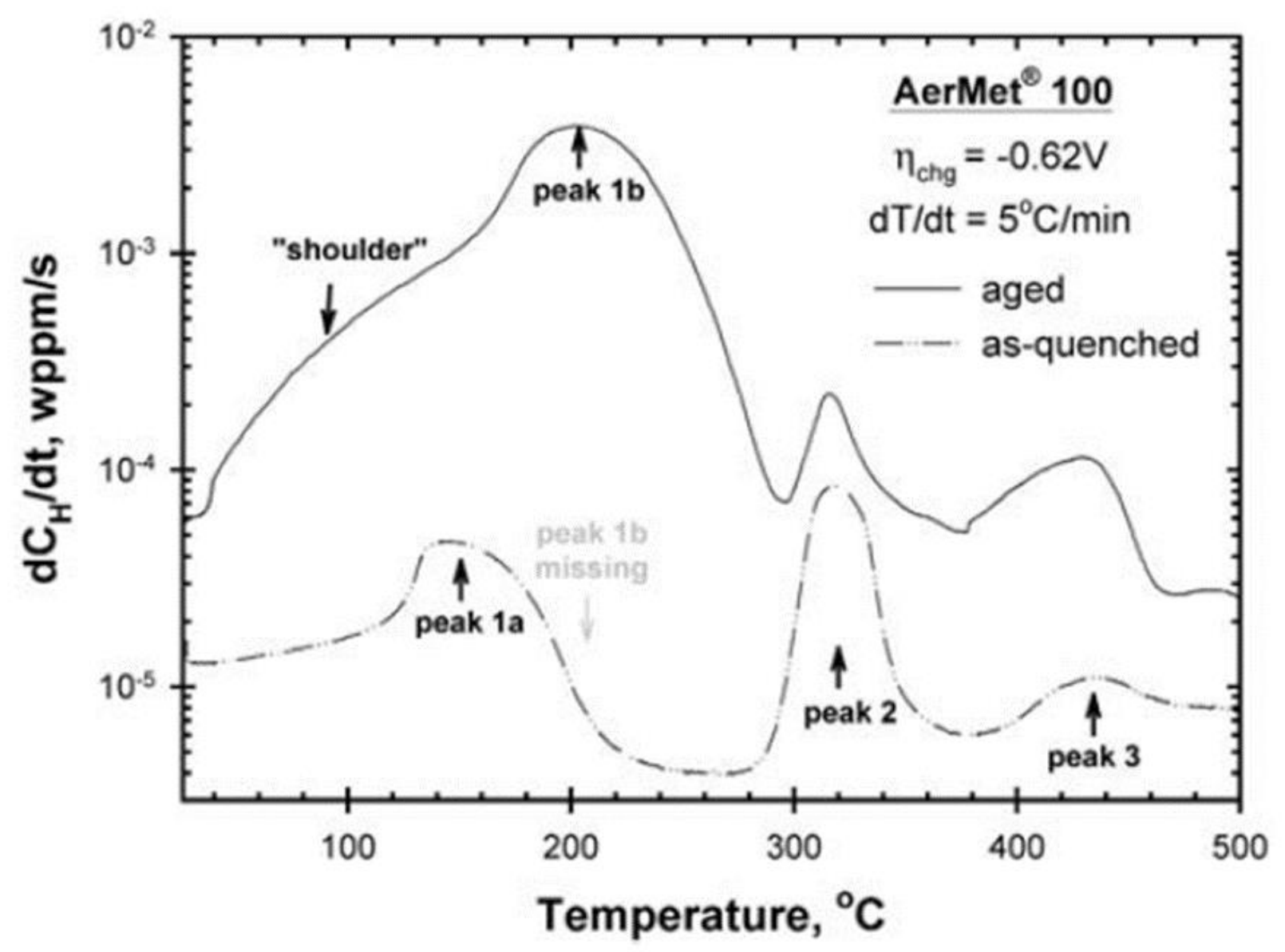

Figure $75 \quad H$-desorption rate $\left(d C_{H} / d t\right)$ versus temperature for as-quenched (dashed line) and aged (solid line) AerMet 100, H-charged at $\eta_{\text {chg }}=-0.62 \mathrm{~V}$ and TDS tested at a heating rate $(d T / d t)$ of $5^{\circ} \mathrm{C} / \mathrm{min}$, taken from [130] 


\subsection{Discussion}

The slow-rising $\mathrm{K}$ method, with precision dcPD monitoring of crack growth, effectively characterized the SCC resistance of two ultra-high strength martensitic stainless steels across a wide range of $\mathrm{E}_{\mathrm{app}}$ and aging conditions/strength levels. Results establish that modern secondary hardening Ferrium PH48S exhibits good resistance to SCC, with exclusively transgranular cracking suggesting a generic HEAC mechanism similar to that encountered in several modern low alloy UHSS alloys (Chapter 3). Further, mild cathodic polarization is demonstrated to confer very high resistance to SCC, which could be used to control SCC. Compared to the Custom 465 at very high strength, crack growth in Ferrium PH48S is TG rather than IG and Stage II crack growth rates are remarkably reduced. However, the potential dependency of $\mathrm{K}_{\mathrm{TH}}$ for the onset of HEAC is identical when these two UHSSS are aged to equal strength.

Measured HEAC kinetics based on fracture mechanics, EBSD-measured microstructure and fractographic observations are correlated and interpreted to improve mechanistic understanding of three key issues: (a) the efficacy of a generic HEAC mechanism in predicting TG HEAC in UHSSS, (b) the origins of reduced SCC-HEAC resistance for Custom 465 aged to ultra-high strength with the H900 temper, and (c) the interaction of steel composition and microstructure with the applied potential dependence of HEAC kinetics in $\operatorname{UHSS}(\mathrm{S})$ alloys. Each issue is examined in the ensuing Discussion sections using the generic HELP/HEDE mechanism and micromechanical models of HEAC properties as an analytical framework for interpretation. This Discussion parallels that presented in Chapter 3 for low alloy martensitic UHSS.

\subsubsection{Hydrogen Trapping and Solubility UHSSS Alloys}

A broad literature has shown a definitive link between the hydrogen trapping behavior of UHSS and HEAC properties [6,130,137,145]. Quantitative characterization of H-trapping phenomena in Custom 465 and Ferrium PH48S provides an opportunity to examine the effect of hydrogen solubility and precipitate trapping on HEAC behavior of stainless UHSS. In a UHSS-environment system, the hydrogen solubility and diffusivity are controlled by the H-trapping in the alloy microstructure. In general, as the number density or binding energy of H-traps in the microstructure increases, H-solubility increases and hydrogen diffusivity decreases $[19,130,178]$. The effect H-trapping on solubility and diffusivity is critical for understanding the SCC-HEAC 
behavior of an alloy due to their direct effect on H-localization to the FPZ where $\mathrm{H}$-damage occurs. This effect is clearly visible in Equations 1 and 2 (reproduced below from Chapter 1), where the effective hydrogen diffusivity and stress enhanced crack tip $\mathrm{H}$ concentration dictate HEAC properties of the equilibrium $\mathrm{K}_{\mathrm{TH}}$ and kinetics based da/dtII.

$$
\begin{gathered}
\text { (1) } K_{T H}=\frac{1}{\beta^{\prime}} \exp \frac{\left(k_{I G}-\alpha C_{H \sigma}\right)^{2}}{\alpha^{\prime \prime} \sigma_{Y S}} \\
\left(\frac{d a}{d t}\right)_{I I}=\frac{4 D_{H-E F F}}{\chi_{c r i t}}\left\{\operatorname{erf}^{-1}\left(1-\frac{C_{H \sigma-c r i t}}{C_{H \sigma}}\right)\right\}^{2}
\end{gathered}
$$

Hydrogen trapping will directly affect the HEAC properties in two primary ways. First, the presence of $\mathrm{H}$-traps enhances the solubility of hydrogen in the microstructure for increasing $\mathrm{C}_{\mathrm{H \sigma}}$. Assuming that a relatively small amount of hydrogen is trapped at lattice sites, the concentration of reversibly trapped hydrogen for a single trap state at equilibrium $\left(\mathrm{C}_{\mathrm{T}, \mathrm{r}}\right)$ is given by [130]:

$$
C_{T, r}=C_{0} \frac{N_{R}}{N_{L}}\left[\exp \left(\frac{E_{B}}{R T}\right)\right]
$$

Where $\mathrm{C}_{0}$ is the equilibrium, perfect lattice $\mathrm{H}$-solubility governed by the heat of solution for an ideal dilute solution, $N_{R}$ is the number of reversible trap sites per volume, $N_{L}$ is the number of interstitial lattice trap sites per unit volume, and $\mathrm{E}_{\mathrm{B}}$ is the binding energy of $\mathrm{H}$ to the single-specific trap site. For an alloy with a single reversible trap state and relatively little lattice solubility $\left(\mathrm{C}_{0}\right.$ $<<1), C_{T, r}$ equals $C_{H-D I F F}$. From this assumption the interactive effect of a single reversible hydrogen trap state and hydrostatic stress can be quantified [20]:

$$
\left.C_{H \sigma}=C_{H-D I F F}\left[\exp \left(\frac{\sigma_{H} V_{H}}{R T}\right)\right]=C_{0} \frac{N_{R, i}}{N_{L}}\left[\exp \left(\frac{E_{B}}{R T}\right)\right]\left[\exp \left(\frac{\sigma_{H} V_{H}}{R T}\right)\right]\right)
$$

Second, the distribution and strength of hydrogen trap states will directly affect the effective hydrogen diffusivity ( $\mathrm{D}_{\text {H-EFF}}$ ). In a trap free microstructure, hydrogen diffusion is governed by random-jump interstitial diffusion, as characterized by the ideal lattice diffusivity $\left(\mathrm{D}_{\mathrm{L}}\right)$ with energy barrier of $\mathrm{E}_{\mathrm{m}}$. Microstructural heterogeneities such as precipitates, grain boundaries, or dislocations are prime sites for reversible $\mathrm{H}$ trapping, with $\mathrm{E}_{\mathrm{B}}>\mathrm{E}_{\mathrm{M}}$, and thus provide a barrier to 
$\mathrm{H}$-diffusion and decrease $\mathrm{D}_{\mathrm{H}-\mathrm{EFF}}$, as is particularly observed for BCC-ferrous alloys. The trap affected hydrogen diffusivity for an alloy with a single dominant trap state is given by [137,178]:

$$
D_{H}=\frac{D_{L} C_{0}}{C_{0}+C_{T, r}\left(1-\theta_{T}\right)}
$$

where $\theta_{\mathrm{T}}$ is the fractional $\mathrm{H}$-trap site coverage and $\mathrm{C}_{\mathrm{T}, \mathrm{r}}$ is given by Equation 11. Thomas et al. proposed that $\theta_{\mathrm{T}} \ll<1$ and $\mathrm{C}_{0} \ll \mathrm{C}_{\mathrm{T}, \mathrm{r}}\left(1-\theta_{\mathrm{T}}\right)$, justified by the low $\mathrm{H}$ solubility of pure iron and high H-solubility of modern UHSS AerMet 100 [137]. Application of these two assumptions to Equation 14 yields the inverse proportionality of reversibly trapped hydrogen concentration and DH-EFF [137]:

$$
\frac{D_{H}}{D_{L}} \approx \frac{C_{0}}{C_{T, r}} \approx \frac{C_{0}}{C_{H-D I F F}}
$$

These literature relationships for the effect of H-trap states on $\mathrm{C}_{\mathrm{H \sigma}}$ (Equation 12) and $\mathrm{D}_{\mathrm{H} \text {-EFF }}$ (Equation 14) will be used to interpret HEAC properties $\left(\mathrm{K}_{\mathrm{TH}}, \mathrm{da} / \mathrm{dt}_{\mathrm{II}}\right)$ in the context of hydrogen trapping and solubility data in ensuing sections.

Despite microstructural similarities, Custom 465 and Ferrium PH48S exhibit a significant departure from literature measurements of hydrogen trapping and solubility in AerMet 100 [130,137]. Departure from baseline AerMet 100 behavior is centered on three notable observations of H-trapping in UHSSS. First, hydrogen solubility in aged Custom 465-H900 is significantly enhanced compared to all aging conditions of Ferrium PH48S (Table 12). Further, the combination of TDS and H-outgassing results indicates that hydrogen is exclusively trapped in room temperature reversible trap states for each of these two steels. Second, there is no evidence of multiple trap states in UHSSS, despite a similar precipitation strengthened martensitic microstructure. This observation does not preclude the presence of multiple overlapping H-trap peaks with a similar-small binding energy. The gaussian nature of the H-desorption peaks in (Figure 73) and the distinct absence of peak 'shoulders' (compare the low temperature peak Figure 73 and Figure 75) imply that this is unlikely. Third, H solubility decreases with aging of Ferrium PH48S, compared to the as-quenched microstructure (Table 12). Each issue will be examined for a possible microstructural origins and impact on HEAC properties. 


\subsubsection{Effect of Increased H-Solubility in Custom 465}

As shown in Figure 71, the H-solubility of UHSSS exhibits a strong alloy and heat treatment dependence. Total hydrogen concentration in Custom 465 - H900 is significantly enhanced compared to aged Ferrium PH48S (72.5 vs. $2.3-8.4$ wppm) when $\mathrm{H}$ is precharged in $0.001 \mathrm{M}$ $\mathrm{NaOH}(\mathrm{pH}=10)$ at a single overpotential for hydrogen production $\left(\eta_{\mathrm{H} / \mathrm{H}+}=-520 \mathrm{mV}\right)$. For Custom 465 - H900 and Ferrium PH48S - H900, $\mathrm{C}_{\text {H-TOTAL }}$ and $\mathrm{C}_{\mathrm{H} \text {-DIFF }}$ are assumed identical based on the presence of a presumably single-reversible H-desorption peak (Figure 73). Further, H-precharged specimens of each steel asymptotically approach the $\mathrm{C}_{\text {H-TOTAL }}$ level of uncharged specimens, indicating that all trapped $\mathrm{H}$ is diffusible at room temperature. Increased $\mathrm{H}$-solubility in the Custom 465 is significant for all charging times and is confirmed by the relative size of $\mathrm{H}$ desorption peaks during TDS testing (Figure 73). Prior work has established the $\eta_{\mathrm{H}^{\mathrm{H}+}}$ dependency

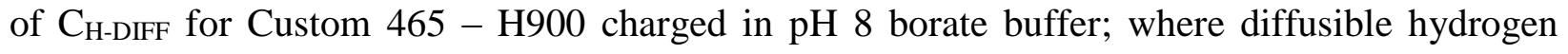
concentration reached a stable value of $16.5-17.5 \mathrm{wppm}$ at $\eta_{\mathrm{H} / \mathrm{H}+}<-500 \mathrm{mV}$ [105]. A similar $E_{\text {app }}$ dependency of $\mathrm{C}_{\mathrm{H}-\mathrm{DIFF}}$ is observed in identically charged AerMet 100, which reaches a stable value of 6 - 10 wppm at $\eta_{\mathrm{H} / \mathrm{H}+}<-500 \mathrm{mV}$ [105]. Notably, $\mathrm{C}_{\mathrm{H}-\mathrm{DIFF}}$ of Ferrium PH48S - H950 (8.4 wppm) in the present work is identical to AerMet 100 charged at similar $\eta$ in $\mathrm{pH} 8$ borate buffer $(6 \mathrm{wppm}$, $\left.\eta_{\mathrm{H} / \mathrm{H}_{+}}=-500 \mathrm{mV}\right)$ and saturated $\mathrm{pH} 12.1 \mathrm{Ca}(\mathrm{OH})_{2}$ at $23^{\circ} \mathrm{C}\left(6.2 \mathrm{wppm}, \eta_{\mathrm{H} / \mathrm{H}_{+}}=-500 \mathrm{mV}\right)[105,137]$.

As characterized by TDS in Figure 74, a single reversible H-trap state $\left(\mathrm{E}_{\mathrm{B}}=12.1-12.2 \mathrm{~kJ} / \mathrm{mol}\right)$ appears to dominate hydrogen trapping in Custom 465 - H900 and Ferrium PH48S - H900 when $\mathrm{H}$ is precharged in $0.001 \mathrm{M} \mathrm{NaOH}(\mathrm{pH}=10)$ for $609 \mathrm{~h}$ at $\eta_{\mathrm{H} / \mathrm{H+}}=-520 \mathrm{mV}$. The reversibility of this single trap state (or combination of similar trap states) is confirmed by the low $\mathrm{E}_{\mathrm{B}}$ and long term H-outgassing experiments, which show that there is sufficient thermal energy at room temperature for essentially all $\mathrm{H}$ to diffuse out of precharged specimens (Figure 71). Prior work has demonstrated that the dominant low $\mathrm{E}_{\mathrm{B}}(11.4-11.6 \mathrm{~kJ} / \mathrm{mol})$ peak in AerMet 100 corresponds to the nano-scale, coherent $\mathrm{M}_{2} \mathrm{C}$ strengthening carbide [130]. This result was confirmed by close matching of the calculated amount of $\mathrm{H}$ necessary for full coverage of all $\mathrm{M}_{2} \mathrm{C}$ precipitates and the total amount of atomic $\mathrm{H}$ measured for the strengthening precipitate peak via TDS, as well as by TDS experiments which showed that this peak was not present in as-quenched AerMet 100 without $\mathrm{M}_{2} \mathrm{C}$ from aging. [74,130]. Congruency of $\mathrm{E}_{\mathrm{B}}$ for the H-desorption peak presented in Figure 73 with $\mathrm{E}_{\mathrm{B}}$ of $\mathrm{M}_{2} \mathrm{C}$ carbides in AerMet 100 indicates that this peak is likely associated with nano- 
scale coherent strengthening precipitates in aged Ferrium PH48S and aged Custom 465. Critically, the lack of multiple resolvable H-desorption peaks in UHSSS demonstrates that essentially all of $\mathrm{C}_{\mathrm{H}-\mathrm{TOTAL}}$ is trapped in a single reversible trap state, or multiple trap states with the same-low binding energy. As such, significantly increased $\mathrm{C}_{\mathrm{H}-\mathrm{DIFF}}$ in Custom 465 - H900 compared to Ferrium PH48S must be linked to a difference in H-trapping at $\eta$ or similar strengthening precipitates.

Four possible microstructural differences between Custom 465 - H900 and Ferrium PH48S $\mathrm{H} 900$ could explain the large change in $\mathrm{H}$-solubility. Close agreement of $\mathrm{C}_{\mathrm{H}-\mathrm{TOTAL}}$ in $\mathrm{H}$-precharged Custom 465 - AQ and Ferrium PH48S - AQ (Figure 71) suggests that an electrochemical or

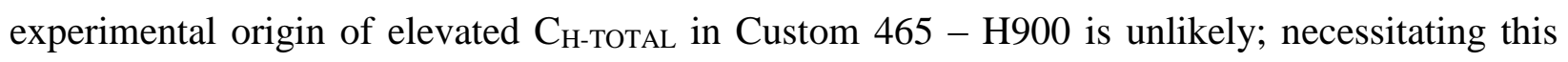
microstructural focus. First, ThermoCalc predictions indicate an increase in the amount of $\eta$ phase precipitates in Custom 465 -H900 relative to Ferrium PH48S -H900 (8.5 versus 3.8 vol\%). At constant precipitate size and coherency this would imply a 2.2 times increase in the H-trap-site density (Equation 11, $\mathrm{N}_{\mathrm{R}}$ ) associated with the surfaces of $\eta$ precipitates. The total amount of hydrogen trapped by the surface of $\eta$ precipitates would be therefore increased by a factor of 2.2 times, and $\mathrm{C}_{\mathrm{H}-\mathrm{DIFF}}$ would proportionately rise.

Second, if multiple layers of hydrogen can be trapped on the interface area of a single $\eta$ precipitate in Custom 465, then it would proportionately raise the total amount of trapped H. Multiple Hlayers on a single precipitate has been suggested by Li et al. to reconcile a large discrepancy between measured and calculated site density of $\mathrm{M}_{2} \mathrm{C}$ carbide traps in conventionally tempered AerMet 100 [130]. Increased interfacial H-trapping would be enabled by a change in $\eta$-matrix interface coherency or the density of dislocations along the precipitate interface. The $\eta$ phase coherency is strongly dependent on precipitate size, as well as composition-lattice parameter differences between the precipitate and matrix [179]. However, no literature information is available on the size/coherency of $\eta$ in Custom 465. Limited atom probe measurements indicate that the rod shaped $\eta$ precipitates present in Ferrium PH48S - H968 are 5-10 nm in diameter, similar to the $\mathrm{M}_{2} \mathrm{C}$ in optimally aged AerMet 100 [24]. Significant addition of cobalt in Ferrium PH48S may refine the $\eta$ phase distribution by preserving martensite dislocation structure for $\eta$ nucleation and reducing the solubility of precipitate forming element(s) in the matrix $[25,42,78]$. Increased $\eta$ precipitate density and surface trapping provides a microstructural explanation for 
enhanced H-solubility in Custom 465, in the context of a single $\mathrm{H}$-desorption peak. This hypothesis must be confirmed through future work to quantify the distribution and morphology of strengthening precipitates in UHSSS through transmission electron microscopy.

Third, the presence of multiple H-trap states in Custom 465-H900 with similar $\mathrm{E}_{\mathrm{B}}$ and apparentsingle H-TDS peak could increase $\mathrm{C}_{\text {H-тотаL }}$ compared to a single H-trap state in Ferrium PH48S. As discussed in Chapter 2, The precipitation sequence, composition, and morphology of intermetallic particles in precipitation strengthened stainless steels is controversial and the presence of multiple strengthening precipitate types cannot be ruled out [80]. Grujicic et al. indicates that intermetallic precipitation in Custom 465 at short aging times is dominated by needle shaped coherent hexagonal $\omega$-phase which gradually transforms into equilibrium orthorhombic $\eta$ phase $\mathrm{Ni}_{3}(\mathrm{Ti}, \mathrm{Mo})[81,82]$. $\omega$-phase precipitates at short aging times as coherent, needle-shaped precipitates with a hexagonal crystal structure, identical to $\mathrm{M}_{2} \mathrm{C}$ precipitates in AerMet 100 $[24,81]$. Good coherence with the BCC matrix and identical hexagonal crystal structure suggests that the $\omega$ precipitate would have a similar $\mathrm{H}$-trap binding energy to $\mathrm{M}_{2} \mathrm{C}$ carbides. The presence of identical $E_{B} \omega$-phase trap states would increase the H-trap-site density (Equation 11, $\mathrm{N}_{\mathrm{R}}$ ) and

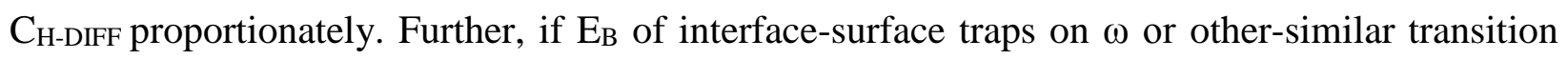
precipitates increased somewhat compared to equilibrium $\eta, \mathrm{C}_{\mathrm{H}-\mathrm{DIFF}}$ would be increased dramatically due to this secondary precipitate distribution. For example, increasing $\mathrm{E}_{\mathrm{B}}$ by $25 \%$ (12 to $15 \mathrm{~kJ} / \mathrm{mol}$ ) would cause a 3 times rise in the equilibrium concentration of $\mathrm{H}$ trapped on that precipitate distribution. It is unknown if such a small rise in $E_{B}$ would be detectable via the TDS method. Speculatively, a secondary fine scale distribution of coherent $\omega$-phase precipitates could explain the large difference in $\mathrm{C}_{\mathrm{H}-\text {-тотAL }}$ in $\mathrm{H}$-precharged Custom 465 and Ferrium PH48S. Confirmation of this hypothesis requires future work to quantify the distribution of precipitates in Custom 465 using TEM or local electron probe techniques. Regardless of the microstructural origin, increased hydrogen solubility in Custom 465 - H900 is significant compared to all heat treatments of Ferrium PH48S (72.5 vs. 2.3 to 8.4 wppm; 8.6 to 34.5 times increased).

Finally, it is well known that certain intermetallic compounds ( $\left.\mathrm{LaNi}_{5}, \mathrm{FeTi}, \mathrm{Ti}_{1.1} \mathrm{CrMn}, \mathrm{Ti}_{1.1} \mathrm{CrV}\right)$ with $\mathrm{AB}_{2}$ Laves, $\mathrm{AB}_{5}$, and $\mathrm{BCC}$ Ti-based crystal structures and reactive elements form hydride phases when exposed to $\mathrm{H}$ at near ambinent temperature $[180,181]$. In this context a large amount of $\mathrm{H}$ dissolves on sites within the intermetallic lattice, constituting a mechanism for volume 
trapping of $\mathrm{H}$ to greatly enhance the concentration of $\mathrm{H}$ which is trapped on precipitate interfaces. This $\mathrm{H}$ storage behavior leads to the speculation that the crystal structure and composition of Tirich $\eta$ in Custom 465-H900 favors volume trapping of $\mathrm{H}$, while this structure and composition is altered for Ferrium PH48S so as to not form the intermetallic hydride [175]. The structure of intermetallic strengthing precipitates in martensitic steels is strongly dependent on alloy composition and heat treatment [78,84-87]. As such, the possibility of a structure change in the strengthing precipitate cannot be ruled out. XRD evidence suggests that the HCP/ordered orthorhombic structure of $\eta$ in Custom 465-H900 changed to an ordered FCC phase (perhaps $\mathrm{Ni}_{3} \mathrm{Al}$ or similar precipitate) in aged Ferrium PH48S which contains increased Al compared to Custom 465; as necessary to support this mechanism for a $\mathrm{H}$ solubility difference. Since intermetallichydride phase transformation is reversible upon removal of the $\mathrm{H}$ source [181], it follows that an effective $E_{B}$ would be relatively low. The large TDS peak for Custom 465-H900, produced at relatively low temperature (Figure 73) and without higher temperature peaks, is consistent with this speculation. Volume trapping of $\mathrm{H}$, enabled by exposure near ambinent temperature, has never been reported for strengthening precipitates in UHSS [39]. Significant work is required to identify the specific strengthening precipiates in Custom 465 and Ferrium PH48S, for high strength aging conditions, and to examine $\mathrm{H}$ trapping associated with each phase [174].

H-trapping has two strong effects on HEAC properties for AerMet 100 [6,20,130,137]. The stress affected crack tip hydrogen concentration $\left(\mathrm{C}_{\mathrm{H \sigma}}\right.$, Equation 12) is proportionately increased with the diffusible hydrogen concentration, assuming that this quantity is in equilibrium with $\mathrm{H}$ in lattice sites which are dilated by hydrostatic stress. Previous work indicates that $\mathrm{C}_{\mathrm{H}-\mathrm{DIFF}}$ reaches a stable value at $\eta_{\mathrm{H} / \mathrm{H}+}<-500 \mathrm{mV}$ in Custom $465-\mathrm{H} 900$ [105]. While not measured, an identical stabilization of $\mathrm{C}_{\mathrm{H}-\mathrm{DIFF}}$ versus $\eta_{\mathrm{H} / \mathrm{H}+}\left(\mathrm{E}_{\mathrm{app}}\right)$ is expected for Ferrium PH48S at $\eta_{\mathrm{H} / \mathrm{H}+}<-500 \mathrm{mV}$. Typical $\eta_{\mathrm{H} / \mathrm{H}+}$ are much lower for HEAC relevant levels of $\mathrm{E}_{\mathrm{app}}$, and the ratio of $\mathrm{C}_{\mathrm{H}-\mathrm{DIFF}}$ in Custom - 465 compared to AerMet 100 was shown to decrease with increasing $\eta_{H / H+}$ in this range [105]. Without characterization of the $\mathrm{E}_{\text {app }}$ dependence of $\mathrm{C}_{\mathrm{H} \text {-DIFF }}$ for Ferrium PH48S, it is not possible to precisely know the change in $\mathrm{C}_{\mathrm{H}-\mathrm{DIFF}}$ between UHSSS at each $\mathrm{E}_{\mathrm{app}}$. However, for HEAC relevant cathodic $\mathrm{E}_{\mathrm{app}}, \mathrm{C}_{\mathrm{H}-\mathrm{DIFF}}$ is increased in Custom 465 - H900, up to a maximum of $~ 30$ times compared to Ferrium PH48S at heavily cathodic $\mathrm{E}_{\mathrm{app}}$. $\mathrm{K}_{\mathrm{TH}}$ predicted by Equation 1, is drastically affected by increasing $\mathrm{C}_{\mathrm{H \sigma}}$. Although the precise values cannot be determined, it is expected that increased $\mathrm{H}-$ 
solubility will decrease $\mathrm{K}_{\mathrm{TH}}$ for Custom 465 relative to Ferrium PH48S. However, Figure 67 shows that $\mathrm{K}_{\mathrm{TH}}$ is essentially equal in Custom 465 and Ferrium PH48S when compared at equal strength. This discrepancy between modeled and observed behaviors will be discussed in ensuing sections.

Following the proposed correlation of $\mathrm{D}_{\mathrm{H}} / \mathrm{D}_{\mathrm{L}}$ to $\mathrm{C}_{0} / \mathrm{C}_{\mathrm{T}, \mathrm{r}}$ (Equation 14) for AerMet 100, Custom 465 is expected to have significantly decreased $\mathrm{D}_{\mathrm{H}}$ compared to Ferrium PH48S. There is no reason to suspect that $\mathrm{D}_{\mathrm{L}}$ or $\mathrm{C}_{0}$ would significantly vary between Custom 465 and Ferrium PH48S. Substitutional solid solution Co atoms in the matrix of Ferrium PH48S may slow H-diffusion through the lattice $\left(D_{L}\right)$, but this effect is likely negligible [182]. As shown in Figure 71, all the precharged hydrogen in both Custom 465 - H900 and Ferrium PH48S is reversibly trapped, indicating that $\mathrm{C}_{\mathrm{H}-\mathrm{DIFF}}=\mathrm{C}_{\mathrm{T}, \mathrm{r}}$. It follows from Equation 14 that $\mathrm{D}_{\mathrm{H}}$ should be inversely proportional in to $\mathrm{C}_{\mathrm{H}-\mathrm{DIFF}}$ in Custom 465 - H900 and Ferrium PH48S. This implies that effective hydrogen diffusivity in Custom 465 - H900 will be decreased 31.5 to 8.6 times compared to the aging conditions of Ferrium PH48S - H950; proportional to the variation in $\mathrm{C}_{\mathrm{H}-\mathrm{DIFF}}$ shown in Figure 71. Regression fit $\mathrm{D}_{\mathrm{H}-\mathrm{EFF}}$ (Table 12) is either constant decreased by 1.5 times or increased 2.2 - 28 times for Custom 465-H900 compared to the aging conditions of Ferrium PH48S, for H-uptake and outgassing, respectively. In the case of $\mathrm{H}$-uptake, the results shown in Table 12 indicate that H-solubility should be on 1.5-times higher for Custom 465-H900 compared to aged Ferrium PH48S. Conversely, reduced $\mathrm{D}_{\mathrm{H}-\mathrm{EFF}}$ for H-outgassing indicates that H-solubility should be increased in Ferrium PH48S; which was not observed. These results stand in direct opposition to the proportionality of $\mathrm{D}_{\mathrm{H}} / \mathrm{D}_{\mathrm{L}}$ to $\mathrm{C}_{0} / \mathrm{C}_{\mathrm{T}, \mathrm{r}}$ proposed for AerMet 100 and based on H-trapping theory [177]. The origins of this anomalous behavior in Custom 465 is unknown.

\subsubsection{Absence of H-Trap States in UHSSS}

Hydrogen desorption spectra for H-precharged UHSSS alloys exhibit a significant departure from near-identical TDS measurements for AerMet 100. As shown in Figure 75, three major Hdesorption peaks are resolved for the precipitation hardened microstructure of AerMet 100. Prior work identified the corresponding microstructural feature for each H-trap state [130]. Peak 1b corresponds to the primary strengthening precipitate, coherent $\mathrm{M}_{2} \mathrm{C}$ carbides. An analysis of literature H-trap binding energies indicated that Peak 2 is associated with martensite interfaces, austenite grain boundaries, and mixed dislocation cores. Peak 3 corresponds to undissolved, incoherent metal carbides retained during thermo-mechanical processing and solution treatment. 
Critically, a single $\mathrm{H}$-desorption peak associated with the $\eta$-phase strengthening precipitate is observed in for two UHSSS alloys (Figure 73). No higher energy peaks are observed and the desorption rate in Figure 73 quickly falls to background levels as temperature is increased above the initial peak. This result indicates that, for H-precharged specimens of Custom 465 - H900 and Ferrium PH48S - H900, the only significant H-trap has a low-reversible binding energy, corresponding to coherent $\mathrm{Ni}_{3} \mathrm{Ti}$ strengthening precipitates.

The features associated with the higher $\mathrm{E}_{\mathrm{B}}$ peaks in AerMet 100 should be present in microstructurally similar martensitic UHSSS. As shown in Table 9, the GBCD of AerMet 100, Custom 465, and Ferrium PH48S contains a variable, but significant amount of high angle interfaces. Strong reversible trapping at such interfaces $\left(\mathrm{E}_{\mathrm{B}}=\sim 60 \mathrm{~kJ} / \mathrm{mol}\right)$ would indicate a high concentration of such trapped $\mathrm{H}$ compared to that at weak reversible traps such as $\eta$ strengthening precipitates $\left(E_{B}=12 \mathrm{~kJ} / \mathrm{mol}\right)[130,183,184]$. Similar strong reversible $\mathrm{H}$-traps $\left(E_{B} \sim 60 \mathrm{~kJ} / \mathrm{mol}\right)$ at mixed dislocation cores and dislocation cell walls in the martensitic UHSSS microstructure are expected to be common [185]. The site density of such traps has been characterized to be $10^{20}$ to $10^{23}$ sites per $\mathrm{m}^{3}$ in cold worked ferritic iron [185]. The unrecovered martensite microstructure of UHSSS Custom 465 and Ferrium PH48S should contain a similarly large site density of H-traps at dislocation cell walls, and the strong binding energy would indicate a high concentration of trapped hydrogen.

No significant difference in the distributions of grain boundary misorientation is observed between AerMet 100 and Ferrium PH48S to explain the discrepancy in UHSSS TDS spectra (Figure 73 vs. Figure 75). Interfacial area of random, high energy interfaces per volume of steel is increased in Ferrium PH48S $\left(1,761 \mathrm{~mm}^{2} / \mathrm{mm}^{3}\right)$ relative to AerMet $100\left(1,456 \mathrm{~mm}^{2} / \mathrm{mm}^{3}\right)$, which is in turn increased relative to Custom $465\left(695 \mathrm{~mm}^{2} / \mathrm{mm}^{3}\right)$ in Table 9 . The expectation from this result is a proportionate increase in the concentration of $\mathrm{H}$ trapped at a high $\mathrm{E}_{\mathrm{B}}(\sim 60 \mathrm{~kJ} / \mathrm{mol})$ martensitebased peak in Ferrium PH48S compared to Aermet 100, which in turn should show a higher highEв peak compared to Custom 465. This is opposite to the TDS observations. At present, there is no hypothesis for the lack of significant $\mathrm{H}$-trapping at the higher $\mathrm{E}_{\mathrm{B}}$ martensite interfaces, austenite grain boundaries, and mixed dislocation cores in Custom 465 and Ferrium PH48S. 
Considering the lower energy CSL interfaces quantified in Table 9, the interfacial area is 2,034 $\mathrm{mm}^{2} / \mathrm{mm}^{3}$ for Ferrium PH48S, $1,016 \mathrm{~mm}^{2} / \mathrm{mm}^{3}$ for AerMet 100 , and $168 \mathrm{~mm}^{2} / \mathrm{mm}^{3}$ for Custom 465. Good atomic fit across a martensite interface with a CSL orientation relationship could speculatively reduce the $E_{B}$ of such interfaces [52,57]. Taken together these results could speculatively suggest a potentially significant concentration of $\mathrm{H}$, trapped in Ferrium PH48S at a lower $\mathrm{E}_{\mathrm{B}}$ and included in the low temperature peak, less so for AerMet 100, and essentially not at all for Custom 465. This expectation is inconsistent with two points. First, the binding energy of grain boundaries in iron is expected to be high $\left(\mathrm{E}_{\mathrm{B}}>50 \mathrm{~kJ} / \mathrm{mol}\right)$ and consistent for two dimensional defects [185]. A hypothesized-severe reduction in $\mathrm{E}_{\mathrm{B}}$ due to good atomic fit at a CSL boundary would yield a $\mathrm{H}$-desorption peak at moderate $\mathrm{E}_{\mathrm{B}}$ (between peak 1a and peak 2 in Figure 75). The lack of a moderate-low $\mathrm{E}_{\mathrm{B}}$ peak in AQ AerMet 100 (Figure 75) is inconsistent with this hypothesis [130]. Second, the density of all martensite interfaces is dramatically increased in AerMet 100 and Ferrium PH48S $\left(2,472\right.$ and $\left.3,795 \mathrm{~mm}^{2} / \mathrm{mm}^{3}\right)$ compared to Custom $465\left(863 \mathrm{~mm}^{2} / \mathrm{mm}^{3}\right)$. Regardless of the relative distribution of CSL and randomly oriented interfaces, increased martensite interfacial density will increase the site density of martensite interface H-traps. If there is no significant difference in the $\mathrm{E}_{\mathrm{B}}$ of CSL and random high angle martensite interfaces, then the site density and concentration of reversibly trapped hydrogen should increase proportionately: 2.8 and 4.4 times for AerMet 100 and Ferrium PH48S compared to Custom 465. This prediction is not consistent with observations which demonstrate that H-solubility in Custom 465-H900 is dramatically higher than that measured for AerMet 100 or Ferrium PH48S (Figure 71 and $[73,105])$. Taken together, the present results indicate that there is relatively little hydrogen trapping at martensite interfaces in UHSSS alloys compared to modern UHSS AerMet 100. At present, there is no fundamental hypothesis why the population of random martensite boundaries in Ferrium PH48S or Custom 465 do not trap significant hydrogen.

Similar to martensite interfaces, high $\mathrm{E}_{\mathrm{B}}$ will promote strong trapping of $\mathrm{H}$ at incoherent MC-type carbides $\left(\mathrm{E}_{\mathrm{B}}=77-94 \mathrm{~kJ} / \mathrm{mol}\right)$ in Custom 465 and Ferrium PH48S [183]. Speculatively, the lack of a very high temperature peak in either UHSSS is explained by the increased size of incoherent carbides in Ferrium PH48S (Figure 54) and Custom 465 (Figure 55) compared to AerMet 100. Hydrogen trapping at such carbides is based on surface area and a coarse distribution of carbides would have a significantly reduced H-trapping capacity compared to a fine distribution [186]. No 
characterization of the incoherent carbide distribution in conventionally tempered AerMet 100 is available in the literature to verify this hypothesis. However, trapping at these features is not relevant to understanding or controlling HEAC.

\subsubsection{Mechanism of HEAC in Modern UHSSS}

As established in Chapter 3, a broad amount of fractographic evidence points toward a common mechanism for TG HEAC in modern UHSS; specifically, the morphology and crystallography of martensite packets and blocks, and the free impurity content that can segregate to interfaces, dictate the HEAC fracture path. Modern martensitic UHSSS alloys examined in this study display a strongly alloy-dependent HEAC fracture morphology and the fracture path has not been previously established. The efficacy of the proposed generic HEAC mechanism in explaining the HEAC fracture morphology of modern UHSSS such as Ferrium PH48S is discussed.

The coupled HELP/HEDE mechanism suggested by Robertson and coworkers provides a set of logical and physically-based mechanistic steps for the nucleation and propagation of HEAC $[11,12,161]$. To review the discussion introduced in Chapter 3, four steps are proposed: (1) Henhanced dislocation glide and interactions are localized along slip planes (typically $\{110\}_{\alpha}$, in martensite) and redistribute $\mathrm{H}$ to martensite boundaries which are parallel to $\{110\}_{\alpha}$, (2) $\mathrm{H}$ enhanced plasticity work hardens the microstructure local to the slip band - boundary intersection to support locally high tensile and hydrostatic stresses, (3) dislocations deposit $\mathrm{H}$ at interaction points of slip bands with microstructural interfaces, and (4) a microcrack initiates and propagates along the block/lath interface by decohesion of the H-embrittled boundary [12]. This sequence could also provide a means to locally nucleate a crystallographic cleavage crack in martensite blocks, perhaps along $\{100\}_{\alpha}$, and due to $\mathrm{H}$ decohesion [116]. The site of fracture initiation is determined by the combination of highest local stress and hydrogen accumulation; a weakest link criterion. Fracture is likely to initiate at microstructural irregularities, such as boundary intersections or precipitates, on lath/block boundaries in the localized FPZ just ahead of the crack tip. 


\subsubsection{TG HEAC of Ferrium PH48S}

Transgranular HEAC of aged Ferrium PH48S exhibits the characteristic quasi-cleavage morphology indicative of cracking along martensite block boundaries, and evidence was obtained of interspersed broad facets which are perhaps crystallographic. The quasi-cleavage fracture morphology was linked in Chapter 3 to $\mathrm{H}$-driven decohesion of martensite block interfaces following the mechanism summarized above. Decohesion of block interfaces, rather than prior austenite grain boundaries, is hypothesized to be a result of slip bands preferentially intersecting the higher areal fraction of martensite block boundaries rather than prior austenite grain boundaries [12]. Close congruency of QC features with previous observations supports the hypothesis that a single HEAC damage mechanism is active both in UHSS (Chapter 3) and UHSSS discussed here.

Direct comparison of QC HEAC appearance and EBSD measured microstructure at high resolution (Figure 76) shows the role of UHSS martensite microstructure in developing the TG QC HEAC morphology. TG HEAC of AerMet 100 (Figure 76a) in 3.5\% $\mathrm{NaCl}$ at $\mathrm{E}_{\mathrm{app}}=-1,000$ $\mathrm{mV}_{\text {SCE }}$ shows an identical appearance to Ferrium PH48S stressed at similar-cathodic $\mathrm{E}_{\text {app }}=-975$ $\mathrm{mV}_{\mathrm{SCE}}$ (Figure 76b). As quantified by stereological methods, the average spacing of martensite block interfaces in Ferrium PH48S - H900 $(0.51 \mu \mathrm{m})$ is reduced compared to AerMet 100 (0.81 $\mu \mathrm{m})$. This reduction is qualitatively seen in comparison of Figure $76 \mathrm{c}$ to Figure $76 \mathrm{~d}$ : the larger size of single IPF-Z color features is increased in AerMet 100 compared to PH48S. Decreased spacing of martensite block interfaces (decreased width of facet features) in Ferrium PH48S correlates to an increased density of fine lath-like features and ridges on the TG QC HEAC fracture surface (Figure 76b), relative to AerMet 100 (Figure 76a). However, despite differences in the spacing of lath-like features on the QC surface, the appearance is near identical to previous observations of QC in lath martensite [11,12,116,120]. This key congruency of QC features across UHSS/UHSSS and low alloy martensitic steels gives strong evidence of an identical HEAC mechanism, which directly interacts with fine scale martensite microstructure.

Single IPF-Z color features are identified as martensite blocks by their exclusive high angle interface with adjacent features and the common $\{111\}_{\gamma}$ habit plane. As shown in Figure 47, no sub-block structure is observed in the microstructure of Ferrium PH48S - H900, despite using an EBSD pixel size of $13 \mathrm{~nm}$. Previous characterization of lath martensite obeying K-S demonstrates that block interfaces are typically parallel to one of the variants of $\{110\}_{\alpha},[112,114]$. As detailed 
in Chapter 3, TG HEAC of martensitic steels has been unambiguously shown to occur along $\{110\}_{\alpha}$ planes [116]. Quasi-cleavage HEAC, identical to that observed in Ferrium PH48S - H900 has been shown to be an inter-lath phenomena in a Ni-alloyed martensitic steel [120]. Cracking along or parallel to $\{110\}_{\alpha^{\prime}}$ lath/block interfaces was confirmed by Shibata et al. in H-embrittled carbon steel [125]. In the context of the present results, this literature implies that transgranular quasi-cleavage in Ferrium PH48S nucleates and propagates predominantly along high angle $\{110\}_{\alpha}$, block boundaries. 


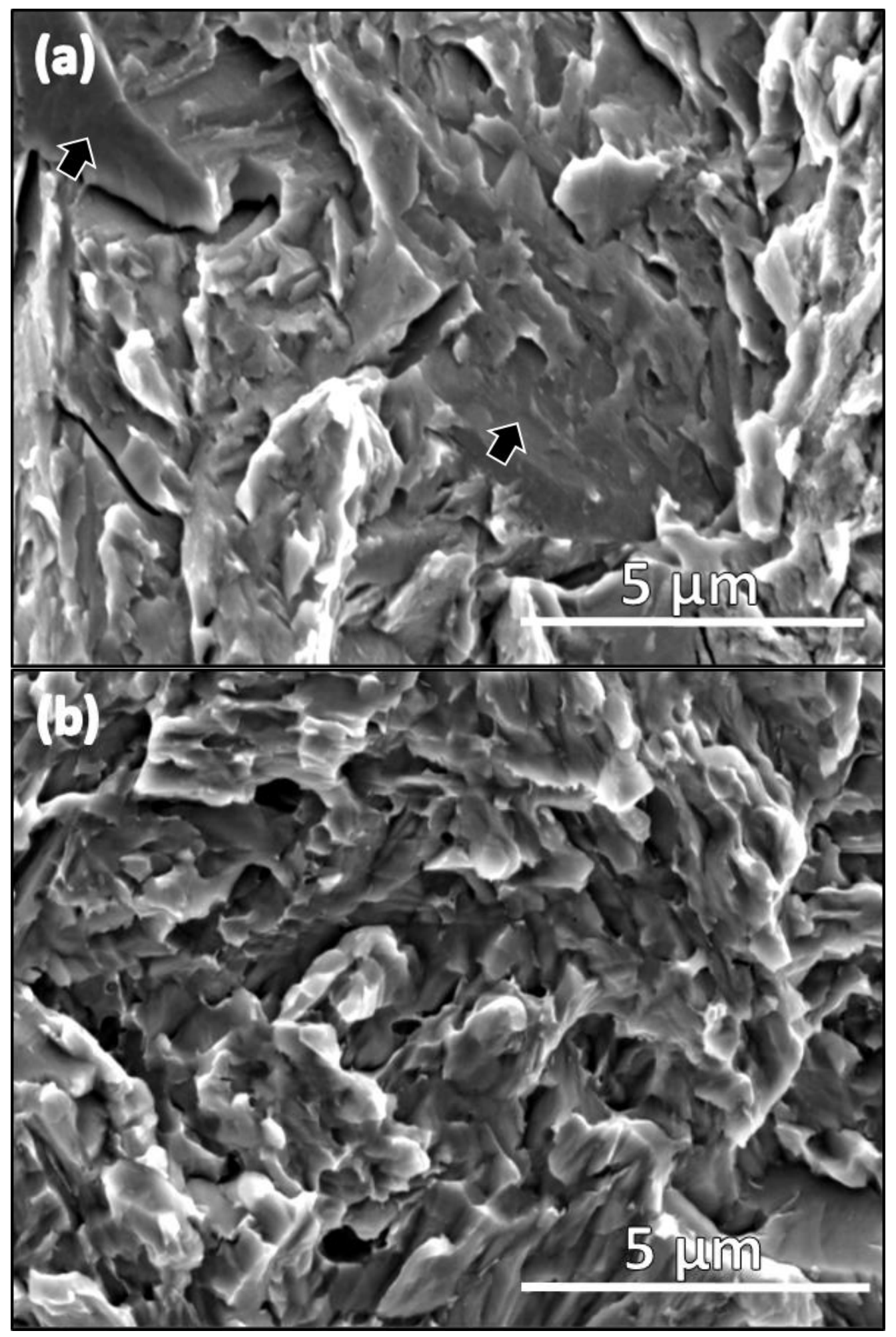



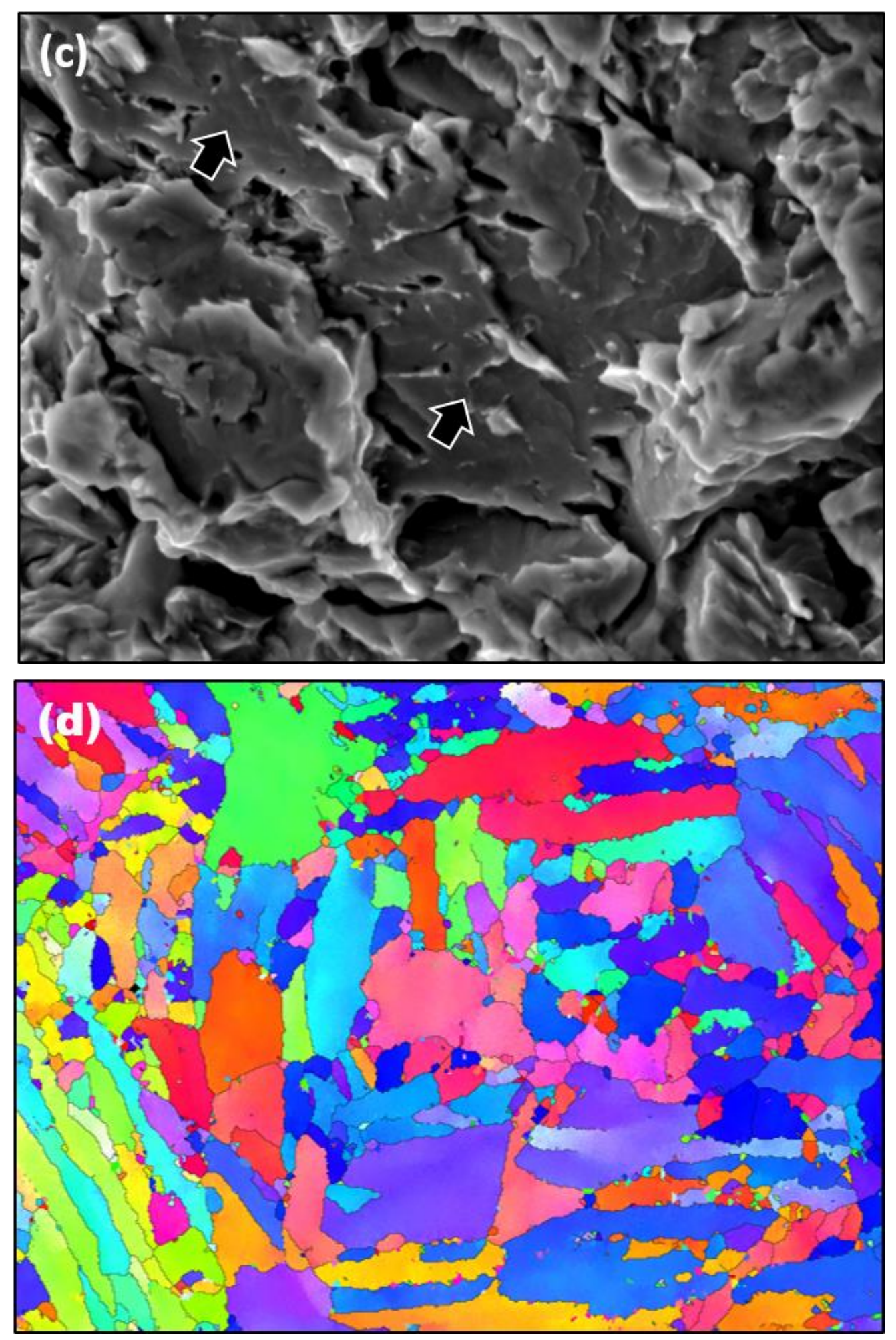


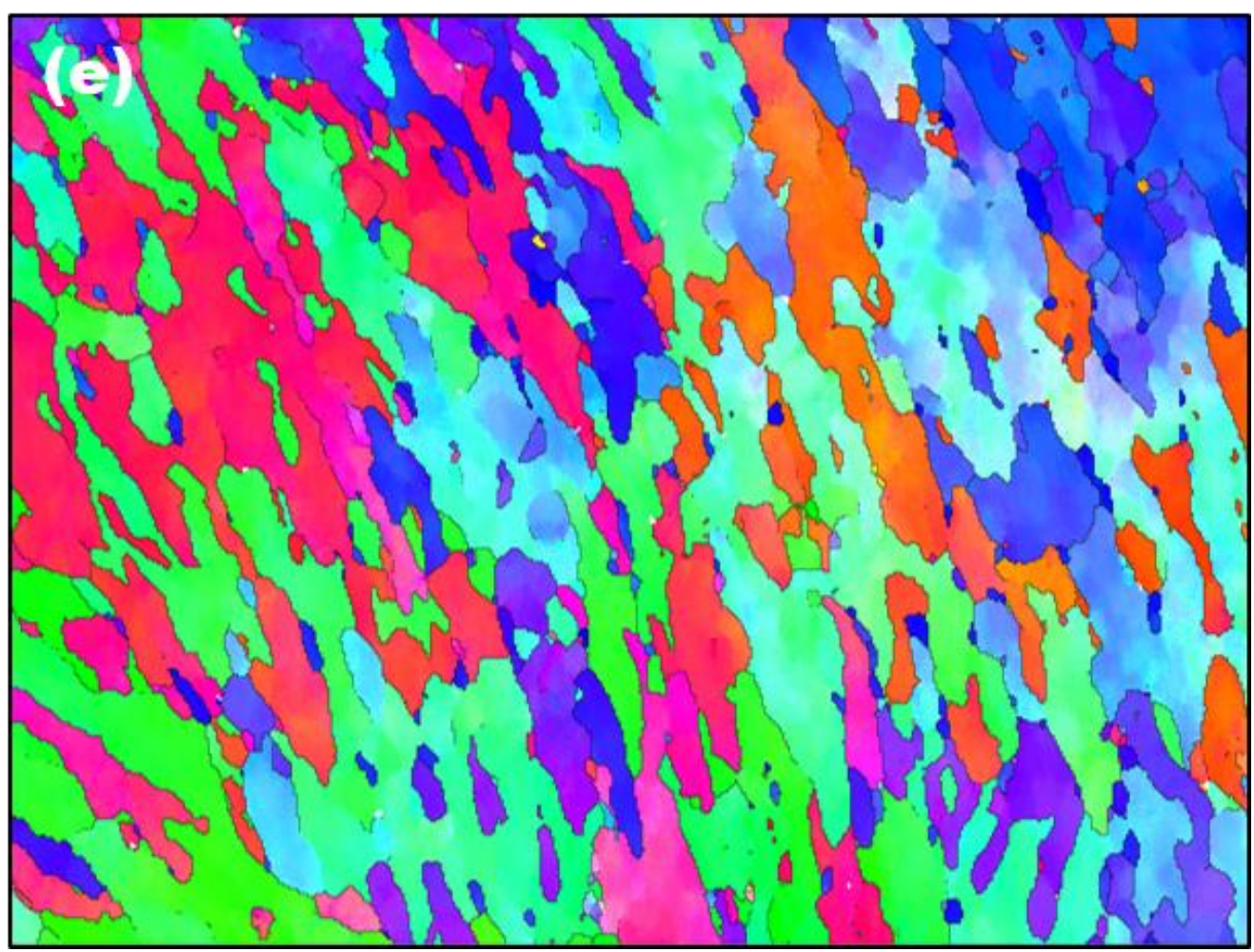

Figure 76 Comparison of QC fracture features for AerMet $100\left(a, E_{a p p}=-1,000 m V_{S C E}\right)$ and Ferrium PH48S-H900 (b and c, $E_{a p p}=-975 \mathrm{mV} V_{S C E}$ with EBSD-measured martensite microstructure. OIM maps of AerMet 100 (d) and Ferrium PH48S (e) are shown at equal magnification to their respective fractographs. Black arrows indicate regions of martensite block cleavage across a small number of block features.

Further comparison of the TG fracture morphology in AerMet 100 (Figure 74a and c) and Ferrium PH48S (Figure 58 and Figure 76b) indicates that the distribution and morphology of intragranular flat cleavage intermixed with QC HEAC is similar between the two alloys. Ferrium PH48S - H900 has a relatively large prior austenite grain size $(\sim 50 \mu \mathrm{m})$ compared to AerMet $100(11 \mu \mathrm{m})$ [73]. In a martensite hierarchy obeying K-S, the relative interfacial area of martensite packet boundaries should be reduced in AerMet 100, compared to Ferrium PH48S and following this austenite grain size reduction. As discussed, there are two possible origins for isolated flat fracture features mixed with the more prevalent QC fracture: (1) trans-block cleavage propagates across unusually large single-variant blocks in a martensite packet, or (2) limited decohesion driven cracking along packet interfaces in the interior of a prior austenite grain. Qualitatively identical amounts of isolated flat fracture in AerMet 100 and Ferrium PH48S, with varying intergranular packet interfacial area, 
indicates that flat fracture likely represents trans-block cleavage. This hypothesis is supported by two pieces of evidence. First, regions of flat fracture commonly have fine river marks on the surface (Figure 77, black arrows), indicative of cleavage fracture. Second, the size and shape of flat fracture features, particularly in Ferrium PH48S, strongly suggest that they represent cleavage of a single large block feature. As shown in Figure 47, single orientation block features are nominally rectangular with rounded surfaces, identical to the shape of flat cleavage facets shown in Figure 77. Further, in most cases it appears that flat cleavage is confined to a single martensite block. As demonstrated by Morris and coworkers for a 9Ni steel; martensite laths, sub blocks, and blocks have a strong tendency to cleave as a unit due to parallel $\{100\}_{\alpha}$, cleavage planes across a single block feature [111]. $\{110\}_{\alpha}$, block boundaries typically act as effective barriers to cleavage fracture, causing the crack to deflect at right angles or bifurcate [111]. There is no reason to expect block boundaries to behave differently in UHSS alloys which have an identical K-S orientation relationship. Morris et al did observe instances of cleavage crack transmission across crystallographically identical blocks from different martensite packets [111]. Speculatively, this transmission of a cleavage crack across a packet boundary could be the source of relatively large sections of flat cleavage such as Figure 76c.

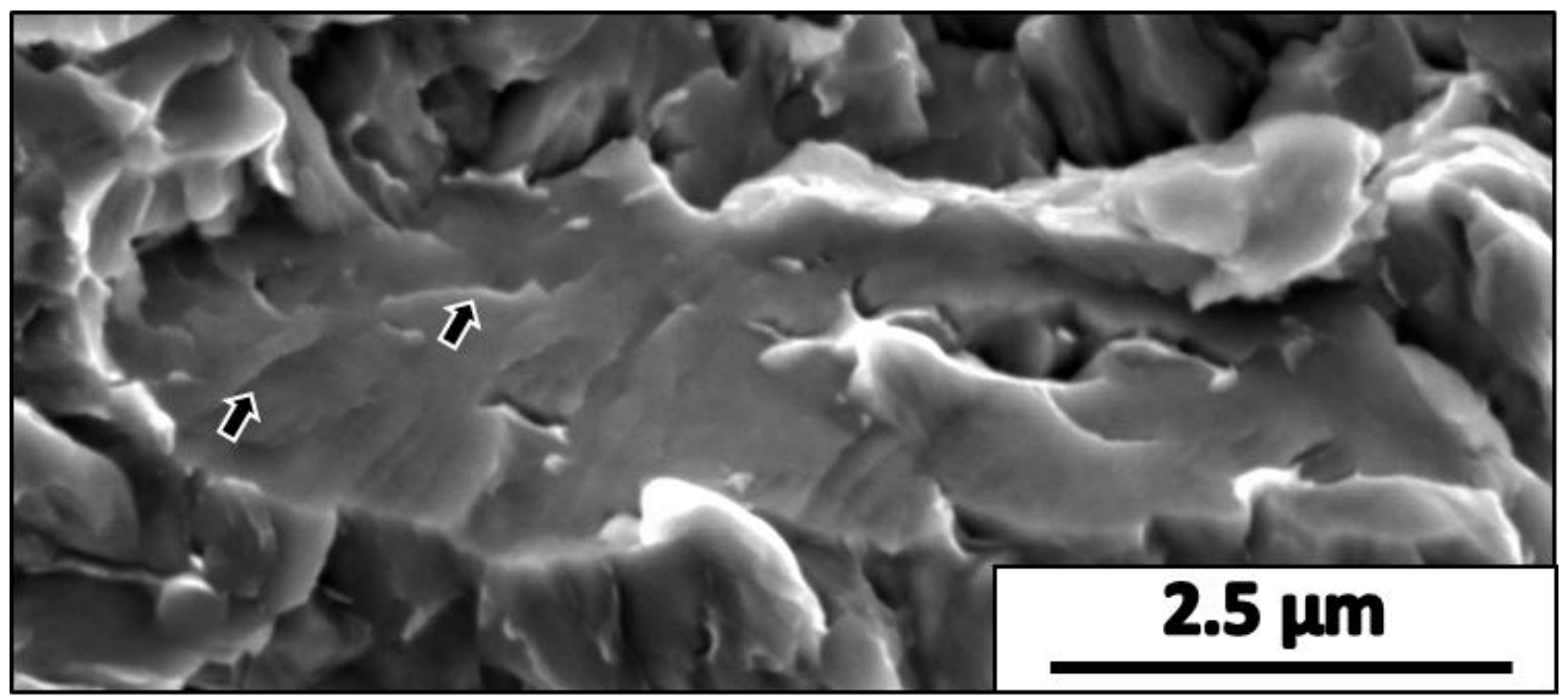

Figure 77 Detail of flat fracture in Ferrium PH48S - H860 stressed in 3.5\% NaCl at $E_{\text {app }}$ $=-975 \mathrm{~m} V_{\text {SCE. }}$. Black arrows indicate fine river marks on the flat cleavage surface. 
The HEAC fracture morphology of Ferrium PH48S is invariant with aging condition (Figures 5860) and polarization applied to a single aging condition (Figure 63). This result has significant implications for the applicability of a generic HELP/HEDE mechanism in UHSSS alloys. Thermo-Calc equilibrium modeling predicts a substantial change in the volume fraction of strengthening precipitates and precipitated austenite in Ferrium PH48S with changing aging temperature as shown in Table 13. For instance, the volume fraction of $\eta$-phase $\left(\mathrm{Ni}_{3} \mathrm{Ti}\right)$ decreases by $14 \%$ (4 to 3.5 vol\%) as aging temperature is increased from $460^{\circ} \mathrm{C}$ to $520^{\circ} \mathrm{C}$. In the absence of significant coarsening, increasing volume fraction of strengthening precipitate should proportionately increase the site density of reversible $\mathrm{H}$-traps located on $\eta$ interfaces, causing $\mathrm{C}_{\mathrm{H} \sigma}$ to rise (Equation 12). While increasing $\mathrm{C}_{\mathrm{H \sigma}}$ has a significant effect on observed HEAC properties, fracture morphology is invariant at all aging conditions. Therefore the HEAC mechanism for transgranular fracture in Ferrium $\mathrm{PH} 48 \mathrm{~S}$ is invariant with changing volume fraction of strengthening precipitates in the range of common aging temperatures. This point is particularly evident when comparison the H900 and H860 aging condition of Ferrium PH48S; the ThermoCalc predicted volume fraction of BCC-Cr changes from nil (H900) to 3.7 vol\% (H860) with no observable change in fracture morphology (compare Figure 58 and Figure 59).

\begin{tabular}{|c|c|c|c|c|}
\hline Ferrium PH48S & H860 & H900 & H950 & H968 \\
\hline $\boldsymbol{\eta}$ (Ni3Ti) & $4.0 \mathrm{vol} \%$ & $3.8 \mathrm{vol} \%$ & $3.7 \mathrm{vol} \%$ & $3.6 \mathrm{vol} \%$ \\
\hline B2 NiAl & 0.9 & 1.0 & 0.9 & 0.7 \\
\hline BCC-Cr & 3.6 & 0.0 & 0.0 & 0.0 \\
\hline $\boldsymbol{\gamma}$ & 13 & 18 & 24 & 28 \\
\hline TiC & 0.005 & 0.005 & 0.005 & 0.005 \\
\hline
\end{tabular}

Table 13 ThermoCalc predictions of the equilibrium volume fraction (\%) of phases in Ferrium PH48S under different aging conditions [43].

Elevated Co content was shown to retard recovery of martensite dislocation structure during aging at elevated temperatures [25,27]. Although the effect of aging temperature on martensite microstructure was not probed for Ferrium PH48S, the significant Co content (7.3 wt \%) suggests that the fine scale martensite structure would not significantly recover or recrystallize as aging temperature increases from $460^{\circ} \mathrm{C}(\mathrm{H} 860)$ to $520^{\circ} \mathrm{C}(\mathrm{H} 968)$. Invariant martensite morphology in this range of aging temperatures allows the HELP/HEDE mechanism to produce the constant TGQC HEAC fracture morphology shown in Figures 58-60. Further, the morphology of TG-QC 
HEAC in Ferrium PH48S is not affected by increasing cathodic polarization; from slightly (-250 $\left.\mathrm{mV}_{\mathrm{SCE}}\right)$ to heavily $(-1,200 \mathrm{mV})$ cathodic levels of $E_{\text {app. }}$ This result indicates that the HEAC mechanism for TG QC of Ferrium PH48S is identical as variable stress-affected crack tip hydrogen concentration $\left(\mathrm{C}_{\mathrm{H \sigma}}\right)$ systematically increases with cathodic polarization (Figure 29).

Within the Nagao HELP/HEDE mechanism, the amount of hydrogen deposited at a martensite block interface should increase as $\mathrm{C}_{\mathrm{H \sigma}}$ increases and more hydrogen is present in the FPZ. During $\mathrm{HEAC}$, a dynamic equilibrium exists between with $\mathrm{H}$ diffusing into the lattice and $\mathrm{H}$ trapped at reversible, low energy sites [184]. As shown in Equation 11, the amount of hydrogen trapped in a given trap state depends exponentially upon $\mathrm{E}_{\mathrm{B}}$. The low binding energy $\left(\mathrm{E}_{\mathrm{B}}=12.1-12.2 \mathrm{~kJ} / \mathrm{mol}\right.$, Figure 74) of H-traps in Ferrium PH48S could provide a mobile reservoir of weakly trapped $\mathrm{H}$ which can redistribute to new dynamically formed dislocation structure that acts as trap sites in the FPZ. HELP-enhanced dislocation movement along $\{110\}_{\alpha}$, slip bands will work harden the area local to its intersection with the martensite interface. New dislocation structure shouold have a strong-reversible trap binding energy $\left(\mathrm{E}_{\mathrm{B}} \sim 60 \mathrm{~kJ} / \mathrm{mol}\right)$, as observed in cold worked ferritic iron and nano-scale voids would have a similar or higher trap binding energy [185]. The creation of new strong $\mathrm{H}$-traps in the martensite microstructure causes redistribution of $\mathrm{H}$ from weakly held strengthening precipitate sites to the dislocation structure. Further, H-trap states in the FPZ are assumed in dynamic equilibrium with the lattice concentration of hydrogen during HEAC, and new strong-reversible traps would be rapidly filled with $\mathrm{H}$. Increasing hydrogen concentration at the intersection of a slip band and martensite interface implies that local stress necessary for initiation of a microcrack should decrease as $\mathrm{E}_{\text {app }}$ becomes more cathodic and $\mathrm{C}_{\mathrm{H \sigma}}$ rises. This hypothesis is consistent with observations of the $\mathrm{E}_{\text {app }}$ dependence of $\mathrm{K}_{\mathrm{TH}}$ in all alloys which exhibit TG HEAC (Figure 24 and Figure 69).

The preceding analysis provides justifies the invariant fracture morphology of Ferrium PH48S with the HELP-HEAC mechanism proposed by Nagao et al [11,12]. There is strong-circumstantial evidence to support this hypothesis, but additional experimentation is needed to verify the mechanism as generic for all UHSS(S) alloys. In the short term, a UHSS similar to Ferrium PH48S, such as AerMet 100, could be aged to variable strength near the peak-aged condition and the fracture morphology tested across cathodic $E_{\text {app. }}$. Fracture morphology obtained from such experiments would verify a universal TG-HEAC mechanism across modern and experimental 
compositions of ultra-high strength alloys. Further, the crystallographic plane(s) of QC HEAC features could be confirmed via EBSD on a polished plane cut perpendicular to the crack plane, verifying the crystallographic orientation of TG QC features in UHSS and UHSSS [187]. Finally, a more fundamental study of the HEAC fracture mechanism could use the FIB lift out technique employed by Nagao et al. to directly examine the microstructure beneath QC HEAC facets in a UHSS and UHSSS aged to identical strength [11,12,161]. Congruency of the sub-surface dislocation structure beneath TG HEAC facets/features would provide direct evidence for a single HELP-HEDE mechanism in microstructurally complex UHSS.

\subsubsection{IG HEAC of Custom 465}

In contrast to TG fracture of Ferrium PH48S, SCC-HEAC of Custom 465 - H900 is dominated by IG fracture almost exclusively along prior austenite grain boundaries. As shown in Figure 65, the IG fracture morphology of Custom 465 - H900 is consistent across all SCC-susceptible $\mathrm{E}_{\text {app. }}$. High magnification imaging (Figure 57) of IG facets reveals a featureless crack surface, devoid of small scale microvoiding or ductile processes. Custom 465 - H900 exhibits a near identical IGSCC fracture morphology to that observed in legacy UHSS 300M, as shown in Figure 78. Facets of IG

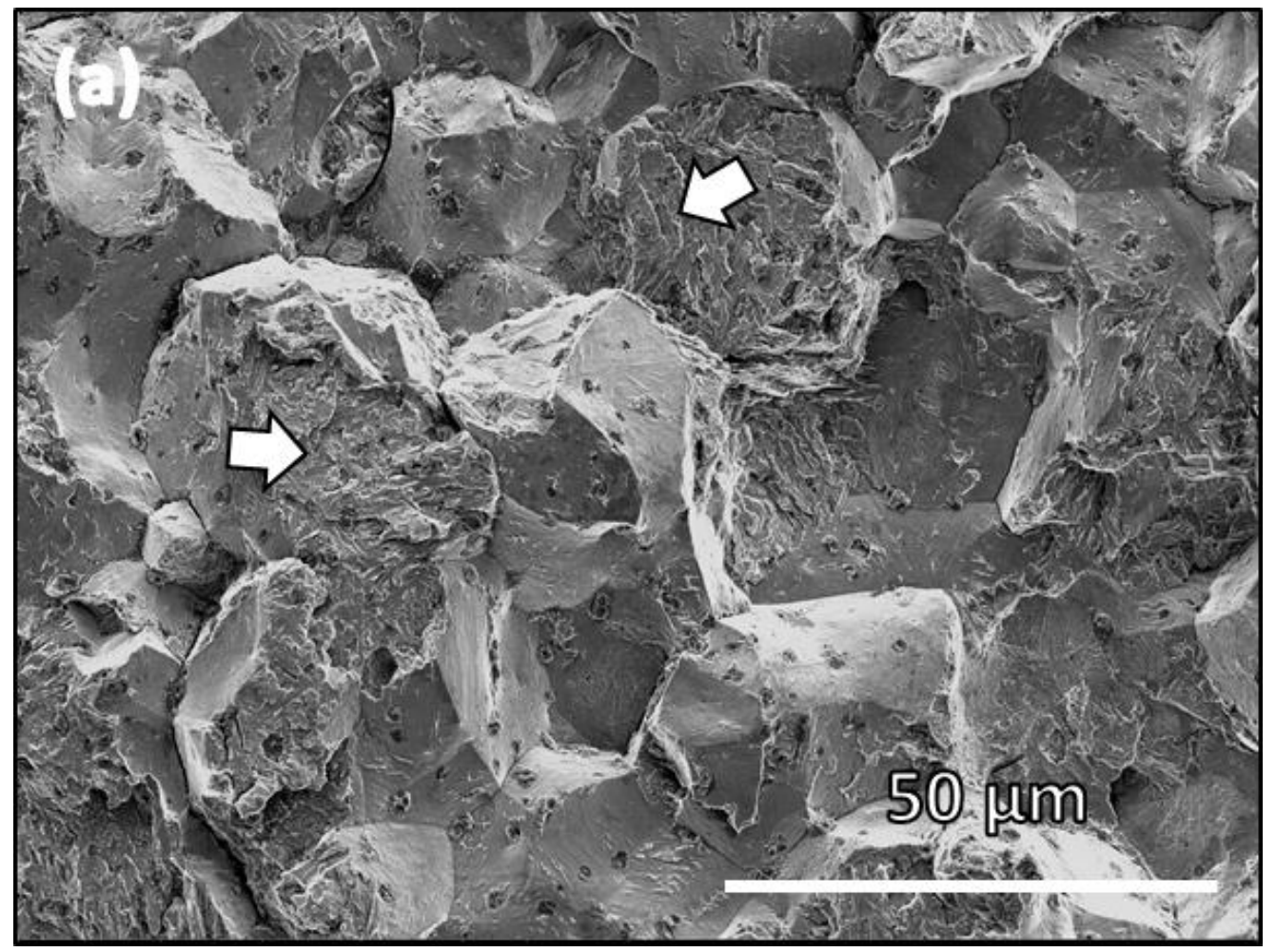



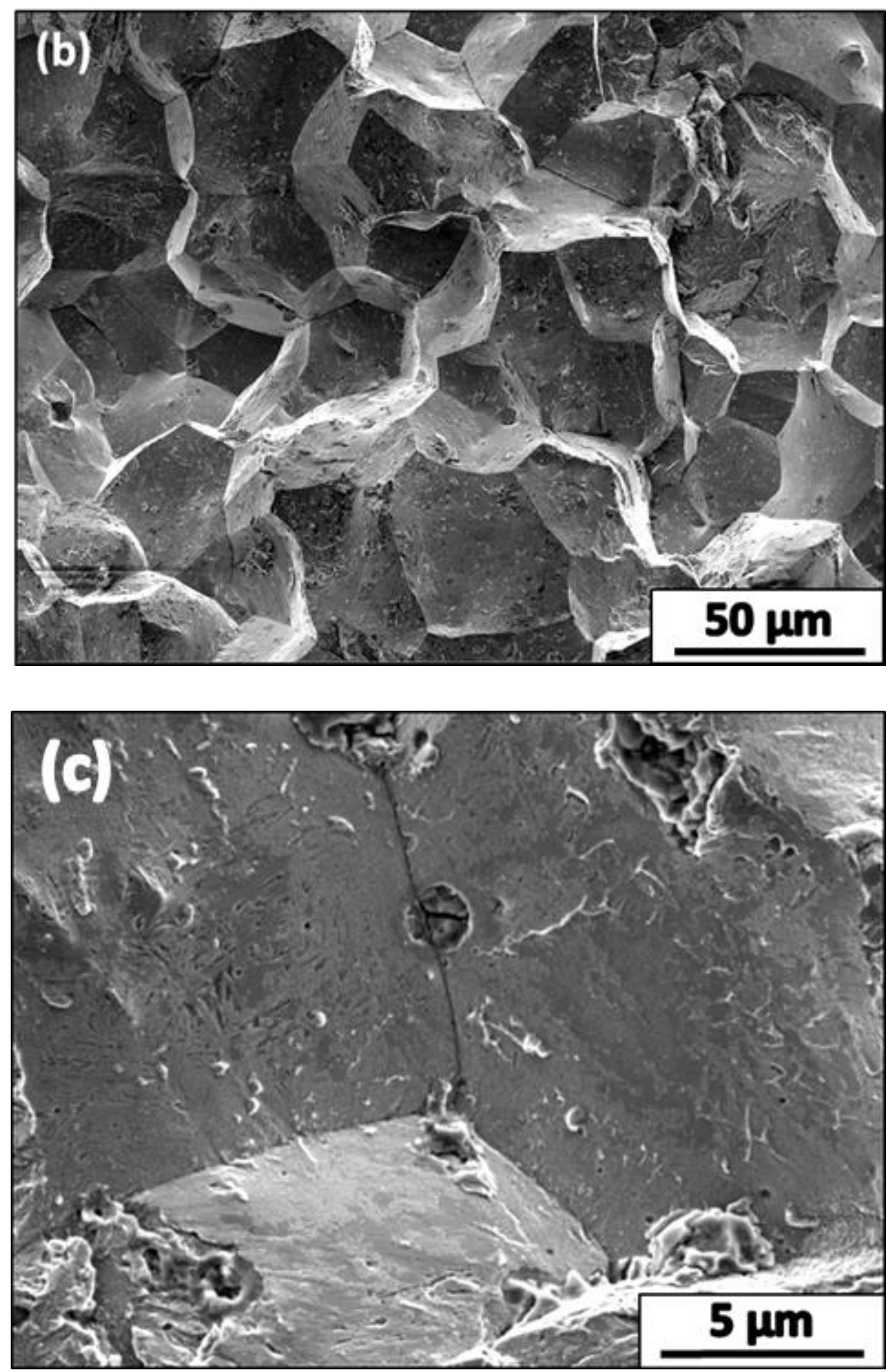


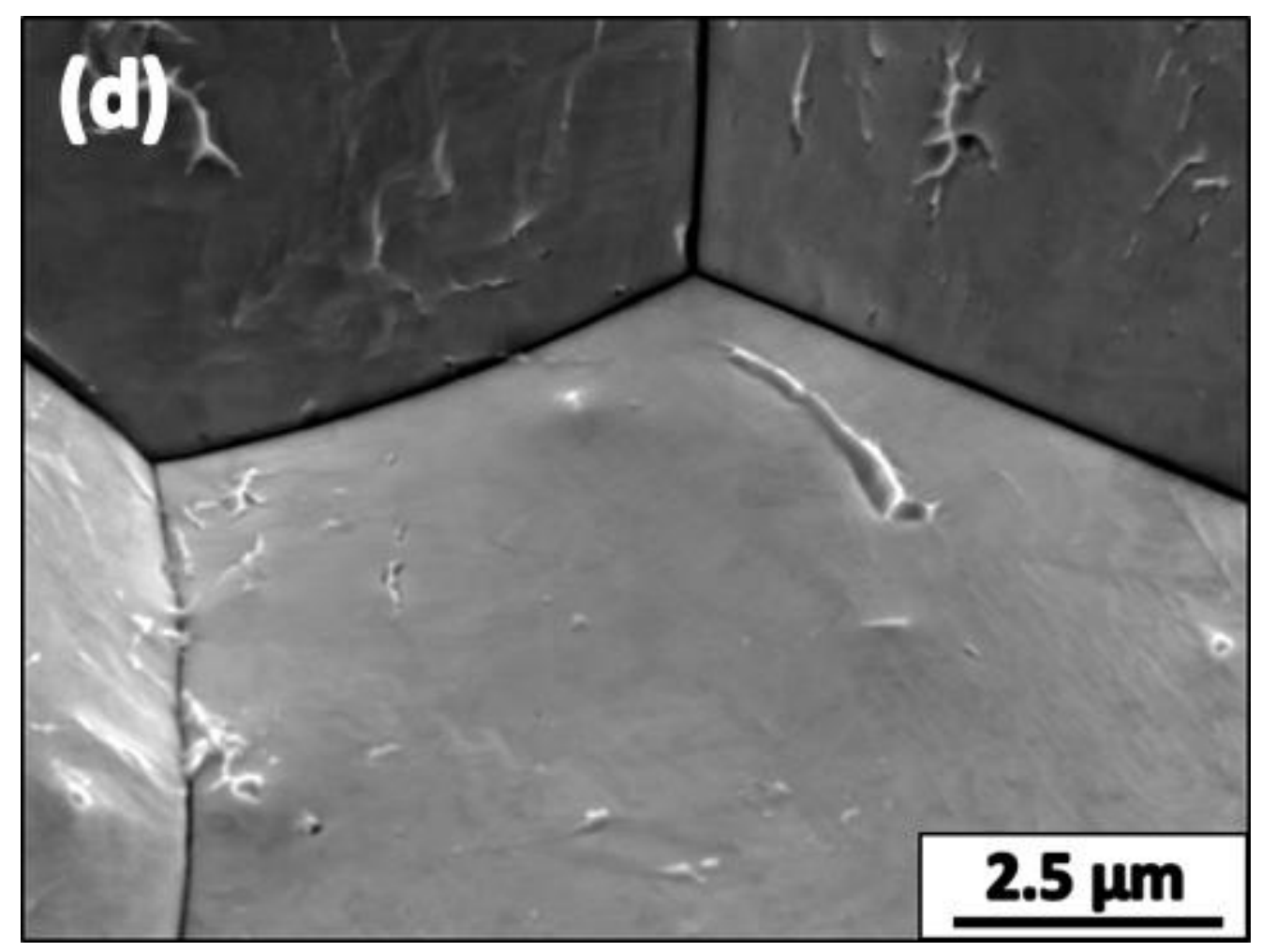

Figure 78 Fracture morphology of $U H S S(S)$ stressed under slow rising displacement in 3.5\% $\mathrm{NaCl}$, (a) IGSCC of 300M at $E_{a p p}=1,000 \mathrm{~m} V_{S C E}$ and, (b) IGSCC of Custom $465-H 900$ at $E_{\text {app }}$ $=1,000 \mathrm{~m} V_{S C E}$. Images $(\mathrm{c})$ and $(\mathrm{d})$ show detail regions of $(a)$ and $(b)$, respectively.

IG fracture in 300M are largely featureless (Figure 78a) and displayg a lack of small scale microvoiding similar to IG surfaces in Custom 465-H900 (compare Figure 78c and Figure 78). The presence of localized corrosion (from post-SCC test solution exposure) and corrosion products on the fracture surface make this assessment difficult for $300 \mathrm{M}$, but small regions of clean IG fracture (Figure 78c) are readily observable. No significant difference in the GBCD (Table 9) or connectivity of random boundaries (Table 10) is observed between 300M and Custom 465.

Critically, IG HEAC of 300M was directly attributed to elevated-bulk impurity concentration, characterized by the parameter $\Psi$ (where $\Psi=[\mathrm{Mn}+0.5 \mathrm{Si}+\mathrm{S}+\mathrm{P}]+\left[10^{4} \mathrm{C}_{\mathrm{H}}\right]$ in at\%), as developed by McMahon and coworkers [131,136]. Fracture mechanics experiments established that IG HEAC is eliminated when the impurity component of $\Psi$ ( $\mathrm{Mn}+0.5 \mathrm{Si}+\mathrm{S}+\mathrm{P}$; without considering $\mathrm{H}$ concentration)) is less than $0.05 \mathrm{wt} \%$ for 4340 steel ( $\left.\sigma_{\mathrm{YS}}=1,450 \mathrm{MPa}\right)$ stressed in 
low pressure $\mathrm{H}_{2}\left(\mathrm{p}_{\mathrm{H} 2}=0.1 \mathrm{MPa}\right)$ [131]. This impurity threshold of $\Psi=0.05 \mathrm{wt} \%$ was shown to effectively predict the HEAC fracture path for UHSS alloys in Chapter 3. Featureless IG HEAC facets (Figure 78c and d) are strongly indicative of impurity-driven IG HEAC in Custom 465H900, identical to 300M. However, $\Psi$ is drastically reduced in Custom $465-\mathrm{H} 900$ ( $\Psi=0.024$ $\mathrm{wt} \%$ ) compared to $300 \mathrm{M}(\Psi=1.8 \mathrm{wt} \%)$, and IG HEAC is not predicted based on the impurity threshold of $0.05 \mathrm{wt} \%$. However, due to the lack of rare earth treatment in Custom 465, some level of impurity driven reduction in grain boundary cohesion is likely active, albeit at a lower level compared to 300M. Comparison between Custom 465 and the 4340 steel in the study by Bandyopadhyay et al. is clouded by yield strength difference between the steels [131]. It is reasonable to speculate that the threshold $\Psi$ for IG $\mathrm{H}$ cracking will decrease with increasing crack tip stresses resulting from increased alloy flow strength. Moreover, the overall threshold composition parameter for IG HEAC should include a $\mathrm{H}$ concentration term, which will be discussed in the remainder of this section.

IG HEAC of CrNiMoWV is hypothesized in Chapter 3 to have a microstructural, rather than impurity origin. To review, increased prior austenite grain size and martensite block length allow slip bands, which run nominally parallel to $\{110\}_{\alpha}$, block interfaces, to pile up significantly more dislocations at the slip band-boundary intersection. Prior austenite grain boundary H-concentration is elevated compared to intragranular block boundaries due to increased slip band length and poor atomic fit of the prior austenite boundary with impinged martensite blocks. The weakest link criterion then favors cracking along the grain boundary interface with lower H-degraded cohesive strength. While hypothetical, this scenario provides a reasonable set of mechanistic steps for IG fracture in the absence of impurity concentration above the McMahon threshold parameter[131].

Speculatively, the IG HEAC morphology of Custom 465 - H900 is produced by the conjoint action of free impurity content, high diffusible hydrogen concentration, and coarse martensite microstructure to produce IG fracture. Custom 465 is low impurity compared to $300 \mathrm{M}$ ( $\Psi_{\text {Impurity }}=$ 0.024 vs $1.8 \mathrm{wt} \%$ ), but has significantly elevated $\mathrm{C}_{\mathrm{H}-\mathrm{DIFF}}$ compared to AerMet 100 when charged under heavy cathodic polarization. The hydrogen uptake behavior of $300 \mathrm{M}$ was assumed identical to AerMet 100 during $\mathrm{K}_{\mathrm{TH}}$ modeling presented Chapter 3. As shown in Figure 71 and previous work [105], $\mathrm{C}_{\mathrm{H}-\mathrm{DIFF}}$ is significantly enhanced in Custom 465. As such, $\mathrm{C}_{\mathrm{H \sigma}}$ in Custom 465 should be proportionately increased, drastically increasing the $\mathrm{H}$-concentration dependent term of the 
McMahon parameter $\left(\Psi\left(\Psi=[\mathrm{Mn}+0.5 \mathrm{Si}+\mathrm{S}+\mathrm{P}]+\left[10^{4} \mathrm{C}_{\mathrm{H}}\right]\right)\right.$ compared to $300 \mathrm{M}$. The conjoint deleterious action of hydrogen and a minor impurity concentration, will force the embrittlement parameter above the threshold for IG fracture. Concurrent with impurity and hydrogen driven decohesion of prior austenite grain boundaries, there is some evidence of a microstructural basis for IG fracture of Custom 465 - H900. Martensite packets in Custom 465 - H900 often span the entire width of a prior austenite grain (Compare Figure 9 and Figure 39). In the context of the generic HELP/HEDE mechanism, this microstructural coarseness would allow slip bands parallel to $\{110\}_{\alpha}$, block interfaces to span the entire length of a prior austenite grain. Increased slip band length would promote IG fracture in an identical manner to CrNiMoWV, but does not explain the largely featureless, rather than plasticity bearing, morphology of IG facets. IG HEAC facets on the fracture surface of CrNiMoWV specimens contain a significant amount of small scale microvoiding (Figure 23d) that is not observed in 300M or Custom $465-\mathrm{H} 900$. The absence of such regions in 300M is understood based upon an overriding impurity effect that severely reduces the cohesive strength of the prior austenite grain boundaries, allowing easy decohesion. Taken together, the simultaneous action of impurity/hydrogen driven reduction in grain boundary cohesive strength and strong hydrogen localization due to slip band length provide the most logical hypothesis for the origins of IG fracture in Custom 465 - H900.

\subsubsection{Measurement of Precipitated Austenite in UHSSS alloys}

As discussed for UHSS alloys in Chapter 3, austenite precipitation during aging is hypothesized to be detrimental to HEAC resistance in UHSS alloys $[119,128]$. Thin-film austenite, present along martensite packet and block interfaces in the FPZ is saturated with H during HEAC. High crack tip stress and plastic strain, in principle, drive the transformation of $\mathrm{H}$-saturated precipitated austenite into H-supersaturated, untempered martensite. Based upon previous connectivity analyses, the transformed untempered martensite would provide an interconnected crack path through the microstructure along martensite block interfaces. This mechanism was hypothesized to have a universal-detrimental effect on HEAC properties by providing an interconnected brittle crack path where thin film austenite is present. High resolution EBSD measurements of Custom 465 and Ferrium PH48S (Figure 39-42) provide the opportunity to examine the applicability of this hypothesis in UHSSS. 
There are no literature measurements of the retained or precipitated austenite content in Custom 465 or Ferrium PH48S in any aging condition. The precipitated austenite content of UHSSS alloys was expected to follow the morphology observed in UHSS, that is, a continuous thin film along block boundaries [24,75], with isolated islands at microstructural heterogeneities (Figure 11), as discussed in Chapter 3. Five techniques were employed to assess the distribution of precipitated austenite in UHSSS: ThermoCalc modeling of equilibrium phase fraction, high resolution EBSD mapping, X-ray diffraction ${ }^{11}$, backscatter electron imaging, and limited transmission electron microscopy. The measured total austenite content from ThermoCalc modeling, XRD measurements, and EBSD measurements (Figures 48-49) is provided in Table 14 for each UHSSS at several aging conditions.

\begin{tabular}{|l|c|c|c|}
\hline \multicolumn{4}{|c|}{ Measured and Calculated Total Austenite Content of UHSSS Alloys (vol\%) } \\
\hline & ThermoCalc & XRD & EBSD \\
\hline Ferrium PH48S AQ & & $7.7 \pm 1.1$ & 0.3 \\
\hline Ferrium PH48S H900 & 18 & $10.7 \pm 1.1$ & ---- \\
\hline Ferrium PH48S H950 & 24 & $10.0 \pm 2.1$ & ---- \\
\hline Ferrium PH48S H968 & 28 & $8.4 \pm 1.1$ & ---- \\
\hline Custom 465 AQ & --- & $4.7 \pm 0.7$ & 0.1 \\
\hline Custom 465 H900 & 16 & $1.8 \pm 0.8$ & \\
\hline
\end{tabular}

Table 14 Measured and Calculated Total Austenite Content of Custom 465 and Ferrium PH48S.

11 XRD measurements were conducted on metallographic sections of each alloy by Lambda Corporation. The volume percent of austenite was determined by the direct comparison method, in accordance with ASTM E975 and SAE SP-453. Four independent volume percent retained austenite values were calculated from the "R" ratios and the total integrated intensities of the austenite (200) and (220), and ferrite/martensite (200) and (211) diffraction peaks. The integrated intensity of each austenite and ferrite/martensite peak was measured using chromium K-alpha radiation. The use of multiple diffraction peaks from each phase minimizes the possible effects of preferred orientation and coarse grain size. The sample was positioned in a Miller fixture, to further minimize the influence of preferred orientation and grain size. The Miller fixture rotates the specimen around the surface normal and oscillates $\pm 45^{\circ}$ perpendicular to the diffracting plane. 
As characterized via XRD, total austenite content in Custom 465 falls from 4.7 to 1.8 vol\% upon aging to the H900 condition, indicating decomposition of retained austenite from the as-quenched and chilled condition. Total XRD measured austenite content after aging (1.8 vol\%) is consistent with AerMet 100 (1.9 vol\%), but the EBSD measured austenite fraction is significantly lower $(0.1$ versus 1.5 vol\%). As was shown for UHSS alloys in Chapter 3, XRD measured austenite content is the sum of thin film austenite and austenite in island morphology (for cryogenically treated microstructures without retained austenite). This comparison implies that a continuous, $12-20 \mathrm{~nm}$ thick film of precipitated austenite is present along all interfaces in Custom 465 to account for the discrepancy between XRD and EBSD measurements shown in Table 14. (EBSD resolution is not sufficient to measure the thin film component of total austenite.) This result assumes that the XRD values are accurate and representative of the average value for multiple replicate measurements. As discussed in Chapter 3, there is significant variability in XRD measurements of small total austenite contents. However, the total calculated-measured volume fraction of precipitated austenite in AerMet 100 (2.2 vol\% as thin film (calculated) plus island morphology (measured)) closely matches the XRD measured total austenite fraction, supporting the accuracy of XRD measurements in UHSS(S). There are no TEM results for Custom 465 to confirm the existence of thin film austenite.

SEM-BSE imaging does not reveal the presence of any secondary phases along prior austenite boundaries or martensite interfaces (Figure 79). Under BSE imaging, FCC austenite would be expected to be visible from compositional and electron channeling contrast with the BCC matrix [188]. While not conclusive, the results presented in Figure 48 and Figure 79 suggest that the XRD measurements of precipitated austenite in Custom 465 - H900 are substantial over-estimates due to the inherent variability in measuring low volume fractions of austenite. Currently, there is no spatially sensitive evidence to suggest the presence of thin film precipitated austenite in Custom $465-\mathrm{H} 900$. 


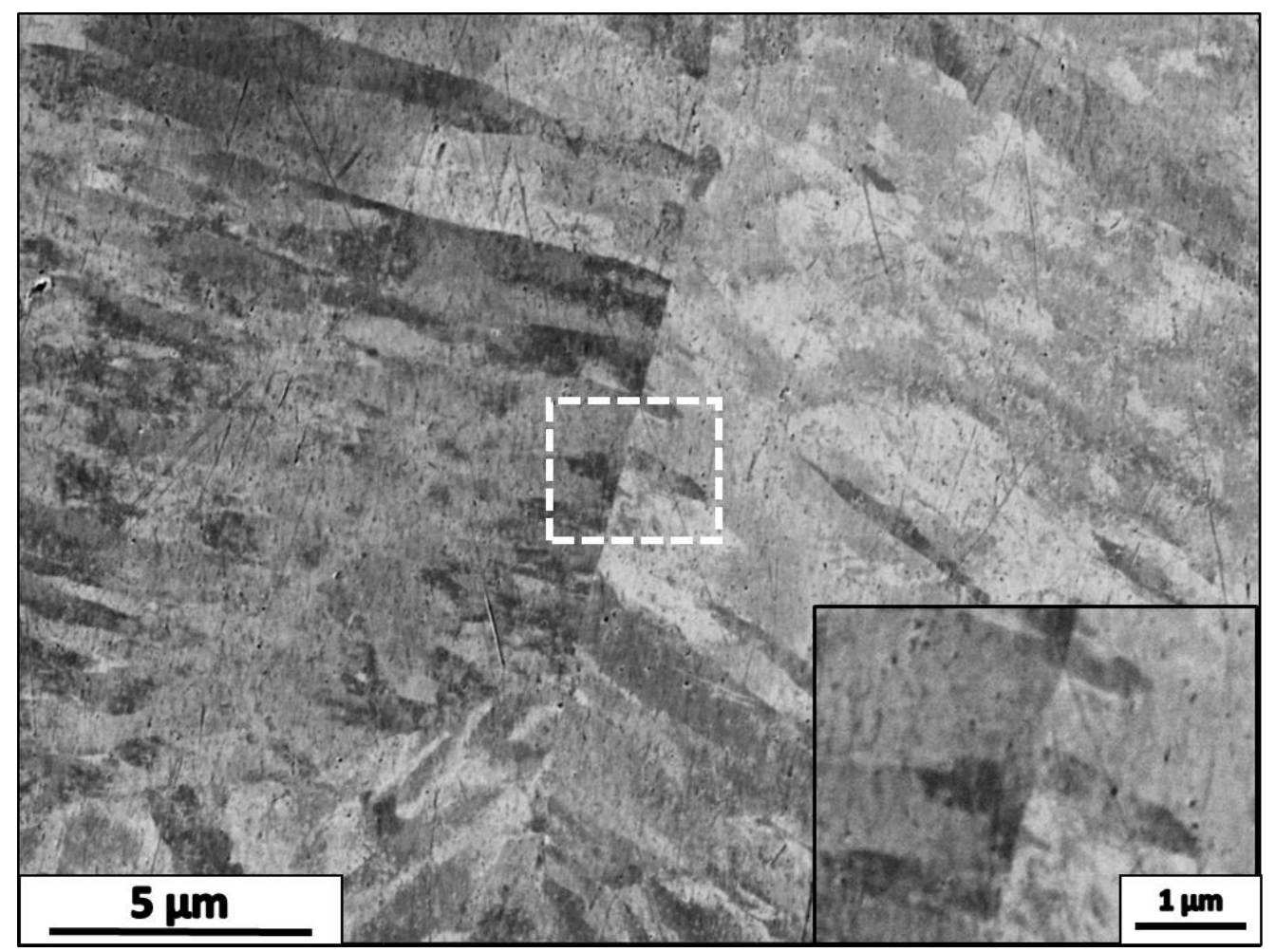

Figure 79 High magnification SEM-BSE imaging of a prior austenite grain boundary in Custom 465. The boxed region is shown magnified in the lower right inserted image.

Total XRD measured austenite content in Ferrium PH48S - H900 is increased compared to Custom 465 - H900 (10.7 vs 1.8 vol\%) and AerMet 100 (1.9 vol\%). Total EBSD measured austenite content is low (0.3 vol\%), comparable to Custom 465 (0.1 vol\%), and significantly reduced from AerMet 100 (1.5 vol\%). If the XRD and EBSD measurements are assumed accurate, then difference of these two measurements must be held in thin film austenite along the martensite interfaces. The upper and lower bounds of the XRD-measured austenite in Ferrium PH48S - H900 (Table 14) dictate a $24-31 \mathrm{~nm}$ thick film of austenite over all martensite interfaces. This prediction is inconsistent with the high resolution EBSD map of austenite islands presented in Figure 49. Without any local variation in film thickness, a $24-31 \mathrm{~nm}$ thick film would be $\sim 2$ times the EBSD pixel step size $(13 \mathrm{~nm})$ wide and at least partially resolvable in Figure 49. Identical to Custom 465 - H900, high magnification SEM-BSE imaging did not reveal the presence of any secondary phases along prior austenite or martensite interfaces (Figure 80). 


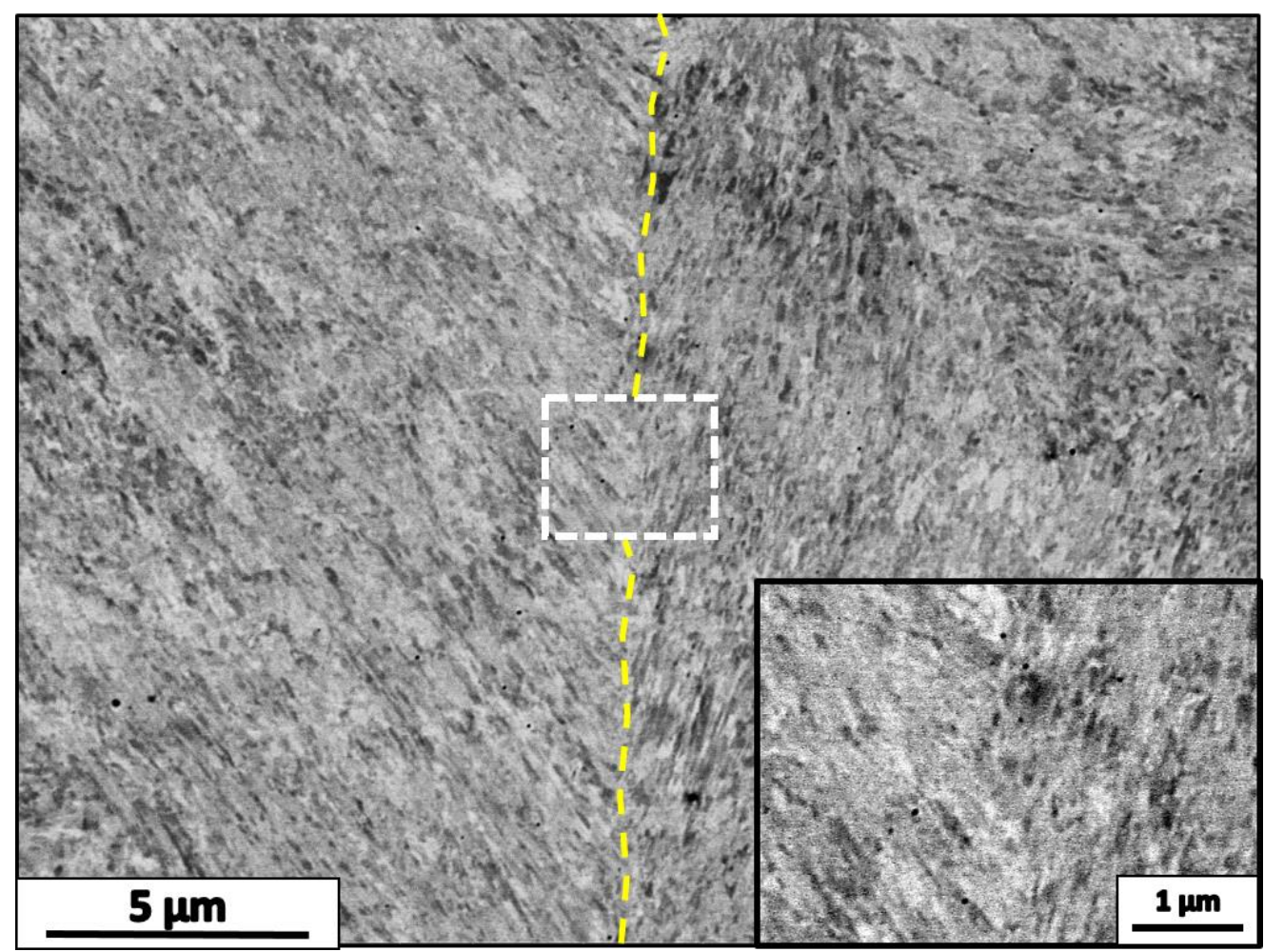

Figure 80 High magnification SEM-BSE imaging of a prior austenite grain boundary in Custom 465. A prior austenite grain or packet boundary is indicated by a yellow dashed line. The boxed region is shown magnified in the lower right image.

To further investigate the phase composition of martensite interfaces in Ferrium PH48S a single TEM specimen was prepared ${ }^{12}$ and examined at high magnification. Two sets of measurements were conducted on this single specimen. First, Evans Analytical Group examined a thin foil of PH48S - H900 in a Hitachi HD2300 scanning transmission electron microscope operating at 200

\footnotetext{
12 Specimen preparation was conduction by Evan's Analytical Group (EAG). A layer of platinum was first sputtered onto a polished specimen surface using a K575X Emitech coating system. The sample was then placed in an FEI 200TEM focused ion beam (FIB) system. An additional layer of platinum was FIB-deposited by injection of an organo-metallic gas and rastering the $30 \mathrm{kV}$ gallium ion beam over the area of interest. A thin cross section measuring approximately 15 um long, 2 um wide and 6 um deep was extracted from the die surface using a proprietary in-situ FIB technique. The cross section was attached to a 200 mesh copper TEM grid using FIB-deposited platinum. An electron transparent window was thinned using the gallium ion beam of the FEI 200TEM FIB. The protective platinum layer was milled away during thinning.
} 
$\mathrm{kV}$. Transmitted electron images were acquired at various magnifications in transmitted electron mode for bright field imaging. Selective area diffraction (SAD) patterns were acquired using a Hitachi HF2000 transmission electron microscope operating at 200kV. A second set of images were obtained at UVa by Mr. Matt Schneider, operating a FEI Titan TEM at $200 \mathrm{kV}$ in transmitted electron mode for bright field imaging. As shown in Figure 81, the martensite block structure observed in TEM bright field imaging is identical to that observed via high resolution EBSD imaging Figure 42. Selected area diffraction of a $3 \mu \mathrm{m}$ diameter circular area in Figure 81 did not yield the expected-unique $\{211\}_{\gamma},\{310\}_{\gamma}$, or $\{312\}_{\gamma}$ diffraction rings for FCC austenite. Identical $\mathrm{SAD}$ measurements conducted at $\pm 5^{\circ}$ tilt on the $x$ and $y$ axis yielded an identical result. Due to the fine block size in Ferrium PH48S - H900, the SAD pattern sampled a large number of martensite block features. A lack of diffraction rings unique to FCC austenite indicates that there is no precipitated austenite along the block boundaries in Ferrium PH48S - H900. To confirm this result, a single block boundary was examined at high resolution (Figure 82, white arrows). As shown in Figure 82b there is no evidence of the predicted 24 - $31 \mathrm{~nm}$ thick layer of austenite along the block boundaries in Ferrium PH48S - H900. While this high magnification imaging was limited to a single boundary, a lack of FCC diffraction rings in the SAD pattern relevant to the image in Figure 81 supports the speculation that examination of additional martensite block boundaries would yield an identical result.

Taken together, the results shown in Figure 49 and Figures 80 to 82 clearly establish that the precipitated austenite content of Ferrium PH48S - H900 is near zero and the XRD measurements shown in Table 14 are dramatic over estimates. There is no evidence to support the presence of a network of thin-film austentite precipitated along martensite block interfaces. However, XRD measurements of total austenite content are based upon the comparison of austenite $\left(\{200\}_{\gamma}\right.$ and $\left.\{220\}_{\gamma}\right)$ and ferrite/martensite $\left(\{200\}_{\alpha}\right.$ and $\left.\{211\}_{\alpha}\right)$ diffraction peaks. The presence of such peaks in the XRD spectra indicates that there must be a microstructural origin for these diffraction peaks. In the context of identical-low EBSD measured precipitated austenite, the large disparity in XRD measured austenite content between Custom 465 - H900 and Ferrium PH48S - H900 indicates that this disparity is unique to Ferrium PH48S. The only microstructural feature which has a similar volume fraction to the false-measured austenite content is a strengthening precipitate. Speculatively, the significant addition of aluminum to Ferrium PH48S has caused a shift in the 
crystal structure of the primary strengthening precipitate from hexagonal $\mathrm{Ni}_{3} \mathrm{Ti}$ towards the ordered FCC phase $\mathrm{Ni}_{3} \mathrm{Al}$, causing the false FCC diffraction peaks to appear in the absence of FCC austenite. Limited atom probe tomography indicates that significant aluminum and cobalt are incorporated into rod shaped strengthening precipitates $\left(\left(\mathrm{Ni}_{0.9} \mathrm{Co}_{0.1}\right)_{3}\left(\mathrm{Ti}_{0.81} \mathrm{Al}_{0.19}\right)\right)$ in Ferrium PH48S - H968, suggesting that a change in structure is possible [43]. It is unknown if TEM analysis of Custom 465 - H900 would yield an identical-nil precipitated austenite content.

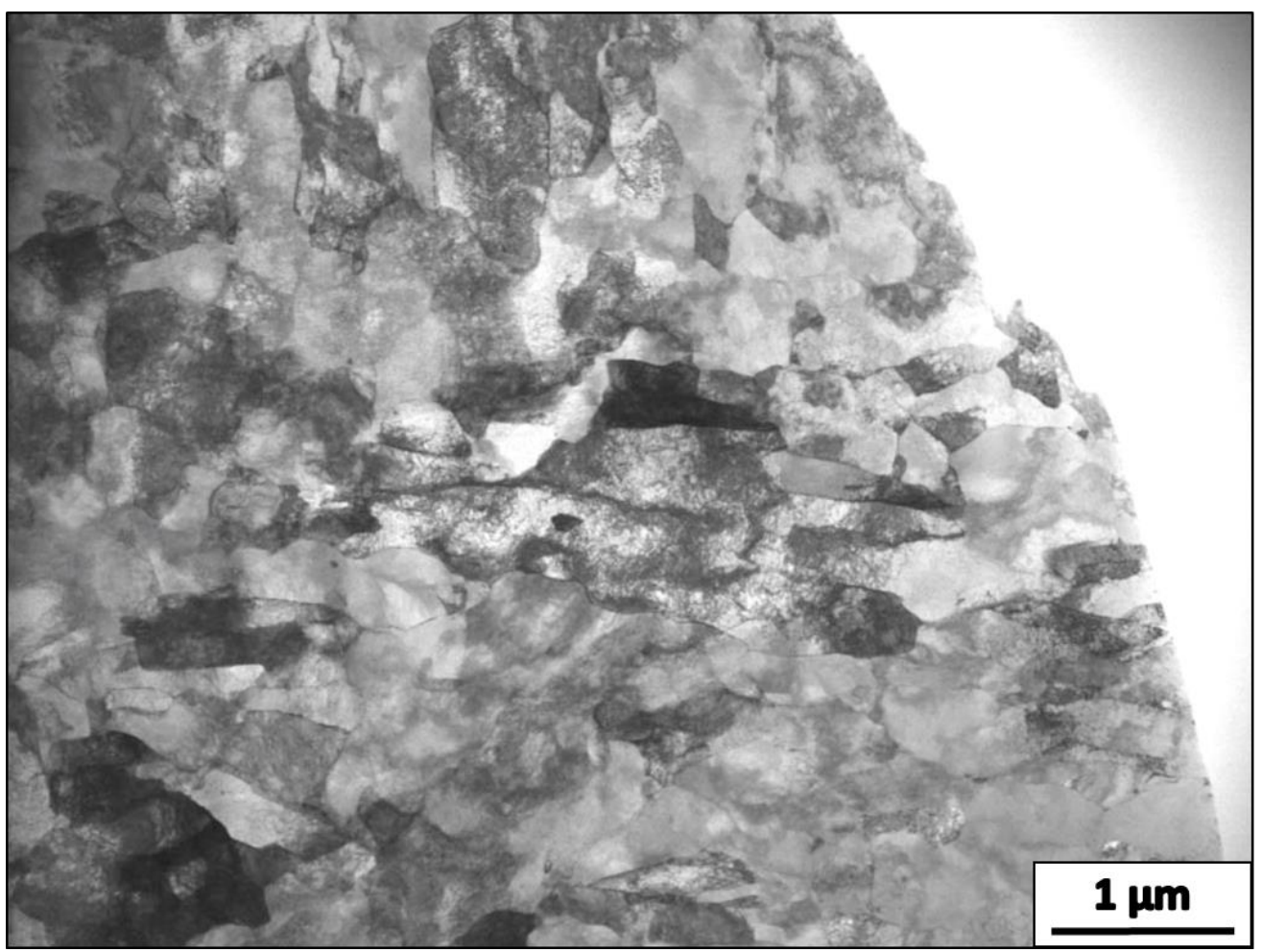

Figure 81

Low magnification TEM micrograph martensite block structure in Ferrium PH48S - H900. 

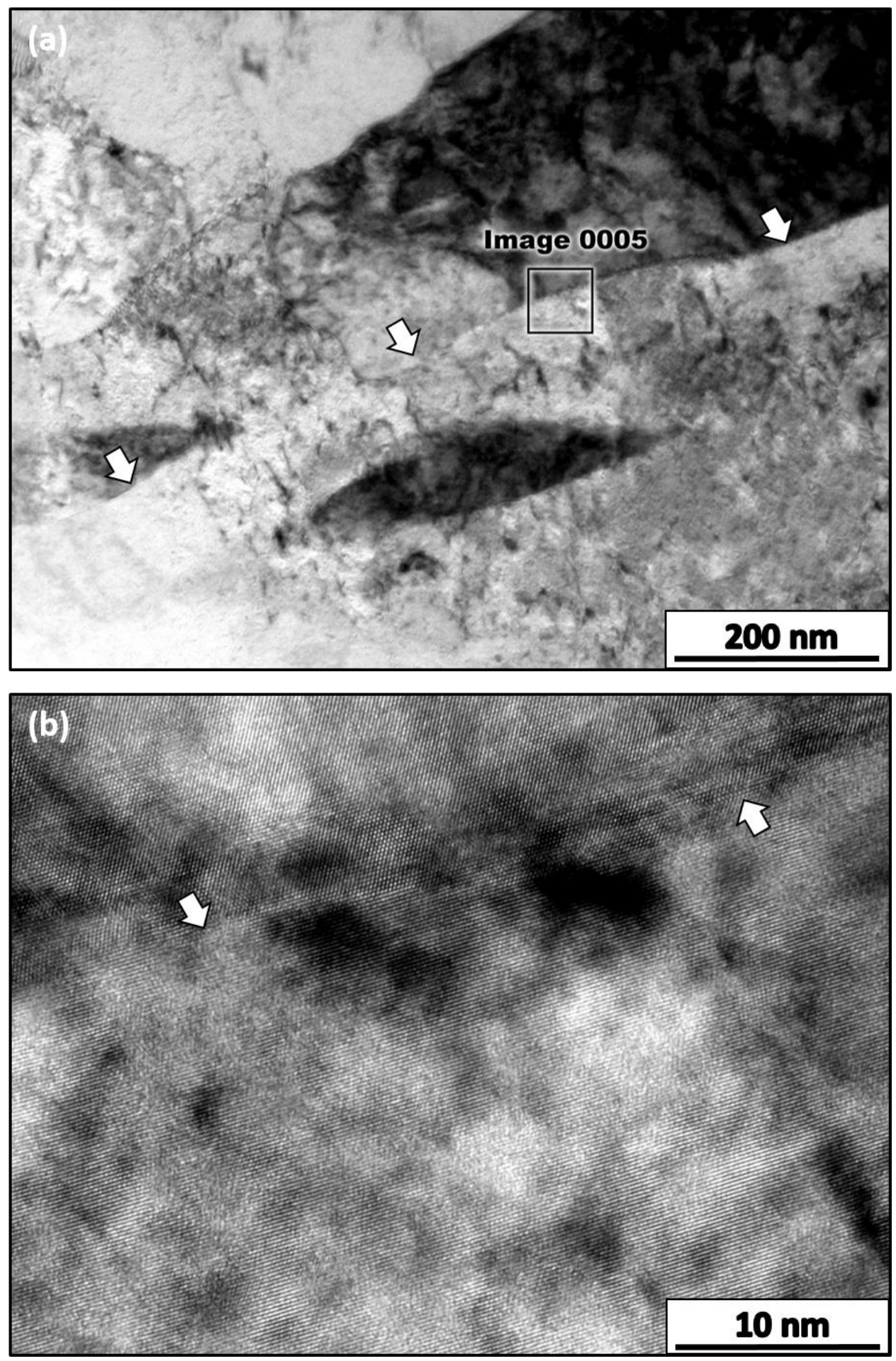

Figure 82 Transmission electron micrographs of Ferrium PH48S - H900: (a) Bright field image of martensite block structure of Ferrium PH48S - H900 and (b) High magnification bright field image of the boxed region in (a). A single, identical block boundary is indicated by white arrows in each image. Both images were taken in collaboration with Matt Schneider at The University of Virginia. 
For UHSS alloys in Chapter 3, thin film austenite along block boundaries was hypothesized to have a universal-detrimental effect on HEAC resistance. Thin film austenite along martensite block boundaries should increase the hydrogen concentration local to the block interface relative to an austenite free structure. During HEAC, high plastic strain should transform $\mathrm{H}$-saturated precipitated austenite into H-supersaturated, untempered martensite along martensite interfaces [128]. Strain transformation of continuous thin film precipitated austenite in UHSS would produce a tensile stress across the entire lath/block boundary to reduce the more global stress necessary to initiate fracture at the block boundary in the HELP/HEDE mechanism for HEAC. In Custom 465 and Ferrium PH48S, all evidence points to a lack of thin film austenite along block boundaries. In Ferrium PH48S - H900, this conclusion is confirmed with SAD and bright field TEM images of martensite block boundaries (Figures 81-82). EBSD and SEM-BSE imaging support this conclusion in Custom 465, but TEM is necessary to confirm the lack of thin film austenite. Both alloys contain a small fraction of precipitated austenite present in a small island morphology, as characterized via fine probe EBSD (Figures 48-49).

In the context of the HELP/HEDE mechanism for HEAC, the lack of thin film austenite in Ferrium PH48S - H900 and Custom 465 - H900 should provide a beneficial effect on HEAC properties. Compared to baseline AerMet 100, which was shown to contain thin film austenite at martensite lath interfaces [24], the local stress needed to fracture a martensite block boundary should be substantially increased in Ferrium PH48S - H900. Within the thin film mechanism outlined above, the absence of thin film austenite along the martensite block boundaries would prevent the formation of $\mathrm{H}$-supersaturated, untempered martensite along the boundary. This effect would limit the easy decohesion based pathway through the microstructure, speculatively increasing $\mathrm{k}_{\mathrm{IG}}$ and reducing $\alpha$ in Equation 1. However, recall the identical HEAC fracture morphology observed for TG QC HEAC in AerMet 100 and Ferrium PH48S when stressed under heavy cathodic polarization (compare Figure 19 and Figure 59). As previously established, TG QC HEAC of UHSS and UHSSS fracture proceeds along $\{110\}_{\alpha^{\prime}}$ martensite block boundaries. Identical HEAC fracture morphology between AerMet 100 and Ferrium PH48S clearly indicates that precipitated austenite has no effect on HEAC fracture morphology. 


\subsubsection{Effect of $E_{\text {app }}$ on $K_{T H}$ of Custom 465 and Ferrium PH48S at Equal Strength}

As shown in Figures 67-70, the HEAC properties in UHSSS alloys strongly depend on aging condition. To understand the effect of H-solubility, impurity content, and grain boundary character distribution on the applied potential dependence of HEAC in UHSSS, it is useful to compare Custom 465 and Ferrium at equal yield strength. An equal strength comparison of the two alloys eliminates the confounding effect of alloy strength on crack tip hydrogen concentration, which can dictate $\mathrm{K}_{\mathrm{TH}}$ and to a lesser extent da/dt $\mathrm{dI}_{\text {II }}$

When aged to equal ultra-high strength, the $\mathrm{E}_{\text {app }}$ dependence of $\mathrm{K}_{\mathrm{TH}}$ in Ferrium PH48S - H980 and Custom 465 - H900 converges to a single unified dependency. In examination of Figure 67, two points are notable. First, both alloys exhibit high $\mathrm{K}_{\mathrm{TH}}$ in a range of $\mathrm{E}_{\text {app }}$ mildly cathodic to OCP. $\mathrm{K}_{\mathrm{TH}}$ is stable and near $\mathrm{K}_{\mathrm{IICi}}$ for $175-225 \mathrm{mV}_{\mathrm{SCE}}$ of cathodic polarization below OCP. However, $\mathrm{K}_{\mathrm{TH}}$ in this range is increased in Ferrium PH48S compared to Custom 465. Second, below $\mathrm{E}_{\mathrm{app}}=$ $-450 \mathrm{mV}_{\mathrm{SCE}}, \mathrm{K}_{\mathrm{TH}}$ precipitously declines and approaches a stable-common value of $\sim 10 \mathrm{MPa} \sqrt{\mathrm{m}}$ for both Custom 465 - H900 and Ferrium PH48S - H860. The precise $E_{\text {app }}$ at which $\mathrm{K}_{\mathrm{TH}}$ begins to decline with decreasing $\mathrm{E}_{\text {app }}$ is uncertain due to the limited number of specimens tested. However, it is assumed that a similar rate of decline in $\mathrm{K}_{\mathrm{TH}}$ would be exhibited by Ferrium PH48S - H860 if sufficient samples were tested. The remainder of this section is devoted to understanding of these two effects in the context of the micromechanical model for decohesion based $\mathrm{K}_{\mathrm{TH}}$ and the results of UHSS modeling of HEAC properties put forth in Chapter 3.

In contrast to Custom 465 and Ferrium PH48S, UHSS alloys (Figure 24) do not exhibit stablehigh $\mathrm{K}_{\mathrm{TH}}$ near $\mathrm{K}_{\mathrm{JICi}}$ across a relatively wide range of $\mathrm{E}_{\mathrm{app}} . \mathrm{K}_{\mathrm{TH}}$ in UHSS appears to spike in a narrow range of $E_{a p p}$ and then sharply decrease with anodic or cathodic polarization. This observation is weakened somewhat by the limited number of samples tested at $E_{a p p}$ in the vicinity of maximum $\mathrm{K}_{\mathrm{TH}}$. However, as shown in Figure 69, when Ferrium PH48S is tempered to higher aging temperatures, the range of cathodic $\mathrm{E}_{\mathrm{app}}$ conferring strong SCC-resistance is dramatically increased. For example, the H950 and H968 aging conditions of Ferrium PH48S exhibit exceptionally high $\mathrm{K}_{\mathrm{TH}}(>90 \mathrm{MPa} \sqrt{\mathrm{m}}$ ) over at least $775 \mathrm{mV}$ of cathodic polarization. Critically, these aging conditions have nearly identical yield strength (Table 1-2) and martensite morphology (compare Figure 9-10 and 40) as CrNiMoWV or Ferrium S53. This key result indicates that the 
wide breadth of the protective window is unique to intermetallic strengthened UHSSS Custom 465 and Ferrium PH48S.

As characterized via EBSD, Custom 465 and Ferrium PH48S have dramatically lower precipitated austenite contents compared to all UHSS alloys. The lack of thin film austenite in Ferrium PH48S - H900 was confirmed via TEM, and similar austenite free martensite interfaces are suggested in Custom 465 - H900. Speculatively, a lack of thin film austenite in Ferrium PH48S - 860 and Custom $465-\mathrm{H} 900$ is the origin of improved SCC-HEAC resistance across the range of protective cathodic $\mathrm{E}_{\text {app. }}$ Thin film austenite could provide a mechanism to enhance the effect of a small amount of crack tip hydrogen produced at mildly cathodic $\mathrm{E}_{\text {app }}$ in UHSS. Dynamic equilibrium between $\mathrm{H}$ residing in reversible trap sites during HEAC dictates that strong reversible trap sites on the surface of austenite interface $\left(\mathrm{E}_{\mathrm{B}} \sim 50 \mathrm{~kJ} / \mathrm{mol},[189]\right)$ would contain a large amount of hydrogen relative to weak-reversible trap states at strengthening precipitates $\left(\mathrm{E}_{\mathrm{B}} \sim 11.5 \mathrm{~kJ} / \mathrm{mol}\right.$ [130]), and moreover, the lattice solubility of $\mathrm{H}$ in FCC-iron austenite is much higher than in bcc martensite [21]. This high hydrogen concentration local to martensite block boundaries will embrittle the interface through strain transformation to $\mathrm{H}$-supersaturated martensite when sufficient crack tip strain is reached. The demonstrated lack of thin film austenite in Custom 465 - H900 and Ferrium PH48S - H860 indicates that this hypothesized-deleterious mechanism is not active. Further, as shown by TDS measurements in Figure 73, there is no evidence of strong reversible H-trap sites with binding energies near $\sim 60 \mathrm{~kJ} / \mathrm{mol}$ that would be expected for the austenite interface $[130,189]$. Note the lack of higher energy peaks in the comparison of TDS desorption spectra in Figure 73 (UHSSS) and Figure 75 (AerMet 100). Taken together, the lack of strong-reversible $\mathrm{H}$-desorption peaks and spatially resolvable thin film austenite provide strong evidence that the amount of precipitated austenite in Custom 465 and Ferrium PH48S is negligible. Within the HELP-HEDE mechanism, reduction of the amount of hydrogen trapped along the HEAC fracture path would logically increase the local stress necessary to initiate fracture of the boundary and raise $\mathrm{K}_{\mathrm{TH}}$. Speculatively, this lack of thin film austenite in Custom 465 and Ferrium PH48S provides the basis for strong-stable $\mathrm{K}_{\mathrm{TH}}$ observed over a range of $\mathrm{E}_{\mathrm{app}}$ slightly cathodic to OCP. In this regime, $\mathrm{C}_{\mathrm{H} \text {-DIFF }}$ is at a minimum and the deleterious effect of $\mathrm{H}$-saturated austenite to hard untempered martensite transformation could enhance the effect of small $\mathrm{C}_{\mathrm{H} \text {-DIFF, which is }}$ insufficient to promote significant HEAC in the absence of precipitated austenite. While 
speculative, the proposed mechanism for deleterious thin film austenite reasonably explains the lack of stable-high $\mathrm{K}_{\mathrm{TH}}$ for AerMet 100 and Ferrium M54 (Figure 24) when compared to Custom 465 and Ferrium PH48S (Figure 69).

When tempered to equal strength, Ferrium PH48S and Custom 465 exhibit a near-identical falloff of $\mathrm{K}_{\mathrm{TH}}$ with decreasing $\mathrm{E}_{\text {app }}$ below the cathodic prevention regime, to reach a common value of $\sim 10 \mathrm{MPa} \vee \mathrm{m}$, which is stable with further-increasing cathodic polarization. As characterized via hot extraction in Figure 71, $\mathrm{C}_{\mathrm{H}-\mathrm{DIFF}}$ in Custom 465 - H900 is increased 8.6 to 31.5 times compared to three aging conditions of Ferrium PH48S (H900, H950, and H968). The hydrogen solubility of Ferrium PH48S - H860 was not probed in the present study. There is no immediate reason to suspect that $\mathrm{C}_{\mathrm{H}-\mathrm{DIFF}}$ in Ferrium PH48S - H860 would show a large departure from this low Hsolubility in Ferrium PH48S at the higher aging temperatures. This speculation assumes that the Al-enriched strengthening precipitate in Ferrium PH48S is similar at all aging tempertures from $860^{\circ} \mathrm{C}$ to $968^{\circ} \mathrm{C}$. Dramatically increased $\mathrm{C}_{\mathrm{H}-\mathrm{DIFF}}$ in Custom 465 should have an overriding effect to reduce $\mathrm{K}_{\mathrm{TH}}$. However, the present work has not fundamentally established the origin of increased H-solubility in Custom 465 and interpretations on HEAC behavior must be metered given this uncertainty. Moreover, the lack of a rare earth treatment in Custom 465 indicates that a significant free impurity content could reduce $\mathrm{k}_{\mathrm{IG}}$ and increase $\alpha$ in Equation 1 . All material based variables indicate that the HEAC resistance of Custom 465 should be reduced compared to Ferrium PH48S at equal strength. Further, increased $\mathrm{k}_{\mathrm{IG}}$ and $\alpha$ for Ferrium PH48S due to rare earth treatment indicates that $\mathrm{C}_{\mathrm{H} \sigma}$ must be enhanced relative to Custom 465 to produce identical $\mathrm{K}_{\mathrm{TH}}$.

A possible source for an identical $\mathrm{E}_{\mathrm{app}}$ dependence of $\mathrm{K}_{\mathrm{TH}}$ in Ferrium PH48S - H860 and Custom 465 - H900 is a change in the hydrogen uptake behavior between the UHSSS specimen charged in the bulk- $\mathrm{NaOH}$ environment compared to $\mathrm{H}$ uptake at the crack tip. For example, Ferrium PH48S charged in $0.001 \mathrm{M} \mathrm{NaOH}(\mathrm{pH}=10)$ could differ from uptake in the aqueous chloride environment at the crack tip during $\mathrm{HEAC}$ at cathodic $\mathrm{E}_{\mathrm{app}}$. If the formation of a passive film limited H-uptake during H-charging of Ferrium PH48S in the bold-surface exposure experiments, then the measured values of $\mathrm{C}_{\mathrm{H}-\mathrm{DIFF}}$ shown in Figure 71 would be substantial underestimates. Dissolution of a surface film in the chloride environment of HEAC testing would lead to enhanced H-uptake and $\mathrm{C}_{\mathrm{H} \sigma}$ similar to Custom 465, driving the identical $\mathrm{E}_{\mathrm{app}}$ dependence of $\mathrm{K}_{\mathrm{TH}}$. Alternately, if $\mathrm{H}$ trapping within an intermetallic phase, as postulated for Ti-rich $\eta$ precipitates in 
Custom 465-H900, requires substantial time, which was available in the long-term bulk charging experiments, then it is possible that such enhanced $\mathrm{H}$ uptake did not occur at the tip of (at least) the propagating crack in this UHSS. As such, the measured values of $\mathrm{C}_{\mathrm{H} \text {-DIFF }}$ shown in Figure 71 would be substantial overestimates for Custom 465-H900, in the context of interpreting HEAC.

\subsubsection{Modeling the effect of $E_{\text {app }}$ on da/dt ${ }_{I I}$ of Custom 465, Ferrium PH48S, and Ferrium S53}

Following the considerations for UHSS cracking in Chapter 3, Stage II HEAC growth rates for UHSSS in $\mathrm{NaCl}$ solution are interpreted through the H-diffusion limited model for discontinuous crack growth presented in Equation $2[2,6,7]$. Provided that $\chi_{\text {crit }}$ is constant for high strength alloys, any variation in observed da/dt is sourced at three parameters in Equation 2: $\mathrm{D}_{\mathrm{H}-\mathrm{EFF}}, \mathrm{C}_{\mathrm{H \sigma}}$, and $\mathrm{C}_{\mathrm{H \sigma}}$ crit, as leveraged or damped by the magnitude of the inverse error function term. The fastest observed da/dt is directly proportional to $\mathrm{D}_{\mathrm{H}-\mathrm{EFF}}$, while the ratio of the remaining two variable parameters influences $\mathrm{da} / \mathrm{dt}_{\text {II }}$ through the inverse error function term. The remainder of this section is devoted to examining the effect of these parameters on $\mathrm{da} / \mathrm{dt}_{\mathrm{II}}$ and evaluating the efficacy of the diffusion limited model for da/dt II (Equation 2) for Custom 465, Ferrium PH48S and Ferrium S53, which each contain a substantial level of Cr. Two fundamental issues hinder this effort. First, literature characterization of $\mathrm{D}_{\mathrm{H}-\mathrm{EFF}}$ in UHSSS is limited for Custom 465 and nonexistent for Ferrium PH48S and Ferrium S53. Second, and more important, there is no independent characterization of the $E_{\text {app }}$ dependence of crack tip hydrogen concentration in UHSSS [19]. Each of these issues will be explored before applying the diffusion limited model for $\mathrm{da} / \mathrm{dt}_{\mathrm{II}}$ to the data shown in Figure 70.

As shown in Figure 70, da/dt II in Ferrium PH48S (of 4 aging conditions) is significantly reduced compared to Custom 465 - H900 at all SCC-susceptible $\mathrm{E}_{\mathrm{app}}$; and maximum observed da/dt II reduced 7 to 25 times in Ferrium PH48S. Literature measurements indicate that $\mathrm{D}_{\mathrm{H}-\mathrm{EFF}}$ in Custom $465-\mathrm{H} 900$ is $6.2 \times 10^{-9} \mathrm{~cm}^{2} / \mathrm{s}$ [175], and is significantly reduced compared to similar UHSSS PH $17-4\left(2.8-9.7 \times 10^{-8} \mathrm{~cm}^{2} / \mathrm{s}\right)$ and UHSS AerMet $100\left(1 \times 10^{-8} \mathrm{~cm}^{2} / \mathrm{s} \mathrm{[16]-} 1 \times 10^{-9} \mathrm{~cm}^{2} / \mathrm{s}\right.$ [22]). No measurements of $\mathrm{D}_{\mathrm{H}-\mathrm{EFF}}$ in Ferrium PH48S have been reported in the literature. However, good agreement of regression fit $\mathrm{D}_{\mathrm{H}-\mathrm{EFF}}$ for Custom $465-\mathrm{H} 900\left(2.6 \times 10^{-9} \mathrm{~cm}^{2} / \mathrm{s}\right.$, Table 12$)$ with TDSoutgassing measurements of Custom $465-\mathrm{H} 900$ by Schaller $\left(6.2 \times 10^{-9} \mathrm{~cm}^{2} / \mathrm{s}\right)$ support the use of regression fit $\mathrm{D}_{\mathrm{H}-\mathrm{EFF}}$ for H-outgassing in modeling of da/dt in Ferrium PH48S [175]; as such, the 
values of $\mathrm{D}_{\mathrm{H}-\mathrm{EFF}}$ for H-outgassing shown in Table 12 will be used for modelling da/dt $\mathrm{II}$. The $\mathrm{D}_{\mathrm{H}-}$ EFF for Ferrium PH48S - H860 is assumed to be the average of regression fit $\mathrm{D}_{\mathrm{H}-\mathrm{EFF}}$ for the remaining aging conditions $\left(5.0 \times 10^{-10} \mathrm{~cm}^{2} / \mathrm{s}\right)$. As discussed in Chapter 1, Ferrium S53 is expected to have a near identical strengthening dispersion of $\mathrm{M}_{2} \mathrm{C}$ carbides as AerMet 100. Furthermore, the martensite morphology (compare Figure 7 to Figure 10) and EBSD-measured austenite content (1.5 and 1.9 vol\% for AerMet 100 and Ferrium S53, respectively) in these alloys are nearly identical. Based upon these similarities, $\mathrm{D}_{\mathrm{H}-\mathrm{EFF}}$ of Ferrium S53 is assumed equal to that for AerMet $100\left(1.0 \times 10^{-9} \mathrm{~cm}^{2} / \mathrm{s}[1]\right)$.

Modeling efforts for HEAC properties $\left(\mathrm{K}_{\mathrm{TH}}\right.$ and $\left.\mathrm{da} / \mathrm{dt}_{\mathrm{II}}\right)$ in Chapter 3 were based upon the independent characterization of the $\mathrm{E}_{\mathrm{app}}$ dependence of occluded crack tip hydrogen concentration $\left(\mathrm{C}_{\mathrm{H} \text {,Diff }}\right.$ in Equation 5 and Figure 29) for AerMet 100 [20]. No prior characterization of $\mathrm{C}_{\mathrm{H}-\mathrm{DIFF}}$ as a function of $E_{\text {app }}$ in UHSSS is available in literature. However, through Equation 1, the $\mathrm{K}_{\mathrm{TH}}$ versus $\mathrm{E}_{\text {app }}$ data presented in Figure 67 and Figure 69 provide a means to back-calculate $\mathrm{C}_{\mathrm{H} \sigma}$ versus $\mathrm{E}_{\text {app }}$ if reasonable assignments of $\beta, \alpha^{\prime}, \alpha$, and $\mathrm{k}_{\mathrm{IG}}$ are made. Conversion of $\mathrm{C}_{\mathrm{H} \sigma}$ to $\mathrm{C}_{\mathrm{H} \text {-DIFF }}$ through Equation 12 yields the $\mathrm{E}_{\text {app }}$ dependence of $\mathrm{C}_{\mathrm{H}-\mathrm{DIFF}}$ for Custom 465, Ferrium PH48S, and Ferrium S53. For each UHSSS alloy, $\beta$ ' and $\alpha$ ', are assumed identical to those calculated for AerMet 100 $\left(\alpha ”=0.00022 \mathrm{MPa}^{-1,} \beta^{\prime}=0.20(\mathrm{MPa} \sqrt{ })^{-1}\right) ; \mathrm{k}_{\mathrm{IG}}$ is constrained such that Equation 1 yields the plane strain fracture toughness of the alloy (Table 1) when no hydrogen is present. (This toughness is a lower bound of the actual Griffith toughness of the prior austenite or martensite block interfaces, which constitute the HEAC path in the steel.) Using the measured values of $\mathrm{K}_{\text {IIci }}$ from Table 11 for Ferrium PH48S - H860, Ferrium PH48S - H900, Ferrium PH48S - H968, Custom $465-\mathrm{H} 900$, and Ferrium S53, $\mathrm{k}_{\mathrm{IG}}$ is $1.060,1.125,1.1701 .025$, and $1.020 \mathrm{MPa} \sqrt{\mathrm{m}}$, respectively. Strong rare earth treatment in Ferrium PH48S and Ferrium S53 indicates that the susceptibility of the microstructure to hydrogen $(\alpha)$ should be identical to that calculated for AerMet $100(0.9$ $\mathrm{MPa} \sqrt{ } \mathrm{m}$ per atom fraction $\mathrm{H})$. Intergranular fracture of Custom 465 - H900 indicates that an impurity and/or slip length effect increases the HEAC susceptibility of the alloy, possibly increasing $\alpha$. However, total impurity concentration is small compared to $300 \mathrm{M}(\alpha=1.2 \mathrm{MPa} \sqrt{\mathrm{m}}$ per atom fraction $\mathrm{H}$ ), and there is no reason to suspect an impurity based increase to $\alpha$ of this magnitude. For Custom 465, $\alpha$ is assumed to be $1.0 \mathrm{MPa} \sqrt{\mathrm{m}} /$ atom fraction $\mathrm{H}$, increased compared to AerMet 100 due to the lack of rare earth treatment (and longer slip length), and reduced from 
$300 \mathrm{M}$ due to the intrinsically low impurity content. As shown in Section 3.3.5, model predictions of HEAC properties are universally improved for UHSS alloys, using the upper bound crack tip hydrostatic stress $\left(6.8 \sigma_{\mathrm{YS}}\right)$, and this value of $\sigma_{\mathrm{H}}$ is used for all UHSSS alloys.

Based upon these assumptions, $\mathrm{C}_{\mathrm{H}-\mathrm{DIFF}}$ was calculated for each measured value of $\mathrm{K}_{\mathrm{TH}}$ using Equation 1 and the results of this analysis are shown in Figure 83. Following the results presented in Figure 29, a second order polynomial was fit to each data set to allow interpolation of $\mathrm{C}_{\mathrm{H}-\mathrm{DIFF}}$ at intermediate $E_{a p p}$. The results of these regression analyses are shown in Equations 15 to 18 for Ferrium PH48S - H860, Ferrium PH48S - H900, Ferrium PH48S - H968, and Ferrium S53, respectively.

$$
\begin{array}{ll}
(P H 48 S-H 860) & C_{H, \text { Diff }}=6.4552 E_{a p p}^{2}+2.8246 E_{a p p}+0.4507 \quad\left(R^{2}=0.76\right) \\
(P H 48 S-H 900) & C_{H, \text { Diff }}=9.9276 E_{a p p}^{2}+6.3789 E_{a p p}+1.2376 \quad\left(R^{2}=0.89\right) \\
(P H 48 S-H 968) & C_{H, \text { Diff }}=17.2080 E_{a p p}^{2}+14.365 E_{a p p}+3.5763 \quad\left(R^{2}=0.67\right) \\
(\text { Ferrium S53 }) & C_{H, \text { Diff }}=82.156 E_{\text {app }}^{2}+116.40 E_{a p p}+0.5494 \quad\left(R^{2}=0.54\right)
\end{array}
$$

For all UHSSS alloys and tempers, except Custom 465, the $\mathrm{E}_{\text {app }}$ dependence of $\mathrm{C}_{\mathrm{H} \text {-DIFF }}$ follows that which is independently established for AerMet 100 (Figure 29) under identical testing conditions. Notably, Custom 465 - H900 does not exhibit rising-calculated $\mathrm{C}_{\mathrm{H} \text {-DIFF }}$ with increasing cathodic polarization (decreasing $\mathrm{E}_{\text {app }}$ ). Diffusible hydrogen concentration exhibits a near-discontinuous step function increase at $\mathrm{E}_{\text {app }}$ of $-475 \mathrm{mV}_{\mathrm{SCE}}$, and is stable at $1.5 \mathrm{wppm}$. Stable $\mathrm{C}_{\mathrm{H}-\mathrm{DIFF}}$ with decreasing $E_{\text {app }}$ has been previously observed by Kehler et all for Custom 465 coupons charged in borate buffer $(\mathrm{pH}=8)$ [105], albeit at a much higher stable value ( $17 \mathrm{wppm})$. This result suggests that there are significant differences between the hydrogen uptake of specimens charged in $0.001 \mathrm{M}$ $\mathrm{NaOH}(\mathrm{pH}=10)$ or borate buffer $(\mathrm{pH}=8$ [105]). Direct characterization of the crack tip diffusible hydrogen concentration is beyond the scope of the present work and, as such, modeling of da/dtII cannot be accurately accomplished in Custom 465 . 


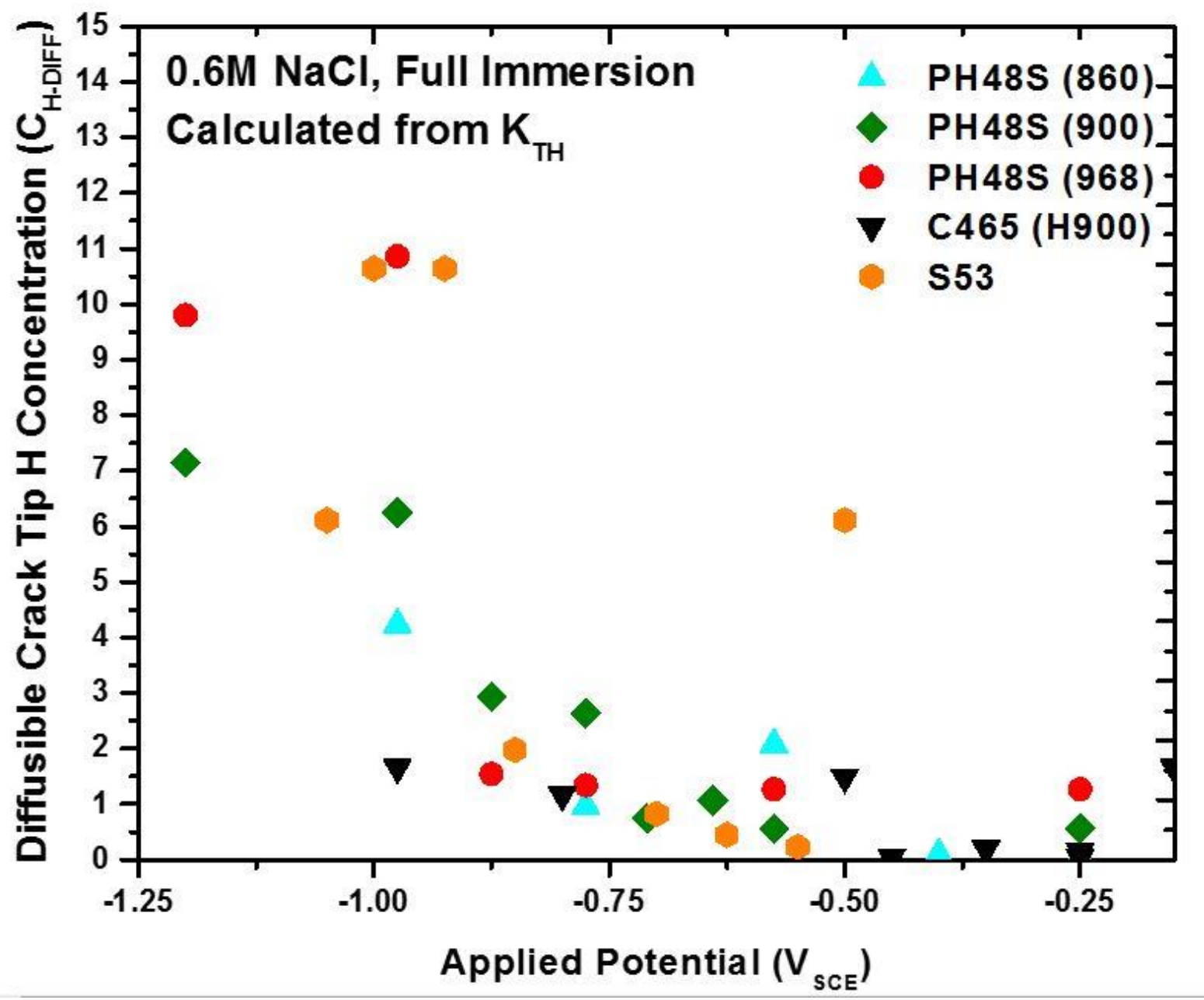

Figure $83 \quad C_{H-D I F F}$ as a function of $E_{a p p}$ for five ultra-high strength stainless steel-aging temperature combinations. Values of $C_{H-D I F F}$ are calculated from $K_{T H}$ versus $E_{a p p}$ measurements (Figures 67 and 69) through Equation 1 and Equation 12.

Using the assumed-reasonable values of $\mathrm{D}_{\mathrm{H}-\mathrm{EFF}}$ and the predicted $\mathrm{C}_{\mathrm{H} \text {-DIFF }}$ verses $\mathrm{E}_{\text {app }}$ from Equations 15-18, $\mathrm{H}$ diffusion limited da/dt $\mathrm{dI}_{\text {II }}$ versus $\mathrm{E}_{\text {app }}$ is calculated from Equation 2 using a single adjustable parameter of $\mathrm{C}_{\mathrm{H \sigma}-\mathrm{CRIT}}$ selected to best-fit da/dtII values measured at $\mathrm{K}$ of $40 \mathrm{MPa} \sqrt{\mathrm{m}}$. The results of this analysis are shown in Figures 84 to 87 . In each plot when the argument for the inverse error function term is negative due to $\mathrm{C}_{\mathrm{H \sigma}} \leq \mathrm{C}_{\mathrm{H \sigma}-\text {-crit, }}$ the predicted da/dtII is equated to $4 \mathrm{x}$ $10^{-5} \mu \mathrm{m} / \mathrm{s}$, essentially the best possible resolution limit of dcPD monitoring of crack growth. 


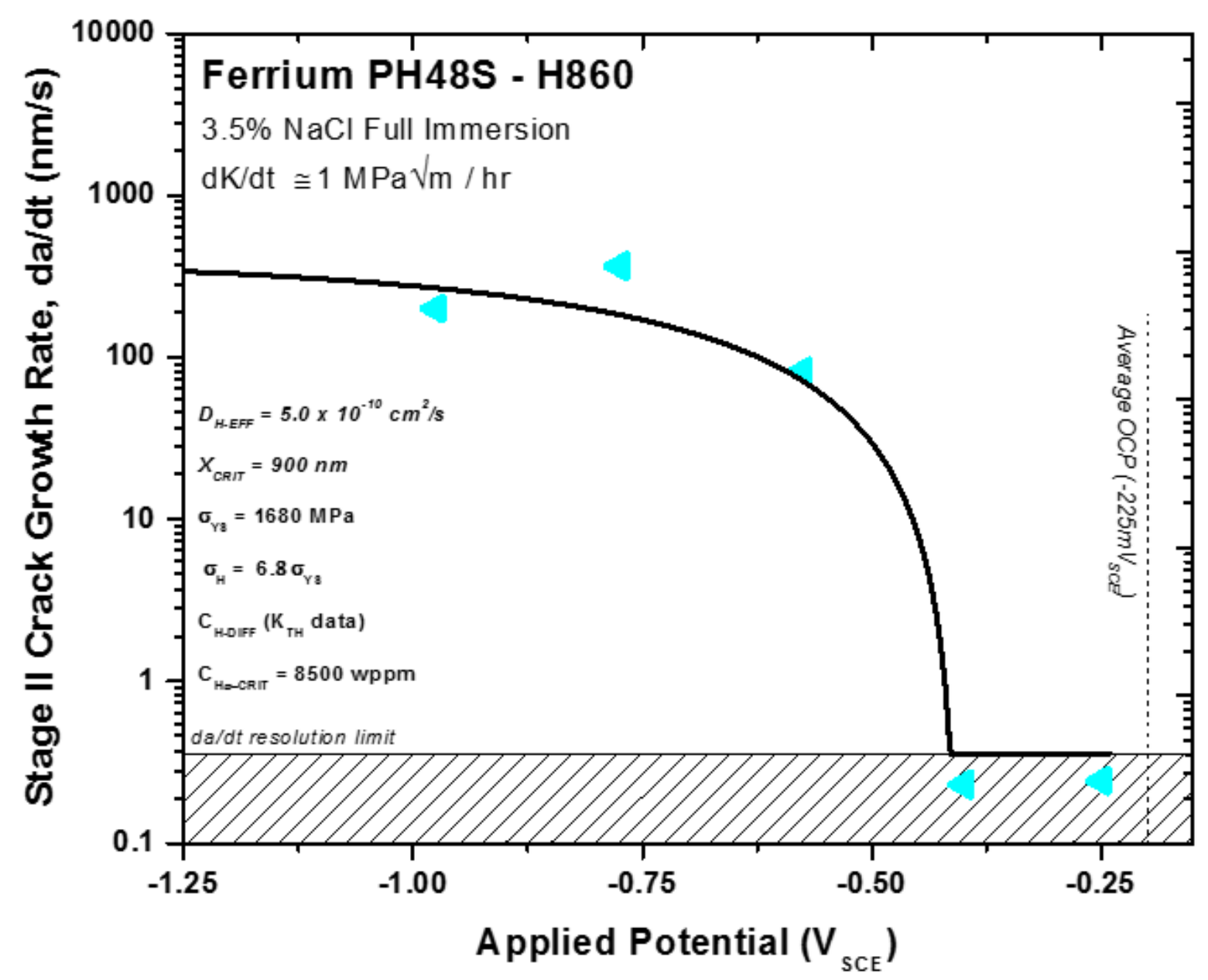

Figure 84 Measured da/dt $t_{I I}$ at $K=40 \mathrm{MPa} \sqrt{\mathrm{m}}(\varangle)$ and the model predicted trend line of the applied potential dependence of da/dt $t_{I}$ for Ferrium PH48S - H860, using the H-diffusion limited model (Equation 2) with inputs of $C_{H-D I F F}$ calculated from Equation 15, average $D_{H-E F F}$ as described in the text, correlated $\chi_{\text {crit }}[2,3]$, best fit $C_{H \sigma-c r i t}$, and the upper bound of $\sigma_{H}=6.8 \sigma_{Y S}$. 


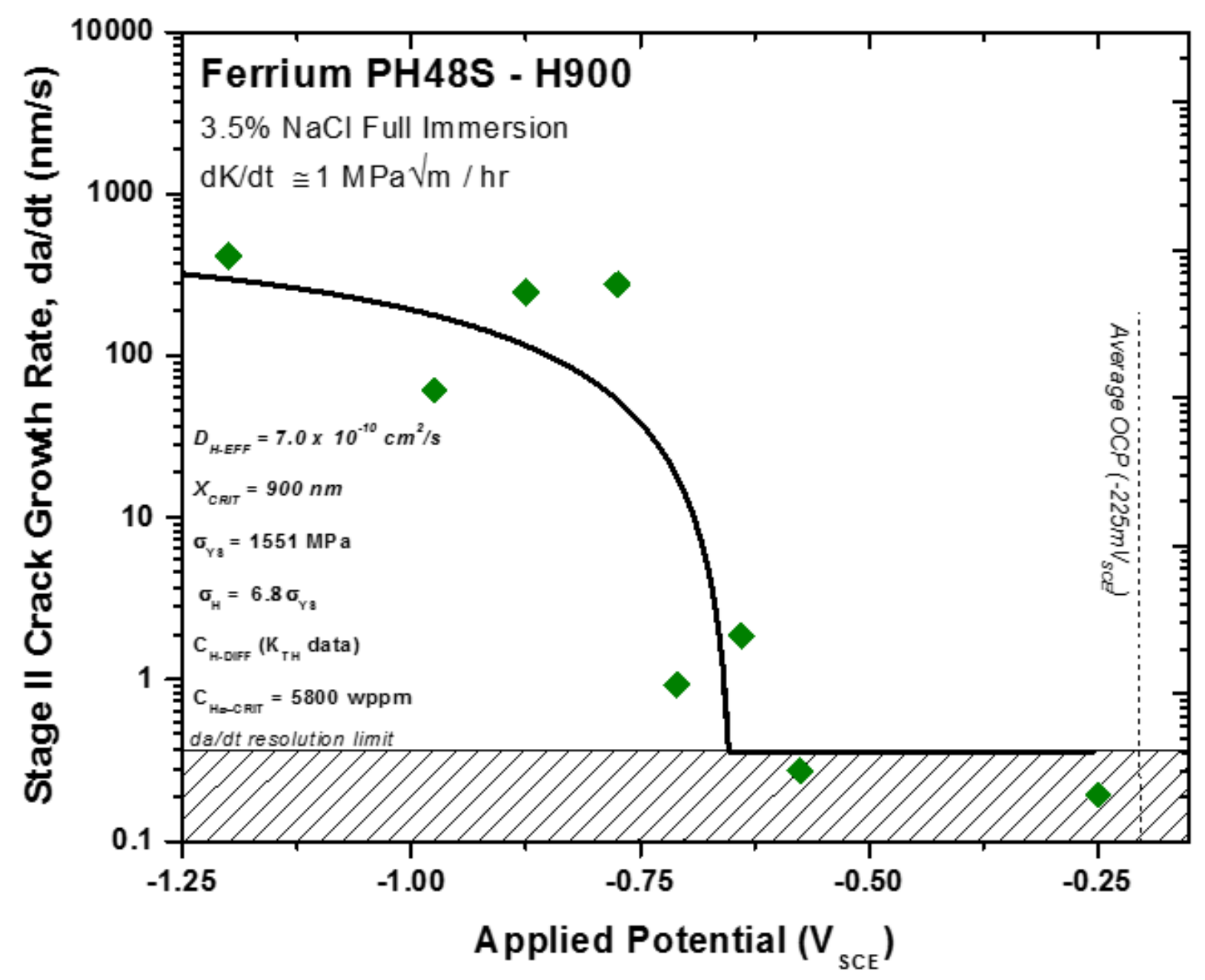

Figure 85 Measured da/dt ${ }_{I I}$ at $K=40 \mathrm{MPa} \mathrm{V}_{\mathrm{m}}$ (४) and the model predicted trend line of the applied potential dependence of da/dtII for Ferrium PH48S - H900, using the H-diffusion limited model (Equation 2) with inputs of $C_{H-D I F F}$ calculated from Equation 16, measured $D_{H-E F F}$, correlated $\chi_{\text {crit }}[2,3]$, best fit $C_{H \sigma-c r i t}$, and the upper bound of $\sigma_{H}=6.8 \sigma_{Y S}$. 


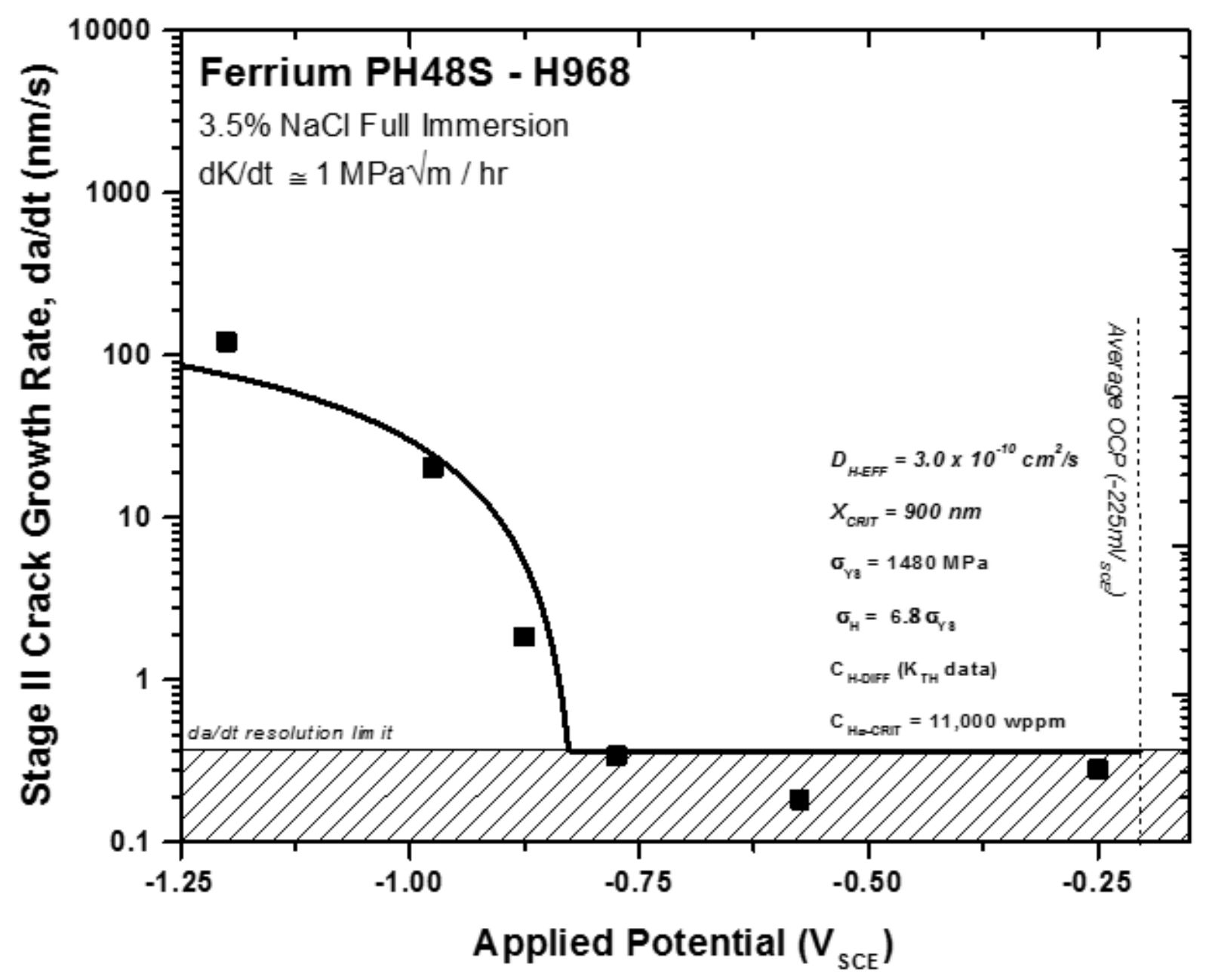

Figure $86 \quad$ Measured da/dt II at $K=40 \mathrm{MPa} \mathrm{V}_{\mathrm{m}}(\square)$ and the model predicted trend line of the applied potential dependence of da/dt $t_{I I}$ for Ferrium PH48S - H968, using the H-diffusion limited model (Equation 2) with inputs of $C_{H-D I F F}$ calculated from Equation 17, measured $D_{H-E F F}$, correlated $\chi_{\text {crit }}[2,3]$, best fit $C_{H \sigma-c r i t}$, and the upper bound of $\sigma_{H}=6.8 \sigma_{Y S}$. 


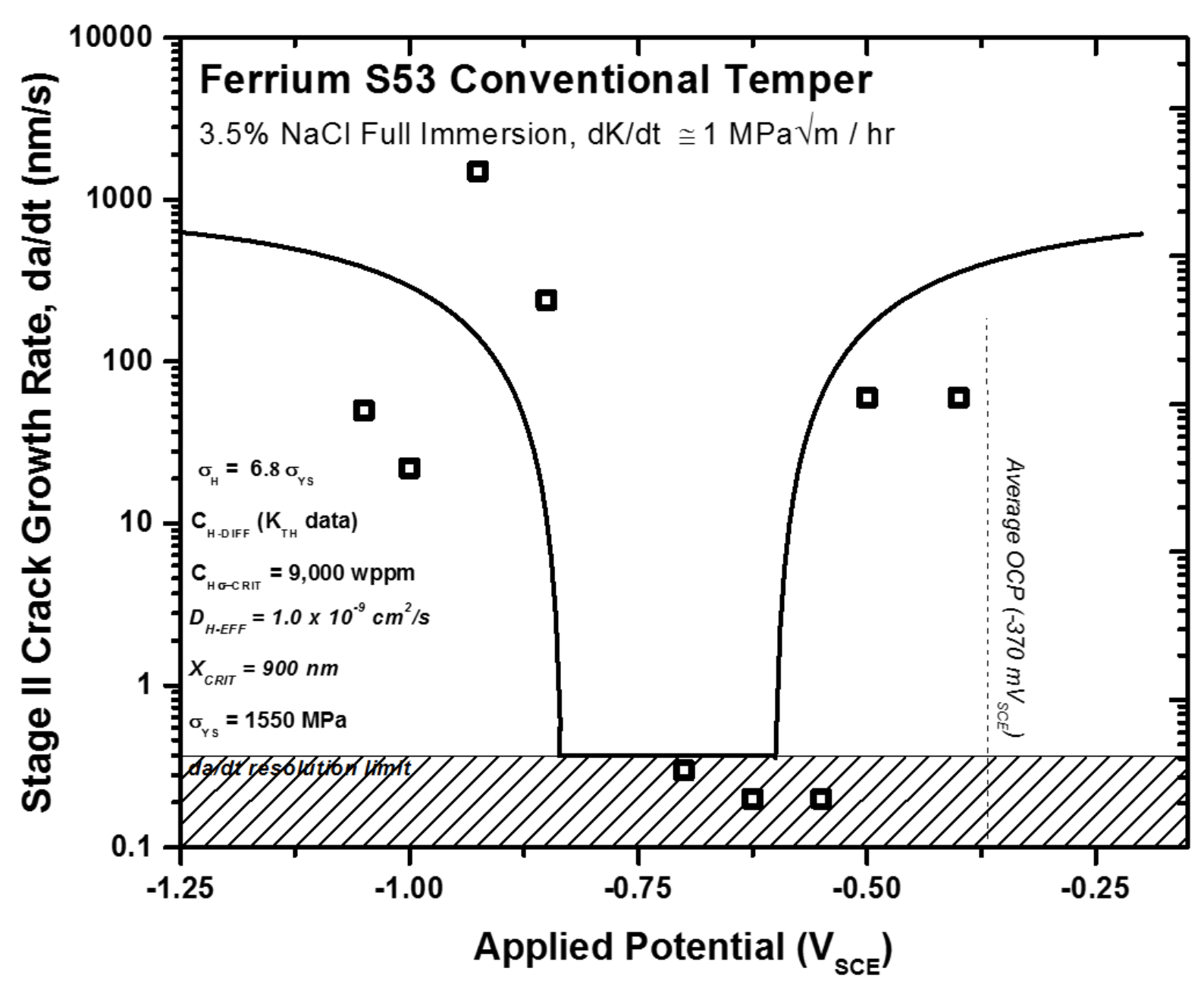

Figure 87 Measured da/dt ${ }_{I I}$ at $K=40 \mathrm{MPa} \backslash \mathrm{Vm}$ (a) and the model predicted trend line of the applied potential dependence of da/dt ${ }_{I I}$ for Ferrium S53, using the H-diffusion limited model (Equation 2) with inputs of $C_{H-D I F F}$ calculated from Equation 18, average $D_{H-E F F}$ for AerMet 100 , correlated $\chi_{\text {crit }}[2,3]$, best fit $C_{H \sigma-c r i t}$, and the upper bound of $\sigma_{H}=6.8 \sigma_{Y S}$.

Comparison of the results presented in Figures 84 to 87 establishes 4 important points. First, the diffusion limited model of $\mathrm{da} / \mathrm{dt}_{\text {II }}$ accurately captures the potential dependence of $\mathrm{da} / \mathrm{dt}_{\text {II }}$ using a set of reasonable assumptions for key parameters, coupled with $E_{\text {app-dependent occluded-crack tip }}$ diffusible $\mathrm{H}$ concentration which is back-calculated from measured $\mathrm{K}_{\mathrm{TH}}$. The predictive strength of the model is significantly improved in Ferrium PH48S (H860, H900, H968) compared to Ferrium S53. This effect is attributed to the quality of the regression fit for Equation 15-18. The quality of the regression fit is significantly degraded in the presence of discontinuous jumps in $\mathrm{C}_{\mathrm{H}}$ DIFF, as is exhibited by Ferrium S53 for $-550<\mathrm{E}_{\mathrm{app}}<-500 \mathrm{mV}_{\mathrm{SCE}}$; subsequently reducing the 
accuracy of the model. This issue is not necessarily caused by a weakness or inaccuracy in the model, but rather appears to be due to the physically real and discontinuous changes in $\mathrm{C}_{\mathrm{H}-\mathrm{DIFF}}$. The electrochemical origins of these changes are not understood, particularly the strong-spiked rise in $\mathrm{H}$ concentration at moderately cathodic values of $\mathrm{E}_{\text {app. }}$ Second, the window of cathodic polarizations conferring exceptional HEAC resistance gradually increases in Ferrium PH48S as the aging temperature is increased from 860 to $968^{\circ} \mathrm{C}$. This effect does not scale with best fit $\mathrm{C}_{\mathrm{H} \sigma-}$ CRIT which is variable with aging condition (8,500, 5,800, and 11,000 wppm for the H860, H900, and $\mathrm{H} 968$ conditions, respectively). This increase in the breadth of protective cathodic polarization appears to be governed by reduced H-uptake at the crack tip, as indicated by Figure 83. Third, $\mathrm{C}_{\mathrm{H \sigma} \text {-CRIT }}$ is nearly equal to, or increased compared to AerMet 100 (6,500 wppm) for all aging conditions of Ferrium PH48S and Ferrium S53. This result confirms the critical contribution of alloy purity and rare earth treatment to HEAC resistance of UHSSS alloys; compared to low purity $300 \mathrm{M}\left(\mathrm{C}_{\mathrm{H \sigma} \text {-CRIT }}=3,500 \mathrm{wppm}\right.$ for $\left.\sigma_{\mathrm{H}}=6.8 \sigma_{\mathrm{YS}}\right), \mathrm{C}_{\mathrm{H \sigma} \text {-CRIT }}$ is increased $1.6-3.2$ times for Ferrium PH48S and Ferrium S53.

Finally, comparison of Figure 36 to Figures 84 to 87 clearly indicates that the accuracy of the diffusion limited model predictions is significantly increased at moderate cathodic polarizations for the UHSSS alloys compared to the predictions for AerMet 100 and Ferrium M54. In Chapter 3 under prediction of $\mathrm{da} / \mathrm{dt}_{\text {II }}$ at moderate cathodic polarizations was attributed to three factors, none of which were conclusively supported. Of the possible factors, surface reaction control of $\mathrm{H}$ uptake was suggested to be the most likely, due to the presence of a passive surface film which reduces $\mathrm{H}$-uptake in this regime. Close agreement of observed and model predicted $\mathrm{da} / \mathrm{dt}_{\text {II }}$ in Figures 84-87 indicates that surface reaction control of H-uptake is not active in UHSSS alloys, and the presence of a passive surface film is unlikely. This result does not preclude the formation of a crack tip passive film or surface reaction control of H-uptake in UHSS alloys AerMet 100 and Ferrium M54, but further research is needed to understand the potential dependence of H-uptake in UHSS(S) alloys. Further, the results shown in Figures 84 to 87 would be significantly strengthened by independent characterization of $\mathrm{D}_{\mathrm{H}-\mathrm{EFF}}$ and H-uptake for an occluded crack tip environment in UHSSS alloys. Simultaneous studies, identical to those conducted by Kehler and Scully [20] and Thomas et al [137] on AerMet 100 would provide a further mechanistic link between applied polarization and H-uptake in UHSS alloys. The efficacy of Equation 2 in 
predicting the HEAC kinetics of Ferrium PH48S and Ferrium S53 strongly validates this diffusion based model as providing a quantitative-foundational framework for interpreting the results of these $\mathrm{H}$ diffusion and crack chemistry studies.

\subsubsection{Metallurgical Design of HEAC-Resistant UHSS and UHSSS Alloys}

The present work provides the opportunity to inform the metallurgical design of next generation HEAC-resistant UHSS and UHSSS alloys. High resolution HEAC kinetics presented in Figures 24 to 25 and Figures 67 to 70 are assessed in the context of alloy composition (Tables 3 and 4), sub-micron microstructure (Figures 6 to 10 and Figures 39 to 42), and HEAC fracture morphology (Figures 19 to 23 and Figures 57 to 60 ) to qualitatively assess the beneficial effect of metallurgical factors on HEAC resistance.

In consideration of the results discussed in previous sections, three points are established for alloy development with strong experimental evidence. First, there is an overriding-beneficial effect of alloy purity and a nano-scale dispersion of strengthening precipitates on HEAC resistance, as demonstrated by micromechanical modeling of the HEAC properties for 300M, AerMet 100, and Ferrium M54 (Sections 3.3.5.4 and 3.3.5.5). High impurity content in 300M reduces $\mathrm{C}_{\mathrm{H \sigma}-\mathrm{CRIT}}$ compared to AerMet 100 and Ferrium M54, eliminating the beneficial effect of mild cathodic $\mathrm{E}_{\text {app }}$ on HEAC resistance. The precipitation of nano-scale $\mathrm{M}_{2} \mathrm{C}$ carbides reduces $\mathrm{D}_{\mathrm{H}^{-} \mathrm{EFF}}$ in AerMet 100

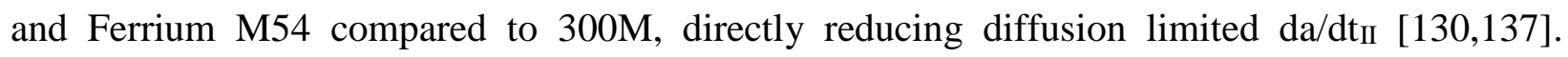
Inclusion of these two key design parameters is critical in establishing damage-tolerant HEAC management, as the difference in observed da/dt II between $300 \mathrm{M}$ and modern UHSS is large, two to four orders of magnitude for the range of $E_{\text {app }}$ examined in the present study.

Second, there is no measurable effect of reduced cobalt and nickel additions on the HEAC resistance of modern UHSS alloys. The alloy sequence of AerMet 100 to Ferrium M54 to CrNiMoWV provides stepwise decreasing Co and Ni from 13 to 0 and 11 to $3 \mathrm{wt} \%$, respectively. SCC-HEAC resistance is essentially equal for AerMet 100 and Ferrium M54 at identical oys (1,720 MPa, Table 1) and speculatively improved in CrNiMoWV (Figures 24 and 25), which shows the lowest total alloy content (10.5 wt\% versus $21.6 \mathrm{wt} \%$ and $28.7 \mathrm{wt} \%$ for Ferrium M54 and AerMet 100, respectively). From an alloy design perspective, if low impurity content and a nano-scale strengthening dispersion can be maintained, then the total alloy content of an UHSS 
may be reduced to a minimum. Both nano-scale carbides and intermetallic precipitates can be employed to reduce $\mathrm{H}$ mobility and thus decrease rates of HEAC. The invariance of HEAC resistance with decreasing alloy content provides a quantified basis for the computational design of low-cost replacements such as Ferrium M54 and CrNiMoWV for modern UHSS.

Third, there is no measureable deleterious effect of increasing chromium content on the HEAC resistance of UHSS(S). The comparison between Ferrium M54, AerMet 100 and Ferrium S53 provides a quantitative assessment of the effect of increasing chromium (1, 3, $10 \mathrm{wt} \%$, respectively) on TG SCC-HEAC in high purity secondary hardening UHSS. Starting with AerMet 100, a large concentration of chromium was added to produce Ferrium S53, which is corrosion resistant via passive film formation and which eliminates the need for Cd electroplating [36,139]. Consideration of the crack tip $\mathrm{H}$ production which governs $\mathrm{HEAC}$, leads to the hypothesis that $\mathrm{Cr}$ (and Co) could improve HEAC resistance for low-to-moderate cathodic polarization due to increase crack tip film passivity [141]. Conversely, elevated chromium is hypothesized to exacerbate SCC-HEAC for $\mathrm{E}_{\mathrm{app}}$ near and anodic to OCP where significant crack tip dissolution and chromium cation acidification may occur. The data summarized in Figures 17-18 and 24-25 for Ferrium M54, AerMet 100, and Ferrium S53 do not provide evidence of either a beneficial or deleterious effect of chromium content on TG SCC-HEAC of modern UHSS(S). Critically, all three alloys in this comparison are modern, high-purity UHSS with a near identical strengthening dispersion of $\mathrm{M}_{2} \mathrm{C}$ carbides. From an alloy design perspective this result extends the conclusions of point (2) on the invariance of strong HEAC resistance to include large additions of Cr. Taken together, these results establish the fundamental UHSS design criteria of exceptional alloy purity and the nano scale strengthening precipitates to improve SCC-HEAC resistance. The specific alloying additions necessary to produce these two microstructural features are irrelevant to design of UHSS optimized for HEAC resistance so long as these features are present.

The results presented in Chapters 3 and 4 further indicate that reducing thin film austenite may improve SCC-HEAC resistance, but this approach is not definitively established in the present work. Comparison of the HEAC properties in Figure 24 and Figure 67 indicates that the breadth of cathodic $\mathrm{E}_{\mathrm{app}}$ conferring elevated HEAC resistance is widened in intermetallic strengthened UHSSS (Custom 465 and Ferrium PH48S) compared to carbide strengthened UHSS(S) (AerMet 100, Ferrium M54, CrNiMoWV, and Ferrium S53). This result is not explained by an impurity 
adjusted $\mathrm{C}_{\mathrm{H} \sigma \text {-CRIT }}$ or $\alpha$ due to the presence of a strong rare earth treatment in both alloys. As detailed in the discussion of Chapters 3 and 4, thin film austenite along martensite block interfaces could provide a mechanistic way to exacerbate the effect of small amounts of crack tip hydrogen present

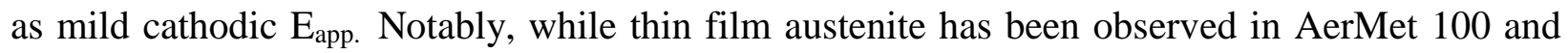
Ferrium M54, there is no evidence to suggest that such films are present in Ferrium PH48S or Custom 465 when aged to ultra-high strength (Figures 79 to 82) [24,75]. Speculatively, such films can contribute to HEAC susceptibility when $\mathrm{C}_{\mathrm{H \sigma}}$ is small relative to $\mathrm{C}_{\mathrm{H} \sigma-\mathrm{CRIT}}$, reducing the HEAC resistance in the window of mildly cathodic $\mathrm{E}_{\mathrm{app}}$ which confer strong HEAC resistance. While not directly probed in the present study, this result indicates that thin film austenite should be avoided in the design of HEAC resistant alloys. Further, the results presented in Figures 79 to 82 indicate that ThermoCalc predictions of total austenite content (Table 14) are substantial over-predictions of physical reality. Future work is needed in this area to improve the quality of ThermoCalc model predictions for computationally designed alloys.

Finally, EBSD results presented in Chapters 3 and 4 provide strong circumstantial evidence that the effect of interface engineering on HEAC kinetics is minor. Although the fraction low energy CSL interfaces was not directly controlled in the present study, no significant correlation to HEAC properties was observed under moderate to heavy cathodic polarizations. For example, total area fraction of low energy CSL interfaces is reduced in AerMet 100 relative to Ferrium PH48S (41 vs $54 \%$, respectively (Table 9)), with identical connectivity. As shown in Figure 24-25 and Figure 69-70, da/dt II is decreased in AerMet 100 compared to near equal strength Ferrium PH48S-H900 and H860. This result is contrary to the hypothesized reduction in $\mathrm{da} / \mathrm{dt}_{\text {II }}$ with increasing CSL fraction, due to reduced $\mathrm{C}_{\mathrm{H \sigma} \text {-CRIT }}$ or significant crack bifurcation. Further, the HEAC fracture morphology is identical between these two alloys, indicating no effect of CSL fraction on fracture path. The lack of a significant CSL fraction effect in UHSS alloys is hypothetically understood based on the potentially dominating effects of interface connectivity and high hydrogen concentrations local to the interfaces during HEAC. While a minor beneficial contribution of increasing CSL fraction on HEAC resistance cannot be ruled out, the present results indicate that it is not a critical component for future $\operatorname{UHSS}(\mathrm{S})$ alloy design. Furture work is needed to examine the effect of random boundary connectivity on HEAC properties in the context of high local hydrogen concentrations and fine scale martensite microstructures of UHSS alloys. 


\subsection{Conclusions}

The stress corrosion cracking resistance two of ultra-high strength stainless steels (at several aging conditions) was characterized for slow-rising $\mathrm{K}$ loading in aqueous chloride solution as a function of widely varying applied-cathodic polarization. High resolution crack growth kinetics and fracture surface morphologies are correlated with a high resolution SEM-based analysis of martensitic microstructure, including feature size/morphology and interface crystallographic characteristics. Observed trends in HEAC properties are interpreted in the context of micromechanical models of hydrogen environment assisted cracking, fracture morphology, and martensite microstructure.

- Custom 465 and Ferrium PH48S, aged to several ultra-high strength levels, are susceptible to severe SCC-HEAC when stressed in $3.5 \% \mathrm{NaCl}$ under moderate-heavy cathodic polarization.

- Custom 465 and Ferrium PH48S, aged to ultra-high strength are highly resistant to SCCHEAC when stressed within a limited range of $\mathrm{E}_{\mathrm{app}}$, mildly cathodic to OCP, which confers elevated $\mathrm{K}_{\mathrm{TH}}$ and reduced da/dt $\mathrm{II}$. Identical to UHSS behavior, the presence of this protective window is correlated to low $\mathrm{C}_{\mathrm{H} \text {-DIFF }}$ owed to a minimum in overpotential for hydrogen production and hydrolytic crack tip acidification. Increased range of cathodic polarizations conferring strong resistance to SCC is increased in UHSSS, possible due to a lack of deleterious thin film austenite along martensite block boundaries.

- The HEAC fracture path in Custom 465 is exclusively IG along prior austenite grain boundaries. IG HEAC facets lack strong indications of small scale grain boundary plasticity observed in IG HEAC of high purity CrNiMoWV. Increased da/dt in Custom 465 is due a significant reduction in grain boundary cohesion sourced at the synergistic interaction of metalloid impurities and hydrogen local to the grain boundary.

- In contrast, all aging conditions of Ferrium PH48S exhibit exclusively TG fracture. Close matching of fracture surface features with EBSD measured martensite microstructure indicates that HEAC primarily proceeds along $\{110\}_{\alpha}$ martensite block boundary planes in Ferrium PH48S, identical to TG HEAC of UHSS. 
- SCC-HEAC resistance of Ferrium PH48S systematically falls as aging temperature is decreased and yield strength rises. When compared to Custom - 465 at equal strength, the potential dependency of $\mathrm{K}_{\mathrm{TH}}$ is identical, but da/dt $\mathrm{dI}$ is substantially reduced for PH48S. Increased da/dt $\mathrm{dI}_{\mathrm{I}}$ in Custom 465 is hypothetically attributed to a significant impurity concentration and lack of rare earth treatment, significantly reducing $\mathrm{C}_{\mathrm{H \sigma} \text {-crit. }}$

- Hydrogen solubility of Custom 465 - H900 is significantly increased compared to three aging conditions of Ferrium PH48S (H900, H950, and H968) when H-charged in 0.001M $\mathrm{NaOH}(\mathrm{pH}=10)$ at $\mathrm{E}_{\mathrm{app}}=-1350 \mathrm{mV}_{\mathrm{SCE}}$. Regression fit $\mathrm{D}_{\mathrm{H}-\mathrm{EFF}}$ for H-outgassing of Custom 465 is increased $\left(2.6 \times 10^{-9} \mathrm{~cm}^{2} / \mathrm{s}\right)$ compared to Ferrium PH48S $\left(2.3-7.0 \times 10^{-10} \mathrm{~cm}^{2} / \mathrm{s}\right)$. Close agreement with recent TDS characterization of $\mathrm{D}_{\mathrm{H}-\mathrm{EFF}}$ in Custom 465 - H900 (6.2 x $10^{-9} \mathrm{~cm}^{2} / \mathrm{s}$ ) strongly supports the validity of these data.

- Close agreement in the morphology of quasi-cleavage facets observed on the TG fracture surfaces of 300M, AerMet 100, Ferrium M54, CrNiMoWV, Ferrium S53, Custom 465, and Ferrium PH48S indicates that an identical HEAC mechanism is active in each alloy. The HELP-HEDE HEAC mechanism proposed by Nagao et al. provides a reasonable set of mechanistic steps to explain the HEAC fracture morphology of UHSS and UHSSS.

- HEAC fracture morphology in Ferrium PH48S is invariant with electrochemical environmental severity and aging condition. This result establishes that the generic HEAC mechanism is not affected by crack tip hydrogen concentration, precipitate composition/morphology, and alloy yield strength.

- When compared to UHSS, Custom 465 - H900 and Ferrium PH48S - H900 exhibit the highest $(80 \%)$ and lowest (46\%) fraction of random high angle boundaries, respectively. No significant difference is observed in the connectivity of random boundaries, despite Ferrium PH48S exhibiting the highest fraction of $\sum 3$ boundaries (34\%), which are expected to significantly disrupt the connectivity of random boundaries. High energy interfaces speculatively trap significantly more hydrogen during HEAC providing an interconnected crack path through the microstructure. However, no alloy-specific beneficial effect on HEAC kinetics can be determined. 


\section{References}

1. W. M. Garrison, J. Mater. 42, 20 (1990).

2. G. B. Olson, in 34th Sagamore Army Mater. Res. Conf., edited by G. B. Olson, M. Azrin, and E. S. Wright (US Army Laboratory Command, Watertown, MA, 1987), pp. 549-593.

3. Y. Lee and R. P. Gangloff, Metall. Mater. Trans. A 38A, 2174 (2007).

4. R. P. Gangloff, in Compr. Struct. Integr., edited by I. Milne, R. O. Ritichie, and B. Karihaloo, Volume 6 (Elsevier Science, New York, NY, 2003), pp. 31-101.

5. S. P. Lynch, Corros. Rev. 30, 63 (2012).

6. S. P. Lynch, Corros. Rev. 30, 105 (2012).

7. S. P. Lynch, in Corros. 2007, Paper No. 07493 (NACE International, Houston, TX, 2007).

8. A. Nagao, C. D. Smith, M. Dadfarnia, P. Sofronis, and I. M. Robertson, Acta Mater. 60, 5182 (2012).

9. A. Nagao, C. D. Smith, M. Dadfarnia, P. Sofronis, and I. M. Robertson, Procedia Mater. Sci. 3, 1700 (2014).

10. R. P. Gangloff, H. M. Ha, J. T. Burns, and J. R. Scully, Metall. Mater. Trans. A 45A, 3814 (2014).

11. W. W. Gerberich, in Gaseous Hydrog. Embrittlement Mater. Energy Technol. Vol. 2 Mech. Model. Futur. Dev., edited by R. P. Gangloff and B. P. Somerday (Woodhead Publishing, Cambridge, UK, 2012), pp. 209-246.

12. R. P. Gangloff, in Hydrog. Eff. Mater., edited by B. P. Somerday, P. Sofronis, and R. H. Jones (ASM International, Metals Park, OH, 2009), pp. 1-21.

13. R. A. Oriani, Berichte Der Bunsengesellschaft Fur Phys. Chemie 76, 848 (1972).

14. R. A. Oriani and P. H. Josephic, Acta Metall. 22, 1065 (1974).

15. R. A. Oriani, Corrosion 43, 390 (1987).

16. R. P. Gangloff, in Hydrog. Eff. Mater. Behav. Corros. Deform. Interact., edited by N. R. Moody, A. W. Thompson, R. E. Ricker, G. W. Was, and R. H. Jones (The Minerals Metals \& Materials Society, Warrendale, PA, 2003), pp. 477-497.

17. R. A. Oriani, L. S. Darken, and J. C. M. Li, Zeitschrift Für Phys. Chemie 49, 271 (1966). 
18. I. M. Bernstein and G. M. Pressouyre, in Hydrog. Degrad. Ferr. Alloy., edited by R. A. Oriani, M. Smialowski, and J. P. Hirth (Noyes Publications, Saddle River, NJ, 1984), pp. 641-685.

19. B. A. Kehler and J. R. Scully, Corrosion 65, 465 (2007).

20. J. P. Hirth, Metall. Trans. A 11A, 861 (1980).

21. T.-Y. Zhang and J. E. Hack, Metall. Mater. Trans. A 30A, 155 (1999).

22. D. Figueroa and M. J. Robinson, Corros. Sci. 52, 1593 (2010).

23. J. Waldman, W. M. Garrison, and R. P. Gangloff, Development of Corrosion-Hydrogen Crack Resistant Aircraft Alloys from Mechanistic Understanding (Phase II, Final Report 003211/CD002, STTR Topic No. N08-T010, Navmar Applied Sciences Corporation, Warminster, PA, 2011).

24. R. Ayer and P. M. Machmeier, Metall. Trans. A 24A, 1943 (1993).

25. G. R. Speich, D. Dabkowski, and L. Porter, Metall. Trans. A 4, 303 (1973).

26. P. M. Novotny, in Fundam. Aging Tempering Bainitic Martensitic Steel Prod. (TMS-AIME, Warrendale, Pa, 1993), pp. 215-236.

27. G. R. Speich and W. C. Leslie, Metall. Trans. 3, 1043 (1972).

28. C. J. McMahon, Eng. Fract. Mech. 68, 773 (2001).

29. S. K. Banerjee, C. J. McMahon, and H. C. Feng, Metall. Trans. 9, 237 (1978).

30. T. V. Philip and T. J. McCaffrey, in Handb. Mater. Sel. Eng. Appl., edited by G. Murray, 1st ed. (CRC Press, Boca Raton, FL, 1997), pp. 149-161.

31. W. Barrett, J. V. Bee, and G. G. Garrett, in Spec. Steels Hard Mater., edited by N. R. Comins and J. B. Clark (Pergamon Press, Pretoria, ZA, 1982), pp. 293-244.

32. W. M. Garrison and J. L. Maloney, Mater. Sci. Eng. A 403, 299 (2005).

33. K. J. Handerhan and W. M. Garrison, Scr. Metall. 22, 409 (1988).

34. L. E. Iorio and W. M. Garrison, Scr. Mater. 46, 863 (2002).

35. J. Grabowski, C. Kuehmann, J. Sebastian, H.-J. Jou, R. Kooy, and C. Kern, in Proc. 2012 Aircr. Airworth. Sustain. Conf. (Baltimore, MD, 2012). http://www.questek.com/filebase/src/ Presentations/FerriumM54PresentationatAA.pdf.

36. C. Kuehmann, B. Tufts, and P. Trester, Adv. Mater. Process. 37 (2008). 
37. M. F. Stevens and I. M. Bernstein, Metall. Trans. A 20A, 909 (1989).

38. H. Asahi, D. Hirakami, and S. Yamasaki, ISIJ Int. 43, 527 (2003).

39. F. G. Wei and K. Tsuzaki, in Gaseous Hydrog. Embrittlement Mater. Energy Technol. Vol. 1 Probl. Its Characterisation Eff. Paticular Alloy Classes, edited by R. P. Gangloff and B. P. Somerday, 1st ed. (Woodhead Publishing Limited, Cambridge, UK, 2012), pp. 493-525.

40. A. Nagao, B. P. Eftink, M. Dadfarnia, B. P. Somerday, and P. Sofronis, in Hydrog. Interact., edited by B. P. Somerday and P. Sofronis (ASME Press, New York, NY, 2012), pp. 127-145.

41. Technical Datasheet: Custom 465 Stainless (Carpenter Technology Corporation, Reading, PA, 2012).

42. J. A. Wright and J.-W. Jung, US Patent No. 7,879,159 B2 (2011).

43. David Snyder, Questek Innovations LLC, Evanston, IL, Private Communication (2014).

44. C. E. Campbell and G. B. Olson, J. Comput. Mater. Des. 7, 145 (2001).

45. C. E. Campbell and G. B. Olson, J. Comput. Mater. Des. 7, 171 (2001).

46. J. Wright and C. Kern, Cast Ferrium PH48S Development of Castable High-Strength Stainless Steel (Questek Innovations, LLC, Evanston, IL,2011).

47. Ferrium PH48S: High-Strength, High-Toughness Stainless Steel Castable and Forgeable Datasheet (Questek Innovations, LLC, Evanston, IL, 2012).

48. L. C. Lim and T. Watanabe, Acta Metall. Mater. 38, 2507 (1990).

49. S. Kobayashi, T. Maruyama, S. Tsurekawa, and T. Watanabe, Acta Mater. 60, 6200 (2012).

50. S. Bechtle, M. Kumar, B. P. Somerday, M. E. Launey, and R. O. Ritchie, Acta Mater. 57, 4148 (2009).

51. T. Watanabe and S. Tsurekawa, Mater. Sci. Eng. A 387-389, 447 (2004).

52. R. W. Balluffi, in Interfacial Segreg., edited by W. C. Johnson and J. M. Blakely (ASM International, Metals Park, OH, 1977), pp. 193-259.

53. T. Watanabe, Res. Mech. 11, 47 (1984).

54. D. Bouchet and L. Priester, Scr. Metall. 20, 961 (1986).

55. T. Watanabe, S. Kitamura, and S. Karashima, Acta Metall. 28, 455 (1980). 
56. W. Swiatnicki, S. Lartigue-Korinek, and J. Y. Laval, Acta Metall. Mater. 43, 795 (1995).

57. S. Jothi, T. N. Croft, and S. G. R. Brown, Int. J. Hydrogen Energy (2014).

58. D. H. Lassila and H. K. Birnbaum, Acta Metall. 34, 1237 (1986).

59. S. Tsurekawa, S. Nakamichi, and T. Watanabe, Acta Mater. 54, 3617 (2006).

60. M. A. Arafin and J. A. Szpunar, Corros. Sci. 51, 119 (2009).

61. M. Seita, J. P. Hanson, S. Gradečak, and M. J. Demkowicz, Nat. Commun. 6, 6164 (2015).

62. R.P. Gangloff, Fracture Mechanics Prediction of Component SCC Using Accelerated Laboratory Testing, DoD 2013-3702, Department of Defense Virtual Corrosion Conference, NACE International, Houston, TX (2013). https://www.corrdefense.org/External/ViewList.aspx? ListID=999.

63. J. L. Youngblood and M. Raghavan, Metall. Trans. A 8A, 1439 (1977).

64. Manufacturing and Thermal Processing of Ferrium M54, Tech. Datasheet 1 (Questek Innovations, LCC, Evanston, IL, 2012).

65. Manufacturing and Thermal Processing of Ferrium S53, Tech. Datasheet 1 (Questek Innovations, LCC, Evanston, IL, 2012).

66. W. Ramberg and W. R. Osgood, NASA Tech. Rep. NACA-TN-902 (1943).

67. Mil. Handbook - MIL-HDBK-5H Met. Mater. Elem. Aerosp. Veh. Struct. (U.S. Department of Defense, 1998), pp. 67-115.

68. Mil. Handb. - MIL-HDBK-5H Met. Mater. Elem. Aerosp. Veh. Struct. (U.S. Department of Defense, Washington, DC, 1998), pp. 116-213.

69. J. Waldman, W. M. Garrison, and R. P. Gangloff, Development of Corrosion-Hydrogen Crack Resistant Aircraft Alloys from Mechanistic Understanding (Phase II, Final Report 003211/CD002, STTR Topic No. N08-T010, Navmar Applied Sciences Corporation, Warminster, PA, 2011).

70. P. M. Machmeier, R. Ayer, T. Matuszewski, and R. Jones, J. Mater. Eng. Perform. 6, 279 (1997).

71. R. Padmanabhan and W. E. Wood, Corrosion 41, 688 (1985).

72. R. Padmanabhan, Heat Treatment, Structure-Property, Stress Corrosion Cracking and Acoustic Emission Studies on 300M Steel, PhD Dissertation, Oregon Health \& Science University, 1982. 
73. R. L. S. Thomas, Internal Hydrogen Embrittlement of a Trap-Rich Ultrahigh-Strength Steel Aermet 100, Master of Science Thesis, University of Virginia, 2000.

74. R. Ayer and P. M. Machmeier, Metall. Mater. Trans. A 27A, 2510 (1996).

75. C. Wang, C. Zhang, and Z. Yang, Micron 67, 112 (2014).

76. G. B. Olson, Acta Mater. 61, 771 (2013).

77. H. M. Lee, A. J. Garratt-Reed, and S. M. Allen, Scr. Metall. 25, 685 (1991).

78. G. D. W. Smith, A. Cerezo, and W. Sha, Metall. Trans. A 24A, 1251 (1993).

79. E. U. Lee, R. Goswami, M. Jones, and A. K. Vasudevan, Metall. Mater. Trans. A 42A, 415 (2010).

80. W. Sha, A. Cerezo, and G. D. W. Smith, Metall. Trans. A 24A, 1251 (1993).

81. M. Grujicic, R. Yavari, J. S. Snipes, S. Ramaswami, C. F. Yen, and B. A. Cheeseman, J. Eng. Manuf. 24, 1 (2014).

82. M. Grujicic, R. Yavari, J. S. Snipes, S. Ramaswami, C. F. Yen, and B. A. Cheeseman, J. Mater. Eng. Perform. 23, 2182 (2014).

83. D. M. Vanderwalker, Metall. Trans. A 18A, 1191 (1987).

84. M. Schober, R. Schnitzer, and H. Leitner, Ultramicroscopy 109, 553 (2009).

85. W. Sha, G. D. W. Smith, and A. Cerezo, Metall. Mater. Trans. A 24A, 1221 (1993).

86. W. Sha, G. D. W. Smith, and A. Cerezo, Metall. Trans. A 24A, 1233 (1993).

87. W. Sha, A. Cerezo, and G. D. W. Smith, Metall. Trans. A 24A, 1241 (1993).

88. S. Morito, H. Yoshida, T. Maki, and X. Huang, Mater. Sci. Eng. A 438-440, 237 (2006).

89. G. F. Vander Voort, Pract. Metallogr. 48, 454 (2011).

90. F. J. Humphreys, Scr. Mater. 51, 771 (2004).

91. F. J. Humphreys, M. Materials, and S. Centre, J. Mater. Sci. 6, 3833 (2001).

92. E. E. Underwood, Quantitative Stereology (Addison-Wesley, Reading, MA, 1970).

93. D. G. Brandon, Acta Metall. 14, 1479 (1966). 
94. P. Lejcek, in Grain Bound. Segreg. Met. (Springer, Prague, 2010), pp. 5-22.

95. E. Charkaluk, M. Bigerelle, and A. Iost, Eng. Fract. Mech. 61, 119 (1998).

96. S. Kobayashi, S. Tsurekawa, T. Watanabe, and G. Palumbo, Scr. Mater. 62, 294 (2010).

97. J. Dolph, The Effect of Microstructure on the HEAC Behavior of Monel K-500, Master of Science Thesis, University of Virginia, 2015.

98. H. Tada, P. C. Paris, and G. R. Irwin, The Stress Analysis of Cracks Handbook, 3rd ed. (ASME Press, New York, NY, 2000).

99. R. P. Gangloff, D. C. Slavik, R. S. Piascik, and R. H. Van Stone, in Small Crack Test Methods, edited by J. M. Larsen and J. E. Allison, 1st ed. (American Society for Testing and Materials, Philadelphia, 1992), pp. 116-168.

100. H. H. Johnson, Mater. Res. Stand. 5, 442 (1965).

101. J. K. Donald and J. Ruschau, in Fatigue Crack Meas. Tech. Appl., edited by K. J. Marsh, R. A. Smith, and R. O. Ritchie (EMAS, West Midlands, UK, 1991), pp. 11-38.

102. ASTM Standard E647, Standard Test Method for Measurement of Fatigue Crack Growth Rates (ASTM International, West Conshohocken, PA, 2008).

103. F. Khodabakhshi and M. Kazeminezhad, Mater. Des. 32, 3280 (2011).

104. T. L. Anderson, Fracture Mechanics Fundamentals and Applications, 3rd ed. (Taylor \& Francis, Boca Raton, FL, 2005).

105. B. A. Kehler, Modeling and Experiments to Explain the Potential Dependency of an UHSS to Hyrogen Environment Assisted Cracking, PhD Dissertation, University of Virginia, 2008.

106. J. Crank, Mathematics of Diffusion, 1st ed. (Oxford University Press, London, 1975).

107. R. O. Ritchie, M. H. Cedeno, V. F. Zackay, and E. R. Parker, Metall. Mater. Trans. A 9A, 35 (1978).

108. ASTM Standard E112-13: Standard Test Methods for Determining Average Grain Size (ASTM International, West Conshocken, PA, 2014).

109. H. Beladi, G. S. Rohrer, A. D. Rollett, V. Tari, and P. D. Hodgson, Acta Mater. 63, 86 (2014).

110. H. Kitahara, R. Ueji, N. Tsuji, and Y. Minamino, Acta Mater. 54, 1279 (2006).

111. J. W. Morris, C. Kinney, K. Pytlewski, and Y. Adachi, Sci. Technol. Adv. Mater. 14, 1 (2013). 
112. S. Morito, X. Huang, T. Furuhara, T. Maki, and N. Hansen, Acta Mater. 54, 5323 (2006).

113. G. . Krauss, in Steels Process. Struct. Preformance (ASM International, Materials Park, OH, 1990), pp. 43-87.

114. S. Morito, H. Tanaka, R. Konishi, T. Furuhara, and T. Maki, Acta Mater. 51, 1789 (2003).

115. P. M. Kelly, A. Jostsons, and R. G. Blake, Acta Metall. Mater. 38, 1075 (1990).

116. M. Gao and R. P. Wei, Acta Metall. 32, 2115 (1984).

117. C. P. Luo and J. Liu, Mater. Sci. Eng. A 438-440, 149 (2006).

118. S. Morito, Y. Adachi, and T. Ohba, Mater. Trans. 50, 1919 (2009).

119. G.L. Pioszak, Yongwon Lee, W.M. Garrison, and R.P. Gangloff, Effect of Liquid Nitrogen Chill on SCC of AerMet ${ }^{\circledR} 100$ Ultra-High Strength Steel, CorrDefense, NACE, Houston, TX (2012). https://www.corrdefense. org/External/ ViewList.aspx?ListID=999.

120. Y. H. Kim and J. W. Morris Jr., Metall. Mater. Trans. A 14A, 1883 (1983).

121. M. Sun, K. Xiao, C. Dong, and X. Li, Acta Metall. Sin. (English Lett.) 23, 301 (2010).

122. E. U. Lee, H. C. Sanders, and B. Sarkar, Stress Corrosion Cracking of High Strength Steels (U.S. Department of Defense, Naval Air Warfare Center Aircraft Division, Patuxent River, MD, 1995).

123. ASTM Standard F1624-12-2014: Standard Test Method for Measurement of Hydrogen Embrittlement Threshold in Steel by the Incremental Step Loading Technique (ASTM International, West Conshocken, PA, 2014).

124. G. L. Pioszak and R. P. Gangloff, Unpublished Research (University of Virginia, Charlottesville, VA, 2014).

125. A. Shibata, H. Takahashi, and N. Tsuji, ISIJ Int. 52, 208 (2012).

126. M. Koyama, C. C. Tasan, E. Akiyama, K. Tsuzaki, and D. Raabe, Acta Mater. 70, 174 (2014).

127. T. Furuhara, T. Shinyoshi, G. Miyamoto, J. Yamaguchi, N. Sugita, N. Kimura, N. Takemura, and T. Maki, ISIJ Int. 43, 2028 (2003).

128. Y. H. Kim, H. J. Kim, and J. W. Morris, Metall. Trans. A 17A, 1157 (1986).

129. C. H. Yoo, H. M. Lee, J. W. Chan, and J. W. Morris, Metall. Mater. Trans. A 27A, 3466 (1996). 
130. D. Li, R. P. Gangloff, and J. R. Scully, Metall. Trans. A 35A, 25 (2004).

131. N. Bandyopadhyay, J. Kameda, and C. J. McMahon, Metall. Trans. A 14A, 887 (1983).

132. X.-Y. Liu, J. Kameda, J. W. Anderegg, S. Takaki, K. Abiko, and C. J. McMahon, Mater. Sci. Eng. A 492, 218 (2008).

133. G. B. Olson, Adv. Mater. Process. 152, 72 (1997).

134. C. J. McMahon, Interface Sci. 12, 141 (2004).

135. N. Bandyopadhyay and C. J. McMahon, Metall. Trans. A 14A, 1313 (1983).

136. C. J. McMahon, Eng. Fract. Mech. 68, 773 (2001).

137. R. L. S. Thomas, D. Li, R. P. Gangloff, and J. R. Scully, Metall. Mater. Trans. A 33A, 1991 (2002).

138. A. Brass, F. Guillon, and S. Vivet, Metall. Trans. A 35A, 1449 (2004).

139. J. Wright, in Proc. 2010 Stainl. Steel Work Am. Conf. (Stainless Steel World, Woodlands, TX, 2010), pp. 1-13.

140. R. Kirchheim, B. Heine, H. Fischmeister, S. Hofmann, H. Knote, and U. Stolz, Corros. Sci. 29, 899 (1989).

141. A. Turnbull, Corros. Rev. 30, 1 (2012).

142. F. Brown, in Stress Corros. Crack. Hydrog. Embrittlement Iron Base Alloy, edited by R. W. Staehle, J. Hochmann, R. D. McCright, and J. E. Slater (NACE International, Houston, TX, 1973), pp. $747-750$.

143. D. P. Dautovich and S. Floreen, in Stress Corros. Crack. Hydrog. Embrittlement Iron Base Alloy, edited by R. W. Staehle, J. Hochmann, R. D. McCright, and J. E. Slater (NACE International, Houston, TX, 1973), pp. 798-815.

144. R. N. Parkins, A. J. Markworth, J. H. Holbrook, and R. R. Fessler, Corrosion 41, 389 (1985).

145. R. L. S. Thomas, J. R. Scully, and R. P. Gangloff, Metall. Trans. A 34A, 327 (2003).

146. Y. D. Park, I. S. Maroef, A. Landau, and D. L. Olson, Weld. J. 81, 27S (2002).

147. M. J. Haynes and R. P. Gangloff, J. Test. Eval. 25, 82 (1997). 
148. W. W. Gerberich, P. G. Marsh, and J. W. Hoehn, in Hydrog. Eff. Mater., edited by N. R. Moody and A. W. Thompson (The Minerals Metals \& Materials Society, Warrendale, Pa, 1996), pp. 539-553.

149. W. W. Gerberich, R. A. Oriani, M. J. Lii, and X. Chen, Philos. Mag. A 363-376, (1991).

150. U. Komaragiri, S. R. Agnew, R. P. Gangloff, and M. R. Begley, J. Mech. Phys. Solids 56, 3527 (2008).

151. M. R. Begley, J. A. Begley, and C. M. Landis, in Gaseous Hydrog. Embrittlement Mater. Energy Technol. Vol. 2 Mech. Model. Futur. Dev., edited by R. P. Gangloff and B. P. Somerday (Woodhead Publishing Limited, Cambridge, UK, 2012), pp. 286-325.

152. Emilio Martinez Pineda, University of Oviedo, Spain, Private Communication (2014).

153. A. J. Griffiths and A. Turnbull, Corros. Sci. 37, 1879 (1995).

154. A. Turnbull, in Gaseous Hydrog. Embrittlement Mater. Energy Technol. Vol. 2 Mech. Model. Futur. Dev., edited by R. P. Gangloff and B. P. Somerday (Woodhead Publishing Limited, Cambridge, UK, 2012), pp. 89-128.

155. A. Taha and P. Sofronis, Eng. Fract. Mech. 68, 803 (2001).

156. J. Toribio and V. Kharin, Mater. Sci. 42, 263 (2006).

157. J. R. Scully and P. J. Moran, J. Electrochem. Soc. 135, 1337 (1988).

158. A. Turnbull and M. Saenz de Santa Maria, Corrosion 44, 176 (1988).

159. M. Guttmann, Surf. Sci. 53, 213 (1975).

160. J. R. Rice and G. F. Rosengren, J. Mech. Phys. Solids 16, 1 (1968).

161. I. M. Robertson, P. Sofronis, A. Nagao, M. L. Martin, S. Wang, D. W. Gross, and K. E. Nygren, Metall. Mater. Trans. A in press (2015).

162. C. Keller, E. Hug, R. Retoux, and X. Feaugas, Mech. Mater. 42, 44 (2010).

163. S. Wang, M. L. Martin, P. Sofronis, S. Ohnuki, N. Hashimoto, and I. M. Robertson, Acta Mater. 69, 275 (2014).

164. D. A. Hughes, Scr. Mater. 47, 697 (2002).

165. M. L. Martin, B. P. Somerday, R. O. Ritchie, P. Sofronis, and I. M. Robertson, Acta Mater. 60, 2739 (2012). 
166. G. M. Pressouyre, Metall. Trans. A 10A, 1571 (1979).

167. R. Ueji, N. Tsuji, Y. Minamino, and Y. Koizumi, Acta Mater. 50, 4177 (2002).

168. B. C. Leslie and R. S. Crouse, in Appl. Metallogr., edited by G. F. Vander Voort (Van Nostrand Reinhold Company, Inc., New York, NY, 1986), pp. 71-88.

169. R. E. Smallman and A. H. W. Ngan, Physical Metallurgy and Advanced Materials Engineering (Elsevier, New York, NY, 2007).

170. S. Morito, H. Saito, T. Ogawa, T. Furuhara, and T. Maki, ISIJ Int. 45, 91 (2005).

171. ASTM Standard E1820: Standard Test Method for Measurement of Fracture Toughness (ASTM International, West Conshocken, PA, 2001).

172. W. M. Garrison and N. R. Moody, J. Phys. Chem. Solids 48, 1035 (1987).

173. Y. Lee, Unpublished Research, University of Virginia (Charlottesville, VA, 2004).

174. R. F. Schaller and J. R. Scully, Electrochem. Commun. 40, 42 (2014).

175. R. F. Schaller and J.R. Scully, Unpublished Research (Charlottesville, VA, 2015).

176. C. San Marchi and B. P. Somerday, Technical Reference for Hydrogen Compatibility of Materials (Sandia National Laboratories, Albuquerque, NM, 2012).

177. S. W. Smith, Hydrogen Trapping in Al-Li-Cu-Zr Alloys, PhD Dissertation, University of Virginia, 1995.

178. R. A. Oriani, Acta Metall. 18, 147 (1970).

179. V. K. Vasudevan, S. J. Kim, and C. M. Wayman, Metall. Trans. A 21A, 2655 (1990).

180. G. Walker, editor, Solid-State Hydrogen Storage: Materials and Chemistry (Elsevier, Cambridge, UK, 2008).

181. B. Sakintuna, F. Lamari-Darkrim, and M. Hirscher, Int. J. Hydrogen Energy 32, 1121 (2007).

182. B. Pound, Acta Mater. 46, 5733 (1998).

183. G. M. Pressouyre and I. M. Bernstein, Metall. Trans. A 9A, 1571 (1978).

184. G. M. Pressouyre and I. M. Bernstein, Acta Metall. 27, 89 (1978).

185. R. Gibala and A. J. Kumnick, in Hydrog. Embrittlement Stress Corros. Crack edited by R. Gibala and R.R. Hehemann (ASM International, Metals Park, OH, 1984), pp. 61-78. 
186. J. Takahashi, K. Kawakami, Y. Kobayashi, and T. Tarui, Scr. Mater. 63, 261 (2010).

187. Y. Ro, S. R. Agnew, and R. P. Gangloff, Metall. Mater. Trans. A 38A, 3042 (2007).

188. G. F. Vander Voort, in Appl. Metallogr., edited by G. F. Vander Voort (Van Nostrand Reinhold Company, Inc., New York, NY, 1986), pp. 139-170.

189. A. Turnbull and R. B. Hutchings, Mater. Sci. Eng. A 177, 161 (1994). 